
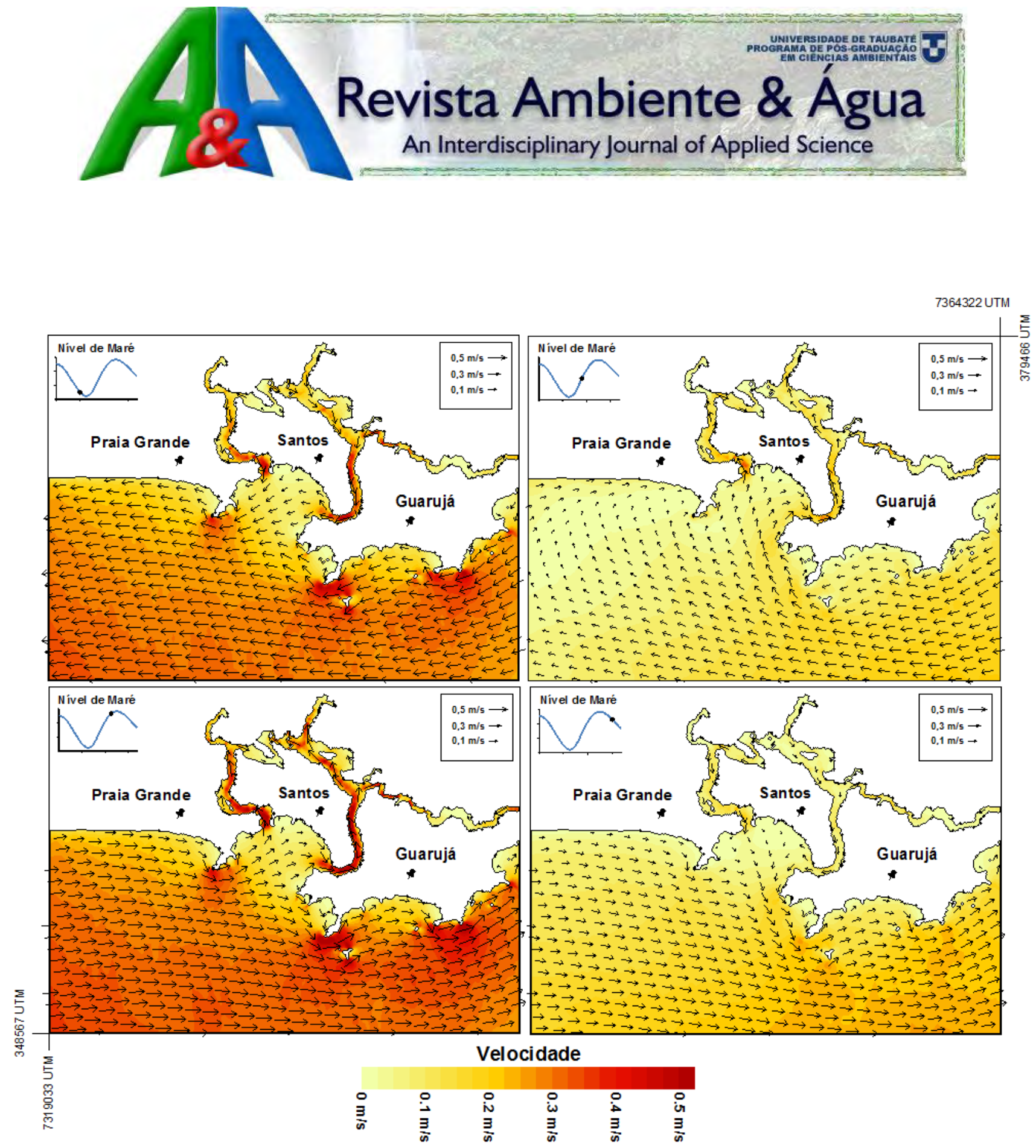

ISSN = 1980-933X (Online)

http://www.ambi-agua.net

Edição 19 da Revista Ambiente \& Água - An Interdisciplinary Journal of Applied Science,

Taubaté, v. 7, n. 3, p. 1-292, dezembro 2012. (http://dx.doi.org/10.4136/ambi-agua.v7.n3) 


\section{EDITORIAL BOARD}

\section{Editor}

\section{Getulio Teixeira Batista}

Instituto de Pesquisas Ambientais em Bacias Hidrográficas (IPABHi), Brasil

\section{Associate Editors}

Amaury Paulo de Souza

Universidade Federal de Viçosa (UFV), Brasil

\section{Antonio Evaldo Klar}

Universidade Estadual Paulista Júlio de Mesquita Filho, UNESP, Brasil

Dar Roberts

Universityof Califórnia at Santa Barbara, US

Hans Raj Gheyi

Universidade Federal de Campina Grande (UFCG), Brasil

\section{Hélio Nóbile Diniz}

Instituto Geológico, Secretaria do Meio Ambiente do Estado de São Paulo (IG/SMA), Brasil

\section{João Vianei Soares}

Instituto Nacional de Pesquisas Espaciais (INPE), Brasil

Editorial Section

Text (EN e ES) Editors

Reference Editor

Layout Editors

Technical Support
Luis A. Bartolucci

Florida International University (FIU), EUA

Marcelo dos Santos Targa

Universidade de Taubaté (UNITAU), Brasil

Nelson Wellausen Dias

Universidade de Taubaté (UNITAU), Brasil

Paul W. Mausel

Indiana State University (ISU), EUA

Paulo Renato Schneider

Universidade Federal de Santa Maria (UFSM), Brasil

\section{Sebastião do Amaral Machado}

Universidade Federal do Paraná (UFPR), Brasil

Silvio Jorge Coelho Simões

Universidade Estadual Paulista Júlio de Mesquita Filho (UNESP), Brasil

Yosio Edemir Shimabukuro

Instituto Nacional de Pesquisas Espaciais (INPE), Brasil

Nelson Wellausen Dias, PPGCA, UNITAU, IBGE, BR

Getulio T. Batista, UNITAU \& Nelson W. Dias IBGE, BR

Liliane Castro, Biblioteca ECA/Civil, UNITAU, BR

Vera L. F. Batista, IPABHi \& Getulio T. Batista, UNITAU, BR

Tiago dos Santos Agostinho, LAGEO, UNITAU, BR

Library catalog entry by

SIBi - Sistema Integrado de Bibliotecas / UNITAU

Revista Ambiente \& Água - An Interdisciplinary Journal of

Applied Science / Instituto de Pesquisas Ambientais em

Bacias Hidrográficas. Taubaté. v. 7, n. 3 (2006)-

Taubaté: IPABHi, 2012.

Quadrimestral

ISSN 1980-993X.

1. Ciências ambientais. 2. Recursos hídricos. I. Instituto de Pesquisas Ambientais em Bacias Hidrográficas. III. Título.

$$
\begin{aligned}
& \text { CDD - 333.705 } \\
& \text { CDU - (03)556.18 }
\end{aligned}
$$




\section{Ad Hoc Peer Reviewers that effectively evaluated submissions in 2012}

\section{(Ambiente \& Agua Journal, volume 7, $n^{\circ} .1,2$ e 3, 2012)}

Adriana Sacioto Marcantonio
Aldo Pacheco Ferreira

Alethea Ernandes M. Sallun

Ana Aparecida da S. Almeida

Ana Teresa Lombardi

Anderson Mululo Sato

Antonio Carlos C. Pignatari

Antonio Jose Teixeira Guerra

Antonio Pedro de Oliveira Netto

Antonio Roberto Formaggio

Antonio Teixeira Matos

Aparecido Osdimir Bertolin

Caetano Chang Dorea

Camilo Daleles Rennó

Carlos Domingos Silva

Carlos Rogério Mello

Catarina da Silva Pedrozo

Claudia Albuquerque Linhares

Claudia Padovesi Fonseca

Claudio Marcio Souza

Claudio Milton C. Montenegro

Cláudio Moisés S. e Silva

Cleber Salimon

Cristiano Christofaro

Cristiano Poleto

Daniele Maia Bila

Danielle Goveia

Danny M. Vaughn

Débora Astoni Moreira

Douglas Francisco M. Gherardi

Edson Rodrigues

Eduardo Bosco Mattos Cattony

Eduardo Rodrigues V. de Lima

Eduardo von Sperling

Evlyn Márcia Novo

Fabio de Oliveira Sanches

Fabrício Berton Zanchi

Getulio Teixeira Batista
Agência Paulista de Tecnologia dos Agronegócios

Escola Nacional de Saúde Pública

Instituto Geológico (IG/SMA-SP)

Universidade de Taubaté (UNITAU)

Universidade Federal de São Carlos (UFSCAR)

Universidade Federal do Rio de Janeiro, RJ

Universidade Federal de São Paulo (UNIFESP)

Universidade Federal do Rio de Janeiro (UFRJ)

Universidade de São Paulo (USP)

Instituto Nacional de Pesquisas Espaciais (INPE)

Universidade Federal de Viçosa (UFV)

Universidade Federal do Tocantins (UFTO)

University of Glasgow, Scotland, UK

Instituto Nacional de Pesquisas Espaciais (INPE)

Universidade Federal Rural do Rio de Janeiro

Universidade Federal de Lavras (UFLA)

Universidade Fed. do Rio Grande do Sul (UFRGS)

Instituto Nacional de Pesquisas Espaciais (INPE)

Universidade de Brasília (UnB)

Univ. Fed. dos Vales do Jequit. e Mucuri (UFVJM)

Universidade Federal de Lavras (UFLA)

Univ. Federal do Rio Grande do Norte (UFRN)

Universidade Federal do Acre (UFAC)

Universidade Federal de Minas Gerais (UFMG)

Universidade Estadual de Maringá (UEM)

Univ. Est. do Norte Fluminense Darcy Ribeiro.

Univ. Est. P. Júlio de Mesquita $\mathrm{F}^{\circ}$. (UNESP Sorocaba)

Indep. Consult., Indiana State University, IN, US

Universidade do Estado de Minas Gerais (UEMG)

Instituto Nacional de Pesquisas Espaciais (INPE)

Universidade de Taubaté (UNITAU)

Instituto Federal do Ceará (IFCE)

Universidade Federal da Paraíba (UFPB)

Universidade Federal de Minas Gerais (UFMG)

Instituto Nacional de Pesquisas Espaciais (INPE)

Universidade Federal da Fronteira Sul (UFFS)

Universidade Federal do Amazonas

Universidade de Taubaté (UNITAU) 


\begin{tabular}{|c|c|}
\hline Gilberto Fisch & Universidade de Taubaté (UNITAU) / CSA-CTA \\
\hline Guilherme Malafaia & Universidade Federal de Ouro Preto (UFOP) \\
\hline Hélcio José Izário Filho & USP, Escola de Engenharia de Lorena - EEL \\
\hline Hélio Nóbile Diniz & Instituto Geo. do Est. de São Paulo (IG-SMA/SP) \\
\hline Herlane Costa Calheiros & Universidade Federal de Itajubá (UNIFEI) \\
\hline Herminia Yohko Kanamura & Universidade de Taubaté (UNITAU) \\
\hline Hugo Abi Karam & Universidade Federal do Rio de Janeiro \\
\hline João Cleps Junior & Universidade Federal de Uberlândia (UFU) \\
\hline Joel Avruch Goldenfum & Univ. Federal do Rio Grande do Sul (UFRGS) \\
\hline José Antonio R. de Souza & Universidade Federal de Viçosa (UFV) \\
\hline José Carlos Mierzwa & Universidade de São Paulo (USP) \\
\hline José Eloi Guimarães Campos & Universidade de Brasília (UnB) \\
\hline José Luís Costa Novaes & Universidade Federal Rural do Semiárido (UFERSA) \\
\hline Jose Luis Ibarra-Montoya & Instituto Tec. de Estudios Sup. de Moterrey, MX \\
\hline José Luiz Stech & Instituto Nacional de Pesquisas Espaciais (INPE) \\
\hline Julieta de Jesus da Silveira Neta & EMBRAPA Amazônia Oriental \\
\hline Julio Cesar Pascale Palhares & EMBRAPA Pecuária Sudeste \\
\hline Luciana Camargo Oliveira & Universidade Federal de São Carlos (UFSCAR) \\
\hline Luis Alberto Basso & Universidade Federal do Rio Grande do Sul \\
\hline Luis Antonio Merino & Inst. Medicina Reg. - Univ. Nacional del Nordeste \\
\hline Luis Nery Rodrigues & Inst. Fed. de Ed., Cien. e Tec. do Pará, IFPA, Brasil. \\
\hline Luiz Fernando C. Oliveira & Univ. Federal de Lavras (UFLA) \\
\hline Luiz Fernando Nascimento & Universidade Estadual Paulista (UNESP)/UNITAU \\
\hline Luiz Gonsaga de Carvalho & Universidade Fed. de Lavras, Dep. de Engenharia \\
\hline Mairo Fabio Camargo & Universidade do Estado de Mato Grosso (UNEMAT) \\
\hline Manoel Alonso Gan & Instituto Nacional de Pesquisas Espaciais (INPE) \\
\hline Manuel Eduardo Ferreira & Universidade Federal de Goiás (UFG) \\
\hline Marçal José Rodrigues Pires & Pontifícia Univ. do Rio Grande do Sul (PUC-RGS) \\
\hline Marcelo dos Santos Targa & Universidade de Taubaté (UNITAU) \\
\hline Marcos Antonio Gomes & Universidade Federal de Viçosa (UFV) \\
\hline Marcus Aurélio Soares Cruz & Embrapa Tabuleiros Costeiros (CPATC) \\
\hline Maria Anita Mendes & Universidade de São Paulo (USP) \\
\hline Maria Claudia Barbosa & Universidade Federal do Rio de Janeiro (UFRJ) \\
\hline Maria Isabel Queiroz & Fundação Univ. Federal do Rio Grande (FURG) \\
\hline Maria Paulete Pereira Martins & Instituto Nac. de Pesquisas Espaciais (INPE) \\
\hline Mariko Ueno & Universidade de Taubaté (UNITAU) \\
\hline Mauricio Almeida Noernberg & Universidade Federal do Paraná (UFPR) \\
\hline Maurício Alves da M. Sobrinho & Universidade Federal de Pernambuco (UFPE) \\
\hline Mauricio José Borges & Instituto Taquaritinguense de Ensino Superior (ITES) \\
\hline Mauro Martinez & Univ. Fed. de Viçosa, Centro de Ciências Agrárias \\
\hline Miguel Mansur Aisse & Universidade Federal do Paraná (UFPR) \\
\hline Mônica Luisa Kuhlmann & Cia. Ambiental do Est. de SP (CETESB) \\
\hline
\end{tabular}




\begin{tabular}{|c|c|}
\hline Monica Maria Pereira da Silva & Universidade Estadual da Paraíba (UFP) \\
\hline Nelson Wellausen Dias & Instituto Brasileiro de Geografia e Estatística (IBGE) \\
\hline Neylor Alves Calasans Rego & Universidade Estadual de Santa Cruz (UESC) \\
\hline Núbia Natália de Brito & Universidade Federal de Goiás (UFG) \\
\hline Paula Benevides de Morais & Universidade Federal do Tocantins (UFTO) \\
\hline Paulo Cesar Sentelhas & Universidade de São Paulo (USP-ESALQ) \\
\hline Paulo Fortes Neto & Universidade de Taubaté (UNITAU) \\
\hline Perola de Castro Vasconcellos & Universidade de São Paulo (USP) \\
\hline Plínio Carlos Alvalá & Instituto Nacional de Pesquisas Espaciais (INPE) \\
\hline Regina Peralta Moreira & Universidade Federal de Santa Catarina (UFSC) \\
\hline Regla Duthit Somoza & Instituto Nacional de Pesquisas Espaciais (INPE) \\
\hline Renato Fontes Guimarães & Universidade de Brasília (UnB) \\
\hline Renato Igor da silva Alves & Universidade de São Paulo (USP) \\
\hline Ricardo de Oliveira Figueiredo & Empresa Brás.de Pesq. Agropecuária (EMBRAPA) \\
\hline Ricardo Valcarcel & Univ. Fed. Rural do Rio de Janeiro (UFRRJ) \\
\hline Richarde Marques Silva & Universidade Federal da Paraíba (UFP) \\
\hline Roberto José Carvalho & Pontifícia Universidade Católica do RJ (PUC-RJ) \\
\hline Ronaldo de Almeida & Universidade Federal do Rio de Janeiro (UFRJ) \\
\hline Rosa Toyoko Shiraishi Frighetto & Empresa Bras. de Pesq. Agropecuária (EMBRAPA) \\
\hline Sandro G. de Castro Britto & $\begin{array}{l}\text { Univ. Est. P. Julio de Mesquita Filho (UNESP) -Lab. de } \\
\text { Biologia e Ecologia de Peixes }\end{array}$ \\
\hline Sergio Zolnier & Universidade Estadual do Oeste do Paraná (UNIOESTE) \\
\hline Sílvia de N. Monteiro Yanagi & Universidade Federal de Lavras, UFLA, Brasil. \\
\hline Silvia Helena Govoni Brondi & EMBRAPA Pecuária Sudeste \\
\hline Silvio Bueno Pereira & Universidade Federal da Grande Dourados (UFGD) \\
\hline Silvio Jorge Coelho Simões & Univ. Est. P. Julio de Mesquita Filho (UNESP) \\
\hline Susana Inés Segura Muñoz & Universidade de São Paulo (USP) \\
\hline Syed Abu Sayeed Mohammed & HKBK College of Engineering, Bangalore, India \\
\hline Tamara Maria Gomes & E. S. de Agric. "Luiz de Queiroz" (USP-ESALQ) \\
\hline Thomaz Correa e Castro Costa & Empresa Bras. de Pesq. Agropecuária (EMBRAPA) \\
\hline Valter José Cobo & Univ. de Taubaté, Inst. Básico de Biociências \\
\hline Vanessa Moraes de Andrade & Univ. do Extremo Sul Catarinense (UNESC) \\
\hline Vicente Paulo Soares & Universidade Federal de Viçosa (UFV) \\
\hline Viviane Moschini-Carlos & Universitat de Barcelona / UNESP \\
\hline Walter de Paula Lima & Universidade de São Paulo (USP) \\
\hline Yosio Edemir Shimabukuro & Instituto Nacional de Pesquisas Espaciais (INPE) \\
\hline
\end{tabular}

Note.: 1) Some of these reviewers evaluated more than one submission.

2) This list includes reviewers that evaluated submissions that were not accepted for publication. 


\section{Peer Reviewer Panel registered in the Journal Ambiente \& Água (Updated in December, 2012)}

Adão Benvindo Luz

Adauto de Souza Ribeiro

Adhemar Watanuki Filho

Adilson Pinheiro

Adonis Moreira

Adriana Sacioto Marcantonio

Adriano Wagner Ballarin

Affonso Celso Gonçalves Junior

Alaide Aparecida F. Gessner

Alcindo dos Santos

Aldo Pacheco Ferreira

Alessandra C. F. A. Campos

Alethea Ernandes M. Sallun

Alex Vladimir Krusche

Alexandre de Avila Leripio

Alexandre Gonçalves Evsukoff

Alexandre Guirland Nowosad

Alexandre Magno Sebbenn

Alexandre Marco da Silva

Alexandre Schiavetti

Alfredo Pereira

Aline Maria Meiguins de Lima

Aline Sueli de Lima Rodrigues

Altemar Amaral Rocha

Ana Aparecida da S. Almeida

Ana Emilia Siegloch

Ana Julia Urias Santos Araujo

Ana Maria Muratori

Ana Teresa Lombardi

Anderson Mululo Sato

Anderson Orestes C. Lobato

André Henrique Rosa

Andréa Bogatti G. Tomazela

Ângela dos Santos Barretto

Anne Hélène Fostier

Annemarie Konig

Antenor Oliveira Aguiar Netto

Antonio Caetano V. Caltabiano
Centro de Tecnologia Mineral

Universidade Federal de Sergipe

Universidade Federal de São Carlos

Universidade Regional de Blumenau

EMBRAPA Pecuária Sudeste

Agência Paulista de Tecnologia dos Agronegócios

Univ. Est. Paulista - Fac. Ciências Agronômicas

Universidade Estadual do Oeste do Paraná

Universidade Federal de São Carlos (USFSCAR)

Universidade Federal de São Carlos

Escola Nacional de Saúde Pública

Universidade Federal de Minas Gerais (UFMG)

Instituto Geológico (IG/SMA-SP)

Universidade de São Paulo (USP-CENA)

Universidade do Vale do Itajaí

Universidade Federal do Rio de Janeiro (COPPE)

Instituto Nacional de Pesquisas Espaciais (INPE)

Instituto Florestal de São Paulo (SMA-IF)

UNESP Sorocaba

Universidade Estadual de Santa Cruz

Instituto Nacional de Pesquisas Espaciais (INPE)

Centro de Ens. Sup. de Porto Alegre (CESUPA)

Instituto Federal Goiano - Campus Urutaí

Universidade Federal da Paraíba (UFPB)

Universidade de Taubaté (UNITAU)

Universidade de São Paulo (USP / FFCLRP)

Universidade de Taubaté (UNITAU)

Universidade Federal do Paraná (UFPR)

Universidade Federal de São Carlos (UFSCAR)

Universidade Federal do Rio de Janeiro, RJ

Universidade Federal de Pelotas

Universidade Estadual Paulista (UNESP Sorocaba)

UNESP Campus Experimental do Litoral Paulista

Universidade de Campinas (UNICAMP)

IQ - Universidade de Campinas (UNICAMP)

Universidade Federal de Campina Grande (UFCG)

Universidade Federal de Sergipe (UFS)

International Clivar Project Office 


\begin{tabular}{|c|c|}
\hline Ant & Universidade Federal de São Paulo (UNIFESP) \\
\hline Antonio Carlos Vitte & Universidade de Campinas (UNICAMP) \\
\hline Antonio César Ortega & Universidade Federal de Uberlândia \\
\hline Antonio Jose Teixeira Guerra & Universidade Federal do Rio de Janeiro (UFRJ) \\
\hline Antonio Pedro de Oliveira Netto & Universidade de São Paulo (USP) \\
\hline Antonio Roberto Formaggio & Instituto Nacional de Pesquisas Espaciais (INPE) \\
\hline Antonio Teixeira Matos & Universidade Federal de Viçosa (UFV) \\
\hline Antonio Evaldo Klar & Universidade Estadual Paulista (UNESP Botucatu) \\
\hline Aparecido Osdimir Bertolin & Universidade Federal do Tocantins (UFTO) \\
\hline Arimatéa Carvalho Ximenes & Instituto Nacional de Pesquisas Espaciais (INPE) \\
\hline Arlindo Tribess & Escola Poli \\
\hline ton R. Ribeir & de Campina Grande \\
\hline attos & Universidade Federal do Rio Grande do Norte \\
\hline Áureo Eduardo M. Ribeiro & Universidade Federal de Lavras (UFLA) \\
\hline Azizah Endut & Faculty of Innovative Design and Technology \\
\hline Rudorff & Instituto Nacional de Pesquisas Espaciais (INPE) \\
\hline Bianca Ferreira da Silva & Universidade Federal de São Carlos (UFSCAR) \\
\hline Brani Rozemberg & Fundação Oswaldo Cruz (FIOCRUZ) \\
\hline Caetano Chang Dorea & University of Glasgow, Scotland, UK \\
\hline Camilo Daleles Rennó & Instituto Nacional de Pesquisas Espaciais (INPE) \\
\hline Camilo de Lelis T. de Andrade & Empresa Bras. de Pesq. Agropecuária (EMBRAPA \\
\hline Carlos Alberto V. de Azevedo & Universidade Federal de Campina Grande (UFCG) \\
\hline Carlos Andre Bulhões Mendes & Instituto de Pesquisas Hidráulicas (UFRGS) \\
\hline Carlos de Oliveira Galvão & Universidade Federal de Campina Grande (UFCG) \\
\hline Carlos Domingos Silva & Universidade Federal Rural do Rio de Janeiro \\
\hline Carlos Edwar Freitas & Universidade Federal do Amazonas \\
\hline Carlos Ernesto Schaefer & Universidade Federal de Viçosa (UFV) \\
\hline Carlos Manoel Pedro Vaz & EMBRAPA - CNPDIA, São Carlos, SP \\
\hline Carlos Rogério Mello & Universidade Federal de Lavras (UFLA) \\
\hline Carmen Lidia A. P. Zottarelli & Instituto de Botânica de São Paulo \\
\hline Carmen Lucia Paiva Silveira & Centro Univers \\
\hline Catarina & do Rio Grande do Sul (UFRGS) \\
\hline Cecília Volkmer Ribeiro & Fundação Zoobotânica do RGS \\
\hline Celso Augusto Guimarães & Universidade Federal da Paraíba (UFPB) \\
\hline César Augusto Moreira & USP - Escola de Engenharia de São Carlos \\
\hline Cesar Koppe Grisolia & Universidade de Brasília (UnB) \\
\hline Cesar Reis & Universidade Federal de Viçosa, UFV, Brasil. \\
\hline César Ulisses Vieira Veríssimo & Universidade Federal do Ceará (UFC) \\
\hline Chigueru Tiba & Universidade Federal de Pernambuco (UFPE) \\
\hline Chinwe Sylvanus Alisi & Federal University of Technology Owerri \\
\hline Christian Okechukwu Nweke & Federal university of Technology, Owerri, Niger \\
\hline ilene Gomes & Universidade Federal do Rio de Janeiro (UFRJ) \\
\hline
\end{tabular}


Clarisse Guimarães Rabelo

Claudia Albuquerque Linhares

Claudia Padovesi Fonseca

Claudinei Fonseca Souza

Cláudio José Cavalcante Blanco

Claudio José Von Zuben

Claudio Luis Frankenberg

Claudio Marcio Souza

Claudio Milton C. Montenegro

Cláudio Moisés S. e Silva

Cleber Salimon

Cléber Silva e Silva

Corina da Costa Freitas

Cristiano Christofaro

Cristiano Poleto

Cyro Barros Rezende-Filho

Dalton Morisson Valeriano

Daniel Diego Costa Carvalho

Daniele Maia Bila

Danielle Goveia

Danny M. Vaughn

Débora Astoni Moreira

Deborah Arnsdorff Roubicek

Denilson Silva Bezerra

Denilson Teixeira

Denise de Castro Pereira

Diego Antonio França Freitas

Diego de Souza Sardinha

Diogenes Salas Alves

Dirceu Luis Herdies

Douglas Francisco M. Gherardi

Durval Dourado

Edmir Daniel Carvalho

Ednaldo Miranda de Oliveira

Edson Rodrigues

Edson Rodrigues Filho

Eduardo Antonio G. Marques

Eduardo Bosco Mattos Cattony

Eduardo Lucas Subtil

Eduardo Marone

Eduardo Pereira Cabral Gomes

Eduardo Rodrigues V. de Lima
Universidade Federal de Goiás

Instituto Nacional de Pesquisas Espaciais (INPE)

Universidade de Brasília (UnB)

Universidade Federal de São Carlos (UFSCAR)

Universidade Federal do Pará (UFPA)

UNESP/Rio Claro

Pontifícia Univ. do Rio Grande do Sul (PUC-RS)

Univ. Fed. dos Vales do Jequit. e Mucuri (UFVJM)

Universidade Federal de Lavras (UFLA)

Univ. Federal do Rio Grande do Norte (UFRN)

Universidade Federal do Acre (UFAC)

Universidade Federal do Pará (UFPA)

Instituto Nacional de Pesquisas Espaciais (INPE)

Universidade Federal de Minas Gerais (UFMG)

Universidade Estadual de Maringá (UEM)

Universidade de Taubaté (UNITAU)

Instituto Nacional de Pesquisas Espaciais (INPE)

Universidade de Brasília (UnB)

Univ. Est. do Norte Fluminense Darcy Ribeiro.

Univ. Est. P. Júlio de Mesquita $F^{\circ}$. (UNESP Sorocaba)

Indep. Consult., Indiana State University, IN, US

Universidade do Estado de Minas Gerais (UEMG)

Companhia Ambiental do Estado de SP (CETESB)

Instituto Maranhense de Meio Amb. e Rec. Hídricos

Centro Universitário de Araraquara (UNIARA)

Pontifícia Univ. Católica de Minas Gerais (PUC-MG)

Universidade Federal de Lavras

Universidade Federal de Alfenas

Instituto Nac. de Pesquisas Espaciais (INPE)

Instituto Nacional de Pesq. Espaciais (CPTEC-INPE)

Instituto Nacional de Pesquisas Espaciais (INPE)

Universidade de São Paulo (USP-ESALQ)

Instituto de Biociências (UNESP Botucatu)

Universidade Federal de Viçosa

Universidade de Taubaté (UNITAU)

Universidade Federal de São Carlos (UFSCAR)

Universidade Federal de Viçosa (UFV)

Instituto Federal do Ceará (IFCE)

Universidade de São Paulo (USP)

Universidade Federal do Paraná (UFPR)

Instituto de Botânica

Universidade Federal da Paraíba (UFPB) 
Eduardo von Sperling

Elaine C. Cardoso Fidalgo

Elisangela de Jesus C. Moraes

Elmo Rodrigues da Silva

Emilia de Rodat F. Moreira

Enio F. F. Silva

Enner Herenio Alcântara

Eny Maria Vieira

Ernani F. da Rosa Filho

Essam Saleh Al Sharabi

Eugenio Avila Pedrozo

Eunice da Costa Machado

Evlyn Márcia Novo

Fabio de Oliveira Sanches

Fábio Kummrow

Fabio Laurindo da Silva

Fábio Marcelo Breunig

Fabrício Berton Zanchi

Fabrício S. C. Oliveira

Flávia Cristina Sossae

Flávia Pinheiro Zanotto

Flávia Talarico Saia

Flavio C. B. Mascarenhas

Flávio Jorge Ponzoni

Flavio Jose Malta

Francisco A. Silva Vecchia

Francisco de Assis de Oliveira

Francisco de Assis S. de Sousa

Francisco de Sousa Ramos

Francisco Gonçalves da Silva

Francisco Marcuzzo

Francisco Roberto Azevedo

Fúlvio Rodriguez Simão

Georgina Bond Buckup

Gerson Cardoso Silva Jr.

Getulio Rincon

Getulio Teixeira Batista

Gianmarco Silva David

Gilberto Fisch

Gilson B. Athayde Júnior

Glicério Trichês

Guilherme Casoni Rocha
Universidade Federal de Minas Gerais (UFMG)

Empresa Br. de Pesquisa Agr., EMBRAPA, Brasil

Faculdade de Roseira, FARO, Brasil.

Universidade do Estado do Rio de Janeiro (UERJ)

Universidade Federal da Paraíba (UFPB)

Universidade Federal Rural de Pernambuco (UFPE)

Instituto Nacional de Pesquisas Espaciais (INPE)

Universidade de São Paulo (USP)

Universidade Federal do Paraná (UFPR)

Taiz University, Yemen

Universidade Federal do Rio Grande do Sul (UFGRS)

Universidade Federal do Paraná (UFPR)

Nacional de Pesquisas Espaciais (INPE)

Universidade Federal da Fronteira Sul (UFFS)

Universidade Federal de São Paulo

Universidade Federal de São Carlos (UFSCAR)

Instituto Nacional de Pesquisas Espaciais (INPE)

Universidade Federal do Amazonas

Inst. Ocean. da Universidade de São Paulo (IO-USP)

Centro Universitário de Araraquara (UNIARA)

Universidade Presbiteriana Mackenzie (UPM)

Wageningen University, The Netherlands

Universidade Federal do Rio de Janeiro (UFRJ)

Instituto Nacional de Pesquisas Espaciais (INPE)

Universidade de Taubaté (UNITAU)

Esc. de Eng. de São Carlos Univ. de SP (EESC-USP)

Universidade Federal do Piauí (UFPI)

Universidade Federal de Campina Grande (UFCG)

Universidade Federal de Pernambuco (UFPE)

Universidade Federal da Paraíba (UFPB)

Ministério de Minas e Energia. Goiânia/GO - Brasil

Universidade Federal do Ceará (UFC)

Empresa de Pesq. Agrop. de Minas Gerais (EPAMIG)

Univ. Federal do Rio Grande do Sul (UFRGS)

Universidade Federal do Rio de Janeiro (UFRJ)

Conselho Nac. de Pesca e Aqüicultura (CONEPE)

Universidade de Taubaté (UNITAU)

Agência Paulista de Tecnologia do Agron. (APTA)

Universidade de Taubaté (UNITAU) / CSA-CTA

Universidade Federal da Paraíba (UFPB)

Universidade Federal de Santa Catarina (UFSC)

Universidade de São Paulo (USP) 


\begin{tabular}{|c|c|}
\hline Guilherme Malafaia & Universidade Federal de Ouro Preto (UFOP) \\
\hline Gustavo Bayma S. da Silva & Embrapa Monitoramento por Satélite \\
\hline Gustavo Ferreira Simões & Universidade Federal de Minas Gerais (UFMG) \\
\hline Gustavo Figueiredo M. Leite & Universidade de Brasília (UnB) \\
\hline Hélcio José Izário Filho & USP, Escola de Engenharia de Lorena - EEL \\
\hline Helena Carvalho Lorenzo & Centro Universitário de Araraquara (UNIARA) \\
\hline Helio Grassi Filho & Universidade Estadual Paulista (UNESP Botucatu) \\
\hline Hélio Nóbile Diniz & Instituto Geo. do Est. de São Paulo (IG-SMA/SP) \\
\hline Henrique Nunes Oliveira & Univ. Est. P. Júlio de Mesquita Fo. (UNESP) \\
\hline Herickson Akihito Sudo Lutif & Universidad Carlos III de Madrid, Espanha \\
\hline Herlane Costa Calheiros & Universidade Federal de Itajubá (UNIFEI) \\
\hline Herly Carlos Teixeira Dias & Universidade Federal de Viçosa (UFV) \\
\hline Hermann Johann Heinrich Kux & Instituto Nacional de Pesquisas Espaciais (INPE) \\
\hline Herminia Yohko Kanamura & Universidade de Taubaté (UNITAU) \\
\hline Homero Giorge Cerqueira & Policia Militar (APMBB) / Univ. Cruzeiro do Sul \\
\hline Horst Frischkorn & Universidade Federal do Ceará (UFCE) \\
\hline Hugo Abi Karam & Universidade Federal do Rio de Janeiro \\
\hline Hugo Carvalho Guerra & Universidade Federal de Campina Grande (UFCG) \\
\hline Hugo Moreira Soares & Universidade Federal de Santa Catarina (UFSC) \\
\hline Humberto Calloni & Fundação Universidade Federal do Rio Grande \\
\hline Ijar Milagre da Fonseca & Instituto Nacional de Pesquisas Espaciais (INPE) \\
\hline Iraê Amaral Guerrini & Universidade Estadual Paulista (UNESP Botucatu) \\
\hline Irineu Bianchini Júnior & Universidade Federal de São Carlos - UFSCar \\
\hline Itamar Alves Martins & Univ. Est. P. Júlio de Mesquita Filho (UNESP) \\
\hline Ivanildo Hespanhol & Universidade de São Paulo (USP) \\
\hline Jacob Binsztok & Universidade Federal Fluminense (UFF) \\
\hline Jaildo Santos Pereira & Universidade Fed. do Recôncavo da Bahia (UFRB) \\
\hline Jaime Cabral & Universidade Federal de Pernambuco (UFPE) \\
\hline Jairo José de Oliveira And & Pontifícia Univ. do Rio Grande do Sul (PUC-RS) \\
\hline Janary Fonseca Pinheiro & Universidade Federal do Pará (UFPA) \\
\hline Jarbas Bonetti & Universidade Federal de Santa Catarina (UFSC) \\
\hline Javier Tomasella & Instituto Nacional de Pesquisas Espaciais (INPE) \\
\hline Jean Carlos Miranda & UERJ - Universidade do Estado do Rio de Janeiro \\
\hline Jean Pierre Henry B. Ometto & Instituto Nacional de Pesquisas Espaciais (INPE) \\
\hline Jeanedy Maria Pazinato & Empresa Agrícola Jandele Big Frango \\
\hline João Carlos Pinheiro Beck & Pontifícia Univ. do Rio Grande do Sul (PUC-RS) \\
\hline João Cleps Junior & Universidade Federal de Uberlândia (UFU) \\
\hline João G. Mendonça Filho & IG da Univ. Federal do Rio de Janeiro (UFRJ) \\
\hline João Henrique Zonta & Universidade Federal de Viçosa (UFV) \\
\hline João Luiz Lani & Universidade Federal de Viçosa (UFV) \\
\hline João Vianei Soares & Instituto Nacional de Pesquisas Espaciais (INPE) \\
\hline oel Avruch Goldenfum & Univ. Federal do Rio Grande do Sul (UFRGS) \\
\hline
\end{tabular}

Guilherme Malafaia

Gustavo Bayma S. da Silva

Gustavo Ferreira Simões

Gustavo Figueiredo M. Leite

Helena Carvalho Lorento

Helio Grassi Filho

Hélio Nóbile Diniz

Herlane Costa Calheiros

Herly Carlos Teixeira Dias

Herminia Yohko Kanamura

Homero Giorge Cerqueira

Horst Frischkorn

Hugo Carvalho Guerra

Hugo Moreira Soares

Humberto Calloni

Ijar Milagre da Fonseca

Iraê Amaral Guerrini

Itamar Alves Martins

Ivanildo Hespanhol

Jacob Binsztok

Jaildo Santos Pereira

Jaime Cabral

Janary Fonseca Pinheiro

Jarbas Bonetti

Javier Tomasella

Jean Pierre Henry B. Ometto

João Carlos Pinheiro Beck

João G. Mendonça Filho
Universidade Federal de Ouro Preto (UFOP)

Donitoramento por Satélite

Universidade de Brasília (UnB)

USP, Escola de Engenharia de Lorena - EEL

(UNIARA)

Instituto Geo. do Est. de São Paulo (IG-SMA/SP)

Univ. Est. P. Júlio de Mesquita $F^{\circ}$ (UNESP)

Universidad Carlos III de Madrid, Espanha

Universidade Federal de Viçosa (UFV)

Universidade de Taubaté (UNITAU)

Policia Militar (APMBB) / Univ. Cruzeiro do Sul

(UFCE)

Universidade Federal de Santa Cataina (UFSC)

Fundação Universidade Federal do Rio Grand

Universidade Estadual Paulista (UNESP Botucatu)

Universidade Federal de São Carlos - UFSCar

Univ. Est. P. Júlio de Mesquita Filho (UNESP)

Universidade Federal Fluminense (UFF)

Universidade Federal de Pernambuco (UFPE)

Universidade Federal do Pará (UFPA)

Universidade Federal de Santa Catarina (UFSC)

Instituto Nacional de Pesquisas Espaciais (INPE)

UERJ - Universidade do Estado do Rio de Janeiro

Instituto Nacional de Pesquisas Espaciais (INPE)

Empresa Agrícola Jandele Big Frang

Pontifícia Univ. do Rio Grande do Sul (PUC-RS)

Universidade Federal de Uberlândia (UFU)

IG da Univ. Federal do Rio de Janeiro (UFRJ)

Universidade Federal de Viçosa (UFV)

Universidade Federal de Viçosa (UFV)

Univ. Federal do Rio Grande do Sul (UFRGS)
Universidade Federal de Campina Grande (UFCG) 
Jorge Ramirez Hernandez

Jorge Xavier da Silva

José Alberto Quintanilha

José Antonio Perrella Balestieri

José Antonio R. de Souza

José Carlos Becceneri

José Carlos Mierzwa

José Dantas Neto

José Dias Corrêa Junior

José Eduardo dos Santos

José Eloi Guimarães Campos

José Euclides Stipp Paterniani

José Geraldo Querido

José Guilherme Chaui-Berlinck

José Luís Costa Novaes

Jose Luis Ibarra-Montoya

José Luiz Stech

José Marinaldo Gleriani

José Marques da Costa

José Renato Boucas Farias

José Rui Camargo

José Tavares Souza

José Vicente Granato Araújo

Josino Costa Moreira

Juliana Cristina Barreiro

Julieta de Jesus da Silveira Neta

Julio Cesar Pascale Palhares

Junior Alexandre Moreira Pinto

Jurandir Zullo

Justina Chibuogwu Orji

Kelly Cristina Tonello

Laércio A. Gonçalves Jacovine

Leandro Redin Vestena

Leonardo Santos Collier

Leonor Almeida Souza-Soares

Lia Osório Machado

Lidriana de Souza Pinheiro

Lino Carlos Borges

Loreley Gomes Garcia

Lucia Maria Sa Antunes Costa

Luciana Camargo Oliveira

Luciana Sarmento
Universidad Autón. de B. California (UABC), MX

Universidade Federal do Rio de Janeiro (UFRJ)

Esc. Politécnica da Univ. de São Paulo (Poli-USP)

Univ. Est. P. J. de Mesq. Fo (UNESP/Guaratinguetá)

Universidade Federal de Viçosa (UFV)

Instituto Nacional de Pesquisas Espaciais (INPE)

Universidade de São Paulo (USP)

Universidade Federal de Campina Grande (UFCG)

Universidade Federal de Minas Gerais (UFMG)

Universidade Federal de São Carlos (UFSCAR)

Universidade de Brasília (UnB)

Universidade de Campinas (UNICAMP)

Universidade de Taubaté (UNITAU)

Universidade de São Paulo (USP)

Universidade Federal Rural do Semiárido (UFERSA)

Instituto Tec. de Estudios Sup. de Moterrey, MX

Instituto Nacional de Pesquisas Espaciais (INPE)

Universidade Federal de Viçosa (UFV)

Instituto Nacional de Pesquisas Espaciais (INPE)

EMBRAPA Soja

Universidade de Taubaté (UNITAU)

Universidade Estadual da Paraíba (UEPB)

Universidade Federal de Goiás (UFG)

Fundação Oswaldo Cruz (FIOCRUZ)

Universidade Federal de São Carlos (UFSCAR)

EMBRAPA Amazônia Oriental

EMBRAPA Pecuária Sudeste

Universidade de Taubaté (UNITAU)

C. Pesq. Met. e Clim. Apl. a Agric. (UNICAMP)

Federal University of Technology, Owerri, Nigeria

Universidade Federal de São Carlos (UFSCAR)

Universidade Federal de Viçosa (UFV)

Universidade Estadual do Centro-Oeste do Paraná

Universidade Federal do Tocantins (UFTO)

Fundação Universidade Fed. do Rio Grande (FURG)

Universidade Federal do Rio de Janeiro (UFRGS)

Universidade Estadual do Ceará (UFCE)

Agência Goiana de Desenv. Rural e Fundiário

Universidade Federal da Paraíba (UFPB)

Universidade Federal do Rio de Janeiro (UFRJ)

Universidade Est. Paulista Júlio de Mesquita Filho

Agência Nacional de Águas (ANA) 
Luciano Brito Rodrigues

Luciano Farinha Watzlawick

Luis A. Bartolucci

Luis Alberto Basso

Luis Antonio Merino

Luis Antônio Serrão Contim

Luis Eduardo Aragon

Luís Fernando Stone

Luis Nery Rodrigues

Luiz Carlos Laureano da Rosa

Luiz Carlos Pinheiro M. Filho

Luiz Eduardo Oliveira

Luiz Fernando C. Oliveira

Luiz Fernando Nascimento

Luiz Gonsaga de Carvalho

Luiz Guerreiro Lopes

Magda Adelaide Lombardo

Mairo Fabio Camargo

Manoel Alonso Gan

Manuel Eduardo Ferreira

Mara Regina Rizzatti

Marçal José Rodrigues Pires

Marcelo de Julio

Marcelo dos Santos Targa

Marcelo Friederichs L. Souza

Marcelo Sandin Dourado

Marcelo Santos Chaves

Marcelo Zaiat

Márcio Balbino Cavalcante

Márcio J. Estefano de Oliveira

Marco Antonio Igarashi

Marcos Adami

Marcos Antonio Gomes

Marcos Esdras Leite

Marcos Gervasio Pereira

Marcos Rivail Silva

Marcos Vinícius Folegatti

Marcus Aurélio Soares Cruz

Marcus Cesar Avezum Castro

Maria Anita Mendes

Maria C. Vidotte B. Tarrega

Maria Cecília P. Rodrigues
Universidade Estadual do Sudoeste da Bahia

Universidade Estadual do Centro-Oeste

Florida International University (FIU), EUA

Universidade Federal do Rio Grande do Sul

Inst. Medicina Reg. - Univ. Nacional del Nordeste

Centro Educacional Adalberto Valle (CEAV)

Universidade Federal do Pará (UFPA)

EMBRAPA Arroz e Feijão

Inst. Fed. de Ed., Cien. e Tec. do Pará, IFPA, Brasil.

Universidade de Taubaté (UNITAU)

Universidade de la Republica de Uruguay, U

Univ. Est. P. J. de Mesq. Fo (UNESP/Guaratinguetá)

Univ. Fed. Lavras / Univ. Fed. Goiás (UFLA/UFGO)

Universidade Estadual Paulista (UNESP)/UNITAU

Universidade Fed. de Lavras, Dep. de Engenharia

University of Madeira, Portugal

Univ. Est. P. J. de Mesq. Fo (UNESP Rio Claro)

Universidade do Estado de Mato Grosso (UEMT)

Instituto Nacional de Pesquisas Espaciais (INPE)

Universidade Federal de Goiás (UFG)

Pontifícia Univ. do Rio Grande do Sul (PUC-RGS)

Pontifícia Univ. do Rio Grande do Sul (PUC-RGS)

Instituto Tecnológico de Aeronáutica - ITA

Universidade de Taubaté (UNITAU)

Universidade Estadual de Santa Cruz (UESC)

Universidade Federal do Paraná, UFPR, Brasil

Universidade Federal da Paraíba (UFPB)

Escola de Engenharia de São Carlos - USP

Universidade Estadual da Paraíba (UEPB)

Universidade de Taubaté (UNITAU)

Universidade Federal do Ceará (UFCE)

Instituto Nacional de Pesquisas Espaciais (INPE)

Universidade Federal de Viçosa (UFV)

Univers. Estadual de Montes Claros (UNIMONTES)

Universidade Federal do Rio de Janeiro (UFRJ)

Universidade Regional de Blumenau

Universidade de São Paulo (ESALQ/ USP)

Embrapa Tabuleiros Costeiros, CPATC, Brasil.

Univ. Estadual Paulista (UNESP rio Claro)

Universidade de São Paulo (USP)

Universidade Federal de Goiás (UFGO)

Universidade Federal Fluminense (UFF) 


Maria Claudia Barbosa
Maria Cristina Crispim
Maria do Carmo C. Gastaldini
Maria do Carmo Calijuri
Maria do Carmo P. Batitucci
Maria do Carmo Vieira
Maria Dolores Alves Cocco
Maria Elaine de Oliveira
Maria Helena Arruda Leme
Maria Hermínia F. Tavares
Maria Inês Salgueiro Lima
Maria Isabel Queiroz
Maria Jesus Robim
Maria Júlia Martins-Silva
Maria Lúcia Ribeiro
Maria Paulete Pereira Martins
Maria Sallydelandia S. Farias
Mariko Ueno
Mário Augusto Jardim
Marisete Dantas Aquino
Marta Bustos Romero
Masato Kobiyama
Mauricio Almeida Noernberg
Maurício Alves da M. Sobrinho
Mauricio Alves Moreira
Mauricio José Borges
Mauro Martinez
Mayesse Aparecida da Silva
Messias Borges Silva
Miguel Mansur Aisse
Milton Kampel
Mra Hata

Mirza Hasanuzzaman

Mohammed Mahabubur Rahman

Mônica Luisa Kuhlmann

Monica Maria Pereira da Silva

Murali Krishna M. P. S.

Napoleão E. de M. Beltrão

Naziano Pantoja Filizola

Nei Kavaguichi Leite

Neida Patias Volpi

Nelson Wellausen Dias

Nestor Aldo Campana
Universidade Federal do Rio de Janeiro (UFRJ)

Universidade Federal da Paraíba (UFPB)

Universidade Federal de Santa Maria

Universidade de São Paulo (USP)

Universidade Federal do Espírito Santo (UFES)

Universidade Federal da Grande Dourados (UFGD)

Universidade de Taubaté (UNITAU)

Universidade Federal Fluminense (UFF)

Universidade Presbiteriana Mackenzie

Universidade Est. do Oeste do Paraná (UNIOESTE)

Universidade Federal de São Carlos (UFSCAR)

Fundação Univ. Federal do Rio Grande (FURG)

Instituto Florestal (IF-SMA-SP)

Universidade de Brasília (UnB)

Univ. Estadual Paulista (UNESP Araraquara)

Instituto Nac. de Pesquisas Espaciais (INPE)

Universidade Federal de Campina Grande (UFCG)

Universidade de Taubaté (UNITAU)

Museu Paraense Emilio Göeldi (MPEG, PA)

Universidade Federal do Ceará (UFCE)

Universidade de Brasília (UnB)

Universidade Federal de Santa Catarina (UFSC)

Universidade Federal do Paraná (UFPR)

Universidade Federal de Pernambuco (UFPE)

Instituto Nacional de Pesquisas Espaciais (INPE)

Instituto Taquaritinguense de Ensino Superior

Univ. Fed. de Viçosa, Centro de Ciências Agrárias

Universidade Federal de Lavras

Universidade de São Paulo (USP)

Universidade Federal do Paraná (UFPR)

Instituto Nacional de Pesquisas Espaciais (INPE)

Faculty of Agriculture, Kagawa University, Japan

Dhaka Imperial College, Dhaka, Bangladesh

Cia. Ambiental do Est. de SP (CETESB)

Universidade Estadual da Paraíba (UFP)

Gov. Polytechnic for Women, Nandigama, India

Univ. Est. da Paraíba, UEPB, Brasil / CNPA, EMBRAPA

Universidade Federal do Amazonas (UFAM)

Universidade Federal de Mato Grosso

Universidade Federal do Paraná (UFPR)

Universidade de Taubaté (UNITAU)

Universidade de Brasília (UnB) 
Néstor Antonio Heredia Zárate

Ney Augusto Nascimento

Neylor Alves Calasans Rego

Núbia Natália de Brito

Orivaldo Brunini

Osmar Alves Lameira

Otávio Coaracy Brasil Gandolfo

Pablo Santana Santos

Patrick Thierry Seyler

Paul W. Mausel

Paula Benevides de Morais

Paulo Cesar Sentelhas

Paulo de Tarso Amorim Castro

Paulo Fortes Neto

Paulo José Adissi

Paulo Renato Schneider

Paulo Roberto Cecon

Paulo Roberto Meneses

Paulo Romera e Silva CTH -

Paulo Sergio G. Magalhães

Pedro R. Jacobi

Pedro Teixeira Lacava

Perola de Castro Vasconcellos

Plínio Carlos Alvalá

Rauquírio A. A. M. da Costa

Regina Peralta Moreira

Regis Alexandre Lahm

Regla Duthit Somoza

Reinaldo José da Silva

Rejane M. de M. Pimentel

Renato Fontes Guimarães

Renato Igor da silva Alves

Renato Mello Prado

Ricardo de Oliveira Figueiredo

Ricardo Santos

Ricardo Valcarcel

Richarde Marques Silva

Roberto Antonio F. de Almeida

Roberto Chiaranda

Roberto de Oliveira

Roberto José Carvalho

Roberto Luiz Carmo
Universidade Fed. da Grande Dourados (UFGD)

Universidade Federal do Paraná (UFPR)

Universidade Estadual de Santa Cruz - UESC

Universidade Federal de Goiás, UFG, Brasil.

Instituto Agronômico de Campinas (IAC)

EMBRAPA Amazônia Oriental

Inst. de Pesquisas Tecnológicas de SP (IPT)

Instituto Nac. de Pesquisas Espaciais (INPE)

Universidade de Brasília (UnB)

Indiana State University (ISU)

Universidade Federal do Tocantins (UFTO)

Universidade de São Paulo (USP-ESALQ)

Universidade Federal de Ouro Preto, UFOP

Universidade de Taubaté (UNITAU)

Universidade Federal da Paraíba (UFP)

Universidade Federal de Santa Maria (UFSM)

Universidade Federal de Viçosa (UFV)

Universidade de Brasília (UnB)

Dept. Águas e Energia Elétrica de SP (DAEE)

Universidade de Campinas (UNICAMP)

Universidade de São Paulo (USP)

Instituto Tecnológico de Aeronáutica (ITA)

Universidade de São Paulo (USP)

Instituto Nacional de Pesquisas Espaciais

Universidade Federal do Pará (UFPA)

Universidade Federal de Santa Catarina (UFSC)

Pontifícia Univ. Cat. do R. G. do Sul (PUC-RS)

Instituto Nacional de Pesquisas Espaciais (INPE)

Universidade Estadual Paulista (UNESP)

Universidade Federal Rural de Pernambuco (UFPE)

Universidade de Brasília (UnB)

Universidade de São Paulo, USP, Brasil.

Universidade Est. Paulista Campus Jaboticabal

Empresa Bras.de Pesq. Agropecuária (EMBRAPA)

Universidade Federal de Viçosa (UFV)

Univ. Fed. Rural do RJ., Inst. de Florestas, RJ

Universidade Federal da Paraíba (UFP)

Centro de Prev. Tempo e Est. Clim. (CPTEC/INPE)

Universidade Federal de Mato Grosso (UFMT)

Universidade Federal de Santa Catarina (UFSC)

Pontifícia Universidade Católica do RJ (PUC-RJ)

Universidade de Campinas (UNICAMP) 
Roberto Naime

Rodrigo F. dos S. Salazar

Rodrigo Medeiros

Rodrigo Nóbrega

Rogério Nora Lima

Ronaldo Angelini

Ronaldo Borges Barthem

Ronaldo de Almeida

Ronaldo Teixeira Pelegrini

Ronaldo Viana Soares

Rosa Maria Formiga Johnsson

Rosa Toyoko Shiraishi Frighetto

Rouverson Pereira da Silva

Rubem La Laina Porto

Sandro Froehner

Sandro G. de Castro Britto

Sebastião do A. Machado

Sérgio Campos

Sérgio Marcos Sanches

Sergio Nascimento Duarte

Sergio Zolnier

Servio Tulio Cassini

Shiguenoli Miyamoto

Shozo Shiraiwa

Sílvia de N. Monteiro Yanagi

Silvia Helena Govoni Brondi

Silvio Bueno Pereira

Silvio Jorge Coelho Simões

Simey Thury Vieira Fisch

Simone Pinheiro Pereira

Solon Jonas Longhi

Sonia Cristina J. G. de A. Perez

Stelio Maia Menezes

Sueli Pércio Quináia

Susana Inés Segura Muñoz

Syed Abu Sayeed Mohammed

Tamara Maria Gomes

Teodorico Alves Sobrinho

Teresa Cristina Tarlé Pissarra

Teresinha Guerra

Thereza C. Almeida Rosso

Thomaz Correa e Castro Costa
Centro Universitário FEEVALE, RS

Univ. Est. do Rio Grande do Sul_UERGS

Universidade Federal Rural do Rio de Janeiro

Mississippi State University, MI, US

Centro Federal de Edu. Tecn. do Piauí (CEFETPI)

Universidade Fed. do Rio Grande do Norte, UFRN

Museu Paraense Emílio Göeldi (MPEG, PA)

Universidade Federal do Rio de Janeiro (UFRJ)

Universidade Federal de São Carlos (UFSCAR)

Universidade Federal do Paraná (UFPR)

Universidade do Estado do Rio de Janeiro (UERJ)

Empresa Bras. de Pesq. Agropecuária (EMBRAPA)

Universidade Estadual Paulista / Jaboticabal

Escola Politécnica da USP (Poli USP)

Universidade Federal do Paraná (UFPR)

Laboratório de Biologia e Ecologia de Peixes

Universidade Federal do Paraná (UFPR)

Universidade Estadual Paulista (UNESP Botucatu)

Escola de Enfermagem de Ribeirão Preto (USP)

E. S. de Agric. "Luiz de Queiroz" (USP-ESALQ)

Universidade Estadual do Oeste do Paraná

Universidade Federal do Espírito Santo (UFES)

Universidade Estadual de Campinas (UNICAMP)

Universidade Federal de Mato Grosso (UFMT)

Universidade Federal de Lavras, UFLA, Brasil.

EMBRAPA Pecuária Sudeste

Universidade Federal da Grande Dourados (UFGD)

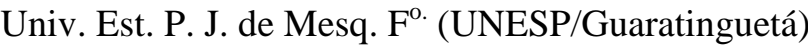

Universidade de Taubaté (UNITAU)

Universidade Federal do Pará (UFPA)

Universidade Federal de Santa Maria (UFSM)

Universidade Federal de São Carlos (UFSCAR)

Universidade Federal de Lavras (UFLA)

Univ. Estadual do Centro-Oeste (UNICENTRO)

Universidade de São Paulo (USP)

HKBK College of Engineering, Bangalore, India

E. S. de Agric. "Luiz de Queiroz" (USP-ESALQ)

Universidade Federal de Mato Grosso do Sul (UFMS)

Univ. Est. Paulista Júlio de Mesquita Filho (UNESP)

Univ. Federal do Rio Grande do Sul (UFRGS)

Universidade do Estado do Rio de Janeiro (UFRJ)

Empresa Bras. de Pesq. Agropecuária (EMBRAPA) 


\begin{tabular}{|c|c|}
\hline Twiggy Cristina Alves Batista & Secretaria de Estado da Saúde, Tocantins \\
\hline Ubirajara Aluizio Mattos & Universidade do Estado do Rio de Janeiro (UFRJ) \\
\hline Ubiratan Ferrucio Faccini & Universidade do Vale do Rio dos Sinos \\
\hline Ulisses Thadeu Vieira Guedes & Instituto Nacional de Pesquisas Espaciais (INPE) \\
\hline Valdemar Luiz Tornisielo & Universidade de São Paulo (USP) \\
\hline Valdira de Caldas Brito Vieira & Centro Federal de Edu. Tecn. do Piauí (CEFETPI) \\
\hline Valter José Cobo & Univ. de Taubaté, Inst. Básico de Biociências \\
\hline Vanessa Moraes de Andrade & Univ. do Extremo Sul Catarinense, UNESC, Brasil. \\
\hline Vera Lucia Antunes Lima & Universidade Federal de Campina Grande (UFCG) \\
\hline Veraldo Liesenberg & Freiberg Univ. of Mining and Technology, Germany \\
\hline Vicente de P. Rodrigues Silva & Universidade Federal de Campina Grande (UFCG) \\
\hline Vicente Paulo Soares & Universidade Federal de Viçosa (UFV) \\
\hline Vinícius Batista Campos & Universidade Federal de Campina Grande (UFCG) \\
\hline Vinicius Ribeiro & Centro Universitário Ritter dos Reis (UniRitter, RS) \\
\hline Viviane Moschini-Carlos & Universitat de Barcelona / UNESP \\
\hline Wagner Cotroni Valenti & Univ. Est. Paulista Júlio de Mesquita Filho (UNESP) \\
\hline Wagner W. A. Alves & Universidade Federal de Campina Grande, UFCG \\
\hline Walter de Paula Lima & Universidade de São Paulo (USP) \\
\hline Washington Franca Rocha & Universidade Estadual de Feira de Santana (UEFS) \\
\hline Wendell de Queiróz Lamas & Universidade de Taubaté (UNITAU) \\
\hline Wesley A. Conde Godoy & Universidade de São Paulo (ESALQ/USP) \\
\hline Yahia Y Mosleh & Suez Canal University, Ismaïlia, Egypt \\
\hline Yanko M. de Alencar Xavier & Univ. Federal do Rio Grande de Norte (UFRN) \\
\hline Yoshiya Nakagawara Ferreira & Universidade Estadual de Londrina (UEL) \\
\hline Yosio Edemir Shimabukuro & Instituto Nacional de Pesquisas Espaciais (INPE) \\
\hline Yuri Tavares Rocha & Universidade de São Paulo (USP) \\
\hline Z.V.P. Murthy & Sardar Vallabhbhai Nat. Instit.of Techn., Surat, India \\
\hline
\end{tabular}




\section{ÍNDICE}

\section{CAPA}

Campos de velocidade gerados pelo modelo H2D para diversos instantes de maré.

Fonte: SUBTIL, E. L; MIERZWA, J. C.; ORTIZ, J. P. Influência do nível de tratamento de esgoto na qualidade da água do mar na região de lançamento do Emissário Submarino de Santos. AmbiAgua, Taubaté, v. 7, n. 3, p. 72-86, 2012. (http://dx.doi.org/10.4136/ambi-agua.975)

\section{EDITORIAL}

Editorial of the twentieth edition of Ambiente \& Agua - An Interdisciplinary Journal of Applied Science. (http://dx.doi.org/10.4136/ambi-agua.1035)

Getulio Teixeira Batista

\section{ARTIGOS}

Determination of the lethal doses LD50 and LD90 of Euphorbia splendens var. hislopii latex on Physa cubensis Pfeiffer (http://dx.doi.org/10.4136/ambi-agua.860)

José Augusto Albuquerque dos Santos; Valdinei Valin; Aloysio da Silva Ferrão Filho

$\mathrm{UV} / \mathrm{H}_{2} \mathrm{O}_{2}$ process performance improvement by ultrafiltration and physicochemical clarification systems for industrial effluent pretreatment (http://dx.doi.org/10.4136/ambiagua.926)

José Carlos Mierzwa; Eduardo Lucas Subtil; Ivanildo Hespanhol

Evaluation of the use of Pycnoporus sanguineus fungus for phenolics and genotoxicity decay of a pharmaceutical effluent treatment (http://dx.doi.org/10.4136/ambi-agua.966)

Renata Alberto de Morais Watanabe ;Paulo de Tarso Ferreira Sales; Luiza Cintra Campos;Telma Alves Garcia1 Marize Campos Valadares;Fernando Schimidt; Mariângela Fontes Santiago

The potential of Zea mays, Commelina bengelensis, Helianthus annuus and Amaranthus hybridus for phytoremediation of waste water (http://dx.doi.org/10.4136/ambi-agua.684)

Oyaro Nathan; Kimenyu Phylis Njeri; Okong'o Eric Rang'ondi; Chacha Joseph Sarima

Concentración de clorofila-a y límite de zona fótica en el embalse Río Tercero (Argentina) utilizando imágenes del satélite CBERS-2B (http://dx.doi.org/10.4136/ambi-agua.847)

Matias Bonansea; Claudia Ledesma; Claudia Rodriguez; Angel Ramon Sanchez Delgado

Influência do nível de tratamento de esgoto na qualidade da água do mar na região de lançamento do Emissário Submarino de Santos (http://dx.doi.org/10.4136/ambi-agua.975)

Eduardo Lucas Subtil; José Carlos Mierzwa; Jayme Pinto Ortiz

Remoção de nitrogênio e fósforo do esgoto sanitário em um sistema de alagados construídos utilizando o capim vetiver (http://dx.doi.org/10.4136/ambi-agua.925)

Fernando Ernesto Ucker; Rogério de Araújo Almeida; Pedro Daniel da Cunha Kemerich

Influência de características físico-químicas da água no transporte de metano para a atmosfera na Lagoa Rodrigo de Freitas, RJ (http://dx.doi.org/10.4136/ambi-agua.972)

Lia Braz; Willian José Ferreira; Marcelo Gomes da Silva; Plínio Carlos Alvalá; Luciano Marani; Getulio Teixeira Batista; Valiya Mannathal Hamza

Determinação de áreas prioritárias para o restabelecimento da cobertura florestal, apoiada no uso de geotecnologias (http://dx.doi.org/10.4136/ambi-agua.964)

Celso de Souza Catelani; Getulio Teixeira Batista; Marcelo dos Santos Targa; Nelson Wellausen Dias 
Consequências hidrológicas da mudança de uso da terra de floresta para pastagem na região da floresta tropical pluvial Atlântica (http://dx.doi.org/10.4136/ambi-agua.927)

Luiz Felippe Salemi1; Juliano Daniel Groppo; Rodrigo Trevisan; Gustavo Bicci Seghesi; Jorge Marcos de Moraes; Silvio Fronsini de Barros Ferraz; Luiz Antonio Martinelli

Avaliação espaço-temporal da qualidade da água e simulação de autodepuração na bacia hidrográfica do córrego São Simão, SP (http://dx.doi.org/10.4136/ambi-agua.894)

Julio César de Souza Inácio Gonçalves; Diego de Souza Sardinha; Antonio Donizetti Gonçalves de Souza; André Luis Bigaram Dibiazi; Letícia Hirata Godoy; Fabiano Tomazini da Conceição

Qualidade físico-química e microbiológica de água mineral e padrões da legislação (http://dx.doi.org/10.4136/ambi-agua.908)

Helenilza Ferreira Albuquerque Cunha; Débora Cristina Isacksson Lima; Priscila Nazaré Freitas Brito; Alan Cavalcanti da Cunha; Arialdo Martins da Silveira Junior; Daímio Chaves Brito.

Caracterização química da água subterrânea em área ocupada por cemitério: uso da técnica de espectrometria de fluorescência de raios-X por energia dispersiva (EDXRF) (http://dx.doi.org/10.4136/ambi-agua.911)

Pedro Daniel da Cunha Kemerich; José Luiz Silvério da Silva; Guilherme Barros; Willian Fernando de Borba; Fernando Ernesto Ucker; Cristian Vargas Foletto

Efeito do uso e ocupação do solo na qualidade da água na micro-bacia do Córrego Banguelo - Contagem (http://dx.doi.org/10.4136/ambi-agua.962)

Patrícia Procópio Pontes; Andréa Rodrigues Marques; Guilherme Fernandes Marques

Análise morfométrica das sub-bacias hidrográficas Perdizes e Fojo no município de Campos do Jordão, SP, Brasil (http://dx.doi.org/10.4136/ambi-agua.945)

Agenor Micaeli dos Santos; Marcelo dos Santos Targa; Getulio Teixeira Batista; Nelson Wellausen Dias

Vazão ecológica e disponibilidade hídrica na bacia das Pedras, Guarapuava-PR (http://dx.doi.org/10.4136/ambi-agua.840)

Leandro Redin Vestena; Éderson Dias de Oliveira; Márcia Cristina da Cunha; Edivaldo Lopes Thomaz

Estudo da qualidade e quantidade da água em microbacia, afluente do rio Paraíba do Sul São Paulo, após ações de preservação ambiental (http://dx.doi.org/10.4136/ambi-agua.987)

Lívia Alves Alvarenga; Maria Paulete Pereira Martins; Luz Adriana Cuartas; Vinicius Alves Penteado; Alexandra Andrade

Adequação de um protocolo de avaliação rápida de rios para ser usado por estudantes do ensino fundamental (http://dx.doi.org/10.4136/ambi-agua.996)

Ariane Guimarães; Aline Sueli de Lima Rodrigues; Guilherme Malafaia

Avaliação espaço-temporal dos parâmetros de qualidade da água do rio Santa Rita (BA) em função do lançamento de manipueira (http://dx.doi.org/10.4136/ambi-agua.880)

Giovanna Porto dos Santos; Neylor Alves Calasans Rego; José Wildes Barbosa dos Santos; Franklin Delano Júnior; Milton Ferreira da Silva Júnior

Biomonitoramento do ar com Tradescantia pallida (Rose) D. R. Hunt var purpurea Boom

(Commelinaceae) (http://dx.doi.org/10.4136/ambi-agua.982)

Mateus Cabral Vasconcellos Teixeira; Agnes Barbério 


ISSN $=$ 1980-993X - doi:10.4136/1980-993X
www.agro.unitau.br/ambi-agua
E-mail: ambi-agua@@agro.unitau.br
Phone.: $+55(12) 3625-4212$

\title{
Editorial of the twentieth edition of Ambiente \& Agua - An Interdisciplinary Journal of Applied Science
}

(doi:10.4136/ambi-agua.1035)

\author{
Getulio Teixeira Batista \\ Professor of the Master Degree Program in Environmental Sciences of the University of Taubaté, SP, Brazil \\ Editor-in-Chief of Ambi-Agua Journal \\ e-mail: ambi-agua@agro.unitau.br
}

\begin{abstract}
We now publishing the $20^{\text {th }}$ edition with 20 articles evaluated and peer reviewed and since the first published issue, all editions were published as scheduled. We continue to practice the complete open access policy and fostering the visibility of articles. We continue to register doi of all articles and publishing the entire issue as a single archive in addition to the individual articles. This editorial shows citation metrics position of Ambiente \& Agua based on Google Scholar Metrics in comparison with other journals.
\end{abstract}

Keywords: Ambi-Agua, Environment, Water Resources, Google Metrics.

\section{Editorial da vigésima edição da revista Ambiente \& Água - An Interdisciplinary Journal of Applied Science}

\section{RESUMO}

Estamos publicando a $20^{\text {a }}$ edição com 20 artigos avaliados e revisados por pares e, desde a primeira edição publicada, todas as edições foram publicadas, no tempo previsto. Continuamos a praticar a política de acesso completamente aberto e a promover continuamente a visibilidade dos artigos. Continuamos a registar o doi para todos os artigos e a disponibilizar cada edição inteira em um único arquivo, além dos artigos individuais. Esse editorial apresenta a posição da revista Ambiente \& Agua em comparação com outras revistas em âmbito mundial com base nas métricas do Google Acadêmico.

Palavras-chave: Ambi-Agua, Meio Ambiente, Recursos Hídricos, Métricas de Citação da Google.

\section{INTRODUCTION}

Ambiente \& Água - An Interdisciplinary Journal of Applied Science (Ambi-Agua) continues to publish electronically via Internet with access based on a very reliable server maintained by the Institute of Environmental Research in Hydrographic Basins (IPABHi), a nonprofit organization.

The journal publishes in this last issue of the year (December) the registered peer review panel member list with their institutional affiliation (http://www.ambiagua.net/seer/index.php/ambi-agua/article/view/1036). In addition, a list of all reviewers that actually evaluated submissions in the year is also published in the complete issue document (http://dx.doi.org/10.4136/ambi-agua.v7.n3). 

Applied Science. Ambi-Agua, Taubaté, v. 7, n. 3, p. 18-20, 2012. (http://dx.doi.org/10.4136/ambi-agua.1035)

In 2012, we met the established goal of publishing 20 original research articles per issue, leading to a total of 60 papers in the year. This was possible due to the increased quality and number of submissions. In fact, at the closing time of this issue, we received the submission number 1034, accumulated from the past six years.

To assess the performance of the journal using a free and worldwide basis, we perused the Google Scholar Metrics (http://scholar.google.com/intl/en/scholar/metrics.html\#metrics), which provides information on the visibility and influence of recent articles in scholarly publications. It allows one to investigate the top 100 publications in several languages, in several thematic topics ordered by their five-year h-index, among other metrics. The h-index is a citation index that can be investigated for any journal or article in a worldwide basis and its method and description can be seen in Harzing and Wal (2008).

Scholar Metrics currently cover articles published between 2007 and 2011, both inclusive. The metrics are based on citations from all articles that were indexed in Google Scholar as of November $15^{\text {th }}, 2012$. This also includes citations from articles that are not themselves covered by Scholar Metrics.

Google Scholar indexes articles from journals in websites that follow inclusion guidelines. On the other hand, it excludes publications with fewer than 100 articles published between 2007 and 2011 or publications that received no citations to articles published between 2007 and 2011. Thus, Google alerts that "Scholar Metrics cover a substantial fraction of scholarly articles published in the last five years. However, they don't currently cover a large number of articles from smaller publications".

The investigation of Google Scholar Metrics based on the words "ambiente", "agua" and "interdisciplinary", which are part of our journal's name, revealed that Ambiente \& Agua - An Interdisciplinary Journal of Applied Science journal is standing at a very distinct position worldwide (Tables 1, 2, and 3).

Table 1. Top 20 publications matching "ambiente" found in http://scholar.google.com/citations? $\mathrm{hl}=\mathrm{en} \& \mathrm{view} \_\mathrm{op}=$ search_venues\&vq=ambiente.

\begin{tabular}{l|c}
\hline \multicolumn{1}{c|}{ Publication } & h5 index \\
\hline 1. Ecosistemas: Revista científica y técnica de ecología y medio ambiente & 12 \\
2. Ambiente \& Sociedade & 11 \\
3. Ambiente \& Água-An Interdisciplinary Journal of Applied Science & $\mathbf{8}$ \\
4. Ambiente Construído & 6 \\
5. Revista Chapingo. Serie ciencias forestales y del ambiente & 6 \\
6. Desenvolvimento e Meio Ambiente & 5 \\
7. Gestión y Ambiente & 5 \\
8. Economia delle fonti di energia e dell'ambiente & 5 \\
9. INTERFACEHS-Revista de Saúde, Meio Ambiente e Sustentabilidade & 4 \\
10. Ambienta: la revista del Ministerio de Medio Ambiente & 4 \\
11. Saúde \& Ambiente em Revista & 4 \\
12. Revista Ciências do Ambiente On-Line & 3 \\
13. Revista em Agronegócio e Meio Ambiente & 3 \\
14. Rivista giuridica dell'ambiente & 3 \\
15. Revista Saúde e Ambiente & 3 \\
16. Cerna: Revista galega de ecoloxía e medio ambiente & 2 \\
17. Energia, ambiente e innovazione & 2 \\
18. Ingeniería de Recursos Naturales y del Ambiente & 2 \\
19. Ingeopres: Actualidad técnica de ing. civil, minería, geología y medio ambiente & 2 \\
20. Revista de derecho urbanístico y medio ambiente & 2 \\
\hline
\end{tabular}


BATISTA, G. T. Editorial of the twentieth edition of Ambiente \& Agua - An Interdisciplinary Journal of Applied Science. Ambi-Agua, Taubaté, v. 7, n. 3, p. 18-20, 2012. (http://dx.doi.org/10.4136/ambi-agua.1035)

Table 2. Publications matching "agua" found in http://scholar.google.com/ citations?hl=en\&view_op=search_venues\&vq=agua.

\begin{tabular}{l|c}
\hline \multicolumn{1}{c|}{ Publication } & $\begin{array}{c}\text { h5 } \\
\text { index }\end{array}$ \\
\hline 1. Ambiente \& Água-An Interdisciplinary Journal of Applied Science & $\mathbf{8}$ \\
2. Tecnología del agua & 3 \\
\hline
\end{tabular}

Table 3. Top 20 publications matching "interdisciplinary” found in: http://scholar.google.com/ citations?hl=en\&view_op=search_venues\&vq=interdisciplinary.

\begin{tabular}{l|r}
\multicolumn{1}{c|}{ Publication } & $\begin{array}{c}\text { h5 } \\
\text { index }\end{array}$ \\
\hline 1. Chaos: An Interdisciplinary Journal of Nonlinear Science & 32 \\
2. Wiley Interdisciplinary Reviews: Nanomedicine and Nanobiotechnology & 25 \\
3. Wiley Interdisciplinary Reviews: Climate Change & 19 \\
4. Wiley Interdisciplinary Reviews: Systems Biology and Medicine & 19 \\
5. Interdisciplinary Perspectives on Infectious Diseases & 17 \\
6. Wiley Interdisciplinary Reviews: Cognitive Science & 14 \\
7. Text \& Talk-An Interdisciplinary J. of Lang. Discourse \& Communication Studies & 14 \\
8. Wiley Interdisciplinary Reviews: Computational Statistics & 11 \\
9. Wiley Interdisciplinary Reviews: RNA & 11 \\
10. Interdisciplinary Science Reviews & 10 \\
11. Southern California Interdisciplinary Law Journal & 9 \\
12. Interdisciplinary Information Sciences & 8 \\
13. Ambiente \& Água-An Interdisciplinary Journal of Applied Science & $\mathbf{8}$ \\
14. Interdisciplinary Sciences: Computational Life Sciences & 8 \\
15. Neuropsychoanalysis: An Interdisciplinary J. for Psychoanalysis and the Neurosciences & 7 \\
16. Journal of Interdisciplinary History & 7 \\
17. Interdisciplinary Toxicology & 7 \\
18. Historical Methods: A Journal of Quantitative and Interdisciplinary History & 7 \\
19. Interdisciplinary Studies in Literature and Environment & 6 \\
20. Environments: a journal of interdisciplinary studies & 5 \\
\hline
\end{tabular}

\section{FINAL REMARKS}

The results of Google Scholar Metrics are very encouraging for Ambi-Agua, especially considering that the comparison is with competing journals of major international publishers. We are grateful to our fellow members of the Ad Hoc Peer Reviewer Panel for their competent, effective and voluntary work, as well as to the authors that have submitted high quality manuscripts. For a more complete description of journal's actions to improve quality, please read a previous version of this editorial (Batista, 2011).

\section{REFERENCES}

BATISTA, G. T. Editorial of the seventeenth edition of Ambiente \& Água - An Interdisciplinary Journal of Applied Science. Ambi-Agua, Taubaté, v. 6, n. 3, p. 23-26, 2011. http://dx.doi.org/10.4136/ambi-agua.802

HARZING, A. W.; WAL, R. van der. Google Scholar as a new source for citation analysis?, Ethics in Science and Environmental Politics, v. 8, no. 1, p. 62-71, 2008. Available in: 〈http://www.int-res.com/articles/esep2008/8/e008pp5.pdf> 


ISSN = 1980-993X - doi:10.4136/1980-993X
www.ambi-agua.net
E-mail: ambi-agua@agro.unitau.br
Tel.: (12) 3625-4212

\title{
Determination of the lethal doses $\mathrm{LD}_{50}$ and $\mathrm{LD}_{90}$ of Euphorbia splendens var. hislopii latex on Physa cubensis Pfeiffer
}

\author{
(http://dx.doi.org/10.4136/ambi-agua.860)
}

José Augusto Albuquerque dos Santos; Valdinei Valin; Aloysio da Silva Ferrão Filho.
Laboratory for Evaluation and Promotion of Environmental Health, Oswaldo Cruz Institute, Oswaldo Cruz Foundation, Av. Brasil 4365, CEP 21045-900, Manguinhos, Rio de Janeiro, RJ, Brazil. e-mail: santosja@ioc.fiocruz.br,nei@ioc.fiocruz.br, aloysio@ioc.fiocruz.br

\begin{abstract}
The genus Physa are among the most common and widespread fresh-water snails and are frequently associated with the genus Lymnaea and Biomphalaria, typically occupying the same habitat and being hosts of trematodes that can cause disease in humans. Several studies have evaluated the control of snails through the use of plant-derived molluscicides; however, few such studies exist regarding the genus Physa. Determining the correct lethal doses of [latex of Euphorbia splendens] is important for the control of the Physa cubensis snail. In one experimental model, 150 snails from Jacarepaguá, Rio de Janeiro, RJ were used. After acclimatization for 72 hours, the snails were divided into groups of 10 and placed into beakers containing different doses, each at a temperature of $26 \pm 1{ }^{\circ} \mathrm{C}$ and a relative humidity of $80 \pm 10 \%$. The results demonstrated $\mathrm{LD}_{50}$ of $1.08 \mu \mathrm{L} / \mathrm{L}$ and $\mathrm{LD}_{90}$ of $3.41 \mu \mathrm{L} / \mathrm{L}$, without significant changes in the physical or chemical parameters of the solutions. We conclude that E. splendens latex had a significant effect on P. cubensis in the laboratory setting.
\end{abstract}

Keywords: Molluscicidal activity, Physa cubensis Pfeiffer, Euphorbia splendens.

\section{Determinação das doses letais $\mathrm{DL}_{50}$ and $\mathrm{DL}_{90}$ do latex da Euphorbia splendens var. hislopii sobre Physa cubensis Pfeiffer.}

\section{RESUMO}

O gênero Physa está entre os mais comuns e difundidos animais de água doce, estando frequentemente associado com os gêneros Lymnaea e Biomphalaria e geralmente ocupando o mesmo habitat, sendo hospedeiros de trematódeos que podem causar doenças ao homem. $\mathrm{O}$ controle de moluscos por meio do uso de moluscicidas de origem vegetal foi avaliado por diversos estudos, no entanto, existem poucos estudos para o gênero Physa. As doses letais do látex da Euphorbia splendens são importantes para o controle dos moluscos Physa cubensis. No modelo experimental foram utilizados 150 moluscos, procedentes de Jacarepaguá, Rio de Janeiro, RJ. Após o processo de aclimatação dos moluscos (72 horas), eles foram divididos em grupos de 10 e colocados em béqueres contendo doses diferentes, na temperatura de $26 \pm 1^{\circ} \mathrm{C}$ e umidade relativa de $80 \pm 10 \%$. Os resultados demonstraram $\mathrm{DL}_{50}$ de $1,08 \mu \mathrm{L} / \mathrm{L}$ e $\mathrm{DL}_{90}$ de 3,41 $\mu \mathrm{L} / \mathrm{L}$, sem alterações significativas nos parâmetros físico-químicos das soluções. Concluímos que o látex da $E$. splendens apresentou atividade sobre $P$. cubensis em laboratório.

Palavras-chave: Atividade moluscicida, Physa cubensis Pfeiffer, Euphorbia splendens. 


\section{INTRODUCTION}

The search for an effective, inexpensive, and environmentally friendly molluscicide has motivated the study of plants as a possible source of such substances. (Mott, 1987; Vasconcellos et al., 2003; Mello-Silva et al., 2007, 2010, 2011). According to Baptista et al. (1994), and as noted by Kloos and McCullough (1987), Kuo (1987) and Jurberg et al. (1989), it is estimated that 1426 plant species with molluscicidal properties have been analyzed to date. One of the most promising is the species Euphorbia splendens var. hislopii (Vasconcellos and Schall, 1986), which has been evaluated for its toxic effects in both field and laboratory studies (Schall et al., 1991) that confirmed its molluscicidal properties. (Schall et al., 1992). In Brazil, this plant is known as Christ's Crown ("Coroa de cristo") and "Bride Mattress" (Joly, 1979).

A study regarding the molluscicidal properties of the latex of E. splendens var. hislopii on Melanoides tuberculata, a snail associated with the habitats of Biomphalaria glabrata, demonstrated that in the presence of thiarid snail the molluscicide demonstrated a synergistic effect on the reduction of Biomphalaria populations (Giovanelli et al., 2001).

Latex of E. splendens was proven to be an effective molluscicide against Lymnaea columella, an intermediate host of Fasciola hepatica. (Vasconcellos et al., 2003), and altered not only the reproductive activity of B. glabrata (Mello-Silva et al., 2007), but also the glucose content B. glabrata's haemolymph and carbohydrate deposits (Mello-Silva et al., 2010), as well as concentrations of proteins and nitrogen products in snails infected with Schistosoma mansoni (Mello-Silva et al., 2011).

Previous studies on phytochemical compounds of the species E. splendens have demonstrated the presence of triterpenes, flavonoids, ingenol, phorbolsters, lasiodiplodin and eight types of milliamines that have molluscicidal properties (Leet et al., 1982; Zani et al., 1993).

Plant-derived molluscicides are important for the control of snail-borne parasitic infections and intestinal helminthiases. However, the use of niclosamida, a synthetic product recommended by the World Health Organization for snail-control in the schistosomiasis control program (WHO, 1998), is a controversial because of its toxic effects on non-targeted organisms, environmental contamination and the tendency of the mollusks to develop resistance.(Andrews et al., 1982).

Experimental infection of Physa cubensis Pfeiffer and L. columella with $F$. hepatica miracidiae has demonstratedthe presence of esporocysts in the ephalopodal mass and mantle in the first days of the infection. A malacological survey of waterways to detect foci of transmission of schistosomiasis and other parasitic diseases found mollusks infected by trematodes, Biomphalaria sp. infected by Schistosoma mansoni, Echinostomatidae, Strigeidae, Derogenidae, and Clinostomatidae cercariae; and Physa sp. infected by Echinostomatidae (Souza et al., 1998). There are records of the genus Physa possibly hosting larval forms of trematodes that may interfere in the mollusks' community structure (Vigueras and Moreno, 1938; Richards, 1964; Anderson and Fried, 1987; Guimarães et al., 1987; Kanev, et al., 1998; McCarthy, 1999; Degueurce et al., 1999; Snyder and Esch, 1993; Kosanke et al., 1988;).

It is believed necessary to determine lethal doses of chemical compounds or vegetal extracts in order to assess the possible environmental impact when used the control of mollusks coexisting other non-targeted aquatic organisms, since the indiscriminate use of molluscicides may lead to changes in local wildlife. For this reason, the test for evaluating molluscicidal properties, used herein, represents an important alternative in the control of mollusk disease vectors. 
Thus, this work aims to investigate the molluscicidal activity and to determine the lethal doses of latex of Euphorbia splendens on Physa cubensis in laboratory conditions.

\section{MATERIAL AND METHODS}

\subsection{Plant}

According to Pio Correa (1931), Euphorbia splendens var. hislopii is an ornamental plant native to Madagascar, introduced and cultivated in gardens for use as hedges. It is commonly known in Rio de Janeiro as "'Christ's Crown"' and is characterized as being a low, contorted shrub, with sprigs and numerous spines, red flowers, and leaves gathered at the apex of its branches.

\subsection{Snails}

Specimes of Physa cubensis Pfeiffer, Jacarepaguá, Rio de Janeiro, RJ, were collected and identified according to Paraense (1983). The specimens were then separated into groups of 10 and placed in 6-liter plastic boxes filled with chorine-free water in order to for acclimatize. They were fed lettuce during this period. (Lactuca sativa L. 1758).

\subsection{Collection of latex}

Latex was collected with covered test tubes by cutting through the stem of Euphorbia splenden plants that were cultivated on the Oswaldo Cruz Foundation campus, Rio de Janeiro, RJ and allowing the latex to drip into the tubes.. The material was then taken to the laboratory for preparation of the dilutions.

\subsection{Preparation of the doses}

From a stock solution with a concentration of $100 \mu \mathrm{L} / \mathrm{L}$ of latex of Euphorbia splendens, aqueous solutions were prepared in the following doses: $0.5 \mu \mathrm{L} / \mathrm{L}, 1.0 \mu \mathrm{L} / \mathrm{L}, 1.2 \mu \mathrm{L} / \mathrm{L}, 1.4$ $\mu \mathrm{L} / \mathrm{L}, 1.5 \mu \mathrm{L} / \mathrm{L}, 1.6 \mu \mathrm{L} / \mathrm{L}, 1.8 \mu \mathrm{L} / \mathrm{L}, 2.0 \mu \mathrm{L} / \mathrm{L}, 2.5 \mu \mathrm{L} / \mathrm{L}, 3.0 \mu \mathrm{L} / \mathrm{L}, 3.5 \mu \mathrm{L} / \mathrm{L}, 4.0 \mu \mathrm{L} / \mathrm{L}, 4.5$ $\mu \mathrm{L} / \mathrm{L}$ and $5.0 \mu \mathrm{L} / \mathrm{L}$.

\subsection{Bioassays}

Groups of 10 snails with a shell size between 5-10 mm were used for each exposure dose of E. splendens' latex plus control, totaling 150 snails, for a period of $24 \mathrm{~h}$. The recently collected latex was used in the bioassays. The groups were kept in $1000 \mathrm{~mL}$ beakers containing a $500 \mathrm{~mL}$ dose of the solution, while a control group was exposed only to the dilutant (distilled water) at a temperature of $26 \pm 1^{\circ} \mathrm{C}$ and a relative humidity of $80 \pm 10 \%$. No food was provided to the animals during that period.

After the exposure period, the animals were removed from the experimental and control solutions, rinsed with distilled water to remove the molluscicide residue and placed in beakers with distilled water, and kept for an additional $24 \mathrm{~h}$.

After this phase, a stereoscopic microscope was used to count the surviving and deceased animals, as determined by the appearance and layout of shells, and the odors of the soft parts of animals. The lethal doses of $\mathrm{LD}_{50}$ and $\mathrm{LD}_{90}$ were determined in accordance with Vasconcellos and Amorim (2003), as recommended by the World Health Organization (1983). The bioassays were carried out in triplicate.

\subsection{Physical and chemical parameters of solutions}

The parameters of alkalinity were determined by the titration method with indicators; total hardness and calcium hardness, titration method by EDTA; parameters of chlorides by the Mohr titration method; conductivity by the electrometric method using a conductivity 
meter; and $\mathrm{pH}$ by the potentiometric method, using a $\mathrm{pH}$ meter (FEEMA, 1982; FUNASA, 2006).

\subsection{Statistical analysis}

Lethal doses $\left(\mathrm{LD}_{50}\right.$ and $\left.\mathrm{LD}_{90}\right)$, were determined probit analysis (Finney, 1971). The plot probit of kill against log of doses $(\mu \mathrm{L} / \mathrm{L})$ provides a simple graphic representation of the doseto- response ratio. The results of physical and chemical analyses were obtained by analysis of variance (ANOVA) to the significance level of 5\% and indexes of correlation and regression were determined (Instat version 1.0).

\section{RESULTS AND DISCUSSION}

We believe that the use of vegetal molluscicides against Physa cubensis should be postponed pending further research in view of the existence of other trematodiosis of medical and veterinary importance.

This study showed the effect of different doses of latex on the significant behavioral changes on lethality of snail in the test. The experimental groups confirmed the molluscicidal effect. Increase in mortality rate due to exposure periods and doses of the latex as well as several factors may be acting the molluscicidal effect of the latex.

There was a relatively dose-dependent response in mortality rates of $P$. cubensis from 0.5 to $1.6 \mu \mathrm{L} / \mathrm{L}$ and the percentage of mortality reached $100 \%$ at latex doses of $1.6 \mu \mathrm{L} / \mathrm{L}$, $3.0 \mu \mathrm{L} / \mathrm{L}, 3.5 \mu \mathrm{L} / \mathrm{L}, 4.5 \mu \mathrm{L} / \mathrm{L}$ and $5.0 \mu \mathrm{L} / \mathrm{L}$, as shown in Table 1 .

Table 1. Mortality rates of Physa cubensis, submitted to various doses of Euphorbia splendens var. hislopii's látex.

\begin{tabular}{ccc}
\hline Dose $(\boldsymbol{\mu l} / \mathbf{L})$ & Dead $($ Mean $\pm \mathbf{S D})$ & Mortality $(\boldsymbol{\%})$ \\
\hline CG & $0 \pm 0$ & 0 \\
0.5 & $3 \pm 1$ & 30 \\
1.0 & $3 \pm 1$ & 30 \\
1.2 & $5 \pm 1$ & 50 \\
1.4 & $8 \pm 1$ & 80 \\
1.5 & $4 \pm 1$ & 40 \\
1.6 & $10 \pm 0$ & 100 \\
1.8 & $9 \pm 1$ & 90 \\
2.0 & $9 \pm 1$ & 90 \\
2.5 & $9 \pm 0$ & 90 \\
3.0 & $10 \pm 0$ & 100 \\
3.5 & $10 \pm 0$ & 100 \\
4.0 & $8 \pm 1$ & 80 \\
4.5 & $10 \pm 0$ & 100 \\
5.0 & $10 \pm 0$ & 100 \\
\hline
\end{tabular}

CG (control grupo); means of three replicats, $\mathbf{S D}=$ standart deviation, $\mathbf{N}=10$.

Table 2, shows the result of probit analysis snail experiments, with doses of $\mathrm{LD}_{50}$ of $1.08 \mu \mathrm{L} / \mathrm{L}$ and $\mathrm{LD}_{90}$ of $3.41 \mu \mathrm{L} / \mathrm{L}$. 
SANTOS, J. A. A.; VALIN, V.; FERRÃO-FILHO, A. S. Determination of the lethal doses LD $_{50}$ and LD $_{90}$ of Euphorbia splendens var. hislopii latex on Physa cubensis Pfeiffer. Ambi-Agua, Taubaté, v. 7, n. 3, p. 21-30, 2012. (http://dx.doi.org/10.4136/ambi-agua.860)

Table 2. $\mathrm{LD}_{50}$ and $\mathrm{LD}_{90}$ of Euphorbia splendens on Physa cubensis Pfeiffer.

\begin{tabular}{c|cc}
\hline \multirow{2}{*}{ Snail } & $\mathbf{L D}_{\mathbf{5 0}}(\boldsymbol{\mu} \mathbf{l} / \mathbf{L})$ & $\mathbf{L D}_{\mathbf{9 0}}(\boldsymbol{\mu l} / \mathbf{L})$ \\
$\mathbf{X} \pm \mathbf{S D}$ & $\mathbf{X} \pm \mathbf{S D}$ \\
\cline { 2 - 3 } Physa cubensis & $1.08 \pm 0.04$ & $3.41 \pm 0.12$ \\
\hline
\end{tabular}

$\mathbf{X}=$ mean of three replicates, $\mathbf{S D}=$ standard deviation. Analysis with $\mathrm{t}$ test unpaired.

Linear regression analysis of the percentage of dead snails in relation to various doses of plant latex found a correlation coefficient of $(\mathrm{r})=0.7688 ; \mathrm{F}$ value $=18.793$ and $\mathrm{p}$-value $=$ 0.0008 , which is considered very significant.

The Euphorbiaceae plants are known to have widespread molluscicidal properties (MacBae et al., 1988), though activity varies greatly with respect to species, plant part and even the method of extraction.

In the present study, the latex of E. splendens was active at a dose of $1.6 \mu \mathrm{L} / \mathrm{L}$. The latex of E. splendens extracted by driping showed a $\mathrm{LD}_{50}$ of $1.08 \mu \mathrm{L} / \mathrm{L}$ and $\mathrm{LD}_{90}$ of $3.41 \mu \mathrm{L} / \mathrm{L}$ against $P$. cubensis in laboratory bioassays (Table 2), which is considerably higher than the minimum level recommended for plants to be considered for use as molluscicidal (WHO, 1983).

The varying environmental factors with changes in $\mathrm{pH}$, hardness of water, conductivity, chlorides, alkalinity and different pollutants including pesticides and molluscicides, suggest that physical and chemical parameters of the aquatic medium must be consistent and controlled.

In the present study, we evaluated the physical and chemical parameters used in the latex test solutions of E. splendens on P. cubensis in laboratory conditions (Table 3). Correlation and regression analysis showed no relationship relationship between the latex doses used and the parameters of total hardness, calcium hardness, $\mathrm{mg} / \mathrm{L} \mathrm{Ca}, \mathrm{pH}$, total alkalinity, conductivity, bicarbonate alkalinity and $\mathrm{mg} / \mathrm{L} \mathrm{HCO}^{-}$, and no significant differences were found (Table 4).

In the experimental model using E. splendens's latex in natura on $P$. cubensis we found a $\mathrm{CL}_{90}$ of $3.41 \mu \mathrm{L} / \mathrm{L}$. According to Mott (1987), to be considered as a molluscicide, the plant's activity must fall in concentrations up to $100 \mathrm{mg} / \mathrm{L}$, and must be able to kill $90 \%$ of the mollusks in the concentration range tested after a 24 hour contact Thus, our results demonstrated that $E$. splendens is an effective molluscicide against these snails, corroborating once again the high potential indicated by Vasconcellos and Schall (1986).

Although this study was performed to evaluate molluscicidal properties in laboratory conditions, previous results demonstrated the activity of $E$. splendens latex under field conditions using both in natura and freeze-dried material (Vasconcellos and Schall, 1986). Toxicity studies were also conducted with latex from plants of the genus (Oliveira-Filho and Paumgartten, 2000), and established their levels of safety and acceptability as molluscicides.

Toxicological studies using E. splendens as molluscicides showed that the latex has no carcinogenic or mutagenic effect, low embryotoxic, teratogenic and ecotoxicological effects (Delgado et al., 2003). 
SANTOS, J. A. A.; VALIN, V.; FERRÃO-FILHO, A. S. Determination of the lethal doses LD $_{50}$ and LD Do $_{90}$ Euphorbia splendens var. hislopii latex on Physa cubensis Pfeiffer. Ambi-Agua, Taubaté, v. 7, n. 3, p. 21-30, 2012. (http://dx.doi.org/10.4136/ambi-agua.860)

Table 3. Values of the chemical and physical parameters and linear regression analysis of aqueous solutions with latex of Euphorbia splendens var hislopii, with Physa cubensis Pfeiffer $(\mathrm{N}=10)$ in laboratory.

\begin{tabular}{|c|c|c|c|c|c|c|c|c|c|c|c|c|c|c|c|}
\hline Parameters & GC & $\begin{array}{c}0.5 \\
\mu \mathrm{L} / \mathrm{L}\end{array}$ & $\begin{array}{c}1.0 \\
\mu \mathrm{L} / \mathrm{L}\end{array}$ & $\begin{array}{c}1.2 \\
\mu \mathrm{L} / \mathrm{L}\end{array}$ & $\begin{array}{c}1.4 \\
\mu \mathrm{L} / \mathrm{L}\end{array}$ & $\begin{array}{c}1.5 \\
\mu \mathrm{L} / \mathrm{L}\end{array}$ & $\begin{array}{c}1.6 \\
\mu \mathrm{L} / \mathrm{L}\end{array}$ & $\begin{array}{c}1.8 \\
\mu \mathrm{L} / \mathrm{L}\end{array}$ & $\begin{array}{c}2.0 \\
\mu \mathrm{L} / \mathrm{L}\end{array}$ & $\begin{array}{c}2.5 \\
\mu \mathrm{L} / \mathrm{L}\end{array}$ & $\begin{array}{c}3.0 \\
\mu \mathrm{L} / \mathrm{L}\end{array}$ & $\begin{array}{c}3.5 \\
\mu \mathrm{L} / \mathrm{L}\end{array}$ & $\begin{array}{c}4.0 \\
\mu \mathrm{L} / \mathrm{L}\end{array}$ & $\begin{array}{c}4.5 \\
\mu \mathrm{L} / \mathrm{L}\end{array}$ & $\begin{array}{c}5.0 \\
\mu \mathrm{L} / \mathrm{L}\end{array}$ \\
\hline Total hardness $\left(\mathrm{mg} / \mathrm{L} \mathrm{CaCO}_{3}\right)$ & 16 & 18 & 12 & 14 & 24 & 14 & 22 & 18 & 34 & 20 & 22 & 18 & 20 & 22 & 18 \\
\hline Calcium hardness $\left(\mathrm{mg} / \mathrm{L} \mathrm{CaCO}_{3}\right)$ & 6 & 6 & 8 & 4 & 4 & 10 & 10 & 10 & 12 & 10 & 8 & 6 & 14 & 8 & 12 \\
\hline Magnesium hardness $\left(\mathrm{mg} / \mathrm{L} \mathrm{CaCO}_{3}\right)$ & 10 & 12 & 4 & 10 & 20 & 4 & 12 & 8 & 22 & 10 & 14 & 12 & 6 & 14 & 6 \\
\hline $\mathrm{mg} / \mathrm{L} \mathrm{Ca}$ & 2.4 & 2.4 & 3.2 & 1.6 & 1.6 & 4 & 4 & 4 & 4.8 & 4 & 3.2 & 2.4 & 5.6 & 3.2 & 4.8 \\
\hline $\mathrm{mg} / \mathrm{L} \mathrm{Mg}$ & 2.4 & 2.9 & 1.0 & 2.4 & 4.8 & 1 & 2.9 & 1.9 & 5.3 & 2.4 & 3.4 & 2.9 & 1.4 & 3.4 & 1.4 \\
\hline Chlorides $\left(\mathrm{mg} / \mathrm{L} \mathrm{Cl}^{-}\right)$ & 3.4 & 4.4 & 3.4 & 2.4 & 3.4 & 3.4 & 2.4 & 3.4 & 5.4 & 5.4 & 3.4 & 3.4 & 3.4 & 3.4 & 3.4 \\
\hline $\mathrm{pH}$ & 6.3 & 6.2 & 6.7 & 6.4 & 6.2 & 6.5 & 6.3 & 6.2 & 6.3 & 6.3 & 6.4 & 6.3 & 6.3 & 6.6 & 6.5 \\
\hline Conductivity & 12.5 & 12.5 & 12.5 & 15 & 15 & 15 & 16.2 & 16.2 & 17.5 & 15 & 15 & 15 & 16.2 & 13.7 & 15 \\
\hline Total alkal. (mg/L $\left.\mathrm{CaCO}_{3}\right)$ & 14.4 & 14 & 14 & 18 & 18 & 18 & 21.6 & 18 & 19.2 & 16.2 & 19.2 & 24 & 16.2 & 14.4 & 14.4 \\
\hline Hydroxide alkal. $\left(\mathrm{mg} / \mathrm{L} \mathrm{CaCO}_{3}\right)$ & 0 & 0 & 0 & 0 & 0 & 0 & 0 & 0 & 0 & 0 & 0 & 0 & 0 & 0 & 0 \\
\hline $\mathrm{mg} / \mathrm{L} \mathrm{OH}^{-}$ & 0 & 0 & 0 & 0 & 0 & 0 & 0 & 0 & 0 & 0 & 0 & 0 & 0 & 0 & 0 \\
\hline Carbona alkal. (mg/L $\left.\mathrm{CaCO}_{3}\right)$ & 0 & 0 & 0 & 0 & 0 & 0 & 0 & 0 & 0 & 0 & 0 & 0 & 0 & 0 & 0 \\
\hline $\mathrm{mg} / \mathrm{L} \mathrm{CO}^{=}$ & 0 & 0 & 0 & 0 & 0 & 0 & 0 & 0 & 0 & 0 & 0 & 0 & 0 & 0 & 0 \\
\hline Bicarb. alkal. (mg/L $\left.\mathrm{CaCO}_{3}\right)$ & 14.4 & 14 & 14 & 18 & 18 & 18 & 21.6 & 18 & 19.2 & 16.2 & 19.2 & 24 & 16.2 & 14.4 & 14.4 \\
\hline $\mathrm{mg} / \mathrm{L} \mathrm{HCO}^{-}$ & 17.6 & 17.1 & 17.1 & 22 & 22 & 22 & 26.3 & 22 & 24.1 & 19.8 & 24.1 & 29.3 & 19.8 & 17.6 & 17.6 \\
\hline
\end{tabular}

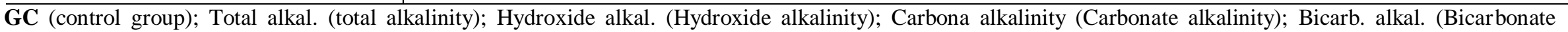
alkalinity). Each value is mean of three replicates. 
SANTOS, J. A. A.; VALIN, V.; FERRÃO-FILHO, A. S. Determination of the lethal doses LD $_{50}$ and LD $_{90}$ of Euphorbia splendens var. hislopii latex on Physa cubensis Pfeiffer. Ambi-Agua, Taubaté, v. 7, n. 3, p. 21-30, 2012. (http://dx.doi.org/10.4136/ambi-agua.860)

Table 4. The linear regression analysis of physical-chemical parameters in the doses of the latex of Euphorbia splendens var hislopii.

\begin{tabular}{l|ccccc}
\hline \multicolumn{1}{c|}{ Parameters } & $\mathbf{r}$ & \multicolumn{1}{c}{$\mathbf{R}^{\mathbf{2}}$} & $\mathbf{F}$ & $\mathbf{P}$ & significance \\
\hline Total hardness & 0.2190 & 0.04796 & 0.6549 & 0.4329 & $\mathrm{~ns}$ \\
Calcium hardness & 0.4959 & 0.2460 & 4.241 & 0.0601 & $\mathrm{~ns}$ \\
Magnesium hardness & 0.0615 & 0.003789 & 0.04944 & 0.8275 & $\mathrm{~ns}$ \\
Chlorides & 0 & 0 & 0 & 1 & $\mathrm{~ns}$ \\
pH & 0.0143178 & 0.000205 & 0.002671 & 0.9596 & $\mathrm{~ns}$ \\
Conductivity & 0.37855 & 0.1433 & 2.175 & 0.1640 & $\mathrm{~ns}$ \\
\hline Total alkalinity & $\mathbf{0 . 0 6 6 8 5}$ & $\mathbf{0 . 0 0 4 4 6 9}$ & $\mathbf{0 . 0 5 8 3 6}$ & $\mathbf{0 . 8 1 2 9}$ & ns \\
\hline
\end{tabular}

$\mathbf{r}=$ correlation coefficient, $\mathbf{R}^{2}=$ regression coefficient, $\mathbf{p}=$ probability, $\mathbf{n s}=$ not significant.

The stability of the molluscicidal property of the in natura latex of E. splendens, collected and stored at room temperaturewas observed in laboratory by Schall et al. (1992). Our results showed that the in natura latex of E. splendens, collected was considered active on Physa cubensis.

Therefore, due the molluscicidal activity of latex on planorbids, such as Physa cubensis, further research should be developed in view of the existence of other trematodiosis of medical and veterinary importance.

\section{CONCLUSION}

Our results showed molluscicidal activity and lethal doses of the latex of Euphorbia splendens on Physa cubensis in laboratory conditions and may be an effective alternative in the reduction of the mollusks disease vectors for humans. However, further studies are needed to better investigate the mechanism of molluscicidal activity of latex of Euphorbia splendens on Physa cubensis.

\section{REFERENCES}

ANDERSON, J. W.; FRIED. B. Experimental infection of Physa heterostropha, Helisoma trivolvis, and Biomphalaria blabrata (Gastropoda) with Echinostoma revolutum (Trematoda) cercariae. The Journal of Parasitology, v. 73, n. 1, p. 49-54, 1987.

ANDREWS, P.; THYSSEN, J.; LORKE, D. The biology and toxicology of molluscicides, Bayluscide. Pharmacology \& Therapeutics, v. 19, n. 2, p. 245-295, 1982.

BAPTISTA, D. F.; VASCONCELLOS, M. C.; LOPES, F. E.; SILVA, I. P.; SCHALL, V. T. Perspectives of using Euphorbia splendens as a molluscicide in schistosomiasis control programs. Southeast Asian Journal Tropical of Medicine Public Health, v. 25, n. 3, p. 419-424, 1994.

DELGADO, I. F.; De-CARVALHO, R. R.; De-OLIVEIRA, A. C. A. X.; KURIYAMA, S. N.; OLIVEIRA-FILHO, E. C.; SOUZA, C. A. M.; PAUMGARTTEN, F. J. R. Absence of tumor promoting activity of Euphorbia milii látex on the mouse back skin. Toxicology Letters, v. 145, n. 2, p. 175-180, 2003. http://dx.doi.org/10.1016/S0378-4274(03)00303-5 
DEGUEURCE, F.; ABROUS, M.; DREYFUSS, G.; RONDELAUD, D.; GEVREY, J. Paramphistomum daubneyi and Fasciola hepatica: the prevalence of natural or experimental infections in four species of freshwater snails in eastern France. Journal of Helminthology, v. 73, n. 3, p. 197-202, 1999. http://dx.doi.org/10.1017/S0022149X 99000311

FUNDAÇÃO ESTADUAL DE ENGENHARIA DO MEIO AMBIENTE - FEEMA (Rio de Janeiro). Métodos de análises físico-químicas da água. Rio de Janeiro, 1982. Sumula n. 8. Vol. 3.

FUNDAÇÃO NACIONAL DE SAÚDE - FUNASA (Brasil). Manual prático de análise de água. 2. ed. rev. Brasília, 2006. 146 p.

FINNEY, D. J. Probit Analysis. 3. ed. Cambrige: Cambridge University Press, 1971. 333p.

GIOVANELli, A.; SILVA, C. L. P. A. C.; MEDEIROS, L.; VASCONCELLOS, M. C. The molluscicidal activity of the látex of Euphorbia splendens var. hislopii on Melanoides tuberculata (Thiaridae), a snail associated with habitats of Biomphalaria glabrata (Planorbidae). Memórias do Instituto Oswaldo Cruz, v. 96, n. 1, p. 123-125, 2001. http://dx.doi.org/10.1590/S0074-02762001000100014

GUIMARÃES, C. T.; SOARES, D. M.; SCHUSTER, L. M. R. The influence of Physa marmorata (Mollusca; Physidae) on the oviposition of several planorbid hosts of Schistosoma mansoni. Memórias do Instituto Oswaldo Cruz, v. 82, n. 3, p. 439-440, 1987. http://dx.doi.org/10.1590/S0074-02761987000300018.

JOLY, A. B. Botânica: introdução à taxonomia vegetal. São Paulo: Nacional, 1979. 777 p.

JURBERG, P. J.; VASCONCELLOS, M. C.; MENDES, N. M. Plantas empregadas como moluscicidas: uma visão crítica. Memórias do Instituto Oswaldo Cruz, v. 84, p. 76-83, 1989. Suplemento 1. http://dx.doi.org/10.1590/S0074-02761989000500008

KANEV, I.; SORENSEN, R.; STERNER, M.; COLE, R.; FRIED, B. The identification and characteristics of Echinoparyphium rubrum (Cort, 1914) comb. new (Trematoda, Echinostomatidae) based on experimental evidence of the life cycle. Acta Parasitologica, v. 43, n. 4, p. 181-188, 1998.

KLOOS, H.; McCULLOUGH, F. S. Plants with recognized molluscicidal activity. In: MOTT, K. E. (Ed.). Plant molluscicides. New York: UNDP/World Bank/WHO, 1987. p. 45108.

KOSANKE, G. J.; SCHWIPPERT, W. W.; BENEKE, T. W. The impairment of mobility and development in freshwater snails (Physa fontinalis and Lymnaea stagnalis) caused by herbicides. Comparative Biochemistry and Physiology, v. 90C, n. 2, p. 373-379, 1988. http://dx.doi.org/10.1016/0742-8413(88)90013-8

KUO, Y. H. Plant molluscicide studies in the people's in Republic of China. In: MOTT, K. E. (Ed.). Plant molluscicides. New York: UNDP/World Bank/WHO, 1987. p. 220-298.

LEET, K. H.; HAYASHI, N.; OKANO, M.; HALL, I. H.; WU, R. Y.; McPHAILTI, A. T. Lasiodiplodin, a potent antileukemic macrolide from Euphorbia splendens. Phytochemistry, v. 21, n. 5, p. 1119-1121, 1982. http://dx.doi.org/10.1016/S00319422(00)82428-0 
MACBAE, W. D.; HUDSON, J. B.; TOWERS, G. H. N. Studies on the pharmacological activity of amazonian euphorbiaceae. Journal of Ethnopharmacology, v. 22, n. 2, p. 143-172, 1988. http://dx.doi.org/10.1016/0378-8741(88)90124-9

McCARTHY, A. M. The influence of second intermediate host species on the infectivity of metacercarial cysts of Echinoparyphium recurvatum. Journal of Helminthology, v. 73, n. 2, p. 143-145, 1999. http://dx.doi.org/10.1017/S0022149X99000219

MELlO-SILVA, C. C.; VASCONCELLOS, M. C.; BEZERRA, J. C. B.; RODRIGUES, M. L. A., PINHEIRO, J. The influence of exposure to Euphorbia splendens var. hislopii latex on the concentrations of total proteins and nitrogen products in Biomphalaria glabrata infected with Schistosoma mansoni. Acta Tropica, v. 117, n. 2, p. 101-104, 2011. http://dx.doi.org/10.1016/j.actatropica.2010.10.007

MELLO-SILVA, C. C.; VILAR, M. M.; BEZERRA, J. C. B.; VASCONCELLOS, M. C.; PINHEIRO, J.; RODRIGUES, M. L. A. Reprodutive activity alterations on the Biomphalaria glabrata exposed to Euphorbia splendens var. hislopii latex. Memórias do Instituto Oswaldo Cruz, v. 102, n. 6, p. 671-674, 2007. http://dx.doi.org/ 10.1590/S0074-02762007005000101

MELlO-SILVA, C. C.; VIlAR, M. M.; VASCONCELlOS, M. C.; PINHEIRO, J.; RODRIGUES, M. L. A. Carbohydrate metabolism alterations Biomphalaria glabrata infected with Schistosoma mansoni and exposed to Euphorbia splendens var. hislopii latex. Memórias do Instituto Oswaldo Cruz, v. 105, n. 4, p. 492-495, 2010. http://dx.doi.org/10.1590/S0074-02762010000400024

MOTT, K. E. Plant Molluscicides. New York: UNDP/World Bank/WHO, 1987.

OLIVEIRA-FILHO, E. C.; PAUMGARTTEN, F. J. R. Toxicity of Euphorbia milii látex and niclosamide to snails and nontarget aquatic species. Ecotoxicology and Environmental Safety, v. 46, n. 3, p. 342-350, 2000. http://dx.doi.org/10.1006/eesa.2000.1924

PARAENSE, W. L. Lymnaea columella in northern Brazil. Memórias do Instituto Oswaldo Cruz, v.78, n. 4, p. 477-482, 1983. http://dx.doi.org/10.1590/S0074-02761983000400011

PIO CORREA, M. Dicionário de plantas úteis do Brasil e das exóticas cultivadas. Rio de Janeiro: Imprensa Nacional, 1931. Vol. 2. p. 408.

RICHARDS, C. S. Studies on Puerto Rican Physidae. Public Health Reports, v. 79, n. 11, p. 1025-1029, 1964.

SCHALL, V. T.; VASCONCELLOS, M. C.; VALENT, G. U.; SATO, M. I.; FURLAN, E. V.; SANCHEZ, P. S. Evaluation of the genotoxic activity and acute toxicity of Euphorbia splendens latex, a molluscicide for the control of schistosomiasis. Brazilian Journal of Medical and Biological Research, v. 24, n. 6, p. 573-582, 1991.

SCHALL, V. T.; VASCONCELLOS, M. C.; VILLAÇA-COELHO, A. L.; FERREIRALOPES, F. E.; SILVA, I. P. Evaluation of temporal, seasonal and geographic stability of the molluscicidal property of Euphorbia splendens latex. Revista do Instituto de Medicinal Tropical de São Paulo, v. 34, n. 3, p. 183-191, 1992. http://dx.doi.org/10.1590/S0036-46651992000300001 
SNYDER, S. D.; ESCH, G. W. Trematode community structure in the pulmonate snail Physa gyrina. The Journal of Parasitology, v. 79, n. 2, p. 205-215, 1993.

SOUZA, C. P.; LIMA, L. C.; JANNOTTI-PASSOS, L. K.; FERREIRA, S. S.; GUIMARÃES, C. T.; VIEIRA, I. B. F. et al. Moluscos límnicos da microrregião de Belo Horizonte, MG, com ênfase nos vetores de parasitoses. Revista da Sociedade Brasileira de Medicina Tropical, v. 31, n. 5, p. 449-456, 1998. http://dx.doi.org/10.1590/S003786821998000500005

SOUZA, M. A. A.; MELO, A. L. Caracterização de larvas de trematódeos emergentes de moluscos gastrópodes coletados em Mariana, Minas Gerais, Brasil. Iheringia. Série Zoológica, v. 102, n. 1, p. 11-18, 2012. http://dx.doi.org/10.1590/S007347212012000100002

VASCONCELLOS, M. C.; AMORIM, A. Molluscicidal action of the latex of Euphorbia splendens var. hislopii N.E.B. ("Christ's Crown") (Euphorbiaceae) against Lymnaea columella (Say, 1817) (Pulmonata: Lymnaeidae), intermediate host of Fasciola hepatica Linnaeus, 1758 (Trematode: Fasciolidae). 1 - test in laboratory. Memórias do Instituto Oswaldo Cruz, v. 98, n. 4, p. 557-563, 2003. http://dx.doi.org/10.1590/S007402762003000400025

VASCONCELlOS, M. C.; SCHALL, V. T. Latex of "Coroa de Cristo" (Euphorbia splendens): an effective molluscicide. Memórias do Instituto Oswaldo Cruz, v. $81, \quad$ n. 4 , p. 475-476, 1986. http://dx.doi.org/10.1590/S007402761986000400017

VIGUERAS, I.; MORENO, A. "Physa cubensis" (Molusca), un nuevo hospedeiro intermediario de "Fasciola hepatica" (Trematoda). Memorias de la Sociedad Cubana de Historia Natural, v. 12, n. 1, p. 74, 1938.

WORLD HEALTH ORGANIZATION - WHO. Report of the scientific working group on plant molluscicides. Geneva, 1983. TDR/SCH-SWG (4)/83.3.

WORLD HEALTH ORGANIZATION - WHO. Report of the WHO informal consultation on schistosomiasis control. Geneva, 1998. WHO/CDS/CPC/SIP/99.2 746.

ZANI, C. L.; MARSTON, A.; HAMBURGER, M.; HOSTETTMANN, K. Molluscicidal milliamines from Euphorbia milli var. hislopii. Phytochemistry, v. 34, n. 1, p. 89-95, 1993. http://dx.doi.org/10.1016/S0031-9422(00)90788-X 


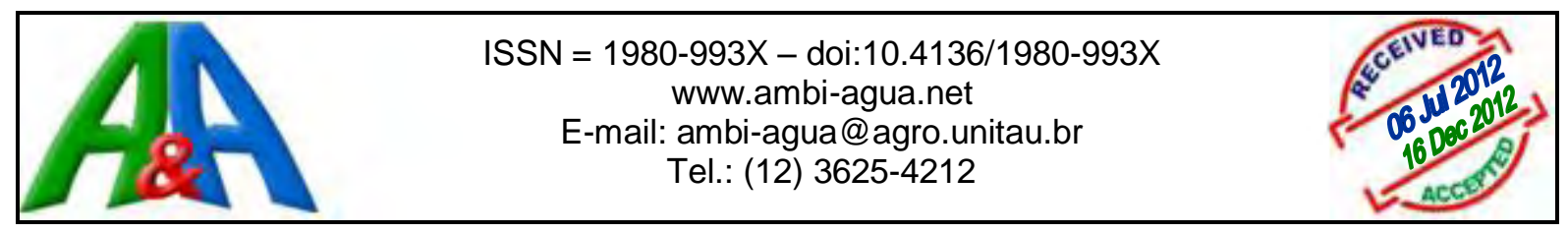

\title{
$\mathrm{UV} / \mathrm{H}_{2} \mathrm{O}_{2}$ process performance improvement by ultrafiltration and physicochemical clarification systems for industrial effluent pretreatment
}

(http://dx.doi.org/10.4136/ambi-agua.926)

\author{
José Carlos Mierzwa; Eduardo Lucas Subtil; Ivanildo Hespanhol \\ Centro Internacional de Referência em Reúso de Água (CIRRA), \\ Departamento de Engenharia Hidráulica e Ambiental (PHA), \\ Escola Politécnica da Universidade de São Paulo (EPUSP), São Paulo - SP, \\ e-mails: mierzwa@usp.br; eduardosubtil@gmail.com; ivanhes@usp.br
}

\begin{abstract}
The present study evaluated the removal of TOC from an effluent with high organic load resulted from the treatment of oil-water emulsion by thermal process. Hollow Fiber Ultrafiltration membrane (HF-UF) and physicochemical clarification process were used as pretreatment options to assess the influence of feed effluent quality on the $\mathrm{UV} / \mathrm{H}_{2} \mathrm{O}_{2}$ oxidation process. Results for TOC removals showed HF-UF and physicochemical clarification processes can significantly improve the efficiency of $\mathrm{UV} / \mathrm{H}_{2} \mathrm{O}_{2}$ oxidation process, when compared with the direct effluent oxidation. Reaction time for obtaining a TOC removal higher than $90 \%$ was reduced to approximately half of the time needed when no pretreatment was applied. Considering both pretreatment processes it was not possible to notice any significant difference on the $\mathrm{UV} / \mathrm{H}_{2} \mathrm{O}_{2}$ oxidation process performance. However, the complexity of physicochemical process due to the use of three different chemicals and sludge production made the HF-UF process the best pretreatment alternative, without increasing the Total Dissolved Solids of the effluent, a very important issue when water reuse is considered.
\end{abstract}

Keywords: $\mathrm{UV} / \mathrm{H}_{2} \mathrm{O}_{2}$, ultrafiltration, physicochemical process.

\section{Melhora do desempenho do processo $\mathrm{UV} / \mathrm{H}_{2} \mathrm{O}_{2}$ usando sistemas de ultrafiltração e de clarificação físico-química como pré-tratamento de efluente industrial}

\section{RESUMO}

O presente trabalho teve por objetivo avaliar a remoção de COT de um efluente com elevada concentração de matéria orgânica proveniente do tratamento de emulsão (água/óleo) por processo térmico. Um processo de separação por membranas de fibra-oca e outro de clarificação físico-químico foram usados como opções de pré-tratamento para avaliar a influência da qualidade do efluente sobre o processo de oxidação $\mathrm{UV} / \mathrm{H}_{2} \mathrm{O}_{2}$. Os resultados de remoção de COT demonstraram que tanto o processo de membranas quanto o físico-químico podem melhorar significativamente a eficiência do processo de oxidação $\mathrm{UV} / \mathrm{H}_{2} \mathrm{O}_{2}$. O tempo de reação necessário para uma remoção de $90 \%$ de COT foi reduzido pela metade quando comprado com a oxidação direta do efluente. Considerando ambos os processos de prétratamento não foi possível observar nenhuma diferença significativa sobre o desempenho do processo de oxidação $\mathrm{UV} / \mathrm{H}_{2} \mathrm{O}_{2}$. Entretanto, a complexidade do processo físico-químico devido ao uso de três diferentes compostos e a produção de lodo faz do sistema de membranas a melhor alternativa de pré-tratamento.

Palavras-chave: $\mathrm{UV} / \mathrm{H}_{2} \mathrm{O}_{2}$; ultrafiltração; processo físico-químico. 
MIERZWA, J. C.; SUBTIL, E. L.; HESPANHOL, I. UV/ $\mathrm{H}_{2} \mathrm{O}_{2}$ process performance improvement by ultrafiltration and physicochemical clarification systems for industrial effluent pretreatment. Ambi-Agua, Taubaté, v. 7, n. 3, p. 31-40, 2012. (http://dx.doi.org/10.4136/ambi-agua.926)

\section{INTRODUCTION}

The industrial development is one of main causes for environmental degradation of urbanized areas in Brazil, mainly of water bodies. Over the past two decades, environmental regulatory requirements have become more stringent because of increased awareness of the human health and ecological risks associated with environmental contaminants.

Metal working fluids are used in many small companies for metal forming and machining process, like cutting, grinding, and milling, however considering the complexity of the effluents produced, most of those companies has no capacity to treat it, then they hire a specialized company to do this job. Companies that provide such kind of service collect effluents from many sources and them treat it by specific ways, charging their service according the volume processed. One of the technologies used for treating such king of effluent is the thermal separation process (Gutiérrez et al., 2011; 2010; Fakhru'l-Razi et al., 2009). The use of thermal separation processes, depending on the inflow effluent characteristics can result in a condensate with a high organic load, because of stripping of volatile and semi-volatile compounds, besides a small fraction of non-volatile substances (Mao et al., 2010), which not allows releasing it to the environment or reusing it. Therefore, a complementary treatment step is necessary in order to cost-effectively meet environmental standards.

Among these technologies the Advanced Photochemical Oxidation Processes (APOP) are very promising, because it could be considered a green technology, involving the generation and use of powerful but relatively nonselective transient oxidizing species, primarily the hydroxyl radical $(\bullet \mathrm{OH})$ for contaminant destruction. The $\bullet \mathrm{OH}$ can be generated by both photochemical and non-photochemical processes to oxidize many contaminants (USEPA, 1998). Among the different available AOP, the one which uses UV light and hydrogen peroxide $\left(\mathrm{UV} / \mathrm{H}_{2} \mathrm{O}_{2}\right)$ was the first one to be used for wastewater treatment (Litter, 2005). This process proceeds in two main steps: hydroxyl radicals production by direct $\mathrm{H}_{2} \mathrm{O}_{2}$ photolysis and oxidation of organic compounds by these radicals (Braun e Oliveros, 1997). In some cases the photon energy is enough to break chemical bounds of organic molecules, resulting on its degradation. The $\mathrm{UV} / \mathrm{H}_{2} \mathrm{O}_{2}$ process has been shown very efficient on the degradation of various organic pollutants. Many successful application of $\mathrm{UV} / \mathrm{H}_{2} \mathrm{O}_{2}$ could be found in the literature, for instance, the works of Beltrán et al. (1997), for phenol oxidation, Cater et al. (2000), for gasoline contaminated groundwater treatment, Arslan et al. (2000), for the treatment of dyes contaminated effluents, Einschlag et al. (2002), for oxidation of nitroaromatic compounds, and more recently the work of Gao et al. (2009), for the treatment of ametryn contaminated waters. The advantage of $\mathrm{UV} / \mathrm{H}_{2} \mathrm{O}_{2}$ processes is that the $\mathrm{H}_{2} \mathrm{O}_{2}$ is the only chemical that needs to be added, and is easily converted to oxygen and water.

Even with the spread use of $\mathrm{UV} / \mathrm{H}_{2} \mathrm{O}_{2}$ process, most of them are applied for degradation of specific contaminants, present in relatively clean matrices, which is not the case for industrial wastewaters. In a recent review developed by Diya'uddeen et al. (2011), it is pointed out that wastewater composition could reduce the UV activated efficiency and even make its application unfeasible. The presence of discoloring substances or suspended solids can reduce the process efficiency by adsorbing or reducing UV light transmittance, which makes necessary the use of pretreatment systems, in order to improve contaminant degradation. Many technologies could be used as a pretreatment for the application of $\mathrm{UV} / \mathrm{H}_{2} \mathrm{O}_{2}$ oxidation process. However the best option should consider not only the improvement on the oxidation process efficiency, but the overall system performance. In some cases AOP are used as a pretreatment for improving the efficiency of biological process for treating complex wastewater focusing water reuse (Oller et al., 2011), where they concluded that is necessary to improve the knowledge about organics degradation kinetics and 
MIERZWA, J. C.; SUBTIL, E. L.; HESPANHOL, I. UV/ $\mathrm{H}_{2} \mathrm{O}_{2}$ process performance improvement by ultrafiltration and physicochemical clarification systems for industrial effluent pretreatment. Ambi-Agua, Taubaté, v. 7, n. 3, p. 31-40, 2012. (http://dx.doi.org/10.4136/ambi-agua.926)

reactor modeling considering the combined process. Considering the contaminants presents in condensates from thermal separation process will probably be partially emulsified, presenting high color and turbidity, the efficiency of $\mathrm{UV} / \mathrm{H}_{2} \mathrm{O}_{2}$ oxidation process will be reduced. To overcome this problem a clarification pretreatment process will be necessary, and for this purpose, physicochemical clarification or membrane filtration processes could be efficiently applied (Yahiaoui et al., 2011; Fakhru'l-Razi et al., 2009).

Focusing the overall performance of a treatment system for industrial effluent treatment, the aim of this study was to evaluate the influence of two pretreatment technologies: (i) physicochemical clarification (ii) Hollow Fiber Ultrafiltration Membrane - on the performance of $\mathrm{UV} / \mathrm{H}_{2} \mathrm{O}_{2}$ oxidation process for treating an industrial effluent with a high organic load. The pretreatment technologies were chosen based on the effluent characteristics.

\section{MATERIAL AND METHODOLOGY}

\subsection{Effluent Composition}

In the experiments an effluent arising from a thermal separation process (vapor compression evaporator), treating oil-water emulsions with a high TOC load was used. Because of evaporation process characteristics, a high level of organic contaminants is still present in the condensate, which not allows its releasing to the environment or even its reuse for industrial purposes. The main composition of raw effluent generated in the thermal separation process is given in Table 1.

Table 1: Mean composition of raw effluent $(\mathrm{N}=7)$.

\begin{tabular}{l|ccl}
\hline \multicolumn{1}{c|}{ Parameter } & Concentration & Unit & Analytical Method or Instrument \\
\hline pH & $7.2 \pm 0.7$ & -- & Electrometric - Quimis pH-meter \\
Turbidity & $141 \pm 51$ & $\mathrm{NTU}$ & Policontrol Turbidimeter \\
Color & $507 \pm 492$ & Color unit & Policontrol Colorimeter \\
COD & $4321 \pm 1015$ & $\mathrm{mg} / \mathrm{L}$ & Standard Methods, 5220 - D* \\
TOC & $827 \pm 109$ & $\mathrm{mg} / \mathrm{L}$ & TOC-V CPH Shimadzu \\
Oil \& Grease & $168 \pm 42$ & $\mathrm{mg} / \mathrm{L}$ & Standard Methods, 5520 - B* \\
Total Dissolved Solids & $106 \pm 19$ & $\mathrm{mg} / \mathrm{L}$ & Standard Methods, 2540 - C* \\
Electrical Conductivity & $1703 \pm 179$ & $\mu \mathrm{S} / \mathrm{cm}$ & Standard Methods, 2510 - B* \\
Total Phosphorus & $12 \pm 2.3$ & $\mathrm{mg} / \mathrm{L}$ & Standard Methods, 4500 - PB* \\
N-Ammonia & $450 \pm 89$ & $\mathrm{mg} / \mathrm{L}$ & Standard Methods, 4500 - NH $\mathrm{N}^{*}$ \\
N-Organic & $64 \pm 16$ & $\mathrm{mg} / \mathrm{L}$ & Standard Methods, 4500 - Norg B* \\
\hline
\end{tabular}

* APHA (1999)

According to the data presented in Table 1, most appropriated pretreatment technologies should be able to remove effluent color and turbidity, because it could significantly affect the $\mathrm{UV} / \mathrm{H}_{2} \mathrm{O}_{2}$ performance, justifying the use of ultrafiltration and physicochemical clarification as pretreatment processes.

\subsection{Membrane Separation Process - HF-UF}

The membrane separation process was carried out using a laboratory scale ultrafiltration unit (Demofilter - Koch Membrane Systems). Prior to the experiment, the effluent was filtered through a $100 \mu \mathrm{m}$ bag filter in order to remove coarse suspended solids, if present. Experiments were carried out using a hollow fiber membrane module from Koch Membrane 
MIERZWA, J. C.; SUBTIL, E. L.; HESPANHOL, I. UV/ $\mathrm{H}_{2} \mathrm{O}_{2}$ process performance improvement by ultrafiltration and physicochemical clarification systems for industrial effluent pretreatment. Ambi-Agua, Taubaté, v. 7, n. 3, p. 31-40, 2012. (http://dx.doi.org/10.4136/ambi-agua.926)

Systems, 1" HF 1.0-45-XM50, with a Molecular Weight Cut-off (MWCO) of 50,000 Daltons. The temperature in all experiments was $20 \pm 0.9{ }^{\circ} \mathrm{C}$ and the transmembrane pressure (TPM) was kept at 1.26 bar. Membrane system was operated in batch, with full concentrate recirculation to the feed tank. Permeate from UF system was used in the oxidation experiments.

\subsection{Physicochemical Clarification Process}

Effluent clarification was carried out in Jar-test equipment (Milan JT 203/6) with the addition of three different chemicals from Procytek Industria e Comercio Ltda. The chemical doses are presented in Table 2. Each chemical was added separately according to the data presented in Table 3. Thirty liters of effluent were treated using chemical clarification. The clarified effluent was used in oxidation experiments with $\mathrm{UV} / \mathrm{H}_{2} \mathrm{O}_{2}$.

Table 2 - Chemical doses used in clarification experiment.

\begin{tabular}{c|cc}
\hline Chemical brand & $\begin{array}{c}\text { Density } \\
(\mathbf{g} / \mathbf{m L})\end{array}$ & $\begin{array}{c}\text { Dosage } \\
(\mathbf{p p m} / \mathbf{L} \text { of effluent })\end{array}$ \\
\hline Procytrat 110B & 1.35 & 200 \\
Procytrat 100C & 1.15 & 700 \\
Procytrat 130C & 0.85 & 500 \\
\hline
\end{tabular}

Table 3 - Operation condition during clarification step.

\begin{tabular}{c|ccc}
\hline Process & $\begin{array}{c}\text { Mixing gradient } \\
\left(\mathbf{s}^{-\mathbf{1}}\right)\end{array}$ & $\begin{array}{c}\text { Time } \\
(\mathbf{s})\end{array}$ & $\begin{array}{c}\text { Chemical } \\
\text { added }\end{array}$ \\
\hline \multirow{3}{*}{ Rapid mix } & 120 & 180 & Procytrat 110B \\
& 120 & 180 & Procytrat 100C \\
Flocculation & 120 & 240 & Procytrat 130C \\
Settling time & 40 & 240 & -------- \\
\hline
\end{tabular}

\section{4. $\mathrm{UV} / \mathrm{H}_{2} \mathrm{O}_{2}$ Process}

Effluents from the ultrafiltration and physicochemical clarification processes, besides the raw effluent were then treated using the $\mathrm{UV} / \mathrm{H}_{2} \mathrm{O}_{2}$ oxidation process in order to obtain the TOC degradation kinetic constants. The photochemical reactor from Germetec UV/IR Technology Ltd. was equipped with a 1,600 watts medium pressure UV lamp. All tests were performed in batch with full effluent recirculation (Figure 1). Samples from the photochemical reactor effluent were taken each hour during the tests for the analysis of TOC and at the end of test for the analysis of the same parameters presented in Table 1. For the evaluation of TOC degradation kinetic constants a pseudo first order reaction was considered according equations 1 and 2, derived from a mass balance based on the diagram of Figure 1 . Since $\mathrm{H}_{2} \mathrm{O}_{2}$ was added in excess a pseudo first order kinetics can be assumed (USEPA, 1998). Equation 1 refers to the TOC degradation in the UV reactor and equation 2 refers to the TOC concentration leaving the storage tank, where no TOC degradation is expected. Oxidation kinetic constant was obtained by plotting the natural logarithm of TOC concentration with time, according the linearization of Equation (2). Operational conditions for the oxidation assays are presented in Table 4. 
MIERZWA, J. C.; SUBTIL, E. L.; HESPANHOL, I. UV/ $\mathrm{H}_{2} \mathrm{O}_{2}$ process performance improvement by ultrafiltration and physicochemical clarification systems for industrial effluent pretreatment. Ambi-Agua, Taubaté, v. 7, n. 3, p. 31-40, 2012. (http://dx.doi.org/10.4136/ambi-agua.926)

$$
\begin{aligned}
& C_{S}=C \cdot e^{-k \cdot \tau} \\
& C=C_{0} \cdot e^{\frac{\left(e^{\left.-k \cdot \tau_{-1}\right)}\right.}{\theta} t}
\end{aligned}
$$

where:

- $\mathrm{C}, \mathrm{C}_{\mathrm{S}}$ and $\mathrm{C}_{0}=\mathrm{TOC}$ concentration in the photochemical reactor influent and effluent and in the effluents from UF and physicochemical processes $(\mathrm{mg} / \mathrm{l})$;

- $\mathrm{k}=$ TOC degradation kinetic constant $\left(\mathrm{h}^{-1}\right)$;

- $\tau=$ photochemical reactor detention time (h);

$-\quad \Theta=$ storage tank detention time $(\mathrm{h})$;

$-\mathrm{t}=$ reaction time $(\mathrm{h})$.

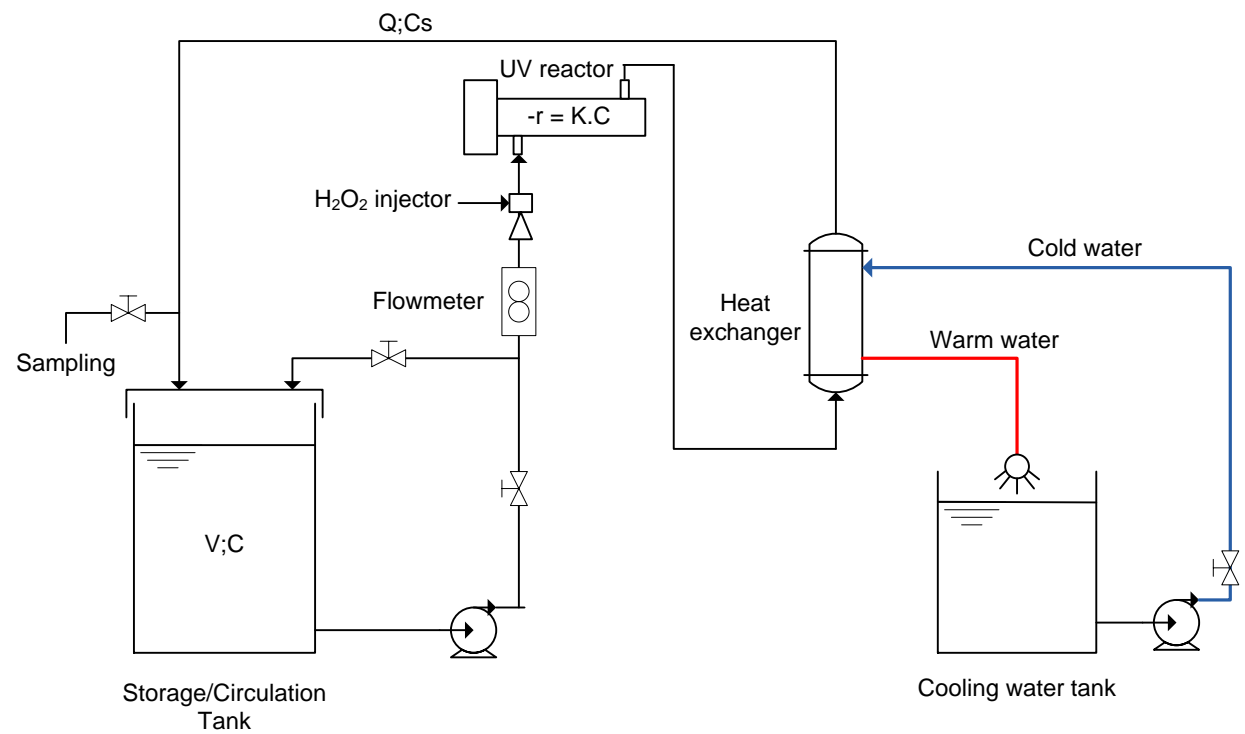

Figure 1: Advanced Oxidation Process diagram.

Table 4: - Operational parameters during $\mathrm{UV} / \mathrm{H}_{2} \mathrm{O}_{2}$ oxidation tests.

\begin{tabular}{l|cl}
\hline \multicolumn{1}{c|}{ Parameters } & Value & Unit \\
\hline Effluent volume per batch & 25 & $\mathrm{~L}$ \\
Effluent circulation flow & 360 & $\mathrm{~L} / \mathrm{h}$ \\
Experiment length & 9,3 & hours \\
UV reactor retention time & 6,7 & $\mathrm{Seconds}\left(1.86 \times 10^{-3} \mathrm{~h}\right)$ \\
$\alpha\left(\mathrm{H}_{2} \mathrm{O}_{2} /\right.$ TOC $)$ & 10 & $\mathrm{mg} \mathrm{H}_{2} \mathrm{O}_{2} / \mathrm{mg} \mathrm{TOC}^{\mathrm{T}}$ \\
UV dose & 2,39 & w.s $/ \mathrm{cm}^{2}$ \\
Operation temperature & $40-50$ & ${ }^{\circ} \mathrm{C}$ \\
\hline
\end{tabular}

\section{5. $\mathrm{H}_{2} \mathrm{O}_{2}$ dosage}

Commercial hydrogen peroxide dosage was determined based on the concentration of TOC fed to the UV reactor according to alpha $(\alpha)$ correlation stated in Table 4, using Equation 3.

$$
V_{\mathrm{H} 2 \mathrm{O2}}=\frac{0.1 \times \propto \times T O C o \cdot V_{\text {effluent }}}{\rho_{\mathrm{HzO}} \cdot \% \mathrm{HzO}_{\mathrm{z}}}
$$


MIERZWA, J. C.; SUBTIL, E. L.; HESPANHOL, I. UV/ $\mathrm{H}_{2} \mathrm{O}_{2}$ process performance improvement by ultrafiltration and physicochemical clarification systems for industrial effluent pretreatment. Ambi-Agua, Taubaté, v. 7, n. 3, p. 31-40, 2012. (http://dx.doi.org/10.4136/ambi-agua.926)

where:

$V_{\mathrm{H} 2 \mathrm{O} 2}: \quad$ hydrogen peroxide volume $(\mathrm{mL})$;

TOCo: total organic carbon in the effluent fed to the UV reactor $(\mathrm{mg} \mathrm{C} / \mathrm{L})$;

$V_{\text {effluent }}$ : effluent volume (L);

$\rho_{\mathrm{H} 2 \mathrm{O2} 2}$ : density of the hydrogen peroxide solution $\left(\mathrm{g} / \mathrm{cm}^{3}\right)$

$\%_{\mathrm{H} 2 \mathrm{O} 2}$ : mass content $(\mathrm{m} / \mathrm{m})$ of hydrogen peroxide solution $(\%)$.

0.1: $\quad$ correction factor.

\section{RESULTS AND DISCUSSION}

\subsection{Pretreatment Performance}

As it was mentioned, two types of pretreatment were applied prior oxidation experiments were initiated: (i) physicochemical clarification using 3 different chemicals and (ii) Hollow Fiber Ultrafiltration Membrane with MWCO of 50,000 Da. Those pretreatments were studied in order to verify the effect of feed water quality on TOC kinetics removal by $\mathrm{UV} / \mathrm{H}_{2} \mathrm{O}_{2}$ oxidation process, mainly with respect of turbidity and color removal which may prevent the penetration of UV light through wastewater during the oxidation experiments.

Thirty eight liters of effluent were treated by ultrafiltration, with a water recovery of $84 \%$. In this experiment, the permeate stream was collected separately for subsequent oxidation experiment. Mean permeate flow rate was $21.6 \pm 9.0 \mathrm{~L} / \mathrm{h}$, which could be considered adequate for effluent treatment. The clarification process resulted in a production of $670 \mathrm{~mL}$ of sludge resulting in a production rate of 22 liters of sludge per cubic meter of treated effluent. Compared to the concentrate produced by the ultrafiltration system, the sludge from the physicochemical process is a drawback because it will be necessary to implement a sludge conditioning process before its final disposal, while the concentrate from UF system could be sent back to the evaporation system.

Table 5 presents the results for contaminant removal obtained by each pretreatment process. Comparing these results it could be observed that HF-UF pretreatment system showed a best performance, except for oil \& grease and N-organic removal. Another point that should be mentioned is the increasing of electrical conductivity (34\%), and Total Dissolved Solids (191\%), in the effluent from clarification process, mainly because chemicals added during treatment. With regard the TOC removals both processes were inefficient, indicating the necessity of one additional treatment step, before its final disposal or reuse. Figure 2 presents the results for turbidity, color, COD, and TOC removal efficiencies.

Table 5. Characteristics of HF-UF and Physicochemical effluent.

\begin{tabular}{l|c|c|c|c}
\hline \multicolumn{1}{c|}{ Parameter } & Raw Effluent & Physical-Chemical & HF-UF & Unit \\
\hline pH & $8.5 \pm 0.7$ & $7.9 \pm 0.5$ & $8.4 \pm 0.3$ & -- \\
Turbidity & $141 \pm 51$ & $2.7 \pm 1.0$ & $1.9 \pm 0.7$ & $\mathrm{NTU}$ \\
Color & $507 \pm 492$ & $59 \pm 41$ & $10 \pm 7.8$ & Color unit \\
COD & $4321 \pm 1015$ & $3283 \pm 786$ & $3076 \pm 565$ & $\mathrm{mg} / \mathrm{L}$ \\
TOC & $827 \pm 109$ & $763 \pm 79$ & $748 \pm 54$ & $\mathrm{mg} / \mathrm{L}$ \\
Oil \& Grace & $168 \pm 67$ & $4.0 \pm 3.8$ & $6.0 \pm 2.5$ & $\mathrm{mg} / \mathrm{L}$ \\
TDS & $106 \pm 19$ & $309 \pm 28$ & $55 \pm 9.2$ & $\mathrm{mg} / \mathrm{L}$ \\
Electrical & $1703 \pm 179$ & $2283 \pm 181$ & $1648 \pm 141$ & $\mu \mathrm{S} / \mathrm{cm}$ \\
Conductivity & $12 \pm 2.3$ & $13 \pm 2.6$ & $7.9 \pm 1.7$ & $\mathrm{mg} / \mathrm{L}$ \\
P-total & $450 \pm 89$ & $296 \pm 55$ & $232 \pm 44$ & $\mathrm{mg} / \mathrm{L}$ \\
N-Ammonia & $64 \pm 16$ & $18 \pm 4.3$ & $21 \pm 4.1$ & $\mathrm{mg} / \mathrm{L}$ \\
N-Organic & \multicolumn{3}{|c}{}
\end{tabular}


MIERZWA, J. C.; SUBTIL, E. L.; HESPANHOL, I. UV/ $\mathrm{H}_{2} \mathrm{O}_{2}$ process performance improvement by ultrafiltration and physicochemical clarification systems for industrial effluent pretreatment. Ambi-Agua, Taubaté, v. 7, n. 3, p. 31-40, 2012. (http://dx.doi.org/10.4136/ambi-agua.926)

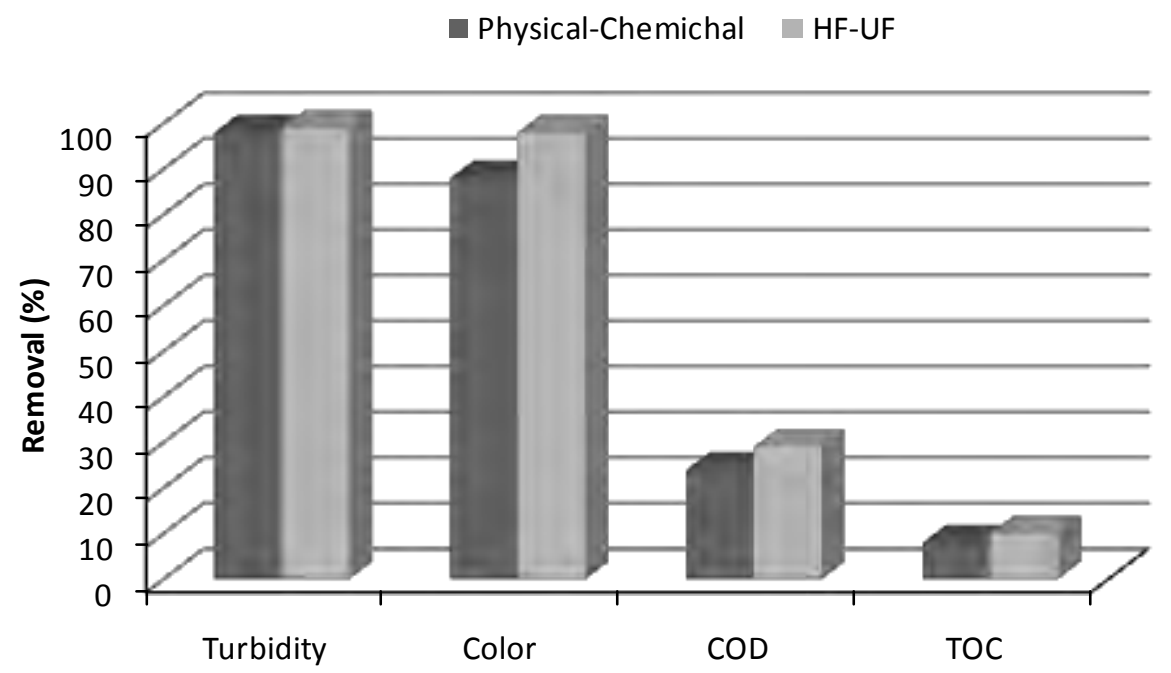

Figure 2: Efficiency of pretreatment systems for turbidity, color, COD, and TOC removal.

\subsection{TOC removal using $\mathrm{UV} / \mathrm{H}_{2} \mathrm{O}_{2}$ oxidation process, and kinetics constants}

TOC removals by $\mathrm{UV} / \mathrm{H}_{2} \mathrm{O}_{2}$ oxidation process were close to $90 \%$ in all experiments (Figure 3). However, when the pretreatment processes were applied the rate of TOC degradation increased, showing that effluent pretreatment is necessary for improving the $\mathrm{UV} / \mathrm{H}_{2} \mathrm{O}_{2}$ oxidation performance (Figure 4 and Table 6). These results are most probably related to the effluent clarification, which resulted in a better UV light transmission in the photochemical reactor, since color and turbidity can absorb UV light. Reaction time for obtaining a TOC removal of $90 \%$ was reduced to approximately 4 hours, half of the time needed when no pretreatment was applied. It is in accordance to the work developed by Benítez et al. (2008).

The results presented in this study are not in agreement the ones obtained by Saquib, Vinckier, and Van der Bruggen (2010), where they concluded that ultrafiltration process has no influence on the performance of an oxidation process using $\mathrm{O}_{3} / \mathrm{H}_{2} \mathrm{O}_{2}$ oxidation process. This could most be associated to the differences on inflow streams characteristics and experimental procedures. It should be mentioned that Saquib et al. (2010), used previously filtered natural water in the experiments, and no mention about water turbidity or color were made.

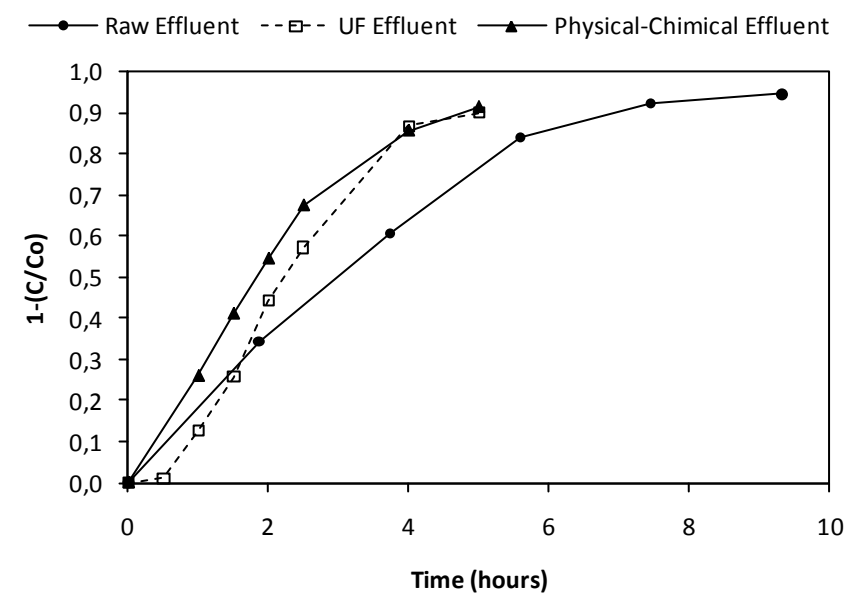

Figure 3. TOC efficiency removal with the $\mathrm{UV} / \mathrm{H}_{2} \mathrm{O}_{2}$ process 
MIERZWA, J. C.; SUBTIL, E. L.; HESPANHOL, I. UV/H $\mathrm{H}_{2} \mathrm{O}_{2}$ process performance improvement by ultrafiltration and physicochemical clarification systems for industrial effluent pretreatment. Ambi-Agua, Taubaté, v. 7, n. 3, p. 31-40, 2012. (http://dx.doi.org/10.4136/ambi-agua.926)
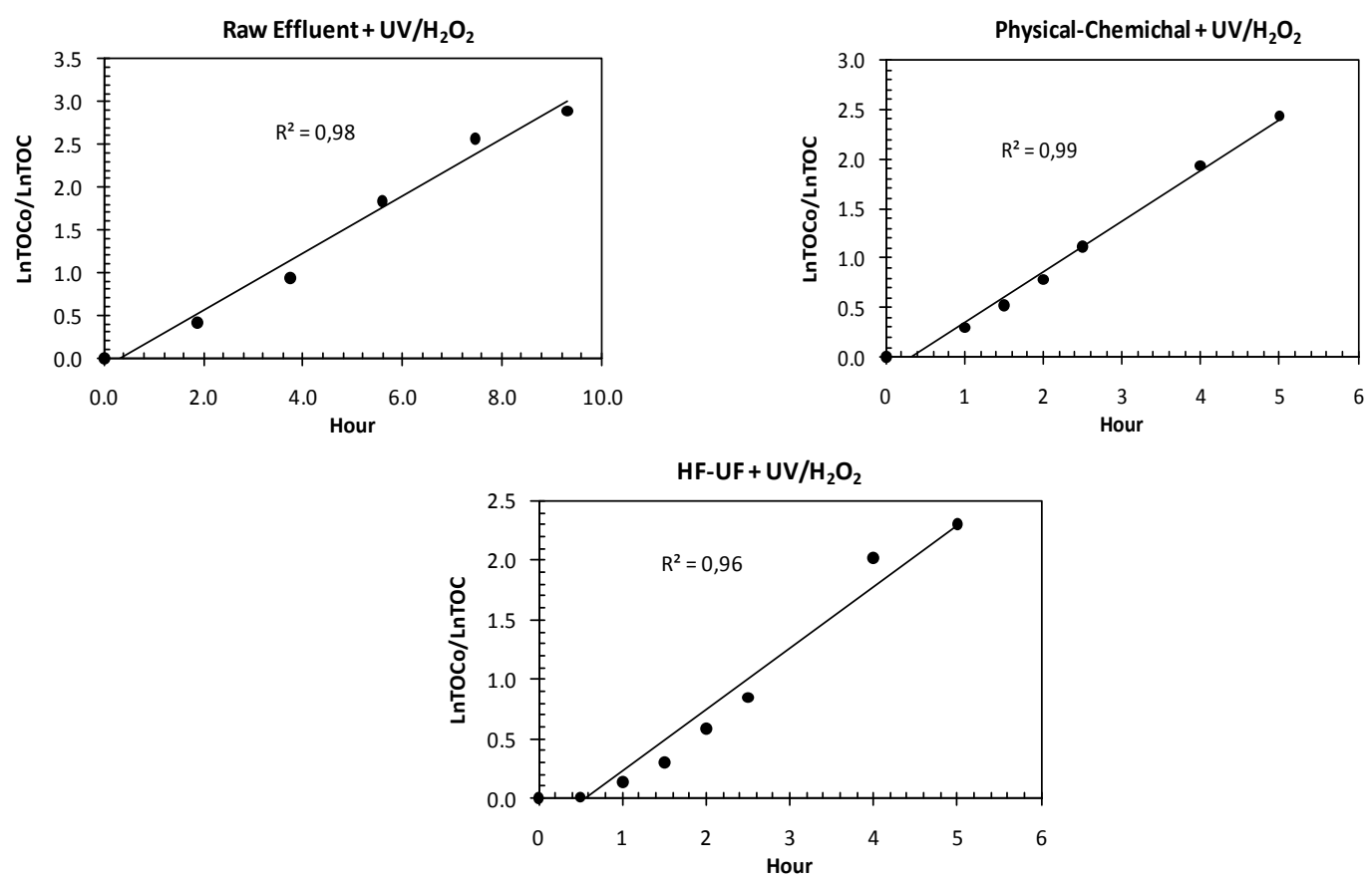

Figure 4. Data linearization for TOC removal by the $\mathrm{UV} / \mathrm{H}_{2} \mathrm{O}_{2}$ oxidation process.

Besides the analyses of the contaminants presented in Table 5, samples of all streams were sent for a private laboratory Bioagri Ambiental, in order to analyze the concentration of volatile and semi-volatile contaminants by gas chromatography. The results of gas chromatography analyses are presented in Figure 5, where it could be seen that the physicochemical clarification process was slightly more efficient than the UF one, and that $\mathrm{UV} / \mathrm{H}_{2} \mathrm{O}_{2}$ oxidation process was very efficient for all conditions evaluated, because the concentration of these contaminants in all samples analyzed were below the equipment detection limit.

Table 6. TOC degradation kinetics constants obtained.

\begin{tabular}{l|c|c}
\hline \multicolumn{1}{c|}{ Effluent } & $\begin{array}{c}\mathbf{K}_{\mathbf{1}} \\
\left(\mathbf{h}^{\mathbf{1}}\right)\end{array}$ & $\begin{array}{c}\mathbf{R}^{2} \\
--\end{array}$ \\
\hline Raw & 12,42 & 0.98 \\
UF & 19,03 & 0.96 \\
Physicochemical & 18,52 & 0.99 \\
\hline
\end{tabular}

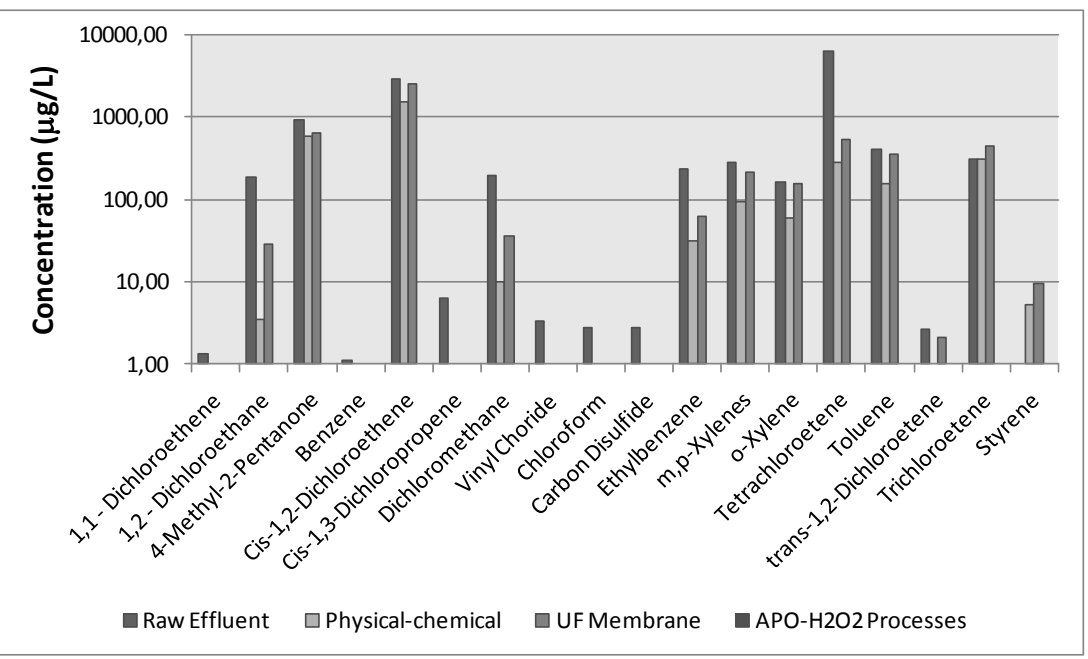

Figure 5. Gas chromatography analyses results for volatile and semivolatile chemicals 
MIERZWA, J. C.; SUBTIL, E. L.; HESPANHOL, I. UV/ $\mathrm{H}_{2} \mathrm{O}_{2}$ process performance improvement by ultrafiltration and physicochemical clarification systems for industrial effluent pretreatment. Ambi-Agua, Taubaté, v. 7, n. 3, p. 31-40, 2012. (http://dx.doi.org/10.4136/ambi-agua.926)

\section{CONCLUSIONS}

In this work a physicochemical clarification process and an ultrafiltration separation system were evaluated as a pretreatment option for industrial wastewater treatment by $\mathrm{UV} / \mathrm{H}_{2} \mathrm{O}_{2}$ oxidation process, in comparison to its direct treatment. The following conclusions can be drawn:

- Results obtained from the treatment of an effluent arising from an evaporation process of oil and water emulsion showed that ultrafiltration and physicochemical process, present no significant differences on contaminants removal, but that ultrafiltration system presents a better performance because there is no need for chemicals use.

- A considerable improvement on the efficiency of TOC removal from the effluents of UF and physicochemical clarification process, compared with the raw effluent, was obtained;

- Considering both pretreatment processes it was not possible to notice any significant difference on the performance of $\mathrm{UV} / \mathrm{H}_{2} \mathrm{O}_{2}$ oxidation process. However, the complexity of physicochemical process, because of the use of three different chemicals, and because of the sludge produced, makes the UF process the best pretreatment option.

\section{REFERENCES}

AMERICA PUBLIC HEALTH ASSOCIATION - APHA. Standard methods for examination of water and wastewater. 20. ed. Washington, DC, 1999.

ARSLAN, I.; BALCIOGLU, I. A.; TUHKANEN, T.; BAHNEMANN, D. $\mathrm{H}_{2} \mathrm{O}_{2} / \mathrm{UV}$ and $\mathrm{Fe}^{2+}$ / $\mathrm{H}_{2} \mathrm{O}_{2} / \mathrm{UV}$-c versus $\mathrm{TiO}_{2} / \mathrm{UV}$-A treatment for reactive dye wastewater. Journal of

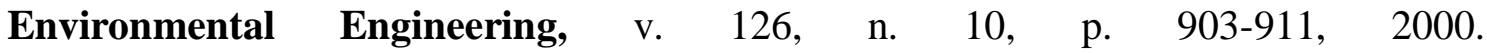
http://dx.doi.org/10.1061/(ASCE)0733-9372(2000)126:10(903)

BENÍTEZ, F. J.; ACERO, J. L.; LEAL, A. I.; REAL, F. J. Ozone and membrane filtration based strategies for the treatment of cork processing wastewaters. Journal of Hazardous Material, v. 152, n. 1, p. 373-380, 2008. http://dx.doi.org/10.1016/ j.jhazmat.2007.07.007

BRAUN, A. M.; OLIVEROS, E. How to evaluate photochemical methods for water treatment. Water Science and Technology. v. 35, n. 4, p. 17-23, 1997. http://dx.doi.org/10.1016/S0273-1223(97)00004-8

BELTRÁN, F. J.; GONZÁLEZ, M.; GONZÁLEZ, J. F. Industrial wastewater advanced oxidation. Part 1. UV radiation in the presence and absence of hydrogen peroxide. Water Research, v. 31, n. 10, p. 2405-2414, 1997. http://dx.doi.org/10.1016/S00431354(97)00077-8

CATER, S. R.; STEFAN, M. I.; BOLTON, J. R.; SAFARZADEH-AMIRI, A. UV/ $\mathrm{H}_{2} \mathrm{O}_{2}$ Treatment of methyl tert-butyl ether in contaminated water. Environmental Science \& Technology, v. 34, n. 4, p. 659-662, 2000. http://dx.doi.org/10.1021/es9905750

DIYA'UDDEEN, B. H.; DAUD, W. M. A. W.; ABDUL AZIZ, A. R. Treatment technologies for petroleum refinery effluents: a review. Process Safety and Environmental Protection, v. 89, n. 2, p. 95-105, 2011. http://dx.doi.org/10.1016/j.psep.2010.11.003

EINSCHLAG, F. S. G.; LOPEZ, J.; CARLOS, L.; CAPPARELLI, A.L.; BRAUN, A. M.; OLIVEROS, E. Evaluation of the efficiency of photodegradation on Nitroaromatics applying the UV/ $\mathrm{H}_{2} \mathrm{O}_{2}$ technique. Environmental Science \& Technology, v. 36, n. 18, p. 3936-3944, 2002. http://dx.doi.org/10.1021/es0103039 
MIERZWA, J. C.; SUBTIL, E. L.; HESPANHOL, I. UV/ $\mathrm{H}_{2} \mathrm{O}_{2}$ process performance improvement by ultrafiltration and physicochemical clarification systems for industrial effluent pretreatment. Ambi-Agua, Taubaté, v. 7, n. 3, p. 31-40, 2012. (http://dx.doi.org/10.4136/ambi-agua.926)

FAKHRU'L-RAZI, A.; PENDASHTEH, A.; ABDULLAH, L. C.; BIAK, D. R. A.; MADAENI, S. S.; ABIDIN, Z. Z. Review of technologies for oil and gas produced wastewater treatment. Journal of Hazardous Materials, v. 170, n. 2/3, p. 530-551, 2009. http://dx.doi.org/10.1016/j.jhazmat.2009.05.044

GAO, N.; DENG, Y.; ZHAO, D. Ametryn degradation in the ultraviolet (UV) irradiation/hydrogen peroxide $\left(\mathrm{H}_{2} \mathrm{O}_{2}\right)$ treatment. Journal of Hazardous Materials, v. 164, n. 2/3, p. 640-645, 2009. http://dx.doi.org/10.1016/j.jhazmat.2008.08.038

GUTIÉRREZ, G.; BENITO, J. M.; COCA, J.; PAZOS, CJ. Vacuum evaporation of surfactant solutions and oil-in-water emulsions. Chemical Engineering Journal, v. 162, n. 1, p. 201-207, 2010. http://dx.doi.org/10.1016/j.cej.2010.05.029

GUTIÉRREZ, G.; LOBO, A.; BENITO, J. M.; COCA, J.; PAZOS, C. Treatment of a waste oil-in-water emulsion from a copper-rolling process by ultrafiltration and vacuum evaporation. Journal of Hazardous Materials, v. 185, n. 2/3, p. 1569-1574, 2011. http://dx.doi.org/10.1016/j.jhazmat.2010.10.088

LITTER, M. I. Introduction to photochemical advanced oxidation processes for water treatment. The Handbook of Environmental Chemistry. v. 2, p. 325-366, 2005. http://dx.doi.org/10.1007/b138188

MAO, W.; MA, H.; WANG, B. Performance of batch vacuum distillation process with promoters on coke-plant wastewater treatment. Chemical Engineering Journal, v. 160, n. 1, p. 232-238, 2010. http://dx.doi.org/10.1016/j.cej.2010.03.053

OLLER, I.; MALATO, S.; SÁNCHES-PÉREZ, J. A. Combination of advanced oxidation processes and biological treatments for wastewater decontamination - a review. Science of the Total Environment, v. 409, n. 10, p. 4141-4166, 2011. http://dx.doi.org/10.1016/j.scitotenv.2010.08.061

SAQUIB, M.; VINCKIER, C.; VAN DER BRUGGEN, B. The effect of UF on the efficiency of $\mathrm{O}_{3} / \mathrm{H}_{2} \mathrm{O}_{2}$ for the removal of organics from surface water. Desalination, v. 260, n. $1 / 3$, p. 39-42, 2010. http://dx.doi.org/10.1016/j.desal.2010.05.003

UNITED STATES. Environmental Protection Agency - USEPA. Handbook of advanced photochemical oxidation processes. Washington, DC, 1998. Report EPA/625/R98/004.

YAHIAOUI, O.; LOUNICI, H.; ABDI, N.; DROUICHE, N.; GHAFFOUR, N.; PAUSS, A.; MAMERI, N. Treatment of olive mill wastewater by the combination of ultrafiltration and bipolar electrochemical reactor processes. Chemical Engineering and Processing: Process Intensification, v. 50, n. 1, p. 37-41, 2011. http://dx.doi.org/ 10.1016/j.cep.2010.11.003 


ISSN = 1980-993X - doi:10.4136/1980-993X
www.ambi-agua.net
E-mail: ambi-agua@agro.unitau.br
Tel.: (12) 3625-4212

\title{
Evaluation of the use of Pycnoporus sanguineus fungus for phenolics and genotoxicity decay of a pharmaceutical effluent treatment
}

\author{
(http://dx.doi.org/10.4136/ambi-agua.966)
}

\author{
Renata Alberto de Morais Watanabe ${ }^{1}$;Paulo de Tarso Ferreira Sales ${ }^{1,2}$; Luiza Cintra \\ Campos $^{3}$; Telma Alves Garcia ${ }^{1}$ Marize Campos Valadares ${ }^{4}$; Fernando Schimidt ${ }^{5}$; \\ Mariângela Fontes Santiago ${ }^{1,2}$ \\ ${ }^{1}$ Department of Enzimology, Federal University of Goiás (UFG), Goiânia-GO-Brazil, \\ e-mails: rewfarm@hotmail.com, paulo@tecpam.com.br, mariangelafs@gmail.com, telma@ farmacia.ufg.br, \\ ${ }^{2}$ Programa de Pós-graduação de Engenharia do Meio Ambiente, \\ Federal University of Goiás, Goiânia-GO-Brazil, \\ ${ }^{3}$ Department of Civil, Environmental and Geomatic Engineering, \\ University College London, United Kingdom, e-mail: 1.campos@ucl.ac.uk, \\ ${ }^{4}$ Laboratório de Farmacologia e Toxicologia Celular, \\ Federal. University of Goiás (UFG), Goiânia-GO-Brazil, \\ e-mail: marizecv@farmacia.ufg.br, \\ ${ }^{5}$ Centro Federal de Educação Tecnológica de Goiás, Goiânia-GO-Brazil, \\ e-mail: schimidt99@bol.com.br
}

\begin{abstract}
If not properly and efficiently treated, wastes produced by the chemical industry can contaminate the environment. Using fungi able to degrade organic compounds (e.g. phenol) seems to be a prominent method to treat pharmaceutical wastewaters, in particular, the whiterot fungus. The aim of this work was to treat pharmaceutical effluent by the Pycnoporus sanguineus fungus. Three effluent samples were collected in a pharmaceutical industry. The production of enzymes such as laccase and manganese peroxidase was determined. Their production increased throughout the treatment with the $P$. sanguineus fungus, reaching maximum concentration of $4.48 \mathrm{U}_{\mathrm{mL}}{ }^{-1}$ (Effluent 1), $8.16 \mathrm{U} \cdot \mathrm{mL}^{-1}$ (Effluent 2), $2.8 \mathrm{U} \cdot \mathrm{mL}^{-1}$ (Effluent 3) and 0.03 Abs. $\min ^{-1}$ (Effluent 2), respectively, during 96 hours of biological treatment. Genotoxic effects of the raw and treated effluents were also investigated using the in vivo mouse bone marrow micronucleus (MN) assay. Results showed the biological treatment reduced the frequency of $\mathrm{MN}$, in a dose-dependent manner, when compared to untreated sample. The decreasing of around $20 \%$ and $45 \%$ of phenolics concentration was observed throughout the treatment, confirming that laccase production can be related to the degradation of toxic compounds present in the effluent. Therefore, the biodegradation by the $P$. sanguineus fungus seems a promising method for the mineralization of recalcitrant compounds present in pharmaceutical effluents.
\end{abstract}

Keywords: pharmaceutical effluent, biodegradation, Pycnoporus sanguineus, laccase, Manganese peroxidase.

\section{Avaliação do uso do fungo Pycnoporus sanguineus no decaimento de compostos fenólicos e genotoxicidade no tratamento de um efluente farmacêutico}

\section{RESUMO}

Se não for tratado de forma adequada e eficientemente, os resíduos produzidos pela indústria química podem contaminar o meio-ambiente. Utilizar fungos capazes de degradar compostos orgânicos (por exemplo, fenol), pode ser um método para tratar efluentes 
WATANABE, R. A. M.; SAlES, P. T. F.; CAMPOS, L. C.; GARCIA, T. A.; VALADARES, M. C.; SCHIMIDT, F.; SANTIAGO, M. F. Evaluation of the use of Pycnoporus sanguineus fungus for phenolics and genotoxicity decay of a pharmaceutical effluent treatment. Ambi-Agua, Taubaté, v. 7, n. 3, p. 41-50, 2012. (http://dx.doi.org/10.4136/ambi-agua.966)

proeminentes de insústrias farmacêuticas, em particular, os fungos da podridão branca. $\mathrm{O}$ objetivo deste trabalho foi tratar um efluente farmacêutico pelo fungo Pycnoporus sanguineus. Três amostras de efluentes foram coletadas em uma indústria farmacêutica. A produção de enzimas como a lacase e manganês peroxidase foi determinado. A sua produção durante o tratamento com o fungo $P$. sanguineus atingiu concentrações máximas de 4,48 U.mL ${ }^{-1}$ (Efluente 1), 8,16 U.mL ${ }^{-1}$ (Efluente 2), 2,8 U.mL ${ }^{-1}$ (Efluente 3) e 0,03 Abs.min ${ }^{-1}$ (efluente 2), respectivamente, durante 96 horas de tratamento biológico. Efeitos genotóxicos dos efluentes brutos e tratados também foram investigados usando o ensaio em que foi avaliada a formação de micronúcleos (MN) na medula óssea de camundongos. Os resultados mostraram que o tratamento biológico reduziu a frequência de $\mathrm{MN}$, de um modo dependente da dose, em comparação com amostras não tratadas. A diminuição de cerca de $20 \%$ e $45 \%$ da concentração de compostos fenólicos foi observada em todo o tratamento, o que confirma que a produção de lacase pode ser relacionada com a degradação de compostos tóxicos presentes no efluente. Por conseguinte, a biodegradação do fungo $P$. sanguineus parece um método promissor para a mineralização de compostos recalcitrantes presentes em efluentes farmacêuticos.

Palavras-chave: efluente farmacêutico, biodegradação, Pycnoporus sanguineus, lacase, manganês peroxidase.

\section{INTRODUCTION}

Pharmaceutical wastewaters comprise a group that, with regard to the environmental issues, has been given little attention for many years (Hirsch et al., 1999; Roberts and Thomas, 2006).

The impact of discharging pharmaceutical effluent without treatment into rivers (Chen, Wang and Ding, 2008), lakes (Boyd et al., 2004), aquifers (Godfrey, Woessner and Benotti, 2007) is quite strong, affecting both aquatic and human lives.

Phenol is one of the main toxic compounds found in pharmaceutical wastewaters and other compounds like that are called phenolics. It belongs to an aromatic organic substances family, by biological or synthetic origin. They can found as a colorless liquid or white solid at ambient temperature, and it has strong and specific odours (Kumaran and Paruchuri, 1997).

Melo et al. (2006) suggested it was necessary to evaluate the efficiency of the decontamination process, and comparing the toxicity of treated and untreated effluents. Bioassays, such as the in vivo micronucleus (MN) assay, have traditionally been used to assess the effluents toxicity, as well as monitoring the wastewater treatment (Wirzinger et al., 2007).

Using fungi able to degrade organic compounds can be a prominent method of pharmaceutical effluent treatment. In particular, the white-rot fungus has got an enzymatic system able to supporting high concentrations of toxic contaminants (Barr and Aust, 1994). These fungus have the ability of degrading recalcitrant organic pollutants such as polyaromatic hydrocarbons, chlorophenols, and polychlorinated biphenyls (Barr and Aust, 1994). This is probably due to the low specificity of their ligninolytic enzymes, such as laccase, lignin peroxidase, and manganese peroxidase (McMullan et al., 2001).

Laccase is a polyphenyloxidase able to oxidise several aromatic compounds (e.g. phenol) coupled with electron reduction of oxygen in water (Durán and Esposito, 1997).

Induced and constitutive laccases are found in many fungal species, but few works have investigated laccases produced by members of the Pycnoporus group (Pointing et al., 2000). The Pycnoporus sanguineus, a white-rot fungus and comprising the Basidiomycota, was 
WATANABE, R. A. M.; SAleS, P. T. F.; CAMPOS, L. C.; GARCIA, T. A.; VAladARES, M. C.; SCHIMIDT, F.; SANTIAGO, M. F. Evaluation of the use of Pycnoporus sanguineus fungus for phenolics and genotoxicity decay of a pharmaceutical effluent treatment. Ambi-Agua, Taubaté, v. 7, n. 3, p. 41-50, 2012. (http://dx.doi.org/10.4136/ambi-agua.966)

recognised by biodegrading some textile dyes (Pointing and Vrijmoed, 2000, Trovaslet et al., 2007) and lignosulphonates (Eugenio et al., 2008). This fungus could mineralize both Lignin (recalcitrant compound present in the lignocellulosic wood matrix) and many resistant pollutants (Durán and Esposito, 2000).

Lignin and Manganese Peroxidases are produced by the majority of white-rot fungi such as Phanerochate chrysosporium, Trametes versicolor and P. sanguineus. Lignin peroxidase catalyzes the one-electron oxidation of various aromatic compounds, with subsequent formation of aryl cation radicals, which are decomposed spontaneously by various pathways. Manganese Peroxidase catalyzes the oxidation of $\mathrm{Mn}(\mathrm{II})$ to $\mathrm{Mn}(\mathrm{III})$, which in turn can oxidize several phenolic substrates (Sayad and Ellouz, 1995). These enzymes were used to decrease phenolic and non-phenolic polluants (Rodríguez et al., 2004).

In Brazil, the country's third largest pharmaceutical and chemical pole is located in the State of Goiás. Within this context, part of the environmental contamination in Goiás is probably attributed to the pharmaceutical industry activities. This work was developed to evaluate the biological treatment of a pharmaceutical effluent by $P$. sanguineus fungi. The genotoxic effects of the raw and treated effluent were also investigated, using the in vivo mouse bone marrow MN assay. Genetic toxicology tests are assays designed to detect direct or indirect genetic damage induced by compounds.

\section{MATERIALS AND METHODS}

\subsection{Effluent}

Three samples of $20 \mathrm{~L}$ each were collected from a pharmaceutical industry in Goiás, Brazil and kept at $4^{\circ} \mathrm{C}$. The sampling technique used was the composite sampling.

\subsection{Fungus and Growth Media}

The fungus used in this work was the Pycnoporus sanguineus CCT-4518 which was provided by the Andre Tosello Foundation, São Paulo, Brazil. The fungus was incubated in potato dextrose agar (PDA) media. The Petri dishes were incubated in $37^{\circ} \mathrm{C}$ for 6 days.

\subsection{Treatment Process}

For the treatment process, it was used $100 \mathrm{ml}$ of the each effluent sample (samples 1, 2 and 3), and the growth media with the fungus. All the samples were incubated in C24KC Refrigerated Incubator Shaker (Edison NJ. USA) for 24, 48, 72, and 96 hours at $150 \mathrm{rpm}$ and $28^{\circ} \mathrm{C}$. The control sample was also treated in the same conditions (as a control, the growth media and the fungus was inoculated to the samples and after that, they were decontaminated at $121^{\circ} \mathrm{C}$ for 15 minutes). After 24, 48, 72 and 96 hours of treatment, all samples were filtered (filter papers) and they were stored in amber glass flasks at $4^{\circ} \mathrm{C}$ (adapted from Santiago, 1999).

\subsection{Total Phenolics Determination}

Phenolis concentrations were determined by the colorimetric method using FolinCiocalteu reagent (APHA, 1992). The reaction mixture was: $1000 \mu \mathrm{L}$ of effluent, $250 \mu \mathrm{L}$ of sodium carbonate solution $\left(12 \mathrm{~g} . \mathrm{L}^{-1}\right)$ and $25 \mu \mathrm{L}$ of Folin-Ciocalteau 2 mol.L ${ }^{-1}$. All samples were kept at $20^{\circ} \mathrm{C}$ for $30 \mathrm{~min}$. The phenolics concentrations were determinated at $700 \mathrm{~nm}$, in a spetrophotometer. The results were expressed in $\mathrm{mg} . \mathrm{L}^{-1}$ of phenolics, using the calibration curve in the range of 0 to $14 \mathrm{mg} . \mathrm{L}^{-1}$ which was obtained using the same procedure, using phenol as a standard. 
WATANABE, R. A. M.; SAleS, P. T. F.; CAMPOS, L. C.; GARCIA, T. A.; VAladARES, M. C.; SCHIMIDT, F.; SANTIAGO, M. F. Evaluation of the use of Pycnoporus sanguineus fungus for phenolics and genotoxicity decay of a pharmaceutical effluent treatment. Ambi-Agua, Taubaté, v. 7, n. 3, p. 41-50, 2012. (http://dx.doi.org/10.4136/ambi-agua.966)

\subsection{Determination of Laccase (Benzenediol: oxygen oxidoreductase, EC 1.10.3.2) Enzymatic Activity}

The enzymatic activities were determined in treated and untreated effluent. The control samples were used to detect likely interferences in the enzymatic methodology. The laccase enzymatic activity was determined in duplicates at $30^{\circ} \mathrm{C}$ for $3 \mathrm{~min}$ and $5 \mathrm{~min}$ using $1 \mathrm{mol.L} \mathrm{L}^{-1}$ Syringaldazine (4-hydroxy-3,5-dimethoxybenzaldehydeazine) as substrate. The reaction mixture consisted of a buffered solution of $300 \mu \mathrm{L}$ of sodium acetate 0.05 mol. $\mathrm{L}^{-1}(\mathrm{pH}=5.0)$, $600 \mu \mathrm{L}$ of filtrate (previously centrifuged at $12,000 \mathrm{rpm}$ during $15 \mathrm{~min}$ at $4^{\circ} \mathrm{C}$ ) and $100 \mu \mathrm{L}$ of Syringaldazine $1 \mathrm{~mol} . \mathrm{L}^{-1}$. The enzymatic activities were determined in $525 \mathrm{~nm}$ (absorbance). The reaction started by the addition Syringaldazine $\left(\varepsilon_{525}=6.5 \times 10^{4} \mathrm{~mol} \cdot \mathrm{L}^{-1} \cdot \mathrm{cm}^{-1}\right)$ and the enzymatic activity was measured after 3 and 5 min later. From each value found, the control value was subtracted in order to remove possible disturbances of other compounds. The arithmetic average of the absorbance values of each treatment period was calculated. One unit laccase activity was defined as the amount of enzyme causing one unit increase in absorbance per min per $\mathrm{mL}$ of the assay (adapted from Szklarz et al., 1989).

\subsection{Determination of Manganese Peroxidase (MnP, EC 1.11.1.7) Enzymatic Activity}

The activity of peroxidase dependent on Mn (II) was determined by the oxidation of red phenol. The reaction mixture $(1.0 \mathrm{~mL})$ consisted of $500 \mu \mathrm{L}$ filtrate juice, $100 \mu \mathrm{L}$ of Sodium Lactate 0.25 mol. $\mathrm{L}^{-1}, 200 \mu \mathrm{L}$ of bovine albumine $0.5 \%, 50 \mu \mathrm{L}$ of Manganese Sulphate 2.0 mmol. $\mathrm{L}^{-1}, 50 \mu \mathrm{L}$ of Hydrogen Peroxide solution 2.0 mol. $\mathrm{L}^{-1}$ prepared in Sodium Succinate buffer 0.2 mol. $\mathrm{L}^{-1}(\mathrm{pH}=4.5)$ and $100 \mu \mathrm{L}$ of red phenol $0.1 \%$. The mixture was incubated at $30^{\circ} \mathrm{C}$ for $5 \mathrm{~min}$ and the reaction was stopped by adding $40 \mu \mathrm{L}$ sodium hydroxide $2.0 \mathrm{~mol} . \mathrm{L}^{-1}$. The absorbance was set at $610 \mathrm{~nm}$ and the enzymatic activity was expressed in Abs. $\mathrm{min}^{-1}$ (Kuwahara et al., 1984).

\subsection{Determination of Lignina Peroxidase (LiP, EC 1.11.1.7) Enzymatic Activity}

Lignina peroxidase activity was determined by the oxidation of veratryl alcohol $\left(\varepsilon_{310}=\right.$ 9,300 mol. $\left.\mathrm{L}^{-1} \cdot \mathrm{cm}^{-1}\right)$. The reaction mixture $(1 \mathrm{~mL})$ consisted of $600 \mu \mathrm{L}$ of filtrate, $200 \mu \mathrm{L}$ of hydrogen peroxide, $2.0 \mathrm{mmol} . \mathrm{L}^{-1}$ and $200 \mu \mathrm{L}$ of veratryl alcohol solution $2.0 \mathrm{mmol} . \mathrm{L}^{-1}$ in sodium tartarate buffer $0.4 \mathrm{~mol} . \mathrm{L}^{-1}(\mathrm{pH}=3.0)$. The reaction was started by adding the hydrogen peroxide and the appearance of veratryl alcohol oxidized was determined by absorbance at $310 \mathrm{~nm}$ (Tien and Kirk, 1984).

\subsection{Toxicity Tests}

The in vivo genotoxicity of the raw and treated pharmaceutical effluent $(0.1 ; 0.2$ or 0.3 mL.day ${ }^{-1}$ per mouse) was investigated in mice $(n=5)$ orally exposed, for 10 days, using the bone marrow cells micronuclei assay. The experiments were carried out on adult male Swiss mice (8-12 week) obtained from the Chemical Industry of Goiás (IQUEGO). All mice weighed between $28 \mathrm{~g}$ and $30 \mathrm{~g}$ and were kept under constant environmental conditions with 12:12 light-dark cycle. All were fed standard granulated chow and given drinking water $a d$ libitum. Animal experiments were done in accordance with Institutional Protocols of Animal Care. The micronuclei frequency was performed 24 hours after the exposure.

\subsubsection{Micronucleus test}

The mutagenicity of the effluents was evaluated using the micronucleus test by scoring 1.000 bone marrow cells per each of 05 animals per treatment group, as described by MacGregor et al. (1987). The cells were stained with Leishman, the slides were coded, and the cells blindly scored by light microscope at 1000 magnification. The frequency of $\mathrm{MN}$ in 
WATANABE, R. A. M.; SAlES, P. T. F.; CAMPOS, L. C.; GARCIA, T. A.; VALADARES, M. C.; SCHIMIDT, F.; SANTIAGO, M. F. Evaluation of the use of Pycnoporus sanguineus fungus for phenolics and genotoxicity decay of a pharmaceutical effluent treatment. Ambi-Agua, Taubaté, v. 7, n. 3, p. 41-50, 2012. (http://dx.doi.org/10.4136/ambi-agua.966)

individual mice was used as the experimental unit, with variability (standard deviation) based on differences among animals within the same group.

\section{RESULTS AND DISCUSSION}

The higher laccase production was determined in 96 hours of treatment (4.48 U.mL-1, 8.16 U.mL-1 and 2.8 U.mL-1) for the effluent samples 1, 2 and 3 respectively. It was not determined laccase production in control samples, showing the relation between fungus growth and enzymatic production, as studied by Garcia (2007). Laccase production during the treatment process is shown in Figure 1.

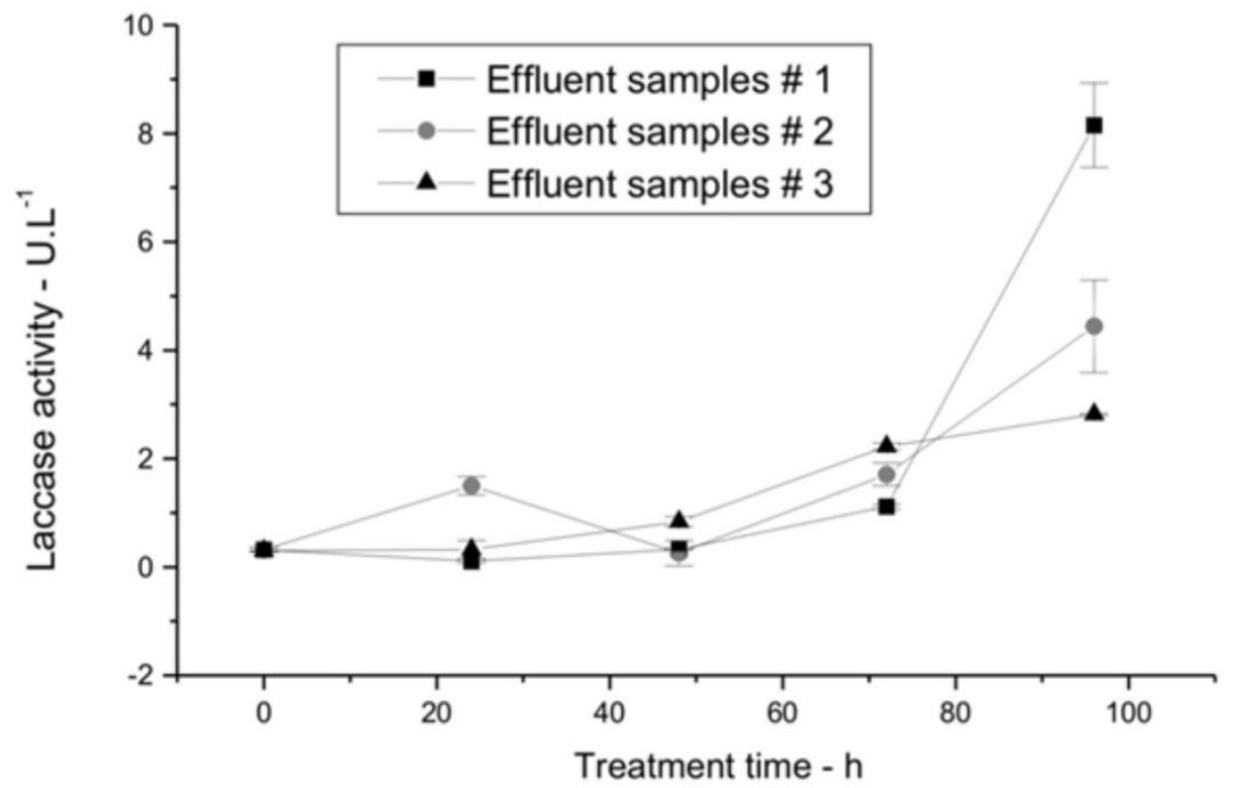

Figure 1. Laccase production for the pharmaceutical effluents 1,2 and 3 treated by Pycnoporus sanguineus.

Manganese Peroxidase enzyme production, also responsible for toxic compound degradation, by the P. sanguineus fungus was determinated in the treated effluent 2 (Figure 2), was also higher in 96 hours of treatment, equal to 0.03 ABS.min-1. The Manganese peroxidase production of was not detected in raw effluent 2 and in control samples. Studies showed the Manganese Peroxidase production is regulated by the nitrogen and carbon substrate concentration and they are inversely proportional to (Linko, 1992). Therefore, the increasing of $\mathrm{MNp}$ production towards the biological treatment indicates the decreasing of organic compounds in the effluent (not shown in Figure 2).

In the samples 1 and 3 and in their controls, the manganese peroxidase production was null. Similar results were found for the production of lignin peroxidase in the effluent samples 1, 2 and 3 treated by the P. sanguineus fungus and their control samples (not treated).

The $\mathrm{pH}$ of the samples 1 and 2 (treated effluent - Figure 3) presents a drop (i.e. acidification) between 24 and 48 hours of treatment and it increased up to its initial value ( $\mathrm{pH}$ $=5.5-6.0$ ). For the effluent 3 , the $\mathrm{pH}$ increased with time during whole treatment time (Figure 3). For the control, the $\mathrm{pH}$ was approximately constant and equal to 4.7, 6.4 and 4.6 respectively for the samples 1,2 and 3 .

According to Garcia et al. (2007), laccase tends to react differently to different substrates depending on the $\mathrm{pH}$. The purified laccase, using syringaldazine as substrate, presented optimum $\mathrm{pH}$ in acid interval (around 4.2) and the same was observed to other laccases from 
WATANABE, R. A. M.; SAlES, P. T. F.; CAMPOS, L. C.; GARCIA, T. A.; VALADARES, M. C.; SCHIMIDT, F.; SANTIAGO, M. F. Evaluation of the use of Pycnoporus sanguineus fungus for phenolics and genotoxicity decay of a pharmaceutical effluent treatment. Ambi-Agua, Taubaté, v. 7, n. 3, p. 41-50, 2012. (http://dx.doi.org/10.4136/ambi-agua.966)

other fungi. $\mathrm{pH}$ range in acid interval is within the interval of optimum $\mathrm{pH}$ for laccase, $\mathrm{MnP}$ and LiP activities (Wesenberg et al., 2003). Figure 3 shows the treatment time that all samples $\mathrm{pH}$ values were below 7.0 (acid). However, the $\mathrm{pH}$ values of all samples were between 5.0 and 6.0 at the end of treatment (at 96 hours).

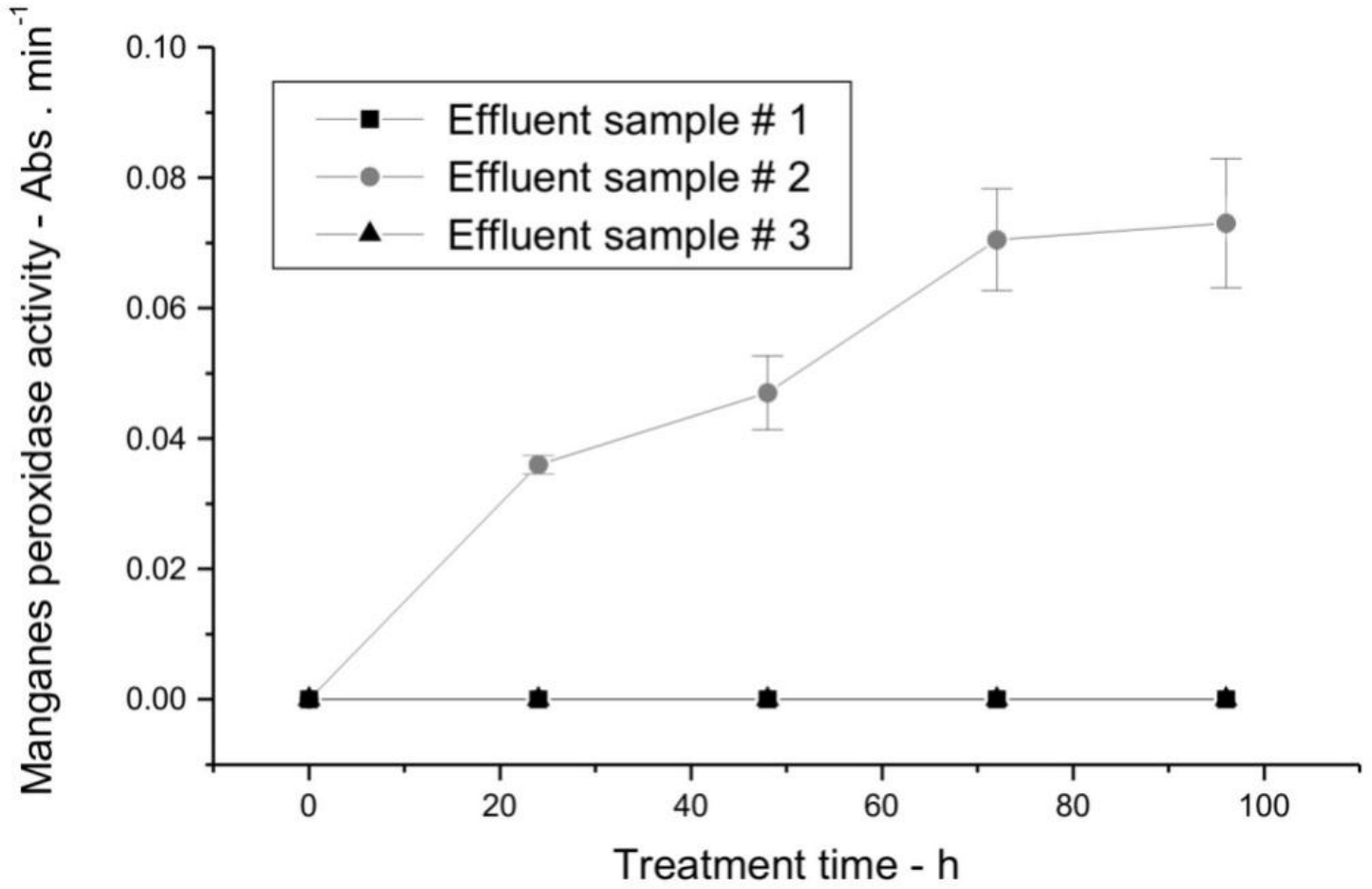

Figure 2. Manganese peroxidase production in the effluent 2 treated by the Pycnoporus sanguineus, during 96 hours.

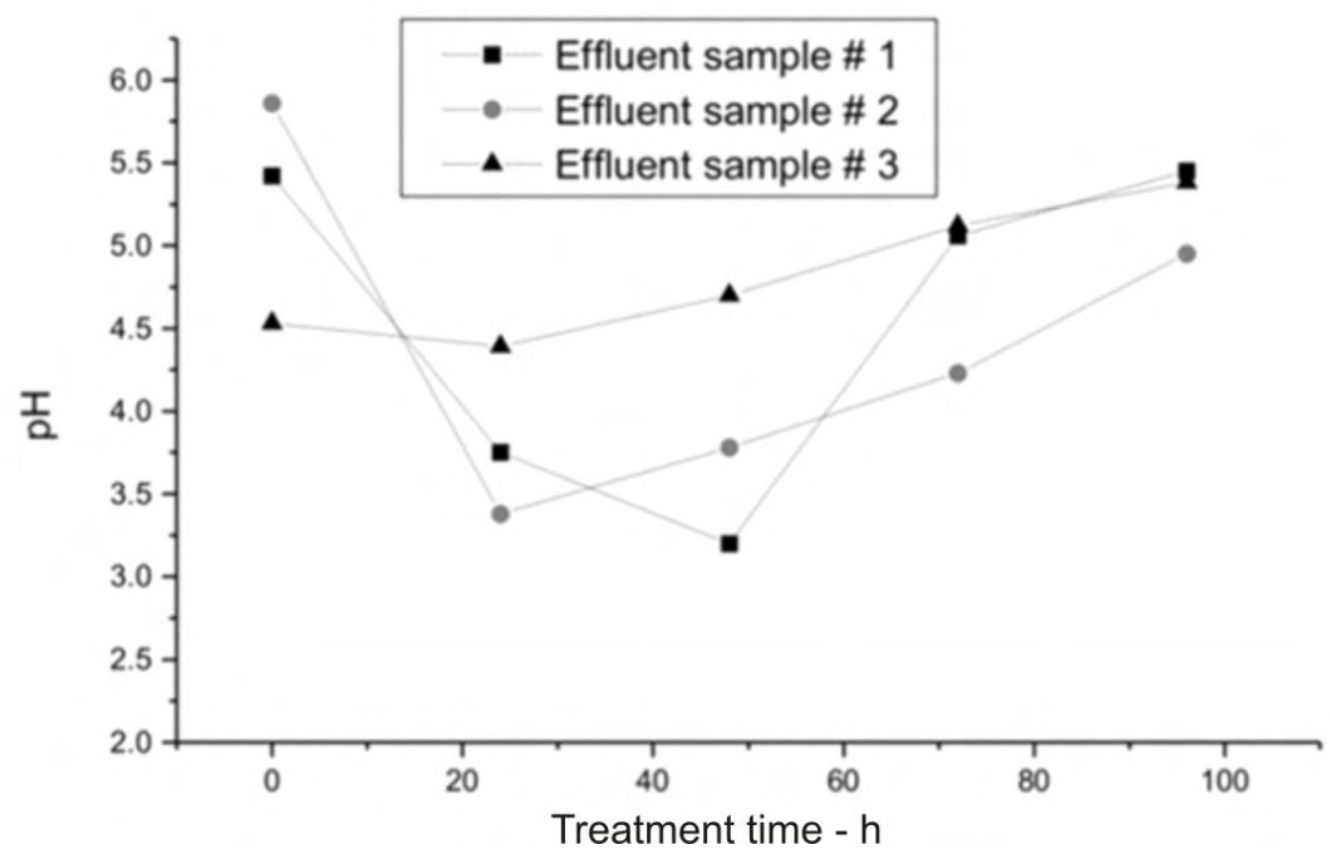

Figure 3. Variation of $\mathrm{pH}$ values during the biological treatment. 
WATANABE, R. A. M.; SAleS, P. T. F.; CAMPOS, L. C.; GARCIA, T. A.; VAladARES, M. C.; SCHIMIDT, F.; SANTIAGO, M. F. Evaluation of the use of Pycnoporus sanguineus fungus for phenolics and genotoxicity decay of a pharmaceutical effluent treatment. Ambi-Agua, Taubaté, v. 7, n. 3, p. 41-50, 2012. (http://dx.doi.org/10.4136/ambi-agua.966)

The phenolics concentration during the biological treatment can be seen in Figure 4, that shows the decreasing around $20 \%$ and $45 \%$ of all samples, comparing to untreated samples.

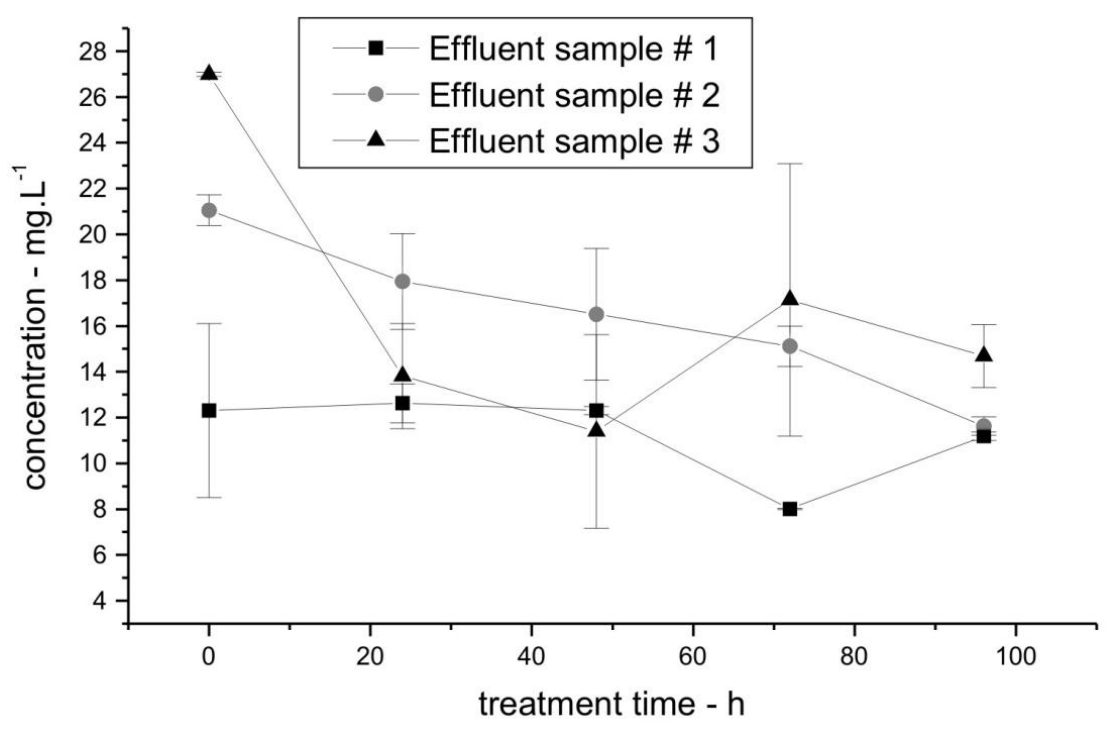

Figure 4. Phenolics concentration for the pharmaceutical effluents 1, 2 and 3 treated by the $P$. sanguineus fungus.

Comparing the enzyme production and the phenolics degradation, it was observed the Laccase and Managese Peroxidase productions increasing, as a result of decreasing of the phenolics, during the biological treatment. This result confirms Laccase production can be related to the degradation of toxic compounds into effluents. In the same way, when the in vivo genotoxicity was investigated it was observed the reduction of frequencies of micronucleated erytrocytes from mice, in a dose-dependent manner, when it was compared to untreated sample (Figure 5).

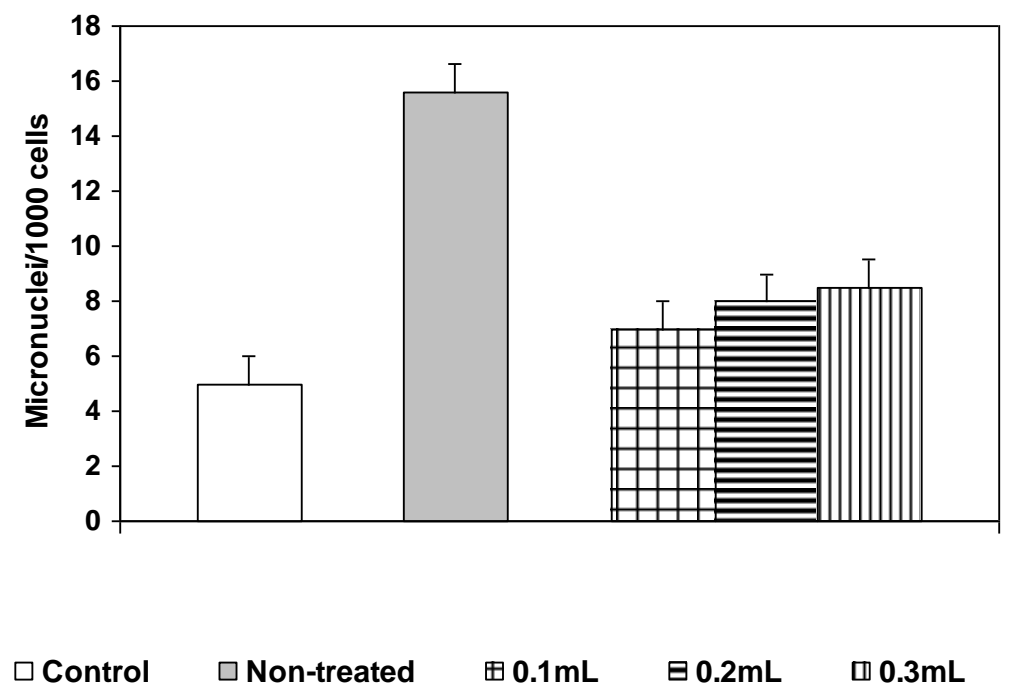

Figure 5. Effects of the raw (non-treated) and treated pharmaceutical effluent $\left(0.1 ; 0.2\right.$ and $0.3 \mathrm{~mL}^{-\mathrm{day}^{-1}}$. mouse $\left.^{1}\right)$, per oral, for 10 days on the frequencies of micronucleated erytrocytes $(\mathrm{MN})$ from mice $(\mathrm{n}=5)$. The control group received saline solution only. 
WATANABE, R. A. M.; SAlES, P. T. F.; CAMPOS, L. C.; GARCIA, T. A.; VALADARES, M. C.; SCHIMIDT, F.; SANTIAGO, M. F. Evaluation of the use of Pycnoporus sanguineus fungus for phenolics and genotoxicity decay of a pharmaceutical effluent treatment. Ambi-Agua, Taubaté, v. 7, n. 3, p. 41-50, 2012. (http://dx.doi.org/10.4136/ambi-agua.966)

Wirzinger et al. (2007) demonstrated that genotoxic assays including the MN test, can be applied routinely for the evaluation of the genotoxic potential of waters.

\section{CONCLUSIONS}

Based on the results, $P$. sanguineus fungus was able to produce high amount of laccase and small amount of manganese peroxidase enzymes for the treatment of the pharmaceutical effluent investigated in this work. The decreasing of phenolics concentration during the biological treatment showed $P$. sanguineus has a high potential of reducing the genotoxic for this kind of effluent, with a high laccase production during the biological treatment.

\section{ACKNOWLEDGEMENTS}

This work was funded by International Foundation for Science (IFS)/W3433-1, CNPQ (Conselho Nacional de Desenvolvimento Científico e Tecnológico), SECTEC-GO (Secretaria de Ciência e Tecnologia do Estado de Goiás) and Edital MCT/CNPq/CT-Hidro n 14/2005.

\section{REFERENCES}

AMERICAN PUBLIC HEALT ASSOCIATION (APHA). Standard Methods for the Examination of Water and Wastewater. 17. ed. Washington DC, USA, 1992.

BARR, D. P.; AUST, S. D. Mechanisms white rot fungi use to degrade pollutants. Environmental Science \& Technology, 28 (2), 1994, 79 - 87.

BOYD, G. R.; PALMERI J. M., ZHANG S.; GRIMM D. A. Pharmaceuticals and personal care products (PPCPs) and endocrine disrupting chemicals (EDCs) in storm water canals and Bayou St. John in New Orleans, Louisiana, USA. Science of the Total Environment, Amsterdan, 333 (1-3), 137-148, 2004.

CHEN, H.; WANG P.; DING W. Using liquid chromatography-ion trap mass spectrometry to determine pharmaceutical residues in Taiwanese rivers and wastewaters. Chemosphere, 6, 72, 863-869, 2008.

DURÁN, N.; ESPOSITO, E. 1997. Lignin biodegradation and effluent treatment by ligninolytic fungi. In: I. S. De Melo, J. L. Acevedo (Eds.). Microbiologia Ambiental, CNPMA/EMBRAPA Publishers. São Paulo, Brazil, Chapter 12, pp. 269.

DURÁN, N.; ESPOSITO, E. Potential applications of oxidative enzymes and phenoloxidaselike compounds in wastewater and soil treatment: a review. Applied Catalysis B: Environmental, 28, 2000, $83-89$.

EUGENIO, M. E.; CARBAJO, J. M.; TERRÓN, M. C.; GONZÁlEZ, A. E.; VILlAR, J. C. Bioremediation of lignosulphonates by lignin-degrading basidiomycetous fungi. Bioresources Technology, 99 (11), 2008, 4929-4934.

GARCIA, T. A.; SANTIAGO, M. F.; ULHOA, C. J. Studies on the Pycnoporus sanguineus CCT-4518 laccase purified by hydrophobic interaction chromatografy. Applied Microbiology and Biotechology, 75 (2), 2007, 311 - 318.

GODFREY, E.; WOESSNER, W. W.; BENOTTI, M. J. Pharmaceuticals in On-Site Sewage Effluent and Ground Water, Western Montana. Groundwater, 45 (3), 2007, 263-271. 
WATANABE, R. A. M.; SAlES, P. T. F.; CAMPOS, L. C.; GARCIA, T. A.; VALADARES, M. C.; SCHIMIDT, F.; SANTIAGO, M. F. Evaluation of the use of Pycnoporus sanguineus fungus for phenolics and genotoxicity decay of a pharmaceutical effluent treatment. Ambi-Agua, Taubaté, v. 7, n. 3, p. 41-50, 2012. (http://dx.doi.org/10.4136/ambi-agua.966)

HIRSCH, R.; TERNES, T.; HABERER K.; KRATZ, K. L. Occurence of antibiotics in the aquatic environment. Science of the Total Environment, Amsterdan, 225, 1999, 109118.

KUMARAN, P.; PARUCHURI, Y. L. Kinetics of phenol biotransformation. Water Resources Research, 31(1), 1997, 11-22.

KUWAHARA, M.; GLEN, J. K.; MORGAN, M. A.; GOLD, M. H. Separation and Characterization of two extracellular $\mathrm{H}_{2} \mathrm{O}_{2}$ dependent oxidases from ligninolytic Cultures of Phanerochaete chrysosporium. FBS Letters, 169, 1984, 247-250.

LINKO, S. Production of Phanerochaete chrysosporium Lignin Peroxidase. Biotechnology Advances, 10 (2), 1992, 191 -236.

MACGREGOR, J. T.; HEDDle, J. A.; HITE, M., MARGOLIN, B. H.; RAMEL, C. SALAMONE, M. F., TICE, R. R., WILD, D. Guidelines for the conduct of micronucleus assay in mammalian bone marrow erythrocytes. Mutatation Research. 189, 1987, 103-112.

MCMULLAN., G.; MEEHAN, C.; CONNEELY, A.; KIRBY, N.; ROBINSON, T.; NIGAM, P.; BANAT I.M., MARCHANT, R.; SMYTH, W. F. Microbial decolourisation and degradation of textile dyes. Applied Microbiology and Biotechology, 56, 2001, 81-87.

MELO, P. S.; FABRIN-NETO, J. B.; MORAES, S. G.; ASSALIN, R.; DURÁN, N.; HAUN, M. Comparative toxicity of effluents processed by different treatments in V79 fibroblasts and the Algae Selenastrum capricornutum. Chemosphere, Oxford, 62, 2006, 1207-1213.

POINTING, S. B.; JONES, E. B. G.; VRIJMOED, L. L. P. Optimization of laccase production by Pycnoporus sanguineus in submerged liquid culture. Mycologia, V 92, 2000, 139-144.

POINTING, S. B.; VRIJMOED L. L. P. Decolorization of azo and triphenylmethane dyes by Pycnoporus sanguineus producing laccase as the sole phenoloxidase. World Journal f. Microbiology and Biotechnology, 16, 2000, 317-318.

ROBERTS P. H., AND THOMAS K.V. The occurrence of selected pharmaceuticals in wastewater effluent and surface waters of the lower Tyne catchment. Science of the Total Environment, Amsterdan, 356 (1-3), 2006, 143-153.

RODRÍGUEZ, E.; NUERO, O.; GUILLEN, F.; MARTINEZ, A.T.; MARTINEZ, M.J.. Degradation of phenolic and non-phenolic aromatic pollutants by four Pleurotus species: The role of laccase and versatile peroxidase. Soil Biology \& Biochemistry, 36 (6), 2004, 909-916.

SANTIAGO, M. F. Estudo de substâncias de baixa massa molar que mimetizam as fenoloxidases com aplicações em tratamento de efluentes industriais. Tese de doutorado, Universidade Estadual de Campinas, São Paulo, Brazil. 1999.

SAYAD, S.; AND ELLOUZ, R. roles of lignin peroxidase and manganese peroxidase from phanerochaete chrysosporium in the decolorization of olive mill wastewaters. Applied Environmental Microbiology, 61(3), 1995, 1098-1103. 
WATANABE, R. A. M.; SAleS, P. T. F.; CAMPOS, L. C.; GARCIA, T. A.; VAladARES, M. C.; SCHIMIDT, F.; SANTIAGO, M. F. Evaluation of the use of Pycnoporus sanguineus fungus for phenolics and genotoxicity decay of a pharmaceutical effluent treatment. Ambi-Agua, Taubaté, v. 7, n. 3, p. 41-50, 2012. (http://dx.doi.org/10.4136/ambi-agua.966)

SZKLARZ, G. D.; ANTIBUS, R. K.; SINSABAUGH, R. L.; LINKINS, A. E. Production of phenoloxidases and peroxidases by wood-rotting fungi. Mycologia. 81 (2), 1989, 234240 .

TIEN, M.; KIRK, K. Lignin degrading enzyme from Phanerochaete chrysosporium: purification characterization and catalytic properties of a unique $\mathrm{H}_{2} \mathrm{O}_{2}$ requiring oxygenase. Proceedings of the National Academy of Sciences of the United States of America. 81, 1984, 2280-2284.

TROVASLET, M.; ENAUD, E.; GUIAVARC'H, Y.; CORBISIER, A. M.; VANHULLE, S. Potential of a Pycnoporus sanguineus laccase in bioremediation of wastewater and kinetic activation in the presence of an anthraquinonic acid dye. Enzyme and Microbial Technology, 41 (3), 2007, 368-376.

WESENBERG, D.; KYRIAKIDES, I.; AGATHOS, S. N. White-rot fungi and their enzymes for the treatment of industrial dye effluents. Biotechnology Advances, 22, 2003, 161187.

WIRZINGER, G.; WELTJE, L.; GERCKEN, J.; SORDYL, H. Genotoxic damage in fieldcollected three-spined sticklebacks (Gasterosteus aculeatus L.): a suitable biomonitoring tool? Mutat. Res. 30,628(1), 2007, 19-30. 


ISSN = 1980-993X - doi:10.4136/1980-993X
www.ambi-agua.net
E-mail: ambi-agua@agro.unitau.br
Tel.: (12) 3625-4212

\title{
The potential of Zea mays, Commelina bengelensis, Helianthus annuus and Amaranthus hybridus for phytoremediation of waste water
}

(http://dx.doi.org/10.4136/ambi-agua.684)

\author{
Oyaro Nathan'; Kimenyu Phylis Njeri²; Okong'o Eric Rang'ondi \\ Chacha Joseph Sarima ${ }^{1}$ \\ ${ }^{1}$ Narok university College, Department of chemistry, Box 861-20500, Narok, Kenya, \\ e-mails: oyarokema@yahoo.com; chachajs@yahoo.com, \\ ${ }^{2}$ Jomo Kenyatta University of Agriculture \& Technology, Department of chemistry, \\ Box 62000-00200, Nairobi, Kenya, Tel. 254 6752223, Fax 254 6752197, \\ e-mails: kimenyupn2012@yahoo.com; erickokongo67@yahoo.com
}

\begin{abstract}
Waste-water from domestic use and from industrial effluent burden the water systems with high levels of heavy metal hence there is need to remove these heavy metals so that the waste water can be recycled for use for household or irrigation. The present study has screened Zea mays (maize), Commelina bengelensis (wondering jew), Helianthus annuus (sunflower) and Amaranthus hybridus (amaranthus) for their ability to bioaccumulate $\mathrm{Pb}, \mathrm{Cu}$, $\mathrm{Cd}$ and $\mathrm{Zn}$ metals. The results obtained show that the $H$. annuus and $C$. bengelensis plant have promising potential for removal of $\mathrm{Pb}, \mathrm{Cu}$ and $\mathrm{Cd}$ from wastewater though their ability to remove $\mathrm{Zn}$ from contaminated solutions is not much different from that of $Z$. mays and $A$. hybridus.
\end{abstract}

Keywords: AAS, lead, copper, zinc, cadmium.

\section{O potencial de Zea mays, Commelina bengelensis, Helianthus annuus $e$ Amaranthus hybridus para fitorremediação de águas residuárias}

\section{RESUMO}

Nas águas residuárias de origem doméstica ou esgoto sanitário, níveis elevados de metais tóxicos poluem os sistemas hídricos e, por conseguinte, há necessidade de eliminar esses elementos para que as águas residuárias possam ser recicladas para uso doméstico ou irrigação. O presente estudo avaliou a capacidade das espécies Zea mays (milho), Commelina bengelensis (trapoeraba), Helianthus annuus (girassol) e Amaranthus hybridus (amaranto) para bioacumulação de $\mathrm{Pb}, \mathrm{Cu}, \mathrm{Cd}$ e $\mathrm{Zn}$. Os resultados revelaram que o $H$. annuus e a $C$. bengelensis têm potencial para remoção do $\mathrm{Pb}, \mathrm{Cu}$ e $\mathrm{Cd}$ de águas residuárias, entretanto, a capacidade delas para remoção de $Z n$ não é muito diferente das espécies $Z$. mays e $A$. hybridus.

Palavras-chave: AAS, chumbo, cobre, zinco, cádmio.

\section{INTRODUCTION}

Heavy metals are major pollutants in marine, ground, industrial and even treated wastewater (Valdman et al., 2001). The heavy metals may come from natural sources, leached from rocks and soils according to their geochemical mobility or come from anthropogenic 
NATHAN, O.; NJERI, K. P.; RANG'ondi, O. E.; SARIMA, C. J. The potential of Zea mays, Commelina bengelensis, Helianthus annuus and Amaranthus hybridus for phytoremediation of waste water. Ambi-Agua, Taubaté, v. 7, n. 3, p. 51-60, 2012. (http://dx.doi.org/10.4136/ambi-agua.684)

sources, as a result of human land occupation and industrial pollution (Espinoza-Quiñones et al., 2005).

Industrial waste constitutes the major source of various kinds of metal pollution in natural waters. The important toxic metals $\mathrm{Cd}, \mathrm{Zn}, \mathrm{Ni}$ and $\mathrm{Pb}$ find their way to the water bodies through waste waters Ajmal et al. (1998). Due to their non- biodegradability and persistence, heavy metals can accumulate in the environment such as in food chains and thus may pose a significant danger to human health (Bakkaloglu et al., 1998).

Rhizofil tration, the use of plants both terrestrial and aquatic to absorb, concentrate and precipitate contaminants from polluted aqueous sources with low contaminant concentration in their roots, can partially be used to treat industrial discharge. It can be used for $\mathrm{Pb}, \mathrm{Cd}, \mathrm{Cu}$, $\mathrm{Ni}, \mathrm{Zn}$ and $\mathrm{Cr}$, which are primarily retained within roots (Chaudhry et al., 1998). For example plants like sunflower, Indian mustard, tobacco, rye, spinach and corn have been studied for their ability to remove $\mathrm{Pb}$ from effluent, with sunflower having the greatest ability (Ghosh and Singh, 2005). Indian mustard has proven to be effective in removing a wide concentration range of lead (4-500 $\mathrm{mg} \mathrm{L}^{-1}$ ) (Raskin and Ensley, 2000).

The technology has been tested in the field with uranium-contaminated water at concentrations of 21-874 $\mu \mathrm{g} \mathrm{L}^{-1}$; the treated uranium concentration reported by Dushenkov et al. (1995) was $<20 \mu \mathrm{g} \mathrm{L}^{-1}$ before discharge into the environment.

This plant-assisted bioremediation is most effective if groundwater is within 10 feet of the surface (Cunningham et al., 1997) and is applicable to sites with large volumes of groundwater with low levels of contamination that have to be cleaned to low (strict) standards (Salt et al., 1997).

In this study, the efficiency of Helianthus annuus (sunflower), Commelina bengelensis (wondering Jew), Zea mays (maize) and Amaranthus hybridus (amaranth) has been studied in the process of heavy metal removing from both single metal solutions and various mixtures. Metals of interest were $\mathrm{Cd}, \mathrm{Pb}, \mathrm{Cu}$ and $\mathrm{Zn}$ based on their industrial applications and potential pollution impact on the environment. $\mathrm{Cd}$ and $\mathrm{Pb}$ are cumulative poisons, highly toxic to humans, plant and animals (Low et al., 2000). Cu and $\mathrm{Zn}$ play major roles in modern industry and in the vicinity of extraction or processing plants, the emissions arising are certainly capable of causing an undesirable contamination of agricultural products and therefore recommended not to omit these metals from scrutiny.

\section{MATERIALS AND METHODS}

This study was conducted in a greenhouse as pot experiments on distilled water at Jomo Kenyatta University of Agriculture and Technology in Kenya. The experiment was carried out during 2008 - 2011 period. The experimental layout for single metal solutions comprised 8 treatments, a control with no metal added, and 7 treatments for which the dose of heavy metal was increasingly higher (Table 1). Heavy metals were added as salts of $\mathrm{Pb}\left(\mathrm{NO}_{3}\right)_{2}$, $\mathrm{Cu}\left(\mathrm{NO}_{3}\right)_{2} 5 \mathrm{H}_{2} \mathrm{O}, \mathrm{Zn}\left(\mathrm{NO}_{3}\right)_{2} .7 \mathrm{H}_{2} \mathrm{O}$ and $\mathrm{Cd}\left(\mathrm{NO}_{3}\right)_{2} \cdot 4 \mathrm{H}_{2} \mathrm{O}$. The experiment was carried out in triplicate and stratified sampling was done to identify the pot but at each pot random sampling was employed in choosing the plant for analysis.

To compare the uptake of these metals from single solutions (uncombined state) and uptake from mixture solutions (combined state), the mixture solutions were prepared by dissolving the salts of the heavy metals in distilled water in order to achieve the same concentrations as those of single solutions as per Table 1 .

Zea mays and Helianthus annuus were germinated on a tray in the green house for 14 days while Amaranthus hybridus seedlings and Commelina bengelensis cuttings were obtained from the university farm, transplanted on tray in the greenhouse for the same number of days. The plants were uprooted and three seedlings per pot were suspended in the 
NATHAN, O.; NJERI, K. P.; RANG'ondi, O. E.; SARIMA, C. J. The potential of Zea mays, Commelina bengelensis, Helianthus annuus and Amaranthus hybridus for phytoremediation of waste water. Ambi-Agua, Taubaté, v. 7, n. 3, p. 51-60, 2012. (http://dx.doi.org/10.4136/ambi-agua.684)

contaminated solutions with the roots completely immersed. Every four days for twelve days, one plant was removed from each pot, put in polyethylene bag immediately sealed and transported to the laboratory for analysis.

Table 1. Dose of heavy metals $\left(\mathrm{mg} \mathrm{L}^{-1}\right)$ added to distilled water for treatments 0 - VII.

\begin{tabular}{c|cccc}
\hline Treatment & Cd & Cu & Zn & Pb \\
\hline Control & 0 & 0 & 0 & 0 \\
I & 10 & 20 & 50 & 30 \\
II & 20 & 40 & 100 & 60 \\
III & 40 & 80 & 200 & 120 \\
IV & 80 & 160 & 400 & 240 \\
V & 160 & 320 & 800 & 480 \\
VI & 320 & 640 & 1600 & 960 \\
VII & 640 & 1280 & 3200 & 1920 \\
\hline
\end{tabular}

In the laboratory, samples were washed with distilled water, bench dried for 5 days then oven dried at $105^{\circ} \mathrm{C}$ for 12 hours and each separately ground to a powder in a porcelain mortar. The powder was then stored in plastic bottles ready for analysis.

The digestion of samples was carried out as follows: $0.1 \mathrm{~g}$ of the oven-dried powder was put in a $100 \mathrm{~mL}$ conical flask and $5 \mathrm{~mL}$ of the tri-acid mixture (nitric, perchloric and sulfuric) in the ratio 3:1:1 was added and let to digest on a hot plate until brown fumes disappeared and white fumes observed. The samples were then cooled, diluted with a $4 \%$ nitric acid and filtered into $100 \mathrm{~mL}$ volumetric flasks. These were then made up to the mark with distilled water and analyzed using Atomic Absorption Spectrophotometer (AAS).

\section{RESULTS AND DISCUSSION}

Table 2 presents the $\mathrm{Pb}$ concentrations in the A. hybridus, Z. mays, C. bengelensis and $H$. annuus were in the range of (0.061-1.211), (0.046-3.921), (1.354-7.632) and (1.168-8.558) $\mathrm{g}$ $\mathrm{kg}^{-1}$, respectively.

These different values show that the uptake of $\mathrm{Pb}$ is plant dependent and also depends on the concentration of $\mathrm{Pb}$ in solution. The $\mathrm{Pb}$ concentration in $C$. bengelensis and $H$. annuus were above the value used to define hyper accumulation $\left(1.0 \mathrm{~g} \mathrm{~kg}^{-1}\right)$, hence these plants in solutions can be referred to as hyperaccumulators of $\mathrm{Pb}$. These values are comparable to values of $0.009-4.561 \mathrm{~g} \mathrm{~kg}^{-1}$ obtained by Lombi et al. (2001) in roots of Z. mays grown in soils with EDTA. In another research by Podar et al. (2004), the concentration of $\mathrm{Pb}$ ranged from $0.001-0.030 \mathrm{~g} \mathrm{~kg}^{-1}$ in $H$. annuus and $0.000-0.018 \mathrm{~g} \mathrm{~kg}^{-1}$ in Z. mays grown in soil contaminated with mine spoils. These values are lower than those obtained in this study. The results obtained in the current study are higher than those of Carlson et al. (1975), who found that the amount of $\mathrm{Pb}$ in leaves of $\mathrm{H}$. annuus and $Z$. mays treated with up to $500 \mathrm{mg} / \mathrm{lof} \mathrm{Pb}$, as $\mathrm{PbCl}_{2}$, averaged $0.004 \mathrm{~g} \mathrm{~kg}^{-1} \mathrm{~Pb}$ and was not statistically different from the $\mathrm{Pb}$ concentration in the control (untreated) plants.

No toxicity was observed for the four plants for all the treatments under investigation. This observation is in agreement with findings of Vyslouzilova et al. (2003) who found out that willow plants grew without any physiological symptoms of phytotoxicity in soil supplied with $2.000 \mathrm{~g} \mathrm{~kg}^{-1}$. The lack of $\mathrm{Pb}$ toxicity is probably due to its immobilization in roots as reported by Vyslouzivola et al. (2003) and Stoltz and Greger (2002). 
NATHAN, O.; NJERI, K. P.; RANG'ondi, O. E.; SARIMA, C. J. The potential of Zea mays, Commelina bengelensis, Helianthus annuus and Amaranthus hybridus for phytoremediation of waste water. Ambi-Agua, Taubaté, v. 7, n. 3, p. 51-60, 2012. (http://dx.doi.org/10.4136/ambi-agua.684)

Table 2. Concentration of lead $\left(\mathrm{g} \mathrm{kg}^{-1}\right)$ in Z. mays, C. bengelensis and A. hybridus grown in contaminated soil for the first, second and third harvests over three months period.

\begin{tabular}{|c|c|c|c|c|c|c|c|c|c|c|c|c|}
\hline $\begin{array}{c}\text { Metal } \\
\mathbf{P b}\end{array}$ & \multicolumn{4}{|c|}{$\mathbf{1}^{\text {st }}$ (Month) } & \multicolumn{4}{|c|}{$2^{\text {nd }}$ (Month) } & \multicolumn{4}{|c|}{$3^{\text {rd }}$ (Month) } \\
\hline Treat. & $\begin{array}{c}\text { A. } \\
\text { hybridus }\end{array}$ & $\begin{array}{c}\boldsymbol{Z} . \\
\text { mays }\end{array}$ & $\begin{array}{c}\boldsymbol{C} . \\
\text { bengelensis }\end{array}$ & $\begin{array}{c}\boldsymbol{H} . \\
\text { annuus }\end{array}$ & $\begin{array}{c}A . \\
\text { hybridus }\end{array}$ & $\begin{array}{c}\boldsymbol{Z} \text {. } \\
\text { mays }\end{array}$ & $\begin{array}{c}C . \\
\text { bengelensis }\end{array}$ & $\begin{array}{c}\boldsymbol{H} . \\
\text { annuus }\end{array}$ & $\begin{array}{c}A . \\
\text { hybridus }\end{array}$ & $\begin{array}{c}\boldsymbol{Z} \text {. } \\
\text { mays }\end{array}$ & $\begin{array}{c}\boldsymbol{C} . \\
\text { bengelensis }\end{array}$ & $\begin{array}{c}\boldsymbol{H} . \\
\text { annuus }\end{array}$ \\
\hline 0 & $\begin{array}{c}0.000 \\
\pm \\
0.000\end{array}$ & $\begin{array}{c}0.000 \\
\pm \\
0.000\end{array}$ & $\begin{array}{c}0.000 \\
\pm \\
0.000\end{array}$ & $\begin{array}{c}0.000 \\
\pm \\
0.000\end{array}$ & $\begin{array}{c}0.000 \\
\pm \\
0.000\end{array}$ & $\begin{array}{c}0.000 \\
\pm \\
0.000\end{array}$ & $\begin{array}{c}0.000 \\
\pm \\
0.000\end{array}$ & $\begin{array}{c}0.000 \\
\pm \\
0.000\end{array}$ & $\begin{array}{c}0.000 \\
\pm \\
0.000\end{array}$ & $\begin{array}{c}0.000 \\
\pm \\
0.000\end{array}$ & $\begin{array}{c}0.000 \\
\pm \\
0.000\end{array}$ & $\begin{array}{c}0.000 \\
\pm \\
0.000\end{array}$ \\
\hline 30 & $\begin{array}{c}0.272 \\
\pm \\
0.039\end{array}$ & $\begin{array}{c}0.046 \\
\pm \\
0.016\end{array}$ & $\begin{array}{c}1.535 \\
\pm \\
0.030\end{array}$ & $\begin{array}{c}1.702 \\
\pm \\
0.014\end{array}$ & $\begin{array}{c}0.167 \\
\pm \\
0.040\end{array}$ & $\begin{array}{c}0.061 \\
\pm \\
0.015\end{array}$ & $\begin{array}{c}1.607 \\
\pm \\
0.037\end{array}$ & $\begin{array}{c}4.250 \\
\pm \\
0.053\end{array}$ & $\begin{array}{c}0.061 \\
\pm \\
0.0305\end{array}$ & $\begin{array}{c}0.101 \\
\pm \\
0.023\end{array}$ & $\begin{array}{c}1.354 \\
\pm \\
0.066\end{array}$ & $\begin{array}{c}4.773 \\
\pm \\
0.045\end{array}$ \\
\hline 60 & $\begin{array}{c}0.231 \\
\pm \\
0.046\end{array}$ & $\begin{array}{c}0.096 \\
\pm \\
0.049\end{array}$ & $\begin{array}{c}1.842 \\
\pm \\
0.051\end{array}$ & $\begin{array}{c}2.767 \\
\pm \\
0.173\end{array}$ & $\begin{array}{c}0.070 \\
\pm \\
0.048\end{array}$ & $\begin{array}{c}0.151 \\
\pm \\
0.027\end{array}$ & $\begin{array}{c}3.293 \\
\pm \\
0.067\end{array}$ & $\begin{array}{c}4.038 \\
\pm \\
0.045\end{array}$ & $\begin{array}{c}0.117 \\
\pm \\
0.018\end{array}$ & $\begin{array}{c}0.222 \\
\pm \\
0.077\end{array}$ & $\begin{array}{c}1.851 \\
\pm \\
0.054\end{array}$ & $\begin{array}{c}5.634 \\
\pm \\
0.046\end{array}$ \\
\hline 120 & $\begin{array}{c}0.222 \\
\pm \\
0.053\end{array}$ & $\begin{array}{c}0.237 \\
\pm \\
0.026\end{array}$ & $\begin{array}{c}2.067 \\
\pm \\
0.018\end{array}$ & $\begin{array}{c}2.067 \\
\pm \\
0.020\end{array}$ & $\begin{array}{c}0.206 \\
\pm \\
0.038\end{array}$ & $\begin{array}{c}0.174 \\
\pm \\
0.050\end{array}$ & $\begin{array}{c}3.456 \\
\pm \\
0.067\end{array}$ & $\begin{array}{c}5.614 \\
\pm \\
0.074\end{array}$ & $\begin{array}{c}0.1917 \\
\pm \\
0.035\end{array}$ & $\begin{array}{c}0.202 \\
\pm \\
0.038\end{array}$ & $\begin{array}{c}2.712 \\
\pm \\
0.093\end{array}$ & $\begin{array}{c}4.865 \\
\pm \\
0.028\end{array}$ \\
\hline 240 & $\begin{array}{c}0.634 \\
\pm \\
0.097\end{array}$ & $\begin{array}{c}0.671 \\
\pm \\
0.046\end{array}$ & $\begin{array}{c}4.321 \\
\pm \\
0.029\end{array}$ & $\begin{array}{c}1.168 \\
\pm \\
0.046\end{array}$ & $\begin{array}{c}0.530 \\
\pm \\
0.046\end{array}$ & $\begin{array}{c}0.771 \\
\pm \\
0.026\end{array}$ & $\begin{array}{c}3.932 \\
\pm \\
0.098\end{array}$ & $\begin{array}{c}4.815 \\
\pm \\
0.010\end{array}$ & $\begin{array}{c}0.827 \\
\pm \\
0.114\end{array}$ & $\begin{array}{c}0.630 \\
\pm \\
0.109\end{array}$ & $\begin{array}{c}3.867 \\
\pm \\
0.022\end{array}$ & $\begin{array}{c}5.084 \\
\pm \\
0.028\end{array}$ \\
\hline 480 & $\begin{array}{c}0.817 \\
\pm \\
0.066\end{array}$ & $\begin{array}{c}0.671 \\
\pm \\
0.023\end{array}$ & $\begin{array}{c}7.236 \\
\pm \\
0.037\end{array}$ & $\begin{array}{c}1.461 \\
\pm \\
0.019\end{array}$ & $\begin{array}{c}0.530 \\
\pm \\
0.031\end{array}$ & $\begin{array}{c}1.295 \\
\pm \\
0.023\end{array}$ & $\begin{array}{c}7.632 \\
\pm \\
0.048\end{array}$ & $\begin{array}{c}4.460 \\
\pm \\
0.038\end{array}$ & $\begin{array}{c}0.832 \\
\pm \\
0.085\end{array}$ & $\begin{array}{c}0.913 \\
\pm \\
0.068\end{array}$ & $\begin{array}{c}5.029 \\
\pm \\
0.148\end{array}$ & $\begin{array}{c}5.360 \\
\pm \\
0.016\end{array}$ \\
\hline 960 & $\begin{array}{c}0.883 \\
\pm \\
0.023\end{array}$ & $\begin{array}{c}3.921 \\
\pm \\
0.070\end{array}$ & $\begin{array}{c}2.043 \\
\pm \\
0.025\end{array}$ & $\begin{array}{c}1.715 \\
\pm \\
0.017\end{array}$ & $\begin{array}{c}0.923 \\
\pm \\
0.015\end{array}$ & $\begin{array}{c}1.789 \\
\pm \\
0.023\end{array}$ & $\begin{array}{c}4.090 \\
\pm \\
0.032\end{array}$ & $\begin{array}{c}4.967 \\
\pm \\
0.054\end{array}$ & $\begin{array}{c}1.211 \\
\pm \\
0.001\end{array}$ & $\begin{array}{c}0.857 \\
\pm \\
0.032\end{array}$ & $\begin{array}{c}6.177 \\
\pm \\
0.070\end{array}$ & $\begin{array}{c}8.558 \\
\pm \\
0.010\end{array}$ \\
\hline 1920 & $\begin{array}{c}0.615 \\
\pm \\
0.057\end{array}$ & $\begin{array}{c}0.217 \\
\pm \\
0.083\end{array}$ & $\begin{array}{c}6.002 \\
\pm \\
0.020\end{array}$ & $\begin{array}{c}3.567 \\
\pm \\
0.045\end{array}$ & $\begin{array}{c}1.064 \\
\pm \\
0.044\end{array}$ & $\begin{array}{c}2.077 \\
\pm \\
0.031\end{array}$ & $\begin{array}{c}3.290 \\
\pm \\
0.017\end{array}$ & $\begin{array}{c}3.881 \\
\pm \\
0.049\end{array}$ & $\begin{array}{c}0.358 \\
\pm \\
0.038\end{array}$ & $\begin{array}{c}1.215 \\
\pm \\
0.061\end{array}$ & $\begin{array}{c}7.187 \\
\pm \\
0.062\end{array}$ & PD \\
\hline
\end{tabular}

Note: PD-Plant died.

From Table 2, it can be seen that $H$. annuus, was the highest accumulator of lead in solutions, followed by $C$. bengelensis, Z. mays and finally A. hybridus. The accumulation of $\mathrm{Pb}$ in $H$. annuus was not statistically different from that of $C$. bengelensis for all the three harvests $(\mathrm{P}=0.5)$ even though $\mathrm{Pb}$ concentration in these plants was statistically different from that of Z. mays and A. hybridus. In our study, H. Annuus and $C$. bengelensis have shown a high ability to remove $\mathrm{Pb}$ from waste water effluent but the ability of $\mathrm{Z}$. Mays and $A$. Hybridus is low. These results are in agreement with findings recorded by Ghosh and Singh (2005), where several plants were investigated for their bioaccumulation of $\mathrm{Pb}$ from waste water; H. annuus was found to have the greatest ability.

\subsection{Cadmium}

Table 3 presents the range of $\mathrm{Cd}$ concentrations as (0.138-19.690), (0.082-22.367), (2.482-9.146) and (0.201-9.972) $\mathrm{g} \mathrm{kg}^{-1}$ for Z. mays, A. hybridus, H. annuus and C. bengelensis respectively. The concentration of $\mathrm{Cd}$ obtained for these treatments were greater than the value used to define $\mathrm{Cd}$ hyperaccumulation $\left(0.1 \mathrm{gkg}^{-1}\right)$. 
NATHAN, O.; NJERI, K. P.; RANG'ondi, O. E.; SARIMA, C. J. The potential of Zea mays, Commelina bengelensis, Helianthus annuus and Amaranthus hybridus for phytoremediation of waste water. Ambi-Agua, Taubaté, v. 7, n. 3, p. 51-60, 2012. (http://dx.doi.org/10.4136/ambi-agua.684)

Table 3. Concentration of cadmium $\left(\mathrm{gkg}^{-1}\right)$ in Z. mays, C. bengelensis and A. hybridus grown in contaminated soil for the first, second and third harvests over three months period.

\begin{tabular}{|c|c|c|c|c|c|c|c|c|c|c|c|c|}
\hline \multirow{2}{*}{$\begin{array}{c}\begin{array}{c}\text { Metal } \\
\text { Cd }\end{array} \\
\text { Treat. }\end{array}$} & \multicolumn{4}{|c|}{$1^{\text {st }}$ (Month) } & \multicolumn{4}{|c|}{$2^{\text {nd }}$ (Month) } & \multicolumn{4}{|c|}{$3^{\text {rd }}$ (Month) } \\
\hline & $\begin{array}{c}\boldsymbol{A} . \\
\text { hybridus }\end{array}$ & Z. & $\begin{array}{c}C . \\
\text { bengelensi }\end{array}$ & $\begin{array}{c}\boldsymbol{H} . \\
\text { annuus }\end{array}$ & $\begin{array}{c}\text { A. } \\
\text { hybridus }\end{array}$ & $\begin{array}{c}Z . \\
\text { mays }\end{array}$ & $\begin{array}{c}\boldsymbol{C} . \\
\text { bengelensis }\end{array}$ & $\begin{array}{c}\boldsymbol{H} . \\
\text { annuus }\end{array}$ & $\begin{array}{c}\text { A. } \\
\text { hybridus }\end{array}$ & $\begin{array}{c}\boldsymbol{Z} . \\
\text { mays }\end{array}$ & $\begin{array}{c}\boldsymbol{C} . \\
\text { bengelensi }\end{array}$ & $\begin{array}{c}\boldsymbol{H} . \\
\text { annuus }\end{array}$ \\
\hline \multirow[t]{3}{*}{0} & 0.000 & 0.000 & 0.000 & 0.000 & 0.000 & 0.000 & 0.000 & 0.000 & 0.000 & 0.000 & 0.000 & 0.000 \\
\hline & \pm & \pm & \pm & \pm & \pm & \pm & \pm & \pm & \pm & \pm & \pm & \pm \\
\hline & 0.000 & 0.000 & 0.000 & 0.000 & 0.000 & 0.000 & 0.000 & 0.000 & 0.000 & 0.000 & 0.000 & 0.000 \\
\hline \multirow[t]{2}{*}{10} & $\begin{array}{c}0.163 \\
+\end{array}$ & $\begin{array}{c}0.273 \\
+\end{array}$ & $\begin{array}{c}0.474 \\
+\end{array}$ & $\begin{array}{c}2.555 \\
+\end{array}$ & $\begin{array}{c}0.082 \\
+\end{array}$ & $\begin{array}{c}0.138 \\
+\end{array}$ & $\begin{array}{c}0.201 \\
+\end{array}$ & $\begin{array}{c}2.626 \\
+\end{array}$ & $\begin{array}{c}0.206 \\
\pm\end{array}$ & $\begin{array}{c}0.326 \\
+0\end{array}$ & $\begin{array}{c}0.289 \\
+\end{array}$ & $\begin{array}{l}2.482 \\
+0\end{array}$ \\
\hline & 0.013 & 0.052 & 0.008 & 0.013 & 0.002 & 0.010 & 0.007 & 0.001 & 0.010 & 017 & 0.006 & .005 \\
\hline \multirow[t]{2}{*}{20} & 0.455 & 0.450 & $\begin{array}{c}0.796 \\
+\end{array}$ & $\begin{array}{c}3.266 \\
+\end{array}$ & $\begin{array}{c}0.439 \\
+\end{array}$ & $\begin{array}{c}0.774 \\
+\end{array}$ & 1.365 & 5.701 & 0.636 & $\begin{array}{c}0.751 \\
+0\end{array}$ & $\begin{array}{c}1.287 \\
+\end{array}$ & 3.159 \\
\hline & $\begin{array}{c} \pm \\
0.013\end{array}$ & $\begin{array}{c} \pm \\
0.007\end{array}$ & $\begin{array}{c} \pm \\
0.009\end{array}$ & $\begin{array}{c} \pm \\
0.011\end{array}$ & $\begin{array}{c} \pm \\
0.008\end{array}$ & $\begin{array}{c} \pm \\
0.007\end{array}$ & $\begin{array}{c} \pm \\
0.008\end{array}$ & $\begin{array}{c} \pm \\
0.030\end{array}$ & $\begin{array}{c} \pm \\
0.011\end{array}$ & $\begin{array}{l} \pm 0 \\
021\end{array}$ & $\begin{array}{c} \pm \\
0.009\end{array}$ & $\begin{array}{c} \pm \\
0.020\end{array}$ \\
\hline \multirow[t]{2}{*}{40} & $\begin{array}{c}0.683 \\
\pm\end{array}$ & $\begin{array}{c}0.602 \\
\pm\end{array}$ & $\begin{array}{c}2.129 \\
\pm\end{array}$ & $\begin{array}{c}5.321 \\
\pm\end{array}$ & $\begin{array}{c}0.435 \\
\pm\end{array}$ & $\begin{array}{c}1.005 \\
\pm\end{array}$ & $\begin{array}{c}2.121 \\
\pm\end{array}$ & $\begin{array}{c}6.414 \\
\pm 0\end{array}$ & $\begin{array}{c}0.395 \\
\pm\end{array}$ & PD & $\begin{array}{c}1.632 \\
\pm\end{array}$ & $\begin{array}{c}5.669 \\
\pm 0\end{array}$ \\
\hline & 0.012 & 0.025 & 0.010 & 0.014 & 0.012 & 0.010 & 0.011 & .007 & 0.018 & & 0.010 & .006 \\
\hline \multirow[t]{2}{*}{80} & 2.576 & 2.734 & 2.964 & 6.402 & 1.538 & 2.632 & 5.912 & 6.965 & 1.369 & PD & 3.950 & 7.046 \\
\hline & $\begin{array}{c} \pm \\
0.093\end{array}$ & $\begin{array}{c} \pm \\
0.100\end{array}$ & $\stackrel{ \pm}{ \pm}$ & $\begin{array}{c} \pm \\
.066\end{array}$ & $\begin{array}{c} \pm \\
0.003\end{array}$ & $\begin{array}{c} \pm 0 \\
.100\end{array}$ & $\stackrel{ \pm}{ \pm}$ & $\stackrel{\stackrel{ \pm}{ }}{0.018}$ & $\stackrel{ \pm}{ \pm}$ & & $\stackrel{\stackrel{ \pm}{ \pm}}{0.004}$ & $\stackrel{ \pm}{ \pm}$ \\
\hline \multirow[t]{2}{*}{160} & 4.419 & 6.001 & 3.668 & 7.513 & 5.246 & 7.754 & 7.337 & 8.887 & 6.560 & PD & 6.811 & PD \\
\hline & $\begin{array}{c} \pm \\
0.014\end{array}$ & 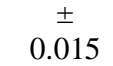 & $\begin{array}{c} \pm \\
0.016\end{array}$ & $\begin{array}{c} \pm \\
0.023\end{array}$ & $\begin{array}{c} \pm \\
0.084\end{array}$ & $\begin{array}{c} \pm \\
0.063\end{array}$ & $\begin{array}{c} \pm \\
0.054\end{array}$ & $\begin{array}{c} \pm \\
0.001\end{array}$ & $\begin{array}{c} \pm \\
0.056\end{array}$ & & $\begin{array}{c} \pm \\
0.009\end{array}$ & \\
\hline \multirow[t]{2}{*}{320} & 10.200 & 10.677 & 5.706 & 8.966 & 16.567 & PD & 8.874 & 9.052 & 15.807 & PD & 7.035 & PD \\
\hline & $\begin{array}{c} \pm \\
0.070\end{array}$ & $\begin{array}{c} \pm \\
0.035\end{array}$ & $\begin{array}{c} \pm \\
0.048\end{array}$ & $\stackrel{ \pm}{ \pm}$ & $\begin{array}{c} \pm \\
0.049\end{array}$ & & $\begin{array}{c} \pm \\
0.064\end{array}$ & $\begin{array}{c} \pm \\
0.006\end{array}$ & $\begin{array}{c} \pm \\
0.119\end{array}$ & & $\begin{array}{c} \pm \\
0.005\end{array}$ & \\
\hline \multirow[t]{2}{*}{640} & 19.233 & 19.690 & 5.901 & 9.132 & 22.367 & PD & 9.972 & 9.146 & PD & PD & 8.729 & PD \\
\hline & $\begin{array}{c} \pm \\
0.179\end{array}$ & $\stackrel{ \pm}{ \pm}$ & $\begin{array}{c} \pm \\
0.019\end{array}$ & $\begin{array}{c} \pm \\
0.004\end{array}$ & $\begin{array}{c} \pm \\
0.248\end{array}$ & & $\begin{array}{c} \pm \\
0.020\end{array}$ & $\begin{array}{c} \pm \\
0.004\end{array}$ & & & $\begin{array}{c} \pm \\
0.007\end{array}$ & \\
\hline
\end{tabular}

Note: PD-Plant died.

In a research done by Antonkiewicz and Jasiewicz (2002) in contaminated soils, the Cd content in Z. mays and A. hybridus was $0.000-0.070 \mathrm{~g} \mathrm{~kg}^{-1}$ and $0.001-0.060 \mathrm{~g} \mathrm{~kg}^{-1}$ respectively while in another study by Lombi et al. (2001), a range of $0.60-0.576 \mathrm{~g} \mathrm{~kg}^{-1}$ was obtained for T. caerulescens. The highest value of Cd concentration of $1.800 \mathrm{~g} \mathrm{~kg}^{-1}$ was recorded in leaves of T. caerulescens in a research carried out by Baker and Walker (1990). The values obtained in the current study are higher than those recorded in these studies. Cd concentrations above $20 \mathrm{mg} / \mathrm{l}, 80 \mathrm{mg} / \mathrm{l}$ and $320 \mathrm{mg} / \mathrm{l}$ were found to be toxic for the growth of Z. mays, H. annuus and $A$. hybridus respectively. $C$. bengelensis did not show any toxicity signs to $\mathrm{Cd}$ which gives the plant an added advantage over the other plants in phytoremediation of wastewater containing higher concentrations of $\mathrm{Cd}$.

From table 3, the high uptake of $\mathrm{Cd}$ by $H$. annuus is comparable to that of $C$. bengelensis while the uptake of $\mathrm{Cd}$ by $Z$. mays is lower than that of A. hybridus. Similar trend was observed by Antonkiewicz and Jasiewicz (2002), where the Cd content in the top parts of $A$. hybridus was found to be higher than that of Z. mays.

$H$. annuus has the highest removal of $\mathrm{Cd}$ from solutions, followed by $C$. bengelensis, then A. hybridus and finally Z. mays.

\subsection{Copper}

Table 4 presents the range of copper concentration $(0.213-35.770) \mathrm{g} \mathrm{kg}^{-1}$ for Z. mays, (0.230-43.273) $\mathrm{g} \mathrm{kg}^{-1}$ for A. hybridus, (1.786-15.995) $\mathrm{g} \mathrm{kg}^{-1}$ for H. annuus and (0.514- 
NATHAN, O.; NJERI, K. P.; RANG'ondi, O. E.; SARIMA, C. J. The potential of Zea mays, Commelina bengelensis, Helianthus annuus and Amaranthus hybridus for phytoremediation of waste water. Ambi-Agua, Taubaté, v. 7, n. 3, p. 51-60, 2012. (http://dx.doi.org/10.4136/ambi-agua.684)

20.807) $\mathrm{g} \mathrm{kg}^{-1}$ for $C$. bengelensis. These values are higher than those obtained in earlier studies where the range of $\mathrm{Cu}$ values indicated as $0.001-0.0075 \mathrm{~g} \mathrm{~kg}^{-1}$ in aquatic macrophytes (Espinoza-Quiñones et al., 2005), $0.0015-0.0189 \mathrm{~g} \mathrm{~kg}^{-1}$ in $Z$. mays and $0.085-0.0327 \mathrm{~g} \mathrm{~kg}^{-1}$ in H. annuus (Podar et al., 2004) and 0.0413- $0.806 \mathrm{~g} \mathrm{~kg}^{-1}$ in Z. mays roots with EDTA (Lombi et al., 2001). The values obtained for all the four plants at treatments of 640 and $1280 \mathrm{ppm}$ are above the value of a $\mathrm{Cu}$ hyperacumulator, Ipomea alpina of $12.300 \mathrm{~g} \mathrm{~kg}^{-1}$ (Baker and Walker, 1990). However the plants were found to suffer from $\mathrm{Cu}$ phytotoxicity at $\mathrm{Cu}$ concentrations higher than $80 \mathrm{ppm}$. This implies that although the plants can be used for phytoremediation of wastewater contaminated with $\mathrm{Cu}$, their remediation potential would be limited by phytotoxicity of $\mathrm{Cu}$. It was also noted that in the uptake of $\mathrm{Cu}$ by $C$. bengelensis, the initial ( $1^{\text {st }}$ harvest), was not as high as in the other plants and this is probably why it was possible to obtain the $2^{\text {nd }}$ harvest for all treatments.

Table 4: Concentration of copper $\left(\mathrm{gkg}^{-1}\right)$ in Z. mays, C. bengelensis and A. hybridus grown in contaminated soil for the first, second and third harvests over three months period.

\begin{tabular}{|c|c|c|c|c|c|c|c|c|c|c|c|c|}
\hline \multirow{2}{*}{$\begin{array}{l}\begin{array}{l}\text { Metal } \\
\text { Cu }\end{array} \\
\text { Treat. }\end{array}$} & \multicolumn{4}{|c|}{$\mathbf{1}^{\text {st }}$ (Month) } & \multicolumn{4}{|c|}{$2^{\text {nd }}$ (Month) } & \multicolumn{4}{|c|}{$3^{\text {rd }}$ (Month) } \\
\hline & $\begin{array}{c}\boldsymbol{A} . \\
\text { hvbridus }\end{array}$ & $\begin{array}{c}Z . \\
\text { mavs }\end{array}$ & $\begin{array}{c}\boldsymbol{C} . \\
\text { bengelensis } \\
\end{array}$ & $\begin{array}{c}\boldsymbol{H} . \\
\text { annuus }\end{array}$ & $\begin{array}{c}\text { A. } \\
\text { hvbridus }\end{array}$ & $\begin{array}{c}Z . \\
\text { mavs }\end{array}$ & $\begin{array}{c}\boldsymbol{C} . \\
\text { bengelensis }\end{array}$ & $\begin{array}{c}\boldsymbol{H} . \\
\text { аппии }\end{array}$ & $\begin{array}{c}\text { A. } \\
\text { hvbridus }\end{array}$ & $\begin{array}{c}Z . \\
\text { mavs }\end{array}$ & $\begin{array}{c}C . \\
\text { bengelensis }\end{array}$ & $\begin{array}{c}\boldsymbol{H} . \\
\text { annuиs }\end{array}$ \\
\hline 0 & $\begin{array}{c}0.000 \\
\pm \\
0.000\end{array}$ & $\begin{array}{c}0.000 \\
\pm \\
0.000\end{array}$ & $\begin{array}{c}0.000 \\
\pm \\
0.000\end{array}$ & $\begin{array}{c}0.000 \\
\pm \\
0.000\end{array}$ & $\begin{array}{c}0.000 \\
\pm \\
0.000\end{array}$ & $\begin{array}{c}0.000 \\
\pm \\
0.000\end{array}$ & $\begin{array}{c}0.000 \\
\pm \\
0.000\end{array}$ & $\begin{array}{c}0.000 \\
\pm \\
0.000\end{array}$ & $\begin{array}{c}0.000 \\
\pm \\
0.000\end{array}$ & $\begin{array}{c}0.000 \\
\pm \\
0.000\end{array}$ & $\begin{array}{c}0.000 \\
\pm \\
0.000\end{array}$ & $\begin{array}{c}0.000 \\
\pm \\
0.000\end{array}$ \\
\hline 20 & $\begin{array}{c}0.230 \\
\pm \\
0.016\end{array}$ & $\begin{array}{c}0.493 \\
\pm \\
0.026\end{array}$ & $\begin{array}{c}0.514 \\
\pm \\
0.009\end{array}$ & $\begin{array}{c}1.786 \\
\pm \\
0.018\end{array}$ & $\begin{array}{c}0.257 \\
\pm \\
0.043\end{array}$ & $\begin{array}{c}0.213 \\
\pm \\
0.047\end{array}$ & $\begin{array}{c}0.699 \\
\pm \\
0.023\end{array}$ & $\begin{array}{c}2.099 \\
\pm \\
0.013\end{array}$ & $\begin{array}{c}0.319 \\
\pm \\
0.009\end{array}$ & $\begin{array}{c}0.371 \\
\pm \\
0.019\end{array}$ & $\begin{array}{c}0.839 \\
\pm \\
0.013\end{array}$ & $\begin{array}{c}2.987 \\
\pm \\
0.020\end{array}$ \\
\hline 40 & $\begin{array}{c}0.596 \\
\pm \\
0.017\end{array}$ & $\begin{array}{c}1.389 \\
\pm \\
0.024\end{array}$ & $\begin{array}{c}1.548 \\
\pm \\
0.009\end{array}$ & $\begin{array}{c}2.664 \\
\pm \\
0.016\end{array}$ & $\begin{array}{c}0.333 \\
\pm \\
0.014\end{array}$ & $\begin{array}{c}1.943 \\
\pm \\
0.119\end{array}$ & $\begin{array}{c}3.425 \\
\pm \\
0.024\end{array}$ & $\begin{array}{c}4.278 \\
\pm \\
0.026\end{array}$ & $\begin{array}{c}0.697 \\
\pm \\
0.020\end{array}$ & $\begin{array}{c}0.866 \\
\pm \\
0.024\end{array}$ & $\begin{array}{c}1.115 \\
\pm \\
0.020\end{array}$ & $\begin{array}{c}3.476 \\
\pm \\
0.022\end{array}$ \\
\hline 80 & $\begin{array}{c}1.900 \\
\pm \\
0.022\end{array}$ & $\begin{array}{c}2.384 \\
\pm \\
0.265\end{array}$ & $\begin{array}{c}2.150 \\
\pm \\
0.023\end{array}$ & $\begin{array}{c}4.502 \\
\pm \\
0.053\end{array}$ & $\begin{array}{c}3.019 \\
\pm \\
0.0136\end{array}$ & $\begin{array}{c}3.504 \\
\pm \\
0.042\end{array}$ & $\begin{array}{c}5.553 \\
\pm \\
0.012\end{array}$ & $\begin{array}{c}6.647 \\
\pm \\
0.007\end{array}$ & $\begin{array}{c}0.958 \\
\pm \\
0.016\end{array}$ & $\begin{array}{c}3.087 \\
\pm \\
0.049\end{array}$ & $\begin{array}{c}6.675 \\
\pm \\
0.013\end{array}$ & PD \\
\hline 160 & $\begin{array}{c}1.918 \\
\pm \\
0.069\end{array}$ & $\begin{array}{c}3.325 \\
\pm \\
0.060\end{array}$ & $\begin{array}{c}2.958 \\
\pm \\
0.027\end{array}$ & $\begin{array}{c}5.851 \\
\pm \\
0.018\end{array}$ & $\begin{array}{c}5.486 \\
\pm \\
0.099\end{array}$ & $\begin{array}{c}4.347 \\
\pm \\
0.346\end{array}$ & $\begin{array}{c}5.876 \\
\pm \\
0.038\end{array}$ & $\begin{array}{c}8.308 \\
\pm \\
0.047\end{array}$ & $\begin{array}{c}1.858 \\
\pm \\
0.028\end{array}$ & PD & PD & PD \\
\hline 320 & $\begin{array}{c}6.072 \\
\pm \\
0.023\end{array}$ & $\begin{array}{c}8.080 \\
\pm \\
0.147\end{array}$ & $\begin{array}{c}5.122 \\
\pm \\
0.126\end{array}$ & $\begin{array}{c}7.025 \\
\pm \\
0.028\end{array}$ & $\begin{array}{c}9.966 \\
\pm \\
0.166\end{array}$ & PD & $\begin{array}{c}12.115 \\
\pm \\
0.074\end{array}$ & $\begin{array}{c}10.782 \\
\pm \\
0.068\end{array}$ & PD & PD & PD & PD \\
\hline 640 & $\begin{array}{c}26.753 \\
\pm \\
0.121\end{array}$ & $\begin{array}{c}24.013 \\
\pm \\
0.085\end{array}$ & $\begin{array}{c}6.581 \\
\pm \\
0.016\end{array}$ & $\begin{array}{c}12.875 \\
\pm \\
0.006\end{array}$ & PD & PD & $\begin{array}{c}11.697 \\
\pm \\
0.049\end{array}$ & PD & PD & PD & PD & PD \\
\hline 1280 & $\begin{array}{c}43.273 \\
\pm \\
0.145\end{array}$ & $\begin{array}{c}35.770 \\
\pm \\
0.185\end{array}$ & $\begin{array}{c}7.960 \\
\pm \\
0.052\end{array}$ & $\begin{array}{c}15.995 \\
\pm \\
0.031\end{array}$ & PD & PD & $\begin{array}{c}20.807 \\
\pm \\
0.147\end{array}$ & PD & PD & PD & PD & PD \\
\hline
\end{tabular}

From Table 4, the $\mathrm{Cu}$ concentration in H. annuus, C. bengelensis, Z. mays and $A$. hybridus was found to increase as the concentration in the solution increased. The first and second harvests did not show much difference in their accumulation for the four plants and by third harvest, most of the plants had died in solutions containing high levels of $\mathrm{Cu}$ contamination. In phytoremediation, due to $\mathrm{Cu}$ phytotoxicity at high concentrations, the plants should be removed from the contaminated aqueous solutions on the $4^{\text {th }}$ day.

Comparison of the uptake of $\mathrm{Cu}$ by the four plants at the end of the first four days reveals that at $\mathrm{Cu}$ concentrations below $640 \mathrm{mg} \mathrm{L}^{-1}, H$. annuus has the highest uptake of $\mathrm{Cu}$, while at concentrations of 640 and $1280 \mathrm{mg} \mathrm{L}^{-1}$, Z. mays and A. hybridus have the highest uptake. 
NATHAN, O.; NJERI, K. P.; RANG'ondi, O. E.; SARIMA, C. J. The potential of Zea mays, Commelina bengelensis, Helianthus annuus and Amaranthus hybridus for phytoremediation of waste water. Ambi-Agua, Taubaté, v. 7, n. 3, p. 51-60, 2012. (http://dx.doi.org/10.4136/ambi-agua.684)

\subsection{Zinc}

Table 5 presents the $\mathrm{Zn}$ concentration in Z. mays, A. hybridus, H. annuus and $C$. bengelensis were found to be in the range (2.282-8.307), (1.141-7.593), (2.674-6.992) and (1.876-7.770) $\mathrm{g} \mathrm{kg}^{-1}$, respectively. These values are lower than the value used to define a $\mathrm{Zn}$ hyper accumulator $\left(10.0 \mathrm{~g} \mathrm{~kg}^{-1}\right)$, (Lasat, 2000), and much lower than a value of $38.055 \mathrm{~g} \mathrm{~kg}^{-1}$ obtained by Hinchman et al. (1996) in the roots of hybrid poplar. The values are comparable to those obtained by Lombi et al. (2001) and Gremion et al. (2004) that is $1.868-9.187 \mathrm{~g} \mathrm{~kg}^{-1}$ and $1.600-10.000 \mathrm{~g} \mathrm{~kg}^{-1}$ respectively for $T$. caerulescens which is a documented $\mathrm{Zn}$ hyperaccumulator. However, the values are lower than the greatest $\mathrm{Zn}$ uptake of $39.600 \mathrm{~g} \mathrm{~kg}^{-1}$ in $T$. caerulescens leaves. This means that the $\mathrm{Zn}$ uptake by these plants is quite significant though they cannot be said to be hyperaccumulative in solutions.

Table 5: Concentration of zinc $\left(\mathrm{g} \mathrm{kg}^{-1}\right)$ in $Z$. mays, C. bengelensis and A. hybridus grown in contaminated soil for the first, second and third harvests over three months period.

\begin{tabular}{|c|c|c|c|c|c|c|c|c|c|c|c|c|}
\hline \multirow{2}{*}{$\begin{array}{c}\begin{array}{c}\text { Metal } \\
\text { Zn }\end{array} \\
\text { Treat. }\end{array}$} & \multicolumn{4}{|c|}{$\mathbf{1}^{\text {st }}$ (Month) } & \multicolumn{4}{|c|}{$2^{\text {nd }}$ (Month) } & \multicolumn{4}{|c|}{$3^{\text {rd }}$ (Month) } \\
\hline & $\begin{array}{c}\text { A. } \\
\text { hybridus }\end{array}$ & $\underset{\text { mays }}{\mathbf{Z}}$ & $\begin{array}{c}\boldsymbol{C} . \\
\text { bengelensis }\end{array}$ & $\begin{array}{c}\boldsymbol{H} . \\
\text { апnиus }\end{array}$ & $\begin{array}{c}\text { A. } \\
\text { hybridus }\end{array}$ & $\underset{\text { mays }}{Z}$ & $\begin{array}{c}\boldsymbol{C} . \\
\text { bengelensis }\end{array}$ & $\begin{array}{c}\boldsymbol{H} . \\
\text { annuus }\end{array}$ & $\begin{array}{c}\text { A. } \\
\text { hybridus }\end{array}$ & $\underset{\text { mays }}{\mathbf{Z}}$ & $\underset{\text { bengelensis }}{\boldsymbol{C} .}$ & $\begin{array}{c}\boldsymbol{H} . \\
\text { апnuиs }\end{array}$ \\
\hline 0 & $\begin{array}{c}0.00 \\
\pm \\
0.00\end{array}$ & $\begin{array}{c}0.00 \\
\pm \\
0.00\end{array}$ & $\begin{array}{c}0.00 \\
\pm \\
0.00\end{array}$ & $\begin{array}{c}0.00 \\
\pm \\
0.00\end{array}$ & $\begin{array}{c}0.000 \\
\pm \\
0.000\end{array}$ & $\begin{array}{c}0.00 \\
\pm \\
0.000\end{array}$ & $\begin{array}{c}0.000 \\
\pm \\
0.000\end{array}$ & $\begin{array}{c}0.000 \\
\pm \\
0.000\end{array}$ & $\begin{array}{c}0.000 \\
\pm \\
0.000\end{array}$ & $\begin{array}{c}0.000 \\
\pm \\
0.000\end{array}$ & $\begin{array}{c}0.000 \\
\pm \\
0.000\end{array}$ & $\begin{array}{c}0.000 \\
\pm \\
0.000\end{array}$ \\
\hline 50 & $\begin{array}{c}1.141 \\
\pm \\
0.006\end{array}$ & $\begin{array}{c}2.417 \\
\pm \\
0.007\end{array}$ & $\begin{array}{c}2.303 \\
\pm \\
0.007\end{array}$ & $\begin{array}{c}2.674 \\
\pm \\
0.020\end{array}$ & $\begin{array}{c}1.327 \\
\pm \\
0.007\end{array}$ & $\begin{array}{c}2.282 \\
\pm \\
0.007\end{array}$ & $\begin{array}{c}1.876 \\
\pm \\
0.005\end{array}$ & $\begin{array}{c}4.456 \\
\pm \\
0.007\end{array}$ & $\begin{array}{c}1.743 \\
\pm \\
0.021\end{array}$ & $\begin{array}{c}3.084 \\
\pm \\
0.016\end{array}$ & $\begin{array}{c}2.014 \\
\pm \\
0.006\end{array}$ & $\begin{array}{c}4.301 \\
\pm \\
0.024\end{array}$ \\
\hline 100 & $\begin{array}{c}1.398 \\
\pm \\
0.002\end{array}$ & $\begin{array}{c}4.246 \\
\pm \\
0.007\end{array}$ & $\begin{array}{c}3.134 \\
\pm \\
0.011\end{array}$ & $\begin{array}{c}4.520 \\
\pm \\
0.012\end{array}$ & $\begin{array}{c}5.293 \\
\pm \\
0.002\end{array}$ & $\begin{array}{c}4.679 \\
\pm \\
0.013\end{array}$ & $\begin{array}{c}4.390 \\
\pm \\
0.051\end{array}$ & $\begin{array}{c}5.552 \\
\pm \\
0.003\end{array}$ & $\begin{array}{c}4.455 \\
\pm \\
0.011\end{array}$ & $\begin{array}{c}4.912 \\
\pm \\
0.013\end{array}$ & $\begin{array}{c}3.056 \\
\pm \\
0.008\end{array}$ & $\begin{array}{c}5.251 \\
\pm \\
0.004\end{array}$ \\
\hline 200 & $\begin{array}{c}3.943 \\
\pm \\
0.006\end{array}$ & $\begin{array}{c}5.967 \\
\pm \\
0.004\end{array}$ & $\begin{array}{c}3.491 \\
\pm \\
0.009\end{array}$ & $\begin{array}{c}5.921 \\
\pm \\
0.027\end{array}$ & $\begin{array}{c}6.181 \\
\pm \\
0.012\end{array}$ & $\begin{array}{c}6.456 \\
\pm \\
0.006\end{array}$ & $\begin{array}{c}5.306 \\
\pm \\
0.024\end{array}$ & $\begin{array}{c}6.141 \\
\pm \\
0.005\end{array}$ & $\begin{array}{c}6.662 \\
\pm \\
0.006\end{array}$ & $\begin{array}{c}6.447 \\
\pm \\
0.014\end{array}$ & $\begin{array}{c}3.22 \\
1 \pm \\
0.009\end{array}$ & $\begin{array}{c}5.704 \\
\pm \\
0.006\end{array}$ \\
\hline 400 & $\begin{array}{c}5.734 \\
\pm \\
0.009\end{array}$ & $\begin{array}{c}6.572 \\
\pm \\
0.007\end{array}$ & $\begin{array}{c}5.170 \\
\pm \\
0.029\end{array}$ & $\begin{array}{c}5.740 \\
\pm \\
0.028\end{array}$ & $\begin{array}{c}6.087 \\
\pm \\
0.007\end{array}$ & $\begin{array}{c}6.813 \\
\pm \\
0.014\end{array}$ & $\begin{array}{c}6.367 \\
\pm \\
0.012\end{array}$ & $\begin{array}{c}6.353 \\
\pm \\
0.075\end{array}$ & $\begin{array}{c}6.509 \\
\pm \\
0.007\end{array}$ & $\begin{array}{c}7.981 \\
\pm \\
0.012\end{array}$ & $\begin{array}{c}7.148 \\
\pm \\
0.004\end{array}$ & PD \\
\hline 800 & $\begin{array}{c}7.114 \\
\pm \\
0.003\end{array}$ & $\begin{array}{c}7.263 \\
\pm \\
0.003\end{array}$ & $\begin{array}{c}5.469 \\
\pm \\
0.027\end{array}$ & $\begin{array}{c}6.137 \\
\pm \\
0.009\end{array}$ & $\begin{array}{c}7.188 \\
\pm \\
0.013\end{array}$ & PD & $\begin{array}{c}7.272 \\
\pm \\
0.012\end{array}$ & $\begin{array}{c}5.986 \\
\pm \\
0.001\end{array}$ & PD & PD & $\begin{array}{c}7.489 \\
\pm \\
0.014\end{array}$ & PD \\
\hline 1600 & $\begin{array}{c}7.730 \\
\pm \\
0.009\end{array}$ & $\begin{array}{c}7.813 \\
\pm \\
0.006\end{array}$ & $\begin{array}{c}6.496 \\
\pm \\
0.021\end{array}$ & $\begin{array}{c}6.559 \\
\pm \\
0.117\end{array}$ & PD & PD & $\begin{array}{c}7.410 \\
\pm \\
0.014\end{array}$ & $\begin{array}{c}6.602 \\
\pm \\
0.011\end{array}$ & PD & PD & PD & PD \\
\hline 3200 & $\begin{array}{c}7.593 \\
\pm \\
0.010\end{array}$ & $\begin{array}{c}8.307 \\
\pm \\
0.010\end{array}$ & $\begin{array}{c}7.121 \\
\pm \\
0.049\end{array}$ & $\begin{array}{c}6.861 \\
\pm \\
0.014\end{array}$ & PD & PD & $\begin{array}{c}7.770 \\
\pm \\
0.003\end{array}$ & $\begin{array}{c}6.992 \\
\pm \\
0.015\end{array}$ & PD & PD & PD & PD \\
\hline
\end{tabular}

Table 5 presents the Zn concentration in Z. mays, A. hybridus, H. annuus and $C$. bengelensis with increase in the metal concentration in solution. The uptake of $\mathrm{Zn}$ by the four plants for the $1^{\text {st }}$ harvest (first 4 days) did not show much difference in concentration.

$H$. annuus and $C$. bengelensis were found to be more tolerant to high $\mathrm{Zn}$ concentration since it was possible to obtain data for all the 8 treatments up to the second harvest ( $8^{\text {th }}$ day) while at high concentration Z. mays and A. hybridus died off by first harvest ( $4^{\text {th }}$ day). Due to their tolerance of high levels of $\mathrm{Zn}$ and high uptake, $C$. bengelensis and $H$. annuus would be the best out of the four plants to be used to remove zinc from solutions contaminated with zinc. 


\section{CONCLUSIONS}

In this study it was found out that the heavy metal uptake by the four plants analyzed was different regarding the metal contamination. In solutions, the uptake of metals by plants may be ranked from the highest to the lowest in the following order:

Lead: $\quad$ Helianthus, Commelina, Zea mays, Amaranthus;

Cadmium: Helianthus, Commelina, Amaranthus, Zea mays;

Copper: Helianthus, Commelina, Zea mays, Amaranthus;

Zinc: $\quad$ Helianthus, Zea mays, Amaranthus, Commelina;

Helianthus, Commelina and Zea mays have shown a high ability to remove $\mathrm{Pb}$ from waste water.

The comparison of individual elements indicates much higher accumulation of $\mathrm{Cd}, \mathrm{Cu}$, and $\mathrm{Zn}$ in the plants than that of $\mathrm{Pb}$.

Helianthus, Commelina, Zea mays and Amaranthus have been found to take up a lot of these heavy metals especially $\mathrm{Zn}, \mathrm{Cd}$ and $\mathrm{Cu}$ as compared to $\mathrm{Pb}$, hence these plants can be used for phytoremediation of these heavy metals at moderate concentration. These can be effectively done if the levels of concentration are below 960, 800, 40 and $20 \mathrm{mg} \mathrm{L}^{-1}$ of solution of $\mathrm{Pb}, \mathrm{Zn}, \mathrm{Cu}$ and $\mathrm{Cd}$, respectively. From the current study it was noted that the period of planting does not really matter since the concentrations of the heavy metals after 4 , 8 and 12 days were not significantly different.

\section{ACKNOWLEDGMENT}

Sincere appreciation goes to Jomo Kenyatta University for all the financial support given in the course of this study, to the technical staff of the Chemistry Department for their help in the analytical work.

\section{REFERENCES}

AJMAL, M.; MOHAMMED, A.; YOUSUF, R.; AHMAD, A. Adsorption behavior of cadmium, zinc, nickel and lead from aqueous solutions by Mangifera Indica seed shell. Indian Journal Environmental Health, v. 40, n. 1, p. 15-26, 1998.

ANTONKIEWICZ, J.; JASIEWICZ, C. The use of plants accumulating heavy metals for detoxification of chemically polluted soils. Electronic Journal of Polish Agricultural Universities, v. 5, n. 1, 2002.

BAKER, A. J. M.; WALKER, P. L. Ecophysiology of metal uptake by tolerant plants. In: SHOW, A. J. (Ed.). Heavy metal tolerance in plants: evolutionary aspects. Boca Raton: CRC Press, 1990. p. 155-157.

BAKKALOGLU, I.; BUTTER, T. J.; EVISON, L. M.; HOLLAND, F. S.; HANCOCKTT, I. C. Screening of various types biomass for removal and recovery of heavy metals ( $\mathrm{Zn}$, $\mathrm{Cu}, \mathrm{Cd})$ by biosorption, sedimentation and desorption. Water Science technology, v. 38, n. 6, p. 269-277, 1998. http://dx.doi.org/10.1016/S0273-1223(98)00587-3

CARLSON, R. W.; BAZZAZ, F. A.; ROLFE, G. L. The effect of heavy metal on plants. Environmental Pollution, v. 7, p. 241-246, 1975. 
NATHAN, O.; NJERI, K. P.; RANG'ondi, O. E.; SARIMA, C. J. The potential of Zea mays, Commelina bengelensis, Helianthus annuus and Amaranthus hybridus for phytoremediation of waste water. Ambi-Agua, Taubaté, v. 7, n. 3, p. 51-60, 2012. (http://dx.doi.org/10.4136/ambi-agua.684)

CUNNINGHAM, S. D.; SHANN, J. R.; CROWLEY, D.; ANDERSON, T. A. In: KRUEGER, E. L.; ANDERSON, T. A.; COATS, J. P. (Ed.). Phytoremediation of soil and water contaminants. Washington, DC: American Chemical Society, 1997.

CHAUDHRY, T. M.; HAYES, W. J.; KHAN, A. G.; KHOO, C. S. Phytoremediation : focusing on accumulator plants that remediate metal-contaminated soils. Australasian Journal of Ecotoxicology, v 4, p. 37-51, 1998.

DUSHENKOV, V.; NANDA KUMAR, P. B. A.; MOTTO, H.; RASKIN, I. Rhizofiltration: the use of plants to remove heavy metals from aqueous streams. Environmental Science \& Technology, v. 29, n. 5, 1239 - 1245, 1995. http://dx.doi.org/10.1021/es00005a015

ESPINOZA-QUIÑONES, F. R.; ZACARKIM, C. M.; PALACIO, S. M.; OBREGON, C. L.; ZENATTI, D. C.; GALANTE, R. M. et al. Removal of heavy metal from polluted river water using aquatic macrophytes Salvinia sp. Brazilian Journal of Physics, v. 55, n. 3b, p.744 -746, 2005. http://dx.doi.org/10.1590/S0103-97332005000500005

GHOSH, M.; SINGH. S. P. A review on phytoremediation of heavy metals and utilization of its byproducts. Applied Ecology and Environmental Research, v. 3, n. 1, p. 1-18, 2005.

GREMION, F.; CHATZINOTAS, A.; KAUFMANN, K.; VON SIGLER, W.; HARMS, H. Impacts of heavy metal contamination and phytoremediation on a microbial community during a twelve-month microcosm experiment. FEMS Microbiology Ecology, v. 48, n. 2, p. 273-283, 2004. http://dx.doi.org/10.1016/j.femsec.2004.02.004

HINCHMAN, R. R.; NEGRI, M. C.; GATLIFF, E. G. Phytoremediation: using green plants to clean up contaminated soil, groundwater and wastewater. In: INTERNATIONAL TOPICAL MEETINGS ON NUCLEAR AND HAZARDOUS WASTE MANAGEMENT, 1996, Seattle. Proceedings... Seattle: American Nuclear Society, 1996.

LASAT, M. M. The use of plants for the removal of toxic metals from contaminated soil. [S.1.]: American Association for the Advancement of Science Environmental Science and Engineering Fellow, 2000.

LOMBI, E.; ZHAO F. J.; DUNHAM, S. J.; MCGRATH, S. P. Phytoremediation of heavy metal-contaminated soils. Journal Environmental Quality, v. 30, n. 6, p. 1919- 1926, 2001. http://dx.doi.org/10.2134/jeq2001.1919

LOW, K. S.; LEE, C. K.; LIEW, S. C. Sorption of cadmium and Lead from aqueous solution by spent grain. Process Biochemistry, v. 36, n. 1/2, p. 59-64, 2000. http://dx.doi.org/10.1016/S0032-9592(00)00177-1

PODAR, D.; DOBROTA, C.; TRIFU, M. Uptake of heavy metals by maize (Zea mays) plants cultivated on mine spoils. Studia Universitatis Babes-Bolyai, Studia Biologia, v. 49, n. 1, p. 45-60, 2004a.

PODAR, D.; RAMSEY M. H.; HUTCHINGS, M. J. Effect of cadmium, zinc and substrate heterogeneity on yield, shoot metal concentration and metal uptake by Brassica juncea: implications for human risk assessment and phytoremediation. New Phytologist, v. 163, n. 2, p. 313-324, 2004b. http://dx.doi.org/10.1111/j.1469-8137.2004.01122.x

RASKIN, I.; ENSLEY, B. D. Phytoremediation of toxic metals: using plants to clean up the environment. New york: John Wiley \& Sons, 2000. p. 53-70. 
NATHAN, O.; NJERI, K. P.; RANG'ondi, O. E.; SARIMA, C. J. The potential of Zea mays, Commelina bengelensis, Helianthus annuus and Amaranthus hybridus for phytoremediation of waste water. Ambi-Agua, Taubaté, v. 7, n. 3, p. 51-60, 2012. (http://dx.doi.org/10.4136/ambi-agua.684)

REEVES, R. D.; BAKER, A. J. M. Metal accumulating plants. In: RASKIN, I.; ENSLEY, B. D. (Ed.). Phytoremediation of toxic metals: using plants to clean up the environment, 2000. p. 193-229.

SALT, E. D.; PICKERING, I. J.; PRINCE, R. C.; GLEBA, D.; DUSHENKOV, S.; SMITH, R. D. et al. Metal accumulation by aquacultured seedlings of Indian mustard. Environmental Science \& Technology, v. 31, n. 6, p. 1636-1644, 1997.

STOLTZ, E.; GREGER, M. Accumulation properties of $\mathrm{As}, \mathrm{Cd}, \mathrm{Cu}, \mathrm{Pb}$ and $\mathrm{Zn}$ by four wetland plant species growing on submerged mine tailings. Environmental and Experimental Botany, v. 47, n. 3, p. 271-280, 2002. http://dx.doi.org/10.1016/S00988472(02)00002-3

VALDMAN, E.; ERIJMAN, L.; PESSOA, F. L. P.; LEITE, S. G. F. Continuous biosorption of $\mathrm{Cu}$ and $\mathrm{Zn}$ by immobilized waste biomass Sargassum sp. Process Biochemistry, v. 36, n. 8/9, p. 869-873, 2001. http://dx.doi.org/10.1016/S0032-9592(00)00288-0

VYSLOUZILOVA, M.; TLUSTOS, P.; SZAKOVA, J.; PAVLIKOVA, D. As, Cd, Pb and Zn uptake by Salix. spp. clones grown in soils enriched by high loads of these elements. Plant, Soil and Environment, v. 49, n. 5, p. 191-196, 2003. 


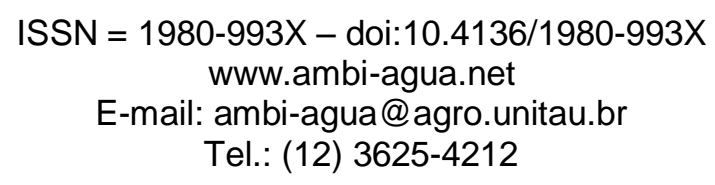

\title{
Concentración de clorofila-a y límite de zona fótica en el embalse Río Tercero (Argentina) utilizando imágenes del satélite CBERS-2B
}

\author{
(http://dx.doi.org/10.4136/ambi-agua.847)
}

\author{
Matias Bonansea ${ }^{1}$; Claudia Ledesma ${ }^{2}$; \\ Claudia Rodriguez ${ }^{3}$; Angel Ramon Sanchez Delgado ${ }^{4}$ \\ Becario Conicet. Universidad Nacional de Rio Cuarto \\ ${ }^{1}$ Becario Conicet. Universidad Nacional de Rio Cuarto, Ecología. Facultad de Agronomía y Veterinaria, \\ Córdoba, Argentina, e-mail: mbonansea@ayv.unrc.edu.ar, \\ ${ }^{2}$ Universidad Nacional de Rio Cuarto, Matemática y Bioestadística, Córdoba, Argentina, \\ e-mail: cledesma@ayv.unrc.edu.ar, \\ ${ }^{3}$ Universidad Nacional de Rio Cuarto, Ecología. Facultad de Agronomia y Veterinária e Programa de Pós- \\ graduação em Ciência, Tecnologia e Inovação Agropecuária; Córdoba, Argentina, \\ e-mail: crodriguez@ayv.unrc.edu.ar, \\ ${ }^{4}$ Universidade Federal Rural do Rio de Janeiro, Programa de Pós-graduação em Ciência, Tecnologia e Inovação \\ Agropecuária; Rio de Janeiro, RJ, Brasil, \\ e-mail: asanchez@ufrrj.br
}

\section{RESUMEN}

Las imágenes satelitales provistas por sensores remotos representan una herramienta importante en el monitoreo, control y gestión de los recursos hídricos. El objetivo del siguiente trabajo fue modelar la distribución espacial de la concentración de clorofila-a y el límite de la zona fótica en el embalse Río Tercero (Córdoba-Argentina), utilizando imágenes del satélite CBERS-2B y un sistema de información geográfica (SIG). Fueron seleccionados 15 sitios de muestreo en donde se midieron parámetros físicos, químicos y biológicos, durante el otoño de 2008. Utilizando una imagen georreferenciada del sensor CBERS-2B y por medio del análisis de componentes principales (ACP), se lograron identificar asociaciones entre variables y valores de reflectancia de las bandas de dicho satélite. En base a estas asociaciones y con modelos de regresión múltiples, se crearon las respuestas estimadas para conocer la distribución espacial de las variables, concentración de clorofila-a $\left(\mathrm{R}^{2}=0,73\right)$ y límite de zona fótica $\left(\mathrm{R}^{2}=0,78\right)$ en todo el reservorio. Podemos concluir que existe una correlación negativa entre la concentración de clorofila-a y la transparencia de la agua en el embalse Rio Tercero; como también que el sensor utilizado, permite evaluar la calidad del agua en un tiempo relativamente corto.

Palabras llaves: sensores remotos, recursos hídricos, ACP.

\section{Concentração de clorofila-a e limite de zona fótica no reservatório Rio Tercero (Argentina) utilizando imagens do satélite CBERS-2B}

\section{RESUMO}

As imagens de satélites com sensores remotos representam uma ferramenta importante no monitoramento, controle e gestão dos recursos hídricos. O objetivo do seguinte trabalho foi modelar a distribuição espacial da concentração de clorofila-a e o limite da zona fótica no reservatório Rio Tercero (Córdoba-Argentina), utilizando imagens do satélite CBERS-2B e um sistema de informação geográfica (SIG). Foram selecionados 15 sítios do reservatório onde foram medidos parâmetros físicos, químicos e biológicos no outono de 2008. Utilizando 
uma imagem georreferenciada do sensor CBERS-2B e com base na análise de componentes principais (ACP), identificou-se associações entre variáveis e valores de reflectância das bandas do satélite. Com base nessas associações e com modelos de regressão múltiplas, alcançaram-se as respostas estimadas para se conhecer a distribuição espacial das variáveis: concentração de clorofila-a $\left(\mathrm{R}^{2}=0,73\right)$ e limite de zona fótica $\left(\mathrm{R}^{2}=0,78\right)$ em tudo o reservatório. Pôde-se concluir que existe uma corelação negativa entre a concentração de clorofila-a e a transparência da água no reservatório Rio Tercero; assim como que o sensor utilizado permite a avaliação da qualidade da água em tempo relativamente curto.

Palavras-chave: sensores remotos, recursos hídricos, ACP.

\title{
Concentration of chlorhophyll-a and the limit of the photic zone in the Third River reservoir (Córdoba-Argentina), using images from CBERS-2B satellite
}

\begin{abstract}
The satellite images provided by remote sensors represent an important tool for monitoring, controlling and administering water resources. The aim of this work was to model the spatial distribution of the concentration of chlorhophyll-a and the limit of the photic zone in the Rio Tercero reservoir (Córdoba-Argentina), using images from CBERS-2B satellite and a geographical information system (SIG). We selected 15 samples places wherefrom we measured physical, chemical and biological parameters during the fall of 2008. By using a georeferenced image from sensor CBERS-2B and performing an analysis of the principal components (ACP), we were able to identify associations between variables and reflectance values of the satellite bands. Based on these associations and with simple regression models, we were able to know the spatial distribution of the variables: concentration of chlorophyll-a $\left(\mathrm{R}^{2}=0,73\right)$ and the limit of the photic zone $\left(\mathrm{R}^{2}=0,78\right)$ in the reservoir as a whole. We may conclude thata there exists a negative correlation between the concentration of chlorophyll-a and the water transparency in reservoir Rio Tercero, as well as that the sensor used allows us to evaluate the quality of the water in a relative short time.
\end{abstract}

Keywords: remote sensors, water resources, ACP.

\section{INTRODUCIÓN}

Históricamente, la preocupación de los países por contar con agua suficiente en cantidad y calidad para sus diferentes actividades es cada vez mayor. Los lagos y embalses proveen el agua para el consumo humano y permiten realizar una serie de funciones ambientales sumamente valiosas. Sin embargo, estos sistemas están expuestos a la degradación ambiental, siendo la eutroficación uno de los problemas más comunes que producen impactos ecológicos, sanitarios y económicos, significativamente negativos a escala local y regional (Rodríguez, 1997). Si bien la eutroficación es parte de un proceso natural de envejecimiento de los lagos, que ocurre en forma lenta e independientemente de la actividad del hombre, la creciente urbanización, el desarrollo agrícola y energético, aceleran este proceso, acortando la vida útil del cuerpo de agua. De esta manera, se genera una "eutroficación artificial o cultural", que es mucho más acelerada y peligrosa que la natural. La importancia que tiene la detección y valorización de las fuentes de nutrientes que ingresan al sistema (principalmente fósforo y nitrógeno), es que a partir de sus análisis, podemos planificar esfuerzos futuros para mantener la calidad del agua. 
BONANSEA, M.; LEDESMA, C.; RODRIGUEZ, C.; DELGADO, A. S. R. Concentración de clorofila-a y límite de zona fótica en el embalse Río Tercero (Argentina) utilizando imágenes del satélite CBERS-2B. AmbiAgua, Taubaté, v. 7, n. 3, p. 61-71, 2012. (http://dx.doi.org/10.4136/ambi-agua.847)

La literatura muestra que a la hora de generar un programa para el análisis de la calidad del agua en un reservorio acuático, los parámetros bio-físicos comúnmente seleccionados son la concentración de clorofila-a, los sedimentos en suspensión y la transparencia del agua (Zhu et al., 2005; Gonzalez et al., 2011). La transparencia del agua, es expresada como el límite de la zona fótica (z-fot). Margalef (2002) define el límite de la zona fótica como la profundidad en la cual la intensidad de la luz queda reducida a un $1 \%$ de la que ha penetrado en la superficie; constituyendo un límite por debajo del cual no queda lugar para los procesos de fotosíntesis. Entre las formas de medir el fitoplancton presente en un cuerpo de agua, la más utilizada es la concentración de clorofila-a, expresada como los pigmentos fotosintéticos por unidad de volumen. Los crecimientos masivos de algas y cianbacterias que se producen por eutroficación, pueden ocasionar mortandades de animales y producir malestares gastrointestinales en los seres humanos, debido a la producción de cianotoxinas (Vincent et al., 2004).

De esta manera, conocer las concentraciones existentes de este compuesto en el agua, así como la profundidad máxima en la cual las comunidades algares pueden subsistir, son de suma importancia en el control y gestión de lagos y embalses (Martini et al., 2006). Los análisis limnológicos se basan en muestras "in sintu" realizados a partir de un número limitado de estaciones, siendo la productividad primaria computada por la extrapolación de medidas puntuales. Muchas veces, tales metodologías no permiten evaluar con precisión la variabilidad espacial y temporal del sistema en estudio. Estas limitaciones metodológicas pueden ser suprimidas con la utilización de sensores remotos en estudios de sistemas acuáticos (Felix 1993; Cestari et al., 1996; Brunkow 2004; Tundisi, 2005; Tundisi et al., 2006; Breunig et al., 2007; Corazza, 2010; Wachholz, 2012). De acuerdo con Novo (1998), sensoriamento remoto es la utilización de sensores para la adquisición de informaciones sobre objetos y fenómenos, sin que haya contacto directo entre ellos. Son equipamientos capaces de colectar energía proveniente del objeto, convirtiéndola en señal adecuadamente registrable para la extracción de informaciones.

El procesamiento de imágenes satelitales provistas por sensores remotos, junto a un sistemas de información geográfica (SIG) y técnicas de modelación, pueden ser utilizadas para evaluar espacial y temporalmente la calidad del agua en una superficie, mediante el estudio de algunos parámetros con tiempo y respuesta relativamente cortos (Sass et al., 2007). Pereira Filho et al. (2011) encontraron una relación de la clorofila-a con la banda verde del sensor Landsat 5 TM en aguas del reservatorio de Passo Real, en Rio Grande del Sur, Brasil; en cuanto, Santos y Dubreuil (2009) consiguieron estimar el material en suspensión a través de las bandas roja e infra-rojo, para el reservatorio de Manso Grosso, Brasil. Rudorft et al. (2007) identificaron una relación entre los datos de los sólidos en suspensión y la clorofila-a, con datos espectrales del sensor Hyperion/E01 en aguas amazónicas.

En mayo del 2010, el Instituto Nacional de Pesquisas Espaciales del Brasil (INPE) anunció el final de la misión del satélite Sino-Brasileiro CBERS-2B (Brasil-China), pero no del programa CBERS; que representa una pieza fundamental para el sector técnico-científico espacial, así como de suma importancia para el Brasil se mantener dentro del selecto grupo de países con tecnología de sensores remotos. Prueba de eso son las renovaciones bilaterales ya realizadas para los CBERS-3 y 4. La familia de satélites de sensoramiento remoto CBERS, le ha dado al Brasil avances científicos, como también una tecnología valiosa para el seguimiento del nuevo código forestal; un ejemplo de eso, es el uso de imágenes para el control de la Amazona, el monitoreo de los recursos hídricos, el crecimiento urbano, la ocupación de la tierra y muchas otras (Santos et al., 2005). El satélite CBERS-2B, contaba con una cámara de alta resolución CCD (Couple Charged Device) que presenta 5 bandas espectrales, las cuales se reparten entre la franja visible e infra-rojo cercano $(450-520 \mathrm{~nm}$; 
BONANSEA, M.; LEDESMA, C.; RODRIGUEZ, C.; DELGADO, A. S. R. Concentración de clorofila-a y límite de zona fótica en el embalse Río Tercero (Argentina) utilizando imágenes del satélite CBERS-2B. AmbiAgua, Taubaté, v. 7, n. 3, p. 61-71, 2012. (http://dx.doi.org/10.4136/ambi-agua.847)

520-590 nm; 630-690 nm; 770-890 nm; 510-730 nm). Una resolución espacial de $20 \mathrm{~m}$ y una resolución temporal de 26 días (Lino et al., 2000; Todt et al., 2006).

En Argentina, las políticas públicas de gestión aplicadas a recursos hídricos carecen de visión, quizás por no establecer planes a largo plazo. Con respecto a la provincia de Córdoba, el estado trófico de diques y embalses, varían entre mesotróficos y eutróficos con altas cargas de materia orgánica (Rodríguez et al., 2006). La mayoría de ellos están altamente influenciados por la agricultura, ganadería, actividad minera, erosión del suelo y descarga de efluentes domésticos e industriales no tratados. Esta problemática no escapa de la observada en el embalse de Rio Tercero en los últimos cuatro años.

Durante el otoño del 2008, realizamos una experiencia de campo y numérica para la determinación de la distribución espacial de la concentración de clorofila-a y el límite de la zona fótica del embalse Río Tercero (Córdoba-Argentina), utilizando imágenes del satélite CBERS-2B; que a pesar de ya no estar en órbita, hallamos interesante presentar una más de sus múltiples contribuciones. En este artículo, modelamos y analizamos la distribución espacial de la concentración de clorofila-a y el límite de la zona fótica del embalse Río Tercero, utilizando imágenes del satélite CBERS-2B, SIG y modelos estadísticos de regresión; como también, algunas comparaciones con los resultados obtenidos en escenarios brasileros que usaron sensoramiento remoto.

\section{MATERIAL Y MÉTODOS}

\section{1. Área de estudio}

El embalse Río Tercero está localizado en el centro de la provincia de Córdoba y abarca una superficie de 4.600 ha, entre los $32^{\circ} 00^{\prime}$ y $32^{\circ} 30^{\prime}$ de latitud sur y los $64^{0} 24^{\prime}$ y $64^{0} 57^{\prime}$ de longitud oeste. Su profundidad media es de $12 \mathrm{~m}$ (máxima $46 \mathrm{~m}$ ) y su tiempo de residencia de 0,84 años (Boltovskoy y Foggetta, 1985; Mariazzi et al., 1992); siendo el cuerpo artificial de agua más grande de la provincia. En las costas de esta represa se encuentran las localidades de Villa del Dique y Villa Rumipal, que aprovechan este recurso para consumo humano, animal, generación de energía eléctrica, riego y actividades recreacionales (náutica y pesca deportiva), como es mostrado en la Figura 1.

\subsection{Toma de muestras}

Se realizó un muestreo durante el otoño de 2008 en fecha coincidente con el paso del satélite CBERS-2B por la zona de estudio. Se seleccionaron 15 sitios de muestreo (Figura 1), en los que se recolectaron muestras a $20 \mathrm{~cm}$ de profundidad. In situ se determinaron las coordenadas de ubicación geográfica con GPS; temperatura del agua, $\mathrm{pH}$, oxígeno disuelto, conductividad y la profundidad del disco de Secchi, que fue utilizada para el cálculo del límite de la zona fótica, definido como el producto entre la profundidad del disco de Secchi y el coeficiente de 2,5 (Ryding y Rast, 1992). En el laboratorio se determinó la concentración de clorofila-a, fósforo y nitrógeno total mediante espectrofotometría. Los lineamientos para la metodología de toma de muestra, almacenamiento conservación y técnicas analíticas se tomaron de APHA et al. (2000).

\subsection{Procesamiento de imágenes}

Se utilizó la imagen del satélite CBERS-2B (correspondiente al 27 de abril de 2008), cedida sin ningún costo, por el Instituto Nacional de Pesquisas Espaciales del Brasil (INPE). Para el análisis, procesamiento e interpretación de la misma se utilizó el software ENVI 3.5 (Versión 2001), desarrollado por ESRI (2001). Se georreferenció la imagen con un RMS (Error Medio Cuadrado) global de 0,84 pixel (20 metros/pixel). 


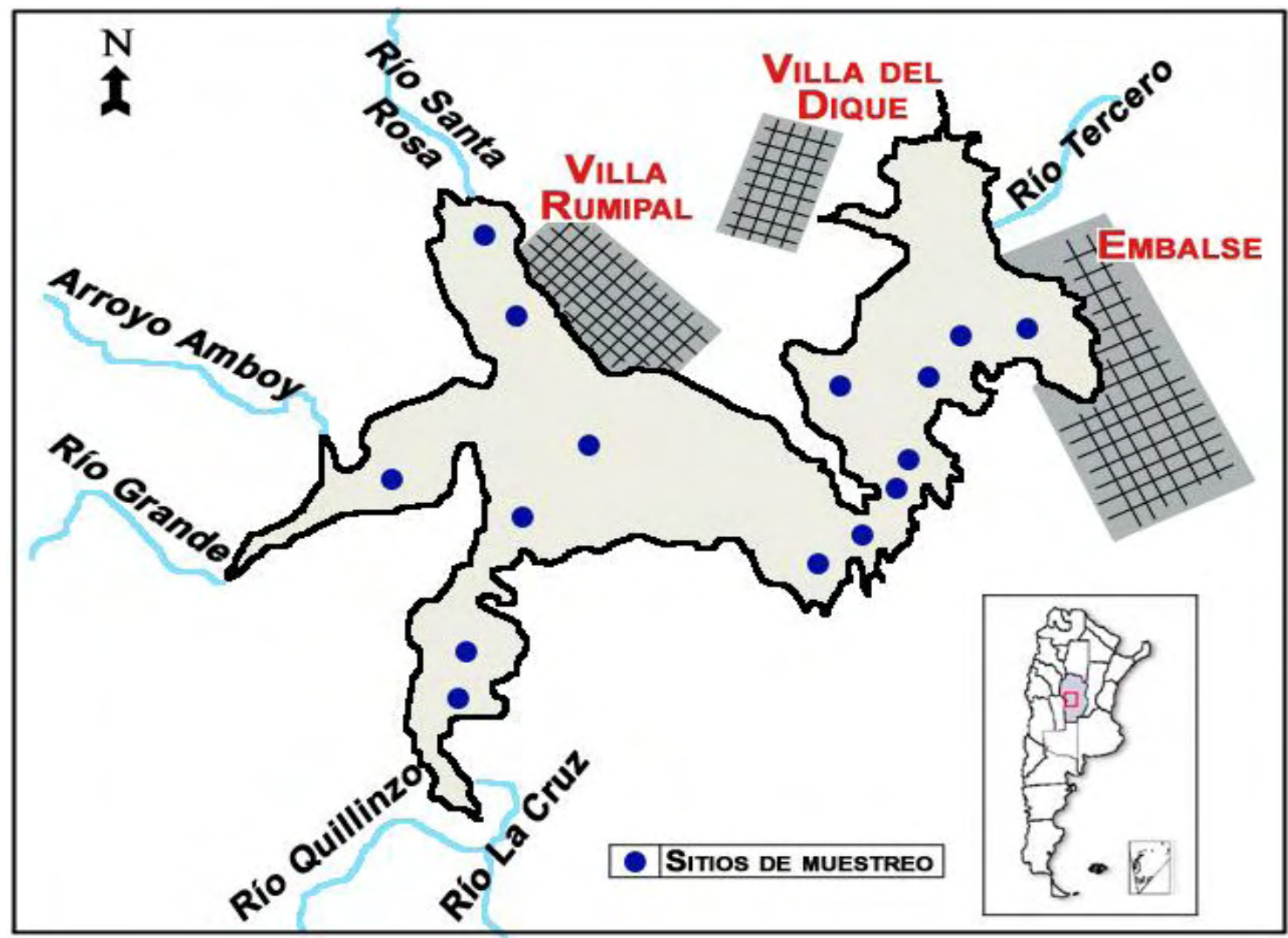

Figura 1: Ubicación de la zona de estudio y sitios de muestreo.

\subsection{Metodología}

Se construyeron los modelos de regresión múltiple para las variables cl-a y z-fot. Respecto a cl-a, se relacionaron las características espectrales de dicho parámetro y una combinación de las bandas 2 (b2) $(0,52-0,59 \mu \mathrm{m})$ y 3 (b3) $(0,63-0,69 \mu \mathrm{m})$ del satélite CBERS$2 \mathrm{~B}$, generando la siguiente respuesta:

$$
c l-a=32,71 * b 2+0,45 * b 3-45,02
$$

Considerando el coeficiente del parámetro Z-fót como variable regresora a los niveles digitales de las bandas $1(0,45-0,52 \mu \mathrm{m})(\mathrm{b} 1)$ y $4(0,77-0,89 \mu \mathrm{m})(\mathrm{b} 4)$, obtuvimos a función respuesta:

$$
z-f o t=0,01 * b 1-1,02 * b 4+40,96
$$

El valor del coeficiente de determinación fue $\mathrm{R}^{2}=0,78$; siendo no significativo para la banda 1 (p>0,05). Con el Software Estadístico InfoStat Profesional (Versión 2009), se realizó un Análisis de Componentes Principales (ACP), que fue utilizado para observar la variabilidad e identificar asociaciones entre los parámetros medidos en el embalse y los datos de reflectancia de las bandas del satélite CBERS-2B.

\subsection{Mapas de distribución}

Para obtener los mapas de distribución de las variables cl-a y z-fot del embalse Río Tercero, se generó una máscara del cuerpo de agua, sobre la cual se aplicaron las respuestas estimadas de las variables cl-a y z-fot. De esta manera se lograron predecir valores de dichos parámetros en toda la superficie del cuerpo de agua, permitiendo estimar variaciones espaciales. 
BONANSEA, M.; LEDESMA, C.; RODRIGUEZ, C.; DELGADO, A. S. R. Concentración de clorofila-a y límite de zona fótica en el embalse Río Tercero (Argentina) utilizando imágenes del satélite CBERS-2B. AmbiAgua, Taubaté, v. 7, n. 3, p. 61-71, 2012. (http://dx.doi.org/10.4136/ambi-agua.847)

\section{RESULTADOS Y DISCUSIÓN}

A continuación se describen los valores medios y el rango de los parámetros: Temperatura, pH, Oxígeno Disuelto, Conductividad, Zona Fótica, Clorofila-a, Nitrógeno Total y Fósforo Total; medidos en el embalse Río Tercero (Córdoba, Argentina) durante el otoño del 2008 (Tabla1).

Tabla 1. Parámetros: Temperatura (T), pH, Oxigeno Disuelto (OD), Conductividad (Cond.), Zona Fótica (ZF), Clorofila-a (Cl-a), Nitrógeno Total (NT), e Fósforo Total (FT); medios, mínimos y máximos, medidos en el embalse Río Tercero durante un muestreo realizado en otoño de 2008.

\begin{tabular}{l|llllllll}
\hline Variable & $\begin{array}{l}\mathbf{T} \\
{ }^{\mathbf{}} \mathbf{C}\end{array}$ & $\mathbf{p H}$ & $\begin{array}{l}\mathbf{O D} \\
\mathbf{m g} / \mathbf{L}\end{array}$ & $\begin{array}{l}\mathbf{C o n d} \\
\mathbf{m S} / \mathbf{c m}\end{array}$ & $\begin{array}{l}\mathbf{Z F} \\
\mathbf{M}\end{array}$ & $\begin{array}{l}\mathbf{C l - a} \\
\mathbf{m g} / \mathbf{L}\end{array}$ & $\begin{array}{l}\mathbf{N ~ T} \\
\mathbf{m g} / \mathbf{L}\end{array}$ & $\begin{array}{l}\mathbf{F} \mathbf{T} \\
\mathbf{m g} / \mathbf{L}\end{array}$ \\
\hline Medio & 21,4 & 7,8 & 10,9 & 1,45 & 6,8 & 24,0 & 1,2 & 0,013 \\
Mínimo & 20,3 & 6,8 & 9,3 & 1,40 & 3,8 & 5,4 & 0,9 & 0,012 \\
Máximo & 25,5 & 8,6 & 13,5 & 1,49 & 10,0 & 49,8 & 1,5 & 0,013 \\
\hline
\end{tabular}

En este período se observó una temperatura media de $21,4{ }^{\circ} \mathrm{C}$; con un valor mínimo y máximo de $20,3{ }^{\circ} \mathrm{C}$ y $25,5^{\circ} \mathrm{C}$ respectivamente, sin diferencias significativas. Respecto a los sitios de muestreo, los valores de temperatura más altos correspondieron a la salida de agua del canal de enfriamiento de la central nuclear, que utiliza $120.000 \mathrm{~m} 3 / \mathrm{h}$ de las aguas de este lago como refrigerante del reactor.

Durante la misma estación (otoño) del año 2009, se registraron temperaturas medias similares en el escenario de la Usina Hidroeléctrica Doña Francisca (UHE-Dona Francisca) (RS-Brasil) (Corazza, 2010) y un año antes (2007); en la Represa de Paraibuna, SP-Brasil (Días et al., 2007), a diferentes niveles de profundidad (0,5 m; $5 \mathrm{~m}$ y $10 \mathrm{~m})$, se observó una variación entre $20^{\circ} \mathrm{C}$ y $22^{\circ} \mathrm{C}$.

Los criterios de protección a la vida acuática fijan la variable $\mathrm{pH}$ (potencial hidrogenito) entre 6 y 9. El pH tiene influencia en diversos equilibrios químicos que ocurren naturalmente durante el tratamiento de aguas. En los ecosistemas acuáticos, posee efectos directos sobre la fisiología de diversas especies e indirectos en relación a la precipitación de elementos químicos tóxicos con metales pesados. También pode ejercer algún efecto en las salubridades de los nutrientes. La media del pH encontrado en el embalse Rio Tercero fue de 7,8; con un rango de 6,8 y 8,6 . No se encontró una tendencia del perfil multivariado de esta variable, ni un sitio de muestreo con características especiales.

La media del pH hallado en la UHE-Doña Francisca fue de 6,9; con un rango de 5,5 y 8,7; en cuanto que en el escenario de la Represa de Paraibuna se encontró una variación entre 5,93 y 7,08 .

La variable oxigeno disuelto (OD) presentó un rango de 9,3 a 13,5 mg/L con una media de 10,9 mg/L. A lo largo del tiempo, se observó una disminución en la concentración de OD que no sería significativa. Aunque no hubo una tendencia entre los sitios de muestreo, ya que los valores de OD varían entre una fecha y la siguiente, se advirtieron grandes desvíos entre los sitios y sus respectivas medias. Para obtener una conclusión más certera de esta variable, se recomendaría generar un gradiente vertical de concentración de oxígeno para conocer su comportamiento en todo el embalse. En la Represa de Paraibuna, el OD varió entre 6,35 y $8,52 \mathrm{mg} / \mathrm{L}$.

La concentración media del fósforo total (FT) encontrada en el embalse fue de 0,013 $\mathrm{mg} / \mathrm{L}$, con tendencia a aumentar a lo largo del tiempo. Esta condición podría deberse a la 
fuerte actividad antrópica que se realizan en algunas zonas del embalse (recreación), sumado con las descargas de efluentes no tratados. No se descarta que en un futuro se observen florecimientos de algas en estos sitios, lo que podría generar una serie de acontecimientos negativos para el funcionamiento del embalse. Siguiendo la clasificación de ITAIPU, el embalse Rio Tercero se encontraba (para la fecha), con una calidad de agua poco degradada. Ya en relación al nitrógeno total (NT), se observó una media de 1,2 $\mathrm{mg} / \mathrm{L}$ y un rango comprendido entre 0,9 y $1,5 \mathrm{mg} / \mathrm{L}$. Esta variable no presentó una tendencia muy marcada a lo largo del tiempo, aunque si se pudo registrar un leve aumento. Al igual que el FT, las máximas concentraciones se hallaron en sitios con alta actividad antrópica.

En relación a la clorofila-a, la concentración media fue de $24 \mathrm{mg} / \mathrm{L}$, en un rango amplio de 5,4 a 49,8 mg/L. Esta variable aumentó a lo largo del tiempo. Considerando que la clorofila-a depende de la concentración de nutrientes (FT y NT), penetración de la luz y temperatura del agua, es posible que estos aumentos se deban a los aumentos hallados en las concentraciones del FT. Entre los sitios de muestreo, se observó que las concentraciones más altas se presentaron en el canal de enfriamiento de la central nuclear y en el murallón del embalse. En el primer caso, el incremento de clorofila-a pudo deberse a la alta temperatura que posee el agua en este sitio, favoreciendo la proliferación de algas. En el segundo caso, podría ser el producto de la alta concentración de nutrientes y de los vientos que generan una deriva del fitoplancton hacia la zona en cuestión. Los vientos predominantes en el embalse Rio Tercero provienen del sector suroeste.

En la UHE-Doña Francisca, la elevada concentración de clorofila-a fue atribuida a la transparencia del agua y a las condiciones ambientales, ya que el reservatorio se encuentra en un valle encajado en un entorno dominado por una cobertura forestal.

En relación a la conductividad, se obtuvo una media de $1,45 \mathrm{mS} / \mathrm{cm}$, con un valor mínimo de $1,40 \mathrm{mS} / \mathrm{cm}$ y un valor máximo de $1,49 \mathrm{mS} / \mathrm{cm}$. También, el valor medio de la zona fótica fue de $6,8 \mathrm{~m}$ y un rango entre 3,8 y $10 \mathrm{~m}$.

A partir del análisis multivariado de componentes principales (ACP) y usan todos componentes que explican el 74,5\% de la variabilidad total de los datos, se generó un grafico biplot (Figura 2), donde se observó correlación positiva entre la variable cl-a y los datos de reflectancia de las bandas 2 y 3 del satélite CBERS-2B, mientras que la variable $\mathrm{z}$-fot se correlacionó en forma positiva con la bandal y negativamente con la banda 4 de dicho satélite.

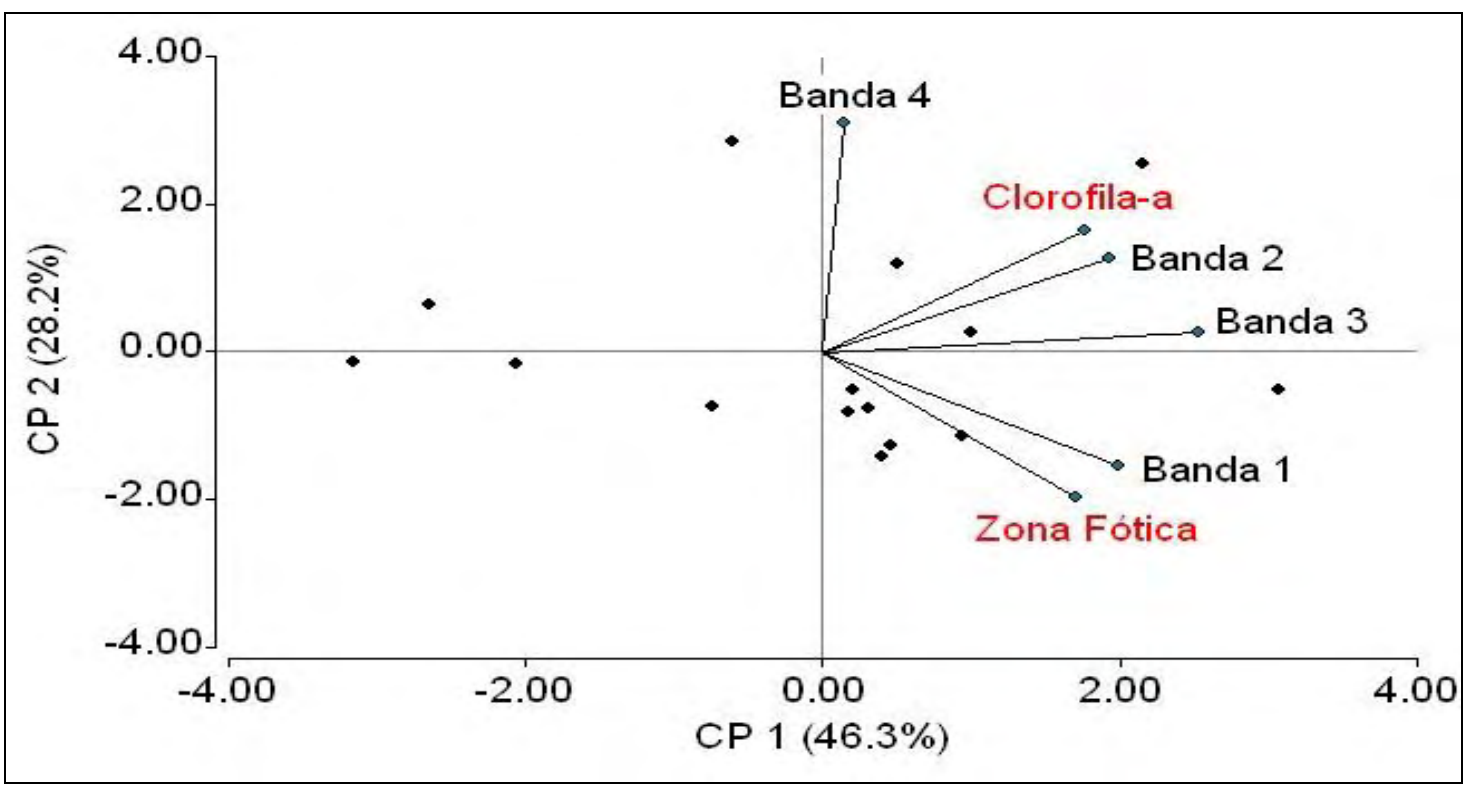

Figura 2. Diagrama de Componentes Principales (CP1/CP2). 


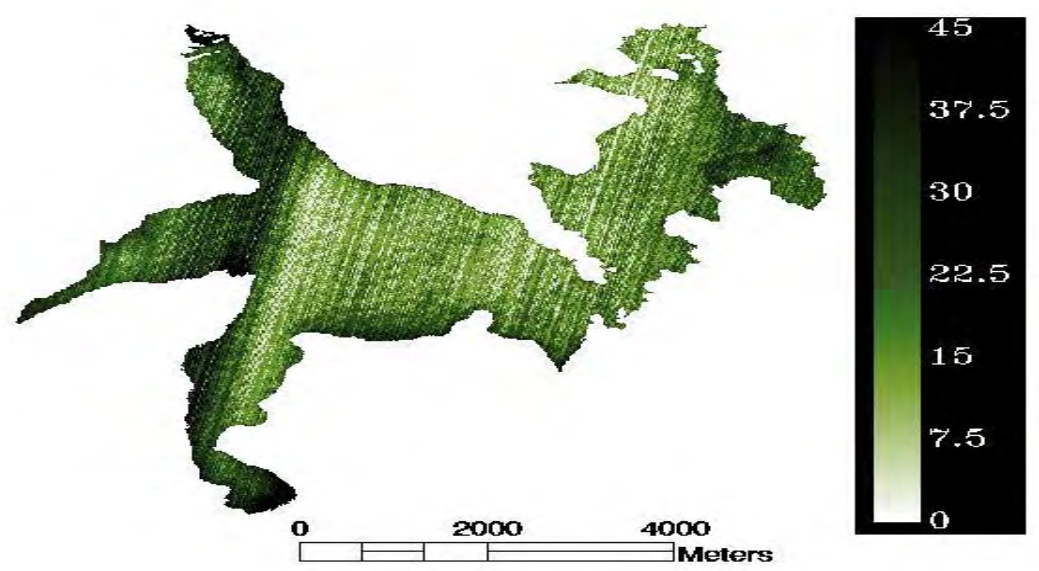

Figura 3. Distribución de la concentración de clorofila-a en Río Tercero.

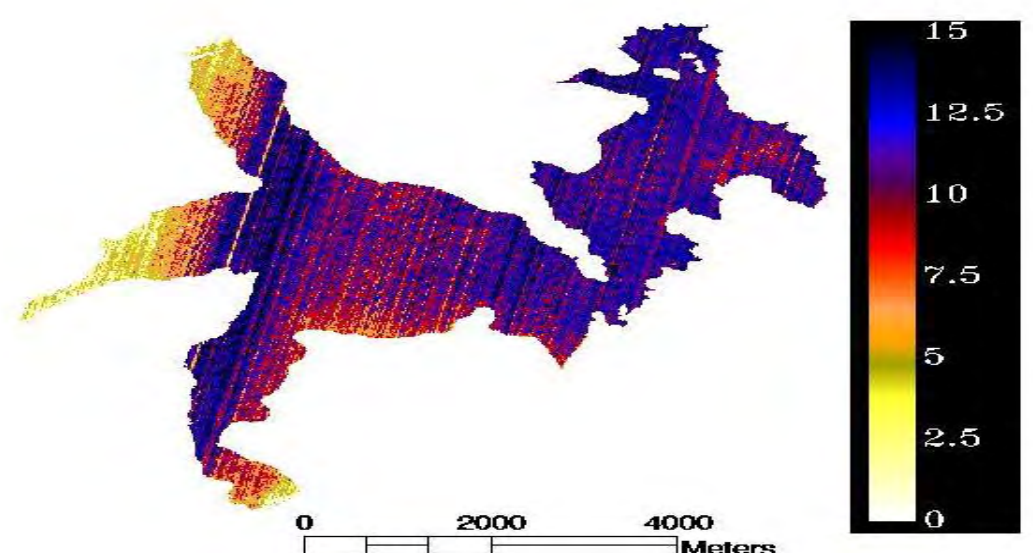

Figura 4. Distribución de la profundidad máxima de zona fótica en Rio Tercero.

Las respuestas estimadas para los modelos de regresión creados fueron aplicadas sobre la máscara del embalse Río Tercero, generada a partir del procesamiento de la imagen CBERS2B en la zona de estudio. De esta manera, se logró mapear la concentración de clorofila-a y el límite de la zona fótica en toda la superficie del embalse Río Tercero (Figura 3 y 4). La franja o línea blanca que se observa en el extremo oeste del mapa de distribución de profundidad máxima de zona fótica, fueron producto de una anomalía radiométrica de la banda 4 del satélite CBERS-2B (Figura 4). Dentro de la evaluación del comportamiento espacial de la cl-a y z-fót en el embalse Río Tercero, y usando teledetección y análisis estadístico, conseguimos relacionar los datos obtenidos en campo con valores de reflectancia provistos por imágenes satelitales. De esta manera se generó una información temática referida a la distribución de los parámetros en toda la superficie del embalse Río Tercero.

A través de los mapas de distribución, podemos observar que existe una correlación negativa entre cl-a y z-fot. No obstante, en las zonas de entrada de ríos, donde se observa con más claridad la relación negativa entre ambas variables, la baja transparencia del agua no estaría dada por una alta concentración de algas, sino por altas cantidades de sólidos en suspensión que descargan en las aguas de los ríos de la cuenca del reservorio en estudio. Esto es demostrado con el ACP, donde se observa una ausencia de correlación entre este par de variables. Si bien las anomalías radiométricas fueron frecuentes en las bandas del satélite CBERS-2B, este sensor resultó un buen instrumento en lo que respecta a la resolución 
espacial, cuando comparamos con otros instrumentos de sensoramiento remoto, como los de la serie LANDSAT.

En la actualidad son conocidas integraciones entre sensores remotos (Santos et al., 2005; Mattews et al., 2010), como también su utilización múltiple (Bentz et al., 2005).

\section{CONCLUSIONES}

A partir de las informaciones obtenidas del embalse Rio Tercero, es posible generar los esfuerzos futuros que deben ser realizados en lo que respecta al manejo y saneamiento del reservatorio. Al mismo tiempo, estas informaciones permiten realizar el HEWS (Sistemas Alerta Temprana de la Salud), para establecer el área de riesgo crítico a la salud pública y animal. En cuanto a la concentración de clorofila-a, se registró un aumento en la concentración de nutrientes, principalmente en fósforo. Se observó un aumento del oxigeno disuelto y el pH no presentó una tendencia particular. Finalmente, fue importante comparar los resultados obtenidos con los conocidos en algunos escenarios brasileros; próximos de la provincia de Córdoba (Rio Tercero), a fin de informar a las autoridades, organizaciones e instituciones públicas y privadas, sobre la situación actual de la calidad del agua en ambos países del Mercosur; así como incitar el monitoramiento y conservación de los embalses acuáticos.

\section{REFERENCIAS}

AMERICAN PUBLIC HEALTH ASSOCIATION - APHA; AMERICAN WATER WORKS ASSOCIATION - AWWA; WATER ENVIRONMETAL FEDERATION - WEF. Standard methods for the examination of water and wastewater. $18^{\text {th }}$ edition. Washington, DC, 2000.

BENTZ, C. M.; POLITANO, A. T.; GENOVEZ, P. Monitoramento ambiental de áreas costeiras e oceânicas com múltiplos sensores orbitais. Revista Brasileira de Cartografia, v. 57, n. 1, p. 43-47, 2005.

BOLtovskoY, A.; FOGGETTA, M. Limnología física del Embalse Rio III. Biología Acuática, v. 7, p. 1-26, 1985.

BREUNIG, F. M.; WACHHOLZ, F.; PEREIRA FILHO, W.; RUDORFF M. de C. Análise das propriedades ópticas da água do reservatório Rodolfo Costa e Silva-Itaara, RS, Brasil, usando dados espectrais. Ambiente \& Água, v. 2, n. 2, p. 88-102, 2007. http://dx.doi.org/10.4136/ambi-agua.28

BRUNKOW, R. F. et al. Monitoramento da qualidade das águas dos reservatórios estado do Paraná, no período de 1999 a 2004. Curitiba: IAP, 2004. 12p.

CESTARI, A. C.; KRUG, T.; NOVO, E. Modelo empírico para a estimativa de concentração da clorofila na zona eufótica em função da concentração de clorofila na superfície. In: SIMPÓSIO BRASILEIRO DE SENSORIAMENTO REMOTO, 8., 1996, Salvador. Anais... São José dos Campos: INPE, 1996. p. 93-98.

CORAZZA, Rosana. Relações entre variáveis espectrais e limnológicas no reservatório da usina hidrelétrica Dona Francisca/RS. 2010. 103 f. Dissertação (Mestrado em Geografia) - Programa de Pós-Graduação em Geografia e Geociências, Universidade Federal de Santa Maria, 2010. 
BONANSEA, M.; LEDESMA, C.; RODRIGUEZ, C.; DELGADO, A. S. R. Concentración de clorofila-a y límite de zona fótica en el embalse Río Tercero (Argentina) utilizando imágenes del satélite CBERS-2B. AmbiAgua, Taubaté, v. 7, n. 3, p. 61-71, 2012. (http://dx.doi.org/10.4136/ambi-agua.847)

DIAS, N. W.; MORAES, E. C.; NOVO, E. M.; MORAES, L.; ARAI, E.; CATELANI, C. S. Caracterização das águas da represa de Paraibuna com o uso de dados hiperespectrais. In: SIMPÓSIO BRASILEIRO DE SENSORIAMENTO REMOTO, 13., 2007, Florianópolis. Anais... São José dos Campos: INPE, 2007. p. 3335-3342.

ENVIRONMENTAL SYSTEMS RESEARCH INSTITUTE - ESRI. ENVI Versión 3.5: programa de análisis y procesamiento de imágenes satelitales. Redlands, 2001.

FELIX, I. M. Monitoramento da qualidade da água utilizando-se modelos empíricos obtidos a partir de sensoriamento remoto Reservatório de Barra Bonita - SP. In: SIMPÓSIO BRASILEIRO DE SENSORIAMENTO REMOTO, 7., 1993, Curitiba. Anais... São José dos Campos: INPE, 1993. p. 119-127.

GONZALEZ, O. S.; ALMEIDA, C. A.; QUINTAR, S.; MALLEA, M. A.; GONZALEZ, P. S. Application of multivariate statistical techniques to evaluate organic pollution on a river in Argentina. Ambiente \& Água, v. 6, n. 3, p. 27-42, 2011. http://dx.doi.org/10.4136/ambi-agua.696

LINO, C. O.; LIMA, M. G. R.; HUBSCHER, G. L. CBERS - An international space cooperation program. Acta Astronáutica, v. 47, n. 2/9, p. 559-564, 2000. http://dx.doi.org/10.1016/S0094-5765(00)00094-1

MARGALEF, R. Teoría de los sistemas ecológicos. Barcelona: Alfa Omega, 2002.

MARIAZZI, A. A.; DONADELLI, J. P.; ARENAS, M. DI SIERVI; BONETTO, C. Impact of a nuclear power plant on water quality of Embalse del Río Tercero reservoir (Córdoba, Argentina). Hidrobiología, v. 246, n. 2, p. 129-140, 1992. http://dx.doi.org/10.1007/BF00014700

MARTINI, L. C.; MATTOS, D. S.; BARBOSA, D. F. P.; RECH, A. I. B. Uso de sensoriamento remoto orbital para avaliação da distribuição espacial de Clorofila_a na Lagoa da Conceição - Florianópolis, SC. Engenharia Sanitária e Ambiental, v. 11, n. 4, p. 318-324, 2006. http://dx.doi.org/10.1590/S1413-41522006000400004

MATTEWS, M. W.; BERNARD, S.; WINTER, K. Remote sensing of cyanobacteriadominant alga blooms and water quality parameters in Zeekoevlei, a small by hypertrophic lake, using MERIS. Remote sensing of environment, v. 114, n. 9, p. 2070-2087, 2010. http://dx.doi.org/10.1016/j.rse.2010.04.013

NOVO, E. M. L. de M. Sensoriamento remoto: princípios e aplicações. 2. ed. São Paulo: Edgard Blucher, 1998. 308p.

PEREIRA FILHO, W. et al. Relação entre clorofila a com perfil espectral e imagem de satélite no reservatório Passo Real - RS. In: SIMPÓSIO BRASILEIRO DE SENSORIAMENTO REMOTO, 15., 2011, Curitiba. Anais... São José dos Campos: INPE, 2011. p. 5433-5440.

RODRÍGUEZ, C. et al. Estudio bacteriológico y químico del embalse Río Tercero (Córdoba). Revista Ciencia Veterinaria, v. 27, p. 3-8, 1997.

RODRÍGUEZ, C. M.; BONANSEA M.; BONATTO, F.; REYNOSO, V.; PROSPERI, C. MANCINI, M. et al. Remote sensing-GIS to predict on the risk of eutrophication in aquatic systems. IN: THE INTERNATIONAL TRADE EVENT AND CONFERENCE FOR EHEALTH, TELEMEDICINE AND HEALTH ICT - Med-e-Tel, 12., 2006, Luxembourg. Proceedings... Luxembourg: Luxexpo, 2006. p. 343-345. 
BONANSEA, M.; LEDESMA, C.; RODRIGUEZ, C.; DELGADO, A. S. R. Concentración de clorofila-a y límite de zona fótica en el embalse Río Tercero (Argentina) utilizando imágenes del satélite CBERS-2B. AmbiAgua, Taubaté, v. 7, n. 3, p. 61-71, 2012. (http://dx.doi.org/10.4136/ambi-agua.847)

RUDORFF, C. M.; NOVO, E. M. L. M.; GALVÃO, L. S.; PEREIRA FILHO, W. Análise derivativa de dados hiperespectrais medidos em nível de campo e orbital para caracterizar a composição de águas opticamente complexas na Amazônia. Acta Amazônica, v. 37, n. 2, p. 269-280, 2007. http://dx.doi.org/10.1590/S004459672007000200014

RYDING, S.; RAST, W. El control de la eutrofización en lagos y pantanos. Madrid: Pirámide, 1992.

SANTOS, J. W. M. C.; DUBREUIL, V. Estimativa da distribuição temporo-espacial de material em suspensão nas águas do reservatório de Manso-MT a partir de imagens Landsat e dados de campo. In: SIMPÓSIO BRASILEIRO DE SENSORIAMENTO REMOTO, 14., 2009, Natal. Anais... São José dos Campos: INPE, 2009. p. 5421-5428.

SANTOS, J. R. dos; MALDONADO, F. D.; ALENCASTRO GRAÇA, P. M. L. Integração de imagens LANDSAT+CBERS-2B para detecção de mudanças em área da Amazônia sob domínio da floresta de transição. Revista Brasileira de Cartografia, v. 57, n. 01, p. 15-27, 2005.

SASS, G.; CREED, I.; BAYLEY, S. E.; DEVITO, K. Understanding variation in trophic status of lakes on the Boreal Plain: a 20 year retrospective using Landsat TM imagery.

Remote Sensing of Environment, v. 109, n. 2, p. 127-141, 2007. http://dx.doi.org/10.1016/j.rse.2006.12.010

VINCENT, R.; QIN, X.; MCKAY, R.; MINER, J.; CZAJKOWSKI, K.; SAVINO, J. et al. Phycocyanin detection from LANDSAT TM data for mapping cyanobacterial blooms in Lake Erie. Remote Sensing of Environment, v. 89, n. 3, p. 381-392, 2004. http://dx.doi.org/10.1016/j.rse.2003.10.014

TODT, V.; RUBERT, C.; SILVA, J. D. S.; FORMAGGIO, A. R.; SHIMABUKURO, Y. E. Classificador adaptativo para o reconhecimento de alvos em imagens. Revista Brasileira de Cartografia, v. 58, n. 3, p. 293-305, 2006.

TUNDISI, J. G. Água no século XXI: enfrentando a escassez. 2. ed. São Carlos: RiMA, 2005. 248p.

TUNDISI, J. G.; TUNDISI, T. M.; ROCHA, O. Ecossistemas de águas interiores. In: REBOUÇAS, A. C.; BRAGA, B.; TUNDISI, J. G. (Orgs.). Águas doces no Brasil: capital ecológico, uso e conservação. 3. ed. São Paulo: Escrituras, 2006. p. 161-202.

UNIVERSIDAD NACIONAL DE CÓRDOBA. InfoStat profesional: statistical Software. Córdoba, 2008.

WACHHOLZ, F. Alterações espectrais nas imagens de satélites nos reservatórios das usinas hidrelétricas Caçu e Barra dos Coqueiros - GO. Revista Geonorte, v. 2, n. 4, p. 11701179, 2012. Edição especial.

ZHU, L.; WANG, S.; ZHOU, Y.; YAN, F.; WANG, L. Determination of chlorophyll a concentration changes in Taihu lake, China using multi-temporal MODIS image data. In: GEOSCIENCE AND REMOTE SENSING SYMPOSIUM, 5., 2005, Seoul. Proceedings... [S.1.]: IEEE International, 2005. Vol. 7. p. 4535- 4538. http://dx.doi.org/10.1109/IGARSS.2005.1526674 


ISSN = 1980-993X - doi:10.4136/1980-993X
www.ambi-agua.net
E-mail: ambi-agua@agro.unitau.br
Tel.: (12) 3625-4212

\title{
Influência do nível de tratamento de esgoto na qualidade da água do mar na região de lançamento do Emissário Submarino de Santos
}

(http://dx.doi.org/10.4136/ambi-agua.975)

\author{
Eduardo Lucas Subtil ${ }^{1}$; José Carlos Mierzwa ${ }^{1}$; Jayme Pinto Ortiz ${ }^{2}$ \\ ${ }^{1}$ Departamento de Engenharia Hidráulica e Ambiental, Escola Politécnica da Universidade de São Paulo, SP, \\ e-mails: eduardosubtil@gmail.com; mierzwa@usp.br, \\ ${ }^{2}$ Departamento de Engenharia Mecânica, Escola Politécnica da Universidade de São Paulo , São Paulo - SP, \\ e-mail: jportiz@usp.br
}

\section{RESUMO}

O presente trabalho teve como objetivo principal avaliar se para o caso do ESS haveria algum beneficio ambiental, em relação a potencial de eutrofização, se ao invés do tratamento preliminar fosse utilizado um TPQA, TS-LA ou um TT-LARN. Neste estudo, o impacto ambiental do lançamento de esgotos pelo ESS, para a vazão de $5,3 \mathrm{~m}^{3} \cdot \mathrm{s}^{-1}$, foi avaliado por um modelo numérico de eutrofização integrado verticalmente. As características da pluma de esgoto no campo próximo foram determinadas pelo software CORMIX, e os resultados acoplados ao modelo de campo distante considerando a conservação do fluxo de massa. Os resultados demonstraram que a utilização de um tratamento secundário com oxidação parcial da amônia promoveria uma redução de apenas $30 \%$ na concentração de clorofila-a, enquanto que a utilização de um TPQA com remoção de fósforo promoveria uma resposta ambiental mais significativa, levando a uma redução de até $69 \%$ na concentração de clorofila-a, resultados semelhantes ao tratamento terciário com remoção de nitrogênio e fósforo.

Palavras-chave: emissário submarino de Santos, nível de tratamento de esgoto, modelo numérico de eutrofização.

\section{Influence of wastewater treatment level on the seawater quality in the discharge region of Santos Submarine Outfall}

\begin{abstract}
This study aimed to evaluate if in the case of the Santos Submarine Outfall (SSO) there was any environmental benefits relative to potential eutrophication if instead of the primary treatment a CEPT, ST-AS or a TT- ASNR was used. The environmental impact of wastewater discharge through the SSO with an average flow of $5,3 \mathrm{~m}^{3} \cdot \mathrm{s}^{-1}$ was evaluated using an eutrophication numerical model which solves the two-dimensional vertically integrated hydrodynamic and transport equation. Wastewater mixing conditions in the near field plume was evaluated using the software CORMIX, and the results were coupled to a far-field model considering the conservation of mass flow. The results demonstrate that the use of a secondary treatment with partial ammonia oxidation promotes a reduction of only $30 \%$ in the chlorophyll-a, while the use of a CEPT with phosphate removal promotes a better environmental response, resulting in chlorophyll-a concentration reduction of $69 \%$, similar results were obtained when tertiary treatment was considered for nitrogen and phosphorus removals.
\end{abstract}

Keywords: Santos submarine outfall; wastewater treatment level; eutrophication numerical model. 
SUBTIL, E. L; MIERZWA, J. C.; ORTIZ, J. P. Influência do nível de tratamento de esgoto na qualidade da água do mar na região de lançamento do Emissário Submarino de Santos. Ambi-Agua, Taubaté, v. 7, n. 3, p. 72-86, 2012. (http://dx.doi.org/10.4136/ambi-agua.975)

\section{INTRODUÇÃO}

Dentre as várias tecnologias disponíveis para o tratamento de águas residuárias em ambientes costeiros, a utilização de emissários submarinos como parte integrante do sistema de tratamento aparece como uma alternativa sustentável para melhorar a qualidade da água (Echavarri-Erasun et al., 2010; Juanes et al., 2005), além de reduzir substancialmente os custos de investimentos, operação e manutenção enquanto alcançam os mesmos objetivos de qualidade ambiental de tratamentos com elevados níveis sem uma otimização do lançamento (Roberts et al., 2010). Apesar desses benefícios, sob determinadas condições ambientais e de lançamento, a Disposição Oceânica de Esgotos Sanitários (DOES) é conhecida por causar diferentes tipos de distúrbios que podem alterar de alguma forma a qualidade da água nas proximidades do lançamento. Aumentos significativos na concentração de nutrientes, principalmente amônia e fosfato, durante períodos de baixa hidrodinâmica (Dhage et al., 2006; Werme e Hunt, 2006), surgimento de fitoplâncton potencialmente tóxicos ou aumento na biomassa de fitoplâncton e turbidez (Thompson e Waite, 2003; Braga et al., 2000) são alguns dos efeitos observados em águas costeiras influenciadas por esse tipo de sistema.

Embora significativos progressos tenham sido feitos visando à melhoria da qualidade da água nos últimos 20 anos, sobretudo em países desenvolvidos, várias regiões costeiras continuam a sofrer com problemas ambientais persistentes, incluindo o lançamento de esgotos sanitários por meio de emissários submarinos mal projetados e/ou operados. As questões relacionadas com esse sistema estiveram no centro do debate recentemente no Brasil, resultando na alteração e complementação da Resolução CONAMA 357/2005, entrando em vigor uma nova resolução a CONAMA 430/2011 sobre emissões de efluentes. Até recentemente, não havia uma legislação nacional que abordasse o tratamento mínimo necessário para lançamento por emissários submarinos. Ficou estabelecido nessa nova resolução que o lançamento de esgotos por sistemas de disposição oceânica deve ser precedido de tratamento que garanta uma remoção mínina de $20 \%$ de Sólidos em Suspensão Totais (SST). Em outras palavras, não será mais possível utilizar apenas o tratamento preliminar como opção de pré-condicionamento para a disposição oceânica, haja vista a baixa eficiência de remoção de SST.

No estado de São Paulo, apesar do processo de tratamento predominante ser o sistema de lodos ativados e a disposição oceânica de esgoto representar apenas $2 \%$ das instalações de tratamento de esgoto da SABESP, em termos de capacidade instalada ela representa $22 \%$ da capacidade total de tratamento, ocupando, assim, o segundo lugar (Ortiz et al., 2011). Dentre os sistemas de DOES existentes na costa paulista o de Santos/São Vicente é o mais antigo em operação sendo responsável por lançar esgotos de aproximadamente 1.322 milhões de habitantes resultando numa vazão média atual de $3,0 \mathrm{~m}^{3} \cdot \mathrm{s}^{-1} \mathrm{com}$ a máxima aprovada de 5,3 $\mathrm{m}^{3} \cdot \mathrm{s}^{-1}$ (Ortiz et al., 2007). A condição atual de lançamento por esse sistema é considerada uma fonte significativa de poluição, uma vez que o nível de tratamento utilizado (preliminar) combinado com condições de lançamento adversas na região (baixa profundidade e diluição) tem resultado em concentrações elevadas na água do mar de poluentes presentes nos esgotos, sobretudo com relação à concentração de fósforo total e nitrogênio amoniacal (CETESB, 2005, 2006; Subtil et al., 2010, 2011, 2012).

Diversos estudos realizados ao longo dos últimos 30 anos demonstraram um aumento na concentração de clorofila-a (Chla) na Baía de Santos (Gianesella-Galvão, 1982; Moser, 2002, CETESB, 2005) indicando um aumento na biomassa de fitoplâncton. CETESB (2005) relataram valores de $10 \mu \mathrm{g}$ Chla. $\mathrm{L}^{-1}$ próximo a região de lançamento do emissário de Santos. Moser et al. (2005) encontraram uma concentração máxima de 97,4 $\mu \mathrm{g}$ Chla.L $\mathrm{L}^{-1}$. Além da concentração de nutrientes, o crescimento do fitoplâncton é limitado por outros fatores ambientais, incluindo luz e temperatura (García et al., 2010). No entanto, entre esses fatores, 
apenas os nutrientes podem ser controlados, por isso eles têm sido o foco da maioria dos esforços para controlar o crescimento de algas responsáveis pela deterioração da qualidade da água (Na e Park, 2006). O nitrogênio controla a produção primária e a eutrofização na maioria dos estuários e águas costeiras temperadas, enquanto o fósforo é o principal motivo de preocupação em águas tropicais (baías e corpos de água semi-fechados) e as vezes em alguns estuários de clima temperado (NRC, 2000). Como consequiência, diferenças ambientais significativas são esperadas se um tratamento com remoção de fósforo for instalado no lugar de tratamento preliminar.

Dentre as várias tecnologias de tratamento de esgoto, o Tratamento Preliminar Quimicamente Assistido tem sido considerado promissor para ser utlizado com emissários submarinos em locais onde é necessário uma qualidade melhor do efluente a ser lançado, pois requer uma baixa demanda de área e menor custo de investimento e operação quando comparado com sistemas convencioanis de tratamento de esgoto (Roberts et al., 2010). Segundo Jordão e Pessoa (2011), o TPQA em condições normais de dosagem de coagulante (10 - $30 \mathrm{mg} / \mathrm{L})$ pode atingir eficiências de 80 - 85\% Sólidos em Suspsensão Totais (SST), 50 - 70\% Demanda Química de Oxigênio $\left(\mathrm{DBO}_{5}\right)$ e de 50 - 90\% de fósforo caso seja aplicado dosagens de coagulante maiores que as convencionais.

Modelos de qualidade de água são ferramentas essenciais para avaliar o impacto das atividades humanas em ambientes costeiros, bem como a resposta ambiental em relação a diferentes configurações sanitárias adotadas, servindo como base no processo de tomada de decisão. O número elevado de fatores que influenciam os processos físicos, químicos e biológicos no ambiente marinho faz necessário considerar o uso de complexas ferramentas matemáticas para estudar e prever o impacto do lançamento de esgotos sanitários através de emissários submarinos. Nesse sentido, os modelos que simulam o processo de eutrofização aparecem como ferramentas computacionais importantes na avaliação do impacto ambiental em ambientes costeiros referente a mudanças na composição do efluente lançado por emissários submarinos. Esses modelos descrevem a interação entre as substâncias presente no esgoto e o ecossistema, incluindo variáveis físicas (batimetria, correntes, luminosidade, etc.) químicas (nutrientes) e biológicas (fitoplâncton). Alguns estudos foram feitos na última década utilizando modelos de eutrofização em diferentes regiões, merecendo destaque os trabalhos realizados por Chau e Jin (1998); Chao et al. (2007) e García et al. (2010).

Nesse contexto, o presente trabalho teve como objetivo principal avaliar se para o caso do emissário submarino de Santos haveria algum beneficio ambiental em relação a potencial de eutrofização se ao invés do tratamento preliminar fosse utilizado um Tratamento Primário Quimicamente Assistido (TPQA), Tratamento Secundário por Lodos Ativados (TS-LA) ou um Tratamento Secundário por Lodos Ativados e Remoção Biológica de Nutrientes (TTLARN).

\section{MATERIAIS E MÉTODO}

\subsection{Emissário Submarino de Santos (ESS)}

O sistema de disposição oceânica de Santos é composto por um tratamento preliminar e um emissário submarino responsável por lançar esgoto a uma vazão média de $3,0 \mathrm{~m}^{3} \cdot \mathrm{s}^{-1}$ e a uma distância de 4000 metros da costa a aproximadamente 11 metros de profundidade (Figura 1 e Tabela 2) (SABESP, 2006). Foi prevista uma reforma do sistema difusor do emissário submarino de Santos, alterando sua configuração. O novo sistema tem um comprimento total de 390 metros com 158 orifícios de diâmetro interno de 0,11 metros, sendo a altura de cada orifício de 1,2 m (SABESP, 2006). Além disso, a vazão máxima de descarga estabelecida é de $5,3 \mathrm{~m}^{3} \cdot \mathrm{s}^{-1}$. Nesse estudo, esta configuração foi adotada. O lançamento ocorre 
SUBTIL, E. L; MIERZWA, J. C.; ORTIZ, J. P. Influência do nível de tratamento de esgoto na qualidade da água do mar na região de lançamento do Emissário Submarino de Santos. Ambi-Agua, Taubaté, v. 7, n. 3, p. 72-86, 2012. (http://dx.doi.org/10.4136/ambi-agua.975)

no meio da baía de Santos sendo o impacto caracterizado pela recirculação da pluma na região da zona de mistura dentro da baía (Ortiz et al, 2007).

Atualmente, na Estação de Condicionamento Prévio, o esgoto é tratado a nível preliminar, sendo utilizadas peneiras rotativas com aberturas de $1,5 \mathrm{~mm}$ visando à remoção de sólidos grosseiros e uma pequena parcela de sólidos em suspensão (CETESB, 2006). As características do esgoto lançado pelo emissário submarino com tratamento preliminar, TPQA, TS-LA e TT-LARN, em termos de Demanda Bioquímica de Oxigênio (DBO), nitrogênio amoniacal $\left(\mathrm{NH}_{4}-\mathrm{N}\right)$ e fosfato $\left(\mathrm{PO}_{4}{ }^{3-}-\mathrm{P}\right)$, estão apresentadas na Tabela 1 , sendo estabelecidas com base em dados de literatura.

Tabela 1: Processos de tratamento simulados com as eficiências consideradas para o emissário submarino de Santos.

\begin{tabular}{l|ccc|ccc}
\hline \multirow{2}{*}{$\begin{array}{l}\text { Processo de } \\
\text { tratamento }\end{array}$} & \multicolumn{3}{|c|}{ Eficiências Esperadas (\%) } & \multicolumn{3}{c}{$\begin{array}{c}\text { Concentração no esgoto } \\
(\mathbf{m g} / \mathbf{L})\end{array}$} \\
\cline { 2 - 7 } & DBO & Amônia & Fósforo & DBO & Amônia & Fósforo \\
\hline Preliminar & - & - & - & 200 & 25,0 & 5,0 \\
TPQA & $60 \%$ & $30 \%$ & $80 \%$ & 80,0 & 17,5 & 1,0 \\
TS-LA & $90 \%$ & $60 \%$ & $35 \%$ & 20,0 & 10,0 & 3,8 \\
TT-LARN & $95 \%$ & $90 \%$ & $80 \%$ & 10,0 & 2,5 & 1,0 \\
\hline
\end{tabular}

Fonte: Metcalf \& Eddy (2003); Roberts et al. (2010).

Tabela 2: Localização do emissário submarino de Santos e das estações amostrais utilizadas nas calibrações do modelo.

\begin{tabular}{l|cc}
\hline \multirow{2}{*}{ Local } & \multicolumn{2}{|c}{ Localização (UTM) } \\
\cline { 2 - 3 } & Latitude & Longitude \\
\hline ESS & 362650 & 7344550 \\
Ponto Cotrole & 357648 & 7343385 \\
P1 & 362650 & 7344600 \\
P2 & 362700 & 7344600 \\
P3 & 362700 & 7344500 \\
P4 & 362600 & 7344500 \\
P5 & 362600 & 7344600 \\
P6 & 362600 & 7344700 \\
P7 & 362700 & 7344700 \\
P8 & 362800 & 7344700 \\
P9 & 362800 & 7344600 \\
\hline
\end{tabular}

\subsection{Métodos Computacionais Utilizados}

Para simulação dos cenários estabelecidos e avaliação do impacto do lançamento de esgoto pelos emissários submarinos foram utilizados o software CORMIX (versão 6.0 GT licença acadêmica) e o modelo computacional AQUALAB. O CORMIX foi utilizado para estabelecer a diluição ao final da zona de mistura para o emissário submarino com vazão de $5,3 \mathrm{~m}^{3} \cdot \mathrm{s}^{-1}$. O resultado do modelo de campo próximo foi acoplado no modelo de campo distante (modelo de qualidade da água - AQUALAB) utilizando a mesma abordagem de Bleninger (2006), o qual assume um acoplamento passivo, i.e., em um único sentido. A fonte (emissário submarino) é introduzida dentro das células do modelo de campo distante como um fluxo de volume que é igual ao fluxo de volume da fonte multiplicado pela diluição 
alcançada no final da zona de mistura, enquanto que a concentração do poluente é igual à concentração na fonte dividida pela mesma diluição.

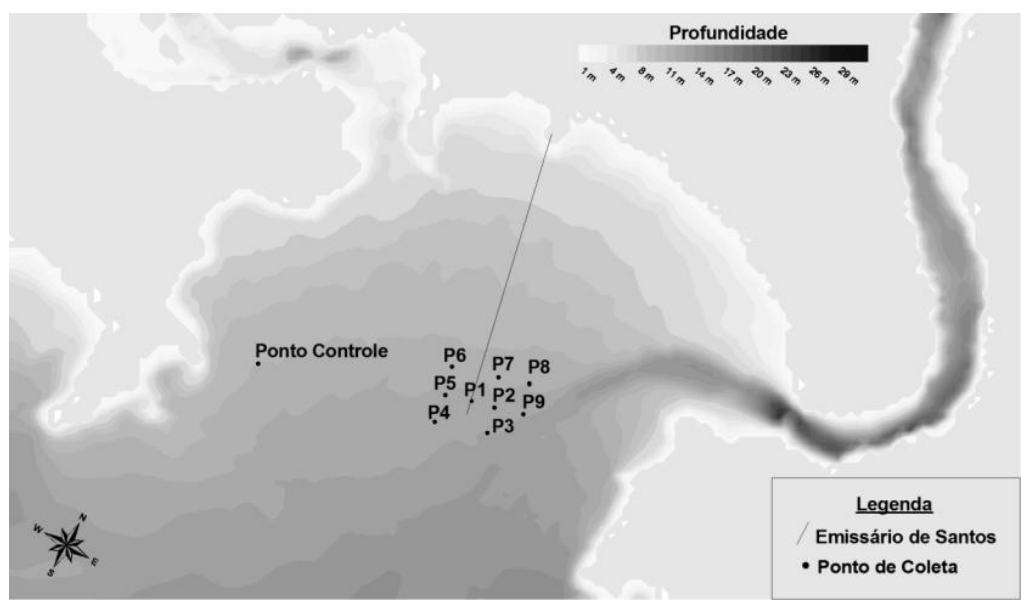

Figura 1: Representação esquemática da localização do emissário submarino com as estações amostrais da CETESB utilizadas no processo de calibração.

\section{Modelo Hidrodinâmico}

O modelo hidrodinâmico e de transporte utilizado nesse estudo resolve as equações de transporte e hidrodinâmica em duas dimensões integradas verticalmente. A computação numérica foi realizada em um domínio espacial através de uma grade de diferenças finitas com espaçamento horizontal de $90 \times 90 \mathrm{~m}$, totalizando 451827 pontos, que representa toda região de estudo. O sistema de equações é expressa em coordenadas cartesianas (x aumenta para leste e y aumenta para o norte) e assume uma aproximação hisdrostática e de Boussinesq. O resultado de uma simulação são os níveis de água e fluxos (velocidades) em cada célula do domínio computacional.

O modelo hidrodinâmico H2D usa a tecnica Alternating Direction Implicit (ADI) para integrar as equações de conservação de massa e da quantidade de movimento no tempo e no espaço, sendo estas expressas como:

$$
\begin{aligned}
& \frac{\partial H}{\partial t}+\frac{\partial(U H)}{\partial x}+\frac{\partial(V H)}{\partial y}=0 \\
& \frac{\partial(U H)}{\partial t}+\frac{\partial\left(U^{2} H\right)}{\partial x}+\frac{\partial(U V H)}{\partial y}=f V H-g H \frac{\partial \eta}{\partial x}-\frac{g H^{2}}{2 \rho_{0}} \frac{\partial \rho_{0}}{\partial x}+\frac{1}{\rho_{0}}\left[\tau_{x z(\eta)}-\tau_{x z(-h)}\right]+H v_{\theta}\left[\frac{\partial^{2} U}{\partial x^{2}}+\right. \\
& \left.\frac{\partial^{2} U}{\partial y^{2}}\right]+2 H \frac{\partial v_{\varepsilon}}{\partial x} \frac{\partial U}{\partial x}+H \frac{\partial v_{\theta}}{\partial y}\left[\frac{\partial U}{\partial y}+\frac{\partial V}{\partial x}\right]
\end{aligned}
$$

$$
\begin{aligned}
& \frac{\partial(V H)}{\partial t}+\frac{\partial(U V H)}{\partial x}+\frac{\partial\left(V^{2} H\right)}{\partial y}=f U H-g H \frac{\partial \eta}{\partial y}-\frac{g H^{2}}{2 \rho_{0}} \frac{\partial \rho_{0}}{\partial y}+\frac{1}{\rho_{0}}\left[\tau_{y z(\eta)}-\tau_{y z(-h)}\right]+H v_{e}\left[\frac{\partial^{2} V}{\partial x^{2}}+\right. \\
& \left.\frac{\partial^{2} V}{\partial y^{2}}\right]+2 H \frac{\partial v_{\varepsilon}}{\partial y} \frac{\partial V}{\partial y}+H \frac{\partial v_{\varepsilon}}{\partial x}\left[\frac{\partial U}{\partial y}+\frac{\partial V}{\partial x}\right]
\end{aligned}
$$


em que:

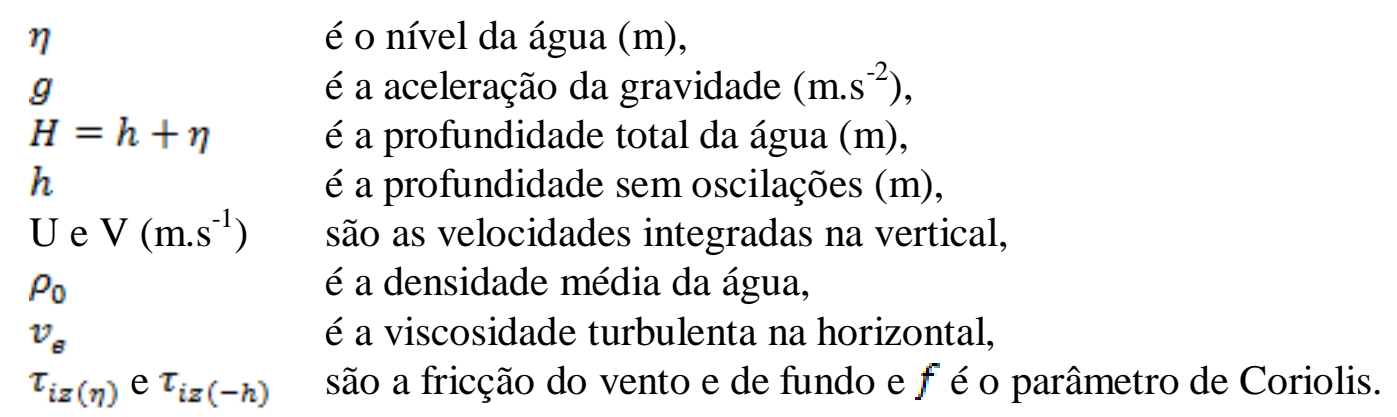

\section{Modelo de Qualidade de Água}

O modelo de qualidade de água resolve o sistema de equações diferencias que descrevem as principais interações químicas e biológicas do processo de eutrofização. O modelo simula o transporte e as reações de transformações de oito variáveis de estados (Figura 2), sendo elas agrupadas em 4 sistemas de interação: cinética do fitoplâncton, ciclo do nitrogênio, ciclo do fósforo e balanço da concentração de oxigênio dissolvido.

A evolução temporal e espacial dessas variáveis no modelo de qualidade de água são influenciadas por fatores externos como: incidência solar, temperatura, descargas de água doce e efluentes urbanos. O modelo de qualidade de água é acoplado com o modelo hidrodinâmico através da equação de transporte (Eq. 4), que integra as propriedades de advecção e difusão do fluxo, bem como os processos básicos que ocorrem na coluna d'água.

$$
\frac{\partial\left(H C_{i}\right)}{\partial t}+\frac{\partial\left(U H C_{i}\right)}{\partial x}+\frac{\partial\left(V H C_{i}\right)}{\partial y}=\frac{\partial}{\partial x}\left(H D_{x} \frac{\partial C_{i}}{\partial x}\right)+\frac{\partial}{\partial y}\left(H D_{y} \frac{\partial C_{i}}{\partial y}\right)+R_{i} H
$$

em que:

$C_{i}$ : concentração média na coluna da água de uma substância de qualidade da água $(i)$ (figura 2);

$R_{i}$ : descreve o termo de reação química, correspondentes às equações de interação para as variáveis de estado (Tabela 3).

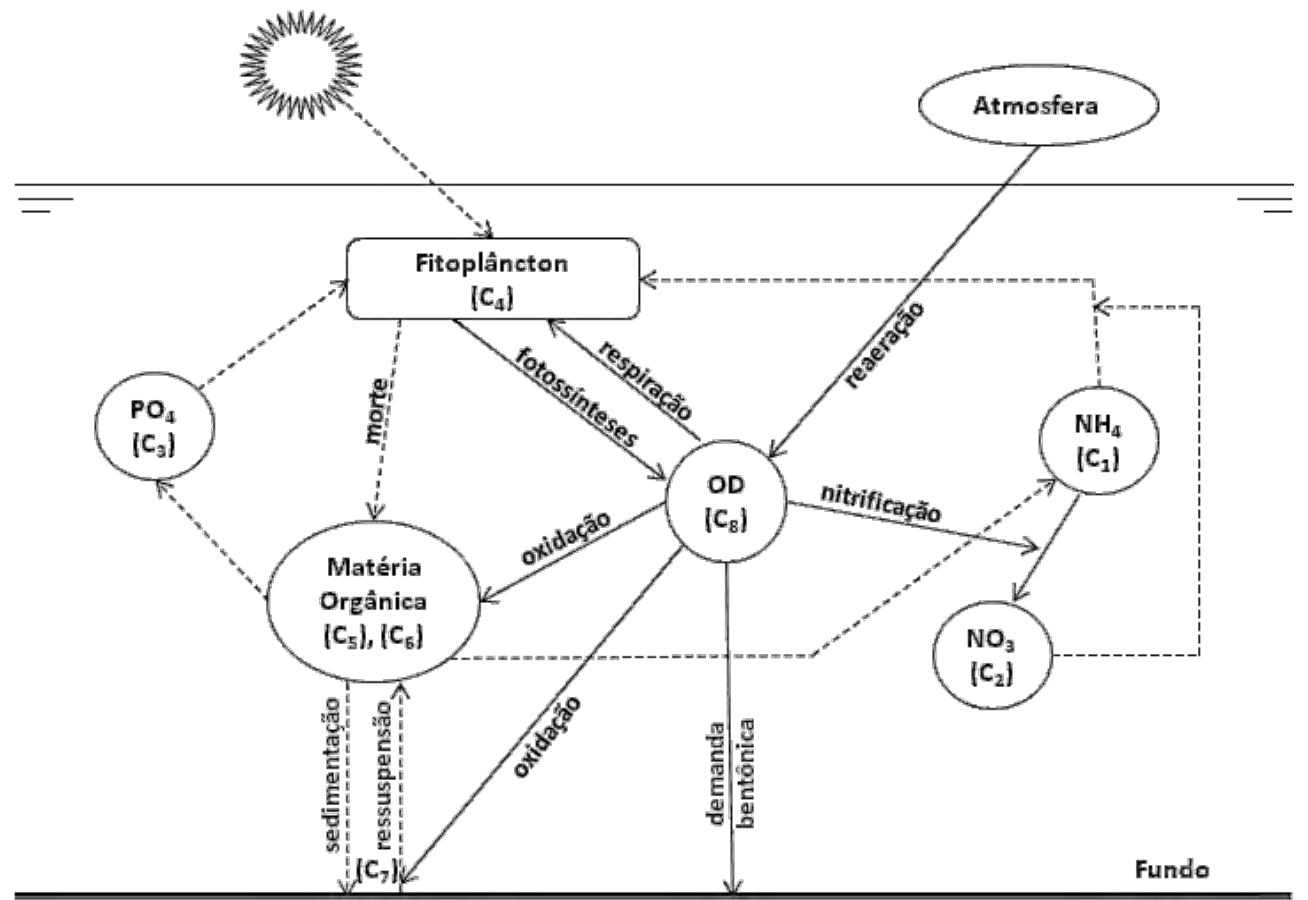

Figura 2: Diagrama conceitual das principais variáveis e processos simulados pelo modelo de eutrofização. 
O modelo desenvolvido permite que a Equação 4 seja resolvida usando um esquema de diferenças finitas explicito, no qual os processos de advecção e difusão são calculados de forma independente para cada passo de tempo (García et al., 2010). Essa abordagem permite o uso de diferentes métodos numéricos para resolver cada processo independentemente (Komatsu et al., 1997). Desta forma, a integração das equações do transporte advectivo é feita utilizando um esquema upwind enquanto que a difusão é resolvida por um esquema centrado. Para assegurar soluções estáveis para a equação de transporte, foi utilizado um passo de tempo de integração $(\Delta t)$ de 20 segundos. O período total simulado foi de 3 meses (01/12/2005 até 28/02/2006) sendo utilizado apenas os dados do mês de fevereiro para avaliação dos resultados.

Tabela 3. Sistema de interação do modelo de qualidade de água.

\begin{tabular}{ll}
\hline Variável & \multicolumn{1}{c}{ Equação de Interação } \\
\hline $\mathrm{NH}_{4}-\mathrm{N}$ & $\frac{d C_{1}}{d t}=Y_{d} K_{d} \theta_{d}^{(T-20)} C_{5}+Y_{s} K_{s} \theta_{s}^{(T-20)} C_{6}+Y_{b} K_{b} \theta_{b}^{(T-20)} C_{7}+\left(k_{r} \theta_{r}^{(T-20)}+K_{d m}\right) a_{n c}\left(1-f_{o n}\right) C_{4}-$ \\
& $G(T) G(I) G(N) P_{N H_{3}} a_{n c} C_{4}-k_{n} \theta_{n}^{(T-20)}\left[\frac{C_{8}}{\left(C_{8}+k_{n i t}\right)}\right] C_{1}$ \\
$\mathrm{NO}_{3}-\mathrm{N}$ & $\frac{d C_{2}}{d t}=k_{n} \theta_{n}^{(T-20)}\left[\frac{C_{8}}{\left(C_{8}+k_{n i t}\right)}\right] C_{1}-G(T) G(I) G(N)\left(1-P_{N H_{3}}\right) a_{n c} C_{4}-k_{d n} \theta_{d n}^{(T-20)}\left[\frac{K_{N o}}{\left(C_{8}+k_{N O}\right)}\right] C_{2}$ \\
$\mathrm{PO}_{4}-\mathrm{P}$ & $\frac{d C_{1}}{d t}=Y_{d 2} K_{d} \theta_{d}^{(T-20)} C_{5}+Y_{s 2} K_{s} \theta_{s}^{(T-20)} C_{6}+Y_{b 2} K_{b} \theta_{b}^{(T-20)} C_{7}+a_{p c}\left(k_{r} \theta_{r}^{T-20}+K_{d m}\right)\left(1-f_{o p}\right) C_{4}-$ \\
& $G(T) G(I) G(N) a_{p c} C_{4}$ \\
Fito-C & $\frac{d C_{4}}{d t}=\left[G(T) G(I) G(N)-\left(k_{r} \theta_{r}^{T-20}+K_{d m}\right)-\frac{V_{p h}}{H}\right] C_{4}$ \\
$\mathrm{DBO}_{\text {dis }}$ & $\frac{d C_{5}}{d t}=-K_{d} \theta_{d}^{(T-20)} C_{5}$ \\
$\mathrm{DBO}_{\text {sus }}$ & $\frac{d C_{6}}{d t}=\frac{V_{r}}{H} C_{7}+\left(k_{r} \theta_{r}^{(T-20)}+K_{d m}\right)\left(a_{n c} f_{o n}+a_{p c} f_{o p}\right) C_{4}-\frac{V_{s}}{H} C_{6}-K_{s} \theta_{s}^{(T-20)} C_{6}$ \\
$\mathrm{DBO}_{\text {sed }}$ & $\frac{d C_{6}}{d t}=\frac{V_{s}}{H} C_{6}-\frac{V_{r}}{H} C_{7}-K_{b} \theta_{b}^{(T-20)} C_{7}+\frac{V_{p h}}{H} C_{4}$ \\
$\mathrm{OD}$ & $\frac{d C_{8}}{d t}=k_{a} \theta_{a}^{(T-20)}\left(C_{s}-C_{8}\right)+G(T) G(I) G(N)\left[\frac{32}{12}+\frac{48}{14} a_{n c}\left(1-P_{N H_{3}}\right)\right] C_{4}-\frac{32}{12} k_{r} \theta_{r}^{(T-20)} C_{4}-$ \\
& $\frac{64}{14} k_{n} \theta_{n}^{(T-20)}\left[\frac{C_{8}}{\left(C_{8}+k_{n}\right)}\right] C_{1}-\left(Y_{d} K_{d} \theta_{d}^{(T-20)} C_{5}+Y_{s} K_{s} \theta_{s}^{(T-20)} C_{6}+Y_{b} K_{b} \theta_{b}^{(T-20)} C_{7}\right)-\frac{S O D}{H} \theta_{S O D}^{(T-20)}$ \\
\hline
\end{tabular}

\section{RESULTADOS E DISCUSSÃO}

\subsection{Calibração do modelo hidrodinâmico}

O modelo hidrodinâmico foi calibrado utilizando-se dados de elevação de superfície coletados por um marégrafo instalado na região de Praia Grande no mês de julho de 2005 durante um período de 18 dias (SABESP, 2006). Devido a escassez de dados de velocidade o ajuste do modelo hidrodinâmico foi focado em reproduzir da melhor forma a onda de maré dentro do domínio modelado. Na Figura 3 são apresentados os resultados do modelo hidrodinâmico para o período de calibração. Pode-se observar uma boa correlação entre os dados de elevação de superfície medidos e modelado. Além da correlação significativa entre os dados do modelo H2D utilizando as constantes da SABESP (2006), pode-se observar na Figura 3 que a onda de maré apresenta co-oscilação com período semi-diurno e desigualdades diárias, cujo resultado é o mesmo reportado por Harari et al. (2008). O mesmo autor cita que as marés em Santos possuem amplitudes de $0,60 \mathrm{~m}$ e $0,14 \mathrm{~m}$, para maré de sizígia e quadratura, respectivamente. Resultado semelhante é apresentado por Picarelli (2001), onde a região denominada Centro-Sul do Estado de São Paulo, que abrange desde o município de Praia Grande até o complexo estuarino lagunar de Iguape e Cananéia, pode ser classificada como possuindo maré-mista, principalmente do tipo semi-diurno, com 2 preamares e 2 baixamares com desigualdades em alturas. 
Além do nível do mar, outra importante caracterização hidrodinâmica da região está relacionada com as correntes. Os campos de velocidades gerados pelo modelo H2D durante maré de sizígia e quadratura são apresentados na Figura 4. Pode-se observar, conforme esperado, maiores velocidades durante maré de sizígia, com velocidades médias máximas na coluna da água de $0,25 \mathrm{~m} / \mathrm{s}$ e $0,36 \mathrm{~m} / \mathrm{s}$ na região do emissário submarino de Santos e Praia Grande 2, respectivamente.
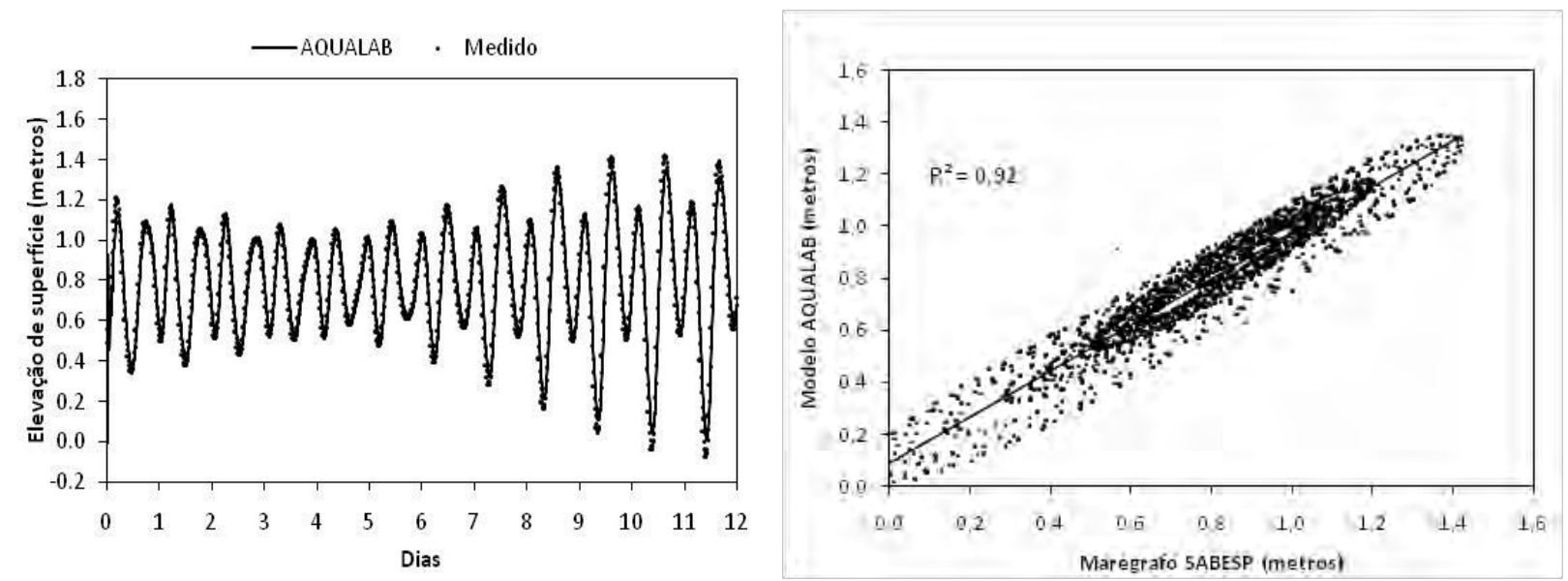

Figura 3: Elevação de superfície (A) e correlação (B) dos resultados do modelo e dos dados de medidos em campo.

\subsection{Calibração do modelo de qualidade}

Um processo de calibração foi desenvolvido baseado em dados coletados durante uma campanha de campo na região de estudo em período de verão. Os dados de qualidade de água utilizados nesse processo foram gerados pela Companhia de Tecnologia de Saneamento Ambiental do Estado de São Paulo (CETESB) e referem-se a um dia de coleta realizada na data de 22 de fevereiro de 2006, na região de influência do emissário submarino de Santos/São Vicente (Figura 1). Os resultados dos parâmetros Nitrogênio Amoniacal, fosfato, Oxigênio Dissolvido e clorofila-a são apresentados na Figura 5. Com base nessas figuras é possível observar que o modelo reproduz a tendência geral dos dados. No caso da amônia, o modelo superestima a concentração nos pontos 3 e 4 , estando os resultados acima do desvio padrão, sobretudo no ponto 3 , enquanto que nos outros pontos, incluindo o ponto controle, os valores estimados pelo modelo encontram-se dentro da faixa de variação e, em alguns casos, bem próximo da média.

Resultados semelhantes aos do nitrogênio amoniacal foram encontrados para o fosfato, onde no ponto 3 o modelo superestima sua concentração e no ponto controle o modelo a subestima. Nos outros pontos os resultados encontram-se dentro da faixa de variação para concentração de fosfato. A diferença entre os valores do modelo e os dados medidos nos pontos 3 e 4 podem estar relacionado com a forma na qual o emissário submarino foi introduzido no modelo de qualidade, haja vista que nessa etapa de calibração o sistema foi considerado uma fonte pontual, pois não havia informações sobre como estava funcionando o emissário submarino para o período que foi realizada a coleta pela CETESB em 2006. Embora exista essa discrepância para os pontos 3 e 4, ao menos para amônia e fosfato, os valores do modelo para o ponto controle estiveram bem próximo para essas duas substâncias.

Outro parâmetro de qualidade da água utilizado no processo de calibração foi a concentração de oxigênio dissolvido (Figura 5). Pode-se observar uma boa concordância entre os dados de medição e do modelo T2D8, com exceção do ponto 3 , onde a concentração de oxigênio dissolvida, computada pelo modelo, encontra-se fora da faixa de variação e abaixo do valor medido por CETESB (2006). 


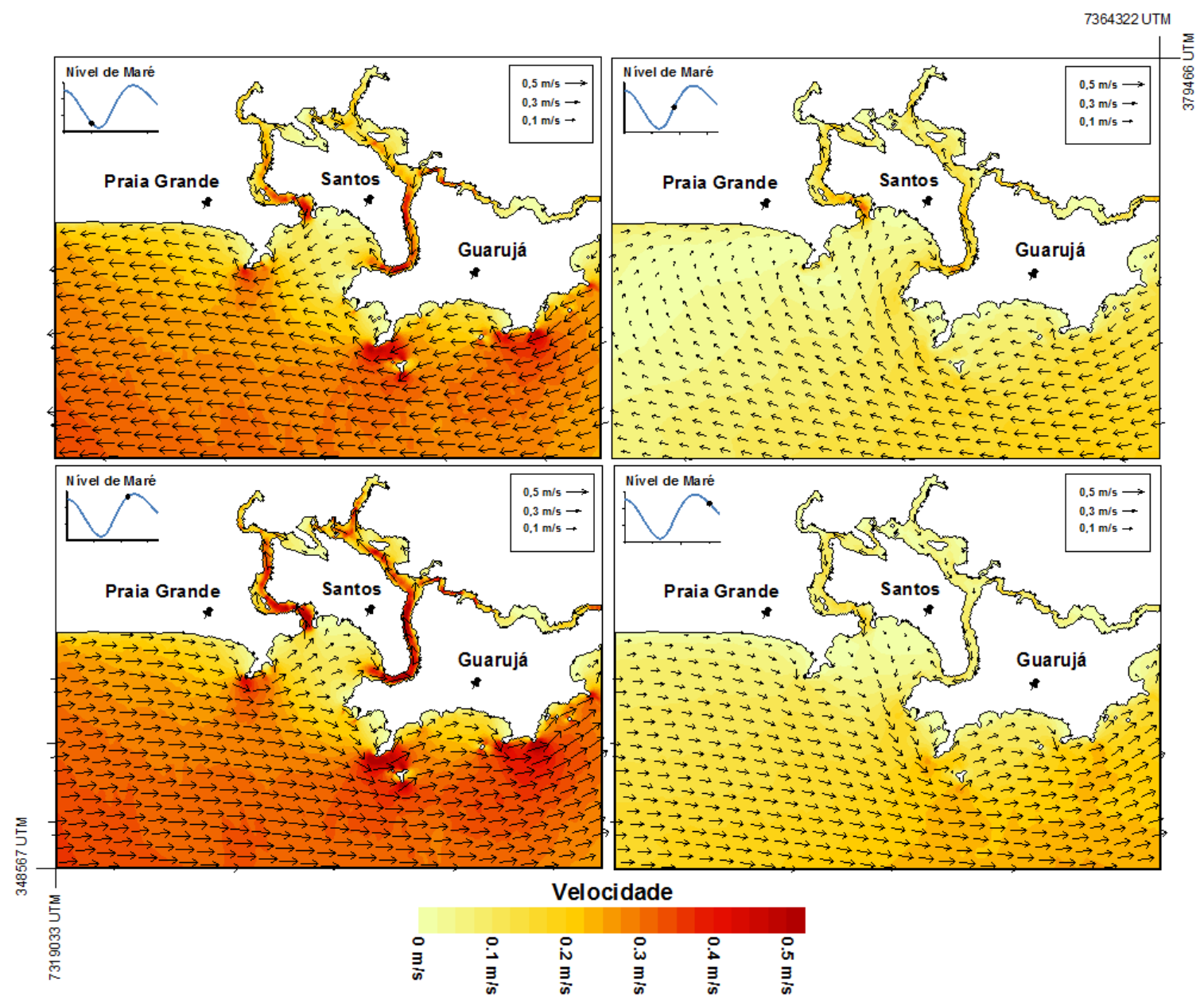

Figura 4: Campos de velocidade gerados pelo modelo H2D para diversos instantes de maré.

Em relação à concentração de fitoplâncton (Figura 5) o erro médio relativo é menor que $10 \%$ para as estações amostrais 1,5 e 7 e de $38 \%$ na estação 9 , todas localizadas na região do emissário submarino de Santos. No entanto, o modelo superestima a concentração de fitoplâncton no ponto controle com um erro médio maior que $200 \%$, sendo justamente nessa região o local que, segundo o modelo, haveria condições favoráveis para o crescimento do fitoplâncton. Esse resultado pode estar relacionado com o fato do modelo T2D8 não levar em consideração o transporte de sedimentos coesivos, o que influi diretamente no coeficiente de extinção da luz e, consequentemente, na Zona Eufótica (ZE) podendo, em alguns casos, limitar o crescimento do fitoplâncton. Contudo, vale ressaltar que valores superiores aos relatados por CETESB (2006) foram encontrados por Ancona (2007), que realizou um monitoramento na região da baía de Santos durante o período de novembro de 2004 e dezembro de 2005, obtendo valores de Chl-a para os meses de Fev/2005 de 15,41 e 22,79 $\mu \mathrm{g}$ $\mathrm{Cl}-\mathrm{a} / \mathrm{l}$ na superfície e fundo, respectivamente. Utilizando a mesma relação $\mathrm{Chl}-\mathrm{a} / \mathrm{C}$ do modelo de qualidade a concentração de fitoplâncton estaria entre 771 - $1140 \mu \mathrm{g} \mathrm{C} / \mathrm{L}$. Isso indica que sob condições ótimas o fitoplâncton nessa região poderia atingir valores superiores ao que foi calculado pelo modelo e reportados por CETESB (2006). Considerando que esses dados representam apenas um dia de coleta é possível dizer que o modelo consegue reproduzir os principais processos do ambiente quando submetido ao lançamento de esgoto, podendo ser utilizado para avaliar o impacto e a resposta ambiental frente a diferentes configurações sanitárias adotadas. 

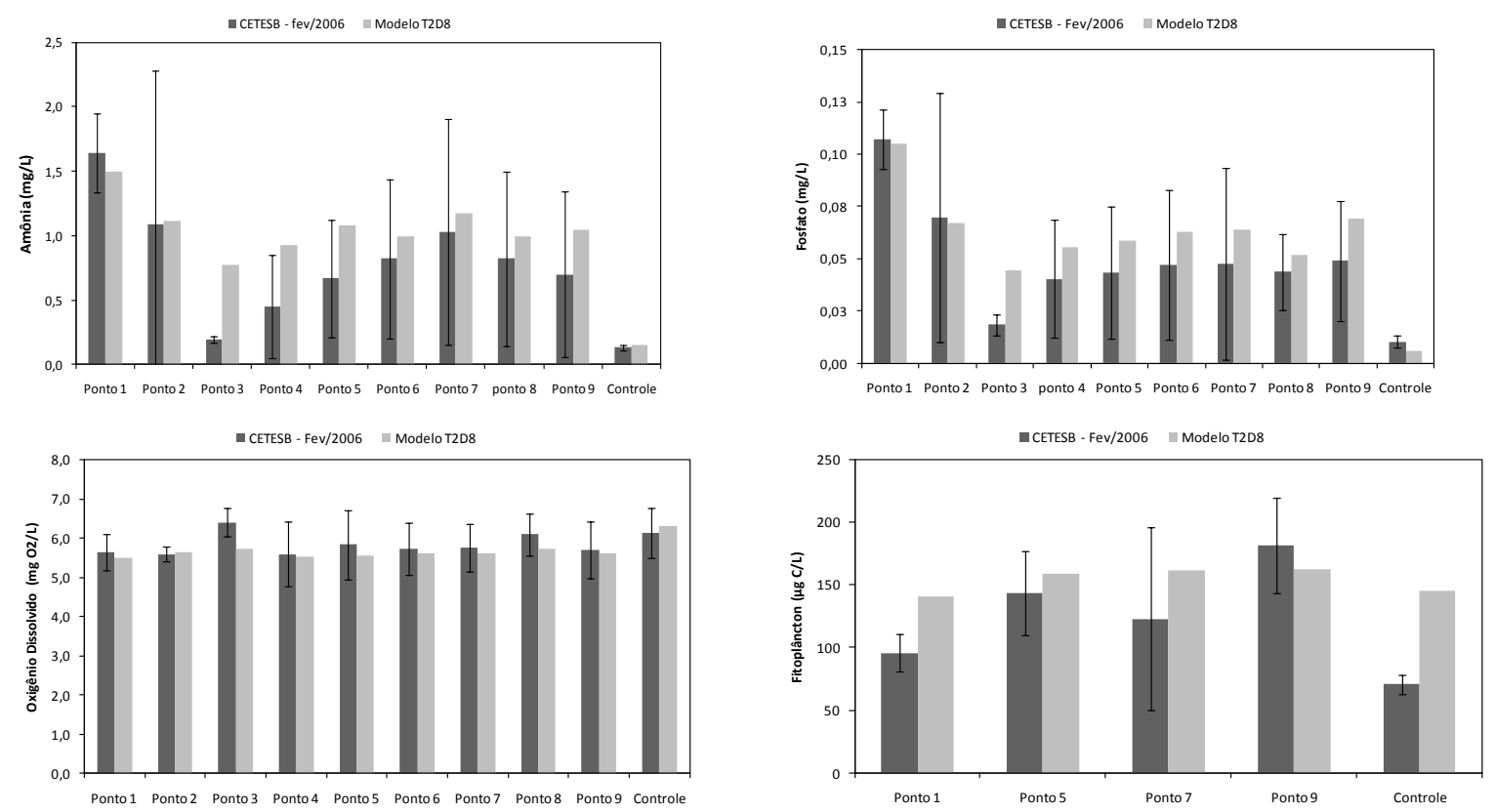

Figura 5: Comparação entre os resultados de qualidade da água do modelo T2D8 e dos dados obtidos pela CETESB no mesmo período simulado (26/02/2006).

\subsection{Influência do processo de tratamento na qualidade da água do mar}

Tendo em vista a necessidade de tratamento do esgoto lançado pelo emissário submarino de Santos em termos de nutrientes, sobretudo fósforo, e o potencial do Tratamento Primário Quimicamente Assistido (TPQA) para utilização com emissários submarinos, foi feita uma comparação para avaliar se haveria algum beneficio ambiental se ao invés do TPQA fosse utilizado um Tratamento Secundário por Lodos Ativados Convencional (TS-LAC) ou um sistema de Lodos Ativados com Remoção de Nutrientes (TT-LARN). Os resultados para o emissário submarino de Santos operado com uma vazão média de $5,3 \mathrm{~m}^{3} \cdot \mathrm{s}^{-1}$ são apresentados na Figura 6. A utilização do tratamento secundário por um sistema de lodos ativados convencional possibilitaria uma redução média de $30 \%$ na concentração de Chl-a quando comparado com o tratamento preliminar, enquanto que o TPQA e o TT-LARN promoveriam uma redução média de 69 e 77\%, respectivamente, mais do que o dobro do tratamento secundário. Nesse sentido, pode-se observar que a o efluente produzido pelo TPQA produz um efeito no crescimento do fitoplâncton e, consequentemente na concentração de clorofila-a, semelhante ao esgoto lançado pelo emissário submarino com tratamento terciário por lodos ativados com remoção de nitrogênio e fósforo (Figura 7).
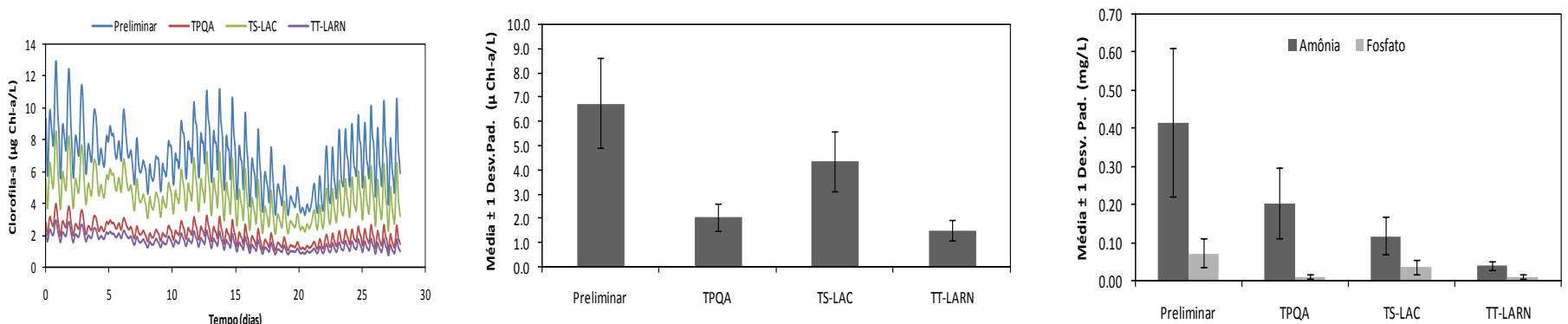

Figura 6: Concentração de clorofila-a, nitrogênio amoniacal e fosfato em função da tecnologia de tratamento para o emissário submarino de Santos com vazão de $5,3 \mathrm{~m}^{3} \cdot \mathrm{s}^{-1}$ 
SUBTIL, E. L; MIERZWA, J. C.; ORTIZ, J. P. Influência do nível de tratamento de esgoto na qualidade da água do mar na região de lançamento do Emissário Submarino de Santos. Ambi-Agua, Taubaté, v. 7, n. 3, p. 72-86, 2012. (http://dx.doi.org/10.4136/ambi-agua.975)

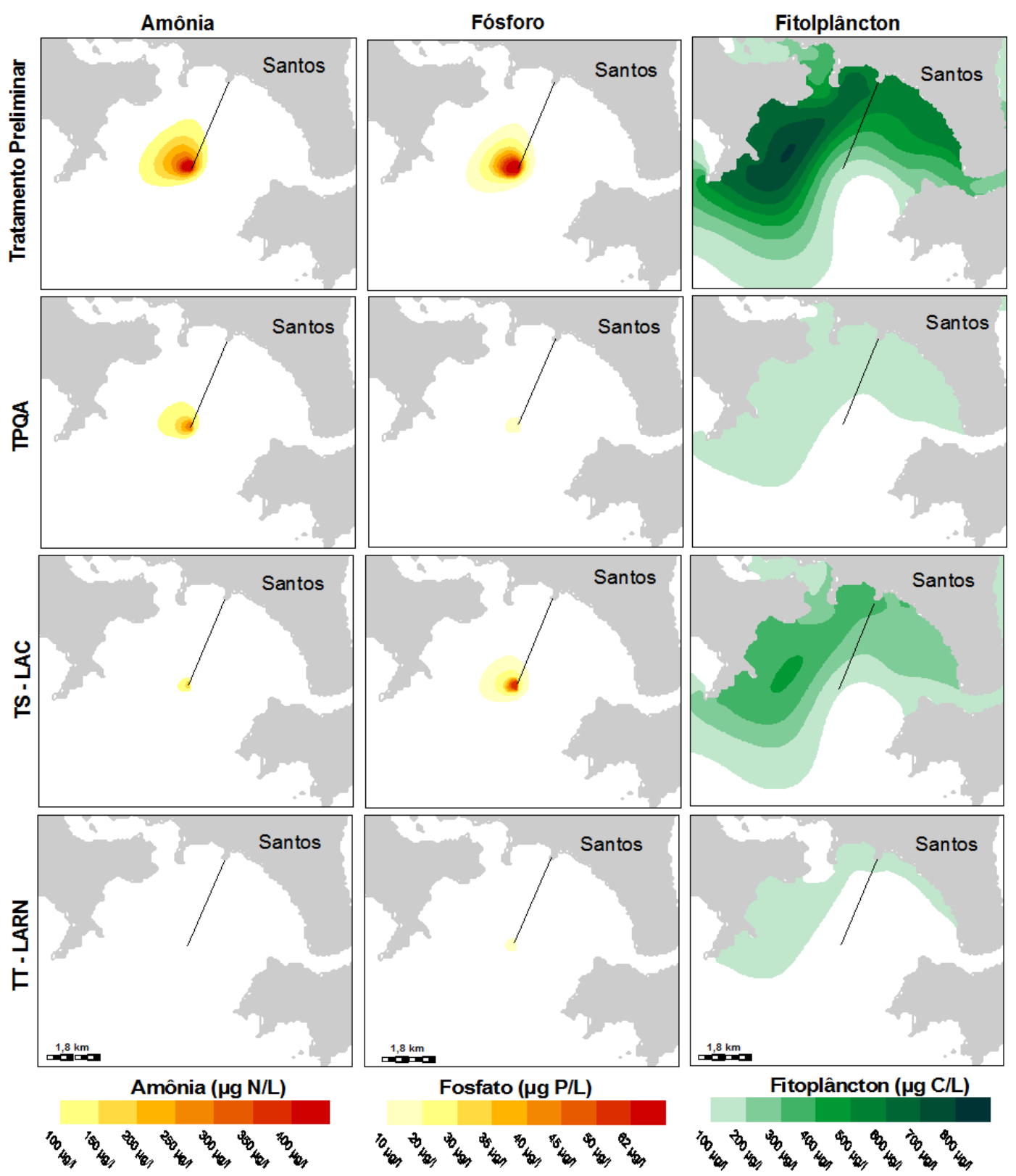

Figura 7: Variação espacial de amônia, fosfato e fitoplâncton na baía de Santos para o emissário submarino de Santos (Lat. 362650 - Long. 7344550) operado com vazão de $5,3 \mathrm{~m}^{3} \cdot \mathrm{s}^{-1}$.

Quando comparados os tratamentos TPQA e TS-LAC não se observa nenhum benefício ambiental em termos de potencial de eutrofização. Na verdade, o efluente proveniente do TPQA, ou seja, baixa concentração de fósforo, apresenta maiores benefícios do que o efluente produzido pelo TS-LAC que possui baixa concentração de DBO $(20 \mathrm{mg} / \mathrm{L})$ e amônia, mas, em contrapartida, possui concentrações significativamente maiores de fósforo do que o efluente do TPQA. Resultados semelhantes foram reportados por (Grigg e Dollar, 1995; Rodgers, 2002), onde no Hawaii e em San Diego (EUA) foi demonstrado que a mudança do tratamento físico-químico por um tratamento secundário não traria nenhum benefício significativo para o meio ambiente marinho. Desta forma, embora a combinação TPQA e emissário submarino apresente valores de amônia acima de 0,4 mg N/L durante períodos de maré de quadratura, limite CONAMA (CONAMA 357/2005), o efeito produzido na concentração de clorofila-a não é significativo, haja vista a diferença dos valores quando 
comparado com o TT-LARN. Nesse sentido, para alguns casos onde não é possível atingir diluição inicial elevada (baixa profundidade e/ou elevadas vazões), o controle da concentração de fósforo apenas apresenta-se como uma medida eficiente para evitar problemas de eutrofização, sendo o TPQA uma tecnologia viável para atender esse critério.

\section{CONCLUSÕES}

Com base nos resultados obtidos pode-se concluir que para o caso do emissário submarino de Santos com vazão de $5,3 \mathrm{~m}^{3} \cdot \mathrm{s}^{-1}$ e tratamento preliminar, associado com a condição atual de descarga, haveria grande potencial de desenvolvimento de condições eutróficas na maior parte da baía de Santos. Além disso, apesar da redução de nitrogênio conjuntamente com fósforo ser uma medida eficiente para evitar o crescimento do fitoplâncton, os resultados demonstraram que apenas a redução de fósforo é necessária para reduzir a concentração de clorofila-a, mesmo que, em alguns casos, a concentração de amônia esteja acima do permitido pela legislação e da concentração no corpo receptor. Nesse sentido, a utilização de um tratamento secundário apenas com remoção de matéria orgânica e oxidação parcial da amônia não teria benefício significativo em relação ao processo de eutrofização na baía de Santos quando comparado com o TPQA e TT-LARN sendo, ainda, que o TPQA promoveu reduções semelhantes na concentração de clorofila-a das obtidas com o TT-LARN.

\section{AGRADECIMENTOS}

Os autores agradecem a Fundação de Apoio a Pesquisa do Estado de São Paulo (FAPESP) pelo suporte financeiro e ao Grupo de Emissários Submarinos e Hidráulica Ambiental (GESHA) da Universidade de Cantabria (Espanha), pela utilização do software AQUALAB.

\section{REFERENCIAS}

ANCONA, C. M. Aspectos da variação espacial e temporal da biomassa e produção fitoplânctônica e parâmetros correlatos no estuário e baía de Santos. 2007. 229 f. Dissertação (Mestrado em Oceanografia Biológica) - Instituto Oceanógrafo, Universidade de São Paulo, São Paulo, 2007.

BLENINGER, T. Coupled 3D hydrodynamic models for submarine outfalls: environmental hydraulic design and control of multiport diffusers. 2006. Tese (Doutorado em Hidromecânica) - University of Karlsruhe, Karlsruhe, 2006.

BRAGA, E. S.; BONETTI, C. V. D. H.; BURONE, L.; BONETTI FILHO, J. Eutrophication and bacterial pollution caused by industrial and domestic wastes at Baixada Santista Estuarine System- Brazil. Marine Pollution Bulletin, v. 40, n. 2, p. 165-173, 2000. http://dx.doi.org/10.1016/S0025-326X(99)00199-X

BRASIL. Conselho Nacional do Meio Ambiente. Resolução 357, de 17 de março de 2005. Disponível em: <http://www.mma.gov.br/port/conama/res/res05/res35705.pdf>. Acesso em: 20 dez. 2012.

BRASIL. Conselho Nacional do Meio Ambiente. Resolução 430, de 13 de maio de 2005. Disponível em: <http://www.mma.gov.br/port/conama/legiabre.cfm?codlegi=646>. Acesso em: 20 dez. 2012. 
COMPANHIA DE SANEAMENTO BÁSICO DO ESTADO DE SÃO PAULO - SABESB. Programa de monitoramento ambiental da área sob influência dos emissários submarinos de esgotos de Santos / São Vicente e Praia Grande: subsistemas 1, 2 e 3. Relatório Final. São Paulo, 2006

COMPANHIA DE TECNOLOGIA DE SANEAMENTO AMBIENTAL - CETESB. Relatório de qualidade das águas litorâneas no estado de São Paulo: balneabilidade das praias 2004. São Paulo, 2005.

COMPANHIA DE TECNOLOGIA DE SANEAMENTO AMBIENTAL - CETESB. Relatório de qualidade das águas litorâneas no estado de São Paulo: balneabilidade das praias 2005. São Paulo, 2006.

CHAO, X.; JIA, Y.; SHIELDS JR., F. D.; WANG, S. Y.; COOPER, C. M. Numerical modeling of water quality and sediment related processes. Ecological Modelling v. 201, n. 3/4, p. 385-397, 2007. http://dx.doi.org/10.1016/j.ecolmodel.2006.10.003

CHAU, K.W.; JIN, H. Eutrophication model for a coastal bay in Hong Kong. Journal of $\begin{array}{lllllll}\text { Environmental Engineering, } & \text { v. 124, n. 7, p. 628-638, }\end{array}$ http://dx.doi.org/10.1061/(ASCE)0733-9372(1998)124:7(628)

DHAGE S. S.; CHANDORKAR, A. A.; KUMAR, R.; SRIVASTAVA, A.; GUPTA, I. Marine water quality assessment at Mumbai West Coast. Environment International, v. 32, n. 2, p. 149-158, 2006. http://dx.doi.org/10.1016/j.envint.2005.08.005

ECHAVARRI-ERASUN, B.; JUANES, J. A.; PUENTE, A. REVILLA, J. A. Coastal outfalls, a sustainable alternative for improving water quality in north-east Atlantic estuaries. Journal of Environmental Monitoring, v. 12, p. 1737-1746, p. 2010. http://dx.doi.org/10.1039/B927243G

GARCÍA, A.; JUANES, J. A.; ÁlVAREZ, C.; REVILLA, J. A.; MEDINA, R. Assessment of the response of a shallow macrotidal estuary to changes in hydrological and wastewater inputs through numerical modelling. Ecological Modelling, v. 221, n. 8, p. 1194-1208, 2010. http://dx.doi.org/10.1016/j.ecolmodel.2009.12.027

GIANESELlA-GALVÃO, S. M. F. Standing-stock and potential of phytoplankton production in the bay of Santos, Brazil. Boletim do Instituto Oceanográfico, v. 31, n. 2, p. 85-94, 1982. http://dx.doi.org/10.1590/S0373-55241982000200009

GRIGG, R. W.; DOLLAR, S. J. Environmental protection misapplied: alleged versus documented impacts of a deep ocean sewage outfall in Hawaii. Ambio, v. 24, n. 2, p. 125-128, 1995. http://www.jstor.org/stable/4314309

HARARI, J.; FRANÇA, C. A. S.; CAMARGO, R. Climatology and hydrography of Santos estuary. In: NEVES, R; BARETTA, J.; MATEUS, M. (Orgs.). Perspective on integrated coastal zone management in south America. Portugal: IST Press, 2008.

JORDÃO, E. P.; PESSOA, C. A. Tratamento de esgotos domésticos. 6. ed. Rio de Janeiro: ABES, 2011. 968 p. 
JUANES, J. A.; REVILlA, J. A.; ÁlVAREZ, C.; GARCÍA, A.; PUENTE, A.; NIKOLOV, K. Environmental design and monitoring of large submarine outfalls: an integrated approach for coastal protection. In: ZIMMERMANN, C.; DEAN, R. G.; PENCHEV, V.; VERHAGEN, H. J. (Eds.). Environmentally friendly coastal protection. Dordrecht: Springer, 2005. p. 243-253. (NATO Science, 53).

KOMATSU, T.; OHGUSHI, K.; ASAI, K. Refined numerical scheme for advective transport in diffusion simulation. Journal of Hydraulic Engineering, v. 123, n. 1, p. 41-50, 1997. http://dx.doi.org/10.1061/(ASCE)0733-9429(1997)123:1(41)

METCALF \& EDDY INC. Wastewater engineering: treatment, disposal, reuse. 4. ed. New York: McGraw-Hill, 2003.

MOSER, G. A. O. Aspectos da eutrofizacão no sistema estuarino de São Vicente-Santos: distribuição espaço temporal da biomassa e produtividade primária fitoplânctonica e transporte instantâneo de sal, clorofila-a, material em suspensão e nutrientes. 2002. 246 f. Tese (Doutorado em Oceanografia) - Instituto Oceanográfico, Universidade de São Paulo, São Paulo, 2002.

MOSER, G. A. O.; GIANESEllA, S. M. F.; ALBA, J. J. B.; BÉRGAMO, A. L.; SALDANHA-CORREAA, F. M. P.; MIRANDA, L. B. et al. Instantaneous transport of salt, nutrients, suspended matter and chlorophyll-a in the tropical estuarine system of Santos. Brazilian journal of oceanography, v. 53, n. 3/4. p. 115-127, 2005. http://dx.doi.org/10.1590/S1679-87592005000200003

NA, E. H.; PARK, S. S. A hydrodynamic and water quality modelling study of spatial and temporal patterns of phytoplankton growth in a stratified lake with buoyant incoming flow. Ecological Modeling, v. 199, n. 3, p. 298-314, 2006. http://dx.doi.org/10.1016/j.ecolmodel.2006.05.008

NATIONAL RESEARCH COUNCIL - NRC; COMMITTEE ON THE CAUSES AND MANAGEMENT OF ENTROPHICATION; OCEAN STUDIES BOARD; WATER SCIENCE AND TECHNOLOGY BOARD. Understanding and reducing the effects of nutrient pollution. Washington, D.C.: National Academy Press, 2000.

ORTIZ, J. P.; ALFREDINI, P.; ARASAKI, E. Santos submarine outfall wastewater dispersion process: computer and physical modeling evaluation. In: INTERNATIONAL SYMPOSIUM ON FLUID CONTROL, MEASUREMENT AND VISUALIZATION FLUCOME, 9., 2007, Tallahassee. Proceedings... Tallahassee: Florida State University, 2007.

ORTIZ, J. P.; SUBTIL, E. L. New design of Santos submarine outfall: hydrodynamic modeling simulation at The Santos Bay. In: INTERNATIONAL SYMPOSIUM ON OUTFALL SYSTEMS, 2011, Mar del Plata. Papers... [S.1.]: OSMGP, 2011. Disponível em: <http://www.osmgp.gov.ar/symposium2011/Papers/70-Ortiz.pdf>. Acesso em: 20 dez. 2012.

PICARELLI, S. S. Modelagem numérica da circulação da maré na região costeira centro-sul do Estado de São Paulo. 2001. 116 f. Dissertação (Mestrado em Oceanografia Biológica) - Instituto Oceanográfico, Universidade de São Paulo, São Paulo, 2001. 
ROBERTS, P. J. W.; SALAS, J. H.; REIFF, F. M.; LIBHABER, M.; LABBE, A.; THOMSON, J. C. Marine wastewater outfalls and treatment systems. London: IWA Publishing, 2010.

RODGERS, T. Treated sewage waiver signed. Union Tribune, San Diego, 2002, 14 Sep. 2002.

SUBTIL, E. L.; MIERZWA, J. C.; ORTIZ, J. P. Improvement in preconditioning treatment system of Santos Submarine Outfall. In: INTERNATIONAL SYMPOSIUM ON ENVIRONMENTAL HYDRAULICS, 6., 2010, Atenas. Proceedings... Atenas: CRC Press, 2010.

SUBTIL, E. L.; MIERZWA, J. C.; ORTIZ, J. P.; GARCIA, R. S.; GARCIA, A.; ALBA, J. G. Environmental response of wastewater inputs through Santos Submarine Outfall (SSO) utilizing an eutrophication numerical model. Journal of Shipping and Ocean Engineering, v. 2, p. 68-75, 2012.

SUBTIL, E. L.; MIERZWA, J. C.; ORTIZ, J. P.; ROBINSON, R. S.; GARCIA, A.; ALBA, J. G. Study of the influence of Chemically Enhanced Primary Tratment (CEPT) in the water quality of Santos Submarine Outfall (SSO) using an eutrophication numerical model. In: INTERNATIONAL SYMPOSIUM ON OUTFALL SYSTEMS, 2011, Mar del Plata. Papers... [S.1.]: OSMGP, 2011. Disponível em: <http://www.osmgp.gov.ar/ symposium2011/Papers/53_Subtil.pdf $>$. Acesso em: 20 dez. 2012.

THOMPSON, P.; WAITE, A. Phytoplankton responses to wastewater discharges at two sites in Western Australia. Marine \& Freshwater Research, v. 54, n. 6, p. 721-735, 2003. http://dx.doi.org/10.1071/MF02096

WERME, C.; HUNT, C. D. 2005 outfall monitoring overview. Boston: Massachusetts Water Resources Authority, 2006. Report ENQUAD 2006-18. 105p. 


\begin{tabular}{ccc|} 
ISSN = 1980-993X - doi:10.4136/1980-993X \\
www.ambi-agua.net \\
E-mail: ambi-agua@agro.unitau.br \\
Tel.: (12) 3625-4212
\end{tabular}

\title{
Remoção de nitrogênio e fósforo do esgoto sanitário em um sistema de alagados construídos utilizando o capim vetiver
}

\author{
(http://dx.doi.org/10.4136/ambi-agua.925)
}

\author{
Fernando Ernesto Ucker ${ }^{1}$; Rogério de Araújo Almeida ${ }^{2}$; \\ Pedro Daniel da Cunha Kemerich ${ }^{3}$ \\ ${ }^{1}$ Universidade Federal de Goiás, Goiânia, GO, e-mail: ferucker@gmail.com, \\ ${ }^{2}$ Universidade Federal de Goiás, Goiânia, GO, e-mail: rogerioufg@ gmail.com, \\ ${ }^{3}$ Universidade Federal de Santa Maria, Frederico Westphalen-RS, \\ e-mail: eng.kemerich@yahoo.com.br
}

\section{RESUMO}

No presente trabalho objetivou-se avaliar a eficiência do capim vetiver (Vetiveria zizanioides L. Nash) na remoção de nitrogênio e fósforo do esgoto sanitário, em um sistema de tratamento do tipo alagados construídos. A unidade experimental possuiu doze módulos de tratamento, cada um preenchido com camadas sobrepostas de substrato. Do fundo até a superfície foram utilizados: $0,15 \mathrm{~m}$ de brita \# 3;0,10 $\mathrm{m}$ de brita \# 1;0,20 $\mathrm{m}$ de areia lavada e $0,05 \mathrm{~m}$ de brita \# 1. No interior dos módulos, o esgoto foi mantido a $0,05 \mathrm{~m}$ ou a $0,25 \mathrm{~m}$ abaixo da superfície do substrato, resultando em tempos de detenção hidráulica de 3,4 dias ou 1,9 dias, respectivamente. O afluente foi captado da entrada da lagoa facultativa de uma Estação de Tratamento de Esgotos e aplicado na superfície de cada módulo, de forma automatizada, em uma taxa de aplicação superficial de $51 \mathrm{~L} \cdot \mathrm{m}^{-2} \cdot \mathrm{d}^{-1}$. O esgoto percolava verticalmente no sistema, em um fluxo sub-superficial descendente, até ser captado em uma tubulação de drenagem no inferior do módulo. Determinaram-se as concentrações de fósforo total e nitrogênio amoniacal do esgoto antes e após passar pelo tratamento. As taxas de evapotranspiração foram medidas e, a partir delas, calcularam-se as eficiências na remoção da carga contaminante. Os resultados foram submetidos aos testes $\mathrm{F}$ e de Tukey, a 5\% de probabilidade. O tratamento com a presença da planta e nível de esgoto a $0,05 \mathrm{~m}$ da superfície apresentou eficiência mais elevada na remoção dos nutrientes, com 90,5\% para fósforo total e 93,9\% para nitrogênio amoniacal.

Palavras-chave: fósforo total, nitrogênio amoniacal, zona de raízes.

\section{Removal of nitrogen and phosphorus from wastewater in a constructed wetland system using vetiver grass}

\begin{abstract}
This study aimed to evaluate the Vetiver grass (Vetiveria zizanioides L. Nash) efficiency in removing nitrogen and phosphorus from the wastewater in a constructed wetlands treatment system. The experimental unit had twelve treatment modules, filled with layers of substrate. From the bottom to the surface, the following materials were placed: $0.15 \mathrm{~m}$ of gravel \# 3; $0.10 \mathrm{~m}$ of gravel \# 1;0.20 $\mathrm{m}$ of washed sand and $0.05 \mathrm{~m}$ of gravel \# 1 . Inside the modules, the wastewater was maintained at $0.05 \mathrm{~m}$ or $0.25 \mathrm{~m}$ below the substrate surface, resulting in hydraulic retention times of 3.4 days and 1.9 days, respectively. The influent wastewater was captured in the entrance of a facultative pond, and it was applied to the surface of each treatment module, automatically, on a surface application rate of $51 \mathrm{~L} \cdot \mathrm{m}^{-2} \cdot \mathrm{d}^{-1}$. The sewage percolated vertically in the system, in a sub-surface flow downward until it was
\end{abstract}


captured in a drain pipe at the bottom of the module. The wastewater concentrations of total phosphorus and ammonium were analyzed before and after passing through the treatment modules. Evapotranspiration rates were measured and the efficiencies in removing the contaminant load were calculated. The results were submitted to $\mathrm{F}$ and Tukey tests, at $5 \%$ of probability. Treatment with the presence of the plant and sewage at $0.05 \mathrm{~m}$ from the surface had higher efficiency in the removal of nutrients reaching $90.5 \%$ of phosphorus removal and $93.9 \%$ for ammonia.

Keywords: total phosphorus, ammonia, root zone system.

\section{INTRODUÇÃO}

O investimento em saneamento traz grandes benefícios ao Estado, dentre eles, a melhoria da saúde da população e a redução dos recursos aplicados no tratamento de doenças de veiculação hídrica. Adicionalmente, há a diminuição dos custos do tratamento de água para abastecimento, a eliminação da poluição estética/visual, o desenvolvimento do turismo e a conservação ambiental (Almeida et al., 2007).

Apesar de serem conhecidos inúmeros sistemas para realizar o tratamento do esgoto, uma estação de tratamento nem sempre é viável economicamente. Esta realidade exige que novos sistemas sejam urgentemente estudados e implementados, a fim de obter-se uma tecnologia capaz de relacionar favoravelmente o custo/benefício, tanto para o meio ambiente quanto para a sociedade (Ucker, 2012).

Segundo Arias e Brix (2003), entre as soluções mais atrativas encontradas atualmente, evidenciam-se aquelas em que no tratamento simulam os fenômenos que ocorrem espontaneamente na natureza, como os verificados nos brejos, em que espécies vegetais purificam as águas. $\mathrm{O}$ tratamento de efluentes pelo sistema de alagados construídos constitui uma opção reconhecida e recomendada, sendo efetivo na redução da matéria orgânica e na retenção ou eliminação de substâncias tóxicas que, de outra maneira, seriam lançadas no meio ambiente.

Além da redução dos contaminantes, os sistemas de alagados construídos possuem comprovados resultados na remoção de nutrientes, como nitrogênio e fósforo. Para Sezerino e Philippi (2000), nesses sistemas de tratamento, aproximadamente $74 \%$ da remoção do nitrogênio amoniacal pode ser associada a uma assimilação por parte das plantas. Sousa et al. (2004) salientam que para a remoção específica de fósforo, o tipo de vegetação e a composição química do material suporte podem ter interferência significativa, já que os principais mecanismos de remoção do atributo no sistema são a adsorção pelos substratos e a assimilação pelos vegetais.

Em âmbito do estado de Goiás, algumas plantas já foram avaliadas nesse sistema de tratamento. Almeida et al. (2007) utilizaram o Lírio-do-brejo (Hedychium coronarium J. König), a Conta-de-lágrima (Coix lacryma-jobi L.), a Taboa (Typha angustifolia L.) e o Capim-de-Angola (Urochloa mutica (Forssk.) T. Q. Nguyen). Abrantes (2009) utilizou a Taboa e o Caniço (Phragmites australis). Já Mendonça (2010) utilizou as espécies de bambu Guadua angustifolia Kunth, Guadua chacoensis e Dendrocalamus giganteus Munro. Pitaluga (2011) avaliou três tipos de substratos no sistema de alagados construídos vegetados com Lírio-do-brejo e Quege (2011) estudou a eficiência de três espécies de bambu (Guadua angustifolia, Phyllostachys aurea e Phyllostachys bambusoides) no tratamento do esgoto sanitário.

Entretanto, em vários países do mundo tem sido utilizada uma outra planta, de sistema radicular profundo e abundante, bastante resistente a variações climáticas e tolerante a contaminantes. Trata-se do capim Vetiver (Vetiveria zizanioides L. Nash), já utilizado no 
Brasil na vegetação de encostas, com o objetivo de controlar processos erosivos (Orozco, 2009).

Assim, no presente trabalho teve-se como objetivo avaliar a eficiência do capim Vetiver na remoção de nitrogênio e fósforo presentes no esgoto sanitário produzido em uma unidade universitária, em um sistema de tratamento do tipo alagados construídos, com fluxo subsuperficial vertical descendente.

\section{MATERIAL E MÉTODOS}

O experimento foi realizado na Estação de Pesquisas em Tratamento de Esgotos por Plantas (EPTEP) da Universidade Federal de Goiás (UFG), localizada em área da Estação de Tratamento de Esgotos (ETE) Samambaia, que é operada pela Saneamento de Goiás S.A. (Saneago), no município de Goiânia, Goiás. A ETE encontra-se no Campus II da UFG, e realiza a captação e tratamento do esgoto produzido unicamente naquele campus universitário.

A unidade experimental possui doze módulos de tratamento, cada um constituído por uma caixa em fibra de cimento amianto, com altura de $0,73 \mathrm{~m}$, e capacidade volumétrica total de $1,0 \mathrm{~m}^{3}$. As caixas foram dispostas linearmente na superfície do solo, equidistantes $1,0 \mathrm{~m}$ umas das outras. Cada módulo foi preenchido com a mesma altura e tipo de substrato: no fundo colocou-se $0,15 \mathrm{~m}$ de brita \# 3; na sequência $0,10 \mathrm{~m}$ de brita \# 1;0,20 $\mathrm{m}$ de areia lavada e $0,05 \mathrm{~m}$ de brita \# 1 .

Em cada módulo, um tubo de cloreto de polivinila (PVC) de $16 \mathrm{~mm}$ de diâmetro foi instalado para a aplicação do esgoto, proveniente da entrada da lagoa facultativa. O esgoto não foi submetido a qualquer tratamento prévio antes de ser aplicado nos módulos experimentais. Na camada mais profunda (composta pela brita \# 3) foi posicionado um tubo de drenagem (também de PVC), com $50 \mathrm{~mm}$ de diâmetro, destinado à coleta do efluente e sua condução para fora da caixa. Na parte externa do tubo de drenagem foi adaptada uma união soldável e um tubo de PVC com 0,60 m de comprimento, posicionado na vertical ou diagonal (dependendo do nível interno de esgoto desejado para o módulo) (Figura 1).

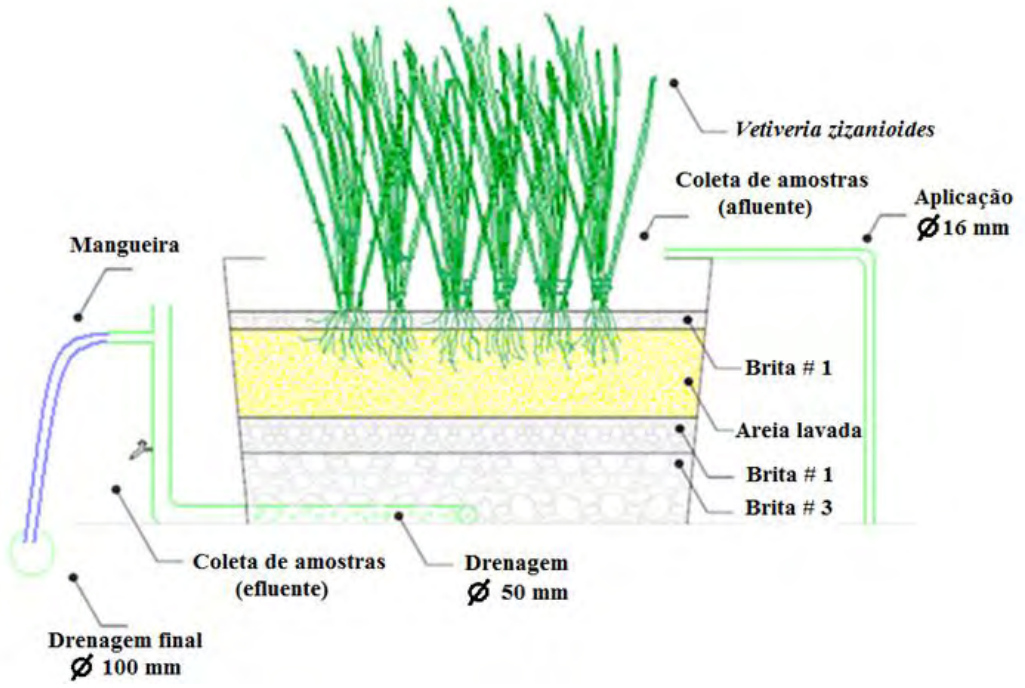

Figura 1. Representação esquemática de um módulo de tratamento de efluente com o capim Vetiver, detalhando o sistema hidráulico e as camadas sobrepostas de substrato.

A $0,15 \mathrm{~m}$ da parte inferior do tubo de $0,60 \mathrm{~m}$ foi instalada uma torneira, e na parte superior do tubo, um "T" com redução para $25 \mathrm{~mm}$ e uma mangueira plástica. A torneira 
possibilita a coleta de amostras de esgoto tratado, para análises. A mangueira destina-se à drenagem do efluente e à manutenção do nível interno de esgoto dentro do reservatório de tratamento, o que é determinado pelo posicionamento do " $T$ ".

Após passar pela unidade de tratamento, o esgoto escoa pela mangueira de drenagem para uma rede coletora (tubo de PVC com $100 \mathrm{~mm}$ de diâmetro) que conduz o efluente dos doze módulos de tratamento para um novo reservatório, de onde o efluente é bombeado de volta à lagoa facultativa.

$\mathrm{O}$ esgoto no interior dos módulos de tratamento foi mantido nas posições $\mathrm{H}_{1}$ e $\mathrm{H}_{2}$, mediante o posicionamento do tubo externo de drenagem. A altura $\mathrm{H}_{1}$ significou um nível de esgoto dentro dos módulos de tratamento mantido a $0,05 \mathrm{~m}$ abaixo da superfície do substrato. Para a altura $\mathrm{H}_{2}$ o nível foi mantido a 0,25 m da superfície.

Os tratamentos utilizados na pesquisa correspondem a uma combinação da presença ou ausência do capim Vetiver com um dos dois níveis de manutenção do esgoto dentro do módulo de tratamento, totalizando quatro tratamentos, conforme apresentado no Quadro 1. Seis módulos de tratamento foram plantados com o capim Vetiver, numa população de sete mudas em cada um. Os outros seis módulos permaneceram sem plantas, com o objetivo de servirem de tratamento testemunha.

Quadro 1. Tratamentos aplicados para avaliação da eficiência do capim Vetiver na remoção de nitrogênio e fósforo do esgoto sanitário.

\begin{tabular}{|c|c|c|c|}
\hline Tratamento & Descrição dos tratamentos & Sigla & Módulos \\
\hline Tratamento 1 & $\begin{array}{c}\text { Módulo de tratamento com a presença da planta }+ \\
\text { nível de esgoto } \mathrm{H}_{1}\end{array}$ & $\mathrm{VH}_{1}$ & 1 , 4 e 11 \\
Tratamento 2 & $\begin{array}{c}\text { Módulo de tratamento com a presença da planta }+ \\
\text { nível de esgoto } \mathrm{H}_{2}\end{array}$ & $\mathrm{VH}_{2}$ & 2,5 e 12 \\
Tratamento 3 & $\begin{array}{c}\text { Módulo de tratamento com a ausência da planta }+ \\
\text { nível de esgoto } \mathrm{H}_{1}\end{array}$ & $\mathrm{SH}_{1}$ & 3,7 e 9 \\
Tratamento 4 & $\begin{array}{c}\text { Módulo de tratamento com a ausência da planta }+ \\
\text { nível de esgoto } \mathrm{H}_{2}\end{array}$ & $\mathrm{SH}_{2}$ & 6,8 e 10 \\
\hline
\end{tabular}

As mudas de capim Vetiver foram plantadas na primeira semana de janeiro de 2011, e a aplicação do esgoto nos módulos teve início no dia primeiro de junho de 2011, após vinte semanas do transplantio das mudas para os módulos de tratamento. No período que compreendeu o transplantio e o início da aplicação de esgoto, a umidade mantida nos módulos se deu apenas pela precipitação pluvial.

O esgoto submetido aos tratamentos foi bombeado diretamente da parte inicial da lagoa facultativa da ETE Samambaia e conduzido aos módulos de tratamento por meio de um tubo de PVC com diâmetro de $50 \mathrm{~mm}$.

As coletas de amostras do esgoto e suas análises (em número de nove) ocorreram entre o primeiro dia de julho e o décimo quinto dia de dezembro de 2011, com amostragem feita nos registros dos módulos de tratamento, representando o esgoto tratado (efluente), e uma amostra retirada da tubulação de alimentação de esgoto proveniente da lagoa facultativa (afluente), totalizando treze pontos de coleta.

Avaliaram-se os teores de nitrogênio amoniacal e fósforo total, utilizando-se métodos padronizados pelo Standard Methods for the Examination of Water and Wastewater (Apha et al., 1998).

A aplicação de esgoto nos módulos se deu três vezes ao dia, em bateladas, (as $7 \mathrm{~h} 00$ min, 12 h $00 \mathrm{~min}$ e 17 h $00 \mathrm{~min}$ ) por períodos de 14 minutos para cada aplicação, resultando 
em um volume de $74 \mathrm{~L}$ diários de esgoto, subdividido nas três aplicações. O tempo de detenção hidráulica (TDH) do esgoto nos módulos de tratamento foi calculado utilizando o volume de espaços vazios ( $\mathrm{Vv}$ ) das camadas de substrato sob efeito do nível de esgoto $\left(\mathrm{Vv} \mathrm{H}_{1}\right.$ $\left.=0,253 \mathrm{~m}^{3} ; \mathrm{Vv} \mathrm{H}_{2}=0,143 \mathrm{~m}^{3}\right)$, a área superficial de cada módulo $\left(\mathrm{Am}=1,45 \mathrm{~m}^{2}\right)$ e a taxa de aplicação $\left(\right.$ Tap $\left.=51 \mathrm{~L} \cdot \mathrm{m}^{-2} \cdot \mathrm{d}^{-1}\right)$. O TDH representa o tempo que o esgoto bruto permaneceu em tratamento dentro de cada reservatório, e resultou em 3,4 dias para o nível de esgoto $\mathrm{H}_{1}$ e 1,9 dias para o nível de esgoto $\mathrm{H}_{2}$. O esgoto era aplicado na parte superior do sistema, e percolava verticalmente.

Após o início da aplicação do esgoto, mensalmente, e em períodos de cinco dias consecutivos (segunda-feira a sexta-feira), foram realizadas medições das taxas de evapotranspiração do sistema de alagados construídos. As saídas das drenagens dos módulos de tratamento foram interligadas, por meio de mangueiras, a tambores de $200 \mathrm{~L}$ de capacidade volumétrica, em um módulo representante de cada tratamento $\left(\mathrm{VH}_{1}, \mathrm{SH}_{1}, \mathrm{VH}_{2}\right.$ e $\left.\mathrm{SH}_{2}\right)$. Durante os cinco dias consecutivos coletaram-se os volumes drenados pelas mangueiras nos tambores.

Diariamente, mediu-se o volume armazenado em cada tambor, e por meio da Eq. (1) estimou-se a taxa de evapotranspiração de cada tratamento. As medições foram realizadas nas semanas de coleta de amostras para determinação dos teores de nutrientes, e seus valores foram utilizados nos cálculos de eficiência do sistema.

$$
\mathrm{ETP}=\mathrm{P}+\frac{\mathrm{I}-\mathrm{D}}{\mathrm{S}}
$$

em que:

$\begin{array}{ll}\text { ETP - } & \text { Evapotranspiração, mm; } \\ \text { P - } & \text { Precipitação efetiva, mm; } \\ \text { I - } & \text { Esgoto aplicado, L; } \\ \text { D - } & \text { Drenagem, L; } \\ \text { S - } & \text { Área superficial do módulo, } \mathrm{m}^{2} .\end{array}$

A eficiência percentual do sistema de tratamento do tipo alagado construído, com a espécie Vetiveria zizanioides (capim Vetiver), na remoção de nitrogênio e fósforo foi determinada pela Eq. (2), conforme sugerido por Almeida e Ucker (2011):

$$
\mathrm{Ef}(\%)=\frac{(\mathrm{Ce} \times \mathrm{Ve})-(\mathrm{Cs} \times \mathrm{Vs})}{(\mathrm{Ce} \times \mathrm{Ve})} \times 100
$$

em que:

Ef (\%) - eficiência percentual de remoção da carga contaminante de determinado atributo;

Ce - concentração do atributo na entrada do sistema;

Ve - volume de esgoto na entrada do sistema;

Cs - $\quad$ concentração do atributo na saída do sistema;

Vs - volume de esgoto na saída do sistema.

Os resultados das eficiências na remoção dos nutrientes foram submetidos à análise de variância (teste F) e ao teste de médias (Tuckey), com 5\% de probabilidade, utilizando a ferramenta SAS (Statistical Analysis System). 


\section{RESULTADOS E DISCUSSÃO}

\subsection{Fósforo Total}

Os valores médios da concentração de fósforo total no afluente (esgoto proveniente da entrada da lagoa facultativa) e no efluente (esgoto proveniente da saída do registro de cada módulo), da evapotranspiração e da eficiência percentual de sua remoção no sistema de alagados construídos são apresentados na Tabela 1.

Tabela 1. Valores médios da concentração de fósforo total no afluente e no efluente, da taxa de evapotranspiração e da eficiência percentual de sua remoção no sistema de alagados construídos.

\begin{tabular}{c|c|ccc}
\hline Tratamento $^{\mathbf{1}}$ & Afluente $^{\mathbf{2}}$ & ETP $(\boldsymbol{\%})^{\mathbf{3}}$ & Efluente $^{\mathbf{4}}$ & Eficiência $^{\mathbf{5}}$ \\
\hline $\mathbf{V H}_{\mathbf{1}}$ & & 57,8 & 1,1 & $90,5 \mathrm{a}$ \\
$\mathbf{V H}_{\mathbf{2}}$ & & 28,5 & 1,9 & $70,2 \mathrm{~b}$ \\
$\mathbf{S H}_{\mathbf{1}}$ & 4,9 & 13,9 & 3,0 & $44,3 \mathrm{c}$ \\
$\mathbf{S H}_{\mathbf{2}}$ & & 10,1 & 3,0 & $44,6 \mathrm{c}$ \\
C.V. (\%) & & & & 14,3 \\
\hline
\end{tabular}

Nota: ${ }^{1} \mathrm{VH}_{1}$ : módulo com Vetiver e esgoto mantido a $0,05 \mathrm{~m}$ da superfície; $\mathrm{VH}_{2}$ : módulo com Vetiver e esgoto mantido a $0,25 \mathrm{~m}$ da superfície; $\mathrm{SH}_{1}$ : módulo sem plantas e esgoto mantido a $0,05 \mathrm{~m}$ da superfície; $\mathrm{SH}_{2}$ : módulo sem plantas e esgoto mantido a $0,25 \mathrm{~m}$ da superfície; ${ }^{2}$ Valor médio da concentração de fósforo total $\left(\mathrm{mg} \mathrm{L}^{-1}\right)$ na entrada da lagoa facultativa; ${ }^{3}$ Evapotranspiração média percentual; ${ }^{4}$ Valor de fósforo total $\left(\mathrm{mg} \mathrm{L}^{-1}\right)$ após passar pelo sistema de alagados construídos; ${ }^{5}$ Eficiência percentual: (Eq. 2).

Durante o período de cinco dias consecutivos em que foram aplicados $370 \mathrm{~L}$ de esgoto em cada módulo de tratamento, o volume médio de saída (esgoto drenado) para o tratamento $\mathrm{VH}_{1}$ foi de $165,5 \mathrm{~L}$, correspondendo a uma evapotranspiração média de 57,8\% do volume aplicado. Para o tratamento $\mathrm{VH}_{2}$ houve a drenagem de 279,7 L (ETP de 28,5\%). Já nos módulos sem a presença da planta, o valor drenado foi de 335,5 L para o tratamento $\mathrm{SH}_{1}$ e de $350,0 \mathrm{~L}$ para $\mathrm{SH}_{2}$, correspondendo a taxas de evapotranspiração de 13,9\% e 10,1\%, respectivamente. Nos módulos em que o esgoto foi mantido a $0,05 \mathrm{~m}$ da superfície do substrato o maior tempo de detenção proporcionou maiores taxas de evapotranspiração. $\mathrm{O}$ mesmo comportamento foi observado quando da presença de plantas.

O valor médio obtido nas análises para o atributo fósforo total no esgoto afluente foi de $4,9 \mathrm{mg} \mathrm{L}^{-1}$, valor este dentro das faixas de referência apresentadas por Von Sperling (2005) e Jordão e Pessôa (2011), que são de 3 a $9 \mathrm{mg} \mathrm{L}^{-1}$ e de 3 a $13 \mathrm{mg} \mathrm{L}^{-1}$, respectivamente, para esgotos considerados domésticos. Após passagem pelo sistema de tratamento de esgoto por alagados construídos, o valor médio encontrado nos módulos submetidos ao tratamento $\mathrm{VH}_{1}$ foi de $1,1 \mathrm{mg} \mathrm{L}^{-1}$, e para os módulos $\mathrm{VH}_{2}$ o valor médio foi de $1,9 \mathrm{mg} \mathrm{L}^{-1}$. Para os módulos sem planta (tratamentos $\mathrm{SH}_{1}$ e $\mathrm{SH}_{2}$ ), o valor médio de saída do atributo foi de $3,0 \mathrm{mg} \mathrm{L}^{-1}$.

O nível de esgoto dentro dos módulos influenciou na eficiência do tratamento onde havia a presença de plantas. A remoção de fósforo total foi de $90,5 \%$ para $\mathrm{VH}_{1}$ e de $70,2 \%$ para $\mathrm{VH}_{2}$, que diferiram estatisticamente entre si. Nos módulos sem a presença da planta os valores foram mais baixos, com 44,3\% de remoção nos módulos de tratamento $\mathrm{SH}_{1}$ e 44,6\% de remoção nos módulos $\mathrm{SH}_{2}$ (Figura 2), que não diferiram estatisticamente entre si, mas diferiram dos demais tratamentos.

Segundo Van de Moortel et al. (2009), a liberação de oxigênio pelas raízes das plantas pode aumentar a capacidade de adsorção do fósforo por meio dos substratos, o que pode ter influenciado para fazer com que os sistemas de tratamento com plantas tivessem uma eficiência de remoção maior do que os sistemas sem a presença das plantas. 
De outro lado, Kadlec e Knight (1996) e Arias et al. (2001) afirmam que os mecanismos de retenção do fósforo incluem processos físicos, químicos e biológicos, envolvendo fenômenos de precipitação, sedimentação e, principalmente, adsorção; e o mecanismo de remoção consiste na incorporação do fósforo na biomassa das macrófitas.

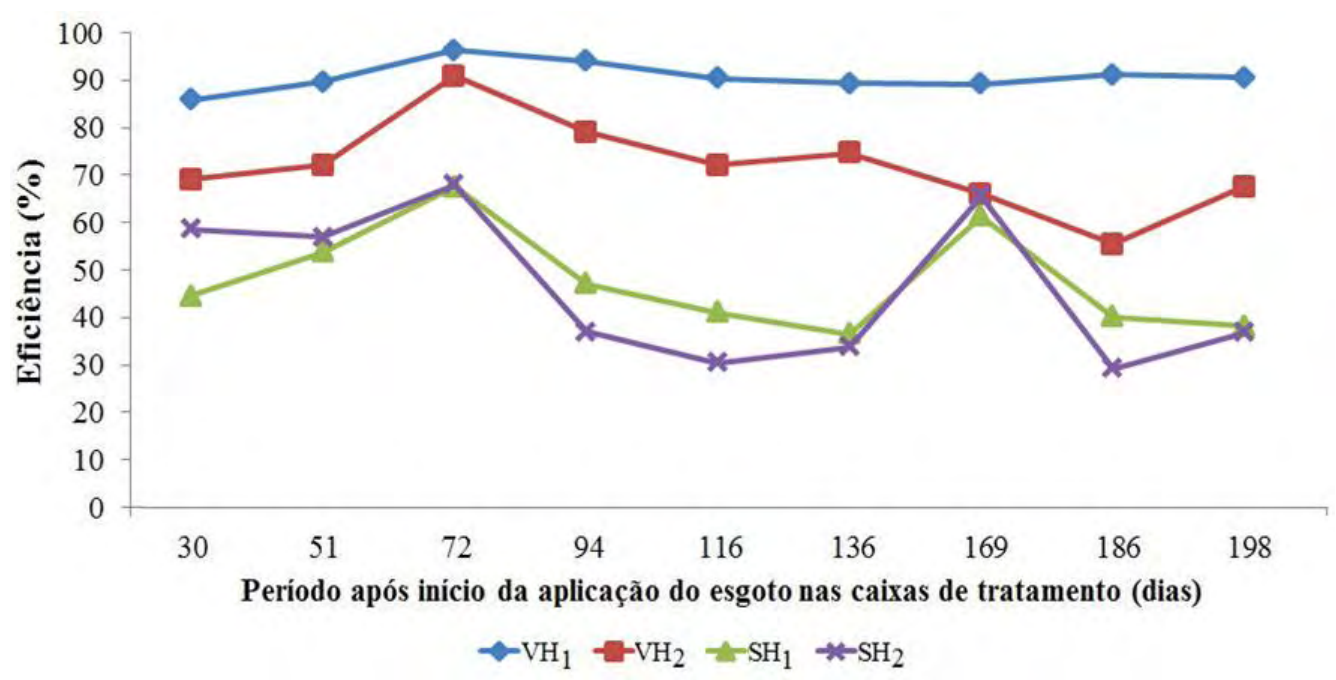

Figura 2. Evolução da eficiência percentual na remoção do fósforo total nos módulos de tratamento de esgoto ao longo do tempo.

Nota: $\mathrm{VH}_{1}$ : módulo com Vetiver e esgoto mantido a $0,05 \mathrm{~m}$ da superfície; $\mathrm{VH}_{2}$ : módulo com Vetiver e esgoto mantido a $0,25 \mathrm{~m}$ da superfície; $\mathrm{SH}_{1}$ : módulo sem plantas e esgoto mantido a $0,05 \mathrm{~m}$ da superfície; $\mathrm{SH}_{2}$ : módulo sem plantas e esgoto mantido a $0,25 \mathrm{~m}$ da superfície.

A eficiência de remoção de fósforo dos módulos $\mathrm{VH}_{1}$ manteve-se praticamente constante durante todo o experimento. Já os módulos $\mathrm{VH}_{2}$ alternaram eficiências altas, como no período de 72 dias após o início da aplicação de esgoto, e baixas. Os módulos $\mathrm{SH}_{1}$ e $\mathrm{SH}_{2}$ tiveram um comportamento bem diferente dos demais, inclusive com uma queda brusca na eficiência de remoção do fósforo a partir do dia 72, com ponto de elevação aos 169 dias.

A mudança de comportamento e redução da eficiência ocorrida após 72 dias, possivelmente foi ocasionada pela saturação do substrato e início da liberação do fósforo adsorvido. De acordo com Arias et al. (2001), os maiores responsáveis pela redução das concentrações de fósforo no efluente são as reações de adsorção e precipitação que ocorrem quando da presença de minerais como o cálcio, alumínio e ferro no material filtrante. Nos módulos com o capim Vetiver a redução na eficiência de remoção de fósforo foi menor, em decorrência da absorção pelas plantas.

Brix et al. (2003) verificaram uma remoção de fósforo da ordem de $98 \%$ ao analisar um alagado construído semeado com caniço para tratar águas residuárias de uma pequena comunidade na Dinamarca. Souza e Bernardes (1996), ao utilizar leitos cultivados com taboa no pós-tratamento de esgotos domésticos, com um tempo de detenção de 2,7 dias, verificaram 40,0\% de remoção de fósforo.

Em estudo realizado na Venezuela, Mayorca (2007) obteve um percentual de remoção do atributo fósforo total de $74 \%$, em um sistema de alagados construídos para tratar o efluente gerado por uma indústria de cerveja. Ash e Truong (2004) avaliaram o potencial do capim Vetiver no tratamento de esgoto em Queensland na Austrália e observaram uma remoção de $85 \%$ do fósforo total. Liao et al. (2003) observaram uma remoção de $40 \%$ a partir da oitava semana de tratamento do esgoto gerado em suinocultura, valor bem abaixo do encontrado neste trabalho (média de 90,5\% de remoção ao final de 198 dias de aplicação). 
Hart et al. (2003) avaliaram o potencial do capim Vetiver no tratamento do efluente do tanque séptico de um hotel, em um sistema de fertirrigação com fluxo de $20 \mathrm{~L} \mathrm{~min}^{-1}$. Em oito dias, um metro quadrado de plantas bem enraizadas removeu $3.575 \mathrm{mg}$ de fósforo do efluente no tanque de recirculação.

Pitaluga (2011) observou uma redução percentual média da concentração de fósforo total de 93,9\% em um alagado construído, de fluxo horizontal, preenchido com areia e vegetado com a espécie Hedychium coronarium, em Goiânia, GO. Na mesma cidade, Quege (2011), utilizando um sistema de tratamento por alagados construídos de fluxo vertical descendente encontrou uma média percentual para a remoção de fósforo no valor de 98,4\% para módulos cultivados com Guadua angustifolia e 94,5\% em módulos não vegetados, além de uma remoção de 87,0\% nos módulos com Phyllostachys bambusoides e 86,4\% em módulos vegetados com Phyllostachys aurea. Na presente pesquisa, o maior valor verificado foi de $90,5 \%$, para o tratamento $\mathrm{VH}_{1}$.

\subsection{Nitrogênio Amoniacal}

Os valores médios da concentração de nitrogênio amoniacal no afluente e no efluente, da evapotranspiração e da eficiência percentual de sua remoção no sistema de alagados construídos são apresentados na Tabela 2.

Tabela 2. Valores médios da concentração de nitrogênio amoniacal no afluente e no efluente, da evapotranspiração e da eficiência percentual de sua remoção no sistema de alagados construídos

\begin{tabular}{c|c|ccc}
\hline Tratamento $^{\mathbf{1}}$ & Afluente $^{\mathbf{2}}$ & ETP $(\boldsymbol{\%})^{\mathbf{3}}$ & Efluente $^{\mathbf{4}}$ & Eficiência $^{\mathbf{5}}$ \\
\hline $\mathbf{V H}_{\mathbf{1}}$ & & 57,8 & 2,2 & $93,9 \mathrm{a}$ \\
$\mathbf{V H}_{\mathbf{2}}$ & \multirow{2}{*}{15,0} & 28,5 & 5,3 & $73,7 \mathrm{~b}$ \\
$\mathbf{S H}_{\mathbf{1}}$ & & 13,9 & 9,6 & $42,1 \mathrm{c}$ \\
$\mathbf{S H}_{\mathbf{2}}$ & & 10,1 & 9,0 & $43,0 \mathrm{c}$ \\
C.V.(\%) & & & & 12,4 \\
\hline
\end{tabular}

Nota: ${ }^{1} \mathrm{VH}_{1}$ : módulo com Vetiver e esgoto mantido a $0,05 \mathrm{~m}$ da superfície; $\mathrm{VH}_{2}$ : módulo com Vetiver e esgoto mantido a $0,25 \mathrm{~m}$ da superfície; $\mathrm{SH}_{1}$ : módulo sem plantas e esgoto mantido a $0,05 \mathrm{~m}$ da superfície; $\mathrm{SH}_{2}$ : módulo sem plantas e esgoto mantido a $0,25 \mathrm{~m}$ da superfície; ${ }^{2}$ Valor médio da concentração de fósforo total $\left(\mathrm{mg} \mathrm{L}^{-1}\right)$ na entrada da lagoa facultativa; ${ }^{3}$ Evapotranspiração média percentual; ${ }^{4}$ Valor de fósforo total $\left(\mathrm{mg} \mathrm{L}^{-1}\right)$ após passar pelo sistema de alagados construídos; ${ }^{5}$ Eficiência percentual: (Eq. 2).

O valor médio da concentração de nitrogênio amoniacal observado durante a pesquisa, foi de $15 \mathrm{mg} \mathrm{L}^{-1}$, valor este que está dentro da faixa referenciada por Jordão e Pessôa (2011), entre 10 a $50 \mathrm{mg} \mathrm{L}^{-1}$, e de Metcalf e Eddy (1981), entre 12 a $50 \mathrm{mg} \mathrm{L}^{-1}$, para esgotos considerados domésticos. Este valor, considerado baixo, corresponde ao esgoto retirado da entrada da lagoa facultativa, tendo este sido diluído ao entrar na lagoa.

Após passar pelo tratamento no sistema de alagados construídos, os valores médios da concentração de nitrogênio amoniacal nos módulos com planta foram de $2,2 \mathrm{mg} \mathrm{L}^{-1}$ para o tratamento $\mathrm{VH}_{1}$ e de $5,3 \mathrm{mg} \mathrm{L}^{-1}$ para $\mathrm{VH}_{2}$. Nos módulos onde não havia a presença da planta Vetiveria zizanioides, os valores de saída foram de $9,6 \mathrm{mg} \mathrm{L}^{-1}$ e $9,0 \mathrm{mg} \mathrm{L}^{-1}$, para $\mathrm{SH}_{1}$ e $\mathrm{SH}_{2}$, respectivamente.

A eficiência média percentual de remoção do nitrogênio amoniacal foi de 93,9\% para $\mathrm{VH}_{1}$, e de $73,7 \%$ para $\mathrm{VH}_{2}$. Nos módulos $\mathrm{SH}_{1}$, houve remoção de $42,1 \%$ do nitrogênio amoniacal presente, e nos módulos $\mathrm{SH}_{2}$ houve remoção de 43,0\%. Estatisticamente, os módulos com planta diferiram entre si, e diferiram também dos módulos sem a presença da 
planta, que por sua vez não diferiram entre si. Os resultados apresentados mostram uma superioridade dos módulos vegetados e com maior nível interno de esgoto.

Observa-se que os módulos de tratamento $\mathrm{VH}_{1}$ mostraram grande potencialidade para remoção de nitrogênio amoniacal, pois além da remoção média final acima de 90\%, apresentaram um comportamento praticamente constante durante todo o experimento (Figura 3). Os módulos $\mathrm{VH}_{2}$, ou seja, com menor nível de esgoto, apresentaram pequenas variações na remoção do atributo. Já os módulos sem a presença da planta mostraram grandes oscilações ao longo do experimento.

Kadlec e Knight (1996) atentam para os diferentes processos de remoção do nitrogênio amoniacal da fração líquida nos sistemas de tratamento por alagados construídos, destacando a incorporação na biomassa vegetal, a adsorção ao material filtrante e, com mais ênfase, a nitrificação seguida da desnitrificação.

Abrantes (2009), utilizando um sistema de fluxo sub-superficial vertical vegetado com taboa (Typha spp) e TDH de 4,6 dias, observou uma eficiência de 69,3\% na redução do nitrogênio amoniacal, em Goiânia, GO. Nos módulos vegetados com caniço, foi verificada uma redução de 53,6\%. De acordo com a autora, uma das ações influenciadoras na remoção de nitrogênio amoniacal é a oxigenação do meio pelas plantas, onde as bactérias nitrificantes utilizam o oxigênio para oxidar o nitrogênio amoniacal no processo de nitrificação.

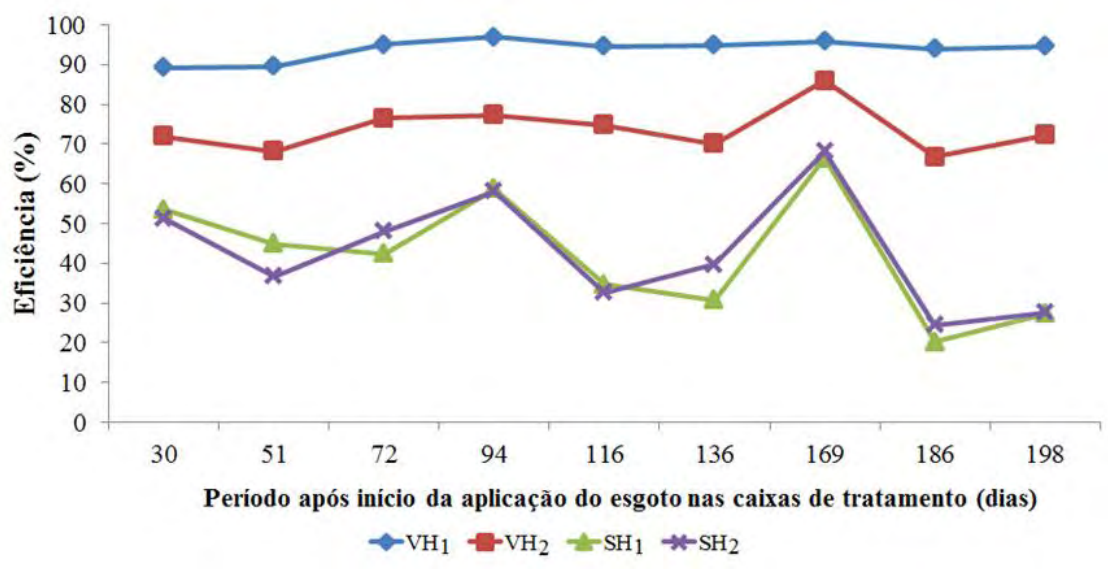

Figura 3. Evolução da eficiência percentual na remoção do nitrogênio amoniacal nos módulos de tratamento de esgoto ao longo do tempo.

Nota: $\mathrm{VH}_{1}$ : módulo com Vetiver e esgoto mantido a $0,05 \mathrm{~m}$ da superfície; $\mathrm{VH}_{2}$ : módulo com Vetiver e esgoto mantido a $0,25 \mathrm{~m}$ da superfície; $\mathrm{SH}_{1}$ : módulo sem plantas e esgoto mantido a $0,05 \mathrm{~m}$ da superfície; $\mathrm{SH}_{2}$ : módulo sem plantas e esgoto mantido a $0,25 \mathrm{~m}$ da superfície.

Estudos com a planta Vetiveria zizanioides comprovam sua eficiência na remoção do nitrogênio presente no esgoto. Segundo Cull et al. (2000), pesquisas na Ásia mostram que o capim Vetiver tem uma rápida, constante e alta capacidade na remoção de nutrientes como nitrogênio e fósforo, além de grandes quantidades de agroquímicos e metais pesados.

Ash e Truong (2003), utilizando a planta para o tratamento de esgoto em Queensland, Austrália, observaram uma eficiência de $99 \%$ na remoção do nitrogênio amoniacal. Na mesma cidade, o capim Vetiver foi plantado para tratar o esgoto gerado em um pequeno aeródromo. Naquela pesquisa o autor chegou a ótimos resultados, ao observar remoção de $99,7 \%$ do atributo em questão (Truong, 2001). Neste trabalho, a remoção do nitrogênio amoniacal foi menor do que nos trabalhos citados (média de 93,9\% ao final de 198 dias de aplicação de esgoto).

Cheng et al. (2008) avaliaram o desempenho dos sistemas de alagados construídos num efluente que é descartado em um dos rios mais poluídos de Taiwan, o rio Wu-Luo. Os autores 
chegaram à conclusão de que o capim Vetiver foi capaz de remover cerca de $93 \%$ do nitrogênio do efluente.

Almeida et al. (2007), num sistema de tratamento do tipo zona de raízes com fluxo subsuperficial descendente, vegetado com lírio-do-brejo (Hedychium coronarium J. König), observaram uma eficiência na redução do teor de nitrogênio amoniacal de 53,5\%. Pitaluga (2011) encontrou redução média percentual do nitrogênio amoniacal de 58,2\% em sistema alagado construído preenchido com areia; $58,3 \%$ no substrato preenchido com brita \# 0 e $59,5 \%$ em substrato preenchido com brita \# 1; todos vegetados com a espécie Hedychium coronarium.

De acordo com Abrantes (2009) e Mendonça (2010), a eficiência na remoção de nitrogênio amoniacal pode ser explicada pela transferência de oxigênio da atmosfera para o tratamento através das raízes das plantas, sendo o oxigênio utilizado pelas bactérias nitrificantes para oxidar o nitrogênio amoniacal durante a nitrificação. A maior média de remoção do nitrogênio amoniacal observada neste estudo foi de 93,7\%, nos módulos onde o esgoto estava constantemente em presença das raízes das plantas (nível $\mathrm{H}_{1}$ ).

\section{CONCLUSÃO}

1. O sistema de tratamento do tipo alagados construídos, de fluxo sub-superficial vertical descendente, vegetado com capim Vetiver mostrou-se eficiente na remoção de nitrogênio amoniacal e fósforo total presentes no esgoto sanitário produzido em uma unidade universitária.

2. Os módulos de tratamento com a presença do capim Vetiver e nível de esgoto $\mathrm{H}_{1}$, ou seja, com tempo de detenção hidráulica de 3,4 dias, foram superiores na remoção dos atributos pesquisados.

3. Os valores encontrados nesta pesquisa (remoção de 90,5\% de fósforo total e 93,9\% de nitrogênio amoniacal) mostram-se, no geral, superiores aos valores encontrados na literatura.

\section{REFERÊNCIAS}

ABRANTES, L. L. M. Tratamento de esgoto sanitário em sistemas alagados construídos utilizando Typha angustifolia e Phragmites australis. 2009. 140 f. Dissertação (Mestrado em Engenharia do Meio Ambiente) - Escola de Engenharia Civil, Universidade Federal de Goiás, Goiânia, 2009.

ALMEIDA, R. A.; UCKER, F. E. Considerando a evapotranspiração no cálculo de eficiência de estações de tratamento de esgoto com plantas. Revista Engenharia Ambiental, Espírito Santo do Pinhal, v. 8, n. 4, p. 39-45, 2011.

ALMEIDA, R. A.; OLIVEIRA, L. F. C.; KLIEMANN, H. J. Eficiência de espécies vegetais na purificação de esgoto sanitário. Pesquisa Agropecuária Tropical, Goiânia, v. 37, n. 1, p. 1-9, 2007.

AMERICAN PUBLIC HEALTH ASSOCIATION; AMERICAN WATER WORKS ASSOCIATON; WATER ENVIRONMENT FEDERATION. Standard methods for examination of water and wastewater. 20. ed. Washington D.C, 1998.

ARIAS, C. A; BRIX, H. Humedales artificiales para el tratamiento de aguas residuales. Revista Ciência e Ingeniería Neogranadina, Bogotá, v.13, p. 17-24, 2003. 
ARIAS, C. A., DEL BUBBA, M., BRIX, H. Phosphorus removal by sands for use as media in subsurface flow constructed reed beds. Water Research, v. 35, n. 5, p. 1159-1168, 2001. http://dx.doi.org/10.1016/S0043-1354(00)00368-7

ASH, R.; TRUONG, P. The use of vetiver grass for sewerage treatment. In: SEWAGE MANAGEMENT QEPA CONFERENCE, 5-7 April, 2004, Cairns. Paper for... Disponível em: <http://www.vetiver.org/AUS_ekeshire01.pdf>. Acesso em: $14 \mathrm{dez}$. 2011.

BRIX, H.; ARIAS, C. A.; JOHANSEN, N. H. Experiments in a two-stage constructed wetlands system: nitrification capacity and effects of recycling on nitrogen removal. In: VYMAZAL, J. (Ed.). Wetlands: nutrients, metals and mass cycling. Leiden: Backhuys Publishers, 2003. p. 237-258.

CHENG, L. L.; TSAI, H. A.; LIN, J. H.; CHOU, M. S. Performance study on the constructed wetlands for clarification of polluted Wu-Luo river water. Jounal of Environmental Engineering and Management, v. 18, n. 5, p. 345-354, 2008.

CULL, R. H.; HUNTER, H.; HUNTER, M.; TRUONG, P. Application of Vetiver Grass Technology in off-site pollution control. II. Tolerance of vetiver grass towards high levels of herbicides under wetlands conditions. In: INTERNATIONAL VETIVER CONFERENCE, 2., 2000, Thailand. Proceedings, Thailand: ICV, 2000.

HART, B.; CODY, R.; TRUONG, P. Hydroponic vetiver treatment of post septic tank effluent. In: INTERNATIONAL CONFERENCE ON VETIVER AND EXHIBITION, 3., 2003, Guangzhou. Proceedings... Guangzhou: China Agriculture Press, 2003. p. 121-131. Disponível em: <http://www.vetiver.org/ICV3-Proceedings/AUS_hydroponic. pdf>. Acesso em: 14 dez. 2011.

JORDÃO, E. P.; PESSÔA, C. A. Tratamento de esgotos domésticos. 6. ed. Rio de Janeiro: ABES, 2011. $1050 \mathrm{p}$.

KADLEC, R. H.; KNIGHT, R. L. Treatment wetlands. Boca Raton: Lewis Publishers, 1996. $893 \mathrm{p}$.

LIAO, X.; LUO, S.; WU, Y.; WANG, Z. Studies on the abilities of Vetiveria zizanioides and Cyperus alternifolius for pig farm wastewater treatment. In: INTERNATIONAL CONFERENCE ON VETIVER AND EXHIBITION, 3., 2003, Guangzhou. Proceedings... Guangzhou: China Agriculture Press, 2003. p. 174-181. Disponível em: <http://www.vetiver.org/ICV3-Proceedings/CHN_pigwaste2.pdf>. Acesso em: $12 \mathrm{dez}$. 2011.

MAYORCA, A. Vetiver system used in wastewater treatment. 2007. Disponível em: <http://www.vetiver.org/VEN_Beer_WW01pdf.pdf>. Acesso em: 12 dez. 2011.

MENDONÇA, E. A. Disposição de efluentes de estação de tratamento de esgoto industrial em solo vegetado com bambu. 2010. 128 f. Dissertação (Mestrado em Engenharia do Meio Ambiente) - Universidade Federal de Goiás, Goiânia, 2010.

METCALF, L.; EDDY, H. P. Tratamiento y depuración de las aguas residuales. Barcelona: Labor, 1981. 
OROZCO, M. M. D. Caracterização da gramínea Vetiveria zizanioides para aplicação na recuperação de áreas degradadas por erosão. 2009. 96 f. Dissertação (Mestrado em Saneamento, Meio Ambiente e Recursos Hídricos) - Universidade Federal de Minas Gerais, Belo Horizonte, 2009.

PITALUGA, D. P. S. Avaliação de diferentes substratos no tratamento de esgoto sanitário por zona de raízes. 2011. 133 f. Dissertação (Mestrado em Engenharia do Meio Ambiente) - Escola de Engenharia Civil, Universidade Federal de Goiás, Goiânia, 2011.

QUEGE, K. E. Tratamento de esgoto sanitário pelo sistema zona de raízes utilizando plantas de bambu. 2011. 85 f. Dissertação (Mestrado em Engenharia do Meio Ambiente) - Escola de Engenharia Civil, Universidade Federal de Goiás, Goiânia, 2011.

SEZERINO, P. H.; PHILIPPI, L. S. Utilização de um sistema experimental por meio de "wetland" construído no tratamento de esgotos domésticos pós tanque séptico. In: SIMPÓSIO LUSO-BRASILEIRO DE ENGENHARIA SANITÁRIA E AMBIENTAL, 9., 2000, Porto Seguro. Anais... Porto Seguro: ABES, 2000. p. 688-697.

SOUSA, J. T.; VAN HAANDEL, A.; LIMA, E. P. C.; HENRIQUE, I. N. Utilização de wetlands construído no pós-tratamento de esgotos domésticos pré-tratados em reatores UASB. Engenharia Sanitária e Ambiental, v. 9, n. 4, p. 285-290, 2004.

SOUZA, L. E. L.; BERNARDES, R. S. Avaliação do desempenho de um RAFA no tratamento de esgotos domésticos, com pós-tratamento através de leitos cultivados. In: SIMPÓSIO ÍTALO-BRASILEIRO DE INGENIERÍA SANITARIA-AMBIENTALE, 1., 1996, Gramado. Anais... Gramado: ABES, 1996.

TRUONG, P. Vetiver grass system for effluent, landfill leachate and seepage control. 2001. Disponível em: 〈http://www.vetiver.org/AUS_effluent.pdf〉. Acesso em: $12 \mathrm{dez}$. 2011.

UCKER, F. E. Eficiência do capim Vetiver (Vetiveria zizanioides L. Nash) no tratamento de esgoto sanitário. 2012. 102 f. Dissertação (Mestrado em Engenharia do Meio Ambiente) - Escola de Engenharia Civil, Programa de Pós-Graduação Stricto Sensu em Engenharia do Meio Ambiente, Universidade Federal de Goiás, Goiânia, 2012.

VAN DE MOORTEL, A. M. K.; ROUSSEAU, D. P. L.; TACK, F. M. G.; PAUW, N. D. A comparative study of surface and subsurface flow constructed wetlands for treatment of combined sewer overflows: a greenhouse experiment. Ecological Engineering, v. 35, n. 2, p. 175-183, 2009. http://dx.doi.org/10.1016/j.ecoleng.2008.08.015

VON SPERLING, M. Princípios do tratamento biológico de águas residuárias: introdução a qualidade das águas e ao tratamento de esgotos. 3. ed. Belo Horizonte: UFMG, 2005. $452 \mathrm{p}$. 


ISSN = 1980-993X - doi:10.4136/1980-993X
www.ambi-agua.net
E-mail: ambi-agua@agro.unitau.br
Tel.: (12) 3625-4212

\title{
Influência de características físico-químicas da água no transporte de metano para a atmosfera na Lagoa Rodrigo de Freitas, RJ
}

(http://dx.doi.org/10.4136/ambi-agua.972)

\author{
Lia Braz'; Willian José Ferreira²; Marcelo Gomes da Silva³; \\ Plínio Carlos Alvalá ${ }^{4}$; Luciano Marani ${ }^{5}$; Getulio Teixeira Batista ${ }^{6}$; \\ Valiya Mannathal Hamza ${ }^{7}$ \\ 1,2,3,4,5 Instituto Nacional de Pesquisas Espaciais - INPE-São José dos Campos, SP, Brasil, \\ e-mails: lia.braz@hotmail.com, will.ferreira@hotmail.com, engmarcgomes@gmail.com, \\ plinio.alvala@inpe.br, lmarani@gmail.com, \\ ${ }^{6}$ Programa de Pós-Graduação, Universidade de Taubaté, SP, Brasil, \\ e-mail: getulio@agro.unitau.br, \\ ${ }^{7}$ Observatório Nacional, Rio de Janeiro, Brasil, \\ e-mail: hamza@on.br
}

\section{RESUMO}

O acúmulo e a decomposição de matéria orgânica em corpos d'água pode incrementar o potencial de emissão de gases de efeito estufa à atmosfera. Neste trabalho, de modo prospectivo e exploratório, foi avaliada a relação entre características físico-químicas da água e o transporte de metano para a atmosfera na Lagoa Rodrigo de Freitas, RJ, em dias típicos de verão fluminense. Em campanhas realizadas em 2011, foram coletadas 200 amostras para análise da emissão superficial do metano e 30 amostras de água para se verificar a dissolução do gás na coluna d'água, medindo simultaneamente os parâmetros físico-químicos ao longo desta coluna. As medidas de fluxo de metano evidenciaram que a Lagoa é fonte emissora do gás para a atmosfera, com fluxo médio de $33 \pm 10 \mathrm{mgCH}_{4} \mathrm{~m}^{-2} \mathrm{~d}^{-1}$. Na avaliação de como alguns parâmetros limnológicos poderiam influenciar as emissões, observou-se que a altura da lâmina d'água e a temperatura foram os fatores de maior influência nesse processo. A análise do perfil de metano na coluna d'água sugere a existência de uma maior concentração do gás na região dos estratos sedimentares do fundo da lagoa. No entanto, esse metano não chega à superfície, possivelmente, devido à diminuição da taxa de difusão vertical do metano em função do aumento da pressão com a profundidade e também devido à presença de bactérias metanotróficas, que consomem o metano ao longo da coluna d'água.

Palavras-chave: Efeito estufa, gases traços, limnologia.

\section{Influence of physicochemical water characteristics in the transport of methane to the atmosphere at Rodrigo de Freitas lagoon, RJ}

\section{ABSTRACT}

The accumulation and decomposition of organic matter in water bodies can increase the potential emission of greenhouse gases to the atmosphere. In this study, in a prospective and exploratory way, we evaluated the relationship between physicochemical characteristics of water on the transport of methane to the atmosphere at Rodrigo de Freitas lagoon, RJ. In 2011, campaigns were carried out in which 200 samples were collected for analysis of superficial methane emission and 30 water samples were collected to verify the dissolution of 
this gas in the water column, measuring simultaneously both the physicochemical water parameters along this column. The methane flow measurements showed that this lagoon is a source methane emission to the atmosphere with an average flux of $33 \pm 10 \mathrm{mgCH}_{4} \mathrm{~m}^{-2} \mathrm{~d}^{-1}$. The evaluation of limnological parameters that could affect the emission flux revealed that the water depth and temperature were the most influential factors in this process. A profile analysis of methane in the water column suggests the existence of a higher concentration of this gas in the region of the sedimentary strata at the bottom of the lagoon. However, the potential emission may not reach the surface, possibly due to the decrease in the rate of vertical diffusion of methane, as a result of the increase of pressure with depth and the presence of methanotrophic bacterias that consume methane throughout the water column.

Keywords: Greenhouse effect, trace gases, limnology.

\section{INTRODUÇÃO}

Em meio à busca por políticas sustentáveis e eficientes, no sentido de conservar os recursos naturais e proporcionar melhorias ao meio ambiente, o acúmulo de matéria orgânica nos corpos d'água surge como enorme desafio à gestão pública moderna.

$\mathrm{O}$ descarte de dejetos e efluentes de sistemas de esgoto com tratamento deficitário em rios e lagos, aliado à contribuição de águas pluviais com carga orgânica oriunda de áreas urbanizadas, aumenta a importância de estudos que avaliam o potencial de incremento de emissão de gases de efeito estufa (GEE) associado a estes processos.

O metano é o hidrocarboneto mais abundante na atmosfera terrestre, com uma concentração global estimada em 1,8 ppmv (partes por milhão por volume) e é responsável por cerca de $20 \%$ do aquecimento global, onde assume importante papel na regulação térmica do planeta e considerável papel no ciclo do carbono (IPCC, 2007). Somente na década passada, a produção de metano $\left(\mathrm{CH}_{4}\right)$ pela decomposição de matéria orgânica contabilizava $80 \%$ da emissão deste gás para a atmosfera (Dalal e Allen, 2008).

A variabilidade das fontes emissoras de metano torna difícil uma avaliação mais precisa do quanto elas contribuem individualmente. Contudo, para Wuebbles e Hayhoe (2002) e Lima (2005), as áreas alagadas respondem por cerca de 30\% das emissões naturais biogênicas, algo entre 100 e $250 \mathrm{Tg} \mathrm{CH}_{4}$.ano ${ }^{-1}$, reforçando ainda mais o potencial emissor destas áreas. A Tabela 1 apresenta a contribuição das fontes naturais de metano para a atmosfera, com base nos dados do IPCC (2007).

Tabela 1. Fontes naturais e variabilidade global do metano atmosférico.

\begin{tabular}{l|c}
\hline \multicolumn{1}{c|}{ Fontes } & Tg $\mathbf{C H}_{\mathbf{4} \cdot \mathbf{a n o}^{\mathbf{- 1}}}$ \\
\hline Áreas Alagadas & $100-231$ \\
Cupins & $20-29$ \\
Oceanos & $4-15$ \\
Hidratos & $4-5$ \\
Fontes Geológicas & $4-14$ \\
Animais Selvagens & 15 \\
Queimadas & $2-5$ \\
Total & $\mathbf{1 4 5 - 2 6 0}$ \\
\hline
\end{tabular}

Fonte: Adaptado de IPCC (2007). 
Reconhecida como a lagoa mais urbanizada do município do Rio de Janeiro, a Lagoa Rodrigo de Freitas é abastecida por rios canalizados que conduzem a ela fluxos residuais e pluviais em quantidade e qualidade não controladas (FEEMA, 1995; Machado, 2009).

Esta observação se acentua nos dias de precipitação intensa, quando a lagoa recebe a descarga do sistema de águas pluviais e o esgoto doméstico que seria lançado ao mar por meio de emissário (Enrich-Prast et al., 2008).

Evidentemente, o eventual acúmulo de carga orgânica na Lagoa Rodrigo de Freitas pode fomentar alterações no fluxo de metano para a atmosfera, além de condições de desequilíbrio e degradação ambiental.

Nota-se, a reflexo disto, a ocorrência de períodos de mortandade de peixes, até então associados ao frágil equilíbrio promovido pelo acúmulo de matéria orgânica na Lagoa, às variações bruscas de sua salinidade (Oliveira et al., 1957) e à estratificação, que ocorre devido a insuficiente circulação e renovação de suas águas (Rosman et al., 1992).

Com relação à circulação das águas, salienta-se que a Lagoa Rodrigo de Freitas possui uma única ligação com o oceano através de um estreito canal com $835 \mathrm{~m}$ de extensão denominado Jardim de Alah, o qual permite a circulação da água entre estes ambientes, fazendo com que o balanço hídrico da Lagoa se dê por esta conexão (Lima, 2010). Todavia, a água do mar penetra na Lagoa somente no período da preamar das marés de sizígia e a troca de massas existente entre ela e o mar dá-se de maneira bastante restrita (Maciel, 2007).

Estudos geológicos realizados na década de 1970 identificaram uma falha disruptiva de grande porte na região da Lagoa Rodrigo de Freitas, denominada como Falha de São João, (Brenner et al.,1980; Rideg et al., 1989; Tupinambá et al., 2010), também indicada por anomalias do campo magnético na parte central da Lagoa, em levantamento realizado por Ponte Neto et al. (2010).

Embasado nestes estudos e em dados observacionais das falhas e lineamentos geológicos na área ao entorno da Lagoa, Hamza (2010) sugere que a ocorrência de depressões tectônicas poderia propiciar a liberação de gases e a descarga de fluídos termais de origem crustal na região, que, mesmo episódicos, dependendo da forma e intensidade a que ocorressem, determinariam a morte dos peixes por anoxia.

Enfim, faltam dados que permitam uma conclusão sobre a questão e os motivos que desencadeiam a morte dos peixes que ainda ocorre, uma vez que as intervenções antrópicas aumentam ano a ano e dificultam a aplicação de soluções sustentáveis para que melhorias na qualidade ambiental da área ocorram.

Neste trabalho apresentam-se os resultados de medidas de emissão de metano na Lagoa Rodrigo de Freitas, que objetivaram, de modo prospectivo, avaliar a relação entre algumas características físico-químicas básicas da água e o transporte de metano para a atmosfera na área da lagoa em dois dias típicos de verão na cidade do Rio de Janeiro.

\section{MATERIAIS E MÉTODOS}

A Lagoa Rodrigo de Freitas está localizada na cidade do Rio de Janeiro, entre as coordenadas $22^{\circ} 57^{\prime}$ e $22^{\circ} 58^{\prime}$ de latitude sul e $43^{\circ} 11^{\prime}$ e $43^{\circ} 13^{\prime}$ de longitude oeste (IBAMA, 1989). Seu formato atual é de um polígono irregular, com um espelho d'água que ocupa a área de $2,5 \mathrm{~km}^{2}$ e profundidade máxima próxima de $11 \mathrm{~m}$ (Andreata et al., 1997). O clima da região se enquadra no grupo "AW" da classificação de Köppen, apresentando verão úmido e inverno seco.

Utilizando-se de uma pequena embarcação que era ancorada nos pontos de coleta, foram realizadas duas campanhas em 2011: a primeira em 02 de fevereiro e a segunda em 15 de março, dias que se mostraram típicos de verão na cidade do Rio de Janeiro. Embora os registros de precipitação acumulada nos dias que antecederam as coletas tenham alcançado, 
BRAZ, L.; FERREIRA, W. J.; SILVA, M. G.; ALVALÁ, P. C.; MARANI, L.; BATISTA, G. T.; HAMZA, V. M. Influência de características físico-químicas da água no transporte de metano para a atmosfera na Lagoa Rodrigo de Freitas, RJ. Ambi-Agua, Taubaté, v. 7, n. 3, p. 99-112, 2012. (http://dx.doi.org/10.4136/ambiagua.972)

respectivamente, $50 \mathrm{~mm}$ e $22 \mathrm{~mm}$ (SINDA, 2012), no momento das observações ambos os dias estavam claros, com céu aberto e poucas nuvens, com a temperatura média de $29^{\circ} \mathrm{C}$, variando de 27 a $32^{\circ} \mathrm{C}$ durante os horários de coleta.

$\mathrm{Na}$ Figura 1 são ilustrados os cinco pontos amostrais deste trabalho, bem como é apresentada a batimetria da Lagoa, segundo dados do Projeto Ambiental Lagoa Limpa (Grupo EBX, 2011).

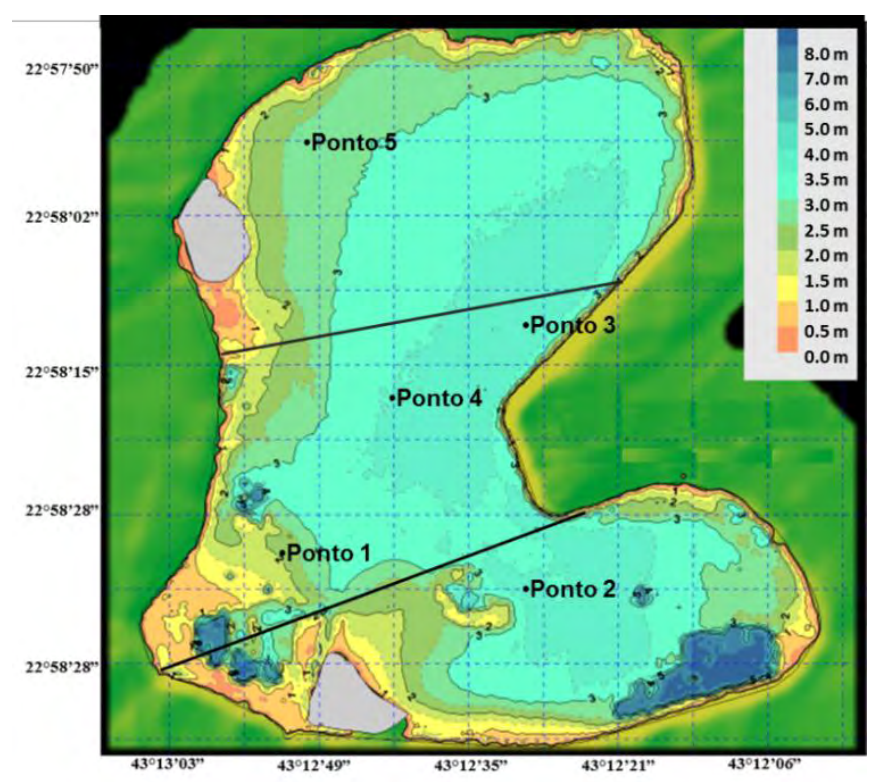

Figura 1. Batimetria da Lagoa Rodrigo de Freitas, RJ, e indicação dos pontos amostrais: as linhas contínuas delimitam a Falha de São João (Ponte Neto et al., 2010). Imagem adaptada do Projeto Ambiental Lagoa Limpa (Grupo EBX, 2011).

Os pontos de amostragem 1, 3 e 4 foram definidos a partir da falha geológica que cruza a Lagoa (Ponte Neto et al., 2010), enquanto os pontos 2 e 5 foram escolhidos por estarem fora desta faixa de delimitação. Registra-se que o ponto 1 foi situado junto à área de atividade de pescadores na Lagoa e o ponto 5 próximo a uma das entradas de dejetos e efluentes do sistema de esgoto.

As amostras foram obtidas por meio da técnica de câmara estática (Khalil et al., 1998; Marani e Alvalá, 2007), onde o metano emitido pela superfície da lagoa foi coletado em seringas plásticas de polipropileno com o uso de cúpulas cilíndricas de PVC, com volume de 26 litros e flutuadores de espuma acoplados em sua base para sustentação na água (Figura 2).

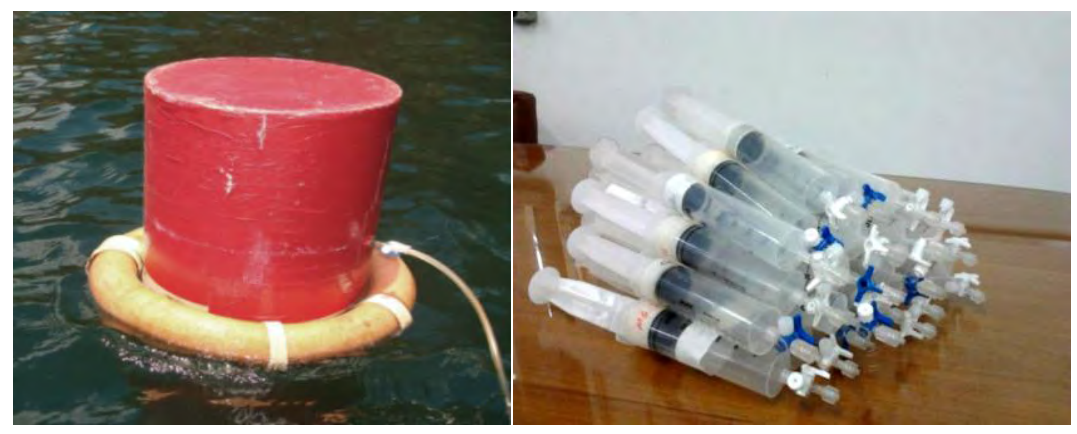

Figura 2. Câmara de PVC e seringas de polipropileno utilizadas nas coletas. 
Amostras de água foram coletadas nos pontos observando a batimetria da Lagoa (Figura 1 por meio de uma garrafa de Van Dorn. Utilizando-se a técnica de headspace (Ioffe e Vitenberg, 1984), a concentração de metano dissolvido foi determinada para estas amostras, sendo possível obter um perfil de metano dissolvido na coluna d'água da Lagoa com medidas em três profundidades: logo abaixo da superfície, no meio da coluna d'água e próximas ao sedimento no fundo da lagoa. Assim, com auxílio de seringas plásticas, foram retirados $30 \mathrm{ml}$ da água coletada em cada um dos níveis, sendo em cada uma delas adicionados $30 \mathrm{ml} \mathrm{de} \mathrm{ar}$ ambiente. Cada seringa foi agitada manualmente por aproximadamente dois minutos e permaneceu em repouso pelo mesmo período. Após isto, o ar de cada seringa foi injetado em um pequeno frasco de vidro previamente evacuado, com volume de $20 \mathrm{ml}$, e acondicionado para análise em laboratório. Destaca-se que o metano é pouco solúvel em água e, no momento da agitação, devido à variação da pressão parcial, o gás dissolvido na água passa para o ar existente na seringa.

As amostras foram enviadas ao Laboratório de Biogeoquímica Ambiental do INPE, São José dos Campos, SP, analisadas por meio da técnica de cromatografia gasosa associada a um detector de ionização de chama e as calibrações realizadas tendo por referência a concentração de um gás padrão confeccionado com $1804 \pm 2$ nanomol.mol ${ }^{-1}$ de $\mathrm{CH}_{4}$.

Nas análises foi injetado o volume total de cada seringa $(60 \mathrm{ml})$, sendo para cada uma delas executadas três injeções de $20 \mathrm{ml}$, considerada a média das três injeções como valor principal e o desvio padrão percentual entre as injeções de no máximo $1 \%$.

O fluxo de metano emitido pela lagoa foi obtido a partir da variação temporal da sua concentração no interior da câmara estática, descrita como a razão entre o número de moles de $\mathrm{CH}_{4}\left(\mathrm{n}_{\mathrm{CH}_{4}}\right)$ e o número total de moles da mistura $\left(\mathrm{n}_{\mathrm{t}}\right)$.

Adotando-se uma variação no número de moles de metano, $\Delta \mathrm{n}_{\mathrm{CH}_{4}}$, dado pela razão entre a massa da substância $\left(\mathrm{m}_{\mathrm{CH}_{4}}\right)$ e sua massa molar $\left(\mathrm{M}_{\mathrm{CH}_{4}}\right)$, ou seja, $\left(\mathrm{n}_{\mathrm{CH}_{4}}\right)=\mathrm{m}_{\mathrm{CH}_{4}} / \mathrm{M}_{\mathrm{CH}_{4}}$, e aplicando-se a equação dos gases ideais $(p V=m R T / M)$, pode-se escrever:

$$
\frac{\Delta \mathrm{n}_{\mathrm{CH}_{4}}}{\mathrm{n}_{\mathrm{t}}}=\frac{\Delta \mathrm{m}_{\mathrm{CH}_{4}} \mathrm{RT}}{\mathrm{M}_{\mathrm{CH}_{4}} \mathrm{pV}}=\Delta \chi_{\mathrm{CH}_{4}}
$$

Consequentemente,

$$
\Delta \mathrm{m}_{\mathrm{CH}_{4}}=\frac{\Delta \chi_{\mathrm{CH}_{4}} \mathrm{pVM}_{\mathrm{CH}_{4}}}{\mathrm{RT}}
$$

em que:

p é a pressão total (atm), $\mathrm{V}$ o volume total (litros), $\mathrm{M}_{\mathrm{CH}_{4}}$ é a massa molar do metano (16, $\left.04 \mathrm{~g} \mathrm{~mol}^{-1}\right)$, R é a constante dos gases $\left(0,082 \mathrm{~atm} \mathrm{~L} \mathrm{~mol}^{-1} \mathrm{~K}^{-1}\right)$ e $\mathrm{T}$ é a temperatura $(\mathrm{K})$. $\mathrm{O}$ termo $\Delta \chi_{\mathrm{CH}_{4}}$ faz referência à razão de mistura do metano.

Admitindo-se que a variação do fluxo por meio da área $\mathrm{A}\left(\mathrm{m}^{2}\right)$ seja constante durante um intervalo de tempo $\Delta \mathrm{t}$, descreve-se o fluxo $\Phi_{\mathrm{CH}_{4}}\left(\mathrm{mgCH}_{4} \mathrm{~m}^{-2} \mathrm{dia}^{-1}\right)$ como:

$$
\Phi_{\mathrm{CH}_{4}}=\frac{\Delta \chi_{\mathrm{CH}_{4}} \mathrm{pVM}_{\mathrm{CH}_{4}}}{\Delta \text { tART }}=\frac{p M_{\mathrm{CH}_{4}}}{\text { ART }} \frac{\Delta \chi_{\mathrm{CH}_{4}}}{\Delta \mathrm{t}}=\frac{\mathrm{pVM}_{\mathrm{CH}_{4}}}{\text { ART }} \frac{\partial \chi_{\mathrm{CH}_{4}}}{\partial \mathrm{t}}
$$


A variável mais importante na Equação 3 é $\partial \chi_{\mathrm{CH}_{4}} / \partial \mathrm{t}$, que é a derivada das medidas de concentração, estando todas as demais variáveis associadas às medições efetuadas diretamente no momento da coleta. Esta grandeza foi estimada a partir de uma regressão linear, considerando-se $\partial \chi_{\mathrm{CH}_{4}} / \partial \mathrm{t}$ como o "b" em $\chi=\chi_{0}+\mathrm{bt}$.

Enfim, para utilizar a regressão linear na obtenção de $\partial \chi_{\mathrm{CH}_{4}} / \partial \mathrm{t}$, considerou-se que o fluxo foi constante durante o tempo de coleta das amostras. Para afirmar a confiabilidade dos dados, devido à possibilidade de ocorrência de erro amostral e contaminação das amostras desde a etapa de coleta até a análise cromatográfica, foram adotados dois critérios de validação dos fluxos:

1. Observância do coeficiente de determinação $\left(\mathrm{r}^{2}\right)$ : foram considerados somente os valores de $\mathrm{r}^{2}$ superiores a 0,9 na reta de regressão para garantir a linearidade requerida dos pontos (Khalil et al., 1998; Alvalá e Kirchhoff; 2000).

2. Comparação entre a concentração inicial de metano no tempo inicial $(t=0)$, obtida a partir da regressão linear, e a concentração do metano em amostras de ar ambiente, coletadas antes de cada coleta: a concentração do metano nas amostras de ar ambiente e no tempo inicial da coleta devem apresentar valores semelhantes.

A fim de caracterizar os parâmetros físico-químicos na coluna d'água e verificar possíveis relações existentes entre estes parâmetros e o fluxo de metano, foram medidas as variáveis potencial hidrogeniônico $(\mathrm{pH})$, oxigênio dissolvido (OD), potencial de óxido redução (ORP) e temperatura do ar e da água com o uso de uma sonda de multiparâmetros de qualidade da água, marca Horiba, modelo U-51, que permitiu a tomada de medidas diretamente submerso na água e eliminou a necessidade de coletar amostras.

A análise dos componentes principais (ACP) foi usada para reduzir os dados físicoquímicos por combinações lineares das variáveis originais e julgar a importância destas próprias variáveis no processo de emissão de metano.

Uma vez que os parâmetros ambientais não variaram significativamente a $95 \%$ de confiabilidade pelo teste $\mathrm{t}$ de Student, na análise, os dados foram agrupados e avaliados por campanha, independentemente do ponto onde foram coletados.

Estes dados foram, então, transformados em um conjunto menor de combinações lineares, que responderam pela maior parte da variância do grupo inicial (Moita e Moita Neto, 1998; Cazar, 2003). Neste novo conjunto, reduziu-se o número de variáveis mantendo a informação original tanto quanto fosse possível, de forma que as variáveis com características semelhantes pudessem ser agrupadas em fatores. Uma matriz de correlação entre as o fluxo de metano e variáveis com maior peso na combinação linear das primeiras componentes principais foi gerada a partir destes fatores, determinando, assim, quais foram as variáveis medidas mais importantes do ponto visto estatístico.

\section{RESULTADOS E DISCUSSÃO}

Os resultados das campanhas realizadas nos dias 2 de fevereiro e em 15 de março de 2011, em que foram coletadas 200 amostras nos pontos interiores da Lagoa e que resultaram em 46 fluxos, dos quais 36 foram validados, indicam a variabilidade no fluxo do gás nos dois dias de amostragem. Os valores registrados nas campanhas 1 e 2 foram de $39 \pm 11$ e $29 \pm 6$ $\mathrm{mgCH}_{4} \mathrm{~m}^{-2} \mathrm{~d}^{-1} \mathrm{em}$ média, respectivamente, caracterizando, portanto, a Lagoa Rodrigues de Freitas como emissora de metano para a atmosfera.

Alterações no padrão de vento podem ter favorecido a dispersão dos valores do fluxo de metano, uma vez que, decorrente da ação dos ventos, a circulação da água tenderia a colocar em suspensão partículas sólidas e trazer parte dos poluentes já sedimentados de volta para a coluna d'água (Enrich-Prast et al., 2008). Contudo, ressalta-se que, em profundidades 
BRAZ, L.; FERREIRA, W. J.; SILVA, M. G.; ALVALÁ, P. C.; MARANI, L.; BATISTA, G. T.; HAMZA, V. M. Influência de características físico-químicas da água no transporte de metano para a atmosfera na Lagoa Rodrigo de Freitas, RJ. Ambi-Agua, Taubaté, v. 7, n. 3, p. 99-112, 2012. (http://dx.doi.org/10.4136/ambiagua.972)

menores do que dois metros, a mistura das massas de água ocorre somente nos casos de vento moderado, predominantemente observados nos dias de coleta.

A emissão média geral da lagoa foi de $33 \pm 10 \mathrm{mgCH}_{4} \mathrm{~m}^{-2} \mathrm{~d}^{-1}$, fluxo médio que é menor, porém condizente com os resultados apresentados por estudos realizados em lagoas em condição semelhante de uso: lagoas urbanas construídas para tratamento de águas residuais em zonas úmidas da Nova Zelândia apresentam valores que variam de 45 a $526 \mathrm{mgCH}_{4} \mathrm{~m}^{-2} \mathrm{~d}^{-1}$ (Tanner et al., 1997), enquanto na Suécia, estima-se que este valor esteja entre 80 e 180 $\mathrm{mgCH}_{4} \mathrm{~m}^{-2} \mathrm{~d}^{-1}$ (Johansson et al., 2004).

$\mathrm{Na}$ Tabela 2 são apresentados os fluxos de metano obtidos por meio das câmaras estáticas na interface entre a água e a atmosfera nestas duas campanhas.

Tabela 2. Fluxo de metano na interface água-atmosfera na Lagoa Rodrigo de Freitas, RJ.

\begin{tabular}{c|c|c|c|c|c|c}
\hline \multirow{2}{*}{ Campanha } & \multirow{2}{*}{ Ponto } & \multirow{2}{*}{ Prof. (m) } & \multicolumn{4}{|c}{ Fluxo de metano $\left(\mathbf{m g C H}_{\mathbf{4}} \mathbf{m}^{-\mathbf{2}} \mathbf{d}^{-\mathbf{1}}\right)$} \\
\cline { 3 - 6 } & & $\mathbf{N}$ & Média & $\mathbf{D P}$ & $\mathbf{D P \%}$ \\
\hline 1 & 1 & 2,0 & 5 & 52,7 & 5,8 & 11,0 \\
1 & 2 & 4,5 & 4 & 27,4 & 2,4 & 8,7 \\
1 & 3 & 3,5 & 5 & 28,7 & 2,7 & 9,5 \\
1 & 4 & 3,5 & 4 & 36,4 & 3,2 & 8,7 \\
1 & 5 & 2,5 & 4 & 47,6 & 2,0 & 4,2 \\
2 & 1 & 2,0 & 3 & 36,6 & 0,6 & 1,6 \\
2 & 2 & 4,5 & 2 & 22,0 & 1,5 & 6,8 \\
2 & 3 & 3,5 & 3 & 29,7 & 1,9 & 6,4 \\
2 & 4 & 3,5 & 3 & 23,5 & 2,2 & 9,2 \\
2 & 5 & 2,5 & 3 & 31,1 & 2,9 & 9,4 \\
\hline
\end{tabular}

*N: número de fluxos válidos; DP: desvio padrão; DP\%: desvio padrão percentual.

Coletado na zona da falha, no Ponto 1 foi registrado o maior fluxo médio de metano entre as campanhas, $44,7 \pm 11,4 \mathrm{mgCH}_{4} \mathrm{~m}^{-2} \mathrm{~d}^{-1}$, ao passo que no Ponto 2, observou-se o menor fluxo médio do experimento, 24,7 $\pm 3,8 \mathrm{mgCH}_{4} \mathrm{~m}^{-2} \mathrm{~d}^{-1}$. Nos outros pontos, independentemente de sua localização dentro ou fora da falha, não houve uma variação significativa na medida do fluxo de metano, tornando impossível caracterizar, neste trabalho, a possível influência da Falha de São João na emanação do metano.

Há de se considerar que os maiores valores de emissão, observados em ambas as campanhas nos Pontos 1 e 5, podem estar relacionados às fontes antrópicas locais, como a atividade pesqueira e a entrada de dejetos urbanos na área lagunar.

É pertinente reforçar que a precipitação observada nos dias de verão ocasionaria um maior aporte de esgoto à Lagoa, mas, no entanto, nos momentos de coleta não se observou esta ocorrência.

Embora fosse importante analisar a influência da variável precipitação no processo de emissão de metano, não foi escopo deste trabalho a avaliação deste aporte, uma vez que este item surgiria como um elemento bastante complexo para uma pesquisa transversal e exploratória.

\subsection{Medidas ambientais}

A Tabela 3 apresenta os valores amostrados de parâmetros físico-químicos medidos na água da Lagoa Rodrigo de Freitas. 
BRAZ, L.; FERREIRA, W. J.; SILVA, M. G.; ALVALÁ, P. C.; MARANI, L.; BATISTA, G. T.; HAMZA, V. M. Influência de características físico-químicas da água no transporte de metano para a atmosfera na Lagoa Rodrigo de Freitas, RJ. Ambi-Agua, Taubaté, v. 7, n. 3, p. 99-112, 2012. (http://dx.doi.org/10.4136/ambiagua.972)

Tabela 3. Parâmetros físico-químicos superficiais medidos na lagoa.

\begin{tabular}{|c|c|c|c|c|c|c|c|}
\hline \multirow{2}{*}{ Campanha } & \multirow{2}{*}{ Ponto } & Profundidade & $\mathbf{T}$ ar & $\bar{T}$ água & pH & ORP & OD \\
\hline & & $\mathbf{m}$ & \multicolumn{3}{|c|}{${ }^{\circ} \mathbf{C}$} & $\mathbf{m V}$ & $\mathrm{mg} / \mathrm{L}$ \\
\hline 1 & 1 & 2 & 29,8 & 31,0 & 7,0 & 105,0 & 2,9 \\
\hline 1 & 2 & 4,5 & 29,4 & 30,8 & 8,0 & 58,0 & 5,4 \\
\hline 1 & 3 & 3,5 & 30,6 & 32,0 & 7,8 & 65,0 & 5,3 \\
\hline 1 & 4 & 3,5 & 31,6 & 32,0 & 8,0 & 66,0 & 5,8 \\
\hline 1 & 5 & 2,5 & 31,9 & 33,1 & 8,3 & 52,0 & 6,3 \\
\hline 2 & 1 & 2 & 27,5 & 29,5 & 7,8 & 65,0 & 4,3 \\
\hline 2 & 2 & 4,5 & 27,6 & 28,8 & 8,1 & 56,9 & 6,2 \\
\hline 2 & 3 & 3,5 & 27,4 & 28,8 & 7,7 & 80,0 & 4,3 \\
\hline 2 & 4 & 3,5 & 27,7 & 28,8 & 7,5 & 88,0 & 3,4 \\
\hline 2 & 5 & 2,5 & 29,8 & 29,1 & 7,7 & 63,0 & 4,1 \\
\hline
\end{tabular}

*T ar: temperatura do ar; T água: temperatura da água; ORP: potencial de óxido redução; OD: oxigênio dissolvido.

Corroborando Marani e Alvalá (2007), observou-se um indicativo de relação existente entre a profundidade, dada como a altura medida do espelho d'água até o fundo da lagoa, e a emissão de metano.

De modo geral, foram observadas menores emissões em áreas mais profundas da Lagoa, fato que foi associado à queda na taxa de difusão vertical do metano decorrente do aumento da pressão com a profundidade na coluna de água e à presença de bactérias metanotróficas, que consomem o metano ao longo da coluna de água (Pellizari et al., 2007).

A partir dos dados da Tabela 3 observou-se que os parâmetros medidos na Lagoa se encontravam dentro da faixa de eficiência de produção de metano em meio aquoso, apresentados, como referência, na Tabela 4.

Tabela 4. Eficiência de produção de $\mathrm{CH}_{4}$ em relação aos parâmetros ambientais.

\begin{tabular}{l|c|l}
\hline Parâmetros & Faixa de variação & \multicolumn{1}{c}{ Referência } \\
\hline T Água & 15 a $37^{\circ} \mathrm{C}$ & Yang e Chang (1998) \\
$\mathrm{pH}$ & 6,4 a 7,8 & Wang et al. (1993) \\
OD & $<10 \mathrm{mg} / \mathrm{L}$ & Silva (2010) \\
ORP & -150 a $440 \mathrm{mV}$ & Wang et al., (1993); Silva (2010) \\
\hline
\end{tabular}

As temperaturas do ar e da água não apresentaram variação significativa, mantendo-se nos dois dias de coleta próxima a $28^{\circ} \mathrm{C}$ e $29^{\circ} \mathrm{C}$, respectivamente. A média do $\mathrm{pH}$ obtida na Lagoa Rodrigo de Freitas foi de 7,8, valor situado no limiar da faixa ótima de produção. Salienta-se que grande parte dos organismos produtores de metano é sensível ao $\mathrm{pH}$ e habitam locais onde, preferencialmente, sua condição é próxima da neutralidade (Wang et al., 1993).

Na primeira campanha, no Ponto 1, ponto de maior emissão de metano, observou-se a menor concentração de oxigênio dissolvido e a neutralidade do $\mathrm{pH}$. Neste ponto, o ORP, parâmetro utilizado como indicador da quantidade de matéria orgânica disponível no meio, mostrou-se mais elevado em relação aos outros pontos. Langmuir (1997) avalia que quanto 
maior o valor do ORP, mais favorecido se torna a produção de metano por arquéias metanogênicas. Sua diminuição, ao contrário, aliada ao aumento gradativo da concentração de oxigênio ao longo da coluna d'água proporcionaria incremento no consumo do metano antes que o mesmo atingisse a superfície.

Silva (2010) afirma que a entrada de matéria orgânica na planície sul pantaneira devido ao regime hídrico local faz com que o $\mathrm{pH}$ decaia de 8 no período de seca para 3 na época de cheia, denotando-a como um dos maiores agentes redutores em áreas alagadas.

Diante disto, quando verificada uma maior quantidade de matéria orgânica no sistema, há uma tendência de o pH projetar-se mais ácido e se observar a elevação do consumo de oxigênio, o que, consequentemente, torna o ORP mais positivo.

Nos dados da Lagoa Rodrigo de Freitas ambas as prospecções foram observadas e, de modo geral, os parâmetros pH, ORP e OD sinalizam uma baixa quantidade de matéria orgânica disponível na Lagoa, uma vez que não houve alteração significativa destes parâmetros na maior parte do experimento. Esta informação é reforçada pelo estudo de Baptista Neto et al. (2011), que verificou que os sedimentos de fundo da Lagoa Rodrigo de Freitas são predominantemente lamosos, estando a concentração de matéria orgânica entre 0 e $35 \%$ do peso total da amostra. Segundo estes autores, percentuais mais baixos são registrados no entorno do Jardim de Alah, que sofre uma maior influência hidrodinâmica e denota a presença de sedimentos arenosos no local.

Outrossim, a análise de componentes principais demonstrou que não seriam as variáveis $\mathrm{pH}$, ORP e OD os fatores mais importantes nos fluxos de metano observados.

\subsection{Análise das componentes principais}

Para a análise dos componentes principais (ACP) tomou-se inicialmente um conjunto de dados com cinco variáveis e 36 amostras, de onde puderam ser retidas as componentes principais com autovalores menores do que 1 .

A CP1 indicou que $57 \%$ da variância dos dados são explicados por três componentes principais (pH, ORP e OD), enquanto a $\mathrm{CP} 2$, representando $87 \%$ da variância das amostras, foi explicada por duas componentes (profundidade e temperatura). A partir da Tabela 5, nos Grupos CP1 e CP2, são apresentados os pesos estatísticos que foram utilizados na diminuição do número de variáveis da análise de componentes principais.

Tabela 5. Análise de Componentes Principais dos parâmetros subsuperficiais medidos na coluna d'água da Lagoa Rodrigo de Freitas.

\begin{tabular}{l|c|c}
\hline Parâmetros & CP1 & CP2 \\
\hline Profundidade & $-0,52$ & $-0,71$ \\
Temperatura & $-0,41$ & 0,81 \\
pH & $-0,97$ & 0,00 \\
ORP & $-0,92$ & 0,01 \\
OD & $-0,98$ & 0,04 \\
\hline
\end{tabular}

Após determinar novos fatores de acordo com os grupos CP1 e CP2, foi gerada uma matriz de correlação entre estes fatores e o fluxo médio de metano obtido por ponto medido. Notou-se que o grupo que melhor poderia explicar a variação do fluxo de metano na Lagoa Rodrigo de Freitas foi o CP2, o qual se fundamenta nas medidas de profundidade e temperatura. A partir destes dados obteve-se a curva de regressão linear entre o fluxo médio de metano e $\mathrm{CP} 2$, cujo $\mathrm{R}^{2}$ mostrou-se significativo, com valor de 0,87 , revelando a influência 
BRAZ, L.; FERREIRA, W. J.; SILVA, M. G.; ALVALÁ, P. C.; MARANI, L.; BATISTA, G. T.; HAMZA, V. M. Influência de características físico-químicas da água no transporte de metano para a atmosfera na Lagoa Rodrigo de Freitas, RJ. Ambi-Agua, Taubaté, v. 7, n. 3, p. 99-112, 2012. (http://dx.doi.org/10.4136/ambiagua.972)

destes parâmetros na emissão de metano pela Lagoa. A Figura 3 ilustra este resultado, bem como o limite de confiabilidade da curva a $95 \%$ de confiança.

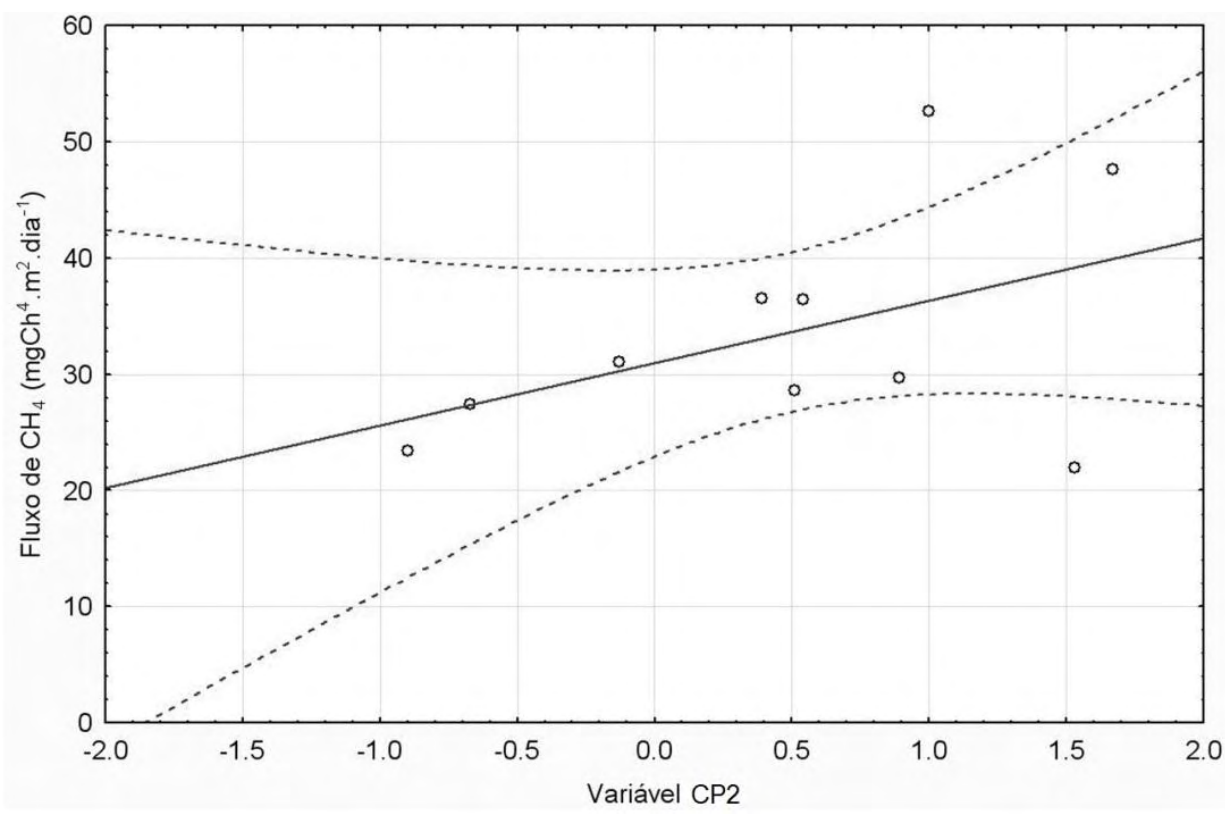

Figura 3. Regressão linear e limite de confiabilidade a $95 \%$ entre o fluxo médio de metano e as variáveis do Grupo $\mathrm{CP} 2$, profundidade e temperatura.

Vale ressaltar que na relação observada, o reduzido número de amostras válidas obtidos em dois dias de avaliação tornou limitada qualquer inferência estatística que pudesse aprofundar a análise destes resultados, sendo necessária uma maior quantidade de fluxos, com novas observações em períodos sazonais diferenciados, para que se seja determinado o quanto estas variáveis poderão influenciar nas emissões de metano.

Assim, ganha importância a organização de um maior número de campanhas para obtenção de dados para o estudo da variação sazonal das emissões, bem como informações sobre a variação climática da região, principalmente, porque ocorrem mudanças nos fluxos residuais e pluviais na área da Lagoa.

\subsection{Metano dissolvido e variáveis ambientais}

Para o estudo do metano dissolvido foram coletadas 30 amostras na coluna d'água da Lagoa Rodrigo de Freitas. Observou-se que o perfil médio do metano dissolvido na Lagoa apresentou valores crescentes partindo da superfície até o fundo (Tabela 6).

Tabela 6. Variabilidade do metano dissolvido na coluna d'água da Lagoa Rodrigo de Freitas.

\begin{tabular}{cc|c}
\hline \multicolumn{2}{c|}{$\begin{array}{c}\text { Profundidade } \\
(\mathbf{m})\end{array}$} & $\begin{array}{c}\text { Metano Dissolvido } \\
\left(\mathbf{m g L}^{-1}\right)\end{array}$ \\
\hline Sub-superfície & $(0,0-0,5)$ & $0,010 \pm 0,002$ \\
Meio & $(0,5-2,0)$ & $0,013 \pm 0,003$ \\
Fundo & $(2,0-4,5)$ & $0,020 \pm 0,009$ \\
\hline
\end{tabular}


BRAZ, L.; FERREIRA, W. J.; SILVA, M. G.; ALVALÁ, P. C.; MARANI, L.; BATISTA, G. T.; HAMZA, V. M. Influência de características físico-químicas da água no transporte de metano para a atmosfera na Lagoa Rodrigo de Freitas, RJ. Ambi-Agua, Taubaté, v. 7, n. 3, p. 99-112, 2012. (http://dx.doi.org/10.4136/ambiagua.972)

$\mathrm{Na}$ Tabela 7 são ilustrados os parâmetros físico-químicos médios na coluna d'água dos cinco pontos amostrais medidos neste ensaio, obtidos na subsuperfície, no meio e no fundo da Lagoa.

Tabela 7. Perfil dos parâmetros ambientais medidos na coluna d'água da Lagoa ( $\mathrm{n}=5$ pontos)

\begin{tabular}{l|c|c|c|c|c}
\hline \multicolumn{1}{c|}{ Posição } & Parâmetros & T água (-C) & $\mathbf{p H}$ & ORP $(\mathbf{m V})$ & OD $\left(\mathbf{m g ~ L}^{-\mathbf{1}} \mathbf{)}\right.$ \\
\hline \multirow{4}{*}{ Sub-superfície } & Média & 29,0 & 7,8 & 70,6 & 4,5 \\
& Dp & 0,3 & 0,2 & 12,9 & 1,0 \\
& Dp\% & 1 & 3 & 18 & 23 \\
\hline \multirow{3}{*}{ Meio } & Média & 28,8 & 7,7 & 73,4 & 3,9 \\
& Dp & 0,5 & 0,2 & 13,0 & 1,0 \\
& Dp\% & 2 & 3 & 18 & 26 \\
\hline \multirow{3}{*}{ Fundo } & Média & 28,6 & 7,6 & 78,4 & 3,2 \\
& Dp & 0,2 & 0,3 & 15,4 & 1,3 \\
& Dp\% & 1 & 4 & 20 & 40 \\
\hline
\end{tabular}

A partir da Tabela 7 verificou-se que a temperatura da água e o $\mathrm{pH}$ se mantiveram constantes em relação à batimetria da lagoa. No entanto, o OD aumentou na medida em que se aproximava da superfície, sendo incrementado pelas trocas gasosas que ocorriam no limiar da interação da água com a atmosfera.

Diferentemente do OD, o ORP apresentou valor mais elevado quando medido próximo ao sedimento, fato que pode ser justificado pela maior concentração de matéria orgânica no fundo da lagoa, o que tende a consumir o oxigênio dissolvido tornando o ambiente mais redutor na interface entre a água e o sedimento.

Ainda por meio da Tabela 7 foi reforçada a ideia de que em ambientes menos oxigenados ocorrem indícios de favorecimento à produção do metano, pois, onde a concentração de oxigênio dissolvido foi menor, consequentemente coexistindo um potencial redutor mais elevado, somente uma fração do gás produzido na interface entre a água e o sedimento foi oxidada por micro-organismos, sendo o saldo residual transferido para atmosfera ao longo da coluna de água.

A partir do estudo do metano dissolvido na Lagoa Rodrigo de Freitas verificou-se que nem todo o metano produzido próximo ao substrato chega a ser transportado para a superfície, justificando os reduzidos valores de fluxo registrados neste trabalho.

\section{CONCLUSÃO}

As medidas de fluxo de metano realizadas na Lagoa Rodrigo de Freitas, resultantes de um estudo prospectivo com referência em dois dias típicos de verão, revelaram-na como uma fonte emissora desse gás para a atmosfera, com fluxo médio de $33 \pm 10 \mathrm{mgCH}_{4} \mathrm{~m}^{-2} \mathrm{~d}^{-1}$. Esse valor é próximo do observado em lagoas de semelhante utilização.

Independentemente da localização dos pontos amostrais, dentro ou fora da falha, não houve uma variação significativa na medida do fluxo de metano, tornando impossível caracterizar, neste trabalho exploratório, a possível influência da Falha de São João na emanação do metano.

A profundidade, medida associada à altura da lâmina d'água até o fundo da lagoa, junto à temperatura revelaram-se como os parâmetros de maior influência no processo de emissão atmosférica do metano pela Lagoa.

A análise do perfil de metano na coluna d'água sugere uma maior concentração do gás nos estratos sedimentares do fundo da Lagoa, apontado por medidas de $\mathrm{pH}$, oxigênio 
dissolvido e ORP ao longo da coluna. Porém, nem todo o metano produzido chega a ser transportado à superfície, sendo consumido na medida em que se aproxima dela.

Deve-se ressaltar a existência de uma complexidade na limnologia da água da Lagoa em razão dos diferentes agentes que podem interferir nas emissões atmosféricas.

\section{AGRADECIMENTOS}

Ao apoio financeiro da Fundação de Amparo à Pesquisa do Estado do Rio de Janeiro FAPERJ, por meio do Projeto "Caracterização Físico-Química das Emanações provenientes das Falhas Geológicas na área Costeira do Estado do Rio de Janeiro" (Processo $n^{\circ}$. 111.342/2010).

\section{REFERÊNCIAS}

ALVALÁ, P. C.; KIRCHHOFF, W. V. J. H. Methane fluxes from the Pantanal floodplain in Brazil: seasonal variation. In: van Ham et al. (Eds.) Non- $\mathrm{CO}_{2}$ Greenhouse gases: scientific understanding, control and implementation. Kluwer Academic Publishers, 2000, p. 95-99.

ANDREATA, J. V.; MARCA, A. G.; SOARES C. L.; SANTOS R. S. . Distribuição mensal dos peixes mais representativos da Lagoa Rodrigo de Freitas. Rio de Janeiro, Brasil. Revista Brasileira de Zoologia, 1997, 14 (1): pp. 121-13.

BAPTISTA NETO, J. A.; SILVA, C. G.; DIAS, G. T. M.; FONSECA, E. M. Distribuição sedimentar da Lagoa Rodrigo de Freitas através de sísmica de alta resolução. Revista Brasileira de Geofísica. [online]. 2011, Vol. 29, n. 1, pp. 187-195. ISSN 0102-261X. http://dx. doi. org/10. 1590/S0102-261X2011000100013.

BRENNER, X; FERRARI, Y; PENHA, Z. Anais do XXXI Congresso Brasileiro de Geologia, 1980, v. 5.

CAZAR, R. A. An exercise on chemometrics for a quantitative analysis course. Journal of. Chemical Education2003, Ed. 80(9): 1026-1029.

DALAL, R. C.; ALLEN, D. E. Greenhouse gas fluxes from natural ecosystems. Australian Journal of Botany, 2008, v. 56, p. 369-407.

ENRICH-PRAST, A.; BENTO, L.; SANTORO, A. Influência das Mudanças Globais sobre as Lagoas da Cidade do Rio de Janeiro. In: Sergio Besserman Vianna; Paulo Pereira Gusmão; Paula Serrano. (Org.). RIO Próximos 100 anos: O aquecimento global e a Cidade. RIO Próximos 100 anos: O aquecimento global e a Cidade. Rio de Janeiro: Instituto Pereira Passos, 2008, p. 176-185.

FEEMA. Lagoa Rodrigo de Freitas - Ações propostas para melhoria da Bacia. Relatório final do grupo de trabalho criado pelo decreto $\mathrm{n}^{\mathbf{0}} 13539.1995$.

GRUPO EBX. Projeto ambiental Lagoa Limpa: uma nova lagoa para o Rio de Janeiro. Disponível em: <http://www.lagoalimpa.com.br/>. Acesso em: 15 nov. 2011.

HAMZA, V. M. Episodes of Biotic Crisis in Coastal Lagoons of Rio de Janeiro: Similarities with Mass Extinctions of end-Permian and end-Cretaceous times, (Extended Abstract), Meeting of the Americas. Anais. 2010, Foz de Iguaçu, Brazil. 
IBAMA. Lagoa Rodrigo de Freitas. Segundo Ministério do interior. Instituto Brasileiro do Meio Ambiente e dos Recursos Naturais Renováveis. IBAMA - RJ, 1989, 177p.

IOFFE, B. V.; VITENBERG, A. G. Headspace Analysis and Related Methods in Gas Chromatography. John Wiley and Sons. 1984, 304p.

IPCC. INTERGOVERNMENTAL PANEL ON CLIMATE CHANGE. Climate Change 2007: The Physical Basis. Contribution of working Group I to the Fourth Assessment Report of the Intergovernmental Panel on Climate Change. Cambridge. 2007.

JOHANSSON, A. E.; GUSTAVSSON, A. M.; ÖQUIST, M. G.; SVENSSON, B. H. Methane emissions from a constructed wetland treating wastewater: Seasonal and spatial distribution and dependence on edaphic factors. Water Research2004, 38:3960-3970.

KHALIL, M. A. K.; RASMUSSEN, R. A.; SHEARER, M.; DALlUGE, R.; REN, L.; DUAN, C. L. Factors affecting methane emissions from rice fields. Journal of Geophysical Research, 1998, v. 103, n. D19, p. 25219-25231.

LANGMUIR, D. Aqueous environmental geochemistry. Upper Saddle River, NJ: PrenticeHall, Inc. 1997, 600p.

LIMA, I. B. T. Biogeochemical distinction of methane releases from two Amazon hydroreservoirs. Chemosphere, 2005, v. 59, p. 1697-1702.

LIMA, L. S. Estudos de hidrodinâmica ambiental e mudanças na qualidade das águas da Lagoa Rodrigo de Freitas após ligação com o mar via dutos afogados. 2010, 116p. Dissertação de Mestrado do Programa de Engenharia Oceânica da COPPE/UFRJ, Rio de Janeiro - RJ, 2010.

MACHADO, L. B. Evolução da degradação ambiental da bacia hidrográfica da Lagoa Rodrigo de Freitas - RJ. 2009, 178p. Dissertação (Mestrado em Engenharia Ambiental) - Universidade do Estado do Rio de Janeiro. Rio de Janeiro, 2009.

MACIEL, G. L. R. Análise de Dados Hidrodinâmicos e Estratificação na Lagoa Rodrigo de Freitas, RJ: Relação Com a Concentração de Oxigênio Dissolvido. 2007. Dissertação de M. Sc., COPPE / UFRJ, Rio de Janeiro, 2007.

MARANI, L.; ALVALÁ, P. C. Methane emissions from lakes and floodplains in Pantanal, Brazil. Atmospheric Environment, 2007, v. 41, p. 1627-1633.

MOITA, G. C.; MOITA NETO, J. M. Uma Introdução à análise exploratória de dados multivariados. Química Nova, 1998, 21(4): 467-469.

OLIVEIRA, L.; NASCIMENTO, R.; KRAU, L; MIRANDA， N. Observações hidrobiológicas e mortandade de peixes na Lagoa Rodrigo de Freitas. Memórias do Instituto Oswaldo Cruz, Rio de Janeiro, 1957, 55 (2):211-275.

PELLIZARI, V. H.; NAKAYAMA, C. R.; ARAUJO, A. C. V.; LIOTTI R. G.; VAZOLLER, R. F. Metano, Mudanças Climáticas Globais e a Microbiologia. Microbiologia In Foco, São Paulo. 2007, p. 4-9.

PONTE NETO, C. F.; VIEIRA, F. P.; HAMZA, V. M. Resultados de levantamentos geomagnéticos da região da Lagoa Rodrigo de Freitas. Relatório Interno Observatório Nacional. 2010. 
RIDEG, P.; STEIN, J.; TOTIS, E. A Falha do Morro de São João em Botafogo - Rio de Janeiro. Simpósio Regional de Geologia, 1, 1989, Rio de Janeiro. Sociedade Brasileira Geologia. Boletim de Resumos: 130-131.

ROSMAN, P. C. C.; NEVES, C. F.; MELO, E. Solução Conjunta dos Problemas de Erosão na Praia de Ipanema-Leblon e Qualidade de Água na Lagoa Rodrigo de Freitas. In: Relatório Final. COPPETEC, Rio de Janeiro-RJ, Brasil. 1992.

SILVA, M. G. Variação interanual e análise da influência de parâmetros ambientais na emissão de metano no Pantanal. 2010, 99p. Dissertação (Mestrado em Geofísica Espacial) - Instituto Nacional de Pesquisas Espaciais. São José dos Campos, 2010.

SINDA. Sistema Nacional de Dados Ambientais. INPE - Centro Regional Nordeste, Natal, RN. Dados disponíveis em <http://sinda.crn2.inpe.br>. Acesso em outubro de 2012.

TANNER, C. C.; ADAMS, D. D.; DOWNES, M. T. Methane emissions from constructed wetlands treating agriculturalwastewaters. Journal of Environmental Quality. 1997, v. 26, 1056-1062.

TUPINAMBÁ, M.; VIEIRA, F. P.; HAMZA, V. M. Resultados de levantamentos geológicos da região da Lagoa Rodrigo de Freitas. Relatório Interno, Observatório Nacional. 2010 .

WANG, Z. P.; DELAUNE, R. D.; MASSCHELEYN, P. H. (1993). Soil redox and pH effects on methane production in a flooded rice. Soil Science Society of America Journal, 1993 , v. 57, n. 2, pp. 382-38.

WUEBBLES, D. J.; HAYHOE, K. Atmospheric methane and global change. Earth-Science Reviews, 2002, v. 57, p. 177-210.

YANG, S. S.; CHANG, H. L. Effect of environmental conditions on methane production and emission from paddy soil. Agriculture Ecosystems \& Environment, Amsterdam, 1998, v. 69, n. 1, p. 69-80. 


ISSN = 1980-993X - doi:10.4136/1980-993X
www.ambi-agua.net
E-mail: ambi-agua@agro.unitau.br
Tel.: (12) 3625-4212

\title{
Determinação de áreas prioritárias para o restabelecimento da cobertura florestal, apoiada no uso de geotecnologias
}

\author{
(http://dx.doi.org/10.4136/ambi-agua.964) \\ Celso de Souza Catelani ${ }^{1}$; Getulio Teixeira Batista ${ }^{2}$; \\ Marcelo dos Santos Targa ${ }^{2}$; Nelson Wellausen Dias ${ }^{3}$ \\ ${ }^{1}$ Programa de pós-graduação em Engenharia Mecânica, UNESP-FEG \\ e-mail: cscatelani@gmail.com, \\ ${ }^{2}$ Programa de pós-graduação em Ciências Ambientais da Universidade de Taubaté (PPGCA) \\ e-mails: getulio@agro.unitau.br,mtarga@unitau.br, \\ ${ }^{3}$ Fundação Instituto Brasileiro de Geografia e Estatística, Diretoria de Geociências \\ e-mail: nwdias@gmail.com
}

\section{RESUMO}

A determinação de áreas prioritárias para o restabelecimento da cobertura florestal nativa em bacias hidrográficas se constitui numa necessidade diretamente associada à probabilidade de sucesso efetivo na recuperação e na maximização dos recursos necessários. No entanto, para atender a essa finalidade, a complexidade no tratamento e o volume de dados ambientais espacializados necessários requerem um aparato tecnológico capaz de processar dados de apoio à análise multicriterial como ferramenta de suporte à decisão, no estado da arte das geotecnologias aplicáveis. Nesse contexto, o presente trabalho desenvolvido para uma área de $476 \mathrm{~km}^{2}$ correspondente à área da bacia hidrográfica do rio Una, no município de Taubaté, SP, baseou-se em uma análise multicriterial usando classificação contínua e na técnica de comparação pareada AHP (Analytical Hierarchy Process), incorporados ao SIG completo denominado SPRING (Sistema de Processamento de Informações Georreferenciadas), para a geração de um mapa de áreas prioritárias para o restabelecimento da cobertura florestal nativa na bacia. Os resultados obtidos com base na análise dos fatores: valor da terra nua; distância de fragmentos; classes de capacidade de uso do solo; áreas de preservação permanente (APPs); dentre outros, revelaram uma grande área, correspondente a 26,6\% da área da bacia, classificada como "Prioridade Extrema". Isso indica a necessidade imediata de recuperação ambiental da bacia uma vez que ela é manancial para o abastecimento de Taubaté, SP. Os resultados permitem de forma prática subsidiar a tomada de decisão na alocação de recursos e projetos dessa natureza nessas áreas prioritárias.

Palavras-chave: floresta nativa, geotecnologia, bacia hidrográfica, AHP.

\section{Determination of priority areas for the re-establishment of forest cover, based on the use of geotechnologies}

\begin{abstract}
The determination of priority areas for the re-establishment of forest cover in watersheds is directly associated to the probability of effective success of restoration processes. However, considering the complexity of the analysis and the large amount of spatial data necessary to accomplish that purpose, state of the art technological tools capable of processing multicriteria analysis to support decision making are necessary. Thus, the current work developed for an area of $476 \mathrm{~km}^{2}$ corresponding to the Una river watershed in the municipal district of
\end{abstract}


Taubaté, SP, used a multi-criteria analysis based on the continuous classification and on Analytical Hierarchy Process (AHP) paired comparisons techniques, available in the complete GIS package named SPRING (Georeferenced Information Processing System) for generating a map of priority areas for the re-establishment of forest cover in that watershed. Results revealed a large area $(26.6 \%$ of the entire watershed) falling in the "Extreme Priority" class for forest cover re-establishment, what indicates the urgent need of environmental recovery of this basin considering that it is used for Taubaté city water supply. Results from this research support the decision making for resource optimization applied to priority areas in an operational way.

Keywords: forest cover, geotechnology, watershed, AHP.

\section{INTRODUÇÃO}

A legislação ambiental brasileira, no que se refere à conservação de maciços florestais e à determinação de áreas de preservação permanente (APP), em especial o Código Florestal (Lei 4771/1965), recentemente revogada pela Lei $\mathrm{N}^{\circ}$ 12.651, de 25 de maio de 2012 (Brasil, 1965, 2012), traz em seu texto condições legais que se aplicadas seriam fundamentais para a preservação da cobertura vegetal florestal e para o estabelecimento de corredores ecológicos importantes para permitir o fluxo gênico e promover a melhoria dos recursos hídricos e demais funções ecológicas. No entanto, o histórico de uso da terra no domínio da Mata Atlântica, um dos mais ameaçados ecossistemas florestais brasileiros, que apresentava em 1998 menos de 9\% de sua cobertura original (Brasil, 1998), mostra que somente no Estado de São Paulo, foram destruídos, entre 1907 e 1934, cerca de $79.500 \mathrm{~km}^{2}$ de floresta nativa, o que corresponde à taxa de $3.000 \mathrm{~km}^{2} / \mathrm{ano}$ (Dean, 1997).

Dados sobre o desmatamento no Estado de São Paulo mostram que entre 1990 e 1995 foram destruídos $674 \mathrm{~km}^{2}$ de Mata Atlântica (Brasil, 1998). Esse desflorestamento e em consequência a degradação dos recursos hídricos, ocasionado pelos diversos usos da terra nos ciclos agro-econômicos que se sucederam na região do Vale do Paraíba paulista determinam a necessidade de recomposição florestal, principalmente das APPs.

O Comitê das bacias Hidrográficas do Rio Paraíba do Sul (CBH-PS) em seu Plano de Bacias (CPTI, 2001) elegeu a bacia do rio Una como prioridade 4, em relação à hierarquização das 128 bacias estudadas, quanto à necessidade de ações imediatas para a recuperação do quadro ambiental. Nesse relatório foram apontadas como ações, que contemplam duas metas, imprescindíveis para a melhoria da qualidade e quantidade de água disponível para o abastecimento de Taubaté e municípios imediatamente à jusante: a primeira visa reduzir a presença de partículas sólidas no rio Una, no ponto de captação para abastecimento de Taubaté, enquanto a segunda meta visa manter a qualidade das águas do rio Una, em seu trecho inferior, nos padrões da Classe 2.

Dados levantados no projeto "Estruturação e Disponibilização do Banco de Dados Ambientais da Bacia do rio Una, Bacia do Rio Paraíba do Sul" (Batista et al., 2005) demonstraram que de um total de $271 \mathrm{Km}^{2}$ de APPs existentes na bacia, $47 \%$ estão em desconformidade de uso, representando uma taxa de 26,6\% em déficit de cobertura florestal em relação à área total da bacia do rio Una. Esse potencial de áreas a serem recuperadas torna-se ainda maior se computados, em adição, a ausência majoritária das áreas de "Reserva Legal", instituída pela Lei Federal n ${ }^{\circ}$ 4.771/65 (Código Florestal), alterada pela Lei Federal $n^{\circ}$ 7.803, de 18 de julho de 1989 e mantida no atual Código aprovado em 2012, e que por si só poderiam representar até $20 \%$ de cada propriedade, que no caso da bacia do rio Una somariam próximo a 9.520 hectares, elevando esse déficit, eventualmente em até $47 \%$ da área total da bacia. 
Nesse contexto, fica evidente a necessidade de esforços de recomposição florestal na bacia do rio Una, o mais importante manancial de Taubaté. Essa bacia abrange $69 \%$ da área do município, que conta com uma população estimada em 278.686 habitantes (IBGE, 2010). Entretanto, é de conhecimento que os custos de recomposição por hectare de reflorestamento com essências nativas são muito altos considerando a economia da maioria das propriedades rurais (IPEF, 2001; Brasil, 2005; Melo, 2005; Barbosa, 2006). Assim, o cenário atual em relação à recomposição florestal, devido à escassez de recursos direcionados para este fim e a grande demanda existente, aponta a necessidade de se desenvolver estudos e métodos de priorização de áreas a serem reflorestadas.

Nesse sentido, o presente trabalho teve como objetivo a identificação de áreas prioritárias e a análise da distribuição espacial dessas áreas por meio do uso de ferramentas computacionais, apoiadas em Sistemas de Informações Geográficas (SIGs) (Eastman, 1995; Câmara et al., 1996) e em técnicas de geoprocessamento aplicadas aos dados pré-existentes e informações de campo (Valente, 2005), cujos resultados podem ser muito importantes para a otimização dos parcos recursos hoje disponíveis para a recuperação florestal.

\section{MATERIAL E MÉTODOS}

\section{1. Área de estudo}

O presente estudo foi realizado na Bacia do rio Una com área de $476 \mathrm{~km}^{2}$ que abrange parte dos municípios de Pindamonhangaba, Redenção da Serra, Taubaté e Tremembé, situados no cone leste do Estado de São Paulo. A área de estudo está delimitada por um retângulo entre as coordenadas UTM dos cantos, inferior esquerdo $E=430.000, N=7.410 .000$ e, superior direito $\mathrm{E}=490.000, \mathrm{~N}=7.460 .000$, Datum horizontal SAD-69 e Meridiano Central $45^{\circ} \mathrm{W}$ (Figura 1).

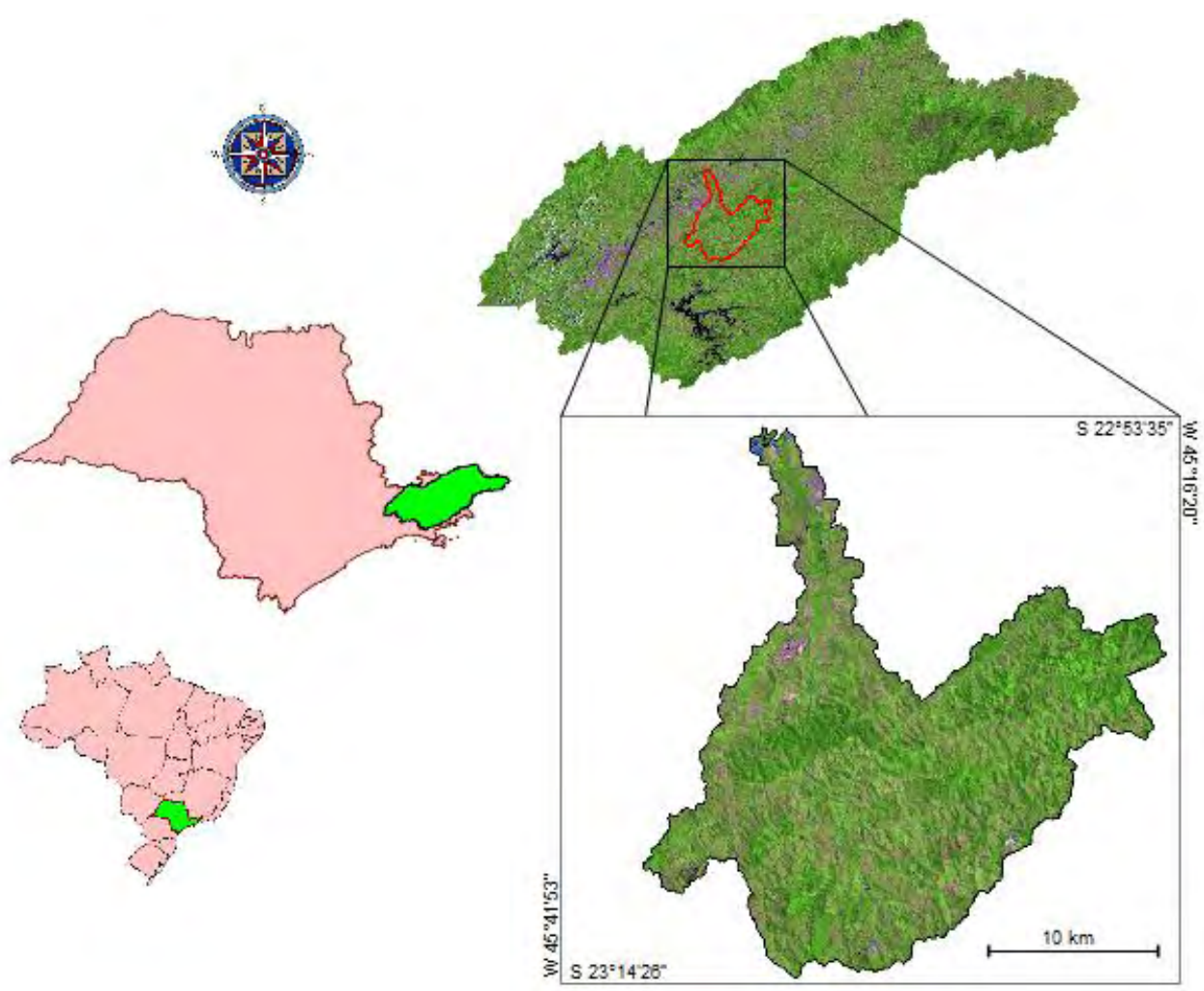

Figura 1. Localização da área de estudo. 


\subsection{Sistemas e técnicas computacionais utilizadas}

O desenvolvimento deste estudo foi baseado na utilização de geotecnologias disponibilizadas em software e linguagem de programação algébrica. O Sistema de Informações Geográficas utilizado foi o SIG completo SPRING - Sistema de Processamento de Informações Georreferenciadas, em sua versão v.4.3.2 (Câmara et al., 1996).

A organização computacional se deu a partir da criação de um banco de dados georreferenciados no SPRING, sobre o qual foram processados os dados de base, os cruzamentos e conversões para compatibilização entre dados matriciais e vetoriais.

A linguagem de programação denominada LEGAL (Linguagem Espacial para Geoprocessamento Algébrico, Cordeiro et al., 1996), foi utilizada para a criação dos programas de transformação de dados temáticos matriciais em dados numéricos matriciais e na discretização de valores numéricos referentes ao intervalo de classificação contínua dos dados no conjunto fuzzy (Klir, 1991), e ainda nos cruzamentos desses dados para a geração dos produtos finais do mapeamento.

As análises de suporte à decisão baseadas em técnicas AHP (Analytical Hierarchy Process, Saaty, 1991) foram processadas utilizando-se um módulo específico do SPRING que possibilita o arranjo das camadas de informação em dupla combinação baseado na "Escala de Valores AHP para Comparação Pareada”.

\subsection{Dados utilizados}

Foram incorporados ao banco de dados georreferenciados, os dados preexistentes produzidos pelo projeto "Estruturação e Disponibilização do Banco de Dados Ambientais da Bacia do rio Una, Bacia do Rio Paraíba do Sul" (Batista et al., 2005), referentes aos temas: Uso da Terra, Malha Viária, Rede de Drenagem, Altimetria, Áreas de Preservação Permanente (APPs), Declividade, Pedologia e Vulnerabilidade a processos erosivos.

\subsection{Mapeamento dos fragmentos florestais}

Com base nos dados de uso da terra oriundos do Projeto Una, foi processado o isolamento da classe "Mata-Capoeira", que corresponde aos fragmentos florestais existentes na bacia. A Figura 2 representa uma amostra de fragmento florestal mapeado como MataCapoeira no Projeto Una, visto no mosaico ortorretificado e sua correspondente foto no campo.
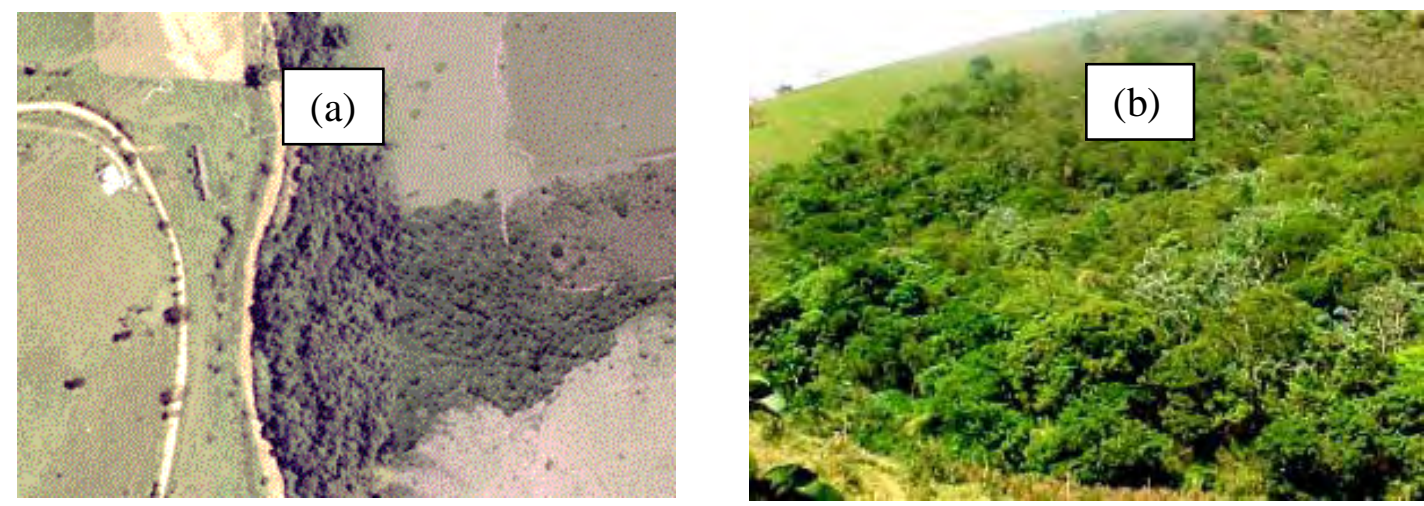

Figura 2. Exemplo da classe Mata-Capoeira a) no Mosaico ortorretificado e b) vista no campo. Essa classe engloba áreas de vegetação natural, pioneira ou secundária em estágio inicial, médio ou avançado de regeneração.

\subsection{Geração do mapa de distância entre fragmentos florestais}

A partir do mapa de fragmentos florestais da Bacia do rio Una foi gerado um mapa de distância no modelo numérico, que corresponde a um mapa matricial no qual as células 
referentes a cada pixel da imagem resultante possuem valores crescentes em metros à medida que se afastam dos fragmentos florestais. $O$ dado numérico resultante desse processo foi agrupado ou fatiado em faixas de $100 \mathrm{~m}$ de largura a partir das bordas dos fragmentos florestais existentes na bacia.

Após a obtenção do mapa de distância entre os fragmentos florestais, foi feita a atribuição de pesos para a normalização dos valores no intervalo $(0,1)$ correspondentes às respectivas faixas de distância entre os fragmentos florestais remanescentes com base no programa escrito em LEGAL.

\subsection{Classes de capacidade de uso e respectivo valor agronômico das terras}

Os dados de Pedologia, Áreas de Preservação Permanente e Declividade, existentes no banco de dados Una (Batista et al., 2005) foram cruzados com base na metodologia desenvolvida pelo Departamento de Agricultura dos Estados Unidos (USDA) e adaptada por Lepsch et al. (1983) para a geração do mapa de classes de capacidade de uso da terra.

Com a informação da classe de capacidade de uso da terra, o valor agronômico pôde ser determinado com base na metodologia utilizada pelo INCRA (2006) que utiliza uma tabela que relaciona a localização e acesso com a capacidade de uso das terras (França, 1983, Tabela 1). No caso em estudo o fator localização e acesso é considerado ótimo, ou seja, a bacia apresenta nos seus diversos cursos, condições de acesso com vias principais asfaltadas, distâncias a vias e centros consumidores próximas e acessos permanentes ao longo do ano. Dessa forma, uma classe de solo associada a uma declividade resulta numa determinada classe de capacidade de uso. Nesse estudo, foram consideradas as classes de capacidade de uso da terra desde a menos restritiva (Classe I) até a mais restritiva (Classe VIII), sem considerar sub-classes relacionadas com a intensidade de restrição descritas por Lepsch et al. (1983). As APPs, por restrição legal, foram agrupadas à classe VIII. Ressalta-se que o mapeamento das APPs envolveu todas as classes de APP, previstas no Código Florestal, 4.771/65 (Brasil, 1965) e Resolução CONAMA 303/2002 (Brasil, 2002). Para cada polígono ou talhão no mapa resultante da classe de capacidade de uso foram atribuídos pesos normalizados no intervalo $(0,1)$ com base na Tabela 1 .

Tabela 1. Fatores de ponderação na obtenção do valor das terras considerando a classe de capacidade de uso e localização.

\begin{tabular}{|c|c|c|c|c|c|c|c|c|c|}
\hline \multirow{2}{*}{\multicolumn{2}{|c|}{$\begin{array}{c}\text { Capacidade } \\
\text { de uso } \\
\text { Localização e } \\
\text { acesso }\end{array}$}} & I & II & III & IV & $\mathbf{V}$ & VI & VII & VIII \\
\hline & & $100 \%$ & $80 \%$ & $61 \%$ & $47 \%$ & $39 \%$ & $29 \%$ & $20 \%$ & $13 \%$ \\
\hline ÓTIMA & $100 \%$ & 1,000 & 0,800 & 0,610 & 0,470 & 0,390 & 0,290 & 0,200 & 0,130 \\
\hline MUITO BOA & $95 \%$ & 0,950 & 0,760 & 0,580 & 0,447 & 0,371 & 0,276 & 0,190 & 0,124 \\
\hline BOA & $90 \%$ & 0,900 & 0,720 & 0,549 & 0,423 & 0,351 & 0,261 & 0,180 & 0,117 \\
\hline REGULAR & $80 \%$ & 0,800 & 0,640 & 0,488 & 0,376 & 0,312 & 0,232 & 0,160 & 0,104 \\
\hline DESFAVORÁ VEL & $75 \%$ & 0,750 & 0,600 & 0,458 & 0,353 & 0,293 & 0,218 & 0,150 & 0,098 \\
\hline MÁ & $70 \%$ & 0,700 & 0,560 & 0,427 & 0,329 & 0,273 & 0,203 & 0,140 & 0,091 \\
\hline
\end{tabular}

Fonte: INCRA (2006).

\subsection{Aplicação do método AHP no processamento do mapa de prioridades}

Após a geração dos produtos base na forma de matrizes numéricas (pesos ponderados e normalizados no intervalo de 0 a 1 correspondentes a cada tema ou fator de peso na determinação de prioridades), os dados foram processados com uso de uma ferramenta de suporte a decisão AHP - Analytical Hierarchy Process, conforme Saaty (1991). O Método baseia-se na "Escala de Valores AHP para Comparação Pareada" (Tabela 2). 
CATElANI, C. S.; BATISTA, G. T.; TARGA, M. S.; DIAS, N. W. Determinação de áreas prioritárias para o restabelecimento da cobertura florestal, apoiada no uso de geotecnologias. Ambi-Agua, Taubaté, v. 7, n. 3, p. 113-126, 2012. (http://dx.doi.org/10.4136/ambi-agua.964)

Tabela 2. Itens da escala de valores AHP para comparação pareada.

\begin{tabular}{c|l}
$\begin{array}{c}\text { Intensidade de } \\
\text { importância }\end{array}$ & \multicolumn{1}{c}{ Definição e Explicação } \\
\hline $\mathbf{1}$ & Importância igual - os dois fatores contribuem igualmente para o objetivo \\
$\mathbf{3}$ & Importância moderada - um fator é ligeiramente mais importante que o outro \\
$\mathbf{5}$ & Importância essencial - um fator é claramente mais importante que o outro \\
$\mathbf{7}$ & $\begin{array}{l}\text { Importância demonstrada - um fator é fortemente favorecido e sua maior relevância foi } \\
\text { demonstrada na prática }\end{array}$ \\
$\mathbf{9}$ & Importância extrema - a evidência que diferencia os fatores é da maior ordem possível \\
& Valores intermediários entre julgamentos - possibilidade de compromissos adicionais \\
\hline
\end{tabular}

O mapa de prioridade para recomposição florestal da Bacia do rio Una numérico e normalizado foi então processado por técnica de fatiamento no módulo de processamento do modelo numérico do SPRING, dando origem a um mapa temático contendo a espacialização das classes de prioridade para recomposição florestal, divididas em:

- Prioridade Extrema, atribuída aos valores normalizados entre 0.9 e 1.0;

- Prioridade Alta atribuída aos valores normalizados entre 0.7 e 0.9 ;

- Prioridade Média atribuída aos valores normalizados entre 0.4 e 0.7 e,

- Prioridade Baixa atribuída aos valores normalizados entre 0 e 0.4.

O limite inferior de cada intervalo é incluído na classe, enquanto o superior é excluído, exceto para a classe $(0,9$ a 1,0$)$.

\section{RESULTADOS E DISCUSSÃO}

\subsection{Mapa fragmentos florestais e de classes de capacidade de uso da terra}

O Mapa de fragmentos e de distância entre fragmentos florestais nativos da bacia do rio Una, gerado em um modelo numérico, trata-se de um mapa matricial, no qual as células referentes a cada pixel da imagem resultante possuem valores crescentes em metros da distância entre fragmentos, como resultado do fatiamento em faixas preestabelecidas a cada $100 \mathrm{~m}$, à medida em que se afastam dos fragmentos florestais, representados pela classe MataCapoeira, originária do mapa de uso do solo (Batista et al., 2005). O mapa resultante demonstra um alto grau de fracionamento e dispersão dos fragmentos florestais remanescentes. A Tabela 3 mostra a área e a percentagem de ocorrência dos remanescentes.

Tabela 3. Resultado da classificação do mapa de distância entre fragmentos florestais nativos da bacia do rio Una.

\begin{tabular}{c|ccc}
\hline Classe & Faixa de Distância & Área (ha) & Área (\%) \\
\hline Mata-Capoeira & 0 & 10083,3 & 21,18 \\
$0-100$ & $0-100$ & 14973,0 & 31,46 \\
$100-200$ & $100-200$ & 10149,4 & 21,32 \\
$200-300$ & $200-300$ & 5924,0 & 12,45 \\
$300-400$ & $300-400$ & 3207,8 & 6,74 \\
$400-500$ & $400-500$ & 1669,7 & 3,51 \\
$500-600$ & $500-600$ & 799,2 & 1,68 \\
$600-700$ & $600-700$ & 400,5 & 0,84 \\
$700-800$ & $700-800$ & 211,6 & 0,44 \\
$800-900$ & $800-900$ & 121,9 & 0,26 \\
900 ou + & 900 ou + & 59,7 & 0,13 \\
\hline \multicolumn{4}{c}{ Total } \\
\hline
\end{tabular}


Os resultados do mapa de distância entre fragmentos da bacia (Tabela 3) demonstraram que cerca de $53 \%$ da área total situa-se a uma distância inferior a $200 \mathrm{~m}$ (faixas de $0-100 \mathrm{~m}$ e $100-200 \mathrm{~m}$ ) em relação ao fragmento florestal nativo mais próximo, o que facilita a conexão entre fragmentos e dessa forma, pode-se indicar a adoção de métodos de restabelecimento baseados em nucleação (Reis et al., 2003). Esse mapa de distância permite visualizar possíveis rotas de ligação de corredores ecológicos e possíveis uniões entre fragmentos e ainda, levando-se em consideração a rede hidrográfica, permite também, observar a configuração da cobertura florestal do conjunto ripário (Lima e Zakia, 2000).

\subsection{Mapa de classes de capacidade de uso da terra e respectivo índice de valor agronômico da terra na bacia do rio una}

Os cruzamentos dos mapas de pedologia, APPs e declividade permitiram a geração do mapa de classes de capacidade de uso do solo (Figura 3). Nesse resultado de considerável complexidade técnica para mensuração (Lepsh et al., 1983) reside uma das mais controversas questões para a indicação de áreas particulares em que devam ser restauradas ou restabelecidas as florestas originais, visto que a adesão de proprietários rurais e a destinação de áreas adequadas a esse fim esbarram no tipo de uso atual dado pelo proprietário, com considerável índice de desistência de projetos de recomposição florestal (Ferretti, 2000). O Mapa de classes de capacidade de uso da terra e respectivo índice de valor agronômico das terras da bacia do rio Una (Figura 3), elaborado com base no sistema adaptado por Lepsch et al. (1983) considera quatro categorias: grupos, classes, subclasses e unidade de restrição à capacidade de uso, definidas em função das características dos solos, declividade e fatores limitantes ao uso da terra. As análises, nesse estudo, se restringiram às classes que são representadas por algarismos romanos (I a VIII), identificadas pelo grau de limitação da capacidade de uso agrícola da terra (Tabela 4).

$\mathrm{Na}$ execução dos processos que resultaram no Mapa de classes de capacidade de uso da terra e respectivo índice de valor agronômico das terras da Bacia do rio Una, em adição aos dados de entrada correspondente ao mapa de solos e de declividade da bacia, foram integrados nesse estudo, os dados do mapa de APPs, que por si só representam restrição legal ao uso da terra, com consequente classificação de todas APPs existentes na bacia como Classe VIII de capacidade de uso da terra.

Tabela 4. Resultado da classificação de capacidade de uso da terra e respectivo índice de valor agronômico das terras, expressos em porcentagem e hectares.

\begin{tabular}{l|crr}
\hline $\begin{array}{l}\text { Classe de } \\
\text { capacidade de } \\
\text { uso do solo }\end{array}$ & $\begin{array}{c}\text { Índice de valor da } \\
\text { terra (INCRA) }\end{array}$ & Área (ha) & Área (\%) \\
\hline Classe_I & 100 & 261 & 0,6 \\
Classe_II & 80 & 6 & 0,0 \\
Classe_III & 61 & 4783 & 10,1 \\
Classe_IV & 47 & 2671 & 5,6 \\
Classe_V & 39 & 0 & 0,0 \\
Classe_VI & 29 & 7316 & 15,4 \\
Classe_VII & 20 & 5805 & 12,2 \\
Classe_VIII & 13 & 26758 & 56,2 \\
\hline Total & & $\mathbf{4 7 6 0 0}$ & $\mathbf{1 0 0 , 0}$ \\
\hline
\end{tabular}


Conforme mostrado na Tabela 4 a Classe VIII ocupa 56,2\% do total da bacia. Mais da metade da área total da bacia possui restrições ao uso da terra, que de acordo com Mendonça et al. (2006), só poderia ser utilizada como abrigo da flora e fauna silvestres, como ambiente para atividades de recreação e educação ambiental ou para fins de armazenamento de água.

No processamento do mapa de classe de capacidade de uso e valor agronômico da terra, por meio de um programa escrito em LEGAL, foram atribuídos pesos normalizados no intervalo $(0,1)$, o que gerou o mapa correspondente no modelo numérico. Observa-se claramente o grau de prioridade indicado pela Classe VIII de capacidade de uso da terra, principalmente pela sua característica restritiva herdada do mapa de APPs do Projeto Una (Dias et al., 2008).

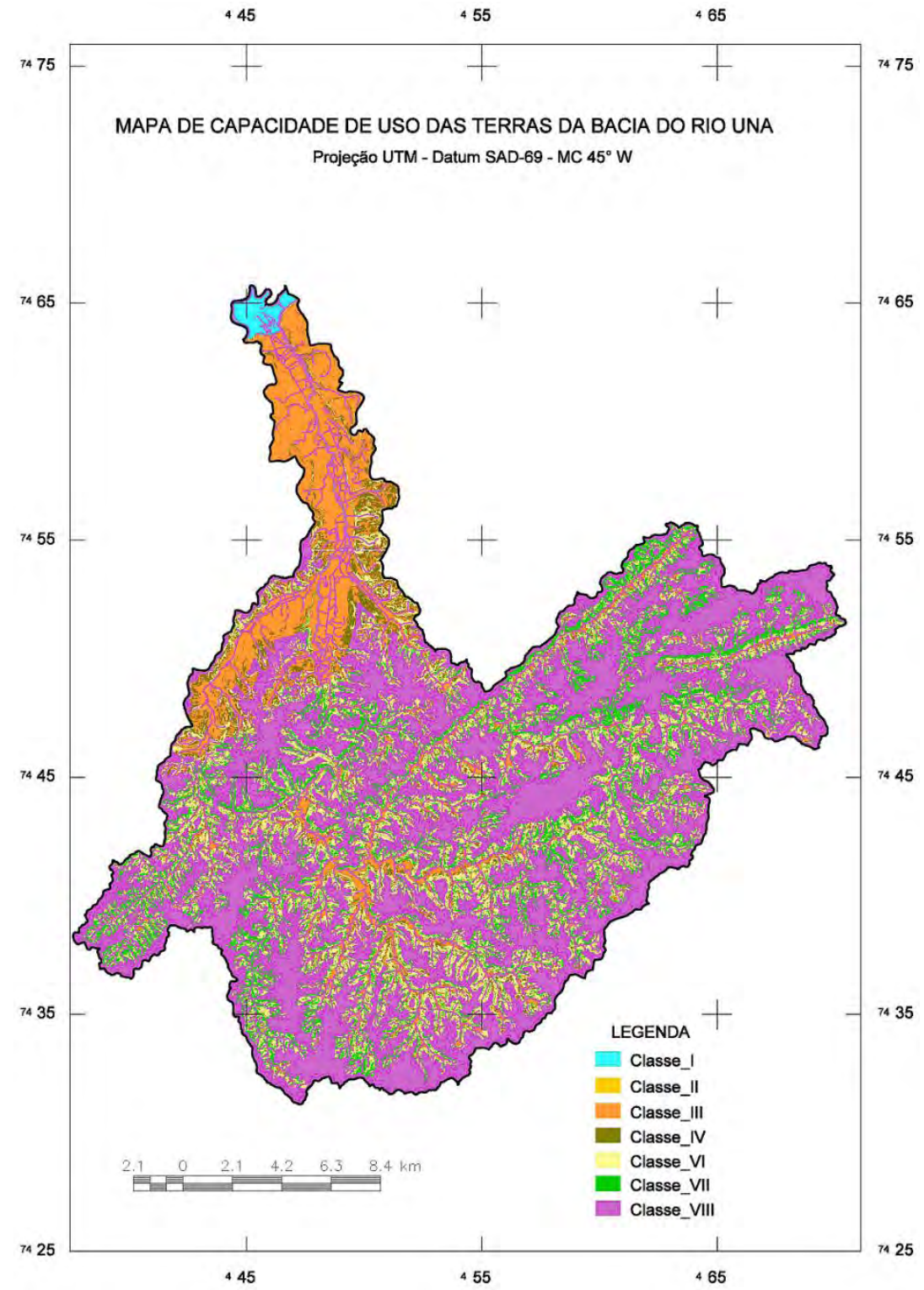

Figura 3. Mapa de classes de capacidade de uso das terras da bacia do rio Una.

O cruzamento do mapa de uso do solo com o mapa de classes de capacidade de uso da terra demonstrou uma intersecção de 6.927 ha da classe Mata-Capoeira com a Classe VIII de capacidade de uso da terra, ou seja, apenas $25,9 \%$ da Classe VIII na bacia apresenta 
conformidade de uso. Tal fato demonstra que o uso predominante está em desacordo com a vocação natural de capacidade de uso da terra (Tabela 5).

Tabela 5. Resultado do cruzamento do mapa de classes de capacidade de uso da terra e respectivo índice de valor agronômico das terras X mapa de uso do solo, em hectares.

\begin{tabular}{l|rrrrrrr}
\multicolumn{1}{c}{ Classes de capacidade } & \multicolumn{1}{c}{\begin{tabular}{c} 
I \\
\cline { 2 - 8 } \multicolumn{1}{c}{ Uso uso do solo }
\end{tabular}} & \multicolumn{1}{c}{ I } & III & IV & VI & VII & VIII \\
\hline Mata-Capoeira & 30,2 & 0,1 & 357,1 & 203,8 & 902,3 & 1662,5 & $\mathbf{6 9 2 7 , 1}$ \\
Agricultura & 41,1 & 0,0 & 169,6 & 66,9 & 97,0 & 19,4 & 210,8 \\
Pasto & 54,1 & 0,4 & 3398,4 & 2038,2 & 5260,7 & 3223,8 & 14925,3 \\
Reflorestamento & 0,6 & 0,0 & 64,4 & 78,2 & 409,6 & 363,5 & 1610,4 \\
Solo Exposto & 0,0 & 0,0 & 94,0 & 56,8 & 65,7 & 14,8 & 136,2 \\
Área Degradada & 0,0 & 0,0 & 0,8 & 6,4 & 4,8 & 2,6 & 21,6 \\
Área Urbanizada & 0,5 & 0,0 & 427,4 & 59,2 & 19,3 & 0,7 & 116,4 \\
Pasto Sujo & 0,0 & 0,0 & 117,9 & 95,7 & 331,1 & 378,0 & 1962,3 \\
Pasto Degradado & 6,6 & 0,0 & 54,4 & 45,1 & 173,1 & 115,0 & 647,7 \\
Corpos d'água & 94,2 & 5,6 & 75,3 & 7,6 & 23,0 & 1,3 & 22,9 \\
Reflorestamento cortado & 0,0 & 0,0 & 0,9 & 3,3 & 18,9 & 17,8 & 144,8 \\
Área Minerada & 31,4 & 0,2 & 12,1 & 5,9 & 6,1 & 3,8 & 32,4 \\
\hline \multicolumn{1}{c}{ Total } & 260,8 & 6,3 & 4782,8 & 2671,3 & 73116,4 & 5804,6 & $\mathbf{2 6 7 5 7 , 8}$ \\
\hline
\end{tabular}

\subsection{Mapeamento das classes de prioridade para recomposição florestal da bacia do rio} Una

Os dados de base relativos à cada camada de informação, pré-processados por média ponderada e normalizados no intervalo ( 0 a 1 ) também denominados conjunto fuzzy, foram submetidos à comparação pareada no módulo de suporte à decisão AHP do SPRING v. 4.3.2, o que retornou um índice de consistência de 0.042 (Figura 4), considerado consistente, uma vez que valores inferiores a 0.1 para a execução do módulo de decisão em técnica AHP são recomendados (Valente, 2005). Com base no programa em LEGAL, utilizando-se as camadas de informação, previamente trabalhadas obteve-se o mapa de prioridade para recomposição florestal da Bacia do rio Una numérico normalizado.

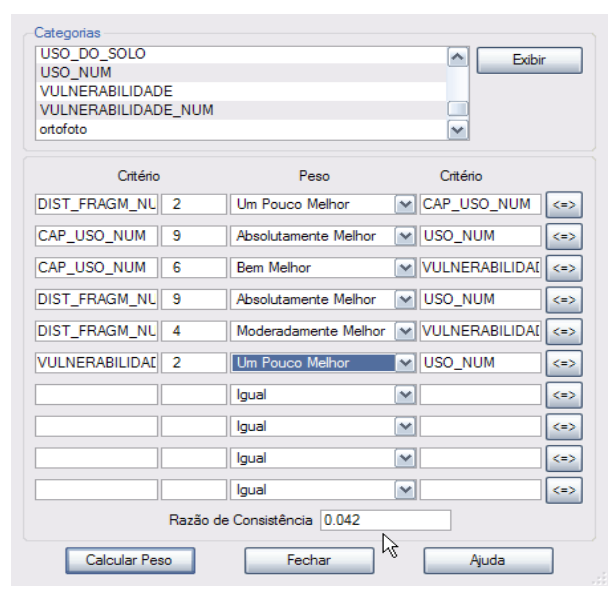

Figura 4. Captura de tela do módulo de tomada de decisão AHP do SPRING, com os respectivos pares e a respectiva razão de consistência. 


\subsection{Mapa Temático de prioridade para o restabelecimento da cobertura florestal nativa da bacia do rio Una}

O mapa de prioridade para o restabelecimento da cobertura florestal nativa da bacia do rio Una numérico e normalizado, após processado por técnica de fatiamento no módulo do modelo numérico do SPRING v.4.3.2, resultou no mapa temático que contém a espacialização das classes de prioridade para recomposição florestal.

Os resultados do mapa temático de prioridade para o restabelecimento da cobertura florestal nativa da Bacia do rio Una demonstram numa avaliação visual que a descontinuidade das classes no modelo numérico permite uma representação não condicionada ao verdadeiro ou falso e não produz polígonos com limites rígidos que se observa quando se trabalha com cruzamentos booleanos. Burrough e McDonnell (1998) já alertavam que os principais problemas relacionados às operações booleanas são os limiares nítidos e rígidos, que nem sempre representam os fenômenos naturais corretamente, visto que na natureza os fenômenos não são representados por limites estáticos ou rígidos.

A fusão do mapa de fragmentos florestais no mapa temático de prioridade se faz necessária uma vez que nos locais onde existe floresta nativa, a prioridade de recomposição deve ser nula.

Os resultados obtidos da inserção dos fragmentos florestais na camada final correspondente ao mapa temático final de prioridade para o restabelecimento da cobertura florestal nativa da bacia do rio Una estão representados na Figura 5 enquanto os dados tabulares referentes às respectivas classes de prioridade expressos em hectares e porcentagem são apresentados na Tabela 6.

Tabela 6. Área e percentagem de cada classe de prioridade para o restabelecimento da cobertura florestal da bacia do rio Una.

\begin{tabular}{|l|cc}
\hline \multicolumn{1}{|c|}{ Classe } & Área (ha) & Área (\%) \\
\hline Mata_Capoeira & 10082 & 21,2 \\
Prioridade baixa & 253 & 0,5 \\
Prioridade média & 6346 & 13,3 \\
Prioridade alta & 18388 & 38,6 \\
Prioridade extrema & 12663 & 26,6 \\
\hline \multicolumn{1}{|c|}{ Total } & $\mathbf{4 7 6 0 0}$ & $\mathbf{1 0 0 , 0}$ \\
\hline
\end{tabular}

Nota: As cores na tabela correspondem às cores do mapa na Figura 5.

Pelos resultados finais do mapeamento das classes de prioridade para o restabelecimento da cobertura florestal da Bacia do rio Una (Tabela 6) demonstra-se que a qualidade ambiental da bacia está ruim, pois uma área correspondente a 26,6\% da área total da bacia encontra-se em situação de "Prioridade Extrema" para a recomposição florestal.

O cruzamento do mapa de prioridade para recuperação com o mapa de APPS (Dias et al., 2008) revelou que $72,7 \%$ das APPs da bacia encontram-se sob prioridades Alta (7473 ha ou $27,8 \%$ ) e Extrema (12051 ha ou 44,9\%) para o restabelecimento da cobertura vegetal nativa (Tabela 7), o que nos leva a duas novas informações relevantes, somente $27,4 \%$ das APPs encontram-se com cobertura vegetal apropriada, e o restante sob condição predominante entre alta e extrema prioridade para recomposição da cobertura florestal nativa. 
CATElANI, C. S.; BATISTA, G. T.; TARGA, M. S.; DIAS, N. W. Determinação de áreas prioritárias para o restabelecimento da cobertura florestal, apoiada no uso de geotecnologias. Ambi-Agua, Taubaté, v. 7, n. 3, p. 113-126, 2012. (http://dx.doi.org/10.4136/ambi-agua.964)

Tabela 7. Resultado do cruzamento do mapa de prioridades para o restabelecimento da cobertura vegetal nativa $\mathrm{X}$ mapa de APPs, em hectares.

\begin{tabular}{l|cccccc}
\hline \multicolumn{1}{c|}{ Prioridades } & Mata Capoeira & Baixa & Média & Alta & Extrema & Total (APPs) \\
\hline APP Lago Lagoa Represa & 4 & 0 & 5 & 50 & 49 & $\mathbf{1 0 8}$ \\
APP Nascente & 487 & 0 & 22 & 558 & 787 & $\mathbf{1 8 5 4}$ \\
APP Topo de Morro & 4058 & 0 & 202 & 4437 & 7907 & $\mathbf{1 6 6 0 3}$ \\
APP Rios ate 10m & 2268 & 0 & 154 & 2386 & 3208 & $\mathbf{8 0 1 5}$ \\
APP Rios entre 10-50 & 109 & 0 & 0 & 42 & 101 & $\mathbf{2 5 2}$ \\
APP Reservatório & 6 & 0 & 5 & 1 & 0 & $\mathbf{1 2}$ \\
APP Cavas & 1 & 0 & 26 & 0 & 0 & $\mathbf{2 8}$ \\
\hline Total (Prioridades) & $\mathbf{6 9 3 3}$ & $\mathbf{0}$ & $\mathbf{4 1 4}$ & $\mathbf{7 4 7 3}$ & $\mathbf{1 2 0 5 1}$ & $\mathbf{2 6 8 7 1}$ \\
\hline
\end{tabular}

Observa-se também que dos 12663 ha de "Prioridade Extrema", 12051 ha situam-se em APPs, o que corresponde a uma intersecção equivalente a 95,2\%, e demonstra a influência das APPs na priorização de áreas para recomposição florestal.

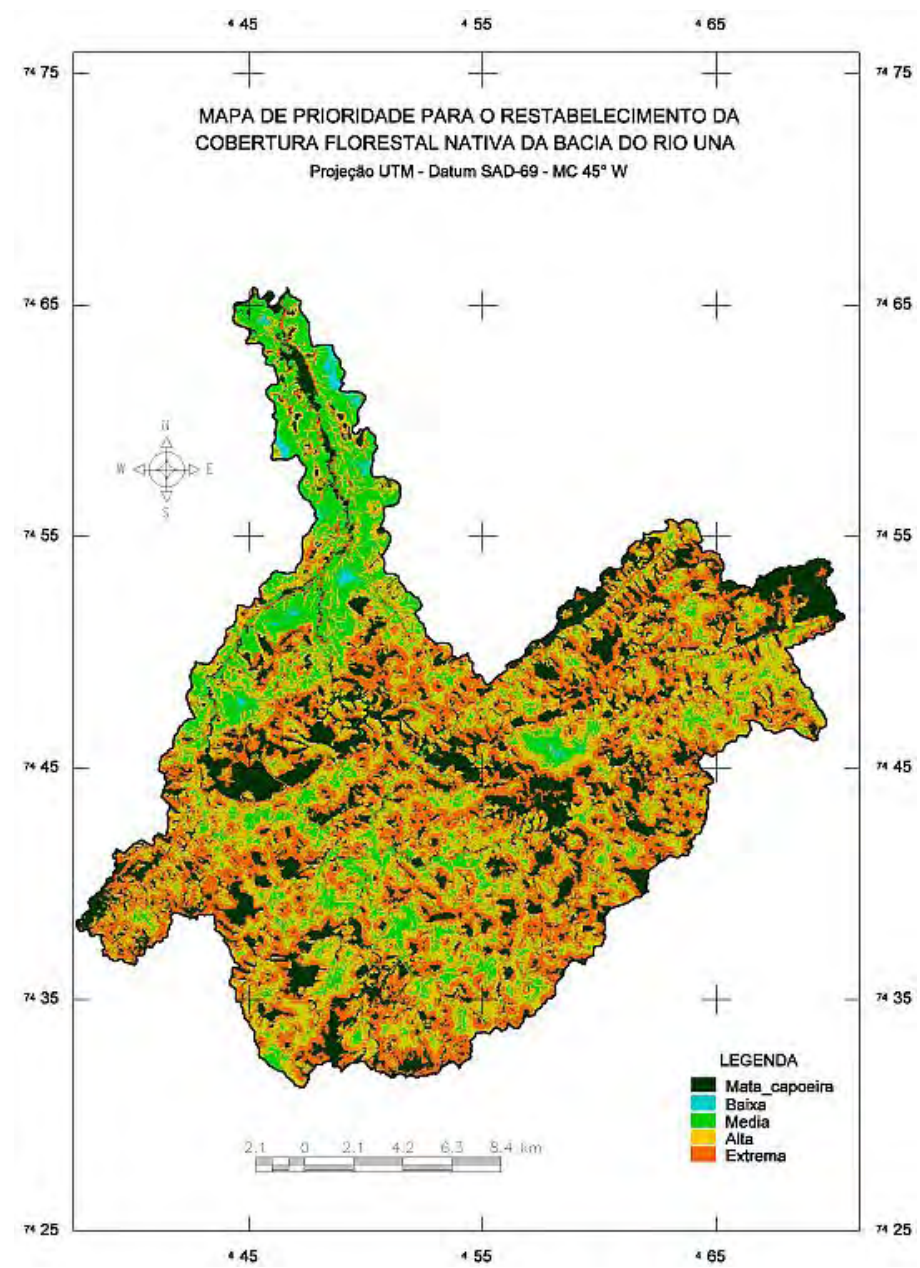

Figura 5. Mapa temático final de prioridade para o restabelecimento da cobertura florestal nativa da bacia do rio Una. 
Santos (1991) classificou os estados alterados da paisagem como rugosidades que expressam o resultado de um comportamento social, nesse sentido, o predomínio absoluto das classes de Prioridade Alta e Extrema, que juntas somam 65,3\% da área da bacia, demonstra em números a gravidade do legado de séculos seguidos de exploração dos recursos naturais, especialmente dos solos, dos recursos hídricos e da flora na região, legado que já foi descrito por Dean (1997).

Como finalidade prática, os resultados desse trabalho podem subsidiar a tomada de decisão na alocação de recursos destinados ao restabelecimento da cobertura florestal nativa da bacia do rio Una, e assim servir como elemento de remediação do quadro de degradação dos recursos hídricos da bacia, apontados no Relatório de Situação da Bacia Hidrográfica do Rio Paraíba do Sul (CPTI, 2001).

\section{CONCLUSÃO}

O presente trabalho permitiu formular algumas conclusões a respeito da priorização de áreas para o restabelecimento da cobertura florestal nativa da bacia do rio Una, que seguem:

A prioridade técnica para a recomposição florestal dessa bacia apresentou uma grande intersecção de área associada às Áreas de Preservação Permanente (APPs), o que ratifica o conceito dessas APPs;

A bacia hidrográfica do rio Una, em Taubaté, SP, apresenta uma situação preocupante, com $26,6 \%$ de sua área detectada como de "prioridade extrema" para o restabelecimento da cobertura florestal nativa;

O mapeamento da prioridade de recuperação da bacia do rio Una em níveis numéricos espacializados permite uma otimização na implantação de projetos de restabelecimento de cobertura florestal nativa nessa bacia, cuja metodologia pode ser replicada para outras regiões.

\section{REFERÊNCIAS}

BARBOSA, L. M. (Coord.). Manual para recuperação de áreas degradadas em matas ciliares do estado de São Paulo. São Paulo: Instituto de Botânica, 2006. 147p. Disponível em: <http://files.pereiraim.webnode.com.br/2000000424f33f502df/Manual\%20RMC-SP.pdf\#page=132>. Acesso em: nov. 2012.

BATISTA, G. T.; TARGA, M. S.; FIDALGO, E. C. C. Banco de dados ambientais da bacia do rio Una, bacia do Rio Paraíba do Sul. Taubaté, 2005. 16p. Disponível em: <http://www.agro.unitau.br:8080/dspace/bitstream/2315/51/1/Projeto_Una_IBICT.pdf>. Acesso em: 26 nov. 2012.

BRASIL. Conselho Nacional do Meio Ambiente - CONAMA. Resolução No 303 de 20 de março de 2002. Dispõe sobre parâmetros, definições e limites de Áreas de Preservação Permanente. Disponível em: <http://www.mma.gov.br/port/conama/res/res02/ res30302.html>. Acesso em: 18 dez. 2012.

Fundo Nacional do Meio Ambiente - FNMA. Edital FNMA No 2/2005. Chamada I - recuperação e proteção de nascentes e áreas que margeiam corpos d'água, adequação ambiental e avaliação qualitativa e quantitativa das águas. Disponível em: <http://www.meioambiente.gov.br/port/fnma/capa/index.html>. Acesso em: 24 ago. 2005.

Lei № 4.771, de 15 de setembro de 1965. Institui o novo Código Florestal. Diário Oficial [da] República Federativa do Brasil, Poder Executivo, Brasília, DF, 16 set. 1995. 
Lei $N^{0}$ 12.651, de 25 de maio de 2012. Dispõe sobre a proteção da vegetação nativa; altera as Leis $\mathrm{n}^{\mathrm{os}}$ 6.938, de 31 de agosto de 1981, 9.393, de 19 de dezembro de 1996, e 11.428, de 22 de dezembro de 2006; revoga as Leis $\mathrm{n}^{\text {os }} 4.771$, de 15 de setembro de 1965, e 7.754, de 14 de abril de 1989, e a Medida Provisória no 2.166-67, de 24 de agosto de 2001; e dá outras providências. Diário Oficial [da] República Federativa do Brasil, Poder Executivo, Brasília, DF, 28 maio 2012.

Ministério do Meio Ambiente. Primeiro relatório nacional para a convenção sobre diversidade biológica - Brasil. Brasília, DF, 1998.

BURROUGH, P. A.; MCDONNELL, R. A. Principles of Geographical Information Systems. Oxford: Oxford University Press, 1998. 333 p.

CÂMARA G.; SOUZA R. C. M.; FREITAS U. M.; GARRIDO J. SPRING: integrating remote sensing and GIS by object-oriented data modelling. Computers \& Graphics, v. 20, n. 3, p. 395-403, 1996. http://dx.doi.org/10.1016/0097-8493(96)00008-8

CORDEIRO, J. P.; AMARAL, S.; FREITAS, U. M.; CÂMARA NETO, G. Álgebra de geocampos e suas aplicações. In: SIMPÓSIO BRASILEIRO DE SENSORIAMENTO REMOTO, 8., 14-19 abr. 1996, Salvador. Anais... São José dos Campos: INPE, 2006. p. 355-361. 1 CD-ROM.

COOPERATIVA DE SERVIÇOS, PESQUISAS TECNOLÓGICAS E INDUSTRIAIS CPTI. Plano das bacias hidrográficas do rio Paraíba do Sul 2000-2003. São Paulo, 2001. 1 CD-ROM.

DEAN, W. A ferro e fogo: a história da devastação da mata atlântica brasileira. São Paulo: Companhia das Letras, 1997.

DIAS, N. W.; BATISTA, G. T.; TARGA, M. S. Water use fund applied to land cover characterization and water quality analysis to support hydrographic basin committee decisions in Brazil. In: INTERNATIONAL WATER CONGRESS AND EXHIBITION, 2008, Viena. Proceedings... Viena: IWA, 2008. Vol. 1. p. 1-8. Disponível em: <http://www.agro.unitau.br:8080/dspace/bitstream/2315/144/1/Artigo_WWC_Una_ Ciencias_Agrarias.pdf $>$. Acesso em: 22 nov. 2012.

EASTMAN, J. R. IDRISI for Windows: user's guide. Massachusetts: Clark University, 1995. 367p.

FERRETTI, A. C. O fomento florestal da CESP/Porto Primavera com propriedades da região de Assis - SP. 2000. 156 f. Dissertação (Mestrado) - Escola Superior de Agricultura “Luiz de Queiroz", Universidade de São Paulo, Piracicaba, 2000.

FRANÇA, G. V. Estudo agrotécnico e avaliações das terras da Fazenda São Sebastião, município de santa Cruz das Palmeiras - SP: levantamento de solos, capacidade de uso e valor relativo das terras. Piracicaba: ESALQ-USP, 1983.

INSTITUTO BRASILEIRO DE GEOGRAFIA E ESTATÍSTICA - IBGE. Cidades@: censo 2010. Disponível em: <http://www.ibge.gov.br/cidadesat/topwindow.htm?1>. Acesso em: 21 nov. 2012.

INSTITUTO DE PESQUISAS E ESTUDOS FLORESTAIS - IPEF. Projeto Corumbataí. Conservação dos recursos hídricos e da cobertura florestal na Bacia do Rio Corumbataí. Piracicaba: IPEF, 2001. 118 p. Relatório do projeto.

INSTITUTO NACIONAL DE COLONIZAÇÃO E REFORMA AGRÁRIA - INCRA. (Brasil). Manual de obtenção de terras e perícia judicial. Brasília, DF, 2006. 123p. 
KLIR, G. J. Generalized information theory. Fuzzy Sets and Systems, v. 40, n. 1, p. 127142, 1991. http://dx.doi.org/10.1016/0165-0114(91)90049-V

LEPSCH, I. F.; BELLINAZZI JR., R.; BERTOLINI, D.; ESPÍNDOLA, C. R. Manual para levantamento utilitário do meio físico e classificação de terras no sistema de capacidade de uso: $4^{\mathrm{a}}$ aproximação. Campinas: SBCS, 1983. 175p.

LIMA, W. P.; ZAKIA M. J. B. Hidrologia de matas ciliares. In: RODRIGUES, R. R.; LEITÃO FILHO, H. F. (Eds.). Matas ciliares: conservação e recuperação. Piracicaba: EDUSP/FAPESP, 2000. p. 33-44.

MENDONCA, I. F. C.; LOMBARDI NETO, F.; VIÉGAS, R. A. Classificação da capacidade de uso das terras da Microbacia do Riacho Una, Sapé, PB. Revista Brasileira de Engenharia Agrícola e Ambiental, v. 10, n. 4, p. 888-895, 2006. http://dx.doi.org/10.1590/S1415-43662006000400016

MELO, A. C. G. de. A legislação como suporte a programas de recuperação florestal no estado de São Paulo. Florestar Estatístico, v. 8, p. 9-15, 2005.

REIS, A.; BECHARA, F. C.; ESPÍNDOLA, M. B.; VIEIRA, N. K.; SOUZA, L. L. Restauração de áreas degradadas: a nucleação como base para incrementar os processos sucessionais. Natureza \& Conservação, v. 1, n. 1, p.28-36, 2003.

SAATY, T. L. Método de análise hierárquica. São Paulo: McGraw-Hill, 1991.

SANTOS, M. Pensando o espaço do homem. 3. ed. São Paulo: Hucitec, 1991.

VALENTE, R. O. A. Definição de áreas prioritárias para conservação e preservação florestal por meio da abordagem multicriterial em ambiente SIG. 2005. $121 \mathrm{f}$. Tese (Doutorado em Recursos Florestais) - Escola Superior de Agricultura Luiz de Queiroz, Universidade de São Paulo, Piracicaba, 2005. 


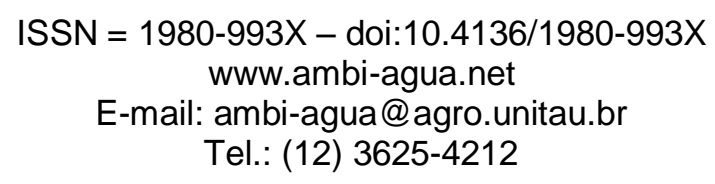

\title{
Consequências hidrológicas da mudança de uso da terra de floresta para pastagem na região da floresta tropical pluvial Atlântica
}

(http://dx.doi.org/10.4136/ambi-agua.927)

\author{
Luiz Felippe Salemi ${ }^{1}$; Juliano Daniel Groppo ${ }^{1}$; Rodrigo Trevisan ${ }^{1}$; \\ Gustavo Bicci Seghesi ${ }^{1}$; Jorge Marcos de Moraes $^{1,2}$; \\ Silvio Fronsini de Barros Ferraz ${ }^{1}$, Luiz Antonio Martinelli ${ }^{1}$ \\ ${ }^{1}$ Laboratório de Ecologia Isotópica - Centro de Energia Nuclear na Agricultura - \\ Universidade de São Paulo, Piracicaba - SP, Brasil, \\ emails: piposalemi@gmail.com, julianogroppo@yahoo.com.br, \\ rtrevisan@yahoo.com.br, gbseghesi@gmail.com, \\ jmmoraes@cena.usp.br, silvio.ferraz@usp.br, zebu@cena.usp.br \\ ${ }^{2}$ Escola de Engenharia de Piracicaba, Piracicaba - SP, Brasil
}

\section{RESUMO}

A floresta tropical pluvial Atlântica é um dos ecossistemas mais ameaçados do Brasil. A degradação deste grande ecossistema ocorre desde o descobrimento do país. Poucos estudos investigaram a hidrologia de microbacias na região deste bioma. A totalidade dos estudos existentes se restringe a ecossistemas cuja cobertura é de florestas naturais. Nesse contexto, este trabalho elucida as consequências hidrológicas da conversão de floresta em pastagem nos domínios da floresta atlântica ombrófila densa. Para tanto, foram medidos o escoamento superficial, a vazão, a condutividade hidráulica do solo, o potencial matricial da água do solo de uma microbacia coberta por pastagem durante um ano. Os resultados indicam que a conversão de floresta ombrófila densa para pastagem promove a redução da condutividade hidráulica próximo à superfície do solo. Entretanto, como as chuvas predominantes são de baixa intensidade, essa redução na permeabilidade do solo à água não implica necessariamente em um aumento substancial de escoamento superficial. Em relação ao potencial matricial da água do solo, a pastagem apresentou valores sempre maiores que os da floresta durante a estação seca. Esse aumento da umidade do solo sobre o uso de pastagens implica em maior drenagem de água rumo ao lençol freático. Este fato explica os maiores valores do coeficiente de deflúvio. Assim, ao converter uma microbacia coberta por Floresta Tropical Atlântica em pastagem, espera-se uma maior conversão de chuva em vazão em termos anuais. Todavia, o aumento do deflúvio ocorre em detrimento da altíssima biodiversidade e da alta proteção do solo quando o solo está coberto por florestas.

Palavras-chave: Floresta Atlântica, água, solo, recursos hídricos.

\section{Hydrological consequences of land-use change from forest to pasture in the Atlantic rain forest region}

\begin{abstract}
The Atlantic rain forest is the most endangered ecosystem in Brazil. Its degradation has started since 1500 when the European settlers arrived. Despite of all land use changes that have occurred, hydrological studies carried out in this biome have been limited to hydrological functioning of rain forests only. In order to understand the hydrological consequences of land-use change from forest to pasture, we described the hydrological
\end{abstract}


SALEMI, L. F.; GROPPO, J. D.; TREVISAN, R.; SEGHESI, G. B.; MORAES, J. M.; FERRAZ, S. F. B.; MARTINELLI, L. A. Consequências hidrológicas da mudança de uso da terra de floresta para pastagem na região da floresta tropical pluvial atlântica. Ambi-Agua, Taubaté, v. 7, n. 3, p. 127-140, 2012. (http://dx.doi.org/10.4136/ambi-agua.927)

functioning of a pasture catchment that was previously covered by tropical rain forest. To reach this goal we measured the precipitation, soil matric potential, discharge, surface runoff and water table levels during one year. The results indicated that there is a decrease in surface soil saturated hydraulic conductivity. However, as low intensity rainfall prevails, the lower water conductivity does not necessarily leads to a substantially higher surface runoff generation. Regarding soil water matric potential, the pasture presented higher moisture levels than forest during the dry season. This increase in soil moisture implies in higher water table recharge that, in turn, explain the higher runoff ratio. This way, land-use change conversion from forest to pasture implies a higher annual streamflow in pasture catchments. Nonetheless, this increase in runoff due to forest conversion to pasture implies in losses of biological diversity as well as lower soil protection.

Keywords: Atlantic Forest, water, soil, water resources.

\section{INTRODUÇÃO}

A Mata Atlântica é o bioma mais ameaçado do Brasil (Schaffer e Prochnow, 2002). A degradação deste grande ecossistema vem ocorrendo desde o descobrimento do país (Dean, 1996; Bueno, 2006) e a supressão da vegetação, que originalmente cobria cerca de 1.300.000 $\mathrm{km}^{2}$, reduziu este sistema natural a uma área descontínua de aproximadamente $98.800 \mathrm{~km}^{2}$, isto é, 7,6\% da sua extensão original (Morellato e Haddad, 2000). Estas florestas foram cortadas para obtenção de madeira, lenha, carvão vegetal, assim como para ceder espaço para a agricultura, a criação de gado e a expansão de centros urbanos (Morellato e Haddad, 2000).

A floresta atlântica é composta principalmente por dois tipos de vegetação: a Floresta Pluvial Atlântica que se estende por quase toda a costa atlântica brasileira e a Floresta Estacional Semidecidual que se estende em direção ao interior do Brasil (Morellato e Haddad, 2000). O primeiro tipo de vegetação também é chamado de floresta ombrófila densa. O termo "ombrófila" caracteriza florestas que se distribuem geograficamente em locais de alta pluviosidade anual. Esta fitofisionomia cobre toda a Serra do Mar e a planície costeira. Poucos estudos investigaram a hidrologia de microbacias nesta região. A maioria das publicações existentes se limitam à região de Cunha - SP devido à existência de infraestrutura e equipamentos para tanto (Arcova e Cicco, 1997; Fujieda et al., 1997; Anido, 2002; Arcova et al., 2003; Ranzini et al., 2004a, b; Donato et al., 2008). Entretanto, exceções são feitas aos trabalhos de Coelho Neto (1987) e Groppo (2010) realizados na Floresta Ombrófila Densa do Rio de Janeiro - RJ e de São Luiz do Paraitinga - SP, respectivamente. Contudo, todos esses estudos se restringem apenas a ecossistemas cuja cobertura é constituída de florestas naturais.

Nesse contexto, buscando contribuir para o entendimento das consequências hidrológicas das mudanças de uso da terra nos domínios da floresta atlântica ombrófila, o presente trabalho pretende descrever o funcionamento hidrológico de uma microbacia coberta por pastagem que outrora fora previamente coberta por floresta ombrófila. Em etapa subsequente, ao se comparar os resultados obtidos aos resultados disponíveis na literatura, tenta-se entender as consequências da mudança de uso da terra de floresta para pastagem nesta região.

\section{MATERIAIS E MÉTODOS}

\subsection{Localização da área}

A microbacia estudada situa-se no bairro Vargem Grande, município de Natividade da Serra - SP e possui área equivalente a 4,7 ha. A altitude média é de 860 metros. A bacia 
SALEMI, L. F.; GROPPO, J. D.; TREVISAN, R.; SEGHESI, G. B.; MORAES, J. M.; FERRAZ, S. F. B.; MARTINELLI, L. A. Consequências hidrológicas da mudança de uso da terra de floresta para pastagem na região da floresta tropical pluvial atlântica. Ambi-Agua, Taubaté, v. 7, n. 3, p. 127-140, 2012. (http://dx.doi.org/10.4136/ambi-agua.927)

constitui uma sub-bacia do rio Paraibuna e o canal fluvial é classificado como de segunda ordem (Figura 1).

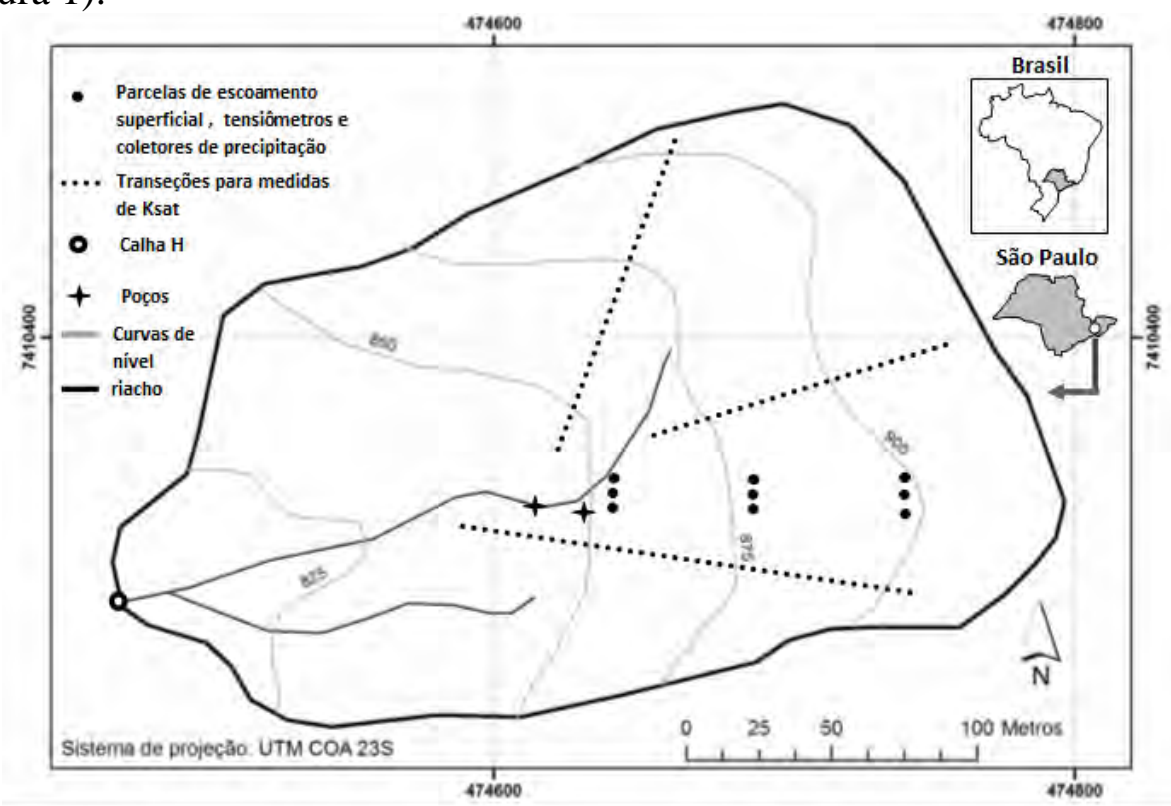

Figura 1. Localização da microbacia utilizada do presente estudo e o delineamento do experimento.

No tocante ao clima, a pluviosidade anual média de Natividade da Serra, distante cerca de $20 \mathrm{~km}$ da área de estudo, é de $1800 \mathrm{~mm}$ (Folhes et al., 2007) podendo haver zonas com menor precipitação $(1300$ a $1500 \mathrm{~mm})$. Em relação à temperatura, as médias mínima e máxima são de, respectivamente, 3 e $26^{\circ} \mathrm{C}$ com média de $17^{\circ} \mathrm{C}$.

Acerca do histórico do uso da terra, este foi obtido por meio de entrevista com os atuais proprietários e moradores locais. De acordo com a descrição, em 1963 houve a conversão de floresta para a cultura de milho e outras, além do cultivo de espécies frutíferas (laranja e limão). Durante este período, não houve preparo do solo com máquinas e implementos. Apenas trabalhos manuais com enxada foram executados. A conversão do uso do solo ocorreu por meio do processo de corte e da queima. De 1968 aos dias atuais, o uso do solo foi convertido para pastagem restando apenas poucas árvores frutíferas do antigo uso da terra. A pastagem possui taxa de lotação de 2,5 cabeças por hectare. A espécie de gramínea predominante é Brachiaria decumbens Stapf. Contudo, há a presença de plantas invasoras herbáceas e arbóreas.

Os solos da microbacia são classificados como cambissolos háplicos distróficos e são derivados da alteração de gnaisses. Contudo, nas regiões adjacentes ao canal fluvial, os solos são classificados como gleissolos.

\subsection{Delineamento experimental}

O delineamento do experimento é ilustrado na Figura 1. Foram usados três coletores de precipitação e nove parcelas de escoamento superficial dispostas de forma que três foram instaladas na porção mais baixa da vertente, três na porção central e três na parte superior. Cada uma das parcelas possuia $2,25 \mathrm{~m}^{2}$ de área e foi delimitada por placas de madeira com um tubo de PVC de 1,5 m na cota mais baixa atuando como coletor de água. Para não sofrer influência direta da precipitação incidente, o coletor foi coberto por uma lona de plástico. Mais detalhes podem ser encontrados em Salemi (2009).

Nas mesmas três porções da encosta foram instalados tensiômetros a 15, 30, 50 e $90 \mathrm{~cm}$ de profundidade para entender o comportamento da umidade do solo. 
SALEMI, L. F.; GROPPO, J. D.; TREVISAN, R.; SEGHESI, G. B.; MORAES, J. M.; FERRAZ, S. F. B.; MARTINELLI, L. A. Consequências hidrológicas da mudança de uso da terra de floresta para pastagem na região da floresta tropical pluvial atlântica. Ambi-Agua, Taubaté, v. 7, n. 3, p. 127-140, 2012. (http://dx.doi.org/10.4136/ambi-agua.927)

Para monitorar o lençol freático, dois poços foram instalados, um em área adjacente ao rio e o outro a cerca de 20 metros de distância do mesmo.

No que tange à vazão, esta foi monitorada por meio de uma calha $\mathrm{H}$. Nesta calha há um poço de tranquilização em que foi instalado um sensor eletrônico de nível de água ("Water Level Sensor", Trutrack) que registrava a altura da lâmina de água da calha H em intervalos de 5 minutos. Deste modo, a descarga foi calculada desde 29 de novembro de 2007 até 29 de outubro de 2008, aplicando a altura da água medida em metros na equação disponível em Gwinn e Parsons (1976).

Todas as variáveis mencionadas, exceto a vazão que foi medida continuamente, foram monitoradas em base semanal de 29 de novembro de 2007 até 29 de outubro de 2008.

\subsection{Densidade do solo e condutividade hidráulica do solo em condição de saturação}

A condutividade hidráulica do solo em condição de saturação $\left(\mathrm{K}_{\text {sat }}\right)$ foi medida com o auxílio de um permeâmetro de carga constante ("Amoozemeter"). As profundidades de medição foram $15,30,50$ e $90 \mathrm{~cm}$. Ao final da medição totalizou-se 25 pontos de medição de $\mathrm{K}_{\mathrm{sat}}$ em cada profundidade.

A densidade do solo foi obtida por meio do uso do trado Uhland. Foram coletadas três amostras não deformadas nas mesmas profundidades que foram realizadas as medidas de $\mathrm{K}_{\text {sat }}$.

\subsection{Precipitação: análise da frequências de intensidades}

A quantificação da precipitação, bem como a análise da frequência de intesidade de chuva foram realizadas através da instalação de um pluviógrafo do tipo "tipping-bucket" (Rain Wise) que registra o volume de chuva $(\mathrm{mm})$ a cada 5 minutos.

\section{RESULTADOS E DISCUSSÃO}

A precipitação total durante o período de estudo foi de aproximadamente $1.433 \mathrm{~mm}$. Deste valor, $77 \%$ dos registros foram considerados de baixa intensidade, ou seja, variando de 0 a $5 \mathrm{~mm} \mathrm{~h}^{-1}$ (Figura 2). Esta classe de intensidade contribui em termos totais com cerca de $48 \%$ da precipitação total (Figura 2). Ao considerar o intervalo de 0 a $15 \mathrm{~mm} . \mathrm{h}^{-1}$, a contribuição aumenta para $70 \%$. Por outro lado, eventos com intensidades altas ocorrem com baixa frequência, sendo, em relação aos eventos de baixa intensidade, muito menos representativos.

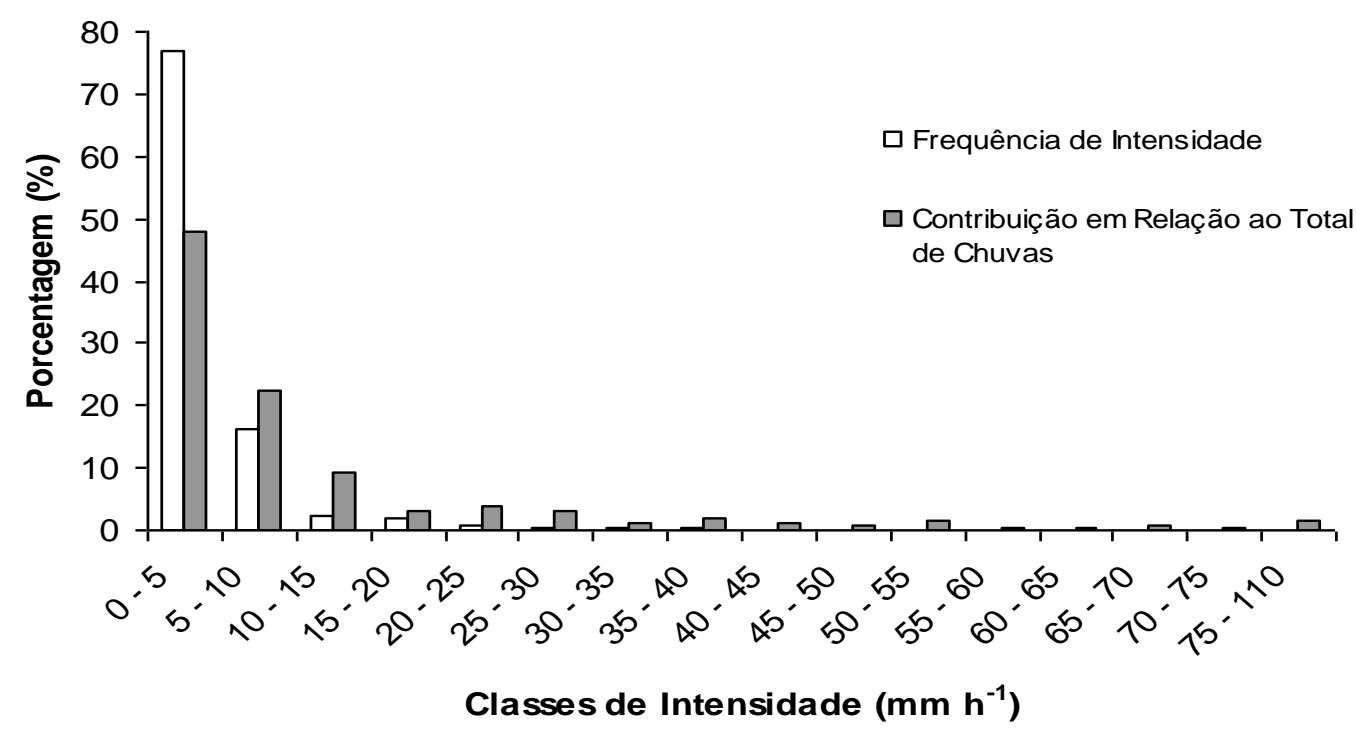

Figura 2. Distribuição de precipitação em classes de intensidade e sua contribuição para o total de chuvas. 
SALEMI, L. F.; GROPPO, J. D.; TREVISAN, R.; SEGHESI, G. B.; MORAES, J. M.; FERRAZ, S. F. B.; MARTINELLI, L. A. Consequências hidrológicas da mudança de uso da terra de floresta para pastagem na região da floresta tropical pluvial atlântica. Ambi-Agua, Taubaté, v. 7, n. 3, p. 127-140, 2012. (http://dx.doi.org/10.4136/ambi-agua.927)

A densidade do solo variou de 1,33 a $1,58 \mathrm{Mg} \mathrm{m}^{-3}$ (Figura 3). A título de comparação, podem-se observar também os dados de densidade obtidos em uma floresta ombrófila densa em área próxima à da pastagem aqui relatada disponível em Groppo (2010).

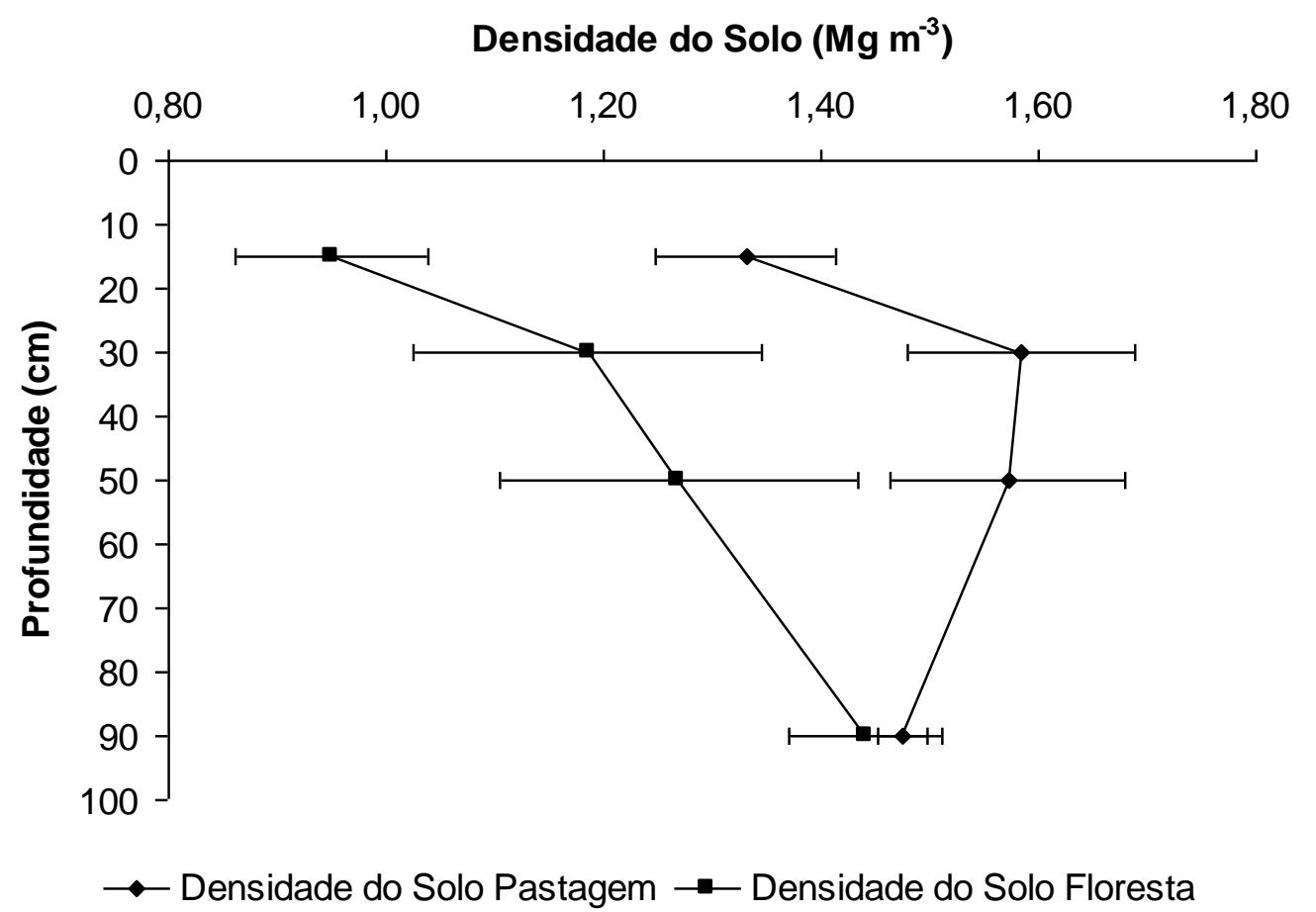

Figura 3. Densidade do solo a 15, 30, 50 e $90 \mathrm{~cm}$ de profundidade na pastagem e floresta.

As medidas de $\mathrm{K}_{\text {sat }}$ apresentaram mediana de $22,6,0,4$ e $0,2 \mathrm{~mm} \mathrm{~h}^{-1}$ respectivamente para 15, 30, 50 e $90 \mathrm{~cm}$ de profundidade (Figura 4).

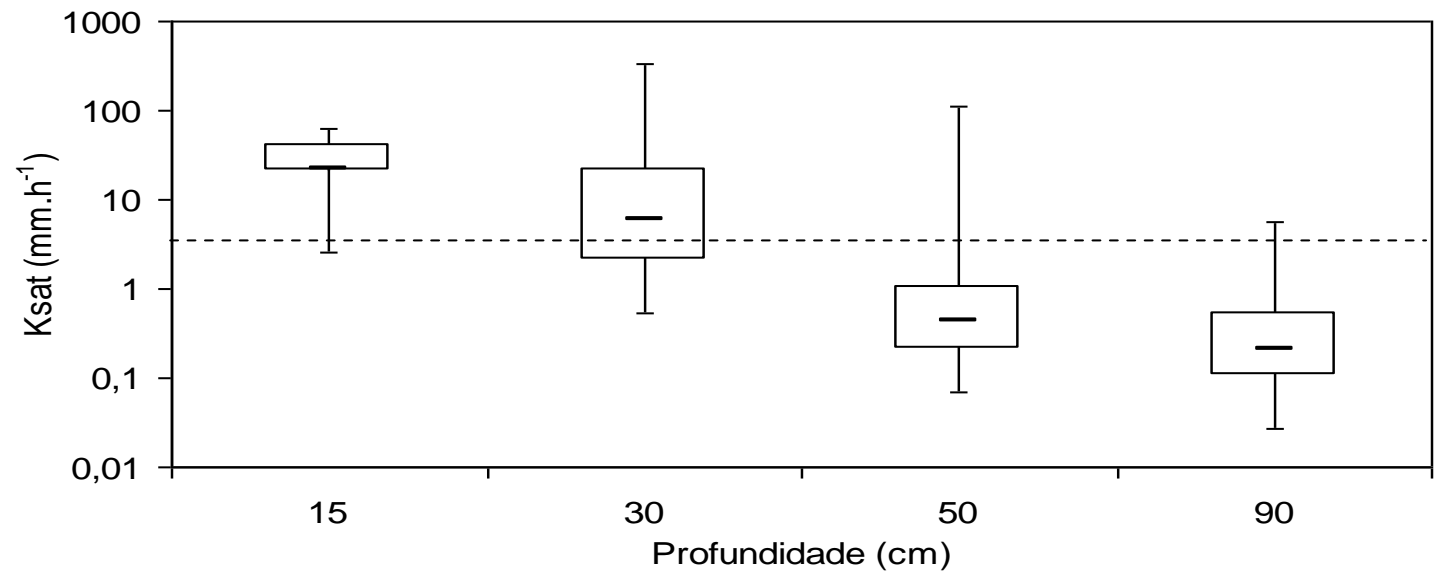

Figura 4. Diagrama de caixas exibindo a condutividade hidráulica do solo em condição de saturação $\left(\mathrm{K}_{\mathrm{sat}}\right)$ na profundidade de $15,30,50$ e $90 \mathrm{~cm}$ na pastagem. A linha pontilhada na horizontal representa a intensidade de chuva de $5 \mathrm{~mm} \mathrm{~h}^{-1}$ que é a mais representativa da região de estudo. 
SALEMI, L. F.; GROPPO, J. D.; TREVISAN, R.; SEGHESI, G. B.; MORAES, J. M.; FERRAZ, S. F. B.; MARTINELLI, L. A. Consequências hidrológicas da mudança de uso da terra de floresta para pastagem na região da floresta tropical pluvial atlântica. Ambi-Agua, Taubaté, v. 7, n. 3, p. 127-140, 2012. (http://dx.doi.org/10.4136/ambi-agua.927)

Nota-se que os valores de $\mathrm{K}_{\text {sat }}$, assim como outros atributos do solo, apresentaram uma grande variabilidade, fato já registrado em outros trabalhos (e.g. Moraes et al., 2006).

Ao comparar os valores de $\mathrm{K}_{\text {sat }}$ da pastagem medidos no presente estudo aos valores obtidos em outras pastagens, nota-se que as medianas de $\mathrm{K}_{\text {sat }}$ são muito próximas entre si, exceção feita aos trabalhos de Elsenbeer et al. (1999) e Moraes et al. (2006) (Tabela 1).

Tabela 1. Comparação dos valores da mediana de $\mathrm{K}_{\mathrm{sat}}\left(\mathrm{mm} \mathrm{h}^{-1}\right)$ em várias profundidades entre vários estudos.

\begin{tabular}{|c|c|c|c|c|}
\hline \multirow{2}{*}{\begin{tabular}{|l} 
Região do estudo \\
Amazônia $^{1}$
\end{tabular}} & \multicolumn{4}{|c|}{ Valores de mediana de Ksat $\left(\mathrm{mm} \mathrm{h}^{-1}\right)$} \\
\hline & $15 \mathrm{~cm}$ & $30 \mathrm{~cm}$ & $50 \mathrm{~cm}$ & $90 \mathrm{~cm}$ \\
\hline Pastagem & 13,00 & 6,00 & 0,80 & 0,70 \\
\hline Floresta & 71,00 & 13,00 & 11,00 & 12,00 \\
\hline Amazônia $^{2}$ & $12,5 \mathrm{~cm}$ & $30 \mathrm{~cm}$ & $50 \mathrm{~cm}$ & \\
\hline Pastagem abandonada & 21,00 & 17,00 & 33,00 & \\
\hline Floresta & 270,00 & 25,00 & 5,00 & \\
\hline Amazônia ${ }^{3}$ & $15 \mathrm{~cm}$ & $30 \mathrm{~cm}$ & $50 \mathrm{~cm}$ & $90 \mathrm{~cm}$ \\
\hline Pastagem & 7,00 & 4,00 & 8,00 & 0,08 \\
\hline Floresta & 310,00 & 35,00 & 8,00 & 1,00 \\
\hline Amazônia ${ }^{4}$ & $12,5 \mathrm{~cm}$ & $20 \mathrm{~cm}$ & & \\
\hline Pastagem & 22,00 & 6,00 & & \\
\hline Floresta & 131,00 & 22,00 & & \\
\hline Mata Atlântica & $15 \mathrm{~cm}$ & $30 \mathrm{~cm}$ & $50 \mathrm{~cm}$ & $90 \mathrm{~cm}$ \\
\hline Pastagem 5 & 22,00 & 6,00 & 0,40 & 0,20 \\
\hline Floresta $^{6}$ & 60,00 & 8,00 & 2,00 & 4,00 \\
\hline
\end{tabular}

Fontes: ${ }^{1}$ Elsenbeer et al. (1999) - Latossolo; ${ }^{2}$ Godsey e Elsenbeer (2002) - Argissolo; ${ }^{3}$ Moraes et al. (2006) Solos plínticos; ${ }^{4}$ Zimmermann et al. (2006) - Latossolo e ${ }^{5}$ presente estudo - Cambissolo; ${ }^{6}$ Groppo (2010) Cambissolo.

Em comparação ao estudo conduzido em área de floresta ombrófila densa na mesma região do presente estudo (Groppo, 2010), a floresta teve seu valor de $\mathrm{K}_{\text {sat }}$ próximo à superfície (15 cm de profundidade) claramente maior que o da pastagem $\left(60\right.$ e $22 \mathrm{~mm} \mathrm{~h}^{-1}$ respectivamente) (Tabela 1). Esse fato decorre do intenso pisoteio do gado que tem um efeito de provocar a compactação do solo (Reiners et al., 1994; Moraes et al., 1996). Em consonância com a literatura, a densidade do solo da pastagem do presente estudo é claramente maior se comparado à da floresta (Figura 3).

Fujieda et al. (1997) relatam também altos valores de $\mathrm{K}_{\text {sat }}$ próximo à superfície do solo de uma floresta ombrófila densa localizada em Cunha - SP variando de 35 a $350 \mathrm{~mm} \mathrm{~h}^{-1}$. Estes valores são próximos aos disponíveis em Groppo (2010) em uma microbacia com o mesmo tipo de floresta em São Luiz do Paraitinga - SP.

$\mathrm{Na}$ profundidade de $50 \mathrm{~cm}$, a pastagem e a floresta (Groppo, 2010) apresentam valores de $\mathrm{K}_{\text {sat }}$ extremamente reduzidos, embora a floresta ainda tenha valores cinco vezes maiores.

O potencial matricial da água do solo medido nas profundidades de 15, 30, 50 e $90 \mathrm{~cm}$ em três locais da vertente (topo, meio e sopé) teve amplitude de 0 a $-87 \mathrm{kPa}$. No entanto, de maneira geral, percebe-se que a maior parte do ano o solo, em todas as porções da encosta, permanece em estados de alto grau de umidade, isto é, com potencial matricial variando de 0 a $-8 \mathrm{kPa}$ (Figura 5). Apesar de haver variabilidade no potencial da água do solo nas quatro profundidades, de uma maneira geral, o solo permaneceu em condições muito úmidas por 
SALEMI, L. F.; GROPPO, J. D.; TREVISAN, R.; SEGHESI, G. B.; MORAES, J. M.; FERRAZ, S. F. B.; MARTINELLI, L. A. Consequências hidrológicas da mudança de uso da terra de floresta para pastagem na região da floresta tropical pluvial atlântica. Ambi-Agua, Taubaté, v. 7, n. 3, p. 127-140, 2012. (http://dx.doi.org/10.4136/ambi-agua.927)

longos períodos (Reichardt, 1988). Foi possível notar que há variabilidade espacial na umidade nas mesmas datas de medição. Tal variação está de acordo com o que outros autores encontraram para pastagens e pode ser atribuído à variação espacial da compactação do solo (Hodnett et al., 1995).

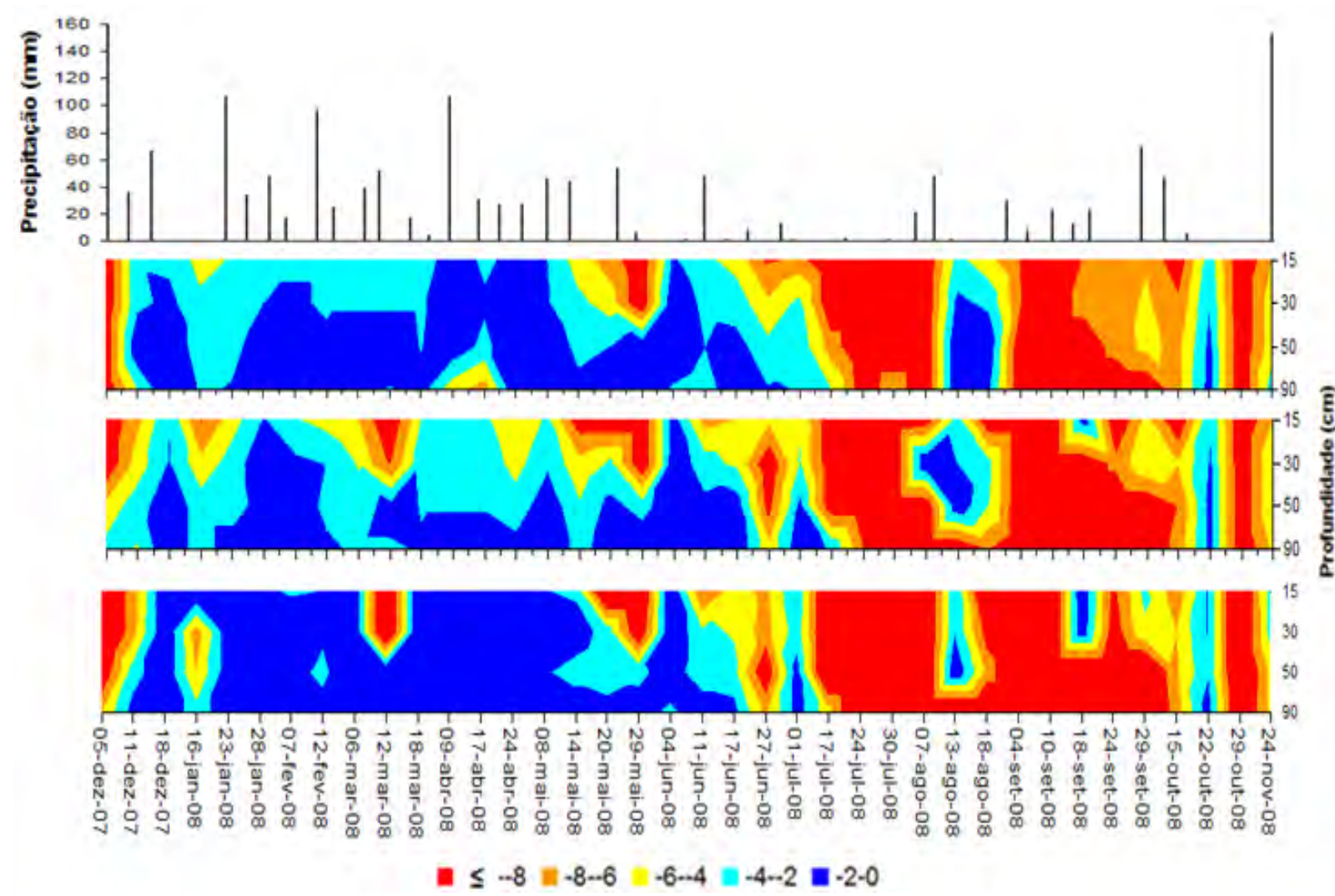

Figura 5. Precipitação semanal e classes de potencial matricial da água no solo $(\mathrm{kPa})$ da vertente (Superior - sopé; intermediário - meio da vertente ; e inferior - porção mais alta da vertente).

Ao comparar o potencial matricial da água do solo na pastagem ao da floresta ombrófila densa estudada por Groppo (2010), nota-se que durante os períodos chuvosos, não há diferença marcante no potencial matricial da água do solo. Esta similaridade permite inferir que a diferença de cobertura vegetal tem pouca influência sobre a umidade do solo que rapidamente é reposta pelas chuvas de baixa intensidade. Porém, se somente o período seco (junho a agosto) for analisado, observa-se que a floresta exibe potenciais matriciais muito mais baixos (negativos). Esse fato também foi observado por vários outros autores comparando florestas e pastagens (Nepstad et al., 1994; Jipp et al., 1998; Moraes et al., 2006). Em todos os estudos mencionados bem como na comparação feita em relação ao trabalho realizado por Groppo (2010), a absorção pelo sistema radicular mostrou-se muito mais vigorosa na floresta do que nas pastagens durante a estação seca, o que reflete as diferenças de uso da água pelas diferentes comunidades vegetais que possuem estrutura e metabolismo distintos.

No que se refere ao coeficiente de escoamento superficial, o valor médio calculados das nove parcelas instaladas (Tabela 2) equivalente a 0,02 , ou seja, cerca de $2 \%$ da precipitação tornou-se escoamento superficial.

Ao utilizar o mesmo delineamento de parcelas de escoamento superficial em floresta ombrófila densa, Groppo (2010) apresentou valores de coeficiente de escoamento superificial inferiores a $1 \%$. Se comparado aos resultados do presente estudo, um pouco mais que o dobro 
SALEMI, L. F.; GROPPO, J. D.; TREVISAN, R.; SEGHESI, G. B.; MORAES, J. M.; FERRAZ, S. F. B.; MARTINELLI, L. A. Consequências hidrológicas da mudança de uso da terra de floresta para pastagem na região da floresta tropical pluvial atlântica. Ambi-Agua, Taubaté, v. 7, n. 3, p. 127-140, 2012. (http://dx.doi.org/10.4136/ambi-agua.927)

do volume da chuva é convertido em escoamento superficial quando se converte floresta para pastagem.

Tabela 2. Média e amplitude do coeficiente de escoamento superficial nas três porções da vertente da microbacia. Os valores se referem às parcelas situadas nas três porções da encosta.

\begin{tabular}{c|cc}
\hline Porção da vertente & Média & Amplitude \\
\hline Inferior & 0,016 & $0-0,44$ \\
Média & 0,006 & $0-0,18$ \\
Superior & 0,017 & $0-0,52$ \\
\hline
\end{tabular}

As chuvas de baixa intensidade não excedem a $\mathrm{K}_{\text {sat }}$ próximo à superfície, o que permite que a água pluvial se infiltre e se redistribua mantendo baixos os valores de potencial matricial (Figura 5). Essa inferência baseia-se nos resultados baixos dos coeficientes de escoamento superficial obtido nas parcelas (Tabela 2). No entanto, nota-se grandes amplitudes já que em alguns eventos cerca de $44 \%$ da precipitação é convertida em escoamento superficial na porção inferior da vertente. Contudo, eventos pluviais que produzem essa magnitude de escoamento superficial são raros (Figura 2).

Para elucidar o conjunto de processos hidrológicos de superfície que ocorrem durante eventos de chuva, foram selecionados dois eventos extremos (Figura 6a e 6b). O primeiro representa a geração de escoamento direto em condições chuvas de baixa intensidade que são típicas da região $\left(0\right.$ a $\left.5 \mathrm{~mm} \mathrm{~h}^{-1}\right)$. $\mathrm{O}$ segundo evento, menos frequente, possui intensidade de chuva máxima que se enquadra na classe de chuvas fortes ou pesadas (35 a $40 \mathrm{~mm} \mathrm{~h}^{-1}$ ) (Hewlett, 1982).

Na Figura 6a, nota-se que há um pequeno aumento da vazão ao longo do evento de chuva. De fato, esse incremento ocorre principalmente devido à geração de escoamento subsuperficial das áreas adjacentes ao canal como descrito por Dunne (1978). Há também, embora em menor proporção, uma contribuição de escoamento superficial hortoniano em trilhas que se dirigem ao canal. O escoamento direto foi obtido por meio da separação de hidrogramas seguindo método da linha reta encontrado em Chow et al. (1988). Desse modo, em relação à Figura $6 \mathrm{a}$, em termos totais, o escoamento direto representou $7 \%$ do escoamento total. De maneira coerente, a resposta hidrológica (volume em $\mathrm{mm}$ de escoamento direto / precipitação do evento medida em $\mathrm{mm}$ ) foi de $1,4 \%$. Ademais, o tempo de pico, entendido como intervalo de tempo entre o máximo valor de intensidade de precipitação e o máximo valor de vazão no hidrograma, é de aproximadamente 1 hora.

Por outro lado, observa-se que as altas intensidades de chuva fazem com que várias áreas da microbacia produzam escoamento superficial hortoniano e de áreas saturadas, produzindo um aumento expressivo da vazão, assim como apresentando tempo de pico de 20 minutos, que é mais rápido do que o apresentado na Figura 6a. No hidrograma apresentado na Figura $6 \mathrm{~b}$, o escoamento direto representou $31 \%$ do escoamento total. Além disso, a resposta hidrológica foi de 6\%. Esse valor, se comparado ao do hidrograma da Figura 6a, é expressivamente mais elevado.

A principal via hidrológica que compõem o deflúvio é o escoamento de base já que a curva de duração de fluxo apresenta-se uma inclinação suave em quase toda a sua extensão (Figura 7). Esta informação está em consonância com os baixos coeficientes de escoamento superficial obtidos a partir das parcelas, bem como os altos valores de umidade do solo. 
SALEMI, L. F.; GROPPO, J. D.; TREVISAN, R.; SEGHESI, G. B.; MORAES, J. M.; FERRAZ, S. F. B.; MARTINELLI, L. A. Consequências hidrológicas da mudança de uso da terra de floresta para pastagem na região da floresta tropical pluvial atlântica. Ambi-Agua, Taubaté, v. 7, n. 3, p. 127-140, 2012. (http://dx.doi.org/10.4136/ambi-agua.927)

a)

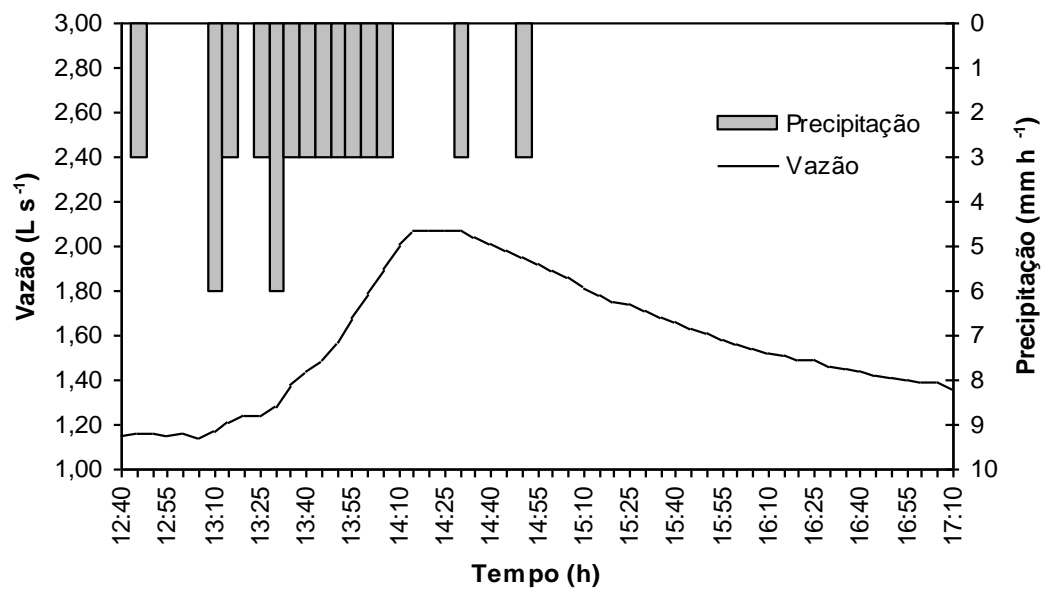

b)

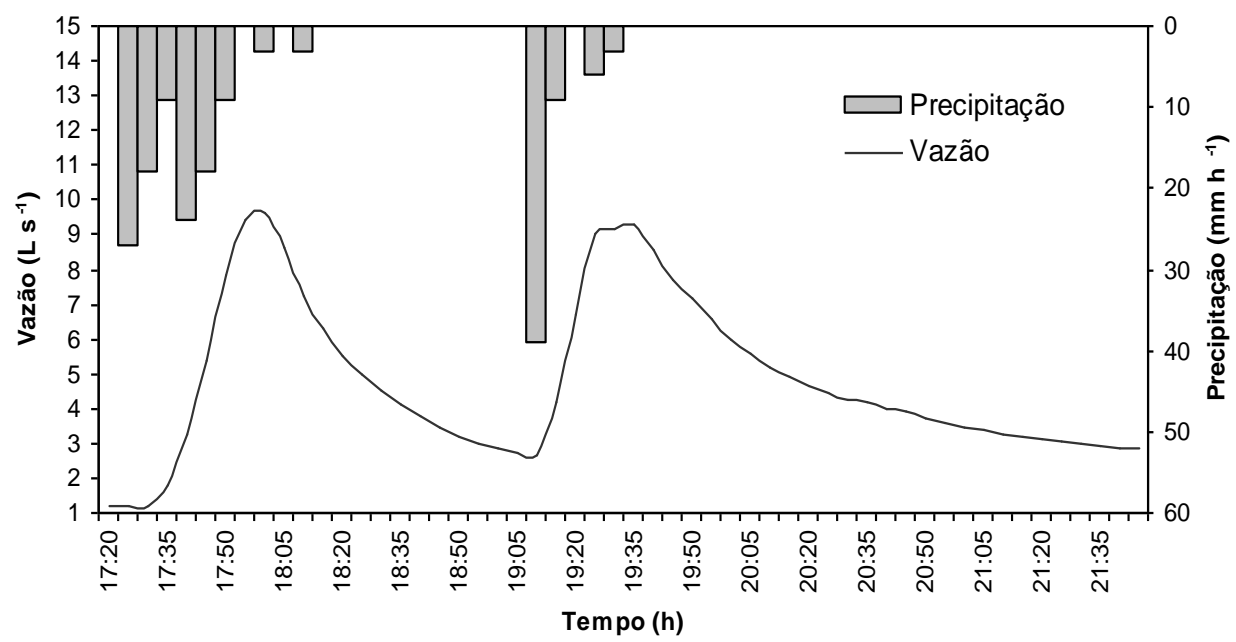

Figura 6. Hidrogramas e hietogramas exibindo geração de escoamento direto (a) evento de precipitação de intensidade leve e (b) evento de precipitação de intensidade forte.

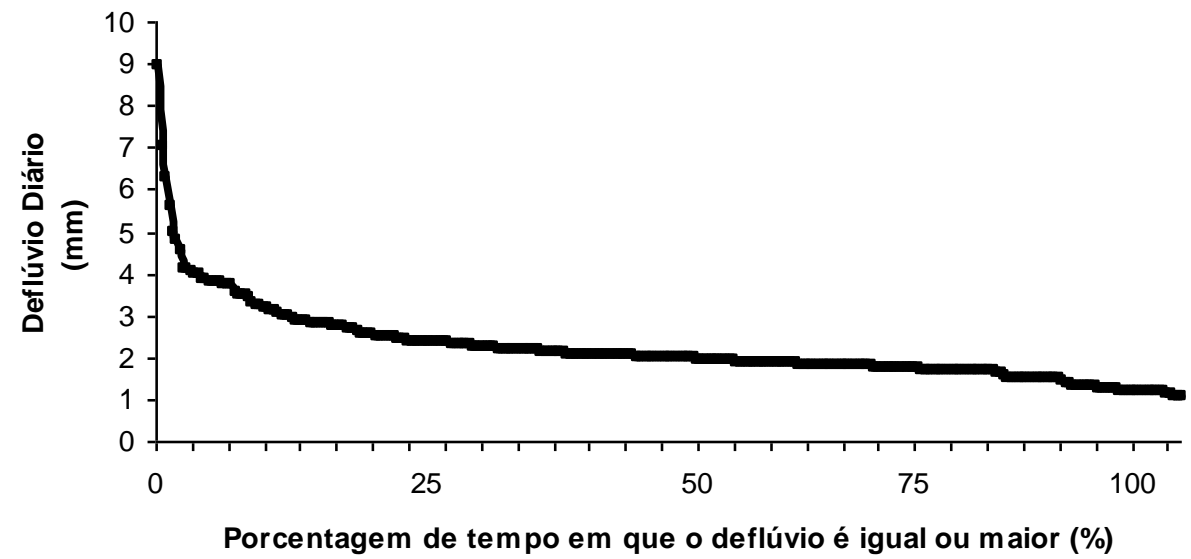

Figura 7. Curva de duração de fluxo. 
SALEMI, L. F.; GROPPO, J. D.; TREVISAN, R.; SEGHESI, G. B.; MORAES, J. M.; FERRAZ, S. F. B.; MARTINELLI, L. A. Consequências hidrológicas da mudança de uso da terra de floresta para pastagem na região da floresta tropical pluvial atlântica. Ambi-Agua, Taubaté, v. 7, n. 3, p. 127-140, 2012. (http://dx.doi.org/10.4136/ambi-agua.927)

O deflúvio anual para o período de estudo totalizou $736 \mathrm{~mm}$. Ao subtrair o valor do deflúvio anual a precipitação anual $(1433 \mathrm{~mm})$, obteve-se a evapotranspiração de $697 \mathrm{~mm}$ $a^{-1}$. Essa estimativa de evapotranspiração anual apresentou um valor muito próximo do que Zhang et al. (2001) modelaram (aproximadamente $700 \mathrm{~mm}^{-1}$ ).

Com a precipitação anual e o deflúvio anual calculou-se o coeficiente de deflúvio entendido aqui como a razão entre a evapotranspiração e a precipitação ambos em base anual. Dessa forma, a razão obtida foi de 0,51 , sugerindo que $51 \%$ da precipitação se transforma em deflúvio sendo o restante perdido por evapotranspiração.

O coeficiente de deflúvio obtido no presente estudo, se comparado ao deflúvio gerado em uma microbacia florestal na mesma região (Groppo, 2010), é substancialmente maior (Tabela 3). De fato, o coeficiente mostra que as árvores das florestas consomem mais água do que as gramíneas das pastagens (Brown et al., 2005). O mesmo foi encontrado, em Paragominas - PA, por Moraes et al. (2006) ao estudar microbacias cobertas por floresta e pastagem drenadas por canais efêmeros.

De maneira diferente, ao comparar estudos como o de Fujieda et al. (1997) realizado na floresta atlântica ombrófila em Cunha - SP, há uma notável divergência. Estes autores encontraram o coeficiente de 0,7 para uma microbacia coberta por floresta nativa. Esse valor, se comparado ao encontrado no presente estudo, é surpreendente já que a floresta teria uma evapotranspiração menor que a de uma pastagem, situação essa que discorda da grande maioria dos estudos de microbacias pareadas (Zhang et al., 2001) e que também difere do resultado encontrado por Groppo (2010). No entanto, Donato et al. (2008) também avaliaram a evapotranspiração das mesmas bacias que Fujieda et al. (1997) e encontraram um coeficiente levemente inferior ao destes autores, mas ainda superior ao da pastagem do presente estudo (Tabela 3).

Tabela 3. Comparação entre os coeficientes de deflúvio obtidos em vários experimentos realizados em microbacias hidrográficas com usos do solo de floresta e pastagem.

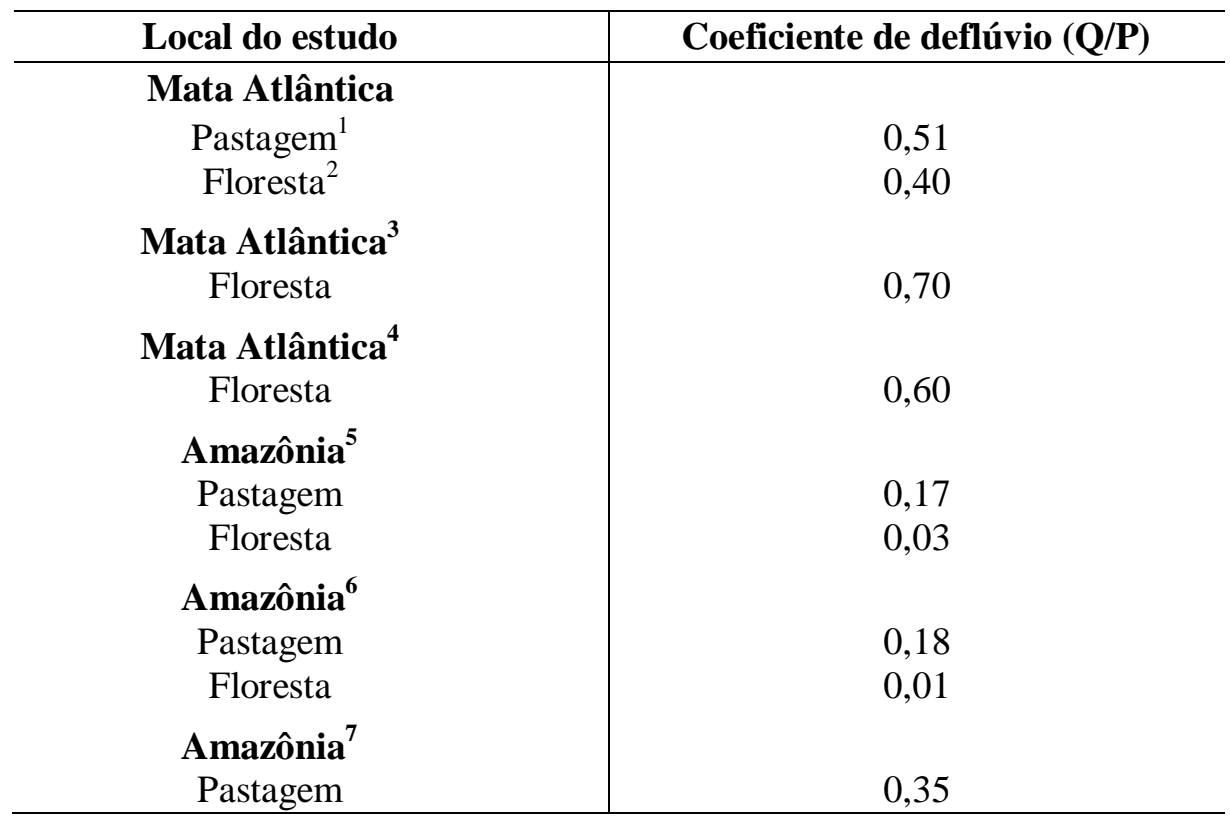

Fontes: ${ }^{1}$ presente estudo; ${ }^{2}$ Groppo (2010); ${ }^{3}$ Fujieda et al. (1997); ${ }^{4}$ Donato et al. (2008); ${ }^{5}$ Moraes et al. (2006); ${ }^{6}$ Germer et al. (2009) e ${ }^{7}$ Biggs et al. (2006). 
SALEMI, L. F.; GROPPO, J. D.; TREVISAN, R.; SEGHESI, G. B.; MORAES, J. M.; FERRAZ, S. F. B.; MARTINELLI, L. A. Consequências hidrológicas da mudança de uso da terra de floresta para pastagem na região da floresta tropical pluvial atlântica. Ambi-Agua, Taubaté, v. 7, n. 3, p. 127-140, 2012. (http://dx.doi.org/10.4136/ambi-agua.927)

\section{CONCLUSÃO}

A conversão de floresta ombrófila densa para pastagem na região da floresta tropical pluvial atlântica promove a redução da condutividade hidráulica próximo à superfície do solo. Entretanto, como as chuvas predominantes são de baixa intensidade, essa redução da permeabilidade do solo à água não implica necessariamente em um aumento substancial de escoamento superficial e de processos erosivos associados a esta via hidrológica.

Em relação ao potencial matricial da água do solo, as pastagens apresentaram valores sempre maiores que os da floresta durante a estação seca. Esse aumento da umidade do solo sobre o uso de pastagens implica em maior percolação de água rumo ao lençol freático. Este fato aliado à maior produção de escoamento superficial nos menos frequentes eventos pluviais de alta intensidade explica os maiores valores do coeficiente de deflúvio. Dessa forma, espera-se uma maior conversão de chuva em vazão em termos anuais, ao converter uma microbacia coberta por floresta tropical atlântica em pastagem. Todavia, o aumento do deflúvio ocorre em detrimento da altíssima diversidade biológica e da alta proteção do solo que ocorre quando o solo está coberto por florestas.

\section{AGRADECIMENTOS}

Os autores gostariam de agradecer ao Fundação de Amparo à Pesquisa no Estado de São Paulo (FAPESP) pelo financiamento da presente pesquisa (processos 2006/54292-9 e 2006/55136-0). Além disso, os autores agradecem João Paulo Villani (gestor do núcleo Santa Virgínia pertencente ao Parque Estadual da Serra do Mar) e todos os seus funcionários cuja dedicação tornou esta pesquisa possível.

\section{REFERENCIAS}

ANIDO, N. M. R. Caracterização hidrológica de uma microbacia experimental visando identificar indicadores de monitoramento ambiental. 2002. 69 f. Dissertação (Mestrado em Recursos Florestais) - Escola Superior de Agricultura Luiz de Queiroz, Universidade de São Paulo, Piracicaba, 2002.

ARCOVA, F. C. S.; CICCO, V. Características do deflúvio de duas microbacias hidrográficas no laboratório de hidrologia florestal Walter Emmerich, Cunha - SP. Revista Instituto Florestal, São Paulo, v. 9, p. 153-170, 1997.

ARCOVA, F. C. S.; CICCO, V. de; ROCHA, P. A. B. Precipitação efetiva e interceptação das chuvas por floresta de mata atlântica em uma microbacia experimental em Cunha São Paulo. Revista Árvore, Viçosa, MG, v. 27, n.2, p. 257-262, 2003. http://dx.doi.org/10.1590/S0100-67622003000200014

BIGGS, T. W.; DUNNE, T.; MURAOKA, T. Transport of water, solutes and nutrients from a pasture hillslope, southwestern Brazilian Amazon. Hydrological Processes, Bristol, v. 20, n. 12, p.2527-2547, 2006. http://dx.doi.org/10.1002/hyp.6214

BROWN, A. E.; ZHANG, L.; McMAHON, T. A.; WESTERN, A. W.; VERTESSY, R. A. A review of paired catchment studies for determining changes in water yield resulting from alteration in vegetation. Journal of Hydrology, Amsterdam, v. 310, n. 1/4, p. 2861, 2005. http://dx.doi.org/10.1016/j.jhydrol.2004.12.010

BUENO, E. Capitães do Brasil: a saga dos primeiros colonizadores. 2. ed. Rio de Janeiro: Objetiva, 2006. 258p. 
SALEMI, L. F.; GROPPO, J. D.; TREVISAN, R.; SEGHESI, G. B.; MORAES, J. M.; FERRAZ, S. F. B.; MARTINELLI, L. A. Consequências hidrológicas da mudança de uso da terra de floresta para pastagem na região da floresta tropical pluvial atlântica. Ambi-Agua, Taubaté, v. 7, n. 3, p. 127-140, 2012. (http://dx.doi.org/10.4136/ambi-agua.927)

CHOW, V. T.; MAIDMENT, D. R.; MAYS, L. W. Applied hydrology. New York: McGraw-Hill Book, 1988. 572p.

COELHO NETO, A. L. Overland flow production in a tropical rainforest catchment: the role of litter cover. Catena, Amsterdam, v. 14, n. 1/3, p. 213-231, 1987. http://dx.doi.org/ 10.1016/S0341-8162(87)80019-X

DEAN, W. A ferro e fogo: a história e a devastação da Mata Atlântica brasileira. São Paulo: Companhia das Letras, 1996. 484p.

DONATO, C. F.; RANZINI, M.; CICCO, V.; ARCOVA, F. C. S.; SOUZA, L. F. S. Balanço de massa em microbacia experimental com Mata Atlântica, na Serra do Mar, CunhaSP. Revista do Instituto Florestal, São Paulo, v. 20, n. 1, p. 1-11, 2008.

DUNNE, T. Field studies of hillslope flow process. In: KIKBY, M. J. (Ed.). Hillslope hydrology. Chichester: John Wiley, 1978. p. 227-293.

ELSENBEER, H.; NEWTON, B. E.; DUNNE, T.; MORAES, J. M. Soil hydraulic conductivities of latosols under pasture, forest and teak in Rondônia, Brazil. Hydrological Processes, Bristol, v. 13, n. 9, p. 1417-1422, 1999. http://dx.doi.org/10.1002/(SICI)1099-1085(19990630)13:9<1417::AID-HYP816>3.0.CO;2-6

FOLHES, M. T.; RENNÓ, C. D.; FISCH, G. Caracterização espaço-temporal do regime pluviométrico na parte paulista da bacia hidrográfica do rio Paraíba do Sul. In: SEMINÁRIO DE RECURSOS HÍDRICOS DA BACIA HIDROGRÁFICA DO PARAÍBA DO SUL: O EUCALIPTO E O CICLO HIDROLÓGICO,1., 7-9 nov., 2007, Taubaté. Anais... Taubaté: IPABHi, 2007. p. 359-361.

FUJIEDA, M.; KUDOH, T.; CICCO, V.; CALVARCHO, J. L. Hydrological processes at two subtropical forest catchments: the Serra do Mar, São Paulo, Brazil. Journal of Hydrology, Amsterdam, v. 196, n. 1/4, p. 26-46, 1997. http://dx.doi.org/10.1016/ S0022-1694(97)00015-2

GERMER, S.; NEILL, C.; VETTER, T.; CHAVES, J.; KRUSCHE, A. V.; ELSENBEER, H. Implications of long-term lad-use change for the hydrology and solute budgets of small catchments in Amazonia. Journal of Hydrology, Amsterdam, v. 364, n. 3/4, p. 349363, 2009.

GODSEY, S.; ELSENBEER, H. The soil hydrologic response to forest regrowth: a case study from southwestern Amazonia. Hydrological Processes, Bristol, v. 16, n. 7, p. 1519-1522, 2002. http://dx.doi.org/10.1002/hyp.605

GROPPO, J. D. Caracterização hidrológica e dinâmica do nitrogênio em uma microbacia com cobertura florestal (Mata Atlântica), no Parque Estadual da Serra do Mar, núcleo Santa Virgínia. 2010. 80 f. Tese (Doutorado em Ciências) Centro de Energia Nuclear na Agricultura, Universidade de São Paulo, Piracicaba, 2010.

GWINN, W. R.; PARSONS, D. A. Discharge equations for HS, H, and HL flumes. Journal of Hydraulics Division, Ithaca, v. 102, n. 1, p.73-88, 1976.

HEWLETT, J. D. Principles of forest hydrology. Athens: The University of Georgia Press, 1982. 183p. 
SALEMI, L. F.; GROPPO, J. D.; TREVISAN, R.; SEGHESI, G. B.; MORAES, J. M.; FERRAZ, S. F. B.; MARTINELLI, L. A. Consequências hidrológicas da mudança de uso da terra de floresta para pastagem na região da floresta tropical pluvial atlântica. Ambi-Agua, Taubaté, v. 7, n. 3, p. 127-140, 2012. (http://dx.doi.org/10.4136/ambi-agua.927)

HODNETT, M. G.; PIMENTEL DA SILVA, L.; ROCHA, H. R.; CRUZ SENNA, R. Seasonal soil water storage changes beneath central Amazonian rainforest and pasture. Journal of Hydrology, Amsterdam, v.170, n. 1/4, p.233-254, 1995. http://dx.doi.org/10.1016/0022-1694(94)02672-X

JIPP, P. H.; NEPSTAD, D. C.; CASSEL, D. K.; REIS DE CARVALHO, C. Deep soil moisture storage and transpiration in forests and pastures of seasonally-dry Amazonia. Climatic Change, Amsterdam, v. 39, n. 2/3, p. 395-412, 1998. http://dx.doi.org/10.1023/A:100508930871

MORAES, J. M.; SCHULER, A. E.; DUNNE, T.; FIGUEIREDO, R. O.; VICTORIA, R. L. Water storage and runoff processes in plinthic soils under forest and pasture in eastern Amazonia. Hydrological Processes, Bristol, v. 20, n. 12, p. 2509-2526, 2006. http://dx.doi.org/10.1002/hyp.6213

MORAES, J. F.; VOLKOFF, B.; CERRI, C. C.; BERNOUX, M. Soil properties under Amazon forest and changes due to pasture installation in Rondônia, Brazil. Geoderma, Amsterdam, v. 70, n. 1, p. 63-81, 1996. http://dx.doi.org/10.1016/0016-7061(95)00072-0

MORELlATO, L. P. C.; HADDAD, C. F. B. Introduction: the brazilian Atlantic Forest. Biotropica, Bristol, v. 32, n. 4b, p. 786-792, 2000. http://dx.doi.org/10.1111/j.17447429.2000.tb00618.x

NEPSTAD, D. C.; CARVALHO, C. R.; DAVIDSON, E. A.; JIPP, P. H.; LEFEBVRE, P. A.; NEGREIROS, G. H. et al. The role of deep roots in the hydrological and carbon cycles of Amazonian forests and pastures. Nature, London, v. 372, p. 666-669, 1994. http://dx.doi.org/10.1038/372666a0

RANZINI, M.; RIGHETTO, A. M.; LIMA, W. P.; GUANDIQUE, M. E. G.; ARCOVA, F. C. S.; CICCO, V. Processos hidrológicos de uma microbacia com Mata Atlântica, na região da Serra do Mar, SP. Scientia Forestalis, Piracicaba, v. 66, p. 108-119, 2004a.

RANZINI, M.; RIGHETTO, A. M.; GUANDIQUE, M. E. G.; MORAES, J. M.; ARCOVA, F. C. S.; CICCO, V. Modelagem hidrológica de uma microbacia florestada da Serra do Mar, SP. Revista Brasileira de Recursos Hídricos, Porto Alegre, v. 9, n. 4, p. 33-44, 2004b.

REICHARDT, K. Capacidade de campo. Revista Brasileira de Ciência do Solo, Viçosa, MG, v. 12, p. 211-216, 1988.

REINERS, W. A.; BOWMAN, A. F.; PARSONS, W. F. J.; KELLER, M. Tropical rain forest conversion to pasture: changes in vegetation and soil properties. Ecological Applications, Ithaca, v. 4, p. 363-377, 1994. http://dx.doi.org/10.2307/1941940

SALEMI, L. F. Balanço de água e de nitrogênio em uma microbacia coberta por pastagem no litoral Norte do Estado de São Paulo. 2009. 87 f. Dissertação (Mestrado em Ecologia Aplicada) - Centro de Energia Nuclear na Agricultura / Escola Superior de Agricultura "Luiz de Queiroz", Universidade de São Paulo, Piracicaba, 2009.

SCHÄFFER, W. B.; PROCHNOW, M. Mata Atlântica: informações gerais. In: SCHÄFFER, W.B.; PROCHNOW, M. (Org.). A Mata Atlântica e você: como preservar, recuperar e se beneficiar da mais ameaçada floresta brasileira. Brasília: APREMAVI, 2002. p. 1213. 
SALEMI, L. F.; GROPPO, J. D.; TREVISAN, R.; SEGHESI, G. B.; MORAES, J. M.; FERRAZ, S. F. B.; MARTINELLI, L. A. Consequências hidrológicas da mudança de uso da terra de floresta para pastagem na região da floresta tropical pluvial atlântica. Ambi-Agua, Taubaté, v. 7, n. 3, p. 127-140, 2012. (http://dx.doi.org/10.4136/ambi-agua.927)

ZHANG, L.; DAWES, W. R.; WALKER, G. R. The response of mean annual evapotranspiration to vegetation changes at the catchment scale. Water Resources Research, New York, v. 37, p. 701-708, 2001. http://dx.doi.org/10.1029 /2000WR900325

ZIMMERMANN, B.; ELSENBEER, H.; MORAES, J. M. The influence of land-use change on soil hydraulic properties: implications for runoff generation. Forest Ecology and Management, Amsterdam, v. 222, n. 1/3, p. 29-39, 2006. http://dx.doi.org/ 10.1016/j.foreco.2005.10.070 


ISSN = 1980-993X - doi:10.4136/1980-993X
www.ambi-agua.net
E-mail: ambi-agua@agro.unitau.br
Tel.: (12) 3625-4212

\title{
Avaliação espaço-temporal da qualidade da água e simulação de autodepuração na bacia hidrográfica do córrego São Simão, SP
}

(http://dx.doi.org/10.4136/ambi-agua.894)

\author{
Julio César de Souza Inácio Gonçalves ${ }^{1}$; Diego de Souza Sardinha²; \\ Antonio Donizetti Gonçalves de Souza ${ }^{2}$; André Luis Bigaram Dibiazi ${ }^{3}$; \\ Letícia Hirata Godoy ${ }^{4}$; Fabiano Tomazini da Conceição ${ }^{4}$

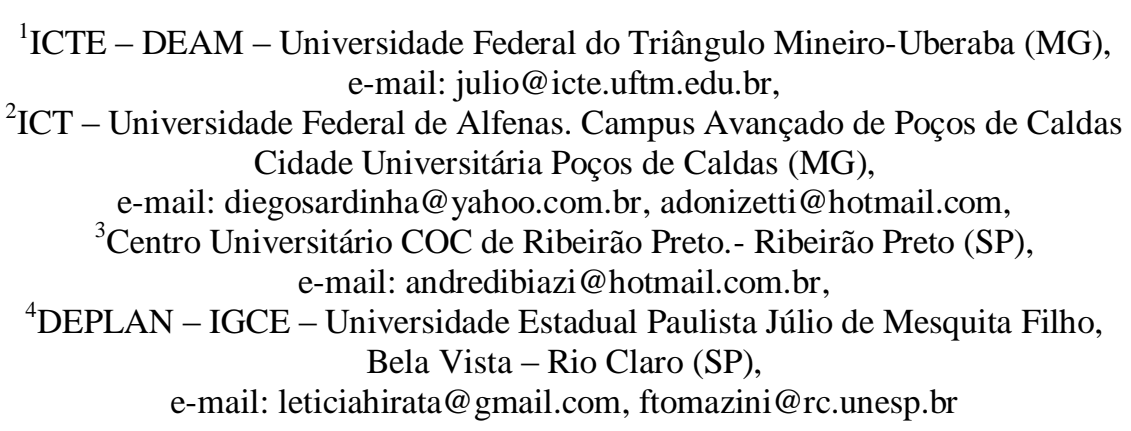

RESUMO

Neste trabalho foram avaliadas as águas fluviais da bacia hidrográfica do córrego São Simão (SP). Para isso, seis pontos de coleta foram estabelecidos e realizaram-se doze coletas de água durante os meses de março, maio, junho, julho, agosto, setembro, outubro, novembro e dezembro de 2005, e janeiro, fevereiro e março de 2006, analisando as seguintes variáveis: vazão, temperatura, turbidez, $\mathrm{pH}$, condutividade, oxigênio dissolvido, alcalinidade, cloreto e sólidos totais em suspensão. A simulação do processo de autodepuração foi realizada com o uso do modelo de Streeter-Phelps, o qual relaciona a variação do déficit de oxigênio dissolvido com o perfil longitudinal do rio. As características do córrego São Simão próximo à sua nascente até a cidade de São Simão permitem concluir que há pouca interferência na sua qualidade. Porém, a falta de tratamento para o esgoto doméstico na cidade de São Simão piora a qualidade da água e intensifica o processo de degradação do córrego São Simão. O modelo de Streeter \& Phelps permitiu identificar as zonas de autodepuração do córrego São Simão e indicou a necessidade de tratamento de esgotos em nível primário, com eficiência de $30 \%$.

Palavras-chave: Avaliação da qualidade da água; esgotos domésticos; modelagem matemática de autodepuração.

\section{Water quality spatial and temporal evaluation and auto-purification simulation from the São Simão stream watershed, SP}

\section{ABSTRACT}

This study evaluated the water of São Simão stream watershed. For this, six sampling points were established and twelve samples of water were collected during the months of March, May, June, July, August, September, October, November, and December of 2005 and January, February, and March of 2006. The following variables were analyzed: discharge, temperature, turbidity, $\mathrm{pH}$, electrical conductivity (EC), dissolved oxygen (DO), alkalinity, chloride and TSS (total suspended solids). The Streter \& Phelps model which correlates the variation of the deficit oxygen with the distance was applied to evaluate the São Simão stream auto-purification. The 
GONÇALVES, J. C. S. I.; SARDINHA, D. S.; SOUZA, A. D. G.; DIBIAZI, A. L. B.; GODOY, L. H.; CONCEIÇÃO, F. T. Avaliação espaço-temporal da qualidade da água e simulação de autodepuração na bacia hidrográfica do córrego São Simão, SP. Ambi-Agua, Taubaté, v. 7, n. 3, p. 141-154, 2012. (http://dx.doi.org/10.4136/ambi-agua.894)

characteristics of the São Simão stream near its spring until it reaches São Simão city allowed concluding that there is low interference in its quality. However, the absence of treatment of domestic wastewater at São Simão city reduced the water quality and increased the degradation process of São Simão stream after passing through the city. The Streeter \& Phelps model allowed identifying the auto-purification zones in this stream and indicated the need for primary wastewater treatment, with an efficiency of $30 \%$.

Keywords: Water quality evaluation; waste water; auto-purification mathematical modeling.

\section{INTRODUÇÃO E OBJETIVO}

Os usos e atividades, irrigação, desmatamento, urbanização, lançamentos pontuais: domésticos e industriais; causam interferências negativas na qualidade e quantidade das águas de um corpo hídrico, consequentemente, a água torna-se indisponível para as necessidades humanas (Souza e Tundisi, 2000; Conceição e Bonotto, 2004; Conceição et al, 2007; Sardinha et al., 2008, 2010; Gonçalves et al., 2009).

Para Pires e Santos (1995), o planejamento e gerenciamento das bacias hidrográficas devem incorporar todos os recursos ambientais da área de drenagem e não apenas o hídrico. Além disso, a abordagem adotada deve integrar os aspectos ambientais, sociais, econômicos e políticos, com ênfase no primeiro, pois a capacidade ambiental de dar suporte ao desenvolvimento possui sempre um limite, a partir do qual todos os outros aspectos serão inevitavelmente afetados. A justificativa para a utilização da bacia hidrográfica como unidade de estudo dá-se pela percepção de que os ecossistemas aquáticos são inteiramente vulneráveis à troca de massa e energia entre si, e principalmente entre os ecossistemas terrestres, sofrendo alterações por consequência da ocupação do solo e atividades neles desenvolvidas (Rocha et al., 2000).

A análise de características físicas, químicas, geológicas e biológicas fornece subsídios relevantes para a caracterização espacial de corpos d'água em bacias hidrográficas. Neste sentido, a análise e interpretação de variáveis de qualidade de água monitoradas no gradiente espaço-temporal, vêm denunciar as condições de uso do solo e as atividades antropogênicas desenvolvidas em uma bacia. Cada bacia hidrográfica deve ter um plano de utilização integrada de recursos hídricos, o qual deve constituir o referencial de todas as decisões e intervenções setoriais nestes recursos (Mota, 1997). O monitoramento da qualidade da água é uma das bases fundamentais de sustentação do gerenciamento integrado (Tundisi, 2005).

No gerenciamento da qualidade da água os modelos matemáticos podem ser utilizados para estimar os focos de poluição existente nas bacias hidrográficas, fornecer estimativas que permitam a tomada de decisões sobre diferentes opções de gerenciamento a longo prazo, e apoiar decisões de gerenciamento a curto prazo. Atualmente há vários estudos que avaliam a qualidade da água e utilizam diversos modelos matemáticos para estimar o déficit da concentração de oxigênio em bacias hidrográficas, dentre eles destacam-se: Gastaldini et al. (2002); USEPA (2004, 2005); Conceição et al. (2007); Sardinha et al. (2008); Gonçalves et al. (2009). Um dos principais modelos matemáticos aplicados à qualidade da água foi desenvolvido por $\mathrm{H}$. S. Streeter e E. B. Phelps em 1925, para o Rio Ohio. Este modelo é utilizado para prever o déficit da concentração de oxigênio em um rio, causado pela descarga de águas residuárias (Von Sperling, 1996).

Atualmente, há grande preocupação em se ter uma abordagem integrada para a gestão de bacias hidrográficas urbanas, pois se tratam de locais que apresentam uma ampla gama de problemas ambientais. Assim, o objetivo deste trabalho consiste na avaliação espaço-temporal da qualidade da água e na simulação de autodepuração na bacia do córrego São Simão, verificando as áreas fortemente degradadas e suas causas, fornecendo subsídios para o seu planejamento e 
GONÇALVES, J. C. S. I.; SARDINHA, D. S.; SOUZA, A. D. G.; DIBIAZI, A. L. B.; GODOY, L. H.; CONCEIÇÃO, F. T. Avaliação espaço-temporal da qualidade da água e simulação de autodepuração na bacia hidrográfica do córrego São Simão, SP. Ambi-Agua, Taubaté, v. 7, n. 3, p. 141-154, 2012. (http://dx.doi.org/10.4136/ambi-agua.894)

gerenciamento, colaborando na busca de soluções para a minimização dos impactos ambientais ocasionados pelas atividades humanas.

\section{MATERIAIS E MÉTODOS}

$\mathrm{Na}$ primeira etapa desse estudo realizou-se a elaboração de um inventário preliminar baseado em pesquisas bibliográficas e em arquivos de órgãos ambientais, técnicos e científicos. A segunda etapa consistiu na caracterização ambiental, com a finalidade de espacializar as informações resultantes da bacia hidrográfica do córrego São Simão. Assim, foram confeccionadas cartas descritivas e interpretativas em meio digital, obtido a partir de fotografias aéreas, imagens de satélite e documentos cartográficos existentes, além de controle em campo utilizando GPS (Global Positioning System). Cartas temáticas foram produzidas através de digitalização em mesa e em tela com posterior edição, usando a seguinte base de dados: cartas 1:50.000 do Instituto Brasileiro de Geografia e Estatística, folhas Cravinhos (IBGE, 1982) e Luis Antônio (IBGE, 1971); cartas 1:100.000 do Instituto Agronômico de Campinas, mapeamento geológico morfoestrutural em parte das folhas de Ribeirão Preto e Descalvado (IAC, 1982); mapas de uso e ocupação do solo (Biota/Fapesp, 2005).

A terceira etapa consistiu na avaliação da qualidade das águas superficiais, onde foram realizadas doze coletas por todo o curso do córrego São Simão (24/03/05 - 07/05/05 - 11/06/05 - 09/07/05 - 09/08/05 - 20/09/05 - 14/10/05 - 27/11/05 - 21/12/05 - 30/01/06 - 28/02/06 31/03/06). A seguinte convenção para os pontos de coleta (Figura 1) será utilizada durante o desenvolvimento deste trabalho: P1 (montante da área urbana); P2 (início da área urbana); P3 (meio da área urbana); P4 (final da área urbana); P5 (jusante da área urbana); e P6 (exutório).

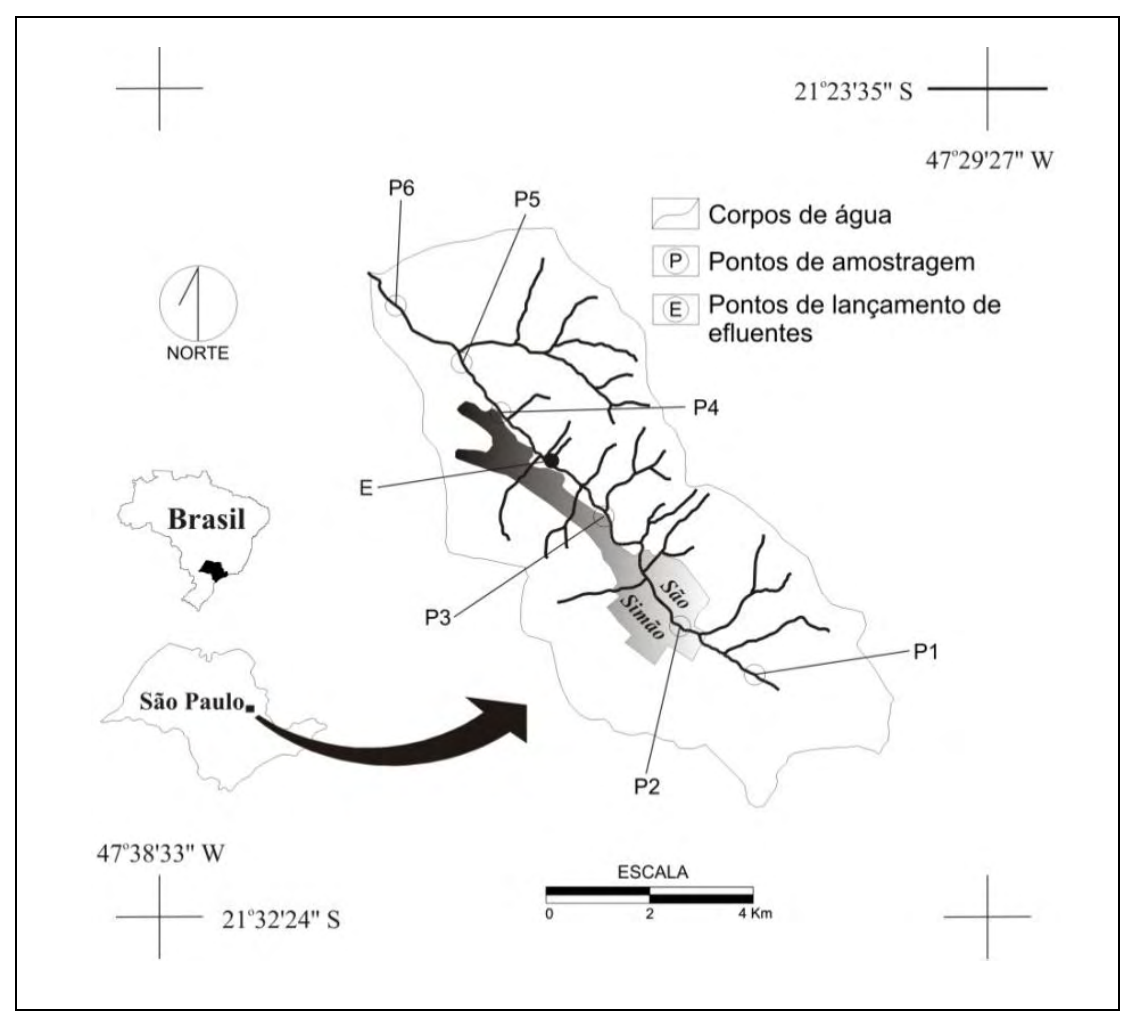

Figura 1. Localização da bacia do córrego São Simão e pontos de amostragem. 
GONÇALVES, J. C. S. I.; SARDINHA, D. S.; SOUZA, A. D. G.; DIBIAZI, A. L. B.; GODOY, L. H.; CONCEIÇÃO, F. T. Avaliação espaço-temporal da qualidade da água e simulação de autodepuração na bacia hidrográfica do córrego São Simão, SP. Ambi-Agua, Taubaté, v. 7, n. 3, p. 141-154, 2012. (http://dx.doi.org/10.4136/ambi-agua.894)

A vazão no ponto de amostragem (P3) foi medida de acordo com a metodologia descrita por (Hermes e Silva, 2004), utilizando-se uma trena, um objeto flutuador e um cronômetro, sendo as variáveis colocadas posteriormente na Equação 1:

$$
Q=\frac{A x D x C}{T}
$$

em que:

$Q=$ vazão $\left(\mathrm{m}^{3} \cdot \mathrm{s}^{-1}\right) ; A=$ área da seção transversal do rio $\left(\mathrm{m}^{2}\right) ; D=$ distância usada para medir a velocidade do escoamento $(\mathrm{m}) ; C=$ coeficiente de correção $(0,8$ para rios com fundo rochoso e 0,9 para rios com fundo lodoso) e $T=$ tempo (s) gasto pelo objeto flutuador para atravessar a distância $D$.

Todas as amostras de águas fluviais foram coletadas no centro da seção transversal, a cerca de $30 \mathrm{~cm}$ de profundidade, com balde previamente lavado com a própria água a ser coletada. Amostras foram armazenadas em vasilhames de polietileno, sendo analisadas as seguintes variáveis físico-químicas: temperatura $\left({ }^{\circ} \mathrm{C}\right), \mathrm{pH}$, oxigênio dissolvido - $\mathrm{OD}\left(\mathrm{mg} \cdot \mathrm{L}^{1-}\right)$, turbidez (NTU), condutividade $\left(\mu \mathrm{S} . \mathrm{cm}^{-1}\right)$, sólidos totais em suspensão - STS (mg. $\mathrm{L}^{-1}$ ), alcalinidade $\mathrm{HCO}_{3}{ }^{-}\left(\mathrm{mg} . \mathrm{L}^{-1}\right)$ e cloreto $\mathrm{Cl}^{-}\left(\mathrm{mg} . \mathrm{L}^{-1}\right)$. As leituras de temperatura, $\mathrm{pH}$, condutividade e oxigênio dissolvido, foram realizadas "in situ" através de métodos ponteciométricos (analisador multiparâmetros Vernier). A variável turbidez foi mensurada com turbidímetro da Vernier (0 a $200 \pm 2 \mathrm{NTU}$ ), assim como cloreto (de 0,01 a $100 \pm 0,02 \mathrm{mg} / \mathrm{L}$ ). Os sólidos totais em suspensão foram quantificados pela metodologia gravimétrica (Standard Methods, 1999). A alcalinidade foi determinada pela titulação com ácido sulfúrico $0,01 \mathrm{~N}$ com concentração entre 1 e $500 \pm 0,2$ mg/L (APHA, 1999).

A modelagem de autodepuração com o uso do modelo de Streeter \& Phelps foi desenvolvida a partir da Equação 2:

$$
\frac{d D}{d t}=K_{1} \cdot L-K_{2} \cdot(D)
$$

em que:

$D=$ déficit de oxigênio dissolvido $\left(\mathrm{mg} . \mathrm{L}^{-1}\right) ; t=$ tempo (dia); $K_{l}=$ coeficiente de desoxigenação $\left(\right.$ dia $\left.^{-1}\right) ; L=$ concentração de DBO remanescente no rio $\left(\mathrm{mg} . \mathrm{L}^{-1}\right) ; K_{2}=$ coeficiente de reaeração $\left(\operatorname{dia}^{-1}\right)$.

A solução analítica da Equação 2 é desenvolvida através da Equação 3:

$$
C(t)=C_{s}-\left[\frac{K_{1} \cdot L_{0}}{K_{2}-K_{1}} \cdot\left(e^{-K_{1} \cdot t}-e^{-K_{2} \cdot t}\right)+\left(C_{s}-C_{0}\right) \cdot e^{-K_{2} \cdot t}\right]
$$

Com uso das equações (4), (5) e (6):

$$
\begin{aligned}
C_{0} & =\frac{Q r \cdot O D r+Q e \cdot O D e}{Q r+Q e} \\
L_{0} & =\left(\frac{Q_{r} \cdot D B O_{r}+Q_{e} \cdot D B O_{e}}{Q_{r}+Q_{e}}\right) \cdot \frac{1}{1-e^{-5 \cdot K_{1}}} \\
C_{s} & =\left(1-\frac{H}{9450}\right) \cdot 14,652-4,1022 \cdot T+7,9910 \times 10^{-3} \cdot T^{2}-7,7774 \times 10^{-5} \cdot T^{3}
\end{aligned}
$$


GONÇALVES, J. C. S. I.; SARDINHA, D. S.; SOUZA, A. D. G.; DIBIAZI, A. L. B.; GODOY, L. H.; CONCEIÇÃO, F. T. Avaliação espaço-temporal da qualidade da água e simulação de autodepuração na bacia hidrográfica do córrego São Simão, SP. Ambi-Agua, Taubaté, v. 7, n. 3, p. 141-154, 2012. (http://dx.doi.org/10.4136/ambi-agua.894)

em que:

$C=$ concentração de oxigênio dissolvido $\left(\mathrm{mg} \cdot \mathrm{L}^{-1}\right) ; C s=$ concentração de saturação do oxigênio dissolvido $\left(\mathrm{mg} . \mathrm{L}^{-1}\right) ; H=$ altitude $(\mathrm{m}) ; T=$ temperatura da água $\left({ }^{\circ} \mathrm{C}\right) ; C_{0}=$ concentração inicial de oxigênio logo após a mistura $\left(\mathrm{mg}^{\mathrm{L}} \mathrm{L}^{-1}\right) ; L_{0}=$ demanda última de oxigênio logo após a mistura $\left(\mathrm{mg} . \mathrm{L}^{-1}\right) ; Q r=$ vazão do córrego São Simão $\left(\mathrm{m}^{3} \cdot \mathrm{s}^{-1}\right) ; Q e=$ vazão dos efluentes domésticos $\left(\mathrm{m}^{3} \cdot \mathrm{s}^{-1}\right) ; O D r=$ concentração de oxigênio dissolvido no córrego São Simão (mg/L); $O D e=$ concentração de oxigênio dissolvido nos efluentes domésticos (mg. $\left.\mathrm{L}^{-1}\right)$; $D B O r=$ concentração de $\mathrm{DBO}_{5}$ no córrego São Simão $\left(\mathrm{mg} . \mathrm{L}^{-1}\right) ; \mathrm{DBOe}=$ concentração de $\mathrm{DBO}_{5}$ no efluente doméstico (mg. $\left.\mathrm{L}^{-1}\right)$.

No modelo de Streeter \& Phelps, o valor de degradação/oxidação da matéria orgânica ou coeficiente de desoxigenação $\left(K_{l}\right)$ é representado por uma taxa que pode variar de 0,08 a 0,45 $\left(\mathrm{dia}^{-1}\right)$ (Von Sperling, 1996), sendo utilizado neste trabalho a taxa de 0,40 ( $\mathrm{dia}^{-1}$ ) para o período seco e para o período chuvoso. O valor indicador da capacidade de autodepuração ou coeficiente de reaeração $\left(K_{2}\right)$ foi utilizado como parâmetro de calibração do modelo, uma vez que as equações empíricas e semi-empíricas usadas para a determinação deste coeficiente, as quais incluem a profundidade e a velocidade do escoamento, produzem resultados divergentes. Considerou-se a população atual de 14.406 habitantes para a cidade de São Simão, a vazão de esgoto doméstico bruto gerado na zona urbana de 2.786,62 $\mathrm{m}^{3} \cdot$ dia $^{-1}$ e a carga orgânica de 696,65 $\left(\mathrm{kg} . \mathrm{DBO}_{5}{ }^{-1} \cdot \mathrm{dia}^{-1}\right)$ (IPT, 2006).

$\mathrm{O} K_{2}$ e o $K_{1}$ são influenciados pela temperatura da água. A correção do valor destes coeficientes é descrita pela Equação 7 . Vale salientar que a temperatura de $20^{\circ} \mathrm{C}$ é utilizada como referência.

$$
K=K(20) \Theta^{(T-20)}
$$

em que: $\mathrm{K}=$ constante cinética da reação à temperatura $\mathrm{T}\left(\mathrm{dia}^{-1}\right) ; \mathrm{K}(20)=$ constante cinética da reação à temperatura de $20^{\circ} \mathrm{C} ; \Theta=$ coeficiente de temperatura para a reação (adimensional). Para $\mathrm{K}_{1}, \Theta$ é igual a 1,047 ; já para $\mathrm{K}_{2}, \Theta$ é igual a 1,024 .

\section{RESULTADOS E DISCUSSÕES}

\subsection{Características da área de estudo}

O córrego São Simão (Figura 1), situa-se entre os paralelos $21^{\circ} 23$ '35' ' e 21 32 '24', de latitude Sul e $47^{\circ} 29^{\prime} 27^{\prime \prime}$ e $47^{\circ} 38^{\prime} 33^{\prime}$ ' de longitude W, na região nordeste do Estado de São Paulo, fazendo parte da Unidade de Gerenciamento de Recursos Hídricos do Pardo (UGRHI 04). Sua nascente está localizada a sudeste do município de São Simão (SP) a uma altitude aproximada de $669 \mathrm{~m}$, e sua foz no Rio Tamanduá, $13 \mathrm{~km}$ a noroeste deste município com altitude aproximada de $575 \mathrm{~m}$.

O clima da região da bacia do córrego São Simão é o do tipo Cwa, segundo o sistema Koppen, ou seja, mesotérmico de verão chuvoso e inverno seco. O verão ocorre entre os meses de outubro a março, sendo intensificado entre dezembro e fevereiro, e o inverno entre os meses de maio a setembro, com temperatura variando entre $7^{\circ} \mathrm{C}$ e $30^{\circ} \mathrm{C}$, com média anual de $19^{\circ} \mathrm{C}$ (IPT, 2006). A precipitação pluviométrica média anual entre os anos de 1950 e 2004 foi de 1420,2 $\mathrm{mm}$, sendo janeiro o mês mais chuvoso (média de $287 \mathrm{~mm}$ ) e agosto o mês mais seco (média de $23 \mathrm{~mm}$ ). Em relação às vazões no córrego São Simão, os meses com maior e menor vazão durante o período de amostragem foram março com $0,68 \mathrm{~m}^{3} . \mathrm{s}^{-1}$ e agosto com $0,32 \mathrm{~m}^{3} . \mathrm{s}^{-1}$, respectivamente. A Figura 2 apresenta a precipitação média mensal (a) e a vazão medida durante o período de amostragem (b), com as quais foram definidos os meses de maio, junho, julho, agosto, setembro e outubro como de período seco, e novembro, dezembro, janeiro, fevereiro, março e abril como de período chuvoso. 


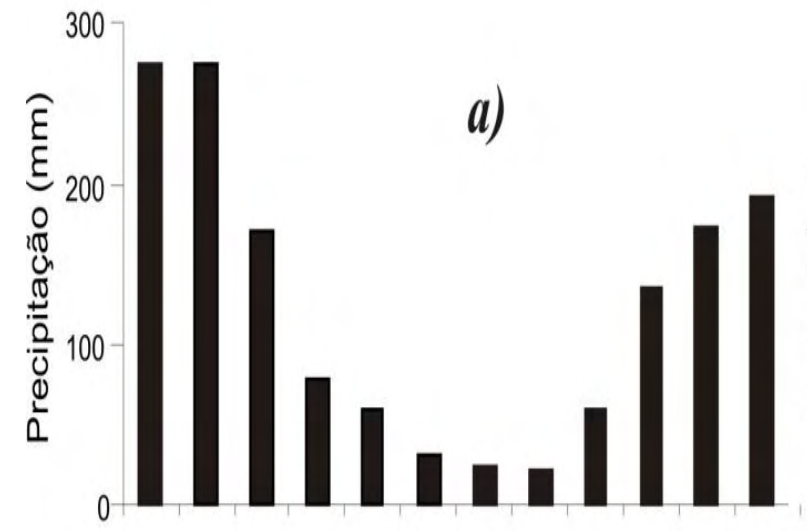

jan fev mar abr mai junh julh ago set out nov dez

\section{Meses}

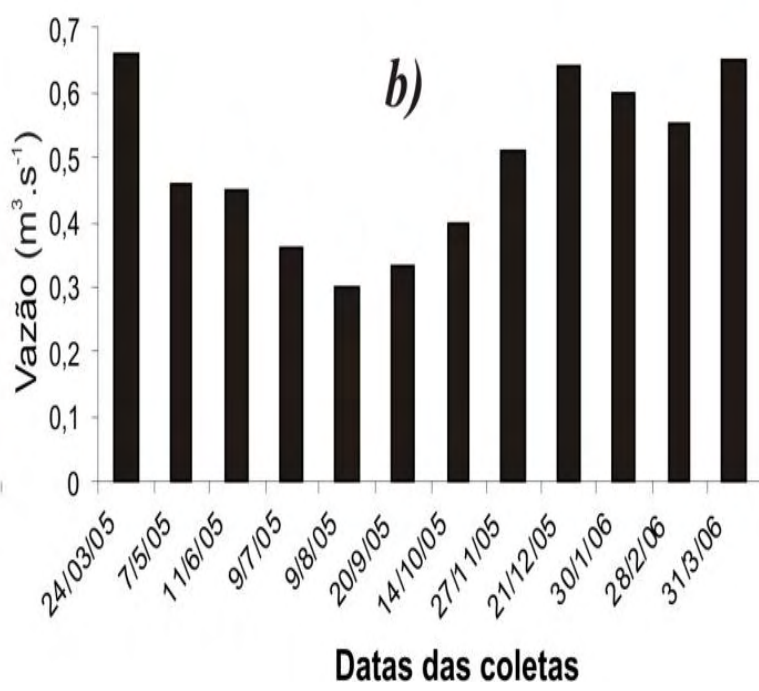

Datas das coletas

Figura 2. (a) Precipitação média mensal (mm) entre os anos de 1950 e 2004 (IPT, 2006). (b) e vazão $\left(\mathrm{m}^{3} \cdot \mathrm{s}^{-1}\right)$ medida no ponto $(\mathrm{P} 3)$.

A vegetação na bacia caracteriza-se pelas matas mesófilas, de formação caracteristicamente descontínua, que ocorre em condições variadas de clima e solo, tendo caráter semicaducifólio, em decorrência de invernos frios e verões quentes e úmidos. Nas áreas mais bem drenadas próximas à calha dos rios, predominam as Matas Ciliares, restritas a uma estreita faixa descontínua às margens do curso do córrego São Simão (Figura 3a). A economia do Município é voltada principalmente para a atividade rural como agrária, avícola e pecuária, dentre as principais se destacam, a cana-de-açúcar, pinos e eucaliptos, gado de corte e granjas.

Em relação à geomorfologia, São Simão encontra-se em sua maior parte na província das Cuestas Basálticas, conforme definida por Almeida (1964), como sendo esta uma das mais marcantes feições da paisagem paulista com relevo de colinas e morros amplos ou arredondados. $\mathrm{Na}$ área de estudo são encontrados, predominantemente na Formação Serra Geral, o sistema de colinas amplas que apresentam, respectivamente, interflúvios de topos extensos e aplainados com vertentes retilíneas ou convexas, vales abertos e planícies inferiores restritas (Sinelli, 1970).

A bacia do córrego São Simão encontra-se em uma região composta por rochas ígneas e sedimentares pertencentes ao Grupo São Bento (Figura 3b). A Formação Pirambóia apresenta arenitos constituídos basicamente por granulação média a fina com matriz silto-argilosa e estratificação cruzada planar de pequeno a médio porte (Soares e Landim, 1974). Já a Formação Botucatu registra uma sequência de eventos de um ciclo desértico que ocorreu durante o Mesozóico (Schneider et al., 1974). Dominou neste ciclo a sedimentação eólica de areias com granulação fina e muito fina, produzindo corpos de arenitos com boa seleção em grandes cunhas, com estratificação cruzada planar tangencial. O contato entre as formações Botucatu e a Serra Geral faz-se por interdigitação, recobrindo-se os arenitos pelos derrames basálticos (IPT, 1981). A Formação Serra Geral apresenta toda uma completa suíte de tipos litológicos, desde basaltos até riolítos que correspondem a um vulcanismo Mesozóico de bacia intracratônica (Schneider et al., 1974). Dos solos que recobrem a área, os dominantes são os Neossolos Quartzarênicos e Latossolos Roxo, ocorrendo também Latossolos Vermelho-Escuro, Latossolos VermelhoAmarelo e Neossolos Líticos (IAC, 1982). 
GONÇALVES, J. C. S. I.; SARDINHA, D. S.; SOUZA, A. D. G.; DIBIAZI, A. L. B.; GODOY, L. H.; CONCEIÇÃO, F. T. Avaliação espaço-temporal da qualidade da água e simulação de autodepuração na bacia hidrográfica do córrego São Simão, SP. Ambi-Agua, Taubaté, v. 7, n. 3, p. 141-154, 2012. (http://dx.doi.org/10.4136/ambi-agua.894)

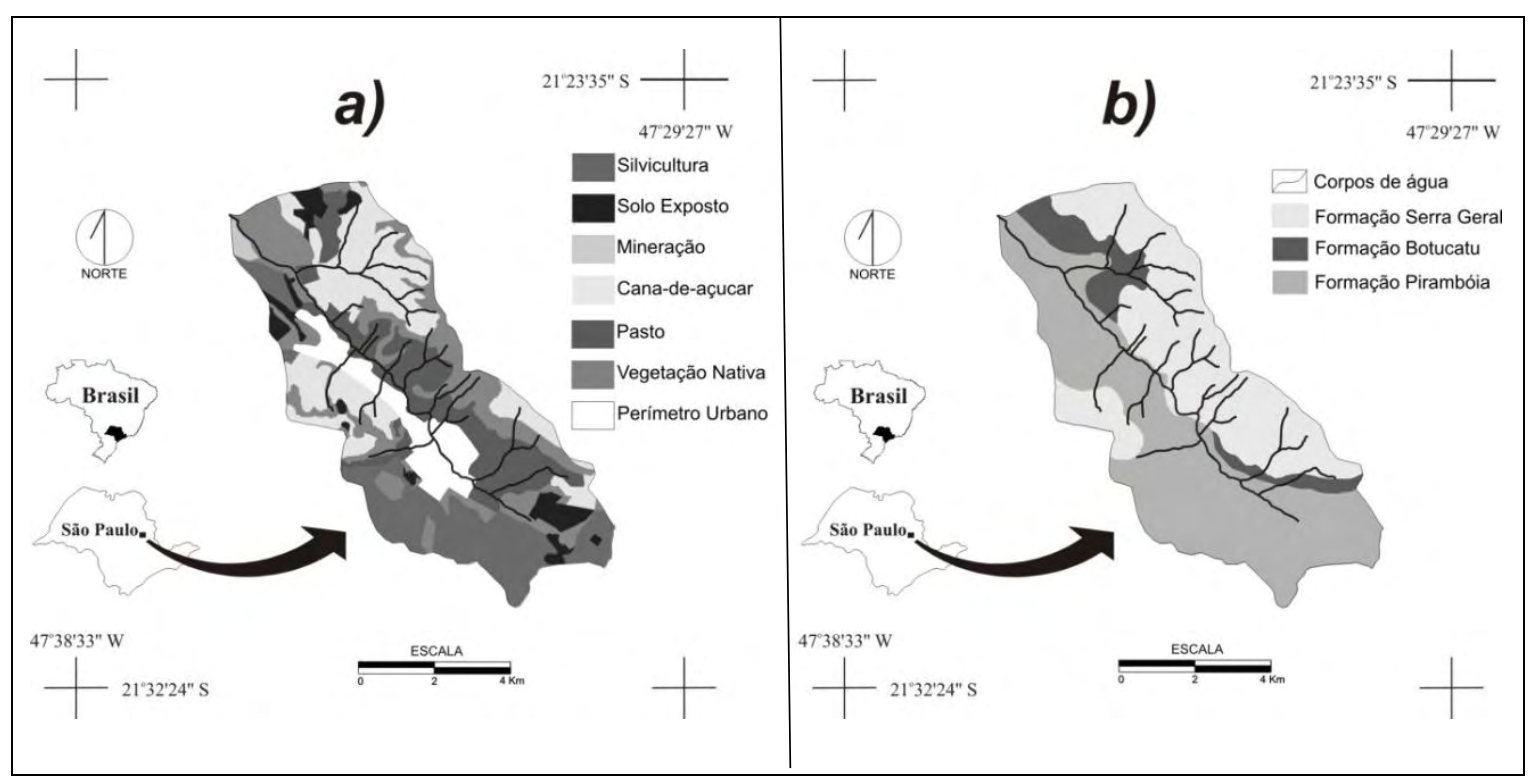

Figura 3. (a) Mapa de uso e ocupação do solo da bacia do córrego São Simão. (b) Mapa geológico da bacia do córrego São Simão.

\subsection{Avaliação espaço-temporal da qualidade da água}

Na Tabela 1 constam os resultados médios obtidos para os parâmetros determinados nas águas fluviais da bacia do córrego São Simão durante o período seco e o período chuvoso. Conforme esperado, a vazão medida no ponto de amostragem (P3) é mais elevada no período chuvoso do que no período seco.

Tabela 1. Média dos parâmetros analisados na bacia do córrego São Simão durante o período seco e chuvoso. P1 (montante da área urbana); P2 (início da área urbana); P3 (meio da área urbana); P4 (final da área urbana); P5 (jusante da área urbana); e P6 (exutório).

\begin{tabular}{|c|c|c|c|c|c|c|c|c|c|}
\hline \multirow[t]{2}{*}{$\begin{array}{l}\text { Ponto de } \\
\text { coleta }\end{array}$} & $\begin{array}{c}\text { Vazão } \\
\left(\mathbf{m}^{3} \cdot \mathbf{s}^{-1}\right)\end{array}$ & $\begin{array}{l}\text { Cond. } \\
\left(\mu \mathrm{S} . \mathrm{cm}^{-1}\right)\end{array}$ & $\begin{array}{c}\text { Temp. } \\
\left({ }^{\circ} \mathrm{C}\right)\end{array}$ & pH & $\begin{array}{c}\text { Turbidez } \\
\text { (NTU) }\end{array}$ & OD & $\begin{array}{l}\mathrm{Cl}^{-} \mathbf{~} \\
(\mathrm{mg} .\end{array}$ & $\begin{array}{l}\mathrm{CO}_{3}^{-} \\
-1)\end{array}$ & STS \\
\hline & \multicolumn{9}{|c|}{ Média do período seco } \\
\hline $\mathrm{P} 1$ & \multirow{6}{*}{0,39} & 22,6 & 20,9 & 4,8 & 1,5 & 6,2 & 0,6 & 1,7 & 4,7 \\
\hline $\mathrm{P} 2$ & & 17,7 & 22,8 & 6,2 & 3,7 & 6,6 & 1,6 & 4,3 & 9,5 \\
\hline P3 & & 37,5 & 23,3 & 6,7 & 5,7 & 6,1 & 3,6 & 9,4 & 5,0 \\
\hline P4 & & 231,1 & 23,4 & 6,4 & 13,5 & 3,3 & 32,7 & 25,4 & 28,7 \\
\hline P5 & & 213,3 & 23,8 & 6,7 & 11,2 & 2,0 & 23,7 & 33,1 & 26,8 \\
\hline \multirow[t]{2}{*}{ P6 } & & 160,7 & 23,3 & 6,5 & 10,0 & 1,7 & 16,4 & 31,6 & 25,2 \\
\hline & \multicolumn{9}{|c|}{ Média do período chuvoso } \\
\hline P1 & \multirow{6}{*}{0,61} & 17,3 & 23,8 & 5,3 & 1,8 & 5,4 & 0,7 & 3,2 & 4,7 \\
\hline P2 & & 24,3 & 25,5 & 6,5 & 15,5 & 5,3 & 1,7 & 9,9 & 14,2 \\
\hline P3 & & 42,9 & 25,4 & 7,1 & 14,7 & 5,3 & 2,0 & 16,2 & 12,1 \\
\hline P4 & & 234,4 & 25,3 & 6,7 & 29,3 & 3,8 & 20,4 & 33,1 & 35,0 \\
\hline P5 & & 166,3 & 25,0 & 6,6 & 22,8 & 3,0 & 13,9 & 36,5 & 26,3 \\
\hline P6 & & 119,1 & 24,3 & 6,8 & 27,8 & 2,7 & 5,9 & 32,8 & 24,8 \\
\hline
\end{tabular}


Os menores valores de condutividade elétrica foram obtidos nos pontos P1 e P2, enquanto que os maiores foram caracterizados sempre no ponto P4 logo após a cidade de São Simão (media anual de 232,7 $\mu \mathrm{S} . \mathrm{cm}^{-1}$ - Figura 4a), valor acima do limite superior esperado para águas naturais, isto é, $100 \mu \mathrm{S} . \mathrm{cm}^{-1}$ (Hermes e Silva, 2004). Apesar de que o intemperismo pode elevar a condutividade, acredita-se que os valores mais acentuados devem ser atribuídos principalmente a descargas de efluentes da cidade de São Simão e às atividades agrícolas realizadas na bacia. No ponto P6, os valores de condutividade já se apresentam menores, no entanto, ainda continuam acima do limite superior permitido.

Dentre os pontos estudados, o valor máximo de temperatura da água foi registrado no ponto (P3) no dia $28 / 02 / 2006\left(29^{\circ} \mathrm{C}\right)$, sendo o menor valor obtido no ponto P1 $\left(19^{\circ} \mathrm{C}\right)$ no dia 20/09/2005. Assim como a condutividade, os menores valores médios anuais de temperatura foram encontrados no ponto P1 (Figura 4b), provavelmente devido à vegetação nativa (Figura 3a) que se encontra na nascente do córrego São Simão. Em geral, há uma variação sazonal de temperatura para as águas fluviais da bacia do córrego São Simão, de aproximadamente $10^{\circ} \mathrm{C}$ entre o verão e o inverno.
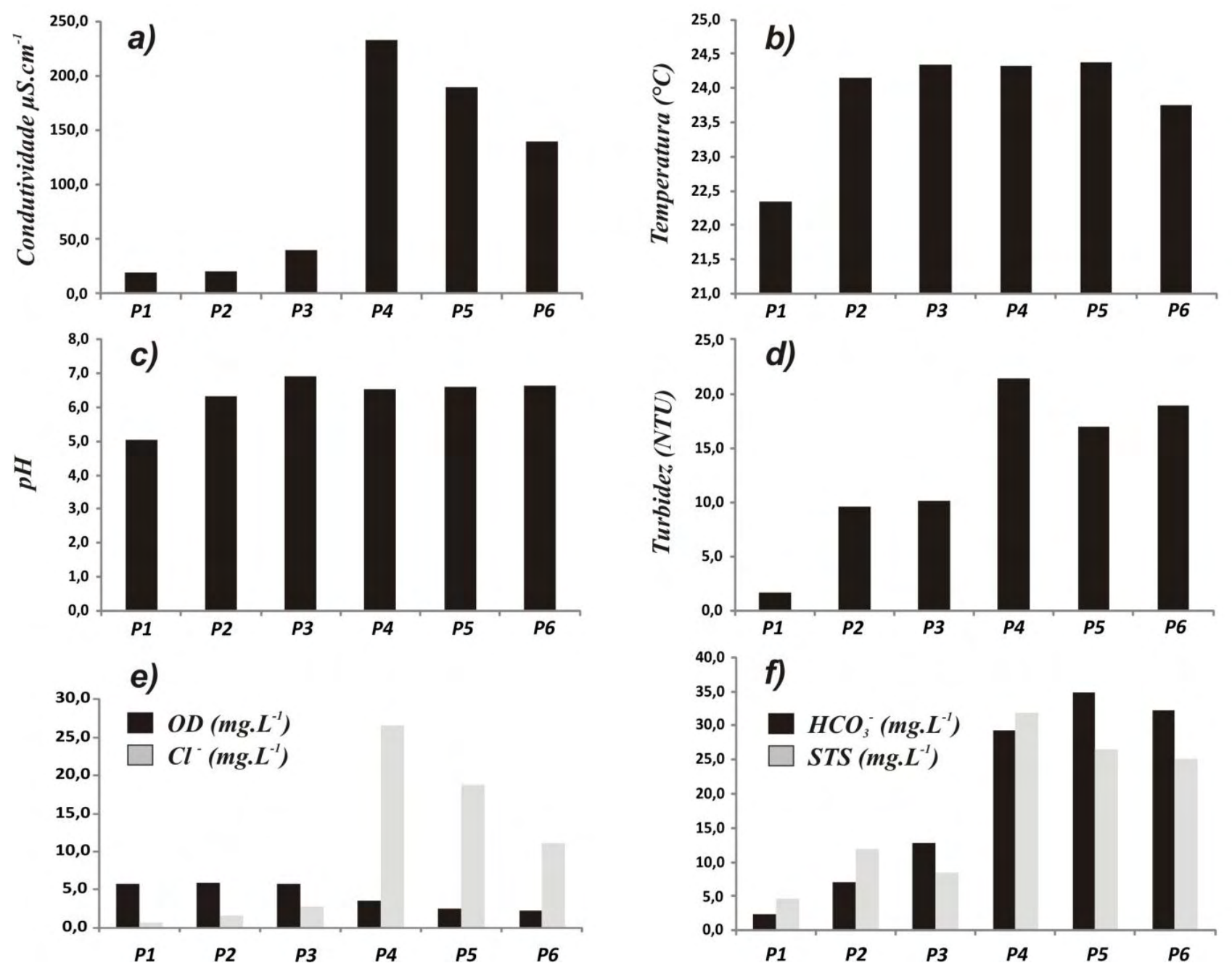

Figura 4. Média dos parâmetros analisados na bacia do córrego São Simão durante todo o período em estudo $(24 / 03 / 2005$ à $31 / 03 / 2006)$. (a) Condutividade $\left(\mu \mathrm{S} . \mathrm{cm}^{-1}\right)$. (b) Temperatura $\left({ }^{\circ} \mathrm{C}\right)$. (c) pH. (d) Turbidez (NTU). (e) Oxigênio dissolvido e cloreto $\left(\mathrm{mg} . \mathrm{L}^{-1}\right)$. (f) Alcalinidade e sólidos totais em suspensão $\left(\mathrm{mg} \cdot \mathrm{L}^{-1}\right)$. 
GONÇALVES, J. C. S. I.; SARDINHA, D. S.; SOUZA, A. D. G.; DIBIAZI, A. L. B.; GODOY, L. H.; CONCEIÇÃO, F. T. Avaliação espaço-temporal da qualidade da água e simulação de autodepuração na bacia hidrográfica do córrego São Simão, SP. Ambi-Agua, Taubaté, v. 7, n. 3, p. 141-154, 2012. (http://dx.doi.org/10.4136/ambi-agua.894)

$\mathrm{O}$ pH é governado pelo equilíbrio do dióxido de carbono-bicarbonato e carbonatos. $\mathrm{Na}$ bacia, os valores obtidos na maioria dos pontos de amostragem indicam que as águas são geralmente neutras, encontrando-se dentro da faixa de proteção da vida aquática, segundo a Legislação Federal (Resolução CONAMA n ${ }^{\circ}$ 357/05) para rios de Classe 4, conforme são enquadrados os rios da bacia do córrego São Simão. Apenas o ponto (P1) encontraram-se valores de $\mathrm{pH}$ abaixo da Legislação (média anual de 5,1 - Figura 4c).

Os valores de turbidez registraram um aumento ao longo do curso principal do córrego São Simão (Figura 4d). No período chuvoso, os valores de turbidez aumentam, em todos os pontos amostrados, em relação ao período seco (variação máxima de 17,8 NTU). O uso do solo (pasto, silvicultura, solo exposto e cana de açúcar), as características geológicas da bacia (principais tributários localizados em rochas da Formação Serra Geral, que conferem um desnível em relação às Rochas areníticas da Formação Botucatu e Pirambóia, onde se encontra a calha principal do córrego São Simão), além da entrada de efluentes ao passar pela área urbana de São Simão, podem estar contribuindo para o aumento nos valores de turbidez.

A atmosfera e a fotossíntese são as principais fontes de oxigênio para as águas fluviais. Por outro lado, as perdas de oxigênio se devem ao consumo pela decomposição da matéria orgânica, trocas para a atmosfera, respiração de organismos aquáticos e oxidação de íons metálicos (Esteves, 1998). Como pode ser observado na Tabela 1, há uma variação dos valores de oxigênio dissolvido em todos os pontos de amostragem em relação à época de chuva e a época seca. Em geral, os maiores valores de oxigênio dissolvido foram obtidos nos pontos P1, P2 e P3, e são mais elevados no período seco do que no período chuvoso. Fato que pode ser atribuído ao aumento da temperatura da água na época de chuva (verão), ocasionando menor solubilidade de gases (oxigênio dissolvido) na água. O inverso ocorre nos pontos P4, P5 e P6, onde há o aumento da vazão na época de chuva e, consequentemente, maior capacidade de diluição dos efluentes (maiores concentrações de OD).

A presença de cloreto nas águas naturais se deve à alteração das rochas, dentre outros fatores (CETESB, 2005). A bacia do córrego São Simão não possui rochas que tenham cloreto como constituinte principal de seus minerais, neste sentido a entrada natural deste elemento pode ser descartada. As concentrações de cloreto aumentam ao longo do curso do córrego São Simão, principalmente após a passagem pela área urbana do município, maiores concentrações no ponto P4 (Figura 4e). No período seco as concentrações de cloreto são maiores que no período chuvoso (variação máxima de $12,3 \mathrm{mg} . \mathrm{L}^{-1}$ ), provavelmente devido ao menor efeito de diluição no período chuvoso.

Na maioria das coletas, os valores de sólidos totais em suspensão (STS) aumentam ao longo do córrego São Simão. Os maiores e menores valores de STS sempre foram encontrados nos pontos P4 e P1, respectivamente. Os valores de STS aumentam na época chuvosa (média de 19,51 mg. $\mathrm{L}^{-1}$ ) comparado com a época de seca (média de $16,65 \mathrm{mg} . \mathrm{L}^{-1}$ ), mesmo comportamento da vazão, indicando uma maior descarga durante esta época, em acordo com a maioria dos rios mundiais (Berner e Berner, 1996; Conceição e Bonotto, 2004; Conceição et al., 2007). O aumento dos valores de STS na época chuvosa pode ser atribuído ao maior arraste de sedimentos pela erosão laminar que é muito acentuada neste período.

O mesmo comportamento para sólidos totais em suspensão (STS) foi observado para as amostras de alcalinidade $\left(\mathrm{HCO}_{3}{ }^{-}\right)$. A alcalinidade decorre da presença de carbonatos, bicarbonatos e hidróxidos, quase sempre alcalinos ou alcalino-terrosos (sódio, potássio, cálcio, magnésio, etc.) (Wetzel, 1983). A atuação dos processos de intemperismo nos minerais formadores das rochas da bacia, e consequente erosão laminar no período chuvoso, pode estar influenciando as concentrações de $\mathrm{HCO}_{3}{ }^{-}$nas águas superficiais da bacia. Em geral, $\mathrm{H}^{+}, \mathrm{Na}^{+} \mathrm{e}$ $\mathrm{Ca}^{2+}$ podem ser liberados pela hidrólise da albita $\left(2 \mathrm{NaAlSi}_{3} \mathrm{O}_{8}+9 \mathrm{H}_{2} \mathrm{O}+2 \mathrm{H}^{+} \rightarrow \mathrm{Al}_{2} \mathrm{Si}_{2} \mathrm{O}_{5}(\mathrm{OH})_{4}\right.$ $\left.+2 \mathrm{Na}^{+}+4 \mathrm{H}_{4} \mathrm{SiO}_{4}\right)$ e anortita $\left(\mathrm{CaAl}_{2} \mathrm{Si}_{2} \mathrm{O}_{8}+\mathrm{H}_{2} \mathrm{O}+2 \mathrm{H}^{+} \rightarrow \mathrm{Al}_{2} \mathrm{Si}_{2} \mathrm{O}_{5}(\mathrm{OH})_{4}+\mathrm{Ca}^{+}\right), \mathrm{H}^{+}$e $\mathrm{K}^{+}$pela 
GONÇALVES, J. C. S. I.; SARDINHA, D. S.; SOUZA, A. D. G.; DIBIAZI, A. L. B.; GODOY, L. H.; CONCEIÇÃO, F. T. Avaliação espaço-temporal da qualidade da água e simulação de autodepuração na bacia hidrográfica do córrego São Simão, SP. Ambi-Agua, Taubaté, v. 7, n. 3, p. 141-154, 2012. (http://dx.doi.org/10.4136/ambi-agua.894)

hidrólise de microclínio $\left(2 \mathrm{KAlSi}_{3} \mathrm{O}_{8}+9 \mathrm{H}_{2} \mathrm{O}+2 \mathrm{H}^{+} \rightarrow \mathrm{Al}_{2} \mathrm{Si}_{2} \mathrm{O}_{5}(\mathrm{OH})_{4}+2 \mathrm{~K}^{+}+4 \mathrm{H}_{4} \mathrm{SiO}_{4}\right)$. Os efluentes gerados pelo município de São Simão também podem estar influenciando nas concentrações deste parâmetro.

\subsection{Autodepuração no Córrego São Simão}

Como se pode observar na Figura 5, o modelo de Streeter \& Phelps de autodepuração mostrou-se bastante acurado neste estudo. Os dados relativos ao período chuvoso, maior volume de água e velocidade de escoamento no córrego São Simão (Figura 5), demonstram um maior prolongamento na curva de autodepuração, bem diferente ao observado para o período seco. As discussões, referentes à aplicação do modelo Streeter \& Phelps neste trabalho, são feitas para o período crítico ou período seco (menor vazão e menor velocidade de escoamento), onde foi possível delimitar as diferentes zonas de autodepuração que envolve o córrego São Simão nesta época do ano (Figura 5), ou seja, a zona de águas limpas, de degradação, de decomposição ativa e de recuperação.

Os valores de $K_{2}$ obtidos pela calibração do modelo, para o período seco e chuvoso, são de 1,0 e $0,8 \mathrm{dia}^{-1}$, respectivamente.

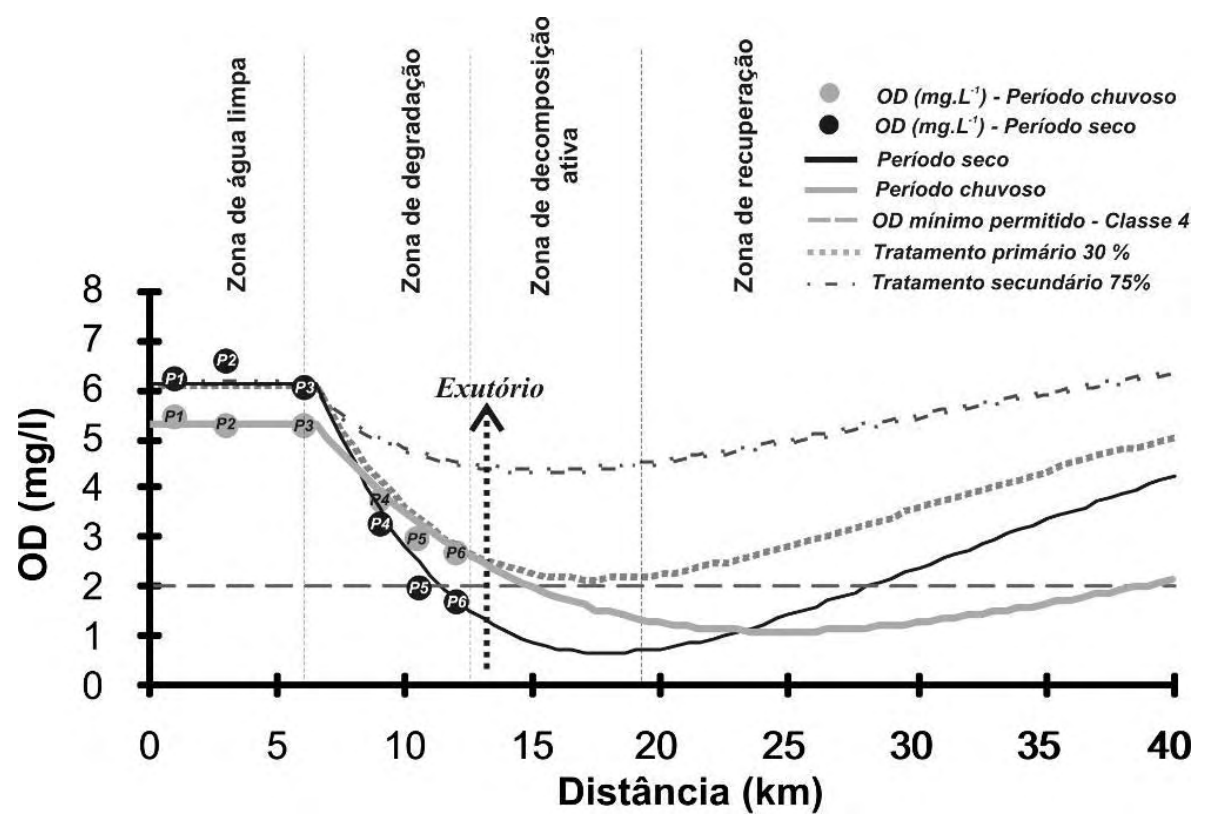

Figura 5. Modelagem de autodepuração no córrego São Simão para o período chuvoso, nas condições atuais, e para o período seco (crítico) nas condições atuais, com tratamento de esgotos primário e secundário com eficiência de $35 \%$ e $75 \%$ respectivamente.

O córrego São Simão, antes da cidade de São Simão, onde se localiza o ponto P1, está situado na zona de águas limpas, onde seu ecossistema encontra-se em estado de equilíbrio e com melhor qualidade da água. A região da nascente do córrego São Simão localiza-se em uma das poucas áreas de vegetação nativa (matas mesófilas - Figura 3a). Conforme suas águas escoam no sentido da área urbana da cidade de São Simão, observa-se uma transição de vegetação nativa para vegetação rasteira, devido à introdução de pastos (pecuária - Figura 3a), proporcionando um aumento da quantidade de areia em seu leito. Os pontos P2 e P3, início e centro da área urbana do município de São Simão, respectivamente, também estão situados na zona de águas limpas, as características da nascente, o desnível topográfico e a ausência de fontes pontuais de efluentes, mantém a boa oxigenação das águas nestes pontos. 
GONÇALVES, J. C. S. I.; SARDINHA, D. S.; SOUZA, A. D. G.; DIBIAZI, A. L. B.; GODOY, L. H.; CONCEIÇÃO, F. T. Avaliação espaço-temporal da qualidade da água e simulação de autodepuração na bacia hidrográfica do córrego São Simão, SP. Ambi-Agua, Taubaté, v. 7, n. 3, p. 141-154, 2012. (http://dx.doi.org/10.4136/ambi-agua.894)

A cidade de São Simão possui 14.406 habitantes e utiliza água para abastecimento de sua população através de captação subterrânea (86\%) e superficial no córrego São Simão (16\%), atendendo $97,5 \%$ da população municipal. A rede de coleta de esgotos atende 95,0\% da população, onde são lançados in natura ao longo do córrego São Simão (vazão de esgoto doméstico de 2,786, $62 \mathrm{~m}^{3} \cdot \mathrm{dia}^{-1}$ ), alterando a qualidade de água, começando aí à zona de degradação (carga orgânica de $696,65 \mathrm{~kg} \mathrm{DBO}_{5}{ }^{-1} \cdot \operatorname{dia}^{-1}$ ), onde o equilíbrio do ecossistema aquático é afetado pela alta concentração de matéria orgânica (Figura 5). Assim, o córrego São Simão entra na zona de degradação (pontos P4, P5 e P6), região onde a qualidade da água apresenta-se em seu estado mais deteriorado (como já caracterizado pelos parâmetros discutidos neste trabalho), provavelmente devido à grande atividade microbiana responsável pela decomposição da matéria orgânica. Nesta região, observou-se que as águas do córrego São Simão possuem forte odor e coloração cinza, com grande quantidade de matéria em suspensão (óleos, espumas e larvas de insetos).

Ao fim da área urbana de São Simão (próximo ao exutório), notou-se a degradação total do córrego São Simão, pois este se encontra na zona de decomposição ativa, com odor muito forte (provavelmente devido há produção de gás sulfídrico gerado pelo lodo de fundo) e aspecto turvo (devido aos sólidos presentes nos esgotos), com alto índice de larvas de insetos, além da presença de óleos e espumas. O córrego São Simão entra na zona de recuperação a aproximadamente $18 \mathrm{~km}$ após o lançamento dos efluentes. Neste caso, a distância necessária para a autodepuração é maior que a distância física do rio, que necessitaria de, pelo menos, mais $18 \mathrm{~km}$ após sua foz para que suas águas voltem a possuir uma concentração de oxigênio dissolvido acima de $2 \mathrm{mg} / \mathrm{L}$, valor recomendado pela Resolução CONAMA 357/05 (Brasil, 2005) para a Classe de seu Enquadramento (Classe 4).

Para que o córrego São Simão possua sempre valores de oxigênio dissolvido acima de 2 $\mathrm{mg} / \mathrm{L}$, torna-se necessário que seja realizado algum tipo de tratamento de efluente antes de seu lançamento no rio. Se a cidade de São Simão possuísse uma estação de tratamento primária de efluentes, com eficiência entre 30 e $40 \%$, a concentração de oxigênio no córrego São Simão ficaria acima de $2 \mathrm{mg} / \mathrm{L}$, como pode ser visto na Figura 5. Assim, como a alternativa de eficiência de 30\% mostrou-se satisfatória, não há necessidade de se investigar alternativas de maior eficiência que, provavelmente, teriam maior custo. Contudo, sempre se deve ter em mente a Resolução CONAMA 357/05 (Brasil, 2005), que preconiza que os efluentes tratados devem possuir DBO máxima de $60 \mathrm{mg} / \mathrm{L}$, eficiência mínima de 80\%, além é claro de não provocarem a alteração da Classe de Enquadramento de um corpo d'água.

\section{CONCLUSÕES}

Os resultados analisados no córrego São Simão levaram em conta parâmetros que permitiram avaliar alterações espaço-temporais na qualidade das águas fluviais e identificar possíveis impactos ambientais que acontecem na bacia. O córrego São Simão até a cidade de São Simão permanece com suas águas limpas, pois a montante da cidade não há fontes poluidoras. A jusante da cidade, o córrego São Simão passa a receber o lançamento de esgoto doméstico in natura, aumentando a concentração da maior parte dos parâmetros estudados. Além disso, há uma diminuição dos valores médios na concentração de condutividade, oxigênio dissolvido e cloreto da época chuvosa em relação ao período seco, opostamente ao observado para o $\mathrm{pH}$, turbidez, alcalinidade e STS, provavelmente devido à erosão laminar, mais atuante no período chuvoso.

A utilização do modelo de autodepuração de Streeter \& Phelps foi compatível com as amostras experimentais feitas no córrego São Simão. Além disso, o modelo conseguiu identificar as várias zonas de autodepuração do córrego São Simão e indicou a necessidade de tratamento de 
GONÇALVES, J. C. S. I.; SARDINHA, D. S.; SOUZA, A. D. G.; DIBIAZI, A. L. B.; GODOY, L. H.; CONCEIÇÃO, F. T. Avaliação espaço-temporal da qualidade da água e simulação de autodepuração na bacia hidrográfica do córrego São Simão, SP. Ambi-Agua, Taubaté, v. 7, n. 3, p. 141-154, 2012. (http://dx.doi.org/10.4136/ambi-agua.894)

esgotos em nível primário, com eficiência de 30\% de remoção de DBO para que as águas sempre permaneçam com a concentração de oxigênio dissolvido acima de $2 \mathrm{mg} / \mathrm{L}$, valor recomendado para seu enquadramento.

Uma política que englobe o desenvolvimento sócio-econômico em harmonia com o meio ambiente deve ser incentivada na bacia do córrego São Simão, de forma a manter uma boa qualidade da água, protegendo os mananciais de abastecimento de água de São Simão.

\section{AGRADECIMENTOS}

Os autores agradecem a FAPESP (Processos no 05/60299-3 e 05/60298-7) e ao Professor Dr. Osmar Sinelli pelo apoio concedido durante a realização deste trabalho.

\section{REFERÊNCIAS}

ALMEIDA, F. F. M. Fundamentos geológicos do relevo paulista. Boletim do Instituto Geográfico e Geológico, São Paulo, v. 41, p. 167-263, 1964.

AMERICAN PUBLIC HEALTH ASSOCIATION - APHA. Standard methods for the examination of water and wastewater. 20. ed. Washington D.C., 1999. 663 p.

BERNER E. K.; BERNER R. A. Global environment: water, air and geochemical cycles. 1. ed. New York: Prentice Hall, 1996. 367 p.

BRASIL. Ministério do Meio Ambiente. Conselho Nacional do Meio Ambiente - CONAMA. Resolução $\mathbf{N}^{\circ}$ 357, de 17 de março de 2005. Dispõe sobre a classificação dos corpos de água e diretrizes ambientais para o seu enquadramento, bem como estabelece as condições e padrões de lançamento de efluentes, e dá outras providências. Disponível em: http://www.mma.gov.br/port/conama>. Acesso em: 09 ago. 2005.

COMPANHIA DE TECNOLOGIA DE SANEAMENTO AMBIENTAL - CETESB. Variáveis de qualidade das águas. Disponível em: <http://www.cetesb.sp.gov.br>. Acesso em: 19 jun. 2005.

CONCEIÇÃO F. T.; BONOTTO D. M. Weathering rates and anthropogenic influences in a sedimentary basin, São Paulo State, Brazil. Applied Geochemistry, v. 19, n. 4, p. 575591, 2004. http://dx.doi.org/10.1016/j.apgeochem.2003.07.002

CONCEIÇÃO, F. T.; SARDINHA, D. S.; SOUZA, A. D. G.; BONOTTO, D. M. Hydrochemical Relationships at Meio Stream Watershed, São Paulo State, Brazil. Revista Brasileira de Geociências, v. 37, n. 2, p. 389-401, 2007.

ESTEVES, F. A. Fundamentos de Limnologia. 2. ed. Rio de Janeiro: Interciência, 1998. 602p.

GASTALDINI, M. C. C.; SEFFRIN, G. F. F.; PAZ, M. F. Diagnóstico atual e previsão futura da qualidade das águas do Rio Ibicuí utilizando o modelo QUAL2E. Engenharia Sanitária e Ambiental, v. 7, n. 3/4, p. 129 -138, 2002.

GONÇALVES, J. C. S. I.; DIBIAZI, A. L. B.; SOUZA, A. D. G.; PENNER, G. C. O uso do modelo QUAL2K como subsídio à seleção de alternativas de tratamento de esgotos Estudo de Caso: Município de São Simão-SP. Revista Uniara, v. 12, n. 2, p. 37-54, 2009.

HERMES, L. C.; SILVA, A. S. Avaliação da qualidade das águas: manual prático. Brasília: Embrapa Informação Tecnológica, 2004. 55p. 
GONÇALVES, J. C. S. I.; SARDINHA, D. S.; SOUZA, A. D. G.; DIBIAZI, A. L. B.; GODOY, L. H.; CONCEIÇÃO, F. T. Avaliação espaço-temporal da qualidade da água e simulação de autodepuração na bacia hidrográfica do córrego São Simão, SP. Ambi-Agua, Taubaté, v. 7, n. 3, p. 141-154, 2012. (http://dx.doi.org/10.4136/ambi-agua.894)

INSTITUTO AGRONÔMICO DE CAMPINAS - IAC. Carta pedológica semidetalhada do Estado de São Paulo: quadrículas de Descalvado e Ribeirão Preto. 1. ed. São Paulo: Aerofoto Cruzeiro S. A., 1982. Escala: 1:100. 000.

INSTITUTO BRASILEIRO DE GEOGRAFIA E ESTATÍSTICA - IBGE. Folha SF-23-V-C-I-4 Cravinhos/SP. 1. reimpr. Rio de Janeiro, 1982.

INSTITUTO BRASILEIRO DE GEOGRAFIA E ESTATÍSTICA- IBGE. Folha SF-23-V-C-IV2 Luis Antonio/SP. 1. reimpr. Rio de Janeiro, 1971.

INSTITUTO DE PESQUISA TECNOLÓGICA DO ESTADO DE SÃO PAULO - IPT. Mapa Geológico do Estado de São Paulo: desenho no 2. São Paulo, 1981. Escala 1:1000. 000.

INSTITUTO DE PESQUISAS TECNOLÓGICAS DO ESTADO DE SÃO PAULO - IPT. Relatório IPT $\mathbf{n}^{\circ} 90635$ - 205: relatório um da unidade de gerenciamento de recursos hídricos do Pardo (UGRHI - 04). São Paulo, 2006. 255p.

MOTA, S. Introdução à engenharia ambiental. 1. ed. Rio de Janeiro: ABES/AIDIS, 1997. $280 \mathrm{p}$.

PIRES, J. S. R.; SANTOS, J. E. Bacias hidrográficas: integração entre meio ambiente e desenvolvimento. Ciência Hoje, v. 19, n. 110, p. 40-45, 1995.

PROGRAMA DE PESQUISAS EM CARACTERIZAÇÃO, CONSERVAÇÃO E USO SUSTENTÁVEL DA BIODIVERSIDADE DO ESTADO DE SÃO PAULO BIOTA/FAPESP. Atlas Biota. 2005. Disponível em: 〈http://www.biota.org.br/index>. Acesso em 23 jan. 2005.

ROCHA, O.; PIRES, J. S. R.; SANTOS, J. E. A bacia hidrográfica como unidade de estudo e planejamento. In: ESPÍNDOLA, E. L. G.; SILVA, J. S.V.; MARINELLI, C. E.; ABDON, M. M. A bacia hidrográfica do rio Monjolinho: uma abordagem ecossistêmica e a visão interdisciplinar. 1. ed. São Carlos: Rima, 2000. p. 1-16.

SARDINHA, D. S.; BONOTTO, D. M. CONCEIÇÃO, F. T. Weathering rates at Alto Sorocaba basin, Brazil, using U-isotopes and major cations. Environmental Earth Sciences, v. 61, n. 5, p. 1025-1036, 2010. http://dx.doi.org/10.1007/s12665-009-0424-7

SARDINHA, D. S.; CONCEIÇÃO, F. T.; SOUZA, A. D. G.; SILVEIRA, A.; DE JÚLIO, M.; GONÇALVES, J. C. S. I. Avaliação da qualidade da água e autodepuração do Ribeirão do Meio, Leme (SP). Engenharia Sanitária e Ambiental, v. 13, n. 3, p. 329-338, 2008.

SCHNEIDER, R. F.; MUHLMANN, H. E.; MEDEIROS, R. A.; DAEMON, R. F. NOGUEIRA, A. A. Revisão estratigráfica da bacia do Paraná. In: CONGRESSO BRASILEIRO DE GEOLOGIA, 28., 1974. Porto Alegre. Anais... Porto Alegre: SBG, 1974. Vol. 1. p. 41-65.

SINELLI, O. Geologia e água subterrânea no município de Ribeirão Preto e adjacências. 1970. 116 f. Tese (Doutorado em Geografia) - Faculdade de Filosofia, Ciências e Letras de Ribeirão Preto, Universidade de São Paulo, Ribeirão Preto, 1970.

SOARES, P. C.; LANDIM, P. M. B. Aspectos da estratigrafia da Bacia do Paraná no seu flanco nordeste. In: CONGRESSO BRASILEIRO DE GEOLOGIA, 28., 1974, Porto Alegre. Anais... Porto Alegre: SBG, 1974. Vol. 1. p. 243-256. 
GONÇALVES, J. C. S. I.; SARDINHA, D. S.; SOUZA, A. D. G.; DIBIAZI, A. L. B.; GODOY, L. H.; CONCEIÇÃO, F. T. Avaliação espaço-temporal da qualidade da água e simulação de autodepuração na bacia hidrográfica do córrego São Simão, SP. Ambi-Agua, Taubaté, v. 7, n. 3, p. 141-154, 2012. (http://dx.doi.org/10.4136/ambi-agua.894)

SOUZA, A. D. G.; TUNDISI, J. G. Hidrogeochemical comparative study of the Jaú and Jacaréguaçu River watersheds, São Paulo, Brazil. Revista Brasileira de Biologia, v. 60, n. 4, p. 563-570, 2000. http://dx.doi.org/10.1590/S0034-71082000000400004

TUNDISI, J. G. Água no século XXI: enfrentando a escassez. 2. ed. São Carlos: Rima, 2005. 248 p.

UNITED STATES. Environmental Protection Agency - USEPA. Total maximum daily load for nutrients Deep Run, Beach Run, and Elizabeth Run Lebanon County, Pennsylvania. Philadelphia, 2004. 77p.

UNITED STATES. Environmental Protection Agency - USEPA. Modeling report for total maximum daily load for Skippack Creek, Montgomery County, Pennsylvania. Philadelphia, 2005. 108p.

VON SPERLING, M. Introdução à qualidade das águas e ao tratamento de esgotos. 2. ed. Belo Horizonte: UFMG, 1996. 246p.

WETZEL, R. G. Limnology. 2. ed. Rochester: Saunders College Publishing, 1983. 767p. 


ISSN = 1980-993X - doi:10.4136/1980-993X
www.ambi-agua.net
E-mail: ambi-agua@agro.unitau.br
Tel.: (12) 3625-4212

\title{
Qualidade físico-química e microbiológica de água mineral e padrões da legislação
}

(http://dx.doi.org/10.4136/ambi-agua.908)

\author{
Helenilza Ferreira Albuquerque Cunha ${ }^{1}$; Débora Cristina Isacksson Lima² \\ Priscila Nazaré de Freitas Brito ${ }^{3}$; Alan Cavalcanti da Cunha ${ }^{4}$; \\ Arialdo Martins da Silveira Junior ${ }^{5}$; Daímio Chaves Brito ${ }^{6}$. \\ Universidade Federal do Amapá_UNIFAP, Macapá-AP, \\ e-mails: helenilzacunha@ unifap.br $^{1}$, debora_isacksson@ hotmail.com $^{2}$, priscila.nfb@ hotmail.com $^{3}$, \\ alancunha@unifap.br ${ }^{4}$, arialdojunior@hotmail.com ${ }^{5}$, daimiobrito@gmail.com ${ }^{6}$
}

\section{RESUMO}

O objetivo deste estudo foi avaliar parâmetros físico-químicos e microbiológicos da água mineral comercializada na cidade de Macapá e verificar sua conformidade com a legislação. Os padrões legais aceitos para potabilidade de água para consumo humano no Brasil são estabelecidos pela Resolução Diretória Colegiada - RDC 274/2005 da Agência Nacional de Vigilância Sanitária (ANVISA) - que regulamenta as águas minerais e a Portaria 518/2004 do Ministério da Saúde, que estabelece a potabilidade para o abastecimento público. As análises mensais foram feitas em amostras de água em garrafões de $20 \mathrm{~L}$ de três marcas diferentes adquiridas em seis locais comerciais. Parâmetros físico-químicos foram comparados com os descritos pela legislação como turbidez, $\mathrm{pH}$, nitrato, ferro, manganês, alumínio e fluoreto, e os microbiológicos como coliformes totais e Escherichia coli. Os resultados indicaram que o pH encontra-se frequentemente abaixo do limite permitido ao abastecimento público. A elevada concentração de alumínio foi considerada grave, com valores significativamente acima dos padrões permitidos em duas marcas. O flúor apresentou concentrações próximas de zero, apesar da legislação sugerir valores entre $1 \mathrm{mg} / \mathrm{L}$ e $2 \mathrm{mg} / \mathrm{L}$. Os parâmetros microbiológicos em todas as marcas apresentaram coliformes totais, mas Escherichia coli foi registrado em apenas uma amostra. A água mineral é consumida por ser considerado um produto seguro em relação a outras fontes, mas este estudo mostra que a água mineral comercializada em Macapá está frequentemente em não conformidade com a legislação. Recomenda-se atenção na fiscalização especialmente em garrafões de $20 \mathrm{~L}$.

Palavras-chave: Água mineral, RDC 274/2005, análises fisico-químicas, coliforme total, saúde pública.

\section{Physicochemical and microbiological quality of mineral water and the legislation standards}

\section{ABSTRACT}

The objective of this study was to evaluate the physicochemical and microbiological mineral water parameters in the city of Macapá and verify their compliance with the legislation. The accepted legal standards for potable water for human consumption in Brazil are established by Board Resolution - RDC 274/2005 of the National Health Surveillance Agency (ANVISA) - which regulates mineral water and Ordinance 518/2004 of the Ministry of Health which defines minimum standards for drinking water. Water samples in $20 \mathrm{~L}$ bottles of three different trademarks acquired in six commercial localities were analyzed monthly. 
Physicochemical parameters were compared with those predicted in the legislation for turbidity, $\mathrm{pH}$, nitrate, iron, manganese, aluminum, fluoride, and microbiological indicators such as total coliform and Escherichia coli. The results indicated that the $\mathrm{pH}$ was often below the limit allowed by public supply. Deviation from the recommended concentration was most exceeded for aluminum, presenting values significantly high to the standards allowed in two trademarks. Fluoride concentrations were close to zero, although the legislation recommends values between $1 \mathrm{mg} / \mathrm{L}$ and $2 \mathrm{mg} / \mathrm{L}$. The microbiological parameters in some localities and all trademarks registered total coliform, but Escherichia coli were present in only one sample. The mineral water consumed has been considered a safe product compared to other sources, but this study showed that the mineral water marketed in Macapá was often not in accordance with the law. Special attention is recommended for monitoring water quality in $20 \mathrm{~L}$ bottles.

Keywords: Mineral water, RDC 274/2005, physical-chemical analysis, total coliform, public health.

\section{INTRODUÇÃO}

A preocupação com a qualidade da água, decorrente da progressiva poluição hídrica, é um dos motivos que levam grande parte da população mundial ao consumo de água proveniente de fontes minerais (Leclerc e Moreau, 2002). A percepção de que a ingestão desta água remete a um estilo de vida saudável tem aumentado seu consumo no Brasil e no mundo (Mavridou, 1992).

Na última década sua produção duplicou nos EUA e na Suíça (Wynn et al., 2009). O Brasil é o sexto maior produtor do mundo, com aproximadamente 3,5 bilhões de litros registrados no ano de 2000. A região Sudeste é responsável pela maior produção (53\%), sendo 36,9\% só no estado de São Paulo. O Nordeste é o segundo, respondendo por 25,1\% (Resende e Prado, 2008). Isso se deve principalmente às propriedades terapêuticas e medicinais atribuídas ao produto. O conceito de pureza associada é, também, responsável por seu aumento de consumo, mas não se tem certeza sobre a superioridade desta em relação à água de abastecimento público (Pontara et al., 2011).

A RDC/274 (ANVISA, 2005) caracteriza as águas minerais como obtidas diretamente de fontes naturais ou artificialmente captadas de origem subterrânea. São determinadas pelo conteúdo definido e constante de sais minerais e presença de oligoelementos. O elemento predominante na sua composição varia com as rochas e terrenos pelos quais percorre enquanto infiltra-se no solo, podendo apresentar alterações devido às condições hidrogeológicas, hidroclimáticas e a biota (Resende e Prado, 2008).

Independente da fonte (superficial ou subterrânea) a água pode servir de veículo para vários agentes biológicos e químicos sendo necessário observar os fatores que podem interferir negativamente na sua qualidade (Di Bernardo, 1993).

Dentre as substâncias encontradas na água, o composto nitrogenado em seus diferentes estados de oxidação (nitrogênio amoniacal, nitrito e nitrato) pode apresentar riscos à saúde humana (Alaburda e Nishihara, 1998). A presença do nitrogênio na água pode ser de origem natural, como matéria orgânica e inorgânica e chuvas; e antrópica, como esgotos domésticos e industriais. $\mathrm{O}$ nitrato, um dos mais encontrados em águas naturais, apresenta-se em baixos teores nas águas superficiais, podendo alcançar altas concentrações em águas profundas, como nas fontes minerais, por ser altamente lixiviante nos solos, contaminando corpos d'água e aquíferos subterrâneos (Alaburda e Nishihara, 1998).

$\mathrm{Na}$ RDC/274 (ANVISA, 2005) o nitrato deve apresentar valor máximo permissível (VMP) de $50 \mathrm{mg} / \mathrm{L}$ em águas minerais naturais. Seu consumo está diretamente relacionado com a caracterização de dois fatores adversos à saúde, como indução à metemoglobinemia e a formação potencial de nitrosaminas e nitrosamidas carcinogênicas. 
O íon fluoreto, encontrado em águas minerais, deve apresentar concentrações abaixo de $1 \mathrm{mg} / \mathrm{L}$, conforme a legislação. Sua ingestão acima de $2 \mathrm{mg} / \mathrm{L}$ é considerada inadequada para lactentes e crianças com até 7 anos de idade, podendo acarretar o surgimento de fluorose dental e óssea. Por outro lado, a presença de fluoreto em água de consumo, em níveis recomendados, é a forma mais eficiente e coletiva para a prevenção de cáries (Brandão e Valsecki Júnior, 1998; Bulcão e Rebelo, 2009).

Metais também podem comprometer a qualidade da água. Silva (1997) afirma que a intoxicação por metais se desenvolve lentamente e muitas vezes só pode ser identificada após anos ou decênios, e sua presença reduz a capacidade autodepurativa da água devido a ação tóxica sobre os microorganismos que realizam esse processo.

Os metais presentes na água, quando ingeridos, são absorvidos pelo organismo humano pelo trato gastrintestinal e a absorção pode ser afetada pelo $\mathrm{pH}$ da água, pelas taxas de movimentação no trato digestivo e pela presença de outros compostos (Freitas et al., 2001). Sua ingestão também pode causar distúrbios gastrointestinais e até disfunção mental com degeneração do sistema nervoso central. Dentre os mecanismos de sua toxicidade estão incluídas as interações com sistemas enzimáticos, interações com membranas celulares e efeitos específicos sobre certos órgãos e sobre o metabolismo celular em geral (Morgano et al., 2002).

Em relação aos microrganismos presentes na água mineral, Pontara et al. (2011) afirmam que a percepção de que a água mineral é segura e isenta de impurezas deve ser repensada, sendo necessário avaliar critérios de monitoramento e análise microbiológica.

A população em geral considera a água mineral segura, mas não existem estudos que comprovem esta hipótese para a maioria das cidades brasileiras. Pesquisas realizadas em etapas curtas podem ser inadequadas para avaliar fontes de água (Alves et al., 2002; Morgano et al., 2002; Pontara et al., 2011). Com o objetivo de estudar parâmetros físico-químicos e microbiológicos da água mineral comercializada na cidade de Macapá, avaliou-se sua conformidade em relação à legislação vigente. A partir desta perspectiva foi definida uma metodologia de monitoramento mensal da qualidade da água num horizonte temporal de dois anos consecutivos.

\section{MATERIAL E MÉTODOS}

Foram analisadas amostras de três marcas de água mineral em garrafões de $20 \mathrm{~L}$, identificadas como A, B e C, adquiridas em diferentes estabelecimentos comerciais escolhidos aleatoriamente na área urbana. Como o objetivo da pesquisa foi apenas quantificar e comparar os parâmetros físico-químicos e microbiológicos com os previstos na legislação vigente, julgamos desnecessário informar as marcas comerciais referenciadas acima.

Os vasilhames originais estavam lacrados, sem possibilidade de contaminação externa no período entre a aquisição e as análises. O período de estudo ocorreu entre julho de 2009 a julho de 2011.

Os parâmetros físico-químicos e microbiológicos foram escolhidos como variáveis explicativas, especialmente a conformidade dos mesmos à RDC 274/2005 (ANVISA, 2005) e Portaria 518/2004 (Brasil, 2004). No total, foram analisadas 150 amostras em duplicatas.

O parâmetro turbidez foi determinado pelo método turbidimétrico (turbidímetro portátil HACH 2100P). É um indicador da transparência física da água e não necessariamente um parâmetro de potabilidade.

Para a determinação de metais, as amostras foram acondicionadas em garrafas plásticas, lavadas em solução de ácido clorídrico 1:9 para a preservação de suas características 
químicas. As concentrações foram determinadas pelos métodos espectrofotométricos: Ferro pelo método FerroVer ${ }^{\circledR}$, Manganês pelo método PAN e Alumínio pelo método AluVer ${ }^{\circledR}$.

$\mathrm{O}$ pH foi analisado pelo método potenciométrico com pH-âmetro Orion. As concentrações das frações de nitrogênio foram determinadas pelos métodos espectrofotométricos: fração nitrato $\left(\mathrm{NO}_{3}{ }^{-}\right)$pelo método NitraVer ${ }^{\circledR}$. A concentração de fluoreto foi determinada pelo método SPADNS ${ }^{\circledR}$, com uso do espectrofotômetro (APHA et al., 2003).

Para os parâmetros microbiológicos, a coleta foi realizada em recipientes assépticos e as análises identificaram a presença ou não de bactérias do grupo de coliformes (coliformes totais) e de E. coli. Ambos foram quantificados pelo método do substrato cromogênico com reagente COLILERT/IDEXX. Posteriormente foram acondicionadas em bolsas estéreis (ThioBag) no interior da qual foi individualmente adicionado um flaconete de substrato para interagir com os microrganismos em análise. Após a diluição do substrato, a amostra foi transferida para uma cartela estéril e depois lacrada em uma seladora Quanti-Tray Sealer. As cartelas foram inseridas em estufa a $35 \pm 0,5^{\circ} \mathrm{C}$ durante $24 \mathrm{~h}$. As leituras foram realizadas com auxílio de câmera escura sob luz ultravioleta (Alves et al., 2002).

Análise de Variância (ANOVA - Dupla Entrada) (Hand, 2008; Magnusson e Mourão, 2009; Wilcox, 2009) foi aplicada para testar significativas diferenças físico-químicas e microbiológicas entre as marcas (3 marcas em duplicatas) e ao longo do eixo temporal (25 meses).

\section{RESULTADOS}

Com base nas informações obtidas dos parâmetros da qualidade da água mineral a Tabela 1 mostra os valores médios e os respectivos intervalos de confiança em torno dessas médias $(95 \%)$.

Os valores médios da turbidez não apresentaram variação significativa entre as seis amostras $\left(F_{[5,24]}=1,74\right.$ e $\left.p=0,13\right)$ nem ao longo do período $\left(F_{[5,24]}=1,01\right.$ e $\left.p=0,46\right)$.

Em relação aos valores médios de $\mathrm{pH}$ observou-se variações significativas, tanto entre períodos $\left(F_{[5,24]}=9,17\right.$ e $\left.p=9,07 \mathrm{E}^{-12}\right)$ quanto entre as marcas $\left(F_{[5,24]}=2,03\right.$ e $\left.p=0,031\right)$, conforme indicado na Figura 1a. Portanto, a variação global é significativa e está em desacordo com um único valor constante normalmente encontrado em rótulos de vasilhames em todas as marcas e por períodos longos, normalmente anuais.

Tabela 1. Média e intervalos de confiança dos parâmetros da qualidade da água.

\begin{tabular}{|c|c|c|c|c|c|c|}
\hline \multirow[t]{2}{*}{ Parâmetros } & \multicolumn{6}{|c|}{$\begin{array}{l}\text { Valores de parâmetros físico-químicos e microbiológicos da água mineral } \\
\qquad(\text { Média } \pm 1,96 * \mathrm{dp}=95 \%)\end{array}$} \\
\hline & Marca A1 & Marca A2 & Marca B1 & Marca B2 & Marca C1 & Marca C2 \\
\hline Turbidez (UT) & $0,13 \pm 0,08$ & $0,13 \pm 0,09$ & $0,14 \pm 0,12$ & $0,15 \pm 0,13$ & $0,21 \pm 0,21$ & $0,30 \pm 1,20$ \\
\hline $\mathrm{pH}$ & $4,47 \pm 0,86$ & $4,43 \pm 0,92$ & $4,04 \pm 0,71$ & $4,01 \pm 0,72$ & $4,82 \pm 1,14$ & $4,82 \pm 1,14$ \\
\hline Nitrato (mg/L) & $2,13 \pm 1,03$ & $2,27 \pm 1,21$ & $29,65 \pm 10,27$ & $29,03 \pm 11,77$ & $1,86 \pm 1,06$ & $1,90 \pm 1,04$ \\
\hline Fluoreto (mg/L) & $0,05 \pm 0,11$ & $0,07 \pm 0,14$ & $0,06 \pm 0,13$ & $0,05 \pm 0,11$ & $0,06 \pm 0,17$ & $0,06 \pm 0,12$ \\
\hline Ferro $(\mathrm{mg} / \mathrm{L})$ & $0,02 \pm 0,02$ & $0,02 \pm 0,03$ & $0,02 \pm 0,03$ & $0,02 \pm 0,02$ & $0,03 \pm 0,03$ & $0,02 \pm 0,04$ \\
\hline Manganês (mg/L) & $0,00 \pm 0,01$ & $0,00 \pm 0,01$ & $0,01 \pm 0,01$ & $0,01 \pm 0,01$ & $0,01 \pm 0,01$ & $0,01 \pm 0,03$ \\
\hline Alumínio (mg/L) & $0,06 \pm 0,03$ & $0,06 \pm 0,05$ & $0,47 \pm 0,29$ & $0,47 \pm 0,25$ & $0,03 \pm 0,03$ & $0,03 \pm 0,04$ \\
\hline \multicolumn{7}{|l|}{ Coliformes Totais } \\
\hline (NMP/100 mL) & $0,08 \pm 0,46$ & $1,54 \pm 13,68$ & $0,08 \pm 0,80$ & $0,30 \pm 2,94$ & $0,81 \pm 6,77$ & $0,41 \pm 2,08$ \\
\hline (E. coli $(\mathrm{NMP})$ & $0,00 \pm 0,00$ & $0,00 \pm 0,00$ & $0,00 \pm 0,00$ & $0,00 \pm 0,00$ & $0,00 \pm 0,00$ & $0,03 \pm 0,26$ \\
\hline
\end{tabular}


Quanto ao íon nitrato todas as marcas apresentaram conformidade com os níveis de concentração exigido pela legislação. Contudo, a marca B (1 e 2) apresentou valores próximos do máximo permitido em duas amostras coletadas (Figura 1b). Foi possível observar na Figura $1 b$ que houve variação significativa entre as médias de concentração do íon nitrato entre as médias das amostras $\left(F_{[525]}=2,14, p=0,0038\right)$ e ao longo do tempo $\left(F_{[5,25]}=548,57\right.$, $p<0,05)$.

As médias das concentrações de ferro não apresentaram variação significativa no eixo temporal $\left(F_{[5,24]}=2,28\right.$ e $\left.p=0,051\right)$, apesar desta se apresentar muito próxima do limite da significância. Por outro lado, foram observadas diferenças significativas entre as médias das três marcas $\left(F_{[5,24]}=3,05\right.$ e $\left.p=3,16 \mathrm{E}^{-05}\right)$.

As médias das concentrações de manganês indicaram variações significativas, tanto entre as marcas $\left(F_{[5,24]}=3,09\right.$ e $\left.p=2,65 \mathrm{E}^{-05}\right)$ quanto no eixo temporal $\left(F_{[5,24]}=10,44\right.$ e $\left.p=2,44 \mathrm{E}^{-08}\right)$.

O flúor apresentou níveis de concentração médios que variaram ao longo do período amostral de 0,00 a 0,19 $\mathrm{mgF}^{-} / \mathrm{L}$ (Figura 1c). Estes níveis estão abaixo do estipulado pela legislação, impedindo que a água sirva como prevenção à cárie, principal função do fluoreto em águas de consumo humano. Assim, estes resultados não indicaram significativa variação entre as médias amostrais ao longo do eixo temporal $\left(F_{[5,24]}=0,378, p=0,86\right)$. Contudo, acusou diferenças significativas entre as marcas $\left(F_{[5,24]}=2.877, p=0,0132\right)$. Neste caso, ressalta-se que as análises para detecção de fluoreto devam ser realizadas com frequência anual ou bimestral na indústria.

Destaca-se que somente a marca C (1 e 2) continha informação em seu rótulo sobre as concentrações de fluoreto, indicando nível igual a $0,02 \mathrm{mgF}^{-} / \mathrm{L}$. Este valor é menor que a metade dos valores médios obtidos nas análises das amostras $\left(0,057 \mathrm{mgF}^{-} / \mathrm{L}\right)$. As demais marcas não apresentaram qualquer informação.

Quanto à concentração média de alumínio houve variações significativas, tanto entre as marcas $\left(F_{[5,24]}=2,88\right.$ e $\left.p=7,83 \mathrm{E}^{-05}\right)$ quanto no período $\left(F_{[5,24]}=240,95\right.$ e $\left.p=8,13 \mathrm{E}^{-61}\right)$. A marca B (1 e 2) apresentou não conformidade com a legislação (Figura 1d), sendo que os elevados valores médios apresentados por esta marca podem incorrer em risco à saúde pública, com média geral das amostras em torno de $0,18 \mathrm{mg} / \mathrm{L}$, e próximo do limite máximo permitido de $0,20 \mathrm{mg} / \mathrm{L}$ pela Portaria MS 518/2005.

Em relação ao parâmetro microbiológico coliforme total, verificou-se que as médias das concentrações não apresentaram diferenças significativas no período $\left(F_{[5,24]}=0,73\right.$ e $p=$ $0,60)$ ou entre as marcas $\left(F_{[5,24]}=1,0\right.$ e $\left.p=0,55\right)$. Contudo, as concentração apresentaram frequentemente não-conformidade microbiológica para todas as marcas no período de análise. Além disso, valores não-zero ocorreram apenas na marca $\mathrm{A} 1$, três vezes no total de vinte e cinco amostras. $\mathrm{O}$ mesmo ocorreu com a marca $\mathrm{A} 2$, com duas ocorrências em vinte e cinco amostras. Nas marcas B1 e B2 foi registrada uma única ocorrência no período e no total de vinte e cinco amostras. Para a marca $\mathrm{C} 1$ foram registradas quatro ocorrências e em $\mathrm{C} 2$ cinco ocorrências de não-conformidade (Figura 1e).

Em relação ao parâmetro microbiológico fecal E. coli foi registrada presença em uma única amostra em outubro de 2010, na marca C2. Portanto, em conjunto, os resultados não apresentaram variações significativas nem no período $\left(F_{[5,24]}=1,00\right.$ e $\left.p=0,42\right)$ nem entre as marcas $\left(F_{[5,24]}=1,0\right.$ e $\left.\mathrm{p}=0,47\right)$. 

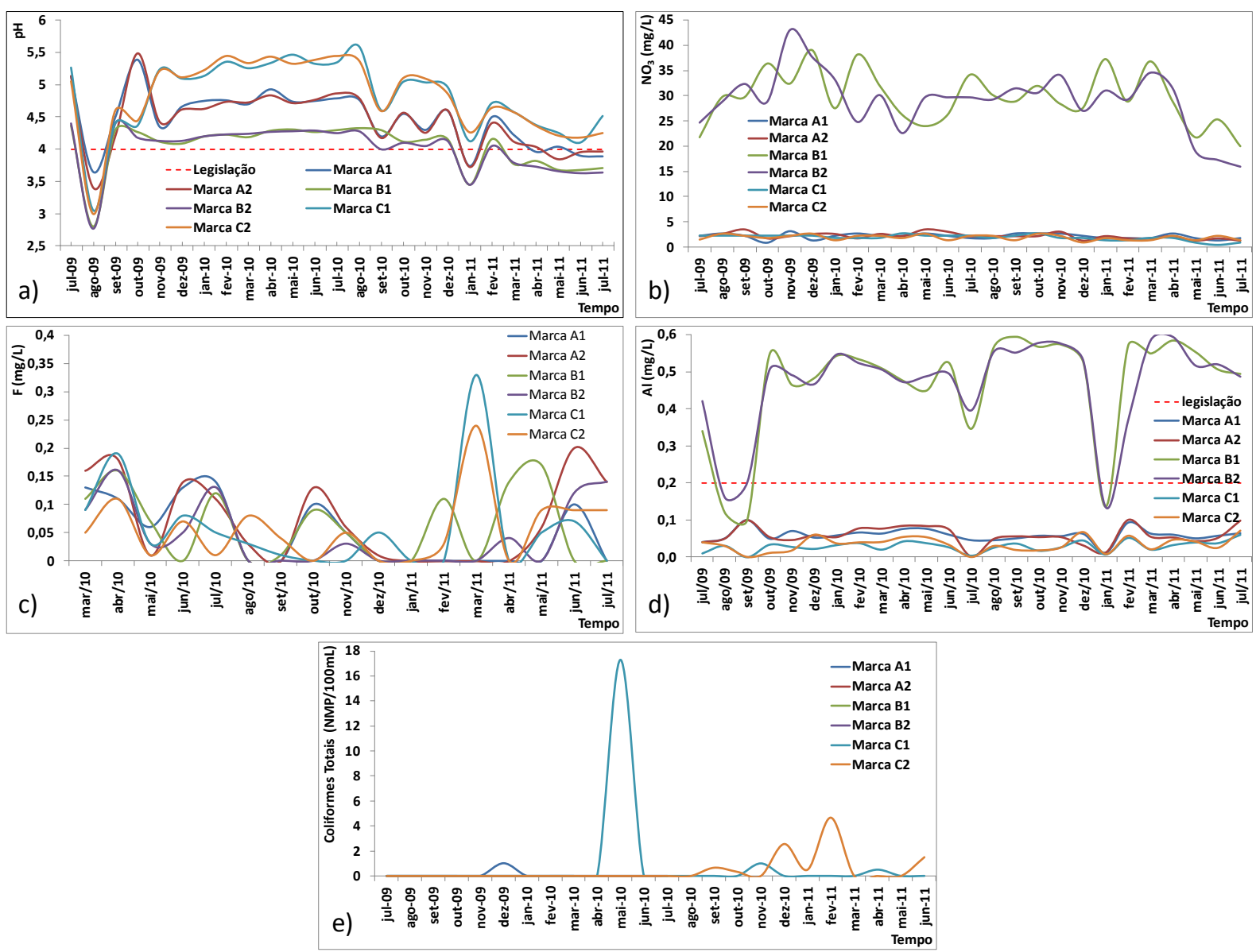

Figura 1. Parâmetros físico-quimicos e microbiológicos da qualidade da água mineral em vasilhames de 20 L. As linhas pontilhadas em vermelho indicam padrões na legislação para as concentrações das amostras: a) variação de $\mathrm{pH}$; b) variação da concentração de $\mathrm{NO}_{3}$; c) variação da concentração de $\mathrm{F}$; d) variação da concentração de $\mathrm{Al}$ e e) variação da concentração de coliformes totais.

\section{DISCUSSÃO}

As amostras de água mineral apresentaram pH médios entre 4,13 e 4,82, sugerindo que as águas não são significativamente mineralizadas. De acordo com Felski et al. (2008) águas ácidas, por serem menos mineralizadas e mais leves, são mais aceitáveis para o consumo do que as águas alcalinas, o que explica o seu elevado consumo. Acidez mais elevada pode advir de fonte natural, sendo mais propícia para as águas minerais, ou pode ocorrer pela dissolução de rochas, por processos de absorção de gases da atmosfera e processos fotossintetizantes.

Apesar de a acidez ser desejável há limites legais estipulados para este parâmetro, entre 4,0 e 9,0. Embora algumas amostras em determinados períodos se apresentaram pouco abaixo de 4,0 houve variações significativas entre as marcas e ao longo do tempo. Portanto, apesar das amostras se apresentarem dentro dos padrões legais, se encontraram frequentemente próximas ou abaixo dos limites (Figura 1a). Tais variações da acidez das águas levam muitos estudiosos a considerarem como característica duvidosa de sua qualidade (ANVISA, 2005).

Segundo Brandão e Valsecki Jr. (1998) geralmente a qualidade das águas subterrâneas tende a variar ao longo do tempo. Mas a legislação só exige análises físico-químicas de controle nas indústrias anualmente. Contudo, as datas das análises não estavam impressas nos rótulos dos garrafões de duas marcas usadas neste estudo.

Os níveis de concentração de nitrato observados foram preocupantes, apesar de ainda estarem em conformidade com a RDC 274/2005 (ANVISA, 2005), a qual estabelece o Valor 
Máximo Permitido (VPM) de $50 \mathrm{mg} / \mathrm{L}$. Mas, este valor quando calculado como fração de nitrogênio na forma de nitrato, converte-se para $11,3 \mathrm{mg} / \mathrm{L}\left(\mathrm{N}_{-} \mathrm{NO}_{3}^{-}\right)$(Nóbrega et al., 2008). Neste caso, está em não-conformidade com a Portaria $\mathrm{n}^{\circ} 518$ (Brasil, 2004) que estabelece o limite máximo de $10 \mathrm{mg} / \mathrm{L}\left(\mathrm{N}_{-} \mathrm{NO}_{3}{ }^{-}\right)$para águas de abastecimento público. Em todo caso, esta concentração pode ser nociva à saúde pela indução de doenças como a metemoglobinemia ou doença do bebê azul.

É importante comentar que as fontes subterrâneas profundas tornam-se um ótimo ambiente para a presença natural deste íon, mas que não excluem o seu efeito adverso à saúde, sobretudo nas crianças que possuem em seu sistema digestivo condições ótimas para a redução bacteriológica do nitrato a nitrito, principal causa da metemoglobinemia (Fernícula e Azevedo, 1981).

Outro aspecto relevante é quanto a importância do íon $\mathrm{NO}_{3}$ para compará-los com valores de concentração ao longo do tempo e em relação à exposição de seus valores nos rótulos comerciais, normalmente constantes. No presente caso, foi observada uma significativa diferença entre as médias das concentrações de nitrato (Figura 1b), cujos valores potencialmente podem alcançar níveis além da margem aceitável de concentração.

A afirmação acima é relevante porque se estima que as análises de controle representadas nos rótulos comerciais de água mineral foram realizadas, em média, há pelo menos um ano antes da pesquisa, ocorrendo o mesmo problema para o parâmetro $\mathrm{pH}$, onde os rótulos não acusaram nenhuma alteração de valor nas concentrações hidrogeniônicas informadas e ao longo do período. Nestes termos, o valor único e constante impressos nos rótulos das embalagens tende a mascarar a variação real observada se o monitoramento fosse mensal (Figura 1a).

O mesmo pode ocorrer com o flúor. Brandão e Valsecki Júnior (1998) revelaram que dezesseis amostras contendo flúor, do total de trinta e duas analisadas, apresentaram valores abaixo do permitido pela legislação, sendo detectados valores da ordem de $0,02 \mathrm{mgF} / \mathrm{L}$. Dentre as amostras observadas pelos referidos autores somente oito apresentavam no seu rótulo a concentração de fluoreto. Isto contraria a legislação, vez que as empresas de água mineral são obrigadas a informar o consumidor sobre a qualidade físico-química do produto. $\mathrm{Na}$ presente pesquisa foram observados valores intermediários de flúor, sendo que apenas uma marca $\mathrm{C}$ (1 e 2) foi detectada informações desta natureza em rótulo (Figura 1c).

A importância do ferro na região amazônica apresenta caráter mais estético do que de potabilidade. As fontes minerais desta região podem apresentar valores que facilmente excedem os limites da legislação sem, contudo, representar riscos reais à saúde. Por outro lado, as concentrações de manganês foram identificadas abaixo do limite máximo de 0,500 $\mathrm{mg} / \mathrm{L}$ estabelecido pela legislação, encontrando-se em conformidade com a legislação.

Por outro lado, o parâmetro alumínio apresentou variação significativa entre as concentrações médias amostrais ao longo do tempo e entre as médias das diferentes marcas. A importância da afirmação está no consumo de altas concentrações de alumínio potencialmente associado aos distúrbios que provocam o mal de Alzheimer (Freitas et al., 2001). Portanto, trata-se de uma preocupação com a comercialização e fiscalização da água com alto teor deste metal, pois as informações referentes aos metais ferro, alumínio e manganês não foram registrados nos rótulos das três marcas monitoradas. Isso porque estas informações não são obrigatórias na legislação para proteger o consumidor do uso exagerado de água mineral com altos teores de alumínio, o que é um contra-senso. Nestes casos, sugerimos que os órgãos fiscalizadores estejam alertas quanto ao potencial consumo deste metal frequentemente observado na água mineral analisada nesta pesquisa.

É importante frisar que, amostras de água mineral alteradas, permitem inferir que podem ter ocorrido contaminações durante a fase de captação e processamento do produto (Alves et 
al., 2002) ou mesmo devido aos efeitos sazonais do clima sobre a composição físico-química ou microbiológica (Silva et al., 2002).

Nestes casos, Silva et al. (2002) avaliaram os efeitos sazonais do clima na composição físico-química de amostras de água mineral provenientes de diferentes fontes em São Paulo (Ba, $\mathrm{Ca}, \mathrm{K} \mathrm{e} \mathrm{Mg).} \mathrm{Os} \mathrm{referidos} \mathrm{autores} \mathrm{afirmaram} \mathrm{que} \mathrm{é} \mathrm{possível} \mathrm{verificar} \mathrm{que} \mathrm{a} \mathrm{constituição}$ da água mineral pode ser utilizada como "impressão digital" para fins de estudo de rastreabilidade das águas minerais por apresentarem variações temporais não-significativas.

Contudo, as afirmações de Silva et al. (2002) podem ser questionadas porque considerou apenas um período curto de três meses. Talvez este prazo seja insuficiente para detectar as variações amostrais, tais como as observadas no presente estudo. Do ponto de vista analítico, acreditamos que um curto prazo de análise não é suficiente para obter uma variação significativa e manter a hipótese de constância das características químicas ao longo de um período anual para a maioria dos parâmetros físico-químicos e microbiológicos.

Quanto aos aspectos microbiológicos, mesmo que não ocorram registros bacteriológicos em amostras de água mineral (coliformes totais e E. coli), na literatura tem sido considerados outros parâmetros além dos normalmente utilizados para analisar o teor microbiano. Por exemplo, a ausência de coliformes ou E. coli não exclui, por exemplo, fungos e filamentosos, independentemente do resultado negativo da análise bacteriológica. Alguns autores afirmam que desconsiderá-los pode representar um problema, em especial para usuários não saudáveis de águas, como pacientes imunocomprometidos (Pontara et al., 2011).

A partir de uma tendência da aplicação da legislação vigente para águas minerais, Alves et al. (2002) afirmam que, após sofrer atualização da Resolução RDC 274 (ANVISA, 2005), houve otimização quanto ao seu espectro microbiológico. Mas ainda é insuficiente para manter o controle de qualidade e está aquém do que exige o padrão da legislação internacional (americana e européia) para a comercialização deste produto (Wynn et al., 2009).

\section{CONCLUSÕES}

No período entre julho de 2009 a junho de 2011 foram determinadas não somente as médias mas as variâncias de cada parâmetro da qualidade da água concernentes às marcas comerciais existentes na cidade de Macapá. Além disso, com base nos poucos estudos da literatura, verificou-se a necessidade de investigações em períodos mais prolongados. Com efeito, foram observadas influências da sazonalidade (entre e inter-ano) onde se constatou significativa variação de alguns parâmetros. Tal efeito corrobora a hipótese de que a rotulagem de valores fixos de qualidade da água não é recomendada.

A água mineral comercializada na cidade de Macapá atendeu apenas parcialmente as especificações previstas em normas de vigilância e saúde, dependendo do período sazonal, pois alguns parâmetros estavam em não-conformidade em relação aos previstos na legislação. Desta forma, podemos resumir da seguinte maneira nossas conclusões:

1. A água comercializada em Macapá frequentemente não atendeu às exigências da legislação, especialmente em relação ao $\mathrm{pH}$, elevado conteúdo de alumínio, frequente presença de coliformes totais em mais de 5\% das amostras analisadas (ou seja 10\% ou mais), não observação das concentrações de flúor no rótulo, e oscilação sazonal das concentrações de nitrato.

2. Os parâmetros microbiológicos regulamentados pela legislação (coliformes totais) são considerados bons ou adequados quando apresentam até três coliformes totais em $100 \mathrm{~mL}$ em $5 \%$ das amostras analisadas no mês e coliformes fecais ausentes (E. coli). Contudo, nas amostras avaliadas, apenas $90 \%$ estavam aptas ao consumo, índice bastante inferior ao recomendado pela legislação. A presença de E. coli em água mineral indica necessidade de melhorar o controle de qualidade do produto. 
3. Observaram-se variações sazonais significativas das amostras de mesma marca e de marcas distintas, com destaque para o metal Alumínio, registradas em níveis acima do permitido pela legislação, comprometendo a qualidade da água. Este parâmetro é um componente neurotóxico, além de indutor ou causador de distúrbios neurológicos. Neste sentido, a legislação atual pareceu inadequada sob vários aspectos, pois o Alumínio não consta como obrigatório no monitoramento e na regulamentação brasileira.

4. O descumprimento da legislação ao se omitir informações corretas nos rótulos, sobre as concentrações dos constituintes da água, impossibilitou comparações entre valores apresentados e para fins de fiscalização ou monitoramento. No processo de rotulagem dos vasilhames de água mineral as informações devem ser adequadamente visíveis ao consumidor e corrigidas com frequência mínima bimestral, para assegurar as variações dos parâmetros no tempo. No entanto, o que se observou nos rótulos foi um valor fixo de sua composição ao longo do tempo ou mesmo entre amostras. Mas este fato foi contestado na presente pesquisa.

5. A significativa variação sazonal da acidez, da concentração de metais e de íons na água mineral, é um fator adicional de risco à saúde humana, em razão de que esta tende a agravar o potencial de certos constituintes que não são previstos na legislação. Por exemplo, ao se excluir os valores máximos da concentração de ferro e alumínio da legislação incorre em potencial prejuízo ao consumidor por este ignorar os riscos associados ao consumo desses metais.

6. Finalmente, a qualidade da água mineral em garrafões de $20 \mathrm{~L}$ apresentou considerável vulnerabilidade físico-química e microbiológica, necessitando ser monitorada em todas as suas etapas produtiva e comercial. Além disso, é necessário inserir na lista de parâmetros de qualidade novos componentes potencialmente perigosos à saúde humana, e que atualmente não são nem sequer exigidos pelos órgãos de fiscalização, a exemplo do metal Alumínio.

\section{AGRADECIMENTOS}

Agradecemos ao CNPq e a Secretaria de Estado da Ciência e Tecnologia (SETEC) pelo auxílio financeiro ao projeto (Convênio CNPq $\mathrm{n}^{\circ}$. 0019/00-07) e concessão de bolsas de iniciação científica (CNPq) e produtividade (CNPq - processo 305657/2009-7).

\section{REFERÊNCIAS}

ALABURDA, J.; NISHIHARA, L. Presença de compostos de nitrogênio em águas de poços. Revista de Saúde Pública, v. 32, n. 2, p. 160-165, 1998. http://dx.doi.org/10.1590/S003489101998000200009

ALVES, N. C.; ODORIZZIA, A. C.; GOULART, F. C. Análise microbiológica de águas minerais e de água potável de abastecimento, Marília, SP. Revista de Saúde Pública, v. 36, n. 6, p. 749-751, 2002. http://dx.doi.org/10.1590/S0034-89102002000700014

AGÊNCIA NACIONAL DE VIGILÂNCIA SANITÁRIA - ANVISA (Brasil). RDC n. 274, de 22 de setembro de 2005. Brasília, 2005. 7 p.

AMERICAN PUBLIC HEALTH ASSOCIATION - APHA; AMERICAN WATER WORKS ASSOCIATION - AWWA; WATER POLLUTION CONTROL FACILITIES - WPCF. Standard methods for the examination of water and wastewater. $20^{\text {th }}$. Edition. Washington, D.C., 2003.

BRANDÃO, I. M. G.; VALSECKI JÚNIOR, A. Análise da concentração de flúor em águas minerais na região de Araraquara, Brasil. Revista Panamericana de Salud Pública, v. 4, n. 4, p. 238-242, 1998. http://dx.doi.org/10.1590/S1020-49891998001000003 
BRASIL. Ministério da Saúde. Portaria 518/GM em 25 de Março de 2004. Estabelece os procedimentos e responsabilidades relativos ao controle e vigilância da qualidade da água para consumo humano e seu padrão de potabilidade, e dá outras providências. Brasília, 2004.

BULCÃO, L. N.; REBELO, M. A. B. Evaluation of the fluoride concentration in mineral water and guaraná-based soft drinks in Manaus, Amazonas. Revista Odonto Ciências, v. 24, n. 3, p. 240-243, 2009.

DI BERNARDO, L. Métodos e técnicas de tratamento de água. Rio de Janeiro: ABES, 1993.

FELSKI, G.; ANAISSI, F. J.; QUINÁIA, S. P. Avaliação da qualidade da água consumida pela população do município de Guarapuava, Paraná. Revista Eletrônica Lato Sensu, ano 3, v. 1, p. 1-25, 2008.

FERNÍCULA, N. G. G.; AZEVEDO, F. A. Metemoglobinemia e nitrato nas águas. Revista de Saúde Pública, v. 15, n. 2, p. 242-248, 1981. http://dx.doi.org/10.1590/S003489101981000200009

FREITAS, M. B.; BRILHANTE, O. M.; ALMEIDA, L. M. Importância da análise de água para a saúde pública em duas regiões do Estado do Rio de Janeiro: enfoque para coliformes fecais, nitrato e alumínio. Cadernos de Saúde Pública, v. 17, n. 3, p. 651660, 2001. http://dx.doi.org/10.1590/S0102-311X2001000300019

HAND, D. J. Statistics: a very short intoduction. Oxford: Oxford University Press, 2008.

LECLERC, H.; MOREAU, A. Microbiological safety of natural mineral water. FEMS Microbiology Reviews, v. 26, n. 2, p. 207-222, 2002. http://dx.doi.org/10.1111/j.15746976.2002.tb00611.x

MAGNUSSON, W. E.; MOURÃO, G. M. Basic statistic in ecological studies: linking the questions to the analyses. Oxford: Oxford University Press, 2009.

MAVRIDOU, A. Study of the bacterial flora of a non-carbonated natural mineral water. Journal of Applied Microbiology, v. 73, n. 4, p. 355-361, 1992. http://dx.doi.org/ 10.1111/j.1365-2672.1992.tb04989.x

MORGANO, M. A.; SCHATTI, A. C.; ENRIQUES, H. A.; MANTOVANI, D. M. B. Avaliação físico-química de águas minerais comercializadas na região de Campinas, SP. Ciência e Tecnologia de Alimentos, v. 22, n. 3, p. 329-343, 2002. http://dx.doi.org/ 10.1590/S0101-20612002000300007

NÓBREGA, M. M. S.; ARAÚJO, A. L. C.; SANTOS, J. P. Avaliação das concentrações de nitrato nas águas minerais produzidas na região da grande Natal. Holos, v. 3, p. 4-25, 2008 .

PONTARA, A. V.; OLIVEIRA, C. D. D.; BARBOSA, A. H.; SANTOS, R. A.; PIRES, R. H.; MARTINS, C. H G. Microbiological monitoring of mineral water commercialized in Brazil. Brazilian Journal of Microbiology, v. 42, n. 2, p. 554-559, 2011. http://dx.doi.org/10.1590/S1517-83822011000200020

RESENDE, A.; PRADO, C. N. Perfil microbiológico da água mineral comercializada no Distrito Federal. Revista de Saúde e Biologia, v. 3, n. 2, p. 16-22, 2008. 
CUNHA, H. F. A.; LIMA, D. C. I.; BRITO, P. N. de F.; CUNHA, A. C.da; SILVEIRA JUNIOR, A. M. da; BRITO, D. C. Qualidade físico-química e microbiológica de água mineral e padrões da legislação. Ambi-Agua, Taubaté, v. 7, n. 3, p. 155-165, 2012. (http://dx.doi.org/10.4136/ambi-agua.908)

SILVA, F. V.; KAMOGAWA, M. Y.; FERREIRA, M. M. C.; NÓBREGA, J. A.; NOGUEIRA, A. R. A. Discriminação geográfica de águas minerais do Estado de São Paulo através da análise exploratória. Eclética Química, v. 27, n. especial, p. 1-9, 2002. http://dx.doi.org/10.1590/S0100-46702002000200008

SILVA, M. O. S. A. Análises físico-químicas para controle das estações de tratamento de esgoto. São Paulo: CETESB; 1997.

WILCOX, R. R. Basic statistics: understanding conventional methods and modern 23. insights. Oxford: Oxford University Press, 2009.

WYNN, E.; RAETZ, E.; BURCKHARDT, P. The composition of mineral waters sourced from Europe and North America: composition of mineral water optimal for bone. Britsh Journal of Nutrition, v. 101, n. 8, p. 1195-1199, 2009. http://dx.doi.org/ 10.1017/S0007114508061515 


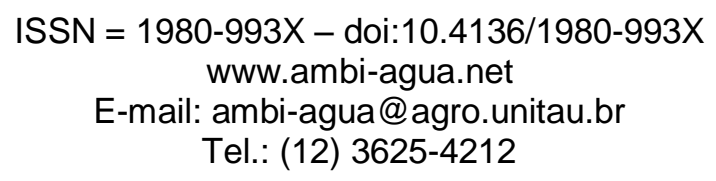

\title{
Caracterização química da água subterrânea em área ocupada por cemitério: uso da técnica de espectrometria de fluorescência de raios-X por energia dispersiva (EDXRF)
}

\author{
(http://dx.doi.org/10.4136/ambi-agua.911) \\ Pedro Daniel da Cunha Kemerich ${ }^{1}$; José Luiz Silvério da Silva ${ }^{2}$; Guilherme Barros ${ }^{3}$; \\ Willian Fernando de Borba ${ }^{3}$; Fernando Ernesto Ucker ${ }^{4}$; Cristian Vargas Foletto ${ }^{5}$ \\ ${ }^{1}$ Universidade Federal de Santa Maria, Frederico Westphalen-RS, \\ e-mail: eng.kemerich@yahoo.com.br, \\ ${ }^{2}$ Universidade Federal de Santa Maria, Santa Maria-RS, \\ e-mail: silverioufsm@gmail.com, \\ ${ }^{3}$ Universidade Federal de Santa Maria, Frederico Westphalen-RS, \\ e-mail: guilherme_barrosp@hotmail.com, borba_willian@hotmail.com, \\ ${ }^{4}$ Universidade Federal de Goiás, Goiânia-GO, \\ e-mail: ferucker@hotmail.com, \\ ${ }^{5}$ Centro Universitário Franciscano, Santa Maria-RS, \\ e-mail: cv.foletto@bol.com.br.
}

\section{RESUMO}

Geralmente, o enterro do ser humano pode contribuir para a poluição da água subterrânea, quando do contato com o lixiviado gerado a partir da decomposição dos corpos na zona não saturada do subsolo. O monitoramento desse tipo de atividade foi realizado neste trabalho, que teve como objetivo a determinação da qualidade da água subterrânea em zona ocupada por cemitério. Para isto, utilizou-se a técnica de Espectrometria de Fluorescência de Raios-X por Energia Dispersiva (EDXRF) para a caracterização química da água subterrânea. Foram construídos cinco poços de monitoramento, de acordo com os padrões brasileiros. Foi estimada a flutuação do lençol freático, a superfície potenciométrica e as concentrações dos elementos cálcio, cobre, ferro, fósforo e silício. O Nível da água apresentou-se bastante superficial, variando entre 0,48 a $0,95 \mathrm{~m}$ no período seco. A concentração de cálcio variou entre 4,65 a $17,85 \mathrm{mg} \mathrm{L}^{-1}$, cobre entre $0,02 \pm 0,29 \mathrm{mg} \mathrm{L}^{-1}$, ferro variou de 0,57 a $15,96 \mathrm{mg} \mathrm{L}^{-1}$, fósforo entre 12 a 13,98 $\mathrm{mg} \mathrm{L}^{-1}$, e por último o silício, variando entre 35,55 a 79,12 $\mathrm{mg} \mathrm{L}^{-1}$. Conclui-se que o uso da técnica de EDXRF demonstrou ser rápida e eficiente para quantificação de alguns constituintes em águas subterrâneas coletadas em poços de monitoramento sob a influência de cemitério em terreno silto-argiloso.

Palavras-chave: lixiviado, poços de monitoramento, constituintes.

\section{Chemical characterization of groundwater in the area occupied by the cemetery: use of fluorescence spectrometry X-ray energy dispersive (EDXRF)}

\begin{abstract}
Generally, the burial of human corpses can contribute to groundwater pollution by the contact of leachate generated from the decomposition of bodies in the unsaturated zone of the subsoil. This process has been investigated in this work that aimed to determine the overall
\end{abstract}


KEMERICH, P. D. C.; SILVA, J. L. S.; BARROS, G.; BORBA, W. F.; UCKER, F. E.; FOLETTO, C. V. Caracterização química da água subterrânea em área ocupada por cemitério: uso da técnica de espectrometria de fluorescência de raios-X por energia dispersiva (EDXRF). Ambi-Agua, Taubaté, v. 7, n. 3, p. 166-182, 2012. (http://dx.doi.org/10.4136/ambi-agua.911)

quality of groundwater in the zone occupied by the cemetery. The fluorescence spectrometry X-ray Energy Dispersive (EDXRF) technique was used for groundwater chemical characterization. Five monitoring wells were constructed according to Brazilian norms. The water level fluctuation, the potentiometric surface and the concentrations of the elements calcium, copper, iron, phosphorus and silicon were estimated. The water level appeared quite shallow, ranging between 0.48 to $0.95 \mathrm{~m}$ in the dry season. The concentrations range for calcium varied from 4.65 to $17.85 \mathrm{mg} \mathrm{L}^{-1}$, for copper $0.02 \pm 0.29 \mathrm{mg} \mathrm{L}^{-1}$, iron 0.57 to 15.96 $\mathrm{mg} \mathrm{L}^{-1}$, phosphorus 12.00 to $13.98 \mathrm{mg} \mathrm{L}^{-1}$, and silicon 35.55 to $79.12 \mathrm{mg} \mathrm{L}^{-1}$. It is concluded that the use of EDXRF techniques proved to be rapid and efficient for monitoring the constituents in the groundwater collected in wells under the influence of graveyard in silt-clay soil.

Keywords: leachate, monitoring wells, constituents.

\section{INTRODUÇÃO}

Dos aproximados 3\% de água doce no planeta, cerca de $22 \%$ são águas subterrâneas. Com a população aumentada em seis vezes e consumo de água doce no planeta triplicou nos últimos 50 anos, os recursos hídricos disponíveis estão com sua qualidade comprometida e, como consequência, a utilização das águas das reservas subterrâneas vem aumentando (Vasconcelos et al., 2006).

Alguns impactos ambientais relacionados às águas subterrâneas podem ser provenientes de cemitérios, considerando que na construção da maioria destas necrópoles não são levados em conta estudos geológicos e hidrogeológicos. Desta forma, estas instalações podem representar certo risco de contaminação durante a decomposição dos corpos. A localização dos cemitérios ocorre, preferencialmente, em áreas afastadas do centro urbano, porém hoje é possível encontrar cemitérios totalmente integrados à malha urbana (Migliorini, 2002).

Os cemitérios, como qualquer outra instalação que afete as condições naturais do solo e das águas subterrâneas, são classificados como atividade com risco de contaminação ambiental. A razão disso é que o solo em que estão instalados funciona como um filtro das impurezas depositadas sobre ele. O processo de decomposição de corpos libera diversas substâncias que constituem o organismo humano, como os metais, somando-se a isso nos caixões são utilizados diferentes utensílios metálicos ou não que podem liberar substâncias para a o solo e a água subterrânea (Kemerich et al., 2012).

Como principal causa de poluição nos cemitérios, durante a decomposição dos cadáveres é liberado um líquido denominado necrochorume. Esta é uma solução aquosa rica em sais minerais e substâncias orgânicas degradáveis, de tonalidade castanho-acinzentada, viscosa, de cheiro forte e com grau variado de patogenicidade (Silva et al., 2006).

Com intuito da preservação do meio ambiente entrou em vigor a Resolução No 335/2003 do Conselho Nacional do Meio Ambiente (CONAMA), sobre as condições mínimas necessárias para o licenciamento ambiental de cemitérios no Brasil, alterada pela Resolução CONAMA N 368/ 2006. (CONAMA, 2003; 2006)

A utilização de técnicas para determinação multielementar tem se desenvolvido muito nos últimos anos devido ao aumento de análises químicas para uma grande variedade de matrizes. Em função disto, a Fluorescência de Raios-X de Energia Dispersiva (EDXRF) mostra-se como uma técnica muito versátil, podendo ser aplicada em diversas amostras, incluindo as de estado sólido e líquidas, sem necessitar de tratamento exaustivo para a preparação destas matrizes, e também oferecendo a grande vantagem de ser um método analítico não-destrutivo (Schimidt et al., 1999). Wastowski et al. (2010) aplicaram o método para caracterização dos níveis de 
KEMERICH, P. D. C.; SILVA, J. L. S.; BARROS, G.; BORBA, W. F.; UCKER, F. E.; FOLETTO, C. V. Caracterização química da água subterrânea em área ocupada por cemitério: uso da técnica de espectrometria de fluorescência de raios-X por energia dispersiva (EDXRF). Ambi-Agua, Taubaté, v. 7, n. 3, p. 166-182, 2012. (http://dx.doi.org/10.4136/ambi-agua.911)

elementos químicos em solos concluindo ser esta técnica como uma importante alternativa, com crescente aplicação na identificação mineralógica de solos, rochas, cerâmica e líquidos, uma vez que permite a determinação simultânea ou seqüencial da concentração de todos os elementos enquadrados entre o Sódio (Na) e o Urânio (U). Como vantagens relatam que não há necessidade de destruição da amostra, ou seja de modo instrumental, sem nenhum prétratamento químico e podendo atingir limites de detecção da ordem de 1 a 20 ppm. Pataca et al. (2005) aplicaram com sucesso a técnica (EDXRF) na determinação de arsênio em águas contaminadas.

Com base no tema exposto, este trabalho tem como objetivo geral determinar a qualidade da água subterrânea em área ocupada por cemitério em Zona de Afloramentos do Sistema Aquífero Guarani/SAG. E como objetivos específicos: (a) Utilizar a técnica de Espectrometria de Fluorescência de Raios-X por Energia Dispersiva (EDXRF) na caracterização química da água subterrânea em poços de monitoramento; (b) Espacializar as concentrações dos parâmetros analisados na área de influência do cemitério; (c) Comparar os resultados com as normas e legislações vigentes Portaria Ministério da Saúde №518/2004, Resoluções CONAMA No396/2008 e No420/2009 (CONAMA, 2008; 2009).

\section{MATERIAIS E MÉTODO}

\subsection{Caracterização Geológica da Área de Estudo}

O local de estudo situa-se no perímetro urbano de Santa Maria, no Estado do Rio Grande do Sul, está localizado na Borda de Bacia do Paraná na Depressão Central. Esta transição com o Planalto é constituída de acordo com Gasparetto et al. (1990) por rochas sedimentares clásticas arenosas pertencentes às Formações Rosário do Sul, Santa Maria (Membro Passo das Tropas e Membro Alemoa), Caturrita e Botucatú.

Essa sequência de rochas sedimentares clásticas é constituída por interdigitações de: arenitos, siltitos, argilitos, concreções calcárias (calcretes), além de apresentar, localmente, em algumas dessas formações um conteúdo de répteis fósseis. Essas unidades hidroestratigráficas podem constituir Aquíferos, Aquitardos ou Aquicludos (Maciel Filho, 1990; CPRM, 1994; Machado, 2005).

Em termos hidrogeológicos o Município de Santa Maria assenta-se sobre importantes aquíferos na área urbana, os quais estão relacionados às unidades Hidroestratigráficas Sanga do Cabral, Santa Maria (Aquífero Passo das Tropas I e II), Caturrita e Botucatu (Machado, 2005). Todos associados com arenitos, com potencial aquífero, mas nem sempre preenchidos por água. Normalmente as águas subterrâneas são doces, potáveis, porém algumas se apresentam salobras com elevado conteúdo de sais dissolvidos (CPRM, 1994; Fachin, 2003 e 2005).

A Unidade Hidroestratigráfica Alemoa é constituída por camadas interdigitadas de siltitosargilosos maciços, concreções carbonáticas e localmente forma sítios de fósseis répteis do Período Triássico. Constitui-se basicamente por lamitos impermeáveis que funcionam como Aquicludo, não armazenando nem transmitindo (Maciel Filho, 1990; CPRM, 1994). Já Böger e Kowalcyk (1993) ainda incluíram nesta unidade a ocorrência de fácies de areias muito finas, as quais podem ser aquíferos. Os argilominerais presentes são principalmente da família das esmectitas expansiva e também formam interestratificados I-S (Silvério da Silva et al., 1990; 2010; Silvério da Silva, 1997). A estrutura originalmente maciça devido à exposição aos agentes intempéricos torna-se fissurada, apresentando fissilidade, o que pode vir a facilitar o fluxo de contaminantes gerados na superfície do terreno. Maciel Filho (1990) 
KEMERICH, P. D. C.; SILVA, J. L. S.; BARROS, G.; BORBA, W. F.; UCKER, F. E.; FOLETTO, C. V. Caracterização química da água subterrânea em área ocupada por cemitério: uso da técnica de espectrometria de fluorescência de raios-X por energia dispersiva (EDXRF). Ambi-Agua, Taubaté, v. 7, n. 3, p. 166-182, 2012. (http://dx.doi.org/10.4136/ambi-agua.911)

considerou esta unidade impermeável em sua capa superior, enquanto que os siltitos e arenitos argilosos da base são semi-permeáveis. Após períodos chuvosos pode haver alguma acumulação de água nas fissuras associadas à zona vadosa, isto não significa o nível freático da unidade, o qual, a rigor não se forma, uma vez que a unidade forma Aquicludo. Machado (2005) estimou uma espessura da ordem de $150 \mathrm{~m}$.

\subsection{Cemitério Santa Rita de Cássia}

O cemitério estudado nesta pesquisa é do tipo parque jardim, denominado Santa Rita de Cássia, fundado no mês de outubro de 1970, junto a rodovia RS 509, km 4 no Município de Santa Maria - RS. Ele conta atualmente com 6000 sepulturas e vem recebendo em média 15 corpos por mês. Situa-se em área de expansão urbana contíguo ao Cemitério Municipal São José, no Bairro Pé de Plátano. Este se localiza em área de afloramentos e zona de recarga do SAG, mais especificamente na Unidade Hidroestratigráfica Alemoa (Machado, 2005).

\subsection{Construção dos Poços de Monitoramento}

A locação dos poços de monitoramento obedeceu à conformação do terreno, à disposição das sepulturas e a direção do fluxo subterrâneo que em condições normais acompanha a superfície do terreno formado por uma vertente suave. Foram construídos cinco poços de monitoramento denominados PM1, PM2, PM3, PM4 e PM5. Sendo que o PM1 encontra-se na cota altimétrica mais elevada, distante das sepulturas sendo por isso considerado o branco ou controle (background), seguido dos demais, em cotas altimétricas decrescentes, em direção a rodovia RS-509.

Os poços de monitoramento foram construídos com o auxílio de um trado mecânico pelo método rotativo direto, com broca de $300 \mathrm{~mm}$ de diâmetro conforme visualizado na Figura 1a, seguindo-se as orientações da NBR 15.495 (ABNT, 2007), conforme ilustra a figura 1b, selo sanitário (Figura 1c), constituído de argamassa de cimento e areia buscando-se evitar a contaminação da água dos poços pelo escoamento superficial. Cada poço recebeu uma tampa e rotulagem contendo sua numeração e data de construção.

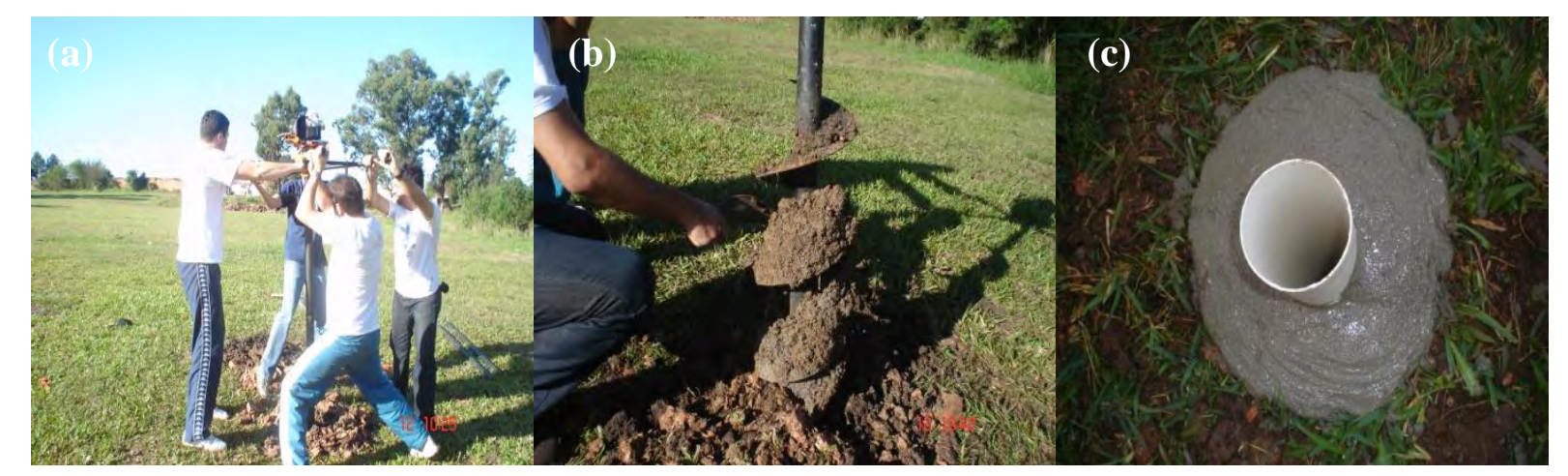

Figura 1. Utilização do trado mecânico para construção dos poços de monitoramento.

\subsection{Método de Amostragem}

Durante a realização das amostragens, as amostras de água foram retiradas dos poços de monitoramento com um amostrador dotado de válvula anti-retorno, previamente esterilizado. Para o acondicionamento foram utilizadas garrafas plásticas de $2000 \mathrm{~mL}$, devidamente rotuladas e identificadas de acordo com o número dos poços estudados. 
KEMERICH, P. D. C.; SILVA, J. L. S.; BARROS, G.; BORBA, W. F.; UCKER, F. E.; FOLETTO, C. V. Caracterização química da água subterrânea em área ocupada por cemitério: uso da técnica de espectrometria de fluorescência de raios-X por energia dispersiva (EDXRF). Ambi-Agua, Taubaté, v. 7, n. 3, p. 166-182, 2012. (http://dx.doi.org/10.4136/ambi-agua.911)

Foram realizadas três coletas, sendo os valores apresentados das médias das campanhas do dia 05/03/2011, 10/03/2011, 17/03/2011. Nesses dias, foram medidos os níveis estáticos dos poços com o auxílio de um freatímetro sonoro dotado de cabo de $100 \mathrm{~m}$. Todas as leituras foram realizadas em triplicata, buscando-se minimização de possíveis erros na manipulação da amostra.

\subsection{Método de Análise}

Após a concentração da amostra, as análises foram realizadas utilizando-se um Espectrômetro de Fluorescência de Raios-X por Energia Dispersiva (EDXRF), do modelo Shimadzu EDX-720. As seguintes condições de operação do equipamento foram selecionadas: tensão do tubo de $15 \mathrm{keV}$ ( $\mathrm{Na}$ a Sc) e $50 \mathrm{keV}$ (Ti a U) com corrente no tubo de $184 \mu \mathrm{A}$ e $25 \mu \mathrm{A}$ respectivamente, colimador de $10 \mathrm{~mm}$, tempo morto do detector de $40 \%$ e $39 \%$, sob vácuo e detector de $\mathrm{Si}(\mathrm{Li})$ refrigerado com nitrogênio líquido, (Pataca et al., 2005), com tempo real de integração de 200 segundos, tempo este diferente do usado por Pataca et al. (2005) e Wastowski et al. (2010) que utilizaram $300 \mathrm{~s}$. Wastowski et al. (2010) sugerem para corrigir os efeitos de absorção e calibrar as linhas espectrais dos elementos analisados, utilizar o padrão A750. Este padrão de calibração utilizado o qual foi fornecido junto com o Modelo EDX-720, trata-se de uma liga metálica composta por Alumínio ( $\mathrm{Al}$ ), Estanho (Sn), Magnésio $(\mathrm{Mg})$, Ferro $(\mathrm{Fe})$ e Cobre $(\mathrm{Cu})$. Desta maneira é possível determinar-se a composição do material analisado.

As amostras foram agitadas por 30 segundos buscando-se a total homogeneização, após transferiu-se $100 \mathrm{~mL}$ com o uso de pipeta volumétrica $(50 \mathrm{~mL})$ para os tubos de evaporação. As amostras foram evaporadas a uma temperatura de $70{ }^{\circ} \mathrm{C}$ em Banho Maria utilizando-se injeção de ar comprimido (25 a $30 \mathrm{lbt} \mathrm{pol}^{-2}$ ) até $1 \mathrm{~mL}$ obtendo-se uma concentração na ordem de 100 vezes, após a amostra concentrada foi transferida para um balão volumétrico de $10 \mathrm{~mL}$ e aferiuse com água destilada.

O método analítico usado é denominado método dos Parâmetros Fundamentais (FP), este método permite a obtenção da curva de sensibilidade do equipamento para cada elemento de interesse, quando uma amostra de composição química conhecida é submetida a parâmetros instrumentais bem definidos (Bona et al., 2007; Wastowski et al., 2010). A curva de sensibilidade do equipamento relaciona a intensidade fluorescente teórica calculada e a medida para cada elemento (Bona et al., 2007). Wastowski et al. (2010) afirmaram que para análises de rotina em massa, se as amostras de calibração estão ausentes, o método (FP) pode fornecer resultados relativamente precisos e quantitativos (Han et al., 2006).

Adotou-se a faixa de concentração esperada para cada constituinte avaliado nesta pesquisa sendo: Maiores os que apresentam concentrações superiores a $5 \mathrm{mg} \mathrm{L}^{-1}\left(\mathrm{Ca}^{2+}\right.$ e $\left.\mathrm{SiO}_{2}\right)$; Menores concentrações entre 0,01 até $10 \mathrm{mg} \mathrm{L}^{-1}(\mathrm{Fe})$ e; os traços com concentração inferior a $0,10 \mathrm{mg} \mathrm{L}^{-}$ ${ }^{1}$ (Cu, Zn, Ti, Mg, P) (Davies e De Wiest, 1966).

\subsection{Espacialização dos Resultados}

Para a confecção dos cartogramas, foi utilizado o programa SURFER 9 (Golden Software, 2004); como método de interpolação matemática foi utilizado krigagem (Landim e Sturaro, 2002). Logo após, foram espacializadas as informações de interesse com o uso da opção Post Map.

O cartograma da superfície potenciométrica recebeu sobreposição com o uso do comando Grid Vector Map, gerando-se, assim, uma representação do fluxo preferencial da água subterrânea (linhas de fluxo). 
KEMERICH, P. D. C.; SILVA, J. L. S.; BARROS, G.; BORBA, W. F.; UCKER, F. E.; FOLETTO, C. V. Caracterização química da água subterrânea em área ocupada por cemitério: uso da técnica de espectrometria de fluorescência de raios-X por energia dispersiva (EDXRF). Ambi-Agua, Taubaté, v. 7, n. 3, p. 166-182, 2012. (http://dx.doi.org/10.4136/ambi-agua.911)

\section{RESULTADOS E DISCUSSÃO}

A Tabela 1 ilustra aspectos relativos à flutuação dos níveis da água subterrânea (N.E.), a superfície potenciométrica (SP obtida pela relação entre a cota altimétrica na boca do poço e o nível da água no poço considerado. A tabela ilustra também as concentrações médias dos elementos analisados na área de estudo.

Tabela 1. Parâmetros dos poços de monitoramento (N.E. e S.P.) e concentrações dos elementos químicos analisados.

\begin{tabular}{|c|c|c|c|c|c|c|c|c|c|c|c|c|}
\hline Ponto & $\mathbf{N E}$ & SP & $\mathrm{Ca}^{2+}$ & $\mathrm{Cu}^{+}$ & $\mathrm{Si}^{4+}$ & $\mathbf{P}^{3+}$ & $\mathrm{Fe}^{+}$ & $\mathbf{S}^{2+}$ & $\mathbf{T i}^{2+}$ & $\mathbf{K}^{+}$ & $\mathrm{Mn}^{2+}$ & $\mathrm{Sc}^{3+}$ \\
\hline & \multicolumn{2}{|c|}{ (m) } & \multicolumn{10}{|c|}{$\left(\mathrm{mg} \mathrm{L} \mathbf{L}^{-1}\right)$} \\
\hline PM1 & 0,48 & 127,52 & 81734,80 & 3016,32 & 455896,67 & 138889,06 & 17498,55 & 100221,91 & <L.D. & 12250,36 & $<$ L.D. & $<$ L.D. \\
\hline PM2 & 0,79 & 120,21 & 46557,40 & 3040,78 & 646481,40 & $<$ L.D. & 36404,71 & 99251,82 & 85,62 & 36975,83 & 47,00 & $<$ L.D. \\
\hline РM3 & 0,95 & 111,05 & 146581,81 & 2863,56 & 355535,73 & 139770,56 & 14076,23 & 101300,86 & $<$ L.D. & 6812,13 & $<$ L.D. & 271,16 \\
\hline PM4 & 0,87 & 106,13 & 178539,36 & 3122,00 & 791280,02 & 122573,97 & 159600,26 & 133147,14 & 182,92 & 71519,82 & 25,09 & $<$ L.D. \\
\hline PM5 & 0,66 & 102,34 & 129715,21 & 2826,37 & 573289,28 & 134651,98 & 5707,05 & 97124,73 & $<$ L.D. & 13346,08 & $<$ L.D. & $<$ L.D. \\
\hline PT & $*<$ L.D. & <L.D. & 48565,28 & 2673,43 & $<$ L.D. & 119972,12 & <L.D. & 124406,14 & <L.D. & <L.D. & $<$ L.D. & <L.D. \\
\hline
\end{tabular}

Nota: $*$ LD = menor do que o Limite de Detecção do aparelho Shimadzu Shimadzu EDX-720.

A Figura 2 (a) apresenta a localização dos poços de monitoramento (PM1, PM2, PM3, PM4 e PM5) e do poço tubular (PT). O poço tubular está localizado na parte mais elevada do empreendimento, em cota altimétrica $134,2 \mathrm{~m}$ e tem uma profundidade de $170 \mathrm{~m}$. Este serve no abastecimento de água para regar flores, de um morador, bem como na construção das carneiras de concreto (Garcia, 2004).

Os poços localizam-se nas cotas altimétricas (PM1) $128 \mathrm{~m}$, (PM2) em $121 \mathrm{~m}$, (PM3) em 112 m, (PM4) em 107 m e o (PM5) a 103 m. O valor médio das cotas altimétricas foi de 117,53 $\mathrm{m}$, variando de $103 \mathrm{~m}$ a $134,2 \mathrm{~m}$, sendo os maiores valores localizados a sudeste, e, representado pelos poços (PM1), (PM2) e (PT).

A Figura 2 (b) ilustra a variabilidade espacial do nível da água dos poços de monitoramento nos valores variou entre $0,48 \mathrm{~m}$ e $0,95 \mathrm{~m}$. O maior valor foi no PM3, e o menor valor no PM1, tendo-se uma média de $0,75 \mathrm{~m}$, apresentando os maiores valores a sudoeste. Estes níveis da água são bastante rasos e podem estar conflitando com o estipulado na Resolução CONAMA N³68/2006, em seu Art. 5, "I- o nível inferior das sepulturas deverá estar a uma distância de pelo menos um metro e meio acima do mais alto nível do lençol freático, medido no fim da estação das cheias. O Estado do Rio Grande do Sul não apresenta estação das cheias, que de acordo com CPRM (1994) à folha de Santa Maria, domina o clima do tipo cfa de Köppen, caracterizado como subtropical, úmido, com chuvas durante todos os meses. A precipitação média anual é de $1769 \mathrm{~mm}$ e os meses de maio, junho e setembro são os mais chuvosos, enquanto que novembro e dezembro são os mais secos.

Os valores de níveis de água subterrânea rasos = foram, também, encontrados em sete poços de monitoramento estudados por Chaves e Silvério da Silva (2008) e Chaves (2009) na cidade de Santa Maria, próximo a área deste estudo. Estes autores encontraram, dentre os poços estudados, uma variação anual da ordem de 0,25 a $3 \mathrm{~m}$, penetrando formações Cenozóicas e a Unidade Hidroestratigráfica Alemoa. 
KEMERICH, P. D. C.; SILVA, J. L. S.; BARROS, G.; BORBA, W. F.; UCKER, F. E.; FOLETTO, C. V. Caracterização química da água subterrânea em área ocupada por cemitério: uso da técnica de espectrometria de fluorescência de raios-X por energia dispersiva (EDXRF). Ambi-Agua, Taubaté, v. 7, n. 3, p. 166-182, 2012. (http://dx.doi.org/10.4136/ambi-agua.911)

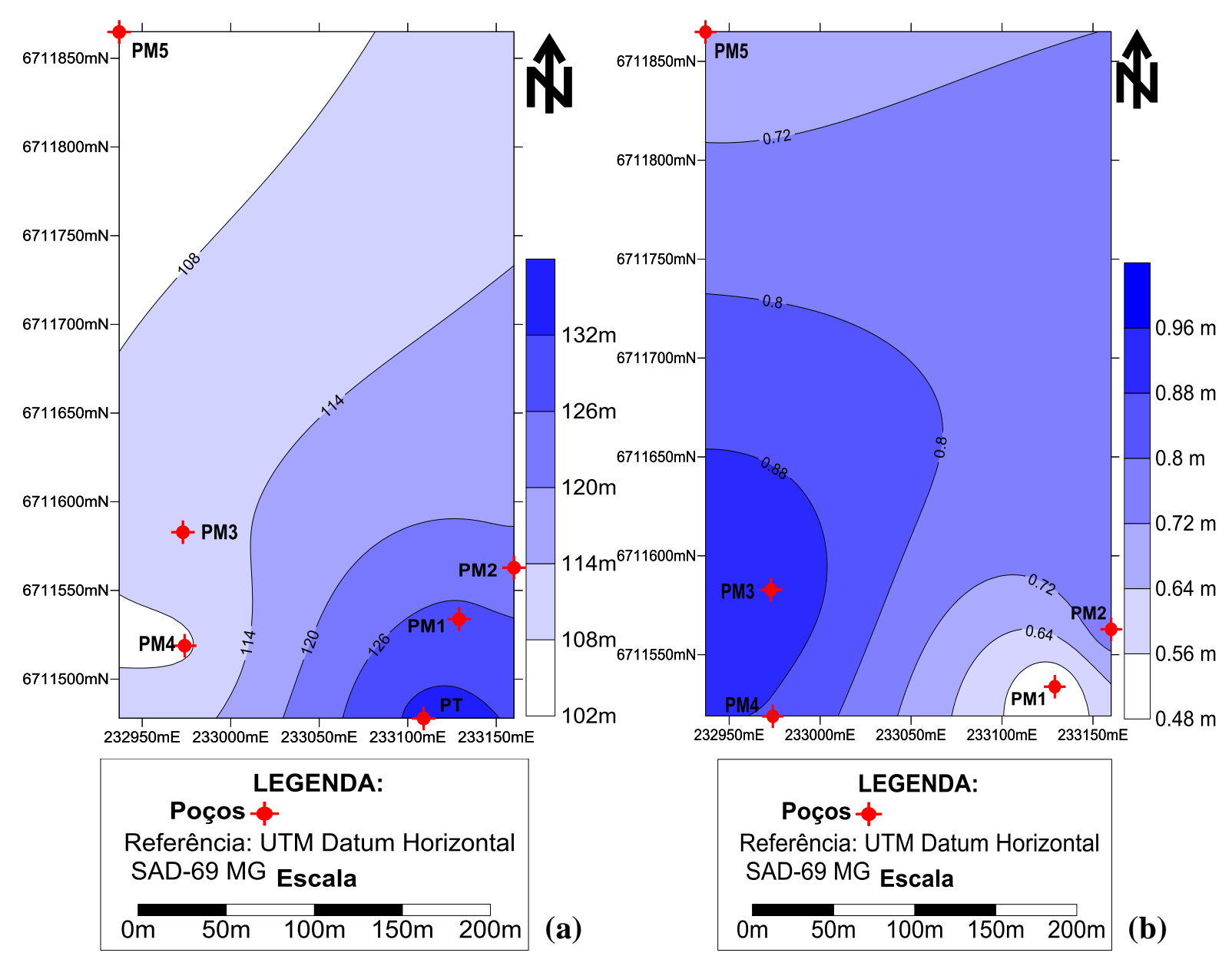

Figura 2. (a) Cartograma da localização e cota altimétrica dos poços e (b) Nível da Água dos poços.

A Figura 3 (a) ilustra a superfície potenciométrica, onde os valores variaram entre 102,3 a 127,5 m próximo da Rodovia Estadual RS-509. Os maiores valores ocorreram na porção sudeste do cemitério (PM1 e PM2), tendo-se uma média entre os poços de 113,4 $\pm 10,3 \mathrm{~m}$. Estima-se que os poços localizados nas cotas altimétricas mais baixas, estiveram sob maior influência do fluxo subterrâneo de direção Sul para Norte e Noroeste. Podendo ter influenciado nas concentrações dos constituintes maiores $\left(\mathrm{Ca}^{2+}\right)$ e $\left(\mathrm{SiO}_{2}\right)$, menores $(\mathrm{Fe})$ e os traços analisados.

Os valores de concentração cálcio $\left(\mathrm{Ca}^{2+}\right)$ variaram entre $4,65 \mathrm{mg} \mathrm{L}^{-1}$ e $17,85 \mathrm{mg} \mathrm{L}^{-1}$ de $\mathrm{Ca}^{2+}$, o valor médio foi de $10,53 \pm 5,46 \mathrm{mg} \mathrm{L}^{-1}$. Os maiores valores de concentração encontrados localizam-se à sudoeste, tendo maior valor no poço PM3, como mostra a Figura 3 (b).

O cálcio é um elemento maior na maioria das águas e na crosta terrestre. É comum precipitar-se como carbonato de cálcio $\left(\mathrm{CaCO}_{3}\right)$ em água com o $\mathrm{pH}$ maior ou igual a 8,2. Comumente apresenta-se sob a forma de bicarbonato $\left(\mathrm{HCO}_{3}{ }^{-}\right)$. A solubilidade do bicarbonato de cálcio aumenta na presença de sais de Sódio $\left(\mathrm{Na}^{+}\right)$e Potássio $\left(\mathrm{K}^{+}\right)$(Mestrinho, 2008).

Verificou-se que tanto a Organização Mundial da Saúde, quanto a Portaria No 518/2004 do Ministério da Saúde, bem como a Resolução No 396/2008, não fazem referência à concentração de cálcio isoladamente, mas este elemento aparece associado à dureza total da água. Alguns autores como Feitosa e Manoel Filho (1997) apresentam a faixa de variação para as águas subterrâneas naturais, com teores variando entre 10 e $100 \mathrm{mg} \mathrm{L}^{-1}$. 
KEMERICH, P. D. C.; SILVA, J. L. S.; BARROS, G.; BORBA, W. F.; UCKER, F. E.; FOLETTO, C. V. Caracterização química da água subterrânea em área ocupada por cemitério: uso da técnica de espectrometria de fluorescência de raios-X por energia dispersiva (EDXRF). Ambi-Agua, Taubaté, v. 7, n. 3, p. 166-182, 2012. (http://dx.doi.org/10.4136/ambi-agua.911)

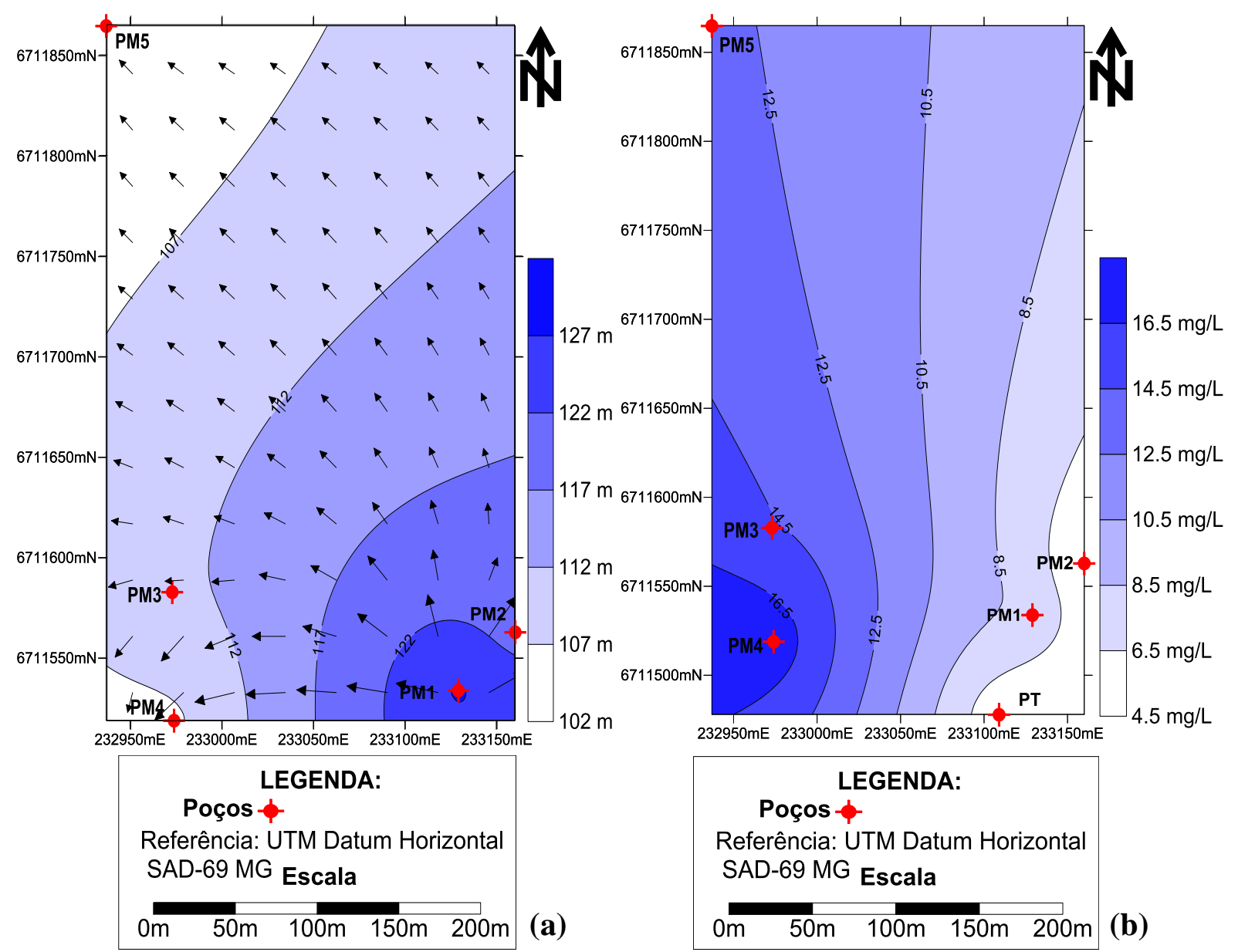

Figura 3. (a) Cartograma da superfície potenciométrica e (b) Concentração de Cálcio na água dos poços.

Kemerich (2008) e Kemerich et al. (2011) relataram em estudo sobre a qualidade da água subterrânea no Bairro Nossa Senhora do Perpétuo Socorro no Município de Santa Maria-RS situado em zona de afloramento do Sistema Aquífero Guarani/SAG. Analisaram cerca de 54 amostras de captações através de poços tubulares, escavados de grande diâmetros e nascentes. Destes, apenas $22,72 \%$ situaram-se entre concentrações 10 e $100 \mathrm{mg} \mathrm{L}^{-1} \mathrm{de} \mathrm{Ca}^{2+}$, sendo que apenas um poço escavado ultrapassou os $100 \mathrm{mg} \mathrm{L}^{-1}$, tendo como valor determinado $241 \mathrm{mg} \mathrm{L}^{-}$ ${ }^{1}$, associado a um $\mathrm{pH}$ do meio levemente ácido de 6,8 .

Os valores de silício variaram entre $35,55 \mathrm{mg} \mathrm{L}^{-1}$ no poço (PM4) e 79,51 $\mathrm{mg} \mathrm{L}^{-1}$ (PM3), tendo-se como valor médio de $56,45 \mathrm{mg} \mathrm{L}^{-1}$. Não foi detectada silício na amostra proveniente do poço Tubular (PT). A Figura 4 (a) ilustra a distribuição das concentrações de silício nos poços amostrados. Silvério da Silva et al. (2006) encontraram valores de concentração da ordem 25 a $98,19 \mathrm{mg} \mathrm{L}^{-1}$ estudando cerca de 22 captações de águas subterrâneas em poços de abastecimento do SAG na fronteira do Brasil e do Uruguai. Na água do Rio Quarai foi obtida uma concentração de $27,95 \mathrm{mg} \mathrm{L}^{-1}$.

Os valores de Ferro variaram entre 0,57 e $15,96 \mathrm{mg} \mathrm{L}^{-1}$, com valor médio 4,67 $\pm 6,41 \mathrm{mg}$ $\mathrm{L}^{-1}$, sendo que os maiores valores apresentaram-se à sudoeste (PM3) conforme ilustrado na Figura 4 (b). 
KEMERICH, P. D. C.; SILVA, J. L. S.; BARROS, G.; BORBA, W. F.; UCKER, F. E.; FOLETTO, C. V. Caracterização química da água subterrânea em área ocupada por cemitério: uso da técnica de espectrometria de fluorescência de raios-X por energia dispersiva (EDXRF). Ambi-Agua, Taubaté, v. 7, n. 3, p. 166-182, 2012. (http://dx.doi.org/10.4136/ambi-agua.911)

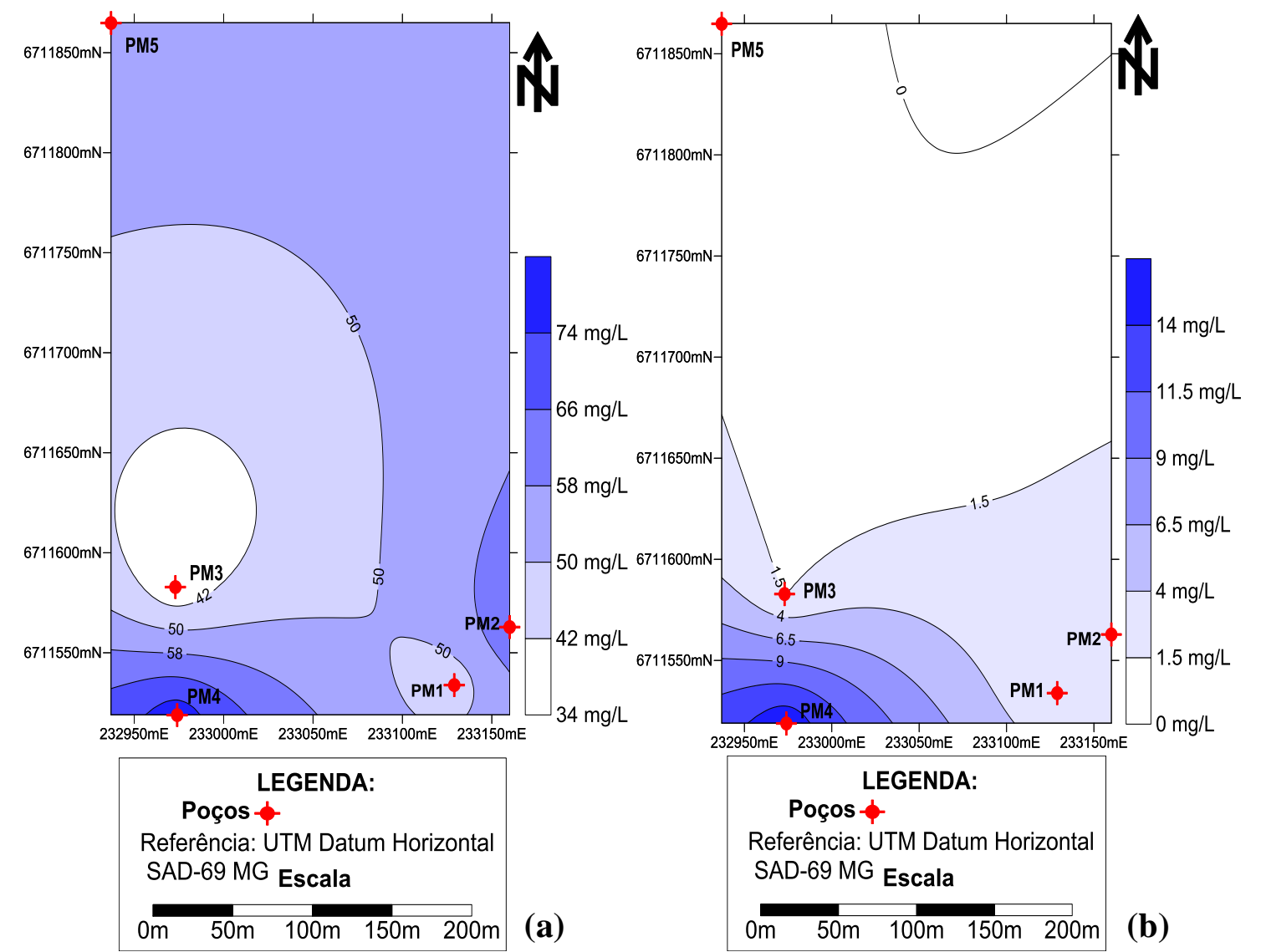

Figura 4. (a) Cartograma da concentração de Silício (Si) e (b) Ferro $\left(\mathrm{Fe}^{2+}\right)$ na água dos poços.

O Ferro é um elemento persistentemente presente em quase todas as águas subterrâneas em teores abaixo de $0,3 \mathrm{mg} \mathrm{L}^{-1}$. Águas com alto conteúdo de Fe, ao saírem da captação por poços são incolores, mas ao entrarem em contato com o oxigênio do ar tornam-se amareladas, o que lhes confere uma aparência nada agradável. Este fenômeno natural ocorre em algumas captações na região de Santa Maria, em zona de afloramento do SAG.

O Ferro, como um importante constituinte das águas subterrâneas, tem origem, principalmente, em compostos minerais tais como os aluminosilicatos (piroxênios, anfibólios, biotita) e/o sulfetos, como a pirita (Davies e De Wiest, 1991; Lemes et al., 2003). Uma água subterrânea onde os níveis de Ferro são elevados, numa primeira avaliação, pode ser preterida a outras águas, até contaminadas com coliformes, em função da cor e do gosto desagradável em decorrência da presença do elemento (Unesco et al., 1992).

A Figura 5 (a) apresenta as concentrações de cobre na água subterrânea coletada nos poços de monitoramento e poço Tubular (PT). O poço PM3 apresentou a maior concentração de $\mathrm{Cu}$, e o menor valor detectado pertence ao poço tubular $0,31,0,26$ e $0,26 \mathrm{mg} \mathrm{L}^{-1}$ respectivamente. Este apresenta um pH alcalino de 8,0, com uma Condutividade Elétrica de cerca de $180 \mu \mathrm{S} \mathrm{cm}^{-}$ ${ }^{1}$ e um concentração de Sólidos Totais Dissolvidos (STD) de cerca de $117 \mathrm{mg} \mathrm{L}^{-1}$, portanto uma água doce (Fachin, 2005).

A média das concentrações foi de $0,29 \pm 0,02 \mathrm{mg} \mathrm{L}^{-1}$, observando-se que os valores de concentração do Cobre da água de todos os poços são muito semelhantes, com valores destaque a valores maiores na porção centro sul do cartograma (Figura 5a).

Todos os poços apresentaram os valores de concentração abaixo das normas brasileiras, sendo que a Portaria do Ministério da Saúde No 518/2004 fixou o Padrão de potabilidade para 
KEMERICH, P. D. C.; SILVA, J. L. S.; BARROS, G.; BORBA, W. F.; UCKER, F. E.; FOLETTO, C. V. Caracterização química da água subterrânea em área ocupada por cemitério: uso da técnica de espectrometria de fluorescência de raios-X por energia dispersiva (EDXRF). Ambi-Agua, Taubaté, v. 7, n. 3, p. 166-182, 2012. (http://dx.doi.org/10.4136/ambi-agua.911)

substâncias químicas que representam risco à saúde como Valor Máximo Permissível - VMP para Cobre $2 \mathrm{mg} \mathrm{L}^{-1}$, o que evidencia os baixos valores encontrados nesta pesquisa.

Davies e De Wiest (1991) encontraram amostras com concentrações de Cobre e com valor máximo de $0,044 \mathrm{mg} \mathrm{L}^{-1}$. Tais valores ficam abaixo dos encontrados, podendo indicar aumento em função de características distintas dos dois locais de estudo ou até mesmo aumento na concentração em função da presença do cemitério.

Os valores de fósforo nas amostras de água subterrânea variaram entre 11,97 e $13,98 \mathrm{mg} \mathrm{L}^{-1}$, com valor médio de $13,26 \mathrm{mg} \mathrm{L}^{-1}$ (Figura $5 \mathrm{~b}$ ). O maior valor encontrado foi na amostra proveniente do poço PM4 (13,98 $\left.\mathrm{mg} \mathrm{L}^{-1}\right)$. A Figura 6 (a) ilustra a espacialização das concentrações de fosfato na água dos poços na região do cemitério. Destacando-se que as maiores concentrações ocorreram nas regiões sudoeste e norte do cartograma.

A forma constituinte responsável pelo fósforo são os sólidos em suspensão e dissolvidos, e sua origem natural é pela dissolução de compostos do solo e da matéria orgânica (Botelho, 2001). O fosfato é um elemento fundamental para o metabolismo dos seres vivos e sua presença em águas naturais depende das características das rochas da região.

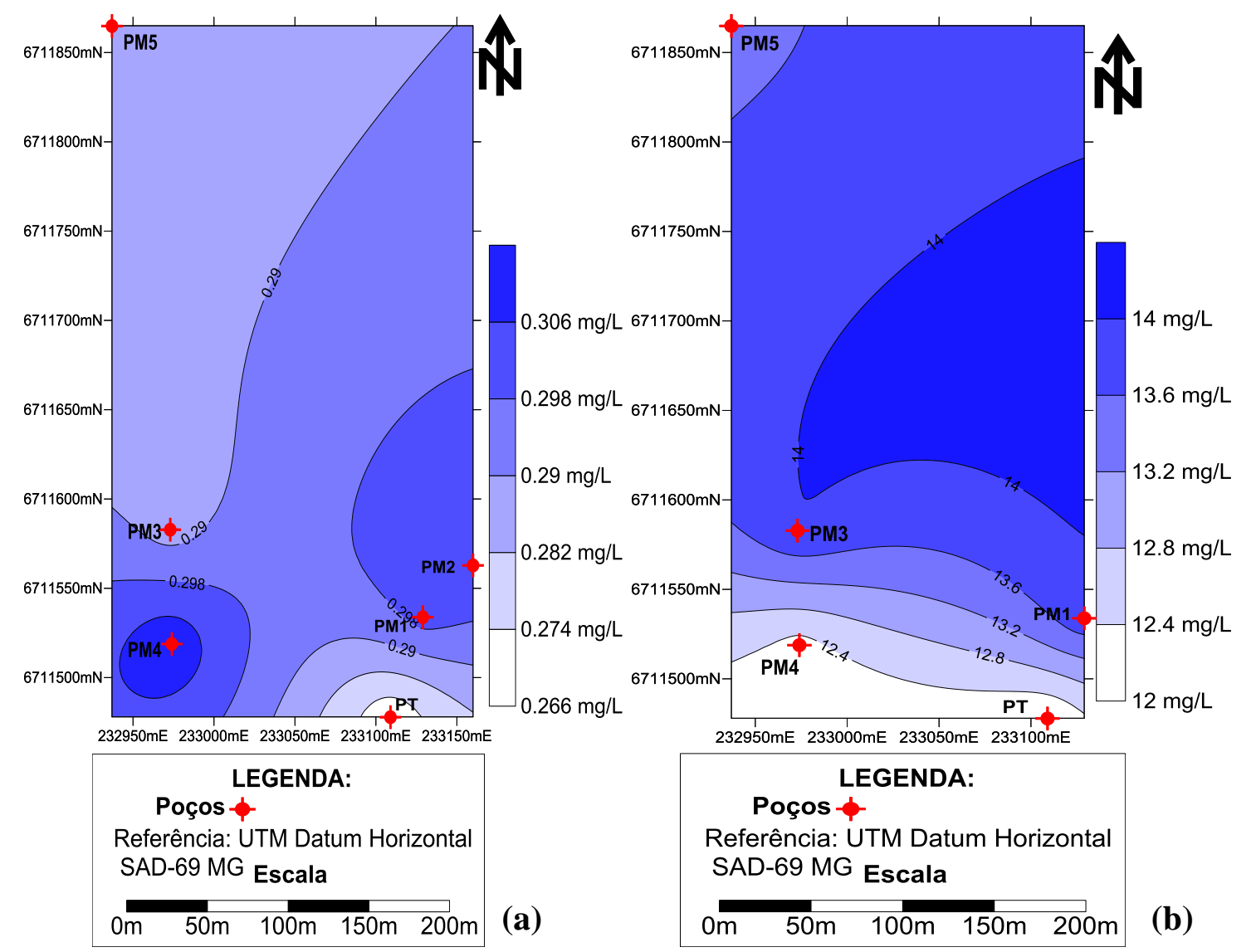

Figura 5. (a) Cartograma da concentração de Cobre $\left(\mathrm{Cu}^{+}\right)$e (b) Fósforo na água dos Poços.

O potássio é um dos dez elementos químicos mais abundantes na crosta terrestre, mas ocorre em pequena quantidade nas águas subterrâneas, pois é facilmente fixado pelos argilominerais e como elemento essencial, é intensivamente consumido pelos vegetais. Seus principais minerais fontes são: Feldspato potássico e a biotita, pouco resistentes aos intemperismo físico e químico. Outra fonte são as micas muscovitas. Este elemento é comum nos argilominerais do tipo Ilitas e interesetraficados I/S comuns na Unidade Hidroestratigráfica Alemoa (Silvério da Silva et al., 2010). Nas águas subterrâneas seu teor médio é inferior a 
KEMERICH, P. D. C.; SILVA, J. L. S.; BARROS, G.; BORBA, W. F.; UCKER, F. E.; FOLETTO, C. V. Caracterização química da água subterrânea em área ocupada por cemitério: uso da técnica de espectrometria de fluorescência de raios-X por energia dispersiva (EDXRF). Ambi-Agua, Taubaté, v. 7, n. 3, p. 166-182, 2012. (http://dx.doi.org/10.4136/ambi-agua.911)

$10 \mathrm{mg} \mathrm{L}^{-1}$. Silvério da Silva et al. (2006) relataram valores entre 0,31 a $3,43 \mathrm{mg} \mathrm{L}^{-1}$, e um valor médio de $1,42 \mathrm{mg} \mathrm{L}^{-1}$, na água do Rio Quarai encontraram $0,78 \mathrm{mg} \mathrm{L}^{-1}$.

Conforme ilustrado na Figura 6, os valores de concentração do potássio variaram de 0,50 a $7 \mathrm{mg} \mathrm{L}{ }^{-1}$. Observa-se que as concentrações mais elevadas ocorreram no setor sul da área estudada. Kemerich (2009) e Kemerich et al. (2011) estudando a qualidade da água subterrânea no Bairro Nossa Senhora do Perpétuo Socorro em Santa Maria - RS encontraram valores entre 0,10 a $11 \mathrm{mg} \mathrm{L}^{-1}$.

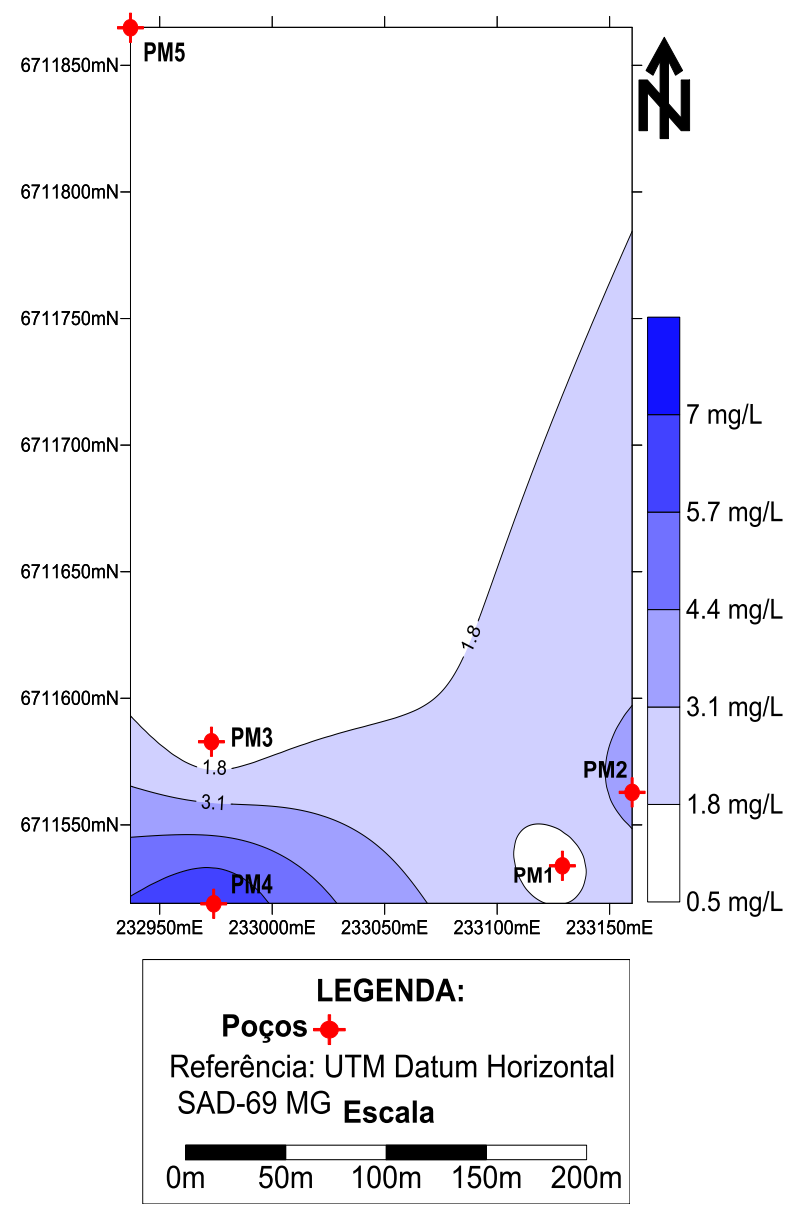

Figura 6. Cartograma da concentração Potássio $\left(\mathrm{K}^{+}\right)$na água dos poços.

A Técnica de Espectrometria de Fluorescência de Raios - X por Energia Dispersiva apresentou resultados extremamente baixos para as concentrações dos elementos de transição avaliados: Titânio, Manganês e Escândio (Tabela 2). Em alguns poços as amostras de água subterrânea não tiveram os elementos detectados pelo equipamento. Hem (1985) relata que os metais de transição geralmente apresentam mais do que um estado de oxidação que é estável termodinamicamente em presença da água. Vários destes elementos estão relacionados na água na forma de complexos aniônicos em $\mathrm{pH}$ neutro.

Krieger (2000) apresentou resultados para o parâmetro de Manganês dissolvido, que variaram na faixa de 11 a 45,6 $\mathrm{mg} \mathrm{L}^{-1}$. O comportamento do $\mathrm{Fe}$ e do $\mathrm{Mn}$, que apresentam dois números de oxidação, é controlado pelos fenômenos de oxidação-redução, que são especialmente importantes em relação a sua solubilidade (Hem, 1985; Mestrinho, 2008). Aqui se deve lembrar que a zona saturada e insaturada do subsolo altera-se durante o ano e pode influenciar diretamente número de oxidação do $\mathrm{Fe}$ e Mn, além do pH do meio. 
KEMERICH, P. D. C.; SILVA, J. L. S.; BARROS, G.; BORBA, W. F.; UCKER, F. E.; FOLETTO, C. V. Caracterização química da água subterrânea em área ocupada por cemitério: uso da técnica de espectrometria de fluorescência de raios-X por energia dispersiva (EDXRF). Ambi-Agua, Taubaté, v. 7, n. 3, p. 166-182, 2012. (http://dx.doi.org/10.4136/ambi-agua.911)

O Manganês $\left(\mathrm{Mn}^{2+}\right)$ é um elemento que acompanha o Ferro em virtude de seu comportamento geoquímico. Ocorre em teores abaixo de $0,20 \mathrm{mg} \mathrm{L}^{-1}$, quase sempre como óxido de manganês bivalente, que se oxida em presença do ar atmosférico, dando origem a precipitados negros.

Dos três elementos metais traços, apenas o $\left(\mathrm{Mn}^{2+}\right)$ tem fixado o VMP de $0,10 \mathrm{mg} \mathrm{L}^{-1}$ Resolução CONAMA No 396/2008 e Portaria No 518/2004 do Ministério da Saúde (Brasil, 2004), sendo que nenhuma das amostras avaliadas superou o VMP. A Resolução CONAMA N ${ }^{\circ}$ 420/2009 indica para valor de investigação $1,05 \mathrm{mg} \mathrm{L}^{-1}$.

Tabela 2. Concentrações de Titânio, Manganês e Escândio nos poços de monitoramento.

\begin{tabular}{c|ccc}
\hline $\begin{array}{c}\text { Poço de } \\
\text { Monitoramento }\end{array}$ & Titânio $\left(\mathbf{T i}^{\mathbf{2 +}}\right)$ & Manganês $\left(\mathbf{M n}^{2+}\right)$ & Escândio $\left(\mathbf{S c}^{\mathbf{3 +}}\right)$ \\
\cline { 2 - 4 } & & $\left(\mathbf{m g ~ L} \mathbf{~ L}^{\mathbf{1}}\right)$ & \\
\hline 1 & 0,009 & 0,005 & $*<$ L.D. \\
2 & <L.D. & $<$ L.D. & $<$ L.D. \\
3 & 0,018 & 0,003 & $<$ L.D. \\
4 & <L.D. & $<$ L.D. & 0,027 \\
5 & <L.D. & <L.D. & $<$ L.D. \\
6 & <L.D. & <L.D. & $<$ L.D. \\
\hline
\end{tabular}

Nota: $*<$ L.D. $=$ menor do que o Limite de Detecção do aparelho Shimadzu Shimadzu EDX-720.

A Tabela 3 ilustra correlação entre os parâmetros analisados, sendo que os valores destacados na cor vermelha apresentaram correlação.

Apresentaram correlação a Superfície Potenciométrica (S.P.) e a Altitude (1), o elemento Enxofre e Ferro $(0,98)$. O elemento Titânio apresentou correlação com os elementos Silício $(0,89)$ e Ferro $(0,95)$. O Cálcio apresentou correlação com Silício $(0,92)$ e Ferro $(0,95)$.

Estas correlações podem indicar que são provenientes da mesma fonte geradora, porém nenhum elemento apresentou correlação com o fluxo de água subterrânea.

Tabela 3. Matriz de correlação dos elementos químicos estudados e características físicas do local em estudo.

\begin{tabular}{|c|c|c|c|c|c|c|c|c|c|c|c|c|c|}
\hline & Alt.(m) & $\mathrm{NE}(\mathrm{m})$ & $\mathbf{S P}(\mathrm{m})$ & $\mathrm{Ca}^{2+}$ & $\mathrm{Cu}^{+}$ & $\mathrm{Si}^{4+}$ & $\mathbf{P}^{3+}$ & $\mathrm{Fe}^{+}$ & $\mathrm{S}^{2+}$ & $\mathbf{T i}^{2+}$ & $\mathbf{K}^{+}$ & $\mathrm{Mn}^{2+}$ & $\mathrm{Sc}^{3+}$ \\
\hline Alt.(m) & 1 & & & & & & & & & & & & \\
\hline NE (m) & $-0,5$ & 1 & & & & & & & & & & & \\
\hline SP (m) & $1 *$ & $-0,51$ & 1 & & & & & & & & & & \\
\hline $\mathrm{Ca}^{2+}$ & $-0,75$ & 0,49 & $-0,75$ & 1 & & & & & & & & & \\
\hline $\mathrm{Cu}^{+}$ & 0,37 & 0 & 0,36 & $-0,09$ & 1 & & & & & & & & \\
\hline $\mathbf{S i}^{4+}$ & $-0,32$ & 0,14 & $-0,32$ & 0,17 & 0,67 & 1 & & & & & & & \\
\hline $\mathbf{P}^{3+}$ & $-0,32$ & $-0,16$ & $-0,31$ & 0,69 & $-0,37$ & $-0,38$ & 1 & & & & & & \\
\hline $\mathrm{Fe}^{+}$ & $-0,29$ & 0,38 & $-0,29$ & 0,54 & 0,76 & 0,8 & $-0,02$ & 1 & & & & & \\
\hline$S^{2+}$ & $-0,34$ & 0,39 & $-0,35$ & 0,66 & 0,68 & 0,7 & 0,15 & $0,98 *$ & $1^{*}$ & & & & \\
\hline $\mathbf{T i}^{2+}$ & $-0,22$ & 0,42 & $-0,23$ & 0,31 & 0,81 & $0,89 *$ & $-0,32$ & $0,95^{*}$ & 0,88 & 1 & & & \\
\hline $\mathbf{K}^{+}$ & $-0,24$ & 0,35 & $-0,24$ & 0,32 & 0,81 & $0,92 *$ & $-0,29$ & $0,95^{*}$ & 0,89 & 1 & 1 & & \\
\hline $\mathrm{Mn}^{2+}$ & 0,16 & 0,31 & 0,15 & $-0,39$ & 0,65 & 0,67 & $-0,91$ & 0,43 & 0,27 & 0,69 & 0,66 & 1 & \\
\hline $\mathrm{Sc}^{3+}$ & $-0,12$ & 0,6 & $-0,13$ & 0,32 & $-0,49$ & $-0,69$ & 0,3 & $-0,28$ & $-0,18$ & $-0,37$ & $-0,44$ & $-0,38$ & 1 \\
\hline
\end{tabular}

Nota: Destacadas em vermelho, as correlações significativas para $\mathrm{p}<, 050$. 
KEMERICH, P. D. C.; SILVA, J. L. S.; BARROS, G.; BORBA, W. F.; UCKER, F. E.; FOLETTO, C. V. Caracterização química da água subterrânea em área ocupada por cemitério: uso da técnica de espectrometria de fluorescência de raios-X por energia dispersiva (EDXRF). Ambi-Agua, Taubaté, v. 7, n. 3, p. 166-182, 2012. (http://dx.doi.org/10.4136/ambi-agua.911)

\section{CONCLUSÃO}

A técnica de Espectrometria Fluorescência de Raios - $\mathrm{X}$ por energia dispersiva demonstrou ser rápida e eficiente para quantificação de vários constituintes em águas subterrâneas sob a influência de cemitério apresentando uma potencial aplicação para o monitoramento ambiental semiquantitativo-qualitativo.

Utilizando-se a técnica EDXRF foi possível determinar as concentrações dos constituintes: maiores $\left(\mathrm{Ca}^{2+}\right.$, silício), menores $\left(\mathrm{Fe}^{+}\right)$e traços $\left(\mathrm{K}^{+}, \mathrm{Cu}^{+}\right.$, fósforo, $\mathrm{Ti}^{+}, \mathrm{Mn}^{+}$e $\mathrm{Sc}^{+}$, facilitando a caracterização química das águas subterrâneas bem como as comparações aos padrões das legislações ambientais vigentes. Estudos futuros em águas subterrâneas comprovarão a eficácia da técnica.

Estimou-se que alguns elementos tiveram seus valores acrescidos em função do fluxo subterrâneo, evidenciando uma possível relação entre a presença de sepulturas e a alteração da qualidade natural da água subterrânea indicando uma poluição difusa, pontual, por substâncias oriundas de processos de alteração caixões, ornamentos mortuários e corpos humanos.

Salienta-se que o terreno apresenta textura silto-argilosa e os argilominerais apresentam elevada capacidade de troca, o que pode ser eficiente na adsorção dos constituintes maiores, menores e traços, na forma catiônica associados ao ambiente de características oxidantes, pelo menos no período de verão.

\section{REFERÊNCIAS}

ASSOCIAÇÃO BRASILEIRA DE NORMAS TÉCNICAS. NBR 15.495: poços de monitoramento de águas subterrâneas em aqüíferos granulados. Rio de Janeiro, 2007.

BOTELHO, C. G. Recursos naturais renováveis e impacto ambiental. Lavras: UFLA/FAEPE, 2001. $136 \mathrm{p}$.

BÖGER, H.; KOWALCZYK, G. Stratigraphische, sedimentologische und Paläoökologische Untersuchungen Im Mesozoikum Der Depressão Periférica in Rio Grande do Sul, Brasilien. Geologisch-Paläontologisches Institut und Museum, Universität Kiel, Berichte-Reports, n. 63, p. 1-72, 2003.

BONA, I. A. T.; SARKIS, J. E. S.; SALVADOR, V. L. R.; SOARES, A. L. R.; KLAMT, S. L. Análise arqueométrica de cerâmica Tupiguarani da região central do Estado do Rio Grande do Sul, Brasil, usando fluorescência de raios X por dispersão de energia (EDXRF). Química Nova, São Paulo, n. 30, p. 785-790, jan./fev. 2007. http://dx.doi.org/10.1590/S0100-40422007000400006

BRASIL. Ministério da Saúde. Portaria $\mathbf{N}^{\circ}$ 518, de 25 de março de 2004. Estabelece os procedimentos e responsabilidades relativos ao controle e vigilância da qualidade da água para consumo humano e seu padrão de potabilidade, e dá outras providências. Brasília, 2004. Disponível em: <http://portal.saude.gov.br/portal/arquivos/ pdf/portaria_518.pdf>. Acesso em: 03 mar. 2011.

CHAVES, A.; SILVÉRIO Da SILVA, J. L. Comportamento do fluxo das águas subterrâneas na área de um empreendimento potencialmente poluidor no Município de Santa Maria. In: CONGRESSO BRASILEIRO DE ÁGUAS SUBTERRÂNEAS, 15., 11 - 14 nov. 2008, Natal. Comunicação Apresentada no... Natal: ABAS, 2008. p. 1-16. 
KEMERICH, P. D. C.; SILVA, J. L. S.; BARROS, G.; BORBA, W. F.; UCKER, F. E.; FOLETTO, C. V. Caracterização química da água subterrânea em área ocupada por cemitério: uso da técnica de espectrometria de fluorescência de raios-X por energia dispersiva (EDXRF). Ambi-Agua, Taubaté, v. 7, n. 3, p. 166-182, 2012. (http://dx.doi.org/10.4136/ambi-agua.911)

CHAVES, A. Monitoramento de águas subterrâneas em um empreendimento potencialmente poluidor no Município de Santa Maria/RS. 2009. 187f. Dissertação (Mestrado em Engenharia Civil/Recursos Hídricos e Saneamento Ambiental) Universidade Federal de Santa Maria, Santa Maria, 2009.

CONSELHO NACIONAL DE MEIO AMBIENTE (Brasil). Resolução $\mathbf{N}^{\mathbf{0}} \mathbf{3 3 5}$, de 3 de abril de 2003. Dispõe sobre o licenciamento ambiental de cemitérios. Brasília, 2003. Disponível em: <http://www.mma.gov.br/port/conama/res/res03/res33503.xml>. Acesso em: 3 mar. 2011.

CONSElHO NACIONAL DE MEIO AMBIENTE. (Brasil) Resolução $\mathbf{N}^{\mathbf{0}} \mathbf{3 6 8}$, de 28 de março de 2006. Altera dispositivos da Resolucção $n^{\circ} 335$, de 3 de abril de 2003, que dispõe sobre o licenciamento ambiental de cemitérios. Brasília, 2006. Disponível em: <http://www.mma.gov.br/port/conama/legiabre.cfm?codlegi=488>. Acesso em: 3 mar. 2011.

CONSELHO NACIONAL DO MEIO AMBIENTE. (Brasil). Resolução No 396, de 03 de abril de 2008. Dispõe sobre a classificação e diretrizes ambientais para o enquadramento das águas subterrâneas e dá outras providências. Brasília, 2008. Disponível em: <http://www.mma.gov.br/port/conama/legiabre.cfm?codlegi=562>. Acesso em: 02 mar. 2011.

CONSELHO NACIONAL DO MEIO AMBIENTE. (Brasil). Resolução No 420, de 28 de Dezembro de 2009. Dispõe sobre critérios e valores orientadores de qualidade do solo quanto à presença de substâncias químicas e estabelece diretrizes para o gerenciamento ambiental de áreas contaminadas por essas substâncias em decorrência de atividades antrópicas. Brasília, 2009. Disponível em: <http://www.mp.rs.gov.br/ambiente/ legislacao/id4830.htm>. Acesso em: 22 out. 2012.

DAVIES, S. N.; De WIEST, R. J. M. Hydrogeology. New York: John Wiley and Sons, 1966. $463 p$.

DAVIES, S. N.; De WIEST, R. J. M. Hydrogeology. Flórida: Krieger Publishing Company, 1991.

FACHIN, E. F. Distribuição espacial dos poços tubulares profundos e informações físicoquímicas das águas subterrâneas no município de Santa Maria/RS. 2003. 72f. Trabalho de Conclusão de Curso (Graduação) - Universidade Federal de Santa Maria, Santa Maria, 2003.

FACHIN, E. F. Avaliação da vulnerabilidade natural dos aquíferos em Santa Maria/RS. 2005. 91f. Monografia (Especialização em Geociências) - Universidade Federal de Santa Maria, Santa Maria, 2005.

FEITOSA, A C. F.; MANOEL FILHO, J. Hidrogeologia: conceitos e aplicações. Fortaleza: LCR, 1997. 812 p.

GARCIA, G. P. Avaliação de pontos potenciais de contaminação de águas subterrâneas em Santa Maria, RS. 2004. 60f. Trabalho de Conclusão de Curso (Graduação em Geografia) - Universidade Federal de Santa Maria, Santa Maria, 2004. 
KEMERICH, P. D. C.; SILVA, J. L. S.; BARROS, G.; BORBA, W. F.; UCKER, F. E.; FOLETTO, C. V. Caracterização química da água subterrânea em área ocupada por cemitério: uso da técnica de espectrometria de fluorescência de raios-X por energia dispersiva (EDXRF). Ambi-Agua, Taubaté, v. 7, n. 3, p. 166-182, 2012. (http://dx.doi.org/10.4136/ambi-agua.911)

GASPARETTO, N. V. L.; MEDEIROS, E. R.; VIEIRA, P.; MACIEL FILHO, C. L.; SARTORI, P. L. P.; MENEGOTTO, E. Mapa geológico da folha de Santa Maria e nota explicativa. Santa Maria: FINEP; UFSM, 1990. 1 Mapa. Escala 1:50.000.

GOLDEN SOFTWARE, Inc. Surfer 8.0: contouring and 3D surface mapping for scientists and engineers: user's guide. Golden, 2004. 640 p.

HAN, X. Y.; ZHUO, S. J.; SHEN, R. X.; WANG, P. L.; JI, A. Comparison of the quantitative results corrected by fundamental parameter method and difference calibration specimens in x-ray fluorescence spectrometry. Journal of Quantitative Spectroscopy and Radiative Transfer, v. 97, n. 1, p. 68-74, 2006. http://dx.doi.org/10.1016/ j.jqsrt.2004.12.018

HEM, J. D. Study and interpretation of the chemical characteristics of natural water. Alexandria: USGS, 1985. 267 p.

KEMERICH, P. D. C. Água subterrânea e a saúde da comunidade em Bairro de Santa Maria- RS. 2008. 108f. Dissertação (Mestrado Recursos Hídricos e Saneamento Ambiental) - Universidade Federal de Santa Maria, Santa Maria, 2008.

KEMERICH, P. D. C.; SILVÉRIO da SILVA, J. L.; DESCOVI FILHO, L. L. V.; VOLPATTO, F.; SAUCEDO, E. M. Determinação da vulnerabilidade natural à contaminação da água subterrânea no Bairro Nossa Senhora do Perpétuo Socorro em Santa Maria, RS. Engenharia Ambiental, Espírito Santo do Pinhal, v. 8, n.3, p. 85-98, jul. /set. 2011.

KEMERICH, P. D. C.; UCKER, F. E.; BORBA, W. F. Cemitérios como fonte de contaminação ambiental. Scientific American Brasil, São Paulo, n. 123, p 78-81, ago. 2012. Disponível em: <http://www2.uol.com.br/sciam/artigos/cemiterios_como_fonte_ de_contaminacao_ambiental.html> Acesso em: 22 out. 2012.

KRIEGER, E. I. F. Avaliação da contaminação das águas subterrâneas na área de influência da usina de tratamento de resíduos S/A - UTRESA, em Estância Velha (RS). 2000. 148f. Dissertação (Mestrado em Biociências) - Universidade Federal do Rio Grande do Sul, Porto Alegre, 2000.

LANDIM, P. M. B.; STURARO, J. R. Krigagem indicativa aplicada à elaboração de mapas probabilísticos de riscos. Rio Claro: DGA;IGCE;UNESP, 2002. 253 p.

LEMES, M. J. DE L.; FIGUEIREDO FILHO, P. M.; PIRES, M. A. F. Mineral influence on chemical composition of drinking water supply from Mogi-Guaçu and Pardo rivers. Química Nova, São Paulo, v. 26, n. 1, p. 13-20, jan./fev. 2003. http://dx.doi.org/10.1590/S0100-40422003000100004.

MACIEL FILHO, C. L. Carta geotécnica de Santa Maria. Santa Maria: Imprensa Universitária UFSM, 1990.

MACHADO, J. L. F. Compartimentação espacial e arcabouço hidroestratigráfico do SAG no Rio Grande do Sul. 2005. 237f. Tese (Doutorado em Geologia) - Universidade do Vale dos Sinos, Porto Alegre, 2005.

MESTRINHO, S. S. Hidrogeologia, conceitos e aplicações. Brasília: CPRM, 2008. 
KEMERICH, P. D. C.; SILVA, J. L. S.; BARROS, G.; BORBA, W. F.; UCKER, F. E.; FOLETTO, C. V. Caracterização química da água subterrânea em área ocupada por cemitério: uso da técnica de espectrometria de fluorescência de raios-X por energia dispersiva (EDXRF). Ambi-Agua, Taubaté, v. 7, n. 3, p. 166-182, 2012. (http://dx.doi.org/10.4136/ambi-agua.911)

MIGLIORINI, R. B. Cemitérios contaminam o meio ambiente: um estudo de caso. Cuiabá: Universitária, $2002.50 \mathrm{p}$.

PATACA, L. C.; BORTOLETO, G. G.; BUENO, M. I. M. S. Determinação de arsênio em águas contaminadas usando fluorescência de raios-X por energia dispersiva. Química Nova, São Paulo, v. 28, n. 4, p. 579-582. jul./ago. 2005. http://dx.doi.org/10.1590/S010040422005000400004

SERVIÇO GEOLÓGICO DO BRASIL - CPRM. Levantamentos geológicos do Brasil. Brasília: CPRM, 1994. 57 p.

SILVA, V. T.; CRISPIM, J. De Q.; GOCH, P.; KUERTEN, S.; MORAES, A. C. Da S. De; OLIVEIRA, M. A.; SOUZA, I. A.; ROCHA, J. A. Da. 2006. Um olhar sobre as necrópoles e seus impactos ambientais. In: ENCONTRO DA ASSOCIAÇÃO NACIONAL DE PÓS-GRADUAÇÃO E PESQUISA EM AMBIENTE E SOCIEDẢDE, 3., 23 - 26 maio, Brasília. Comunicação Apresentada no Brasília, 2006. Vol. 1. 1 CDROM.

SILVÉRIO da SILVA, J. L.; MENEGOTTO, E.; MEDEIROS, E. R. Avaliação preliminar dos argilominerais das folhas de Santa Maria e Camobi, Rio Grande do Sul, Brasil. Ciência e Natura, Santa Maria, v. 12, p. 127-132, jan./fev. 1990.

SILVÉRIO da SILVA, J. L. Estudos dos processos de silicificação e calcificação em rochas sedimentares mesozóicas do estado do Rio Grande do Sul. 1997. 157f. Tese (Doutorado em Geologia) - Universidade Federal do Rio Grande do sul, Porto Alegre, 1997.

SILVÉRIO Da SILVA, J. L.; CAMPONOGARA, I.; FRANTZ, L. C.; DRESSLER, V. L.; FLORES, E. M. M.; BESSOUAT, C. et al. Caracterização de áreas de recarga e descarga do SAG em Rivera-Livramento e Artigas-Quaraí. In: FUNDO DAS UNIVERSIDADES. Projeto Sistema Aquífero Guarani - PSAG. No 10. Relatório Final. Montivideo, 2006. 2 vol. p. 94.

SILVÉRIO Da SILVA, J. L.; PEDRON, F. de A.; WERLANG, M. K.; DESCOVI FILHO, L. V.; CABRAL, D. R. Características morfológicas do Membro Alemoa, Formação Santa Maria, RS. In: REUNIÃO SUL-BRASILEIRA DE CIENCIA DO SOLO, 8., 25-28 abr., Santa Maria. Comunicação Apresentada no... Santa Maria: SBCS, 2010. v. 1. p. 1 - 6.

SCHIMIDT, F.; BUENO, M. I. M.; EINZWEILLER, J. Determinação de alguns metais em solos por fluorescência de raios-X de energia dispersiva e redes neurais. In: CONGRESSO BRASILEIRO DE REDES NEURAIS, 4., 2009, São José dos Campos. Comunicação apresentada no... São José dos Campos: SBRN. 1999. p. 396-399.

UNESCO; OMS; WHO. The selection of water quality variables. London: Chapman and Hall, 1992. 60 p.

VASCONCELOS, U.; CALAZAND, G. M. T.; ANDRADE, M. A. G. De; MEDEIROS, L. V. Evidência do antagonismo entre Pseudomonas aeruginosa sobre bactérias indicadoras de contaminação fecal da água. Revista Higiene Alimentar, São Paulo, v. 20, n. 140, p. 127-131, 2006. 
KEMERICH, P. D. C.; SILVA, J. L. S.; BARROS, G.; BORBA, W. F.; UCKER, F. E.; FOLETTO, C. V. Caracterização química da água subterrânea em área ocupada por cemitério: uso da técnica de espectrometria de fluorescência de raios-X por energia dispersiva (EDXRF). Ambi-Agua, Taubaté, v. 7, n. 3, p. 166-182, 2012. (http://dx.doi.org/10.4136/ambi-agua.911)

WASTOWSKI A. D.; DA ROSA G. M.; CHERUBIN M. R.; RIGO J. P G. Caracterização dos níveis de elementos químicos em solo, submetido a diferentes sistemas de uso e manejo, utilizando espectrometria de fluorescência de raios-x por energia dispersiva (EDXRF). Química Nova, São Paulo, v. 33, n.7, p. 1449-1452, mar./abr. 2010. http://dx.doi.org/10.1590/S0100-40422010000700005 


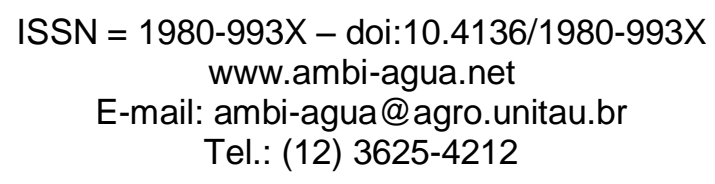

\title{
Efeito do uso e ocupação do solo na qualidade da água na micro- bacia do Córrego Banguelo - Contagem
}

\author{
(http://dx.doi.org/10.4136/ambi-agua.962) \\ Patrícia Procópio Pontes ; Andréa Rodrigues \\ Marques $^{2}$; Guilherme Fernandes Marques ${ }^{3}$ \\ ${ }^{1}$ Departamento de Química, Centro Federal de Educação Tecnológica de Minas Gerais, Belo Horizonte, MG, \\ e-mail: patricia@des.cefetmg.br, \\ ${ }^{2}$ Departamento de Ciência e Tecnologia Ambiental, Centro Federal de Educação Tecnológica de Minas Gerais, \\ Belo Horizonte, MG, e-mail: andrearmg@ gmail.com, \\ ${ }^{3}$ Departamento de Engenharia Civil, Centro Federal de Educação Tecnológica de Minas Gerais, Belo \\ Horizonte, MG, e-mail: gmarques@ civil.cefetmg.br
}

\section{RESUMO}

A micro-bacia do córrego Banguelo da sub-bacia Bom Jesus contribui, junto com outras, para formação do reservatório da Pampulha, cuja poluição é considerada um dos grandes problemas ambientais do município de Belo Horizonte. O presente trabalho teve como objetivo avaliar o efeito do uso e ocupação do solo na qualidade da água num ciclo sazonal em diferentes pontos da micro-bacia. Para o monitoramento da qualidade da água, os parâmetros analisados foram: $\mathrm{pH}$, turbidez, oxigênio dissolvido, fosfato, nitrato, nitrogênio total, temperatura, $\mathrm{DBO}_{5}, \mathrm{DQO}$, sólidos totais e coliformes termotolerantes. Foi realizado, ainda, um levantamento florístico e das condições de ocupação do solo nas margens do córrego. A partir dos resultados do monitoramento, foram calculados os índices de qualidade da água (IQA ${ }_{\mathrm{NSF}}$ ) e identificados os pontos com maior grau de poluição. A análise do IQA indicou que a qualidade da água oscilou entre média e ruim no período seco, tendo-se observado a melhoria da qualidade da água para boa e média no período chuvoso. A deterioração da qualidade das águas ocorreu devido ao lançamento de efluente industrial e esgoto das áreas urbanizadas, com ocorrência de níveis baixos de oxigênio dissolvido, alta concentração de matéria orgânica e nutrientes. Nas áreas com vegetação, observou-se diminuição na concentração de poluentes (principalmente nitrato e fosfato), indicando a importância da manutenção da vegetação ripária como medida de melhoria da qualidade das águas na região. A minimização da poluição neste córrego pode melhorar a qualidade da água da sub-bacia e, consequentemente, contribuir para deseutrofização do reservatório da Pampulha.

Palavras-chave: Qualidade da água, uso e ocupação do solo, índice de qualidade da água.

\section{Effect of land use and occupation in water quality in the micro-basin of Banguelo Creek - Contagem}

\section{ABSTRACT}

The Banguelo creek micro-basin, in the sub-basin Bom Jesus contributes for the formation of Pampulha reservoir, along with other contributors. The pollution in this reservoir is considered one of the major environmental problems of the city of Belo Horizonte. This paper evaluated the effect of land use and occupation in Banguelo Creek on its water quality, considering the seasonal cycle in several points of the micro-basin. The 
PONTES, P. P.; MARQUES, A. R.; MARQUES, G. F. Efeito do uso e ocupação do solo na qualidade da água na micro-bacia do Córrego Banguelo - Contagem. Ambi-Agua, Taubaté, v. 7, n. 3, p. 183-194, 2012. (http://dx.doi.org/10.4136/ambi-agua.962)

analyzed parameters for monitoring the water quality were: $\mathrm{pH}$, turbidity, dissolved oxygen, phosphate, nitrate, total nitrogen, temperature, $\mathrm{BOD}_{5}, \mathrm{COD}$ and thermotolerant coliforms. The results were analyzed based on a floristic survey and on land occupation conditions in the micro-basin. Water quality index $\left(\mathrm{WQI}_{\mathrm{NSF}}\right)$ was calculated and the points with the highest degree of pollution were identified. Analysis of WQI indicated that the water quality fell in the classes "average" and "bad" during dry season, with improvement for "good" and "average" in the rainy season. The deterioration of water quality was attributed to the discharge of industrial effluent and sewage that caused low levels of dissolved oxygen, high concentration of organic matter and nutrients. In areas with vegetation, a decrease in the concentrations of pollutants (mainly nitrate and phosphate) was observed, indicating the importance of maintaining riparian vegetation to improve the water quality in the region. The decrease in the pollution level of this creek could improve the water quality in the region, contributing to the eutrophication reduction of Pampulha reservoir.

Keywords: Water quality, land use and occupation, water quality index.

\section{INTRODUÇÃO}

A qualidade das águas depende de vários aspectos, dentre os quais se destacam os usos antrópicos (retiradas e lançamentos nos corpos d'água) e a variabilidade natural das vazões. O seu estudo é fundamental para a avaliação das suas possibilidades de utilização. A região metropolitana de Belo Horizonte localiza-se em partes altas das bacias dos rios das Velhas e do Paraopeba. O rio das Velhas é um dos mais importantes afluentes em termo de volume de água do rio São Francisco, que é um dos maiores do Brasil. Segundo dados do IBGE (2000), o rio das Velhas foi considerado o mais poluído do estado de Minas Gerais, em parte porque sua bacia possuia uma população humana total maior que 4,5 milhões de pessoas, e o esgoto doméstico e resíduos industriais das cidades eram apenas parcialmente tratados. O esgoto de 3,2 milhões de pessoas nesta região era coletado, mas apenas $27,5 \%$ eram essencialmente tratados. Em muitas áreas rurais e suburbanas, a população que vive no entorno do rio usa a água para fins domésticos, higiene e lazer. A consequência da deterioração da qualidade da água é a restrição ao uso potencial de um manancial. No caso de uso para a irrigação, a utilização de uma água de baixa qualidade pode acarretar problemas de operacionalização em sistemas de irrigação, neste caso, ocorre o risco de contaminação dos alimentos irrigados, comprometendo a qualidade do produto e principalmente a saúde humana (Franco et al., 2006).

A bacia hidrográfica do rio das Velhas tem como seus principais afluentes, responsáveis pelas maiores cargas poluidoras, os ribeirões Arrudas e Onça. Suas bacias hidrográficas compreendem parte dos territórios de Belo Horizonte, de Contagem e uma parte do município de Sabará, na porção de jusante da bacia Arrudas. A bacia do ribeirão do Onça em unidades menores destaca-se num terceiro nível de drenagem na bacia que contribui para o reservatório da Pampulha. A poluição da bacia da Pampulha é considerada um dos grandes problemas ambientais do município de Belo Horizonte. Inúmeras iniciativas têm ocorrido para recuperação da região, entretanto o processo de expansão urbana sem planejamento nas micro-bacias contribui para o assoreamento do reservatório da Pampulha, eutrofização de suas águas e destruição das matas ripárias (PROPAM, 2001). Segundo Friese et al. (2010), o reservatório da Pampulha apresenta estratificação permanente, baixos teores de oxigênio e elevada concentração de nutrientes, culminando em elevada degradação ecológica. A presença de outros contaminantes, incluindo metais pesados é também verificada em índices considerados alarmantes por Friese et al. (2010), sendo ainda relatada 
PONTES, P. P.; MARQUES, A. R.; MARQUES, G. F. Efeito do uso e ocupação do solo na qualidade da água na micro-bacia do Córrego Banguelo - Contagem. Ambi-Agua, Taubaté, v. 7, n. 3, p. 183-194, 2012. (http://dx.doi.org/10.4136/ambi-agua.962)

em Sabino et al. (2004) e Rietzler et al. (2001), dentre outros. As sub-bacias de contribuição ao reservatório da Pampulha possuem cerca de $94 \mathrm{~km}^{2}$, estendendo-se pelos municípios de Belo Horizonte (44\%) e Contagem (56\%) e tendo como principais tributários diretos do reservatório os córregos Mergulhão, Tijuco, Bandeirantes, Ressaca, Bom Jesus ou Água Funda, Baraúna, Garças, AABB, Olhos d'Água ou Paracatu.

Embora alvo de grande número de estudos, o reservatório da Pampulha ainda carece de avaliações acerca do efeito sazonal das atividades antrópicas. O entendimento da variação sazonal da qualidade da água dos tributários do reservatório da Pampulha é importante no planejamento de medidas de intervenção. Nesse contexto, o presente trabalho teve como objetivo avaliar o efeito do uso e ocupação do solo na qualidade da água num ciclo sazonal em diferentes pontos da micro-bacia do córrego Banguelo na sub-bacia do Bom Jesus ou Água Funda. A avaliação da qualidade da água no córrego irá contribuir para um maior conhecimento de suas características e na identificação das principais fontes de degradação ambiental e de poluição, contribuindo para o banco de dados ambientais da Bacia da Pampulha.

\section{MATERIAIS E MÉTODOS}

\section{1. Área de estudo}

O córrego Banguelo (entre $19^{\circ} 51^{\prime} \mathrm{S} 44^{\circ} 03^{\prime} \mathrm{O}$ e $19^{\circ} 50^{\prime} \mathrm{S} 44^{\circ} 02^{\prime} \mathrm{O}$ ) alimenta a sub-bacia do córrego Bom Jesus ou Água Funda (Figura 1), na região de Contagem, integrando-se ao Córrego Ressaca em Belo Horizonte na bacia do Ribeirão do Onça - Minas Gerais. Os corpos d'água das sub-bacias da Pampulha foram classificados na Classe 2, segundo a resolução 357/05 do CONAMA, no entanto, devido à suas condições, incluindo-se a lagoa, encontraram-se fora dos padrões fixados para aquela Classe (PROPAM, 2001).

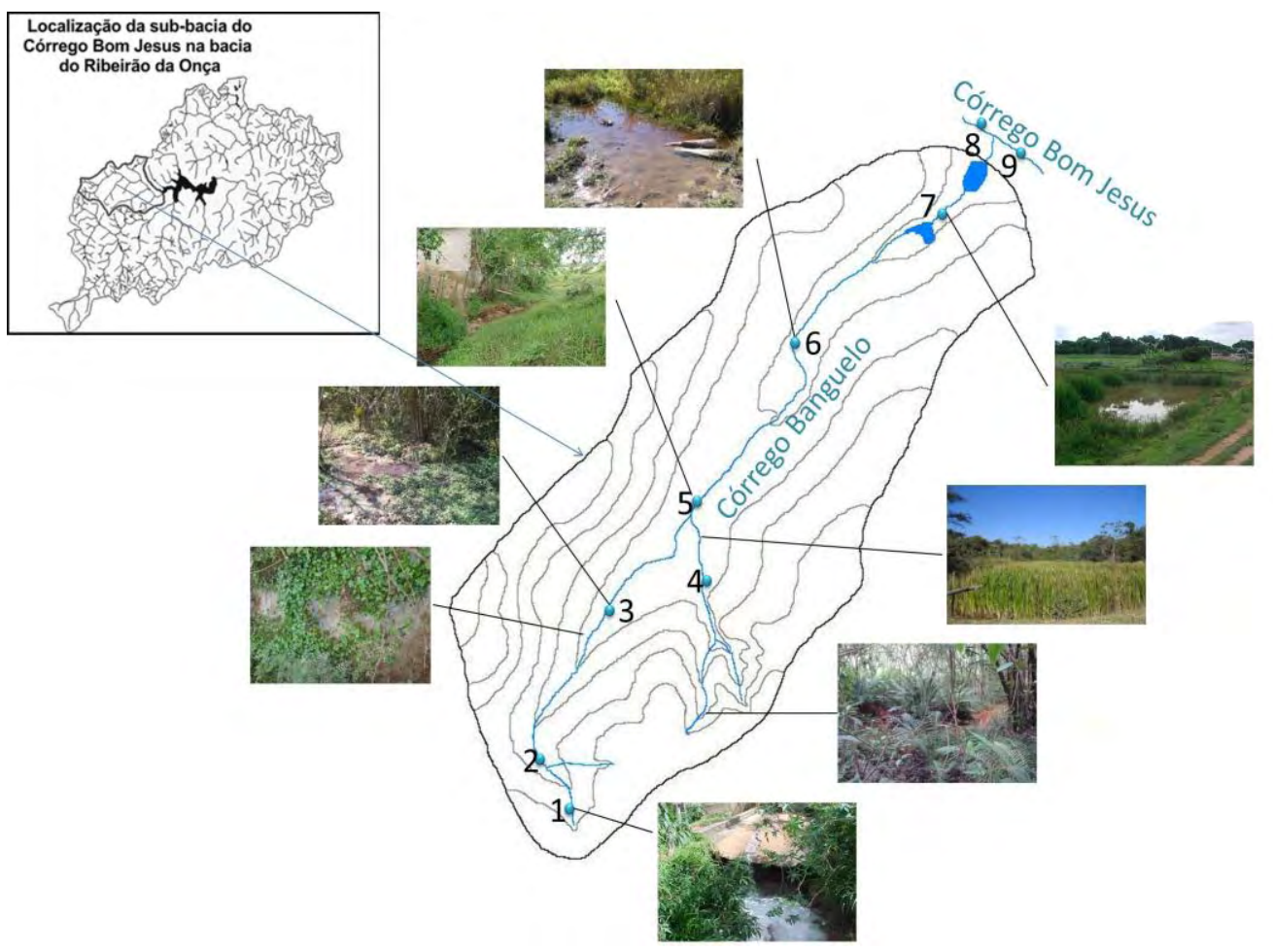

Figura 1. Localização dos pontos de monitoramento da água ( 1 a 9) na microbacia do córrego Banguelo - Contagem $\left(19^{\circ} 51^{\prime} \mathrm{S} 44^{\circ} 03^{\prime} \mathrm{O}\right.$ e $\left.19^{\circ} 50^{\prime} \mathrm{S} 44^{\circ} 02^{\prime} \mathrm{O}\right)$ na sub-bacia do córrego Bom Jesus. As fotos caracterizam alguns pontos de amostragem no curso d'água. 
Os solos residuais constituintes da superfície da bacia são provenientes de rochas granitognaissicas, o que lhes confere homogeneidade morfológica e susceptibilidade à erosão. A altitude média da bacia é de $850 \mathrm{~m}$, estando à superfície do reservatório da Pampulha a $801 \mathrm{~m}$; o ponto mais elevado a $1089 \mathrm{~m}$, no divisor de águas com o Córrego Água Branca (bacia do Ribeirão Arrudas). A declividade média da bacia situa-se na faixa de 10 a $20 \%$. Verificam-se as maiores declividades médias nas sub-bacias dos Córregos Sarandi, Mergulhão e Ressaca, com ocorrência de taxas superiores a 25\% em suas cabeceiras, e as menores na sub-bacia do Córrego Bom Jesus ou Água Funda, cuja predominância é inferior a $10 \%$. A região apresenta um clima mesotérmico (CWB de acordo com o sistema Köppen), com verões quentes e estação seca bem definida. A precipitação é maior no período de Setembro a Abril (primavera e verão) e as médias mínimas das temperaturas variam entre 13 e $19^{\circ} \mathrm{C}$ e as máximas entre 24 e $29^{\circ} \mathrm{C}$. Os dias mais frios coincidem com os meses de Maio a Agosto (inverno na região) (Figura 2).

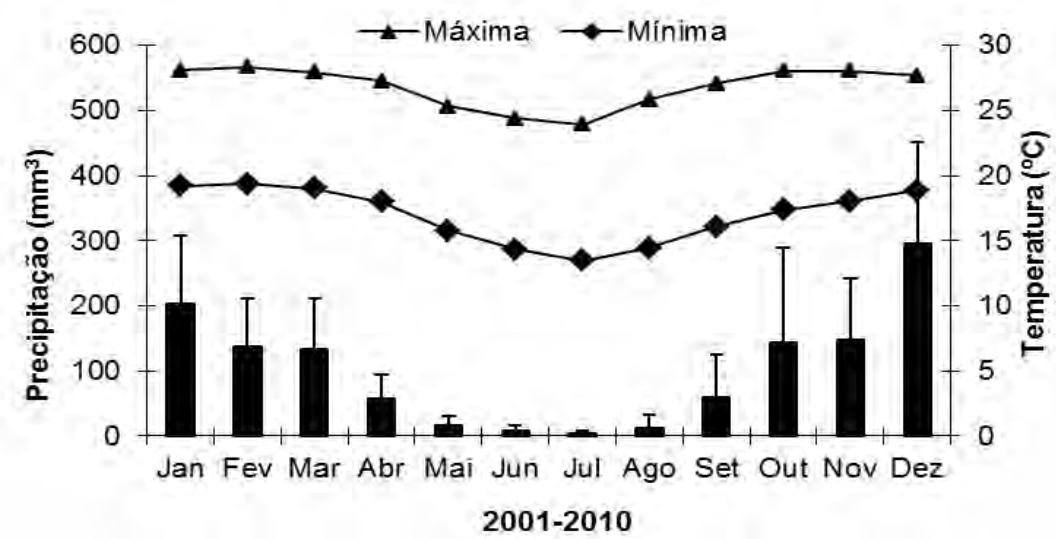

Figura 2. Diagrama do clima próximo à área de estudo entre os anos de 2001 e 2010: temperaturas médias, máximas e mínimas (linhas) e precipitação (barras), com dados obtidos da Estação Meteorológica da Pampulha - Belo Horizonte.

\subsection{Monitoramento da qualidade da água}

Para avaliação da qualidade da água, foram realizados trabalhos de campo com a identificação dos pontos mais adequados para a coleta de amostras. Foram selecionados nove pontos de monitoramento: seis pontos no próprio córrego Banguelo, um em seu afluente (ponto 4) e dois no córrego Bom Jesus (pontos 8 e 9) ( Figura 1).

Para o monitoramento da qualidade da água, que ocorreu durante um período de quase um ano (setembro de 2008 a julho de 2009), os parâmetros analisados foram: pH, turbidez, oxigênio dissolvido (OD), fosfato, nitrato, nitrogênio total, temperatura, demanda bioquímica de oxigênio $\left(\mathrm{DBO}_{5}\right)$, demanda química de oxigênio (DQO), sólidos totais e coliformes termotolerantes. As metodologias para as análises realizadas se encontram descritas no Standard Methods for Examination of Water and Wastewater (AWWA et al., 2005). A partir dos resultados do monitoramento, foram calculados os índices de qualidade da água e identificados os pontos com maior grau de poluição na micro-bacia.

\section{3. Índice de Qualidade da Água (IQA)}

Para determinar o índice de qualidade da água, foram estabelecidos alguns parâmetros pela National Sanitation Foundation (NSF) nos Estados Unidos, através de pesquisa com especialistas da área ambiental. Para cada parâmetro atribuiu-se um peso (wi) de acordo 
PONTES, P. P.; MARQUES, A. R.; MARQUES, G. F. Efeito do uso e ocupação do solo na qualidade da água na micro-bacia do Córrego Banguelo - Contagem. Ambi-Agua, Taubaté, v. 7, n. 3, p. 183-194, 2012. (http://dx.doi.org/10.4136/ambi-agua.962)

com sua importância para o índice de qualidade da água. Assim nove parâmetros foram considerados mais representativos: oxigênio dissolvido (wi $=0,17$ ), coliformes termotolerantes (wi $=0,15), \mathrm{pH}(\mathrm{wi}=0,12)$, demanda bioquímica de oxigênio (wi $=0,10)$, nitrato (wi $=0,10)$, fosfato total (wi $=0,10)$, variação de temperatura da água $(w i=0,10)$, turbidez (wi $=0,08)$ e sólidos totais $($ wi $=0,08)$. No caso do oxigênio dissolvido, determinase a porcentagem de oxigênio dissolvido em relação à concentração de saturação. Assim o IQA é calculado a partir do produto ponderado das qualidades da água correspondentes aos parâmetros.

\subsection{Caracterização do uso e ocupação do solo das margens do córrego}

A caraterização das diferentes formas de uso e ocupação do solo das margens do córrego e nascentes foi realizada através de observações in loco e registros fotográficos. Foram catalogadas as espécies vegetais observadas, e verificada a existência de possíveis focos de erosão e lançamentos de esgotos e efluentes industriais.

\section{RESULTADOS E DISCUSSÃO}

\subsection{Análise sazonal da qualidade da água}

Os resultados obtidos para a qualidade da água foram analisados baseando-se no atendimento aos valores recomendados pela resolução 357/05 do CONAMA para rios classe 2. Os valores médios de oxigênio dissolvido (OD) (Figura 3a), para os vários pontos de monitoramento apresentaram concentrações fora do recomendado, que é igual ou superior a 5 mg. $\mathrm{L}^{-1}$, indicando a presença de poluição no córrego. Pode-se observar que durante o período seco (maio a agosto) cerca de 6 pontos de monitoramento estiveram de acordo com o padrão da Resolução CONAMA ou muito próximos desse valor, enquanto no período chuvoso (setembro a abril) somente um ponto de monitoramento (ponto 7) apresentou valor acima de $5 \mathrm{mg} . \mathrm{L}^{-1}$. As maiores concentrações de OD foram nos pontos de monitoramento 4 e 7 (Figura 3a).

Os valores de $\mathrm{DBO}_{5}$ (Figura $3 \mathrm{~b}$ ) estiveram acima do padrão máximo de $5 \mathrm{mg} \cdot \mathrm{L}^{-1} \mathrm{em}$ todos os pontos de amostragem, tanto no período seco quanto no período chuvoso, tendo-se observado valores de $\mathrm{DBO}_{5}$ um pouco mais elevados no período chuvoso, provavelmente devido à contaminação proveniente de escoamento e drenagem pluvial que se reflete também nos valores de OD (Figuras 3a e 3b). Os valores de DQO (Figura 3c) foram mais elevados no ponto 1 que corresponde ao ponto de lançamento de efluente industrial no córrego. A partir do ponto de monitoramento 2, ocorre uma grande redução no valor de DQO no córrego. Em relação ao pH (Figura 3d), observou-se que os valores estiveram próximos de 7,0 no período seco e no período chuvoso e, na maioria dos pontos de monitoramento, apresentaram uma pequena redução no período de chuvas.

Para a turbidez (Figura 4a), foram observados valores inferiores a 100 UNT em todos os pontos nos meses de monitoramento, ou seja, em termos de $\mathrm{pH}$ e turbidez, o córrego está dentro da faixa padrão para a classe 2, segundo a resolução 357/05 do CONAMA. Não foi observada uma tendência de aumento da turbidez no período chuvoso, provavelmente devido à baixa vazão no córrego que deve ter favorecido a sedimentação de sólidos. Os resultados mostraram que foi possível atender ao limite máximo estabelecido pela Resolução CONAMA 357/05 para contagem de coliformes fecais ou termotolerantes para rio classe 2, que é de $1 \times 10^{3} \mathrm{NMP} .100 \mathrm{~mL}^{-1}$. No ponto 1 (período seco), que recebe lançamento de efluente industrial, e nos pontos 5 e 8 (período chuvoso), que se localizam próximos a áreas parcialmente urbanizadas, os valores médios estiveram acima desse valor (Figura 4b). 
A concentração de nitrato foi mais elevada no ponto de monitoramento 1, principalmente, nos meses secos, local que estava recebendo efluente industrial. Pode-se observar que os valores médios de nitrato no período chuvoso foram inferiores aos do período seco (Figura 4c). No entanto, os resultados obtidos para a concentração de nitrato (Figura4c) estiveram abaixo do limite máximo desejável (45 mg. $\mathrm{L}^{-1}$ de nitrato) em todos os pontos de monitoramento. A presença de baixas concentrações de nitrato pode indicar que o nitrogênio esteja presente na forma orgânica ou amoniacal. Foram observadas elevadas concentrações para o nitrogênio total em alguns pontos de amostragem, principalmente nos pontos 1,3 e 5 , com valores entre 150 e $330 \mathrm{mg} . \mathrm{L}^{-1}$. Os resultados indicam uma contaminação do córrego por esgotos domésticos e industriais, com concentrações de nitrato pequenas em comparação à de nitrogênio total, demonstrando a predominância das formas intermediárias do ciclo geoquímico (orgânica, de nitrito e de amônia), provenientes de dejetos orgânicos lançados ao ambiente.

Notou-se que praticamente todos os valores médios para a concentração de fosfato estavam acima do permitido segundo a resolução 357/05 do CONAMA $\left(\leq 0,025 \mathrm{mg}\right.$. $\left.\mathrm{L}^{-1}\right)$ para rios classe 2. Durante o período chuvoso foram obtidas as concentrações mais elevadas de fosfato (Figura 4d). Este aumento foi esperado considerando-se que o escoamento de áreas urbanas, agrícolas ou de matas pode contribuir para o aumento na sua concentração em cursos d'água (Xavier, 2005). A presença de concentrações de fosfato acima do valor estabelecido na resolução 357/05 do CONAMA no córrego Banguelo e no córrego Bom Jesus pode contribuir para o aumento da sua concentração no reservatório da Pampulha, contribuindo para a sua eutrofização.

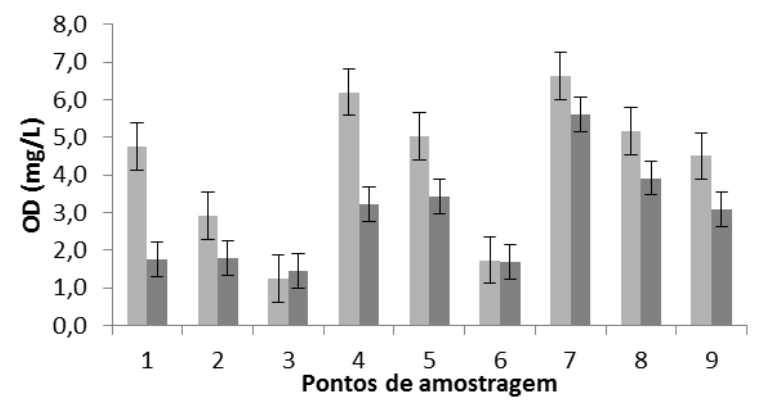

(a)

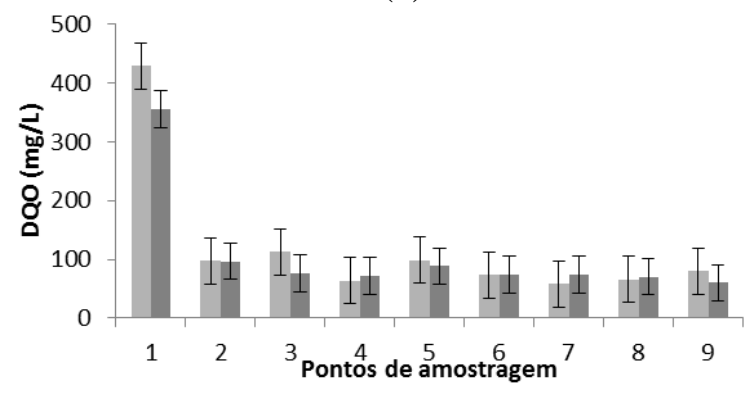

(c)

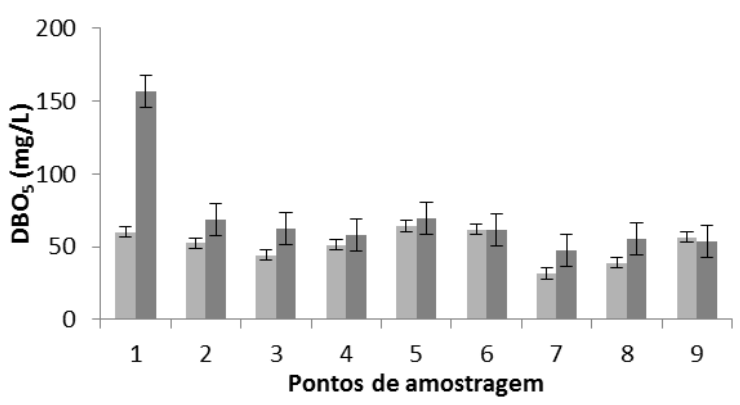

(b)

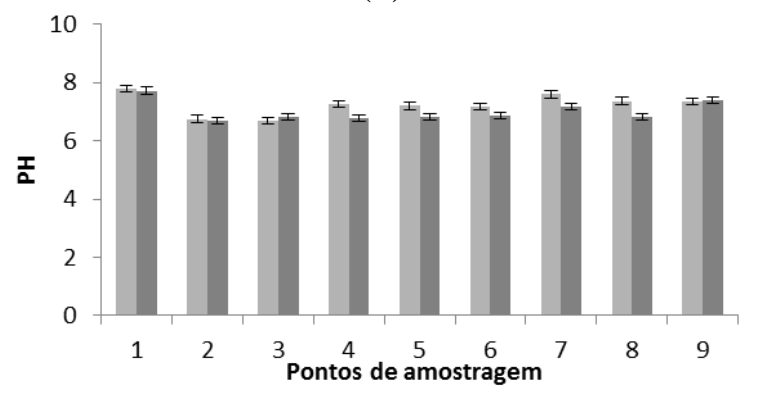

(d)

\section{Período seco Período chuvoso}

Figura 3. Valores médios de (a) OD, (b) $\mathrm{DBO}_{5}$, (c) DQO e (d) $\mathrm{pH}$ nos diferentes pontos de monitoramento na micro-bacia do Córrego Banguelo. 
PONTES, P. P.; MARQUES, A. R.; MARQUES, G. F. Efeito do uso e ocupação do solo na qualidade da água na micro-bacia do Córrego Banguelo - Contagem. Ambi-Agua, Taubaté, v. 7, n. 3, p. 183-194, 2012. (http://dx.doi.org/10.4136/ambi-agua.962)

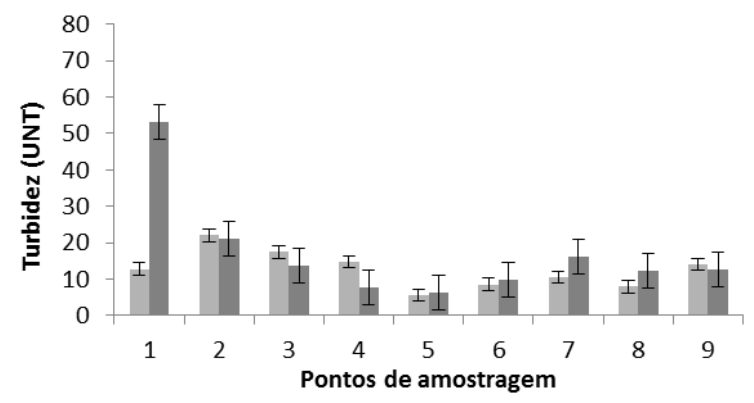

(a)

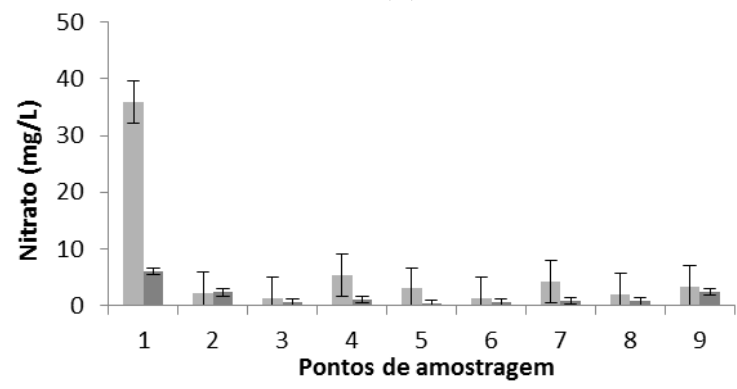

(c)

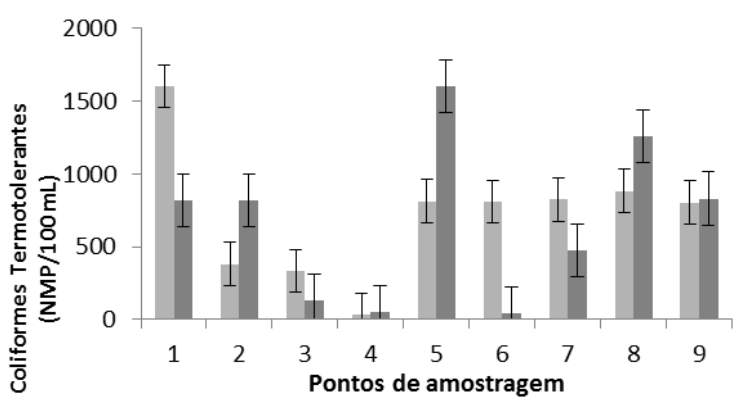

(b)

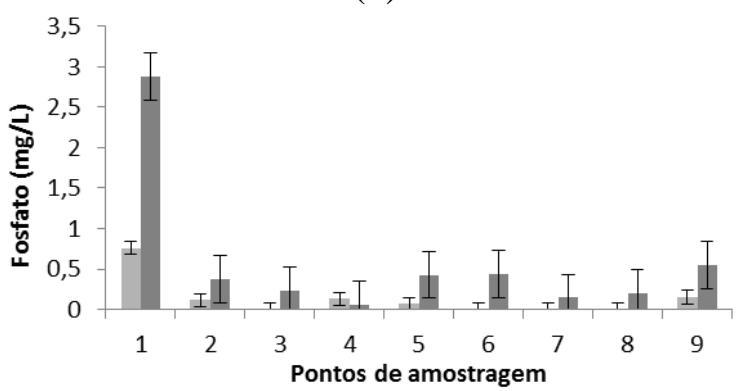

(d)

Período seco Período chuvoso

Figura 4. Valores médios de (a) turbidez, (b) coliformes, (c) nitrato e (d) fosfato nos diferentes pontos de monitoramento na micro-bacia do Córrego Banguelo.

A análise do IQA $A_{N S F}$ indicou que a qualidade da água foi melhor no período chuvoso, sendo observada apenas no ponto 8 uma qualidade da água ruim (Córrego Bom Jesus Figura 1). Nos demais pontos de monitoramento, a qualidade foi média ou boa (Tabela 1).

Tabela 1. Resultados médios para o IQA $A_{\mathrm{NSF}}$ da água coletada no córrego Banguelo no período de setembro de 2008 a julho de 2009.

\begin{tabular}{c|cccccc}
\hline $\begin{array}{c}\text { Ponto de } \\
\text { monitoramento }\end{array}$ & $\begin{array}{l}\text { IQA }_{\text {NSF }} * \\
\text { período } \\
\text { seco }\end{array}$ & $\begin{array}{l}\text { Qualidade } \\
\text { da Água }\end{array}$ & $\begin{array}{l}\text { IQA }_{\text {NSF }} * \\
\text { período } \\
\text { chuvoso }\end{array}$ & $\begin{array}{l}\text { Qualidade } \\
\text { da Água }\end{array}$ & $\begin{array}{l}\text { IQA }_{\text {NSF }}^{*} \\
\text { Médio }\end{array}$ & $\begin{array}{l}\text { Qualidade } \\
\text { da Água }\end{array}$ \\
\hline 1 & 28,4 & Ruim & 56,8 & Médio & 28,4 & Ruim \\
2 & 38,9 & Ruim & 57,1 & Médio & 48,1 & Ruim \\
3 & 37,8 & Ruim & 60,1 & Médio & 48,9 & Ruim \\
4 & 56,2 & Médio & 71,2 & Bom & 63,7 & Médio \\
5 & 39,6 & Ruim & 73,7 & Bom & 56,7 & Médio \\
6 & 33,9 & Ruim & 58,1 & Médio & 46,0 & Ruim \\
7 & 42,6 & Ruim & 62,3 & Médio & 52,5 & Médio \\
8 & 33,9 & Ruim & 44,6 & Ruim & 37,4 & Ruim \\
9 & 36,3 & Ruim & 60,7 & Médio & 36,3 & Ruim \\
\hline
\end{tabular}

* Valores de Índice de Qualidade da Água: Excelente $\left(90<\mathrm{IQA}_{\mathrm{NSF}}<100\right)$, Bom $\left(70<\mathrm{IQA}_{\mathrm{NSF}}<90\right)$, Médio $\left(50<\mathrm{IQ} A_{\mathrm{NSF}}<70\right)$, Ruim $\left(25<\mathrm{IQ} A_{\mathrm{NSF}}<50\right)$, Muito Ruim $\left(0<\mathrm{IQA}_{\mathrm{NSF}}<25\right)$. 
PONTES, P. P.; MARQUES, A. R.; MARQUES, G. F. Efeito do uso e ocupação do solo na qualidade da água na micro-bacia do Córrego Banguelo - Contagem. Ambi-Agua, Taubaté, v. 7, n. 3, p. 183-194, 2012. (http://dx.doi.org/10.4136/ambi-agua.962)

No período seco, a qualidade da água foi ruim em quase todos os pontos de monitoramento. Assim, embora alguns parâmetros tenham apresentado uma pequena deterioração no período chuvoso (devido ao OD e fosfato, principalmente), foi possível observar uma melhoria do IQA $\mathrm{NSF}_{\mathrm{N}}$ nesse período.

\subsection{Efeito do uso e ocupação do solo na qualidade da água}

A maior parte das margens do córrego Banguelo encontrou-se ocupada, particularmente por moradias distribuídas em loteamentos tradicionais ou em chácaras. Próximos aos pontos 1 e 2 de monitoramento da água, foram observadas duas nascentes, sendo que uma delas estava canalizada e dentro de um estabelecimento industrial (Figura 1). Atividades pecuárias e agrícolas em pequena escala foram observadas no entorno do córrego, sendo os pontos 2 , 3, 4 e 5 localizados dentro de uma fazenda. Foram observados diversos focos de erosão próximos ao córrego, consequência do desmatamento, e lançamento de esgoto devido à expansão urbana sem planejamento. Fragmentos florestais remanescentes foram observados na cabeceira do córrego, com diversas áreas de clareiras desmatadas para utilização rural, principalmente próximo às margens do curso d'água. $\mathrm{O}$ ponto 4 , no afluente do córrego estava recebendo água das nascentes ainda protegidas por vegetação arbórea de um destes fragmentos florestais (Figura 1). O ponto 7 , recebendo água de uma pequena lagoa (Figura 1), e os pontos 8 e 9, no córrego Bom Jesus canalizado, estavam em áreas mais urbanizadas.

O levantamento florístico (Tabela 2) mostrou que a maior parte da margem do córrego se encontrava constituído por plantas pioneiras do estrato herbáceo-arbustivo, e por secundárias e clímaxes do estrato arbustivo e arbóreo. Na nascente do afluente do córrego também se observou duas espécies de pteridófitas, Blechnum brasiliensis Desv. (Blechnaceae) e Cyathea delgadii Sternb. (Cyatheaceae), além das espécies arbóreas. Nas áreas de baixadas, bem úmidas ou alagadas, foram comuns as macrófitas das famílias Polygonoceae, Pontederiaceae e Thyphaceae (Tabela 2). Nas margens do córrego das áreas mais urbanizadas as gramíneas (Poaceae) foram predominantes e invasoras como Ricinus communis (mamona).

Tabela 2. Espécies do estrato herbáceo, arbustivo e arbóreo encontradas as margens e nascentes do córrego Banguelo (Contagem) e suas respectivas formas de vida.

\begin{tabular}{l|l|l}
\hline \multicolumn{1}{c|}{ Famílias } & \multicolumn{1}{|c}{ Espécies } & Formas de vida \\
\hline APOCYNACEAE & Asclepias curassavica $\mathrm{L}$ & Erva daninha \\
\hline & Artemisia vulgaris $\mathrm{L}$ & Erva \\
& Baccharis crispa Spreng & Erva \\
& Bidens alba $(\mathrm{L}.) \mathrm{DC}$ & Erva daninha \\
ASTERACEAE & Cyrtocymura scorpioides $($ Lam.) H. Rob & Erva \\
& Pterocaulon virgatum $(\mathrm{L}$.$) DC$ & Erva daninha \\
\hline BURSERACEAE & Protium heptaphyllum (Aubl.) Marchand & Árvore \\
\hline
\end{tabular}


PONTES, P. P.; MARQUES, A. R.; MARQUES, G. F. Efeito do uso e ocupação do solo na qualidade da água na micro-bacia do Córrego Banguelo - Contagem. Ambi-Agua, Taubaté, v. 7, n. 3, p. 183-194, 2012. (http://dx.doi.org/10.4136/ambi-agua.962)

\begin{tabular}{|c|c|c|}
\hline $\begin{array}{l}\text { CYPERACEAE } \\
\text { EUPHORBIACEAE }\end{array}$ & $\begin{array}{l}\text { Rhynchospora corymbosa }(\mathrm{L} \text {.) Britton } \\
\text { Ricinus communis } \mathrm{L}\end{array}$ & $\begin{array}{l}\text { Erva daninha } \\
\text { Arbusto invasor }\end{array}$ \\
\hline FABACEAE & $\begin{array}{l}\text { Dalbergia nigra (Vell.) Alemão ex Benth. } \\
\text { Mimosa bimucronata (DC.) Kuntze } \\
\text { Ormosia arborea (Vell.) Harms } \\
\text { Piptadenia gonoacantha (Mart.) J.F. Macbr }\end{array}$ & $\begin{array}{l}\text { Árvore } \\
\text { Arbusto } \\
\text { Árvore } \\
\text { Árvore }\end{array}$ \\
\hline LAURACEAE & Nectandra oppositifolia Nees & Árvore \\
\hline MELASTOMATACEAE & Miconia chamissois Naudin & Arbusto \\
\hline ONAGRACEAE & Ludwigia elegans (Cambess.) H. Hara & Erva daninha \\
\hline PERACEAE & Pera glabrata (Schott) Poepp. ex Baill & Árvore \\
\hline POACEAE & $\begin{array}{l}\text { Echinochloa cru-sgalli (L.) P.Beauv } \\
\text { Homolepis glutinosa (Sw.) Zuloaga \& Soderstr } \\
\text { Ichnanthus pallens (Sw.) Munro ex Benth. } \\
\text { Lasiacis sorghoidea (Desv. ex Ham.) Hitchc. \& Chase }\end{array}$ & $\begin{array}{l}\text { Erva } \\
\text { Erva } \\
\text { Erva } \\
\text { Erva }\end{array}$ \\
\hline POLYGALACEAE & $\begin{array}{l}\text { Polygala paniculata } \mathrm{L} \\
\text { Polygala monninoides Kunth }\end{array}$ & $\begin{array}{l}\text { Erva } \\
\text { Erva }\end{array}$ \\
\hline POLYGONACEAE & $\begin{array}{l}\text { Polygonum acuminatum Kunt } \\
\text { Polygonum ferrugineum Wedd } \\
\text { Polygonum punctatum Elliott }\end{array}$ & $\begin{array}{l}\text { Macrófita } \\
\text { Macrófita } \\
\text { Macrófita }\end{array}$ \\
\hline PONTEDERIACEAE & $\begin{array}{l}\text { Heteranthera reniformis Ruiz \& Pav } \\
\text { Eichhornia crassipes (Mart.) Solms }\end{array}$ & $\begin{array}{l}\text { Macrófita } \\
\text { Macrófita }\end{array}$ \\
\hline $\begin{array}{l}\text { RUBIACEAE } \\
\text { SOLANACEAE } \\
\text { TYPHACEAE }\end{array}$ & $\begin{array}{l}\text { Borreria capitata (Ruiz \& Pav.) DC. } \\
\text { Solanum americanum Mill. } \\
\text { Typha domingensis Pers }\end{array}$ & $\begin{array}{l}\text { Erva } \\
\text { Erva } \\
\text { Macrófita }\end{array}$ \\
\hline URTICACEAE & $\begin{array}{l}\text { Cecropia hololeuca Miq. } \\
\text { Cecropia pachystachya Trécul }\end{array}$ & $\begin{array}{l}\text { Árvore } \\
\text { Árvore }\end{array}$ \\
\hline VERBENACEAE & $\begin{array}{l}\text { Lantana camara } \mathrm{L} . \\
\text { Stachytarpheta canescens Kunth }\end{array}$ & $\begin{array}{l}\text { Subarbusto } \\
\text { Erva }\end{array}$ \\
\hline
\end{tabular}

A relação entre uso e ocupação do solo e qualidade da água pode ser observada através da análise dos diferentes locais de monitoramento, a exemplo dos pontos 1 e 4 , que estavam próximos às duas nascentes da micro-bacia do córrego Banguelo. $\mathrm{O}$ efeito da poluição causada pelo efluente industrial lançado na nascente próxima do ponto 1 foi constatado pela deterioração da qualidade da água. Observou-se que o lançamento de um efluente industrial contribuiu para um baixo valor de IQA $\mathrm{NSF}_{\mathrm{NS}}$ e elevados valores para $\mathrm{DBO}_{5}, \mathrm{DQO}$, coliformes, 
PONTES, P. P.; MARQUES, A. R.; MARQUES, G. F. Efeito do uso e ocupação do solo na qualidade da água na micro-bacia do Córrego Banguelo - Contagem. Ambi-Agua, Taubaté, v. 7, n. 3, p. 183-194, 2012. (http://dx.doi.org/10.4136/ambi-agua.962)

nitrato e fosfato em relação aos outros pontos de monitoramento do córrego. No entanto, a nascente próxima ao ponto 4 , que está protegida por vegetação, contribuiu para uma melhor

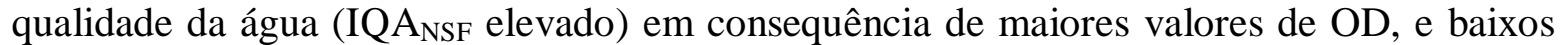
valores para fosfato, nitrato, $\mathrm{DBO}_{5}, \mathrm{DQO}$ e concentração de coliformes termotolerantes. A vegetação ripária pode contribuir para remoção de nitrato, fósforo, pesticidas dentre outros poluentes (Evans et al., 1996; Souza et al., 2004), devendo ser mantida ou recuperada.

O efeito do uso e ocupação do solo na qualidade da água pode ser observado ao longo

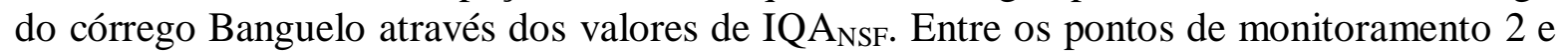
3 observou-se que a presença de macrófitas às margens do córrego, principalmente da espécie Typha domingensis (taboa), Eichhornia crassipes (aguapé) e das espécies Polygonum (erva-de-bicho), que contribuíram junto ao afluente, para melhorar a qualidade da água do córrego Banguelo no ponto 5 (Tabela 1). A partir desses pontos ocorreu um aumento no valor do $\mathrm{IQA}_{\mathrm{NSF}}$ e melhoria geral da qualidade da água. Tal resultado aponta para o potencial benefício no emprego de wetlands e pequenos alagados para melhoria na qualidade da água em regiões parcialmente urbanizadas, especialmente para reduzir a poluição causada pelo escoamento de drenagem pluvial. As regiões de transição solo/água com macrófitas aquáticas, também chamadas wetlands ou terras úmidas são ecótonos e têm papel significativo em processar nutrientes, adsorver e absorver substâncias tóxicas, e em regular o fluxo hidráulico (Marques, 1999; Diniz et al., 2005).

Entre os pontos 5 e 6, as margens do córrego Banguelo apresentaram-se parcialmente urbanizadas, observando-se a maior parte da região ocupada por moradias. A substituição de áreas recobertas por vegetação por áreas impermeabilizadas ocasiona um aumento do volume de água que escoa no período de chuvas. Além disso, o lançamento de esgotos e lixo nos córregos agrava o problema de qualidade das águas, como pode ser observado através dos valores baixos de IQA $A_{N S F}$. Nota-se a redução no OD do ponto 5 para o ponto 6 , resultado do processo de autodepuração do córrego, porém, tal efeito é mais significativo na estação seca, em função das menores vazões nessa época.

Do ponto 4 para o ponto 5 verificou-se um pico na presença de coliformes, consequência de um provável lançamento de efluentes domésticos naquele trecho. Entretanto, percebe-se que o aumento nos coliformes é maior na estação chuvosa, indicativo da presença de escoamento pluvial com contaminação de dejetos, além de uma possível influência de fossas sépticas ali presentes. Esse trecho é caracterizado por residências sem coleta de esgotos, e presença de quintais com criação de animais.

A ocorrência de vegetação herbácea, especialmente gramínea às margens do córrego, e de um lago entre os pontos 6 e 7, pode ter sido um fator que contribuiu para o aumento no valor de IQA $A_{N S F}$. As maiores concentrações de $\mathrm{OD}$ e os menores valores de $\mathrm{DBO}_{5}$ no ponto 7 se deveram, provavelmente, à presença do lago, que pode funcionar como um wetland natural, apresentando uma contribuição mais significativa no processo de autodepuração (Ceballos et al., 2001; Von Sperling e Chernicharo, 2006). Nos pontos de monitoramento 8 (córrego Bom Jesus) e 9 (córrego Bom Jesus após receber a contribuição do córrego Banguelo) observou-se uma diminuição no IQA $\mathrm{NSF}_{\mathrm{N}}$ e deterioração na qualidade da água, possivelmente devido à maior presença de residências nessa área. Esse resultado também indica uma possível contribuição negativa do Banguelo para a qualidade da água do córrego Bom Jesus, e em último caso para o reservatório da Pampulha.

\section{CONCLUSÕES}

A análise do Índice de Qualidade da Água ( $\mathrm{IQA}_{\mathrm{NSF}}$ ) na micro-bacia do córrego Banguelo indicou que a qualidade da água oscilou entre média e ruim no período seco nos 
pontos de monitoramento avaliados. A qualidade da água foi melhor no período chuvoso, tendo-se observado apenas um ponto de monitoramento com qualidade da água ruim no córrego Bom Jesus. Os resultados indicaram que a poluição do córrego Banguelo tem início na região próxima à sua nascente (ponto 1), com lançamento de efluente industrial e ocorrência de níveis baixos de oxigênio dissolvido, presença de matéria orgânica e nutrientes na água monitorada. A poluição das áreas urbanizadas contribuiu para uma maior deterioração da qualidade das águas na micro-bacia do Banguelo, prejudicando não só a população, pelo aspecto e qualidade de suas águas, como também o córrego, que passa a contribuir para a poluição do reservatório da Pampulha, acelerando a eutrofização e deterioração da qualidade da água.

Apesar dos resultados de qualidade da água encontrados, foi possível verificar melhorias nas áreas onde a vegetação ripária encontrou-se menos degradada, destacando a contribuição de espécies como macrófitas na depuração da água e de outras espécies terrestres na proteção contra erosão e aporte de sedimentos ao córrego. Tal contribuição produziu uma recuperação do córrego para melhores patamares de qualidade da água de forma relativamente rápida, o que é percebido comparando os pontos $1 \mathrm{e} 2$. Tais resultados permitem concluir que, apesar dos impactos ambientais presentes no córrego do Banguelo, ainda existem processos naturais (físico-químicos e biológicos) capazes de absorver parte da carga de poluentes lançada. Nesse sentido, ações de coleta e tratamento dos efluentes industriais e domésticos, aliados à preservação da vegetação ripária restante, se apresentam como medidas de grande potencial na melhoria da qualidade das águas do córrego Banguelo e no próprio reservatório da Pampulha. O estudo e aplicação de medidas similares nos demais cursos d'água que drenam para o reservatório da Pampulha constitui, dessa forma, o ponto de partida para a solução do problema de eutrofização ali presente.

\section{AGRADECIMENTOS}

Os autores agradecem à FAPEMIG pelo financiamento do projeto (processo APQ4016-6.01/07) e pelas bolsas concedidas, à Secretaria do Meio Ambiente da Prefeitura de Belo Horizonte, Fundação Zoo-Botânica e ao PROPAM (Programa de Recuperação e Desenvolvimento Ambiental da Bacia da Pampulha) pelo auxílio à pesquisa.

\section{REFERÊNCIAS}

AWWA; APHA; WEF. Standard methods for the examination of water and wastewater. $21^{\text {th }}$ edition. Washington, 2005. $1496 \mathrm{p}$.

CEBAllOS, B. S. O.; KONIG, A.; ROLIM, H. O.; ARAÚJO, M. L.; GUIMARAES, A.O. Efeito do represamento na qualidade da água de um rio impactado por esgotos - rio bodocongó, campina grande, PB. In: CONGRESSO BRASILEIRO DE ENGENHARIA SANITÁRIA E AMBIENTAL, 21., 2001, Joao Pessoa. Proceedings... Joao Pessoa: ABESA, 2001.

DINIZ, C. R.; CEBAllos, B. S. O. de; BARBOSA, J. E. L.; KONIG, A. Uso de macrófitas aquáticas como solução ecológica para melhoria da qualidade de água. Revista Brasileira de Engenharia Agrícola e Ambiental, v. 9, suplemento, p. 226230, 2005.

EVANS, R.; GILLIAM, J. W.; LILLY, J. P. Wetlands and water quality. North Carolina Cooperative Extension Service, 1996. Disponível em: <http://www.bae.ncsu.edu/ programs/extension/evans/ag473-7.html>. Acesso em: ago. 2012. 
FRANCO, R. A. M.; VANZELA, L. S.; HERNANDEZ, F. B. T. Avaliação biológica da qualidade da água para irrigação do Córrego três barras, Marinópolis, SP. In: CONGRESSO NACIONAL DE IRRIGAÇÃO E DRENAGEM (CONIRD), 16. , 2006, Goiânia. Proceedings. Goiânia: ABID, 2006.

FRIESE, K.; SCHMIDT, G.; LENA, J. C.; NALINI, JR., H. A.; ZACHMANN, D. W. Anthropogenic influence on the degradation of an urban lake - The Pampulha reservoir in Belo Horizonte, Minas Gerais, Brazil. Limnologica - Ecology and Management of Inland Waters, v. 40, n. 2, p. 114-125, 2010. http://dx.doi.org/10.1016/j.limno.2009.12.001

INSTITUTO BRASILEIRO DE GEOGRAFIA E ESTATÍSTICA - IBGE. Departamento da População e Indicadores Sociais. Características da população e dos domicílios: resultados do universo. Rio de Janeiro, 2000. 520 p.

MARQUES, D. M. Terras úmidas construídas de fluxo subsuperficial. In: CAMPOS, J. R. (Coord.). Tratamento de esgotos sanitários por processo anaeróbio e disposição controlada no solo. Rio de Janeiro: ABES, 1999. p. 409-435.

PROPAM - PROGRAMA DE RECUPERAÇÃO E DESENVOLVIMENTO AMBIENTAL DA BACIA DA PAMPULHa. Relatório técnico. Belo Horizonte, 2001.

RIETZLER, A. C.; FONSECA, A. L.; LOPES, G. P. Heavy metals in tributaries of Pampulha Reservoir, Minas Gerais. Brazilian Journal of Biology, v. 61, n. 3, p. 363 370, 2001.http://dx.doi.org/10.1590/S1519-69842001000300004

SABINO, C. V. S.; KASTNER, G. F.; AMARAL, A. M.; LEITE, S. C. A.; CARVALHO, R. P.; SILVA, I. S. Estudo da biodisponibilidade de metais nos sedimentos da Lagoa da Pampulha. Química Nova, v. 27, n. 2, p. 231-235, 2004. http://dx.doi.org/10.1590/S0100-40422004000200011

SOUZA, J. T.; VAN HAANDEL, A.; LIMA, E. P. C.; HENRIQUE, I. N. Utilização de wetland construído no pós-tratamento de esgotos domésticos pré-tratados em reator UASB. Engenharia Sanitária e Ambiental, v. 9, n. 4, p. 285-290, 2004. http://dx.doi.org/10.1590/S1413-41522004000400004

VON SPERLING, M.; CHERNICHARO, C. A. L. Biological wastewater treatment in warm climate regions. London: IWA Publishing, 2006. 810p. Vol. 1.

XAVIER, C. F. Avaliação da influência do uso e ocupação do solo e de características geomorfológicas sobre a qualidade das águas de dois reservatórios da região metropolitana de Curitiba - Paraná. 2005. 167f. Dissertação (Mestrado em Ciências do Solo) - Universidade Federal do Paraná, Curitiba, 2005. 


ISSN = 1980-993X - doi:10.4136/1980-993X
www.ambi-agua.net
E-mail: ambi-agua@agro.unitau.br
Tel.: (12) 3625-4212

\title{
Análise morfométrica das sub-bacias hidrográficas Perdizes e Fojo no município de Campos do Jordão, SP, Brasil
}

(http://dx.doi.org/10.4136/ambi-agua.945)

\author{
Agenor Micaeli dos Santos ${ }^{1}$; Marcelo dos Santos Targa ${ }^{1}$; Getulio Teixeira Batista ${ }^{1}$; \\ Nelson Wellausen Dias ${ }^{2}$ \\ ${ }^{1}$ Departamento de Ciências Agrárias, Programa de Pós-Graduação em Ciências Ambientais \\ e-mails: agenormicaeli@yahoo.com.br,targa.marcelo@gmail.com,getulio@agro.unitau.br, \\ ${ }^{2}$ Instituto Brasileiro de Geografia e Estatística - IBGE \\ e-mail: nwdias@gmail.com
}

\section{RESUMO}

A Política de Recursos Hídricos do Estado de São Paulo estabeleceu em 1991 o Sistema Integrado de Gerenciamento de Recursos Hídricos (SIGRHI) e ao definir os princípios básicos para a gestão da água, adotou a bacia hidrográfica como unidade territorial para estudos, planejamento integrado para o desenvolvimento sustentável. O presente trabalho objetivou caracterizar a morfometria das sub-bacias hidrográficas Fojo e Perdizes, no município de Campos do Jordão, SP. A análise morfométrica envolveu a caracterização de parâmetros geométricos, do relevo, da rede de drenagem, e a análise do uso e ocupação do solo das sub-bacias. Na caracterização morfométrica foi encontrada para sub-bacia Perdizes a área de $12,70 \mathrm{~km}^{2}$, o perímetro de $19,85 \mathrm{~km}$ e o comprimento do eixo da bacia de $6,86 \mathrm{~km}$, enquanto para a sub-bacia Fojo, a área de drenagem encontrada foi de $13,97 \mathrm{~km}^{2}$, o perímetro de $19,74 \mathrm{~km}$ e o comprimento do eixo da bacia de $6,94 \mathrm{~km}$. Esses resultados indicam semelhanças entre as duas sub-bacias. O coeficiente de compacidade (Kc) encontrado, 1,56 para a sub-bacia Perdizes e 1,41 para a sub-bacia Fojo, associados aos respectivos fatores de forma, $\mathrm{F}=0,27$ e $\mathrm{F}=0,29$ indicam que estas sub-bacias, em condições normais de precipitação, são pouco suscetíveis a enchentes. $\mathrm{O}$ resultado desses índices é reforçado pelo índice de circularidade encontrado, IC $=0,41$ para Perdizes e IC $=0,45$ para Fojo, pois o afastamento da unidade indica que as sub-bacias não tendem à forma circular, ou seja, possuem forma mais alongada e, portanto, possuem menor concentração de deflúvio. Os resultados obtidos para o Coeficiente de manutenção $(\mathrm{Cm})$ indicam que para manter cada metro de canal, são necessários 286,5 $\mathrm{m}^{2}$ para Perdizes e 243,9 $\mathrm{m}^{2}$ para Fojo. A análise do uso e ocupação do solo revelou que dos quatro tipos de coberturas vegetais existentes: a cobertura vegetal dominante nas duas sub-bacias é de Floresta com 649 ha $(51,1 \%)$ na Perdizes e 608,8 ha $(43,6 \%)$ na Fojo; a cobertura Reflorestamento aparece em segundo lugar, ocupa área muito semelhante nas duas sub-bacias, 218 ha $(17,2 \%)$ na Perdizes e aproximadamente 214 ha $(15,3 \%)$ na Fojo. Em termos de conservação, a sub-bacia Fojo apresenta-se melhor conservada, pois além de menor área urbanizada, apresenta ainda, maior área com cobertura do tipo campo e uma área de floresta apenas um pouco menor que da sub-bacia Perdizes.

Palavras-chave: drenagem, uso do solo, escoamento superficial, manejo de bacias.

\section{Morphometric analysis of sub-basins Fojo and Perdizes in the city of Campos do Jordão, SP, Brazil}

\section{ABSTRACT}

The State of São Paulo Water Resources Policy established, in 1991, the Integrated Water Resources Management System (SIGRHI) and defined the basic principles for water 
management, adopting the watershed as the basic unit for studies, integrated planning for sustainable development. This study characterized the morphology of sub-basins Fojo and Perdizes in the city of Campos do Jordão, SP. Morphometric analysis involved the characterization of geometric parameters, topography and drainage network, and the analysis of land use and land cover of the sub-basins. The morphometric characterization revealed that Perdizes sub-basin has an area of $12.70 \mathrm{~km}^{2}$, a perimeter of $19.85 \mathrm{~km}$, and a main channel length of $6.86 \mathrm{~km}$, while Fojo sub-basin has a drainage area of $13.97 \mathrm{~km}^{2}$, a perimeter of $19.74 \mathrm{~km}$, and a main channel length of $6.94 \mathrm{~km}$. These results indicate similarities between the two sub-basins. The compactness coefficient $(\mathrm{Kc})$ for Perdizes was 1.56 and for Fojo 1.41. These values associated with the respective form factors, $F=0.27$ and $F=0.29$, indicate that these sub-basins, under a normal precipitation regime are not susceptible to flooding. The results of these indices are consisted with the circularity index $(\mathrm{CI})$ found: $\mathrm{CI}=0.41$ for Perdizes and $\mathrm{CI}=0.45$ for Fojo, respectively. As CI values found are far from one, they indicate that these sub-basins tend to have a more elongated shape and, therefore, lower flow concentration tendency. The results obtained for the maintenance coefficient indicate that in order to maintain the flow of each channel meter, Perdizes sub-basin needs $286.5 \mathrm{~m}^{2}$ and Fojo sub-basin needs $243.9 \mathrm{~m}^{2}$. Land cover and land use analysis revealed that among the four existing vegetation cover types, Forest is dominant in both sub-basins with 649 ha $(51.1 \%)$ in Perdizes, and 608.8 ha (43.6\%) in Fojo; Reforestation cover ranks second with similar areas in both sub-basins, Perdizes with 218 ha (17.2\%) and Fojo with approximately 214 ha $(15.3 \%)$. In terms of conservation, Fojo sub-basin can be considered better conserved with a smaller urban area, larger rangeland natural coverage area, and only slightly smaller forest coverage area than Perdizes.

Keywords: drainage, land use, runoff, watershed management.

\section{INTRODUÇÃO}

A Política de Recursos Hídricos no Estado de São Paulo (Lei Estadual 7663/91) estabeleceu uma divisão hidrográfica composta por 21 Unidades de Gerenciamento de Recursos Hídricos (UGRHIs) e o abastecimento público como prioridade no uso da água (São Paulo, 1991). A unidade Serra da Mantiqueira (UGRHI I) é composta pelos municípios de São Bento do Sapucaí, Santo António do Pinhal e Campos do Jordão que integram o Comitê das Bacias Hidrográficas da Serra da Mantiqueira (CBH-SM).

No município de Campos do Jordão, a bacia mais importante é a do rio Capivari, integrada pelas sub-bacias dos afluentes: rio Abernéssia, os ribeirões Imbiri, Perdizes, Fojo e os córregos Mato Grosso e Homem Morto (CBH-SM, 2009). Destes os principais mananciais de captação de água para o abastecimento público para o município de Campos do Jordão são as sub-bacias dos ribeirões Perdizes e Fojo.

O comportamento hidrológico de uma bacia hidrográfica é função de suas características geomorfológicas (forma, relevo, área, geologia, rede de drenagem, solo, dentre outros) e do tipo da cobertura vegetal (Lima, 1986). As características físicas e também as bióticas, principalmente àquelas relacionadas com a cobertura vegetal do solo desempenham importante papel nos processos relacionados à fase terrestre do ciclo hidrológico, influenciando, dentre outros, a infiltração, a evapotranspiração e os escoamentos superficial e subsuperficial e a quantidade de água produzida como deflúvio.

Segundo Kobiyama (2000), os ecossistemas florestais constituídos por parte aérea (árvores) e parte terrestre (solos florestais) desempenham a seguintes funções: (1) mitigação do clima (temperatura e umidade); (2) diminuição do pico do hidrograma (redução de enchentes e aumento da recarga para os rios); (3) controle de erosão; (4) melhoramento da 
qualidade da água no solo e no rio; (5) atenuação da poluição atmosférica; (6) fornecimento do oxigênio $(\mathrm{O} 2)$ e absorção do gás carbônico $(\mathrm{CO} 2)$; (7) prevenção contra ação do vento e ruídos; (8) recreação e educação; (9) produção de biomassa e (10) fornecimento de energia.

O município de Campos do Jordão foi em 2000 caracterizado como o que possuía mais áreas com risco de desabamentos e, portanto, foi classificado em termos de presença de áreas de risco, na faixa entre alto e muito alto risco (IPT, 2003).

A cobertura vegetal nativa do Estado de São Paulo, em especial por ser a mais afetada pelas atividades antrópicas, diminuiu consideravelmente, encontrando-se perto dos $15 \%$ da sua extensão territorial original (Kronka et al., 2005). Nesse sentido, o aumento da população, os processos de urbanização e agricultura entre outras atividades antrópicas se constituem nas principais causas da redução das florestas, redução da água dos mananciais e pode colocar em risco a garantia de oferta de água para as gerações atuais e futuras.

Com relação a atividades antrópicas em bacias, Curvello et al. (2008) em estudo sobre o rio Batedor na Serra da Mantiqueira no município de Cruzeiro, SP, concluíram que sua bacia apresentava características físicas favoráveis ao abastecimento hídrico por ser bem drenada, com grande disponibilidade hídrica, não apresentar risco de enchentes e estar situada em área de muitas nascentes, na encosta da Serra da Mantiqueira, e possuir cursos jovens, com pouca sinuosidade.

As sub-bacias Fojo e Perdizes, no município de Campos do Jordão, SP, na região Serrana do Vale do Paraíba, devido aos problemas advindos do uso e ocupação do solo desordenados, têm recebido recomendações de cuidados relativos à contaminação por efluentes domésticos e criticidade para abastecimento público (CBH-SM, 2009).

As características físicas de uma bacia constituem elementos de grande importância para avaliação de seu comportamento hidrológico, pois, ao se estabelecerem relações e comparações entre elas e os dados hidrológicos conhecidos, pode-se determinar, indiretamente, os valores hidrológicos em locais em que esses dados são desconhecidos (Villela e Mattos, 1975). Por outro lado, para estudos das características de relevo importantes nas bacias hidrográficas deve-se priorizar o uso de métodos quantitativos, pois qualitativamente, os aspectos de forma são insuficientes para a identificação de fatores que influenciam as formas de relevo (Alves e Castro, 2003).

O estudo morfométrico de bacias hidrográficas é definido como a análise quantitativa das relações entre a fisiografia da bacia e a sua dinâmica hidrológica. A análise de parâmetros morfométricos tem grande importância nesses estudos, pois por meio da abordagem quantitativa, pode-se ter uma melhor noção do comportamento hidrológico, uma vez que, os parâmetros morfométricos são bons indicadores da capacidade de escoamento superficial (Nunes et al., 2006).

A área da bacia hidrográfica, por exemplo, tem influência sobre a quantidade de água produzida como deflúvio. A forma e o relevo, no entanto, atuam sobre a taxa ou sobre o regime dessa produção de água, assim como sobre a taxa de sedimentação. O caráter e a extensão dos canais (padrão de drenagem) afetam a disponibilidade de sedimentos, bem como a taxa de formação do deflúvio (Lima, 1986).

Neste contexto, o presente trabalho objetivou analisar a morfometria das sub-bacias dos ribeirões Perdizes e Fojo, afluentes do rio Capivari, utilizados na captação e abastecimento de água para consumo humano no município de Campos do Jordão. 


\section{MATERIAL E MÉTODOS}

\subsection{Caracterização da área de estudo}

Campos do Jordão, com uma área de $290 \mathrm{~km}^{2}$, localiza-se no estado de São Paulo, em um platô a $1.600 \mathrm{~m}$ acima do nível do mar, na Serra da Mantiqueira, distante $167 \mathrm{~km}$ da capital paulista. O município pertence à região administrativa de São José dos Campos, região de governo de Taubaté, no médio Vale do Paraíba, com uma população, em 2010, de 47.504 habitantes (IBGE, 2010). É um dos sete municípios do estado de São Paulo que integram a APA da Mantiqueira. A topografia é bastante acidentada com cerca de $85 \%$ do município composto por regiões onduladas, $10 \%$ por encostas de serra e $5 \%$ por áreas escarpadas. A área urbana da cidade está localizada em um vale com uma parte plana que não ultrapassa 500 metros de largura, contudo, alguns pontos o relevo atinge mais de 2.000 metros de altitude.

A área do presente estudo contempla as sub-bacias hidrográficas dos ribeirões Perdizes e Fojo, localizadas as coordenadas UTM dos cantos, inferior esquerdo E=430.000, $\mathrm{N}=7.480 .000$ e, superior direito $\mathrm{E}=460.000, \mathrm{~N}=7.550 .000$, Datum horizontal SAD-69 e Meridiano Central $45^{\circ} \mathrm{W}$, no município de Campos do Jordão, SP.

As sub-bacias Perdizes e Fojo são pareadas e a demarcação de suas áreas com o estabelecimento dos divisores de águas para a realização da análise morfométrica foi feita com base em fotografias aéreas, mapas temáticos e cartas do Instituto Brasileiro de Geografia e Estatística (IBGE).

Os dados pluviométricos da região de estudo são originários de uma série de dados de 30 anos do posto meteorológico de prefixo D2-096, situado no município de Campos do Jordão, SP e foram obtidos junto ao Banco de Dados Hidrometeorológicos do Estado de São Paulo (São Paulo, 1999). A partir desses dados foi possível estabelecer, para o período de 1972 a 2002, as precipitações mensais máxima, mínima e média (Figura 1), bem como os totais anuais (Figura 2).

Segundo Setzer (1966), o clima dessa região é do tipo Cfb caracterizado pela Classificação Climática do Sistema Internacional de Köeppen, e apresenta um período menos úmido de abril a setembro e um período chuvoso de outubro a março.

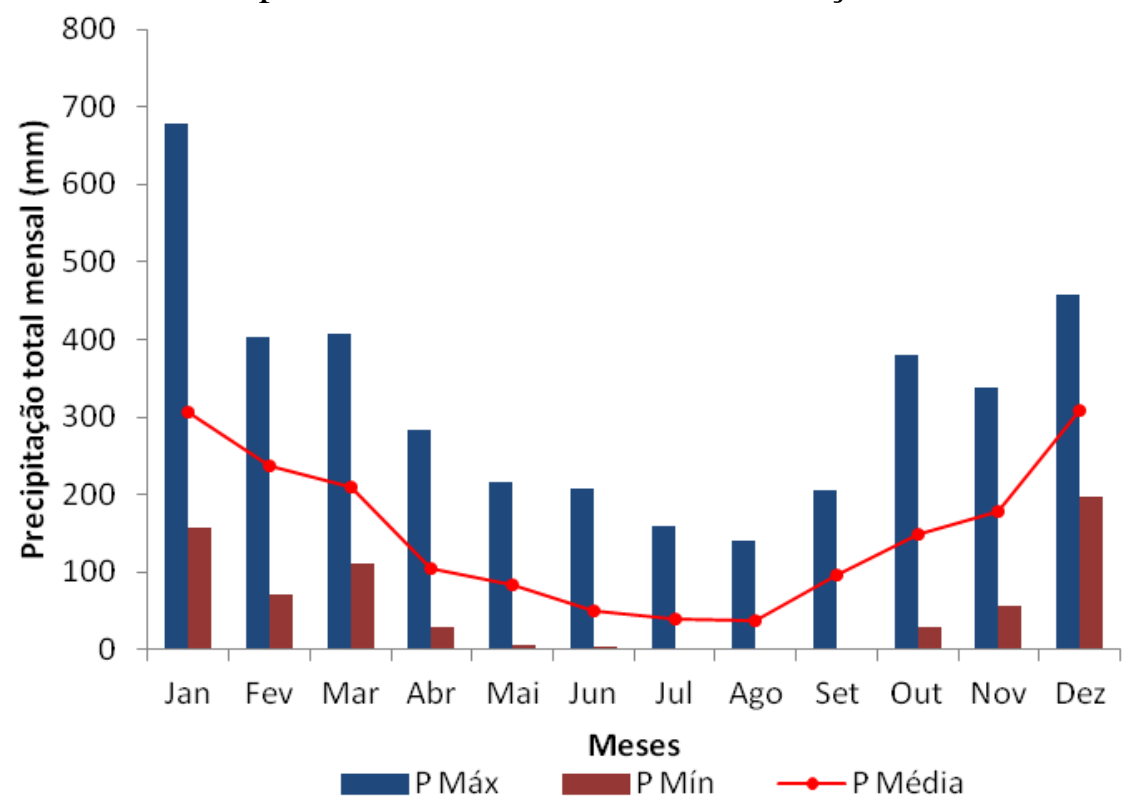

Figura 1. Precipitação mensal mínima, máxima e média para Campos do Jordão, SP, compiladas para o período de 1972 a 2002.

Fonte: (São Paulo, 1999). 


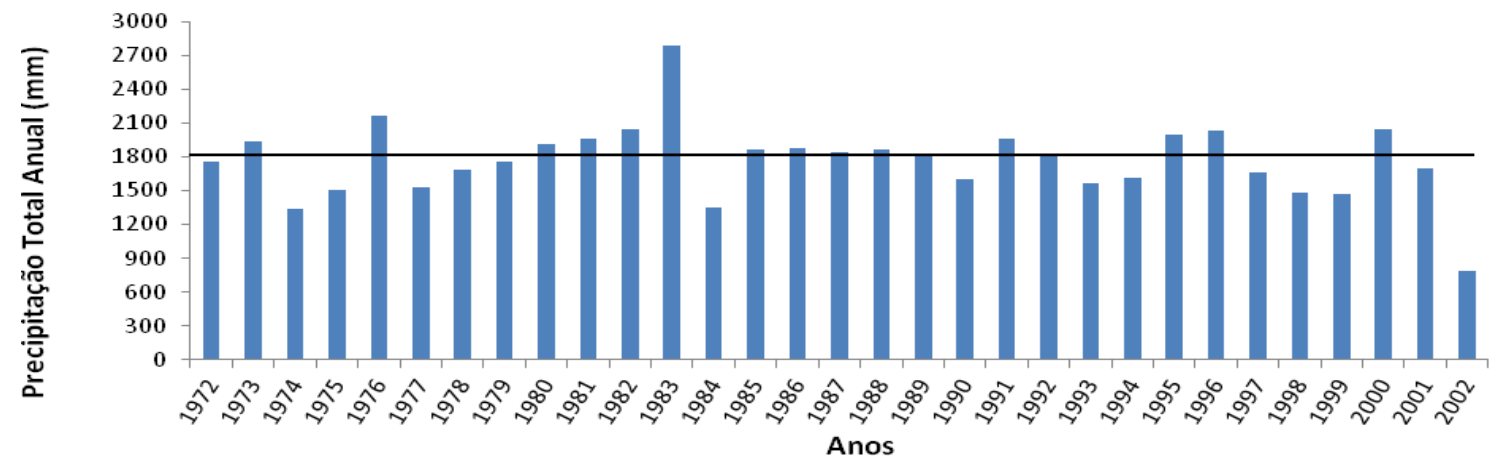

Figura 2. Precipitação Total Anual (mm) de Campos do Jordão, SP, no período de 1972 a 2002.

Fonte: (São Paulo, 1999).

\subsection{Análise morfométrica}

A análise morfométrica das sub-bacias hidrográficas foi feita a partir das características geométricas, de relevo, e das características da rede de drenagem.

$\mathrm{Na}$ caracterização geométrica foram determinados a área, o perímetro, o coeficiente de compacidade (Kc), o fator de forma (F), o índice de circularidade (IC), a densidade hidrográfica $(\mathrm{Dh})$, o número de canais de $1^{\mathrm{a}}$ ordem e o comprimento do eixo da bacia.

O coeficiente de compacidade $(\mathrm{Kc})$, que relaciona a forma da bacia com um círculo, é um número adimensional que varia com a forma da bacia, independente de seu tamanho. De modo que quanto maior a irregularidade da bacia maior será o coeficiente de compacidade. Como o coeficiente de compacidade igual à unidade corresponde a uma bacia circular, ele possibilita a indicação de maior ou menor ocorrência de cheias à medida que se aproxima ou afasta da unidade (Villela e Mattos, 1975). A determinação do Kc pode ser feita por meio da Equação [1]:

$$
K c=0,28 x \frac{P}{\sqrt{A}}
$$

em que:

$\mathrm{Kc}=$ Coeficiente de compacidade adimensional;

$\mathrm{P}=$ Perímetro da bacia em $\mathrm{km}$;

$\mathrm{A}=$ Área da bacia em $\mathrm{km}^{2}$.

O fator de forma (F) relaciona a forma da bacia com um retângulo, correlacionando a razão entre a largura média e o comprimento axial da bacia da foz até o ponto mais distante do espigão. Villela e Mattos (1975) citam que uma bacia com fator de forma baixo é menos suscetível a enchentes.

Para determinar o fator de forma foi utilizada a Equação [2]:

$$
F=\frac{A}{L^{2}}
$$

em que:

$\mathrm{F}=$ Fator de forma, adimensional;

$\mathrm{A}=$ Área da bacia em $\mathrm{km}^{2}$;

$\mathrm{L}=$ comprimento axial da bacia em $\mathrm{km}$. 
O índice de circularidade (IC) relaciona a área da bacia com a área de um círculo de perímetro igual ao da área da bacia. Este valor tende para unidade à medida que a bacia se aproxima da forma circular e diminui conforme a mesma se torna mais alongada (Cardoso et al., 2006).

Para se determinar o índice de circularidade utilizou-se a Equação [3]:

$$
I C=\frac{12,57 x A}{P^{2}}
$$

em que:

IC = Índice de Circularidade, adimensional;

$\mathrm{A}=$ Área da bacia em $\mathrm{km}^{2}$;

$\mathrm{P}=$ Perímetro da bacia em $\mathrm{km}$.

A densidade hidrográfica $(\mathrm{Dh})$ relaciona o número de rios ou canais com a área da bacia. Este índice expressa a grandeza da rede hidrográfica da bacia, indicando a capacidade de gerar novos cursos d'águas (Christofoletti, 1969).

Conforme Silva et al. (2002), o sistema de drenagem de uma bacia pode ser facilmente alterado, bastando uma pequena mudança de gradiente de descarga do rio motivada, por exemplo, por pequenos ajustes neotectônicos e/ou a elevação súbita da descarga da bacia, em razão de enxurradas associadas ao desmatamento em grande escala, na montante da bacia, os quais podem levar à erosão basal das margens côncavas, até a formação de barras de sedimentos, nas margens convexas, a jusante.

Para se determinar a densidade hidrográfica, foi utilizada a seguinte Equação [4]:

$$
D h=\frac{N}{A}
$$

em que:

$\mathrm{Dh}=$ Densidade hidrográfica;

$\mathrm{N}=$ Número de rios ou canais;

$\mathrm{A}=$ Área da bacia $\mathrm{em} \mathrm{km}^{2}$.

Christofoletti (1969) sugere a utilização de N como o número de canais de primeira ordem, conforme classificação de Strahler (1952), com o argumento de que isso conduz a resultados mais realistas sobre o comportamento hidrológico da bacia.

Lollo (1995) cita uma classificação da densidade hidrográfica em faixas de valores (Tabela 1).

Tabela 1. Classificação da densidade hidrográfica $(\mathrm{Dh})$ de bacias.

\begin{tabular}{c|c}
\hline $\begin{array}{c}\text { Dh } \\
\left(* \mathbf{N}_{\mathbf{1}} / \mathbf{k m}^{2}\right)\end{array}$ & Denominação \\
\hline$<3$ & Baixa \\
$3-7$ & Média \\
$7-15$ & Alta \\
$>15$ & Muito alta \\
\hline
\end{tabular}

$* \mathrm{~N}_{1}=$ Comprimento dos rios de primeira ordem conforme Strahler (1952).

Fonte: Lollo (1995). 
Para caracterização de relevo das sub-bacias foram determinados as altitudes máxima, mínima e média, a amplitude altimétrica, o índice de sinuosidade, o gradiente de canais e a relação de relevo.

A variação de altitude média é um fator muito importante, pois a temperatura e precipitação estão relacionadas com a altitude. Variações de temperatura influenciam as perdas de águas que ocorrem na forma de evaporação e transpiração, já variações na precipitação atuarão diretamente no escoamento superficial e infiltração (Villela e Mattos, 1975).

Para avaliação da altitude média foi utilizado o método descrito por Wisler e Brater (1964), no qual se calcula as subáreas existentes entres os pares de curvas de nível, depois se avalia as porcentagens destas subáreas em relação a área total da bacia. Por fim, por simples soma, calcula-se a porcentagem da área total que fica abaixo ou acima de determinada altitude. Com os dados de variação de altitude pode-se determinar a curva hipsométrica da bacia, que é a representação gráfica da variação de altitude em relação à área da bacia.

$\mathrm{O}$ índice de sinuosidade (Is) é a relação entre o comprimento do canal principal e a distância vetorial do canal principal. Este índice expressa a velocidade de escoamento do canal principal. Quanto maior a sinuosidade, maior será a dificuldade de se atingir o exutório do canal, portanto, a velocidade de escoamento será menor.

Segundo Freitas (1952), valores de Is próximos a unidade indicam canais retilíneos, valores de Is superiores a 2 indicam canais sinuosos e os valores intermediários indicam formas transicionais.

Para se determinar o índice de sinuosidade utilizou-se a Equação [5]:

$$
I s=\frac{L c}{L v}
$$

em que:

Is = Índice de sinuosidade, adimensional;

$\mathrm{Lc}=$ comprimento do canal principal em $\mathrm{km}$;

$\mathrm{Lv}=$ comprimento vetorial do canal principal em $\mathrm{km}$.

O gradiente de canais $(\mathrm{Gc})$ é dado pela relação entre a altitude máxima da bacia e o comprimento do canal principal. Este índice tem por finalidade indicar a declividade dos cursos d'água da bacia (Horton, 1945; Freitas, 1952).

Para se determinar o gradiente de canais, foi utilizada a Equação [6]:

$$
G c=\frac{A \max }{L c}
$$

em que:

$\mathrm{Gc}=$ Gradiente de canais em $\mathrm{m} / \mathrm{km}$;

Amax = altitude máxima da bacia em m;

$\mathrm{Lc}=$ comprimento do canal principal em $\mathrm{km}$.

A relação de relevo $(\mathrm{Rr})$ é a relação entre a amplitude altimétrica da bacia e o comprimento do canal principal. Segundo Schumm (1956), quanto maior o valor de Rr, maior será o desnível entre a cabeceira e o exutório, consequentemente maior será a declividade média da bacia.

Para o cálculo da relação de relevo foi utilizada a Equação [7]: 


$$
R r=\frac{H m}{L c}
$$

em que:

$\mathrm{Rr}=$ Relação de relevo em $\mathrm{m} / \mathrm{km}$;

$\mathrm{Hm}=$ amplitude altimétrica em $\mathrm{m}$;

$\mathrm{Lc}=$ comprimento do canal principal em $\mathrm{km}$.

A densidade de drenagem (Dd ) é a relação entre o comprimento total de canais e a área da bacia e para seu cálculo, devem-se considerar todos os rios tanto os perenes como os temporários (Horton, 1945). Para caracterização da rede de drenagem, foram determinados o comprimento do canal principal, o comprimento total dos canais, o comprimento vetorial do canal principal, a densidade de drenagem e a ordem da bacia.

Segundo Christofoletti (1969), valores elevados de Dd indicam áreas com pouca infiltração e melhor estruturação dos canais.

A densidade de drenagem (Dd) foi obtida por meio da Equação [8]:

$$
D d=\frac{L t}{A}
$$

em que:

$\mathrm{Lt}=$ comprimento total dos canais;

$\mathrm{A}=$ Área da bacia.

Beltrame (1994) sugeriu uma classificação em faixas para a densidade de drenagem (Tabela 2).

Tabela 2. Classificação da densidade de drenagem (Dd) de uma bacia.

\begin{tabular}{c|c}
\hline $\begin{array}{c}\text { Dd } \\
\left(\mathbf{k m} / \mathbf{k m}^{2}\right)\end{array}$ & Denominação \\
\hline$<0,50$ & Baixa \\
$0,50-2,00$ & Mediana \\
$2,01-3,50$ & Alta \\
$>3,50$ & Muito Alta \\
\hline
\end{tabular}

Fonte: Beltrame (1994).

A partir da densidade de drenagem é possível se calcular o Coeficiente de manutenção $(\mathrm{Cm})$, que representa a área necessária que a bacia deve ter para manter perene cada metro de canal de drenagem. O Coeficiente de manutenção $(\mathrm{Cm})$ é calculado pela Equação [9]:

$$
C m=\frac{1}{D d}
$$

em que:

$\mathrm{Cm}=$ Coeficiente de manutenção;

$\mathrm{Dd}=$ Densidade de drenagem.

A ordem dos cursos d'água da bacia foi determinada utilizando-se a metodologia descrita por Strahler (1952), em que os canais sem tributários são denominados de primeira ordem, os 
canais de segunda ordem são originados na confluência de canais de primeira ordem, os canais de terceira surgem da confluência de dois canais de segunda ordem, os canais de quarta ordem são originados da confluência de dois canais de terceira ordem e assim sucessivamente. Dessa forma, quanto maior a ordem da bacia, maior será a ramificação do sistema de drenagem.

\subsection{Uso e ocupação do solo}

Na definição do uso e ocupação do solo das sub-bacias Perdizes e Fojo foram utilizadas fotografias aéreas verticais do município de Campos do Jordão, provenientes de aerolevantamento fotográfico na escala 1: 25.000 realizado em abril de 2003 e ortofotos da coleção de fotos do Comitê de Bacias Hidrográficas da Serra da Mantiqueira - CBH-SM, disponíveis em modo digital, com resolução espacial de $60 \mathrm{~cm}$.

A partir das ortofotos e utilizando o aplicativo de Sistema de Informação Geográfica (SIG) da ESRI, ArcGis 9.0, Santos et al. (2011) geraram um mapa temático de uso do solo para campos do Jordão, SP, a partir de recorte feito no mapa de uso e ocupação do solo da região elaborado pelo Instituto Florestal de São Paulo (Figura 3). Para este estudo foi feito um recorte desse mapa utilizando os limites das sub-bacias definidos anteriormente. As classes originais foram reagrupadas em floresta, reflorestamento, campo, pasto, área urbanizada e outros para melhor atender às necessidades deste estudo.

\subsection{Caracterização do solo}

No município de Campos do Jordão, os latossolos LVA9 e LVA18 encontram-se em pequenas quantidades, enquanto o cambissolo CX14 ocupa a maior e principal área do município, incluindo a área das duas sub-bacias em estudo (Santos et al., 2011).

A classificação do solo da área de estudo foi feita a partir de um recorte no Mapa de Solos do Brasil, o qual é resultado da parceria entre o Instituto Brasileiro de Geografia e Estatística - IBGE e o Centro Nacional de Pesquisas de Solos da Embrapa (IBGE, 2002). A classe dos Latossolos constitui o agrupamento de solos mais extenso do Estado de São Paulo. Em geral, são solos com boas propriedades físicas e situados, na maioria dos casos, em relevo favorável ao uso intensivo de máquinas agrícolas, com exceção dos solos em regiões serranas. Segundo Reatto et al. (1999), os Latossolos possuem, geralmente, elevada permeabilidade e a sua capacidade máxima de armazenamento de água pode, em média, variar de 260 a $330 \mathrm{~mm}$ para solos de textura média, de 300 a 500mm para solos de textura argilosa e de 500 a 760 mm para os solos de textura muito argilosa.

Já os Cambissolos, segundo Oliveira (1999), situam-se em relevo forte ondulado a escarpado e apresentam severas restrições quanto ao uso agrícola. Aqueles localizados em terrenos escarpados apresentam maior limitação de uso, mesmo ao uso pastoril e florestal, devido à sua capacidade de desagregação. Tais solos possuem elevada erodibilidade e forte limitação à trafegabilidade, à qual é aumentada com a pedregosidade e afloramentos de rocha e pela presença de solos rasos representados pelos Neossolos Litólicos. É comum a presença de solos apresentando horizonte $\mathrm{Cr}$ (saprolito) constituído por rocha parcialmente intemperizada a profundidades inferiores a $1,5 \mathrm{~m}$.

\section{RESULTADOS E DISCUSSÃO}

A quantidade das áreas de ocorrência dos diferentes tipos de cobertura vegetal e das áreas de ocupação urbanas nas sub-bacias Perdizes e Fojo, com a definição das classes de uso utilizadas nesse trabalho, foram adaptados do mapa de uso e ocupação do solo elaborado por Santos et al. (2011). 
A análise do uso e ocupação do solo das sub-bacias em estudo, revelou que dos quatro tipos de coberturas vegetais existentes: Campo, Floresta, Pasto e Reflorestamento, a cobertura vegetal dominante nas duas sub-bacias é de Floresta (vegetação secundária em estágio médio) com 649 ha $(51,1 \%)$ na Perdizes e 608,8 ha $(43,6 \%)$ na Fojo; a cobertura Reflorestamento aparece em segundo lugar, ocupa área muito semelhante nas duas sub-bacias, 218 ha $(17,2 \%)$ na Perdizes e aproximadamente 214 ha $(15,3 \%)$ na Fojo; o Campo (campo natural com/sem perturbação antrópica) não aparece como uso e cobertura na sub-bacia Perdizes. Por outro lado o Campo ocupa uma área de 265 ha, quase 19\% da sub-bacia Fojo. A cobertura vegetal denominada de Pasto apresenta uma área de 79,8 ha $(6,3 \%)$ na Perdizes e 47 ha $(3,4 \%)$ na Fojo.

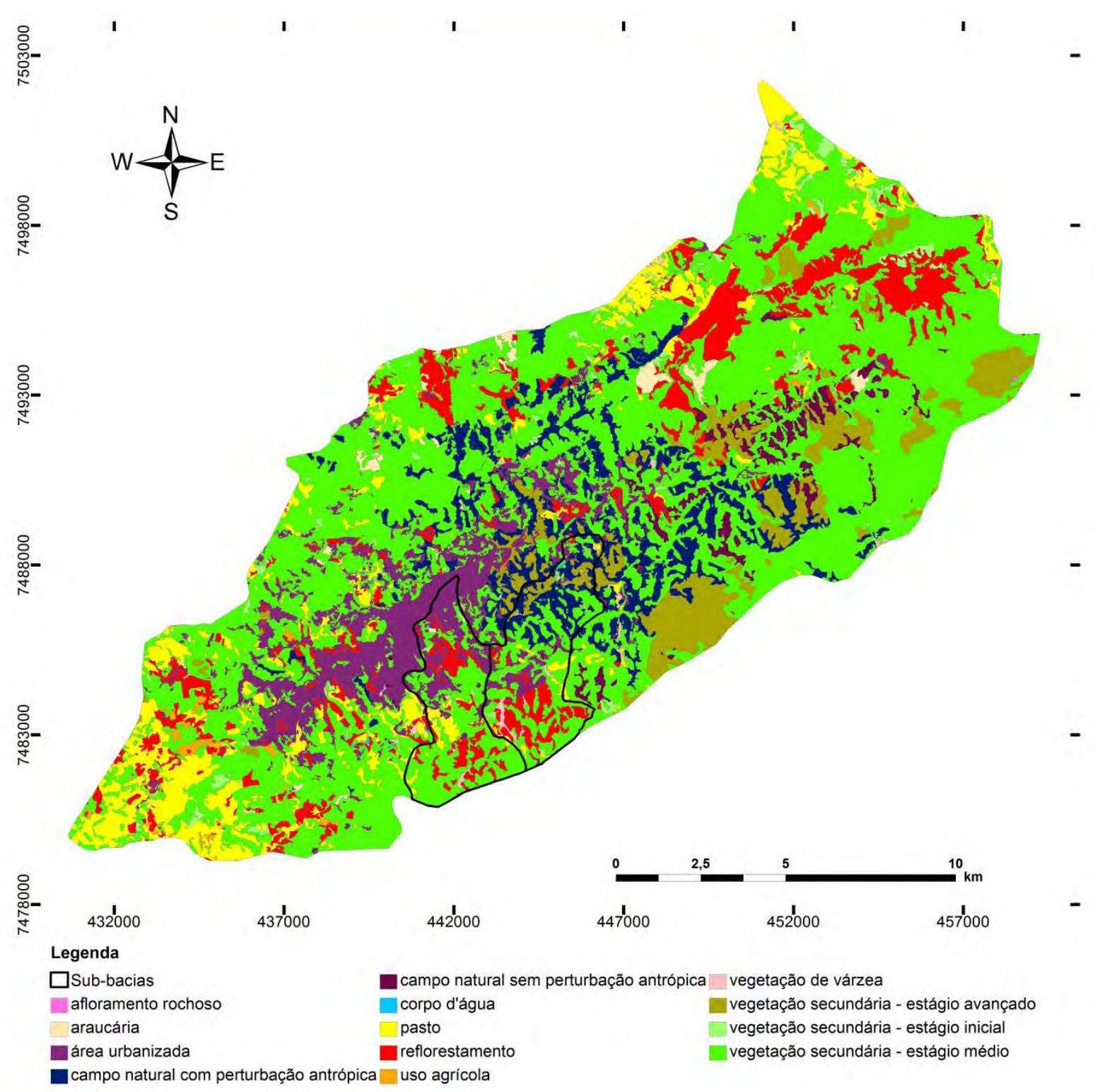

Figura 3. Mapa temático de uso e ocupação do solo do município de Campos do Jordão.

Fonte: Adaptado por Santos et al. (2011).

Atenção especial deve ser dada à classe de uso do solo Área Urbanizada, a qual possui o maior significado antrópico nas sub-bacias, e que no total ocupa 434 ha, sendo que na subbacia Perdizes é de aproximadamente 300 ha $(23,8 \%)$ e na sub-bacia Fojo está acima de 130 ha $(9,5 \%)$. 
Segundo o Instituto de Pesquisa Tecnológica (IPT, 2003), a área do município de Campos do Jordão é de $28.790,35$ ha, enquanto a área total das sub-bacias em estudo corresponde a 2.666 ha $(9,26 \%)$, sendo $4,41 \%$ para a sub-bacia Perdizes e $4,85 \%$ para a subbacia Fojo. Conforme pode ser observado na Figura 4, que apresenta a rede de drenagem, as duas sub-bacias integram o sistema de abastecimento público de Campos do Jordão e são utilizadas pela SABESP. A captação de água é feita em três locais, dois na sub-bacia Perdizes (sendo um mais a montante na bacia, no ribeirão do Salto e outro mais a jusante no próprio ribeirão Perdizes) onde é captada uma vazão de $72 \mathrm{~L} / \mathrm{s}$, e a terceira na sub-bacia Fojo, onde é captada uma vazão de $136 \mathrm{~L} / \mathrm{s}$ em um local mais a jusante conhecido como represa do Fojo (CBH-SM, 2009). As áreas dessas sub-bacias têm sido submetidas a processos diferenciados de uso e ocupação que interferem na dinâmica da água na bacia, o que reforça os resultados obtidos por Santos et al. (2011) para essas bacias com relação as suas capacidades de infiltração e de perdas por escoamento superficial.

Com essas duas captações de água para abastecimento do município, torna-se necessário a implementação de ações de conservação em médio e curto prazo para minimizar possíveis problemas de desabastecimento no município, pois a sub-bacia Perdizes apresenta um menor estado de conservação, uma vez que possui área urbanizada 2,3 vezes maior e não apresenta cobertura vegetal do tipo campo.

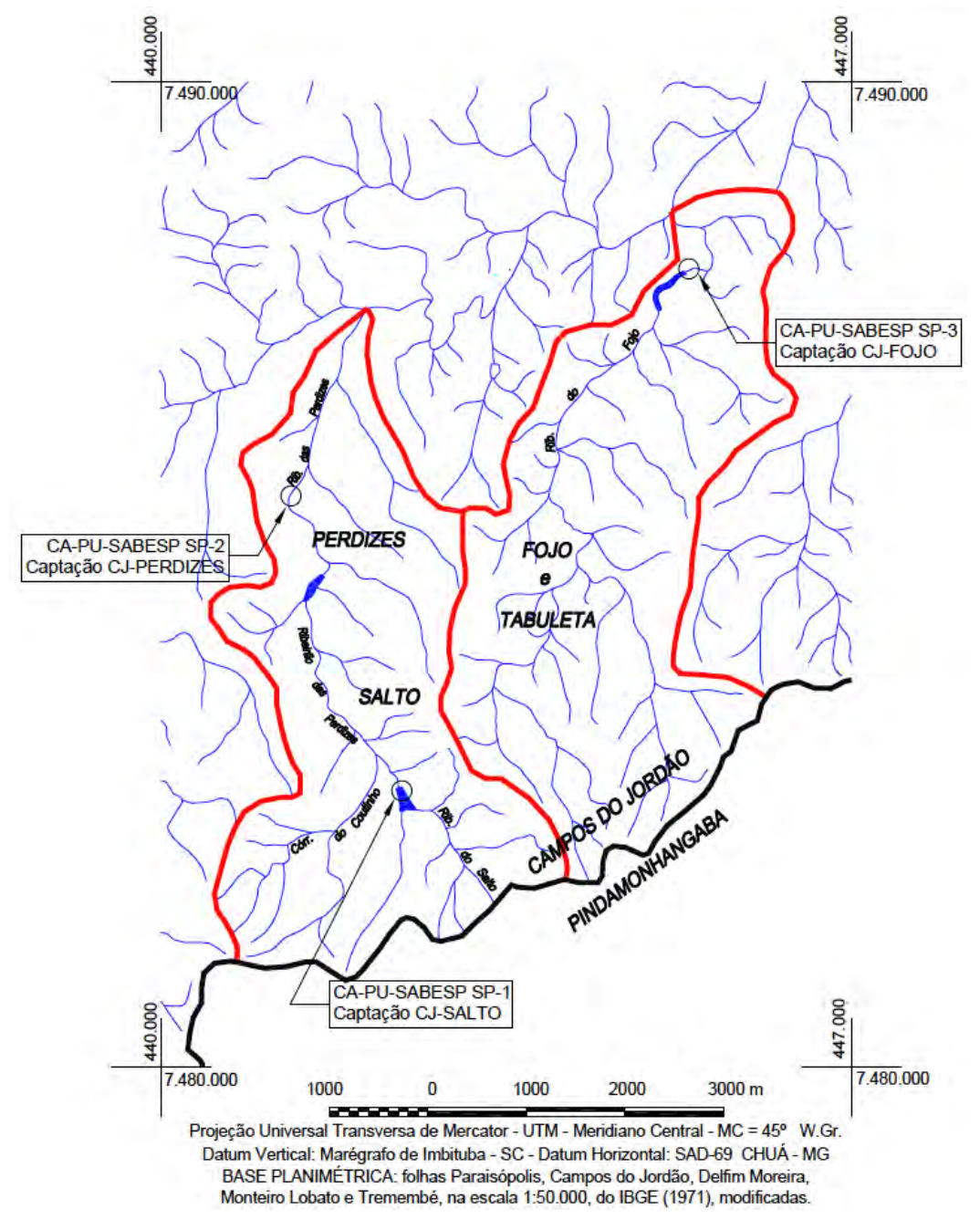

Figura 4. Rede de drenagem das sub-bacias Perdizes e Fojo com destaque para os pontos de captação de água para abastecimento público.

Fonte: Santos et al. (2011). 
SANTOS, A. M.; TARGA, M. S.; BATISTA, G. T.; DIAS, N. W. Análise morfométrica das sub-bacias hidrográficas Perdizes e Fojo no município de Campos do Jordão, SP, Brasil. Ambi-Agua, Taubaté, v. 7, n. 3, p. 195-211, 2012. (http://dx.doi.org/10.4136/ambi-agua.945)

A caracterização morfométrica das sub-bacias Perdizes e Fojo é apresentada na Tabela 3.

Tabela 3. Características morfométricas das sub-bacias Perdizes e Fojo.

\begin{tabular}{|c|c|c|c|c|}
\hline \multirow[b]{3}{*}{$\begin{array}{l}\text { Características } \\
\text { geométricas }\end{array}$} & \multirow{2}{*}{ Características morfométricas } & \multirow{2}{*}{ Unidades } & \multicolumn{2}{|c|}{ Sub-bacias } \\
\hline & & & Perdizes & Fojo \\
\hline & $\begin{array}{l}\text { Área } \\
\text { Perímetro } \\
\text { Número de canais de } 1^{\text {a }} \text { ordem } \\
\text { Comprimento do eixo da bacia } \\
\text { Coeficiente de compacidade (Kc) } \\
\text { Fator de forma (F) } \\
\text { Índice de circularidade (IC) } \\
\text { Densidade hidrográfica (Dh) }\end{array}$ & $\begin{array}{c}\mathrm{km}^{2} \\
\mathrm{~km} \\
- \\
\mathrm{km} \\
- \\
- \\
- \\
\text { canais/km² }\end{array}$ & $\begin{array}{r}12,70 \\
19,85 \\
78 \\
6,86 \\
1,56 \\
0,27 \\
0,41 \\
6,14\end{array}$ & $\begin{array}{r}13,97 \\
19,74 \\
116 \\
6,94 \\
1,48 \\
0,29 \\
0,45 \\
8,30\end{array}$ \\
\hline $\begin{array}{l}\text { Características } \\
\text { do relevo }\end{array}$ & $\begin{array}{l}\text { Altitude máxima } \\
\text { Altitude média } \\
\text { Altitude mínima } \\
\text { Amplitude altimétrica (Hm) } \\
\text { Índice de sinuosidade (Is) } \\
\text { Gradiente dos canais }(\mathrm{Gc}) \\
\text { Relação de relevo (Rr) }\end{array}$ & $\begin{array}{c}\mathrm{m} \\
\mathrm{m} \\
\mathrm{m} \\
\mathrm{m} \\
\mathrm{m} / \mathrm{m} \\
\% \\
\mathrm{~m} / \mathrm{km}\end{array}$ & $\begin{array}{r}1.910 \\
1.720 \\
1.580 \\
330 \\
1,33 \\
22 \\
37,5\end{array}$ & $\begin{array}{r}1.950 \\
1.734 \\
1.520 \\
430 \\
1,43 \\
20 \\
44,6\end{array}$ \\
\hline $\begin{array}{l}\text { Características } \\
\text { da rede de } \\
\text { drenagem }\end{array}$ & $\begin{array}{l}\text { Comprimento do canal principal } \\
\text { Comprimento total dos canais } \\
\text { Comprimento vetorial do canal principal } \\
\text { Densidade de drenagem (Dd) } \\
\text { Coeficiente de Manutenção }(\mathrm{Cm}) \\
\text { Ordem da bacia }\end{array}$ & $\begin{array}{c}\mathrm{km} \\
\mathrm{km} \\
\mathrm{km} \\
\mathrm{km} / \mathrm{km}^{2} \\
\mathrm{~m}^{2} / \mathrm{m} \\
-\end{array}$ & $\begin{array}{r}8,81 \\
44,27 \\
6,63 \\
3,49 \\
286,5 \\
4\end{array}$ & $\begin{array}{r}9,64 \\
57,34 \\
6,73 \\
4,10 \\
243,9 \\
4\end{array}$ \\
\hline
\end{tabular}

Em termos de caracterização geométrica (Tabela 3), foi encontrada para a sub-bacia Perdizes a área de $12,70 \mathrm{~km}^{2}$, o perímetro de $19,85 \mathrm{~km}$ e o comprimento do eixo da bacia de $6,86 \mathrm{~km}$, enquanto para a sub-bacia Fojo, a área de drenagem encontrada foi de $13,97 \mathrm{~km}^{2}$, o perímetro de $19,74 \mathrm{~km}$ e o comprimento do eixo da bacia de $6,94 \mathrm{~km}$. Esses resultados de morfometria indicam que há semelhanças entre as duas sub-bacias.

O coeficiente de compacidade (Kc) encontrado, 1,56 para a sub-bacia Perdizes e 1,41 para a sub-bacia Fojo, associados aos respectivos fatores de forma, $F=0,27$ e $F=0,29$ indicam que estas sub-bacias, em condições normais de precipitação, são pouco suscetíveis a enchentes. O resultado desses índices é reforçado pelo índice de circularidade (IC) encontrado, (IC $=0,41)$ para Perdizes e $(\mathrm{IC}=0,45)$ para Fojo, pois o afastamento da unidade indica que as sub-bacias não tendem à forma circular, ou seja, possuem forma mais alongada e, portanto, segundo Villela e Mattos (1975), possuem menor concentração de deflúvio.

A densidade hidrográfica indica a capacidade das sub-bacias gerarem novos canais e de acordo com Lana et al. (2001), se esse índice estiver acima de 2,00 canais $/ \mathrm{km}^{2}$, a bacia teria grande capacidade de gerar novos cursos d'água. No presente estudo foi encontrada densidade hidrográfica de 6,14 canais $/ \mathrm{km}^{2}$ para a sub-bacia Perdizes e 8,30 canais $/ \mathrm{km}^{2}$ para a sub-bacia Fojo, portanto, de acordo com os parâmetros (Tabela 1) estabelecidos em Lollo (1995), a subbacia Perdizes possui média densidade hidrográfica e a sub-bacia Fojo possui densidade hidrográfica considerada alta. O resultado maior na sub-bacia Fojo é reforçado por apresentar parâmetros de comprimento do canal principal e comprimento total de canais superiores ao da sub-bacia Perdizes, o que deve possibilitar maior facilidade de uma partícula de água que cai na superfície do solo encontrar rapidamente um canal e escoar.

Em termos de características do relevo (Tabela 3), a sub-bacia Perdizes apresenta altitude 
máxima e mínima de 1910 m e 1580 m, respectivamente, enquanto na sub-bacia Fojo esses valores atingem $1950 \mathrm{~m}$ e $1520 \mathrm{~m}$. Dessa forma, os valores encontrados para a Amplitude altimétrica $(\mathrm{Hm})$ foram de 330 para Perdizes e 430 para Fojo, o que indica que estas subbacias possuem relevo montanhoso, o que influencia na quantidade de radiação que a bacia hidrográfica recebe e, consequentemente, na evapotranspiração, na temperatura e na precipitação (Tonello et al., 2006). Os altos valores de amplitude altimétrica, como os observados, tendem a favorecer o escoamento rápido. Esses resultados são reforçados pelos elevados valores encontrados para o Gradiente de canais $(\mathrm{Gc})$ e para a Relação de relevo (Rr) nas duas sub-bacias.

A altitude média obtida, segundo a metodologia de Wisler e Bratter (1964), foi de 1.720 m para a sub-bacia Perdizes e de 1.734 m para a sub-bacia Fojo. Com os resultados da variação altimétrica, foi possível traçar a curva hipsométrica e obter-se, assim, um valor também importante que é a altitude mediana. Para a sub-bacia Perdizes a altitude mediana encontrada foi de $1.724 \mathrm{~m}$ (Figura 5) e para sub-bacia Fojo foi de $1.727 \mathrm{~m}$ (Figura 6).

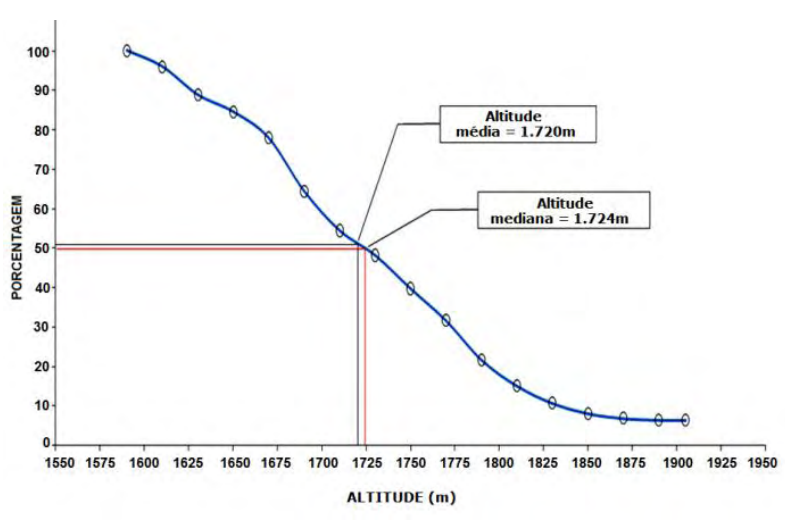

Figura 5. Curva hipsométrica da sub-bacia Perdizes.

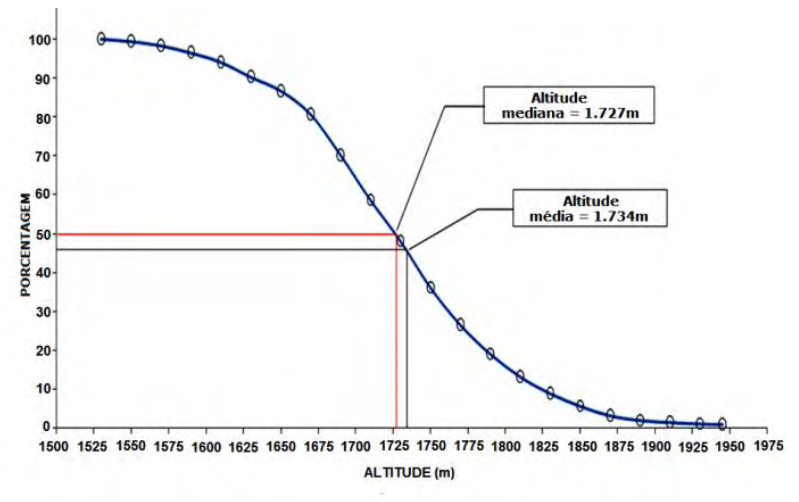

Figura 6. Curva hipsométrica da sub-bacia Fojo.

O índice de sinuosidade dos canais está relacionado à velocidade do escoamento nos canais de drenagem e, em síntese, sua relação com o solo em produzir erosão. Os índices de sinuosidade (Is) encontrados foram 1,33 para Perdizes e 1,43 para Fojo, os quais indicam que os canais de drenagem destas sub-bacias não têm forma retilínea, mas também, não podem ser considerados como sinuosos, ou seja, os canais têm uma forma transitória. Considerando que os solos dessas bacias são Cambissolos que possuem elevada capacidade de desagregação devido a elevada erodibilidade e forte limitação à trafegabilidade (Oliveira, 1999), é indicado o cuidado no uso desses solos principalmente quando à cobertura, especialmente para a urbanização e agricultura.

Em termos das características da rede de drenagem (Tabela 3) da área de estudo, os comprimentos totais dos canais principais foram de 8,81 km (Perdizes) e 9,64 km (Fojo). Ambas sub-bacias são de quarta ordem, sendo que a sub-bacia do Fojo possui 116 canais que apresentam comprimento total de $57,34 \mathrm{~km}$, enquanto a sub-bacia Perdizes possui 78 canais com um comprimento total de $44,27 \mathrm{~km}$.

A densidade de drenagem, que é uma das variáveis mais importantes para a análise morfométrica das bacias hidrográficas, representa o grau de dissecação topográfica, em paisagens elaboradas pela atuação fluvial, ou expressa a quantidade disponível de canais para o escoamento e o controle exercido pelas estruturas geológicas (Christofoletti, 1981).

Segundo Villela e Mattos (1975), a densidade de drenagem de bacias hidrográficas varia de $0,5 \mathrm{~km} / \mathrm{km}^{2}$ (para bacias com drenagem pobre) a $3,5 \mathrm{~km} / \mathrm{km}^{2}$ ou mais (para bacias excepcionalmente bem drenadas). Considerando os resultados obtidos nesse trabalho, a sub- 
bacia Perdizes apresenta densidade de drenagem alta $\left(D d=3,49 \mathrm{~km} / \mathrm{km}^{2}\right)$ enquanto que a Fojo enquadrou-se na classificação muito alta $\left(\mathrm{Dd}=4,1 \mathrm{~km} / \mathrm{km}^{2}\right)$, conforme classificação proposta por Beltrame (1994). Assim, os valores encontrados sugerem que há um elevado escoamento superficial associado a uma alta dissecação. Essa variável se relaciona diretamente com os processos climáticos atuantes na área estudada, os quais influenciam o fornecimento e o transporte de material detrítico ou indicam o grau de manipulação antrópica. Em outras palavras, para um mesmo tipo de clima, a densidade de drenagem depende do comportamento hidrológico dos solos e rochas. Assim, nos mais impermeáveis, as condições para ocorrer escoamento superficial são melhores, possibilitando a formação de canais e, consequentemente, aumentando a densidade de drenagem. O contrário acontece com rochas de granulometria grossa (Horton, 1945).

Outro importante parâmetro da qualidade da rede de drenagem de uma bacia é o Coeficiente de manutenção $(\mathrm{Cm})$, calculado pela relação inversa da densidade de drenagem e que fornece a área mínima necessária para a manutenção de um metro de canal de escoamento (Schumm, 1956). Esse dado permite representar qual seria a área necessária para a manutenção de um metro de curso fluvial perene. Assim, em termos de balanço hidrodinâmico das duas sub-bacias, os resultados obtidos indicam que são necessários 286,5 $\mathrm{m}^{2}$ de área para manter perene cada metro de canal na Perdizes contra $243,9 \mathrm{~m}^{2}$ de área para manter perene cada metro de canal da sub-bacia Fojo. Conforme se pode inferir a partir da Figura 1, a precipitação média mensal é de aproximadamente $150 \mathrm{~mm}$ e a precipitação média anual de aproximadamente $1.800 \mathrm{~mm}$ (Figura 2). Esses valores indicam que a área de estudo é bastante úmida durante o ano. No período menos chuvoso, de abril a setembro (Figura 2), a média mensal de chuva está em torno de $70 \mathrm{~mm}$, enquanto que no período mais úmido, de outubro a março, a média mensal de chuva atinge $230 \mathrm{~mm}$. Isso faz com que no período chuvoso, e em momentos de chuva intensas, haja condições mais propícias ao escoamento do que à infiltração. Dessa forma, o tipo de uso e cobertura pode influenciar na capacidade de produção de água da bacia, sendo importante o seus estados de conservação.

\section{CONCLUSÃO}

Com base na análise morfométrica pôde-se concluir que: as sub-bacias Perdizes e Fojo possuem, respectivamente, média e alta capacidade de formar novos cursos d'água; pelos valores do índice de circularidade não há concentração do deflúvio; e, em função dos valores do fator de forma associados ao coeficiente de compacidade, provavelmente as duas subbacias não são sujeitas a enchentes em condições normais de precipitação.

Com relação às características de relevo, conclui-se que: as sub-bacias Perdizes e Fojo possuem altas altitudes; canais com baixa sinuosidade transitória (nem retos, nem sinuosos); e valores elevados de amplitude altimétrica, da relação de relevo e do gradiente de canais o que indica que o relevo é montanhoso e que favorece o escoamento.

Com relação às características da rede de drenagem, pôde-se concluir que: as sub-bacias Perdizes e Fojo são respectivamente de alta e muito alta densidade de drenagem, sugerindo haver, em momentos de precipitação, um elevado escoamento superficial e uma alta dissecação associada e que ambas as bacias têm área suficiente para manter perenes os cursos d'água.

A área em estudo apresenta características físicas favoráveis ao abastecimento hídrico por ter clima úmido e por localizar-se em altitude elevada, por ter precipitação média mensal alta e por ser bem drenada. A sub-bacia Perdizes apresenta um estado de conservação preocupante por possuir área urbanizada 2,3 vezes superior à sub-bacia Fojo, possuir dois pontos de captação de água para abastecimento localizados praticamente em área urbanizada e não apresentar cobertura vegetal do tipo campo. 


\section{REFERÊNCIAS}

ALVES, J. M. P.; CASTRO, P. T. A. Influência de feições geológicas na morfologia da bacia do rio Tanque (MG) baseada no estudo de parâmetros morfométricos e análise de padrões de lineamentos. Revista Brasileira de Geociências, v. 33, n. 2, p. 117-127, 2003.

BELTRAME, A. V. Diagnóstico do meio ambiente físico de bacias hidrográficas: modelo de aplicação. Florianópolis: UFSC, 1994. 112 p.

CARDOSO, C. A.; DIAS, H. C. T.; SOARES, C. P. B.; MARTINS, S. M. Caracterização morfométrica da bacia hidrográfica do rio Debossan, Nova Friburgo - RJ. Revista Árvore, Viçosa, MG, v. 30, n. 2, p. 241-248, 2006. http://dx.doi.org/10.1590/S010067622006000200011

Comitê das bacias hidrográficas da Serra da Mantiqueira - CBH-SM. Relatório técnico preliminar zoneamento ambiental da unidade de gerenciamento de recursos hídricos - Mantiqueira (UGRHI 01). São Paulo, 2009. 143p.

CHRISTOFOLETTI, A. Análise morfométrica de bacias hidrográficas. Notícia Geomorfológica, v. 18, n. 9, p. 35-64, 1969.

CHRISTOFOLETTI, A. Geomorfologia fluvial: o canal fluvial. São Paulo: Edgard Blücher, $1981.313 \mathrm{p}$.

CURVELlO, R. T.; BATISTA, G. T.; TARGA. M. dos S. Estudo dos impactos da ocupação humana na microbacia do rio Batedor na serra da Mantiqueira no município de Cruzeiro, SP, Brasil. Revista Ambiente \& Água, Taubaté, v. 3, n. 1, p. 91-107, 2008. http://dx.doi.org/10.4136/ambi-agua.45

FREITAS, R. O. Textura de drenagem e sua aplicação geomorfológica. Boletim Paulista de Geografia, v. 11, p. 53-57, 1952.

HORTON, R. E. Erosional development of streams and their drainage basins: hydrophysical approach to quantitative morphology. Geological Society of America Bulletin, v. 56, n. 3, p. 275-370, 1945. http://dx.doi.org/10.1130/0016-7606(1945)56[275:EDOSAT]2.0.CO;2

INSTITUTO BRASILEIRO DE GEOGRAFIA E ESTATÍSTICA - IBGE. Cidades: censo 2010. Disponível em: <http://www.ibge.gov.br/cidadesat/topwindow.htm?1>. Acesso em: 21 nov. 2012.

INSTITUTO BRASILEIRO DE GEOGRAFIA E ESTATÍSTICA - IBGE. Mapa de solos do Brasil. Rio de Janeiro, 2002. Disponível em: <ftp://geoftp.ibge.gov.br/mapas/tematicos/ mapas_murais/solos.pdf $>$. Acesso em: 20 abr. 2008.

INSTITUTO DE PESQUISA TECNOLÓGICA - IPT. Relatório técnico sobre Campos do Jordão-SP. Relatório no 64.399. São Paulo, 2003.

KOBIYAMA, M. Ruralização na gestão de recursos hídricos em área urbana. Revista OESP Construção, São Paulo, ano 5, n. 32, p. 112-117, 2000. 
KRONKA, F. J. N.; NALON, M. A.; MATSUKUMA, M. M.; KANASHIRO, M. M.; YWANE, M. S. S.; LIMA, L. M. P. R.; et al. Monitoramento da vegetação natural e do reflorestamento no Estado de São Paulo. In: Simpósio Brasileiro de Sensoriamento Remoto, 12., 16-21 abril 2005, Goiânia. Anais... São José dos Campos: INPE, 2005. p. 1569-1576. Disponível em: <http://marte.dpi.inpe.br/col/ltid.inpe.br/sbsr/2004/ 11.01.10.06/doc/1569.pdf>. Acesso em: mar. 2008.

LANA, C. E.; ALVES, J. M. de P.; CASTRO, P. T. A. Análise morfométrica da bacia do Rio do Tanque, MG - BRASIL. REM: Revista Escola de Minas, Ouro Preto, v. 54, n. 2, p. 121-126, 2001. http://dx.doi.org/10.1590/S0370-44672001000200008

LIMA, W. P. Princípios de hidrologia florestal para o manejo de bacias hidrográficas. Piracicaba: ESALQ, 1986. 242p.

LOLLO, J. A. O uso da técnica de avaliação do terreno no processo de elaboração do mapeamento geotécnico: sistematização e aplicação na quadrícula de Campinas. 1995. Tese (Doutorado em Geotecnia) - Escola de Engenharia de São Carlos, Universidade de São Paulo, São Carlos, 1995.

NUNES, F. G.; RIBEIRO, N. C.; FIORI, A. P. Propriedades morfométricas e aspectosfísicos da bacia hidrográfica do Rio Atuba: Curitiba-Paraná. In: Simpósio Nacional de Geomorfologia, 6., 2006, Goiânia. Artigos... Goiânia: UFG, 2006.

OLIVEIRA, J. B. Solos do estado de São Paulo: descrição das classes registradas no mapa pedológico. Campinas: Instituto Agronômico, 1999. 112p. (Boletim Científico, 45).

REATTO, A.; SPERA, S. T.; CORREIA, J. R.; MILHOMEM, A. S. Caracterização dos solos e sua associação com as fitofisionomias em uma bacia hidrográfica: aspectos pedológicos e químicos. Planaltina: Embrapa Cerrados, 1999. 23p. (Boletim de Pesquisa, 8)

SÃO PAULO (Estado). Departamento de Águas e Energia Elétrica - DAEE. Banco de Dados Hidrometeorológicos do estado de São Paulo. Dados pluviométricos de Campos do Jordão. São Paulo, 1999. Disponível em: <http://www.sigrh.sp.gov.br/cgibin/bdhm2.exe/plu>. Acesso em: 18 dez. 2012.

SÃO PAUlO (Estado). Política Estadual de Recursos Hídricos. Lei $N^{\circ} 7663$, de 30 de dezembro de 1991. São Paulo, 1991. 19p. Disponível em: 〈http://www.daee.sp.gov.br〉. Acesso em: 04 jul. 2012.

SANTOS, A. M.; TARGA, M. S.; BATISTA, G. T.; DIAS, N. W. Florestamento compensatório com vistas à retenção de água no solo em bacias hidrográficas do município de Campos do Jordão, SP, Brasil. Revista Ambiente \& Água, Taubaté, v. 6, n. 3, p. 110-126, 2011. http://dx.doi.org/10.4136/ambi-agua.490

SETZER, J. Atlas climático e ecológico do estado de São Paulo. [S.1.]: Comissão Interestadual da Bacia Paraná-Uruguai, 1966. 61p.

SCHUMM, S. A. Evolution of drainage systems and slopes in badlands at Perth Amboy, New Jersey. Geological Society of America Bulletin, v. 67, n. 5, p. 597- 646, 1956. http://dx.doi.org/10.1130/0016-7606(1956)67[597:EODSAS]2.0.CO;2 
SILVA, L. G. T.; SILVA, B. N. R. da; RODRIGUES, T. E. Análise fisiográfica das várzeas do baixo Tocantins: uma contribuição ao manejo e desenvolvimento dos sistemas de uso da terra. Belém, PA: EMBRAPA Amazônia Oriental, 2002. 34 p. (Documentos, 149).

STRAHLER, A. N. Hypsometric (area-altitude) analysis of erosional topography. Geological Society of America Bulletin, v. 63, n. 11, p. 1117-1142, 1952. http://dx.doi.org/10.1130/0016-7606(1952)63[1117:HAAOET]2.0.CO;2

TONELlO, K. C.; DIAS, H. C. T.; SOUZA, A. L.; RIBEIRO, C. A. A. S.; LEITE, F. P. Morfometria da bacia hidrográfica da Cachoeira das Pombas, Guanhães - MG. Revista Árvore, Viçosa, MG, v. 30, n. 5, 2006. http://dx.doi.org/10.1590/S010067622006000500019

VILLELA, S. M.; MATTOS, A. Hidrologia aplicada. São Paulo: Mc Graw-Hill do Brasil, 1975.

WISLER, C. D.; BRATER, E. F. Hidrologia. Rio de Janeiro: Livro Técnico, 1964. 484 p. 


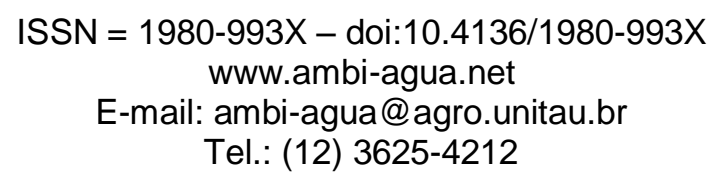

\title{
Vazão ecológica e disponibilidade hídrica na bacia das Pedras, Guarapuava-PR
}

\author{
(http://dx.doi.org/10.4136/ambi-agua.840)
}

\section{Leandro Redin Vestena ${ }^{1}$; Éderson Dias de Oliveira ${ }^{1}$; Márcia Cristina da Cunha ${ }^{1}$; Edivaldo Lopes Thomaz}

\author{
Programa de Pós-Graduação em Geografia, Universidade Estadual do Centro-Oeste \\ Unicentro, Guarapuava, Paraná, Brasil, \\ e-mails: vestena@unicentro.br, edersonjandaia@hotmail.com, \\ marcia1cunha@yahoo.com.br, thomaz@unicentro.br
}

\section{RESUMO}

O conhecimento da vazão ecológica é de suma importância para se determinar a disponibilidade hídrica, visando ao gerenciamento dos recursos hídricos. O presente estudo estimou a vazão ecológica e avaliou a disponibilidade hídrica em um trecho do rio das Pedras, manancial da cidade de Guarapuava, região Centro-Sul do Estado do Paraná. Para tal, aplicaram-se diferentes métodos empregados no Brasil para obtenção da vazão ecológica, uma vazão de referência à concessão de outorgas, e avaliou-se o regime fluvial. Os métodos utilizados foram média mínima de sete dias com período de retorno de dez anos, vazões associadas às permanências de $95 \%$ e $90 \%$, vazões mínimas anuais de sete dias e vazão aquática de base. Os dados de vazão utilizados foram da estação fluviométrica localizada na ETA (Estação de Captação de Água de Guarapuava). Conclui-se que o débito fluvial anual, entre os anos de 1985 e 2009, apresentou uma média diária de $9,12 \mathrm{~m}^{3} \mathrm{~s}^{-1}$ e uma mediana de $9,16 \mathrm{~m}^{3} \mathrm{~s}^{-1}$. A vazão ecológica estimada pelos métodos utilizados para o trecho do rio das Pedras variou de 1,72 a $2,74 \mathrm{~m}^{3} \mathrm{~s}^{-1}$, com uma média de $2,20 \mathrm{~m}^{3} \mathrm{~s}^{-1}$, e coeficiente de variação de $19,5 \%$. A vazão ecológica estimada para o trecho foi de $0,91 \mathrm{~m}^{3} \mathrm{~s}^{-1}$, conforme os critérios adotados no Estado do Paraná. No trecho avaliado, a relação entre a vazão diária e o volume outorgado indica a ineficiência dos métodos avaliados na determinação da vazão ecológica, ou seja, eles não garantiram o volume de água mínimo necessário à conservação do ecossistema fluvial.

Palavras-chave: vazão ambiental, regime fluvial, bacia hidrográfica, ecossistema fluvial.

\section{Instream flow and water availability in the Rio das Pedras basin, Guarapuava-PR, Brazil}

\begin{abstract}
Knowledge of instream flow is of paramount importance to determine water availability for water resources management. This study estimated instream flow and evaluated water availability in the stretch of the Rio das Pedras, which supplies water to Guarapuava, a town in the mid-southern region of the state of Paraná, Brazil. Several different methods were employed to obtain instream flow, a reference discharge for water consumption permit, and the river regime. Methods comprised 7-day mean minimum with a 10-year return period, discharges associated to $95 \%$ and $90 \%$ permanence, yearly 7 -day mean minimum discharge and basic water discharge. Discharge data were obtained from the meteorological station at
\end{abstract}


VESTENA, L. R.; OLIVEIRA, E. D.; CUNHA, M. C.; THOMAZ, E. L. Vazão ecológica e disponibilidade hídrica na bacia das Pedras, Guarapuava-PR. Ambi-Agua, Taubaté, v. 7, n. 3, p. 212-227, 2012. (http://dx.doi.org/10.4136/ambi-agua.840)

the Water Station of Guarapuava (ETA). Results show that yearly river debit between 1985 and 2009 had a daily mean of $9.12 \mathrm{~m}^{3} \mathrm{~s}^{-1}$ and a median discharge of $9.16 \mathrm{~m}^{3} \mathrm{~s}^{-1}$. Estimated instream flow, measured by methods used for the Rio das Pedras stretch, ranged from 1.72 to $2.74 \mathrm{~m}^{3} \mathrm{~s}^{-1}$, with an average of $2.20 \mathrm{~m}^{3} \mathrm{~s}^{-1}$ and a coefficient of variation of $19.5 \%$. Discharge for the stretch was estimated as $0.91 \mathrm{~m}^{3} \mathrm{~s}^{-1}$, following criteria used in the state of Paraná. The relationship of the evaluated stretch between daily flow and the intake volume granted by the government revealed the inefficiency of the applied methods for instream flow assessment. In fact, they failed to warrant a minimum water volume required for the conservation of the river ecosystem.

Keywords: instream flow, river regime, hydrographic basin, river ecosystem.

\section{INTRODUÇÃO}

Os aspectos qualitativos e quantitativos dos recursos hídricos são essenciais à manutenção do equilíbrio ambiental de qualquer sistema fluvial. A variabilidade temporal e espacial da água, a demanda mundial crescente e o avanço da poluição hídrica vêm agravando as condições dos mananciais hídricos. Nos ambientes urbanos e rurais, essa relação é ainda mais dependente, sendo que a demanda por água só tem aumentado nas últimas décadas.

Mesmo com a captação de água se deve garantir nos corpos hídricos uma quantidade mínima de água, vital para a manutenção dos ecossistemas fluviais. Esta quantidade mínima é denominada de vazão ecológica ou ambiental, como também de águas residuais, águas remanescentes, vazão reduzida, dentre outras denominações. A vazão ecológica é a quantidade de água que deve permanecer no leito dos rios para atendimento das demandas do ecossistema aquático, para preservação da flora e da fauna relacionada ao corpo hídrico (Cruz, 2005; Medeiros et al., 2011).

Os métodos para determinação da vazão ecológica são vários. Eles são classificados de modo geral em função dos procedimentos metodológicos adotados para determinação da vazão ecológica, em hidrológicos (histórico do fluxo), hidráulicos (geometria hidráulica), hábitats e holísticos (Jowett, 1997, Sarmento, 2007).

$\mathrm{O}$ volume total de água utilizado de um curso fluvial para atender usos externos como abastecimento público, industrial, dessedentação animal, irrigação, energia elétrica etc. não devem afetar a vazão ecológica (Benetti et. al., 2003). No Brasil, as autorizações de uso de recursos hídricos são concedidas utilizando-se de um valor único de vazão ecológica, obtido geralmente por métodos hidrológicos que não consideram as limitações hídricas nos regimes naturais de vazões (a escala espaço-temporal e a variabilidade hidrológica) e os padrões dos hábitat (aspectos dos micro-hábitats ao longo do curso d'água com as mudanças de vazões) (Medeiros et al., 2011). Apesar das limitações destes métodos tradicionais, já apontadas por Collischon et al. (2005), Moliere et al. (2009) e Medeiros et al. (2011), no presente trabalho, eles serão utilizados para definição da vazão ecológica, por serem os métodos atualmente usados e definidos em legislação para a cessão de outorga no Brasil.

Diante disso, o planejamento e o gerenciamento de recursos hídricos são fundamentais para prevenir e minimizar problemas ambientais, à medida que garante uma quantidade mínima de vazão ecológica, fundamental à conservação do ecossistema fluvial. A vazão ecológica também possibilita identificar a disponibilidade de água real nos mananciais de uma determinada região, com potencialidade de serem captados e aproveitados.

No Brasil, para o aproveitamento dos recursos hídricos, são estabelecidos os valores máximos de retirada de volumes de água de um corpo hídrico, outorgados a usuários, a partir da disponibilidade hídrica real. "A Outorga é o ato administrativo que expressa os termos e as 
VESTENA, L. R.; OLIVEIRA, E. D.; CUNHA, M. C.; THOMAZ, E. L. Vazão ecológica e disponibilidade hídrica na bacia das Pedras, Guarapuava-PR. Ambi-Agua, Taubaté, v. 7, n. 3, p. 212-227, 2012. (http://dx.doi.org/10.4136/ambi-agua.840)

condições mediante as quais o Poder Público permite, por prazo determinado, o uso de recursos hídricos", visa assegurar o controle quantitativo e qualitativo dos usos da água e disciplinar o exercício dos direitos de acesso à água (Paraná, 2002; Instituto das Águas do Paraná, 2012).

A Outorga é dada a usuários após definir-se a vazão ecológica. Contudo, cada Estado da federação brasileira estabelece critérios de outorga e direito de uso da água, ou seja, possui um método, um critério para a definição da vazão ecológica. No Brasil, os critérios para definição da vazão ecológica basicamente são fundamentados em dados de séries históricas de vazão. No Quadro 1 é apresentado um resumo destes critérios.

Quadro 1. Critérios de outorga de direito de uso da água em alguns Estados brasileiros.

\begin{tabular}{|c|c|c|c|}
\hline Estado & $\begin{array}{c}\text { Vazão } \\
\text { referencial }\end{array}$ & Critério de Outorga (1) & $\begin{array}{c}\text { Vazão ecológica } \\
\text { indiretamente } \\
\text { estabelecida }\end{array}$ \\
\hline PR & $\mathrm{Q}_{7.10}$ & $50 \%$ da vazão referencial. & $50 \%$ da $Q_{7.10}$ \\
\hline \multirow{2}{*}{ MG } & \multirow{2}{*}{$\mathrm{Q}_{7.10}$} & $\begin{array}{l}30 \% \text { da vazão referencial em cursos d'água usuais. } \\
\text { Quando o interessado promover a regularização, o } \\
\text { limite poderá ser superior desde que seja mantida } \\
\text { uma vazão residual de } 70 \% \text { da vazão referencial. }\end{array}$ & $\mathrm{Q}_{7.10}$ \\
\hline & & $\begin{array}{l}\text { Poderão ser adotadas vazões residuais inferiores a } \\
70 \% \text { da vazão de referência, quando for de interesse } \\
\text { público e não causar prejuízos a terceiros. }\end{array}$ & $\begin{array}{l}\text { Exceção à regra } \\
\text { quando for de } \\
\text { interesse público }\end{array}$ \\
\hline \multirow[t]{2}{*}{$\mathrm{PE}$} & \multirow[t]{2}{*}{$\mathrm{Q}_{90}$ diário } & $\begin{array}{l}80 \% \text { da vazão referencial quando não houver } \\
\text { barramento, ou quando houver barramento em cursos } \\
\text { d'água perenes. }\end{array}$ & $20 \%$ da $Q_{90}$ \\
\hline & & $\begin{array}{l}\text { 95\% da vazão referencial quando houver barramento } \\
\text { em curso d'água intermitente. }\end{array}$ & $5 \%$ da $Q_{90}$ \\
\hline \multirow{3}{*}{ BA } & \multirow{3}{*}{$\mathrm{Q}_{90}$ diário } & $\begin{array}{l}80 \% \text { da vazão referencial quando não houver } \\
\text { barramento, ou quando houver barramento em cursos } \\
\text { d'água perenes. }\end{array}$ & $20 \%$ da $Q_{90}$ \\
\hline & & $\begin{array}{l}95 \% \text { da vazão referencial quando houver barramento } \\
\text { em curso d'água intermitente. } \\
\text { Quando o suprimento for para abastecimento } \\
\text { humano, o percentual pode atingir } 95 \% \text { da vazão } \\
\text { referencial. }\end{array}$ & $5 \%$ da $Q_{90}$ \\
\hline & & $\begin{array}{l}\text { No caso de vazões regularizadas por reservatórios, a } \\
\text { vazão residual de } 20 \% \text { da vazão referencial deve } \\
\text { escoar a jusante por descargas de fundo ou por } \\
\text { qualquer outro dispositivo que não inclua bombas de } \\
\text { recalques. Nenhum usuário individualmente receberá } \\
\text { outorga superior a } 210 \% \text { da vazão referencial em um } \\
\text { dado manancial. }\end{array}$ & $20 \%$ da $Q_{90}$ \\
\hline $\mathrm{PB}$ & \multirow{3}{*}{$\begin{array}{l}\text { Vazão } \\
\text { regularizada } \\
\text { com } 90 \% \text { de } \\
\text { garantia }\end{array}$} & $90 \%$ da vazão referencial & $10 \%$ da $Q_{90}$ \\
\hline $\mathrm{RN}$ & & $90 \%$ da vazão referencial & $10 \%$ da $Q_{90}$ \\
\hline $\mathrm{CE}$ & & $\begin{array}{l}90 \% \text { da vazão referencial em cursos d'água com } \\
\text { barramento; em lagos ou lagoas, } 33 \% \text { da vazão } \\
\text { referencial. }\end{array}$ & $\begin{array}{c}10 \% \text { ou } 67 \% \text { da } Q \\
90\end{array}$ \\
\hline
\end{tabular}

(1) Limite de autorização de retirada de água acumulada até a seção fluvial.

Fonte: Benetti et al. (2003). 
VESTENA, L. R.; OLIVEIRA, E. D.; CUNHA, M. C.; THOMAZ, E. L. Vazão ecológica e disponibilidade hídrica na bacia das Pedras, Guarapuava-PR. Ambi-Agua, Taubaté, v. 7, n. 3, p. 212-227, 2012. (http://dx.doi.org/10.4136/ambi-agua.840)

No caso do Estado do Paraná, a vazão ecológica é o volume de $50 \%$ da vazão de referência obtido da média mínima de sete dias com período de retorno de dez anos $\left.{ }_{7} \mathrm{Q}_{10}\right)$ (Paraná, 1999; Suderhsa, 2006). Entretanto, para a definição da vazão ecológica existem diversos procedimentos técnicos. De modo geral, os procedimentos fundamentam-se em dados de séries históricas de vazão, nas relações entre parâmetros hidráulicos e a vazão e nas relações entre o hábitat e a vazão.

A unidade territorial básica para o planejamento e o gerenciamento dos recursos hídricos é a bacia hidrográfica (Brasil, 1997). Ela é uma área da superfície terrestre delimitada topograficamente, cuja configuração resulta de uma série de elementos (geomorfológicos, pedológicos, hidrológicos, climáticos, fauna, flora e ocupação antrópica) que se sobrepõem no espaço, constituindo uma dinâmica própria, um sistema natural.

A quantificação da disponibilidade hídrica na bacia hidrográfica do rio das Pedras (BHRP) é de extrema importância por ser área de manancial da cidade de Guarapuava, principalmente no trecho do rio das Pedras onde é realizada a captação de água pela Companhia de Saneamento do Paraná (Sanepar). Portanto, o planejamento de uso e ocupação do solo é fundamental para o equilíbrio qualitativo e quantitativo dos recursos hídricos na BHRP.

O objetivo do presente estudo foi determinar a vazão ecológica e avaliar preliminarmente a disponibilidade hídrica na BHRP, manancial da cidade de Guarapuava, região Centro-Sul do Estado do Paraná, utilizando-se de diferentes métodos e critérios de referência para a vazão ecológica: a média mínima de sete dias com período de retorno de dez anos, a curva de permanência de vazões $\left(\mathrm{Q}_{90}\right.$ e $\left.\mathrm{Q}_{95}\right)$, a vazão aquática de base e as vazões mínimas anuais de sete dias.

Desse modo, a determinação da vazão ecológica por mais de um método e critério fornecerá informações com mais credibilidade como parâmetro orientador de outorga de uso de água, pois o conhecimento da quantidade mínima de água necessária à conservação do ecossistema fluvial possibilita o uso mais correto e racional dos recursos hídricos.

\section{MATERIAIS E MÉTODO}

\section{1. Área de estudo}

A bacia hidrográfica do rio das Pedras (BHRP) com área estimada em $332 \mathrm{~km}^{2}$ localizase no município de Guarapuava, região Centro-Sul do Estado do Paraná, entre as coordenadas UTM 22, 7.210.725 m e 7.186.234 $\mathrm{m} \mathrm{N}$ de latitude e $477.895 \mathrm{~m}$ e $451.960 \mathrm{~m}$ E de longitude, no meridiano central $51^{\circ}$ WGr (Figura 1).

Guarapuava está sob o domínio da zona extratropical, o que resulta em temperaturas com caráter mesotérmico, com médias anuais entre $16^{\circ}$ e $20^{\circ} \mathrm{C}$, inverno frio e verão amenizado pelas altitudes (Thomaz e Vestena, 2003). O clima do município de Guarapuava, segundo Köppen, classifica-se como $\mathrm{Cfb}$, o que significa que o clima é pluvial, sempre úmido, com verões chuvosos e frescos (Maack, 1981).

O rio das Pedras é um dos principais formadores do rio Jordão, que por sua vez é um dos maiores afluentes da margem direita do rio Iguaçu. As principais nascentes que formam o rio das Pedras encontram-se na Área de Proteção Ambiental (APA) da serra da Esperança. O seu sistema de drenagem, de acordo com a hierarquia de Strahler (1957), apresenta ramificação de $5^{\mathrm{a}}$ ordem.

A bacia se desenvolve no reverso da escarpa basáltica, localmente denominada serra da Esperança, sendo que, para Oeste, limita-se com o perímetro urbano de Guarapuava, com altitude que varia de $1.280 \mathrm{~m}$ na serra da Esperança até $950 \mathrm{~m}$ na desembocadura (Santos e Kobiyama, 2003). 
VESTENA, L. R.; OLIVEIRA, E. D.; CUNHA, M. C.; THOMAZ, E. L. Vazão ecológica e disponibilidade hídrica na bacia das Pedras, Guarapuava-PR. Ambi-Agua, Taubaté, v. 7, n. 3, p. 212-227, 2012. (http://dx.doi.org/10.4136/ambi-agua.840)

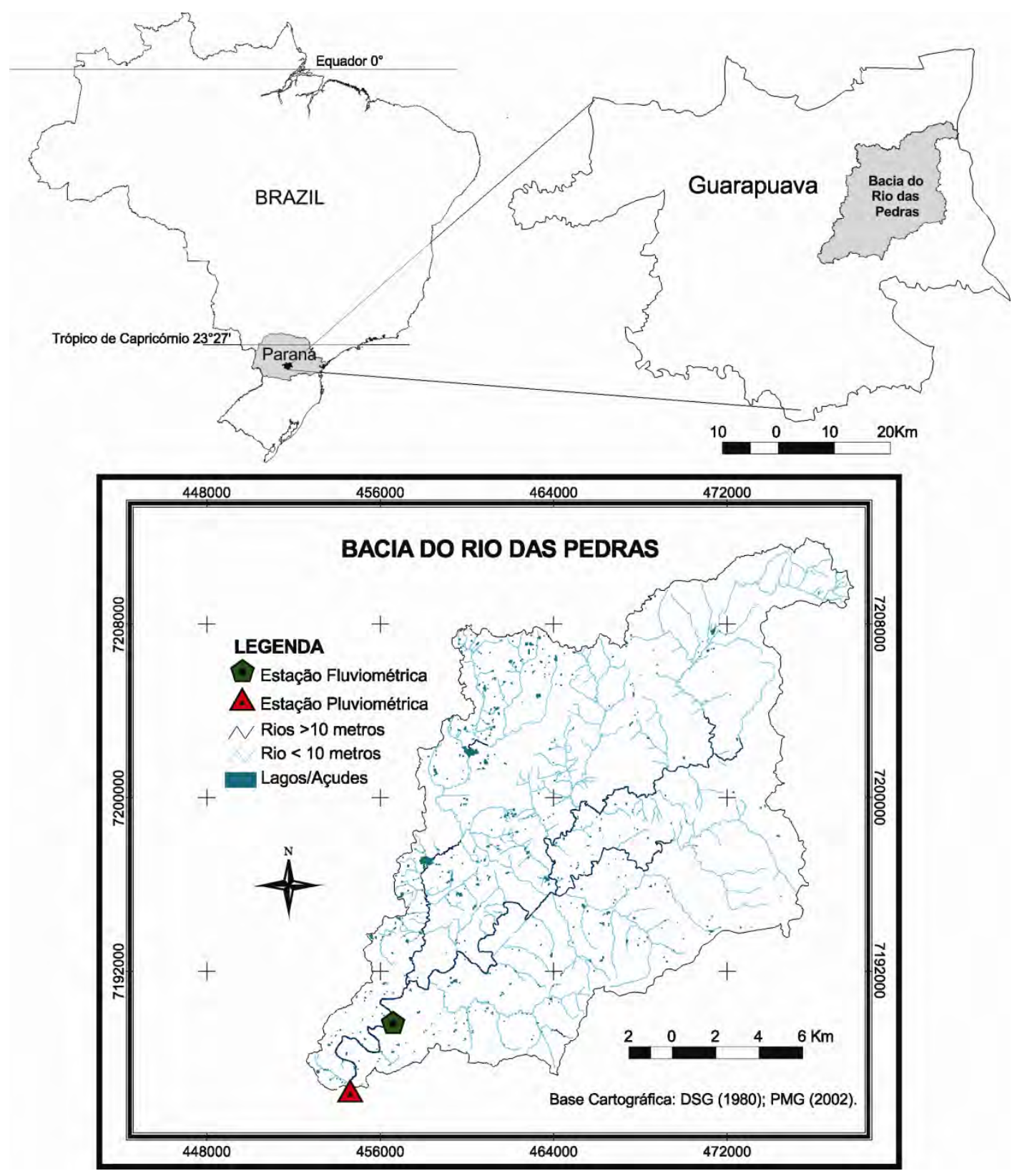

Figura 1. Localização da bacia hidrográfica do rio das Pedras e da estação fluviométrica (local de captação de água pela Sanepar) e pluviométrica utilizada.

O relevo da BHRP varia de plano a montanhoso, onde predominam os tipos suave ondulados e ondulados (declividades de até $12^{\circ}$ ) em $77 \%$ da área. Destaca-se ainda que mais de $20 \%$ da bacia apresenta relevos de forte ondulado a montanhoso (declividades maiores que $12^{\circ}$ ), que potencializa significativas energias aos fluxos superficiais.

Os principais tipos de solos que prevalecem na bacia são: Latossolo, Cambissolo, Neossolo (litólico) e Gleissolo (hidromórfico). Em algumas unidades, ocorrem inclusões de afloramento de rocha, pedregosidade e rochosidade em grau variado (Vestena e Thomaz, 2006).

A vegetação dominante da bacia é constituída de arbustos, florestas secundárias, que aparecem geralmente depois da terra ter sido cultivada, floresta subtropical perenifólia, reflorestamentos, vegetação campestre, campos alagados e pastagens (Santos e Kobiyama, 2003). 
VESTENA, L. R.; OLIVEIRA, E. D.; CUNHA, M. C.; THOMAZ, E. L. Vazão ecológica e disponibilidade hídrica na bacia das Pedras, Guarapuava-PR. Ambi-Agua, Taubaté, v. 7, n. 3, p. 212-227, 2012. (http://dx.doi.org/10.4136/ambi-agua.840)

O uso da terra é bastante diversificado, com atividades relacionadas à agricultura, mata, campo, pecuária, indústria entre outros. Porém, o uso da terra que mais se destaca é a associação de mata e campo que recobrem mais de $65 \%$ da área.

A cidade de Guarapuava passou por uma expansão física territorial urbana expressiva desde a década de 1970, quando surge grande número de loteamentos. A população cresceu significativamente, enquanto que a população rural manteve sua tendência de queda, que se acelerou após os anos de 1980. O município conta com uma população de 166.195 habitantes, dos quais 153.098 residem na área urbana, segundo dados obtidos pelo último censo (Brasil, 2010). Esse desenvolvimento urbano significativo se deve às várias mudanças na forma de apropriação da natureza, como a introdução de novas técnicas na agricultura, via de transporte, relações sociais e de trabalho, no campo e na cidade, que transformaram significativamente sua paisagem (Loboda, 2008).

\subsection{Procedimentos metodológicos}

A vazão de um determinado rio tem como característica básica uma grande variabilidade temporal e espacial. Dessa forma, para analisar uma determinada série histórica de dados fluviométricos de um rio é necessário utilizar alguns valores estatísticos que resumem, em grande parte, o seu comportamento hidrológico. No presente estudo, a série histórica de vazões considerou o ano civil, a fim de garantir a independência dos valores amostrais de vazão, como apontado por Tucci (2000). Dessa forma, a avaliação estatística dos dados de vazão do rio das Pedras e a vazão ecológica foram determinadas por meio da aplicação dos seguintes métodos hidrológicos:

I - Vazão média mínima de sete dias com período de retorno de dez anos $\left({ }_{7} \mathrm{Q}_{10}\right)-\mathrm{A}_{7} \mathrm{Q}_{10}$ constitui um importante instrumento da Política Nacional dos Recursos Hídricos do Brasil, fornecendo estimativa estatística da disponibilidade hídrica dos escoamentos naturais de água. $\mathrm{O}$ valor da ${ }_{7} \mathrm{Q}_{10}$ foi obtido calculando-se as médias móveis das vazões diárias com período de sete dias ao longo de um ano hidrológico, sendo a vazão média mínima destas médias móveis retida. O processo foi repetido para cada ano da série histórica, obtendo-se uma série de valores mínimos de vazões médias em sete dias consecutivos. Estas vazões foram tabeladas em ordem crescente de magnitude juntamente com a frequência de ocorrência $(f)$ e o tempo de retorno, utilizando da equação de Weibull, $f=m / n-1$, em que $m$ é a ordem da vazão amostral e $n$ é o tamanho da amostra (Tucci, 1984), para a determinação da vazão mínima de sete dias com período de retorno de dez anos. $\mathrm{O}$ valor da ${ }_{7} \mathrm{Q}_{10}$ também foi obtido para conferência a partir de um gráfico com abcissas formadas pelo tempo de retorno e ordenadas correspondentes ao logaritmo das vazões. $\mathrm{A}_{7} \mathrm{Q}_{10}$ estimada a partir da equação de Weibull é uma das mais usadas (Sarmento, 2007) e precisa (Pinto et al., 2010).

II - Curva de permanência de vazões - a série histórica de vazões médias diárias por esse método é disposta em um gráfico, as quais são ordenadas de forma crescente. A permanência de cada vazão é o percentual de vezes em que ela foi equiparada ou extrapolada. Para caracterizar as descargas mínimas, foram consideradas as vazões associadas às permanências de $95 \%\left(\mathrm{Q}_{95} \%\right)$ e $90 \%\left(\mathrm{Q}_{90} \%\right)$, frequentemente utilizadas em projetos de outorga para uso da água (Pereira, 2004).

III - Vazões mínimas anuais de sete dias - esta vazão é obtida computando a média móvel das vazões mínimas com período de sete dias ao longo do ano hidrológico, durante a série considerada de dados (Benetti et al., 2003).

IV - Vazão aquática de base - este método calcula por meio da média da série histórica, considerando a vazão mediana do mês de menor débito fluvial do ano como valor mínimo de vazão ecológica a ser estabelecido (Kulik, 1990). Esta vazão corresponde ao fluxo de base do rio, sendo que na BHRP foi determinada calculando a mediana da vazão mensal mínima de cada ano (Longhi e Fomiga, 2011). 
VESTENA, L. R.; OLIVEIRA, E. D.; CUNHA, M. C.; THOMAZ, E. L. Vazão ecológica e disponibilidade hídrica na bacia das Pedras, Guarapuava-PR. Ambi-Agua, Taubaté, v. 7, n. 3, p. 212-227, 2012. (http://dx.doi.org/10.4136/ambi-agua.840)

Os dados de vazão diários utilizados foram os da estação fluviométrica (Figura 1) localizada na ETA (Estação de Tratamento de Água) - Guarapuava, nas coordenadas 2523'52"de latitude Sul e 51 26'09" de longitude Oeste, a $950 \mathrm{~m}$ de altitude, identificada pelo código Aneel (Agência Nacional de Energia Elétrica), de número 65809000.

Estes dados foram mensurados pela Agência Nacional das águas (ANA), tendo como recorte temporal neste estudo o período entre janeiro de 1985 a dezembro de 2009. Os dados foram tabulados, comparados e analisados, por meio de gráficos e tabelas.

A partir dos dados de vazão de referência, obtidas pelos quatros métodos anteriores foi determinada a vazão ecológica específica por meio da área de drenagem $\left(\mathrm{L} \cdot \mathrm{s}^{-1} \cdot \mathrm{km}^{-2}\right)$, pela relação entre a vazão ecológica determinada por cada um dos métodos pela área de drenagem da estação fluviométrica $\left(306 \mathrm{~km}^{2}\right)$.

A vazão máxima outorgável na seção do rio das Pedras para abastecimento público foi quantificada de forma expedita, da seguinte forma (Suderhsa, 2006):

$$
\begin{aligned}
& \mathrm{Q}_{\text {outorgável i }}=0,5 .\left(\mathrm{Q}_{95 \%}\right)_{\mathrm{i}}-\mathrm{Q}_{\text {não-disponível i }} \\
& \mathrm{Q}_{\text {não-disponível i }}=\sum \mathrm{Q} \text { outorgadas m }+\sum \mathrm{Q} \text { outorgadas } \mathrm{j}
\end{aligned}
$$

em que:

Qoutorgável i é a vazão máxima que pode ser outorgada na seção i do corpo hídrico; $\left(\mathrm{Q}_{95 \%}\right)_{\mathrm{i}}$ é a vazão natural com permanência de $95 \%$ do tempo na seção i; $\sum Q$ outorgadas m é o somatório das vazões outorgadas a montante da seção i; $\sum$ Q outorgadas j é o somatória das vazões outorgadas a jusante, que dependem da vazão na seção i. Uma vez que não existem outorgas cedidas nem a montante, nem a jusante da seção estuda pela Suderhsa, a $\mathbf{Q}_{\text {não-disponível i é igual }}$ a zero.

\section{RESULTADOS E DISCUSSÃO}

\subsection{Vazão fluvial}

O débito fluvial anual na BHRP, entre os anos de 1985 e 2009, apresentou uma média de 9,12 $\mathrm{m}^{3} \mathrm{~s}^{-1}$ e mediana de 9,16 $\mathrm{m}^{3} \mathrm{~s}^{-1}$. Os anos de $1998\left(17,07 \mathrm{~m}^{3} \mathrm{~s}^{-1}\right)$ e $1992\left(13,22 \mathrm{~m}^{3} \mathrm{~s}^{-1}\right)$ foram os que apresentaram as maiores vazões e os de $1985\left(3,64 \mathrm{~m}^{3} \mathrm{~s}^{-1}\right)$ e $2006\left(4,04 \mathrm{~m}^{3} \mathrm{~s}^{-1}\right)$ os de menores débitos fluviais (Tabela 1).

Tabela 1. Série de vazões médias anuais e vazões adimensionais (1985-2009).

\begin{tabular}{ccc|ccc|ccc}
\hline \multicolumn{7}{c}{ Estação Fluviométrica de Guarapuava - Código 65809000 } \\
\hline Ano & $\begin{array}{c}\text { Média anual } \\
\left(\mathbf{m}^{\mathbf{3}} \mathbf{~ s}^{-\mathbf{1}}\right)\end{array}$ & $\mathbf{Q}_{\mathbf{a} / \mathbf{Q}_{\mathbf{m}}}$ & $\mathbf{A n o}$ & $\begin{array}{c}\text { Média anual } \\
\left(\mathbf{m}^{\mathbf{3}} \mathbf{s}^{-\mathbf{1}}\right)\end{array}$ & $\mathbf{Q}_{\mathbf{a} / \mathbf{Q}_{\mathbf{m}}}$ & Ano & $\begin{array}{c}\text { Média anual } \\
\left(\mathbf{m}^{\mathbf{3}} \mathbf{~ s}^{-\mathbf{1}}\right)\end{array}$ & $\mathbf{Q}_{\mathbf{a}} \mathbf{Q}_{\mathbf{m}}$ \\
\hline 1985 & 3,64 & 0,39 & 1994 & 7,080 & 0,77 & 2002 & 8,64 & 0,94 \\
1986 & 5,08 & 0,55 & 1995 & 10,50 & 1,15 & 2003 & 6,59 & 0,72 \\
1987 & 8,04 & 0,88 & 1996 & 10,54 & 1,15 & 2004 & 8,02 & 0,87 \\
1988 & 4,99 & 0,54 & 1997 & 12,50 & 1,37 & 2005 & 10,14 & 1,11 \\
1989 & 12,93 & 1,41 & 1998 & 17,07 & 1,87 & 2006 & 4,04 & 0,44 \\
1990 & 12,03 & 1,31 & 1999 & 7,09 & 0,77 & 2007 & 9,05 & 0,99 \\
1991 & 5,57 & 0,61 & 2000 & 10,02 & 1,09 & 2008 & 9,16 & 1,00 \\
1992 & 13,22 & 1,44 & 2001 & 10,19 & 1,11 & 2009 & 11,06 & 1,21 \\
1993 & 10,97 & 1,20 & & & & & & \\
\hline
\end{tabular}

Nota: $\mathrm{Q}_{\mathrm{a}} \mathrm{Q}_{\mathrm{m}}$ - vazão adimensional determinada pela razão entre o total de vazão anual $\left(\mathrm{Q}_{\mathrm{a}}\right.$ e a média $\left(\mathrm{Q}_{\mathrm{m}}\right)$ das vazões da série histórica. 
VESTENA, L. R.; OLIVEIRA, E. D.; CUNHA, M. C.; THOMAZ, E. L. Vazão ecológica e disponibilidade hídrica na bacia das Pedras, Guarapuava-PR. Ambi-Agua, Taubaté, v. 7, n. 3, p. 212-227, 2012. (http://dx.doi.org/10.4136/ambi-agua.840)

Na Tabela 1, observam-se os valores de vazões médias anuais e as vazões adimensionais, determinada a partir da razão entre a vazão anual $\left(\mathrm{Q}_{\mathrm{a}}\right)$ e a vazão média $\left(\mathrm{Q}_{\mathrm{m}}\right)$ da série histórica. Esses dados foram plotados num gráfico (Figura 2) onde o eixo da abscissa representa os anos e o da ordenada a variação média das vazões, sendo que os valores acima de 1 representam anos com vazão acima da média e menor que 1 , abaixo da média.

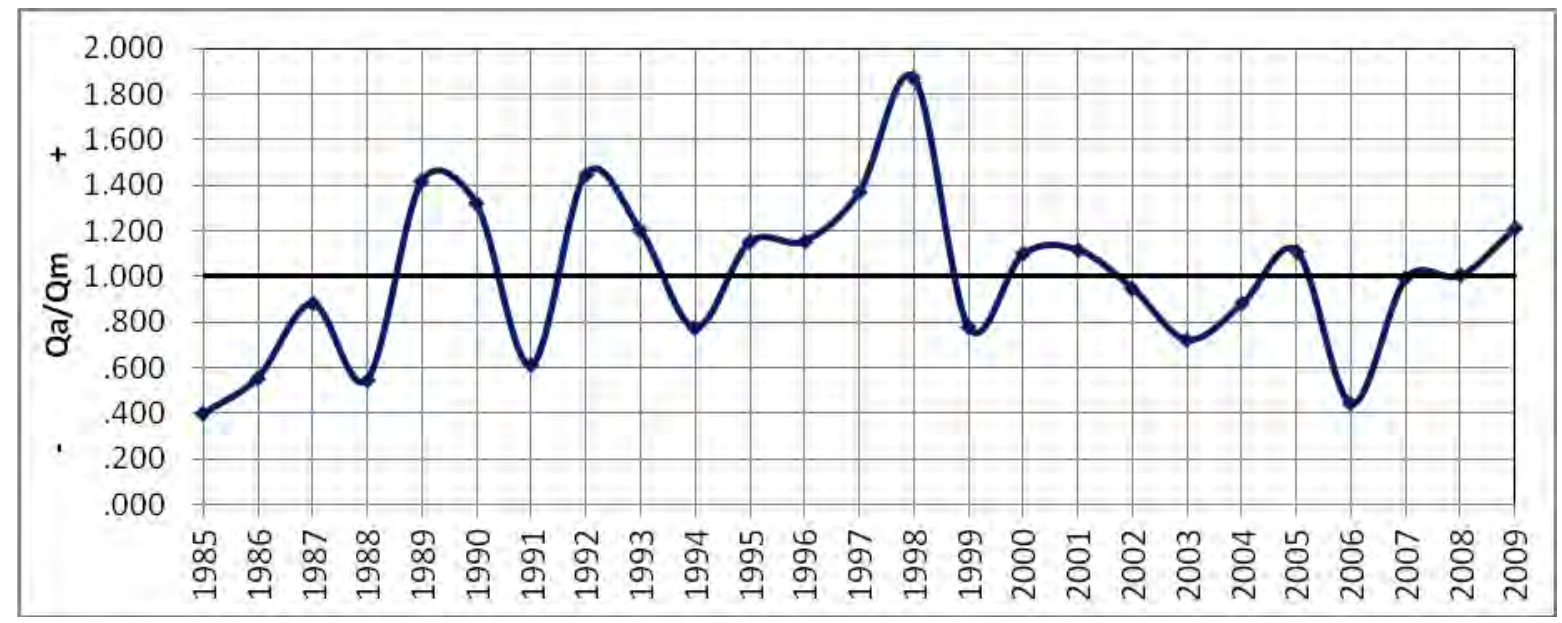

Figura 2. Variação da vazão adimensional no período de 1985 a 2009.

Pelos dados da Tabela 1 e da Figura 2, nota-se que a variação adimensional positiva e negativa do período esteve bem equilibrada, sendo que vazões acima da média ocorreram em 13 anos (52\% do período).

Como um dos principais fatores que influenciam a variação das vazões médias anuais, é a pluviosidade, ela também esteve nesse período muito influenciada pelos fenômenos El Niño e La Niña. Esta relação foi definida por Azevedo et al. (2005) que por meio de estudos na bacia do rio Iguaçu concluíram que a variabilidade pluvial da área está fortemente vinculada a estes fenômenos. Portanto, as anomalias das vazões médias estão em parte relacionadas à variação das entradas (precipitação) na bacia, com anos mais e menos secos.

Na Tabela 2 são apresentados os dados referentes à frequência das séries de vazões anuais, em classes intervalares de $4 \mathrm{~m}^{3} \mathrm{~s}^{-1}$, para melhor visualização dos dados. Nota-se que o intervalo de vazão compreendido entre 8 e $12 \mathrm{~m}^{3} \mathrm{~s}^{-1}$ abrangem quase metade (48\%) das vazões anuais da série histórica, sendo a mesma bem representativa do regime hidrológico anual na BHRP.

Tabela 2. Frequência das vazões anuais no rio das Pedras.

\begin{tabular}{c|ccc}
\hline $\begin{array}{c}\text { Classe } \\
\left(\mathbf{m}^{\mathbf{3}} \mathbf{s}^{-\mathbf{1}}\right)\end{array}$ & Fr. Absoluta & $\begin{array}{c}\text { Fr. Relativa } \\
(\boldsymbol{\%})\end{array}$ & $\begin{array}{c}\text { Fr. Acumulada } \\
(\boldsymbol{\%})\end{array}$ \\
\hline$<4$ & 02 & 08 & 8 \\
$4-8$ & 06 & 24 & 32 \\
$8-12$ & 12 & 48 & 80 \\
$12-16$ & 04 & 16 & 96 \\
$>16$ & 01 & 04 & 100 \\
\hline Total & $\mathbf{2 5}$ & $\mathbf{1 0 0}$ & $\mathbf{1 0 0}$ \\
\hline
\end{tabular}

Os dados fluviométricos mensais apresentaram uma média de $9,10 \mathrm{~m}^{3} \mathrm{~s}^{-1}$ e mediana de $8,80 \mathrm{~m}^{3} \mathrm{~s}^{-1}$. No entanto, há variações significativas ao longo do ano como se pode observar na Figura 3 que mostra um resumo mensal da série histórica. 
VESTENA, L. R.; OLIVEIRA, E. D.; CUNHA, M. C.; THOMAZ, E. L. Vazão ecológica e disponibilidade hídrica na bacia das Pedras, Guarapuava-PR. Ambi-Agua, Taubaté, v. 7, n. 3, p. 212-227, 2012. (http://dx.doi.org/10.4136/ambi-agua.840)

As menores médias mensais das vazões mínimas ocorreram nos meses de maio, junho e agosto. Entretanto, observaram-se as maiores médias mensais das vazões máximas também em maio, além de outubro e setembro, o que demonstra marcante variabilidade interanual no comportamento das vazões máximas e mínimas (Figura 3).

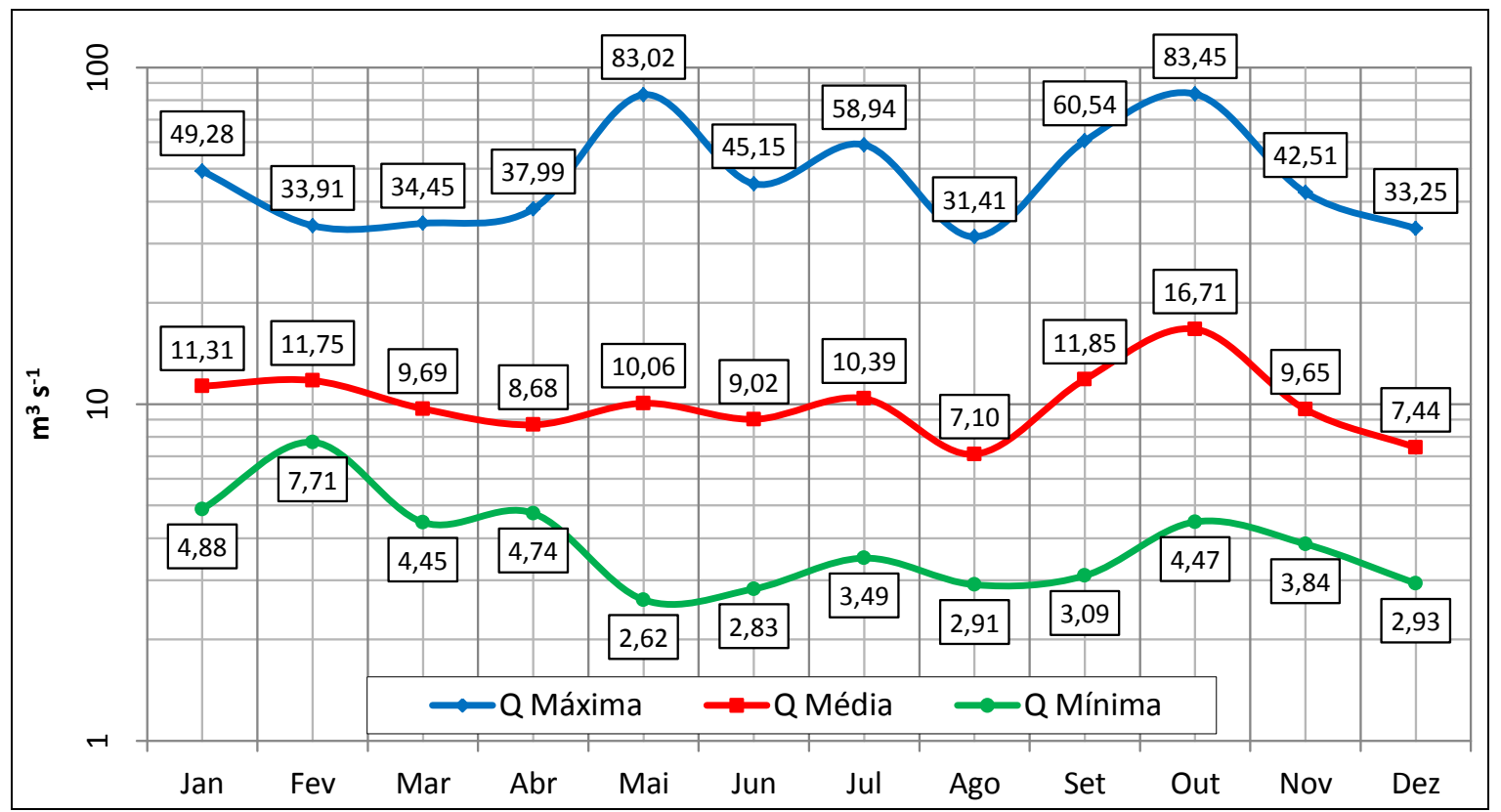

Figura 3. Valores médios das vazões mensais máximas, médias e mínimas do posto fluviométrico do rio das Pedras, no período de 1985 a 2009.

Fonte: Adaptado de ANA (2008).

\subsection{Vazão de referência a vazão ecológica}

\subsubsection{Vazão - ${ }_{7} \mathbf{Q}_{10}$}

Na Tabela 3 é mostrado o tempo de retorno ao longo de 25 anos da média das vazões mínimas obtidas ao longo deste período.

Tabela 3. Vazões mínimas anuais e o seu tempo de retorno (TR).

\begin{tabular}{|c|c|c|c|c|c|c|c|c|}
\hline \multicolumn{9}{|c|}{ Posto Fluviométrico de Guarapuava - Código 65809000} \\
\hline Ano & $\begin{array}{c}\text { Vazão } \\
{ }_{7} \mathbf{Q}_{10} \\
\left(\mathrm{~m}^{3} \mathbf{s}^{-1}\right) \\
\end{array}$ & $\begin{array}{c}\text { TR } \\
(\text { anos) }\end{array}$ & Ano & $\begin{array}{c}\text { Vazão } \\
{ }_{7} \mathbf{Q}_{10} \\
\left(\mathbf{m}^{3} \mathbf{s}^{-1}\right) \\
\end{array}$ & $\begin{array}{c}\text { TR } \\
(\text { anos) }\end{array}$ & Ano & $\begin{array}{c}\text { Vazão } \\
{ }_{7} Q_{10} \\
\left(m^{3} s^{-1}\right) \\
\end{array}$ & $\begin{array}{c}\text { TR } \\
\text { (anos) }\end{array}$ \\
\hline 1992 & 3,11 & 01 & 2002 & 1,83 & 10 & 2006 & 1,49 & 18 \\
\hline 1998 & 2,95 & 02 & 2005 & 1,83 & 11 & 2004 & 1,47 & 19 \\
\hline 2008 & 2,76 & 03 & 1997 & 1,78 & 12 & 1989 & 1,12 & 20 \\
\hline 2001 & 2,56 & 04 & 1987 & 1,69 & 13 & 1994 & 0,98 & 21 \\
\hline 2009 & 2,34 & 05 & 1995 & 1,59 & 14 & 1986 & 0,87 & 22 \\
\hline 2007 & 2,16 & 06 & 2000 & 1,54 & 15 & 1991 & 0,79 & 23 \\
\hline 1996 & 2,10 & 07 & 1999 & 1,52 & 16 & 1988 & 0,72 & 24 \\
\hline 1993 & 2,01 & 08 & 1990 & 1,50 & 17 & 1985 & 0,54 & 25 \\
\hline 2003 & 1,85 & 09 & & & & & & \\
\hline
\end{tabular}


VESTENA, L. R.; OLIVEIRA, E. D.; CUNHA, M. C.; THOMAZ, E. L. Vazão ecológica e disponibilidade hídrica na bacia das Pedras, Guarapuava-PR. Ambi-Agua, Taubaté, v. 7, n. 3, p. 212-227, 2012. (http://dx.doi.org/10.4136/ambi-agua.840)

Com os dados da tabela acima, em um gráfico foram plotados o tempo de retorno (TR) e as vazões mínimas anuais com intervalo de sete dias, sendo confirmado o valor da vazão ecológica de $1,83 \mathrm{~m}^{3} \mathrm{~s}^{-1}$ para ${ }_{7} \mathrm{Q}_{10}$ (Figura 4).

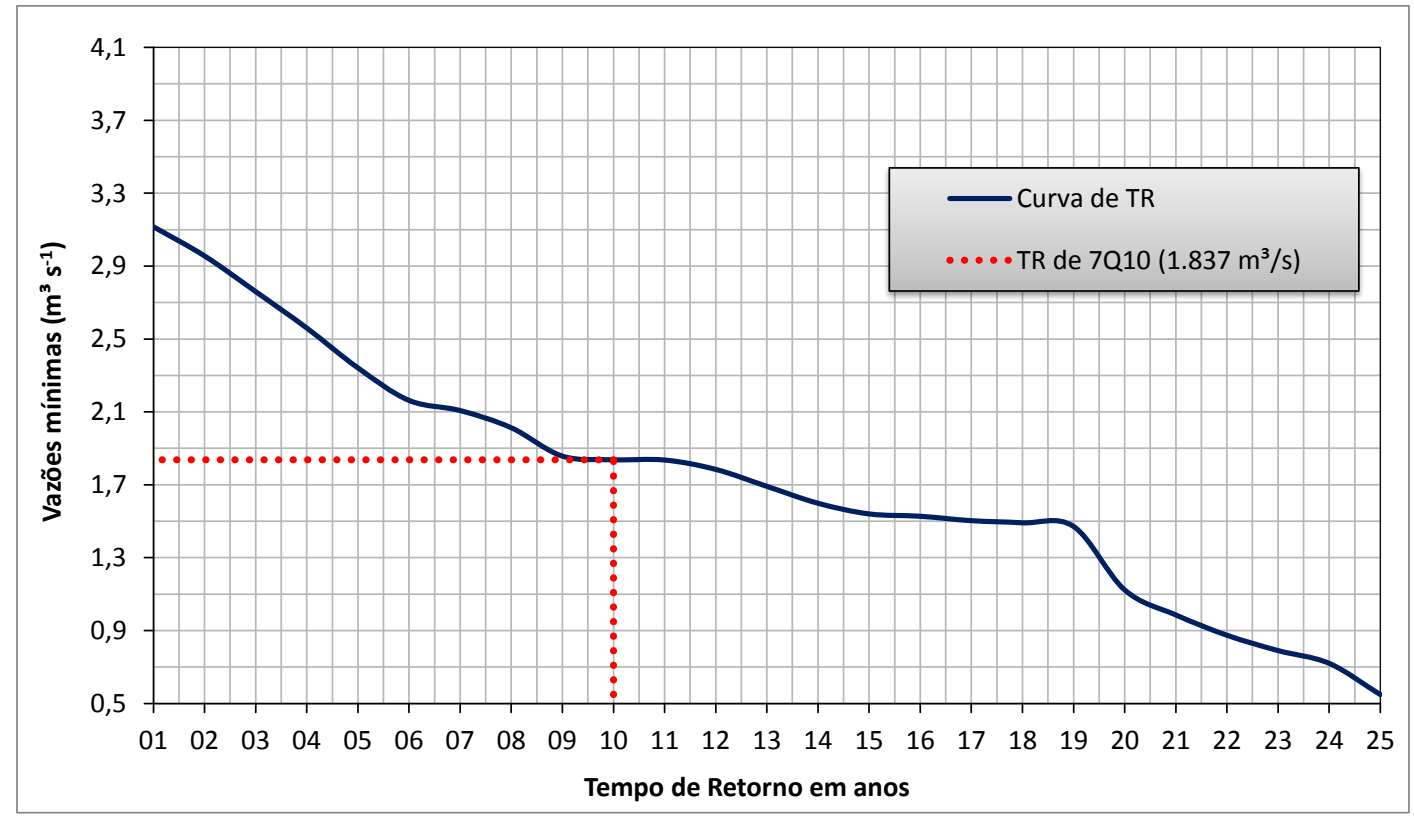

Figura 4. Tempo de retorno das vazões mínimas.

\subsubsection{Curva de permanência}

A curva de permanência permite visualizar de imediato a potencialidade natural do rio, destacando a vazão mínima e o grau de permanência das diferentes magnitudes de vazão. No gráfico da Figura 5 é apresentada a curva de permanência mensal na estação fluviométrica de Guarapuava. As vazões de permanência de $95 \%$ e $90 \%$ correspondem ao valor de vazão de referência à vazão ecológica de $2,22 \mathrm{e} 2,74 \mathrm{~m}^{3} \mathrm{~s}^{-1}$, respectivamente.

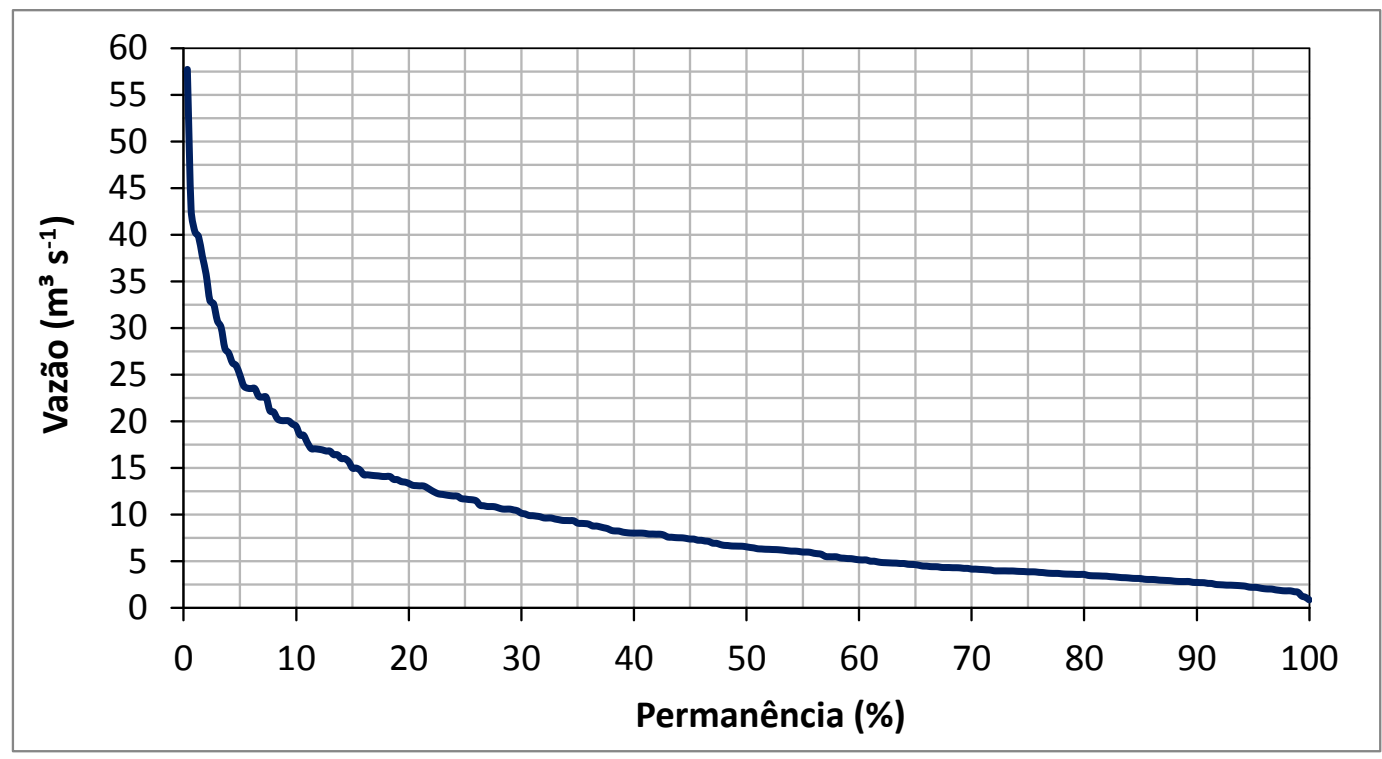

Figura 5. Permanência dos valores mensais de vazões do rio das Pedras (1985-2009). 
VESTENA, L. R.; OLIVEIRA, E. D.; CUNHA, M. C.; THOMAZ, E. L. Vazão ecológica e disponibilidade hídrica na bacia das Pedras, Guarapuava-PR. Ambi-Agua, Taubaté, v. 7, n. 3, p. 212-227, 2012. (http://dx.doi.org/10.4136/ambi-agua.840)

\subsubsection{Média das vazões anuais mínimas de sete dias}

A vazão determinada pela média móvel das vazões diárias mínimas com intervalo de sete dias ao longo do ano corresponde ao valor de $1,72 \mathrm{~m}^{3} \mathrm{~s}^{-1}$, ou seja, a vazão de referência à concessão de outorga.

\subsubsection{Vazão aquática de base}

Pelos dados da Tabela 4 foi determinada a vazão de $2,50 \mathrm{~m}^{3} \mathrm{~s}^{-1}$ como a vazão de referência à vazão ecológica da seção do rio das Pedras na qual se tem aproveitamento da água para abastecimento da cidade de Guarapuava.

Tabela 4. Mediana das vazões mensais mínimas.

\begin{tabular}{cc|cc|cc}
\hline Ano & $\begin{array}{c}\text { Vazão } \\
\left(\mathbf{m}^{\mathbf{3}} \mathbf{s}^{-\mathbf{1}}\right) \\
\text { mediana }\end{array}$ & Ano & $\begin{array}{c}\text { Vazão } \\
\left(\mathbf{m}^{\mathbf{3}} \mathbf{s}^{\mathbf{- 1}}\right) \\
\text { mediana }\end{array}$ & Ano & $\begin{array}{c}\text { Vazão } \\
\left(\mathbf{m}^{\mathbf{3}} \mathbf{s}^{-\mathbf{1}}\right) \\
\text { mediana }\end{array}$ \\
\hline 1985 & 0,78 & 1994 & 1,77 & 2002 & 2.19 \\
1986 & 2,39 & 1995 & 1,78 & 2003 & 2.53 \\
1987 & 2,06 & 1996 & 2,85 & 2004 & 3.01 \\
1988 & 1,14 & 1997 & 2,12 & 2005 & 2.25 \\
1989 & 2,81 & 1998 & 3.54 & 2006 & 1.92 \\
1990 & 2,89 & 1999 & 1.80 & 2007 & 2.57 \\
1991 & 1,67 & 2000 & 3.83 & 2008 & 3.61 \\
1992 & 4,31 & 2001 & 2.39 & 2009 & 2.83 \\
1993 & 3,43 & \multicolumn{7}{l}{} \\
\hline \multicolumn{7}{l}{ Média da série histórica $\left(\mathbf{m}^{\mathbf{3}} \mathbf{s}^{-\mathbf{1}}\right)$} \\
\hline
\end{tabular}

\subsubsection{Vazão ecológica na BHRP}

A vazão ecológica específica foi determinada (relação entre a vazão ecológica e a área de drenagem) por possibilitar a comparação desta em diferentes áreas (bacias hidrográficas) ( $\mathrm{L} \mathrm{s}^{-}$ ${ }^{1} \mathrm{~km}^{-2}$ ) (Tabela 5).

Tabela 5. Vazão ecológica específica da BHRP.

\begin{tabular}{|c|c|c|c|c|c|c|c|c|}
\hline \multirow[b]{2}{*}{ Vazão } & \multirow[b]{2}{*}{ Unidade } & \begin{tabular}{|c|} 
Método \\
1
\end{tabular} & \multicolumn{2}{|c|}{$\begin{array}{c}\text { Método } \\
2\end{array}$} & \multirow{2}{*}{\begin{tabular}{|l|}
\multicolumn{1}{|c|}{$\begin{array}{c}\text { Método } \\
\mathbf{3}\end{array}$} \\
$\begin{array}{l}\text { Média anual } \\
\text { das Q mín. } \\
\text { de } 7 \text { dias }\end{array}$ \\
\end{tabular}} & \multirow{2}{*}{$\begin{array}{c}\begin{array}{c}\text { Método } \\
4\end{array} \\
\text { Vazão } \\
\text { aquática } \\
\text { de base }\end{array}$} & \multirow[b]{2}{*}{ Média } & \multirow{2}{*}{$\begin{array}{c}\text { Coeficiente de } \\
\text { variação } \\
(\%)\end{array}$} \\
\hline & & ${ }_{7} \mathbf{Q}_{10}$ & $\mathbf{Q}_{90}$ & $\mathbf{Q}_{95}$ & & & & \\
\hline $\begin{array}{c}\text { Vazão } \\
\text { ecológica }\end{array}$ & $\mathrm{m}^{3} \mathrm{~s}^{-1}$ & 1,83 & 2,74 & 2,22 & 1,72 & 2,50 & 2,20 & 19,66 \\
\hline $\begin{array}{c}\text { Vazão } \\
\text { ecológica } \\
\text { específica }\end{array}$ & $\mathrm{L} \mathrm{s}_{2}^{-1} \mathrm{~km}^{-}$ & 6,00 & 8,95 & 7,25 & 5,64 & 8,16 & 7,20 & 19,47 \\
\hline
\end{tabular}

A seção da estação fluviométrica drena uma área de $306 \mathrm{~km}^{2}$, sendo que a partir dos quatro métodos hidrológicos aplicados, a vazão de referência à vazão ecológica específica variou de 8,95 a $5,64 \mathrm{~L} \mathrm{~s}^{-1} \mathrm{~km}^{-2}$, com uma média de 7,20 $\mathrm{L} \mathrm{s}^{-1} \mathrm{~km}^{-2}$ e um coeficiente de variação média de 19,47\% (Tabela 5). Os menores volumes foram obtidos pelos métodos da média anual das vazões mínimas de sete dias e da ${ }_{7} \mathrm{Q}_{10}$, com 1,72 e $1,84 \mathrm{~m}^{3} \mathrm{~s}^{-1}$, 
VESTENA, L. R.; OLIVEIRA, E. D.; CUNHA, M. C.; THOMAZ, E. L. Vazão ecológica e disponibilidade hídrica na bacia das Pedras, Guarapuava-PR. Ambi-Agua, Taubaté, v. 7, n. 3, p. 212-227, 2012. (http://dx.doi.org/10.4136/ambi-agua.840)

respectivamente. Enquanto, as vazões ecológicas obtidas pelos métodos da curva de permanência de vazões e vazão aquática de base foram bem maiores, 2,74 e $2,50 \mathrm{~m}^{3} \mathrm{~s}^{-1}$, respectivamente.

\subsection{Vazão ecológica e outorga: disponibilidade hídrica}

O Instituto de Águas do Paraná com base em estudos de 1985 estabeleceu a ${ }_{7} \mathrm{Q}_{10} \mathrm{em}$ bacias de até $5.000 \mathrm{~km}^{2}$ de área para todas as regiões do Estado do Paraná (ÁGUAS PARANÁ, 2011). Com base nesses dados, a região abrangida pela BHRP apresentou uma ${ }_{7} \mathrm{Q}_{10}$ de aproximadamente $2,50 \mathrm{~L} \mathrm{~s}^{-1} \mathrm{~km}^{-2}$. Ela divergiu das ${ }_{7} \mathrm{Q}_{10}$ estimada para a BHRP, com valor de $6,00 \mathrm{~L} \mathrm{~s}^{-1} \mathrm{~km}^{-2}$. Tal valor subestimado deve estar relacionado ao fator escala relacionado à extrapolação da ${ }_{7} \mathrm{Q}_{10}$ para a área da BHRP, dado que a área da bacia abrange pouco mais de $300 \mathrm{~km}^{2}$, enquanto que a média estadual foi obtida para bacias de até 5.000 km² (ÁGUAS PARANÁ, 2011).

Dados fornecidos por técnicos da Companhia de Saneamento do Paraná - Sanepar, unidade de Guarapuava, mostram que a outorga de exploração de água no rio das Pedras pela companhia para o abastecimento urbano é de $1.800 \mathrm{~m}^{3} / \mathrm{h}\left(0,5 \mathrm{~m}^{3} \mathrm{~s}^{-1}\right)$, com regime de bombeamento de $15 \mathrm{~h}$ por dia. Contudo, os mesmos já solicitaram um acréscimo do valor da outorga, alegando que a vazão do rio tem aumentado, assim como a demanda da cidade de Guarapuava. Cabe ressaltar, que esta outorga tem validade de 20 anos com término para 17 de junho de 2013.

Pelos dados de bombeamento d'água, foi definida uma vazão de exploração (captação) média diária de $0,31 \mathrm{~m}^{3} \mathrm{~s}^{-1}$, valor que está abaixo do valor outorgado. No entanto, destaca-se que o valor é médio, e que se tratando de vazão ecológica é necessário considerar os valores extremos mínimos no rio.

De acordo com os critérios adotados no Estado do Paraná, a vazão ecológica é $50 \%$ da ${ }_{7} \mathrm{Q}_{10}$, estimada no presente estudo em $0,91 \mathrm{~m}^{3} \mathrm{~s}^{-1}\left(3.306,60 \mathrm{~m}^{3} \mathrm{~h}^{-1}\right)$. Um dos métodos avaliados que apresentou o valor de vazão ecológica menor (Tabela 5).

A vazão máxima outorgável para abastecimento público, de acordo com o método empregado pelo Instituto de Águas do Paraná (equação 1 e $2-50 \%$ da $\mathrm{Q}_{95}$ ), é de $1,11 \mathrm{~m}^{3} \mathrm{~s}^{-1}$, na seção do rio das Pedras, bem acima do valor atualmente outorgado a Sanepar, que é de $0,50 \mathrm{~m}^{3} \mathrm{~s}^{-1}$.

Diante dos resultados, subtraiu-se da vazão diária monitorada a vazão outorgada $\left(0,5 \mathrm{~m}^{3} \mathrm{~s}^{-1}\right)$ para verificar a vazão ecológica. Os dados foram plotados em um gráfico para comparação (Figura 6). Nela verifica-se que a vazão no trecho fluvial em determinados períodos fica abaixo da vazão ecológica estabelecida oficialmente, que é de $0,91 \mathrm{~m}^{3} \mathrm{~s}^{-1}$ no Estado do Paraná, assim como da vazão ecológica média dos métodos avaliados $\left(2,20 \mathrm{~m}^{3} \mathrm{~s}^{-1}\right)$.

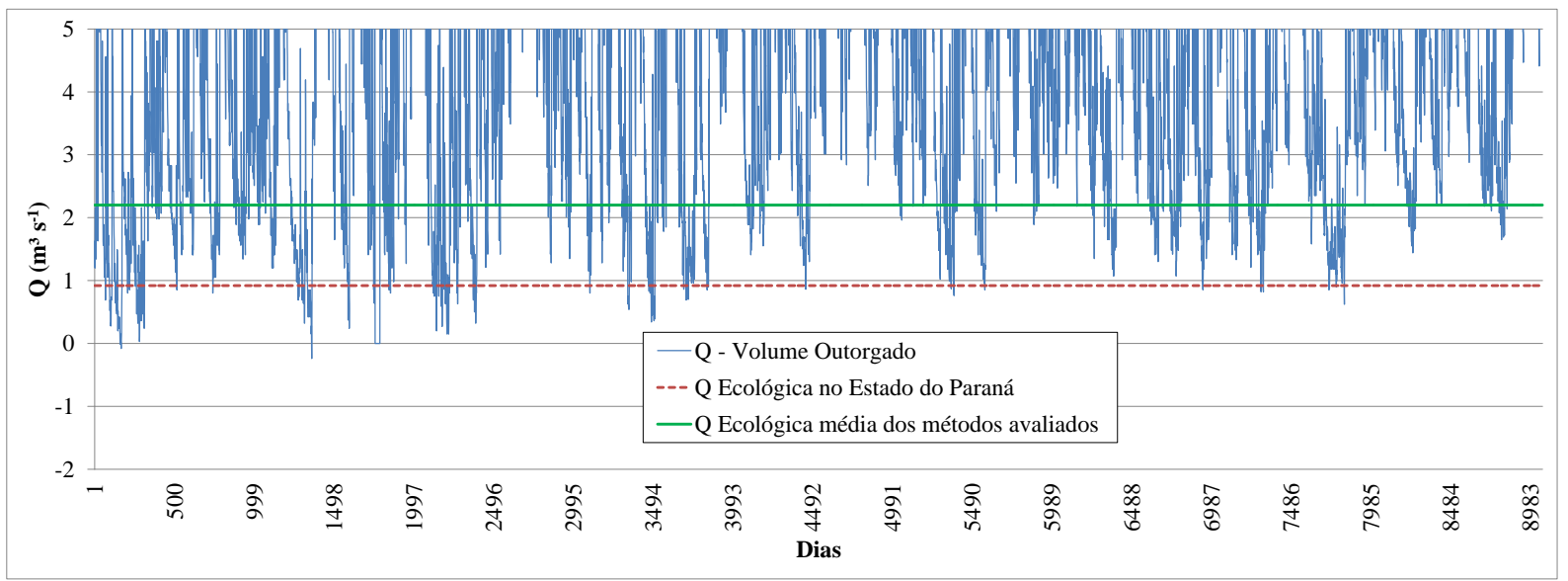

Figura 6. Dados de vazão diária menos o volume outorgado. 
VESTENA, L. R.; OLIVEIRA, E. D.; CUNHA, M. C.; THOMAZ, E. L. Vazão ecológica e disponibilidade hídrica na bacia das Pedras, Guarapuava-PR. Ambi-Agua, Taubaté, v. 7, n. 3, p. 212-227, 2012. (http://dx.doi.org/10.4136/ambi-agua.840)

Constata-se, pela Figura 6, que a variabilidade temporal da vazão impõe condições diferenciadas ao aproveitamento hídrico, dispondo de maiores volumes nos períodos de cheias e menores nos de estiagem. Ela também demonstra a limitação da metodologia adotada para definição da vazão ecológica no Estado do Paraná, como também das demais metodologias empregadas no estudo, uma vez que a vazão ecológica deve conservar os níveis mínimos de fluxo de base especialmente nos períodos de secas, por considerar as prioridades ecológicas, o comportamento do fluxo e a limitação para explotação de água, como destacaram Deitch et al. (2009). Destaca-se também que estudos realizados por Stalnaker et al. (1995) já apontavam que a vazão ${ }_{7} \mathrm{Q}_{10}$ é excessivamente baixa para a manutenção de hábitats aquáticos.

Com base nos resultados obtidos, resume-se que a BHRP apresenta uma série de dados hidrológicos com poucas falhas o que dá confiabilidade aos dados monitorados, a saber:

- a vazão de referência à vazão ecológica na BHRP variou de 1,7 a 2,7 $\mathrm{m}^{3} \mathrm{~s}^{-1}$, de acordo com as especificidades de cada um dos quatro métodos aplicados;

- a vazão ecológica no trecho do rio das Pedras, segundo critérios estabelecidos no Estado do Paraná, que é $50 \%$ da ${ }_{7} \mathrm{Q}_{10}$ é de $0,92 \mathrm{~m}^{3} \mathrm{~s}^{-1}$;

- a vazão de referência específica à vazão ecológica variou de 5,64 a $8,10 \mathrm{~L} \mathrm{~s}^{-1} \mathrm{~km}^{-2}$, com uma média de $6,97 \mathrm{~L} \mathrm{~s}^{-1} \mathrm{~km}^{-2}$, considerando as quatro metodologias;

- a vazão de outorga média utilizada na bacia é de $0,31 \mathrm{~m}^{3} \mathrm{~s}^{-1}$ e a cedida de $0,50 \mathrm{~m}^{3} \mathrm{~s}^{-1}$, com perspectivas de aumentar dado o crescimento da cidade e consequentemente da demanda.

Os resultados reforçam a afirmação de Collischonn et al. (2005) que demostram a insuficiência do tradicional critério de vazão ecológica como um valor único, válido para todas as épocas e estações do ano. Esses autores também apresentam uma metodologia alternativa que vem sendo aplicada em alguns casos nos EUA, focada em vários aspectos do regime hidrológico (Hidrograma Ecológico) e não apenas em vazões mínimas.

Apesar de o valor médio estar abaixo da Outorga, o crescimento urbano e o consequente aumento de demanda por quantidade de água, o volume de água retirado tende a aumentar, o que requer novos estudos hidrológicos dos impactos ambientais, bem como a procura por outras alterativas potenciais para o abastecimento de Guarapuava, a fim de minimizar os impactos tanto de ordem social, econômica e ecológica.

\section{CONCLUSÃO}

Os resultados desse estudo indicaram a ineficiência dos métodos de determinação da vazão ecológica, ou seja, não garantem o volume de água mínimo necessário à conservação do ecossistema fluvial. Portanto, com a manutenção do sistema e a evolução nas técnicas de monitoramento fluviométrico, novas análises devem ser implementadas para avaliar com mais precisão as alterações espaciais e também temporais dos processos hidrológicos.

Os métodos utilizados para definição das vazões de referências à vazão ecológica mostraram-se de fácil aplicação, entretanto, baseiam apenas em dados de séries históricas de vazão, na quantidade de água, sem considerar a biota do rio e do ecossistema do seu entorno. Portanto, novos estudos sobre a vazão ecológica devem ter por base também o conhecimento da biota do curso d'água e suas relações.

Os conhecimentos prévios das vazões ecológicas e dos processos envolvidos proporcionam subsídios que fundamentam a tomada de decisões e ajudam num planejamento e manejo do uso racional dos recursos hídricos, permitindo adequar os fatores socioeconômicos aos ecológicos. 
VESTENA, L. R.; OLIVEIRA, E. D.; CUNHA, M. C.; THOMAZ, E. L. Vazão ecológica e disponibilidade hídrica na bacia das Pedras, Guarapuava-PR. Ambi-Agua, Taubaté, v. 7, n. 3, p. 212-227, 2012. (http://dx.doi.org/10.4136/ambi-agua.840)

\section{AGRADECIMENTOS}

Os autores agradecem a CAPES - Coordenação de Aperfeiçoamento de Pessoal de Nível Superior, pela concessão de bolsas de estudo em nível de mestrado a dois dos autores.

\section{REFERÊNCIAS}

AZEVEDO, L. C.; NERY, J. T.; MARTONI, A. M. Análise da precipitação pluvial associada ao fenômeno El-Niño na bacia hidrográfica do Rio Iguaçu - Paraná, In: SIMPÓSIO BRASILEIRO DE GEOGRAFIA FÍSICA APLICADA, 11, 04-09 Set 2005, São Paulo. Anais... São Paulo: USP, 2005, p. 1904-1913.

BENETTI, A. D.; LANNA, A. E.; COBALCHINI, M. S. Metodologias para determinação de vazões ecológicas em rios. Revista Brasileira de Recursos Hídricos, Porto Alegre, v. 8, n. 2, p. 149-160, abr./jun. 2003.

BRASIL. Lei Federal no 9.433, de 08 de janeiro de 1997, Institui a Política Nacional de Recursos Hídricos, cria o Sistema Nacional de Gerenciamento de Recursos Hídricos. Diário Oficial da República Federativa do Brasil, Brasília, DF, 08 jan. 1997.

BRASIL. Resultado do Censo do Instituto Brasileiro de Geografia e Estatísticas (IBGE) de 2010. Governo Federal. Diário Oficial da República Federativa do Brasil, Brasília, DF, 04 nov. 2010.

COLliSCHONN, W.; AGRA, S. G.; FREITAS, G. K.; PRIANTE, G. R.; TASSI, R.; SOUSA, C. F. Em busca do Hidrograma Ecológico. In: XVI SIMPÓSIO BRASILEIRO DE RECURSOS HÍDRICOS, 16, 20-24 Nov. 2005, João Pessoa. Anais... João Pessoa: ABRH, 2005. 1 CD-ROM.

CRUZ. R. C. Prescrição de vazão ecológica: aspectos conceituais e técnicos para bacias com carência de dados. 2005. 135f. Tese (Doutorado em Ecologia) - Instituto de Biociências, Universidade Federal do Rio Grande do Sul, Porto Alegre, 2005.

DEITCH, M. J.; KONDOLF, G. M.; MERENLENDER, A. M. Hydrologic impacts of small scale instream diversions for frost and heat protection in the California wine country. River Research and Applications, v. 25. p. 118-134, 2009.

INSTITUTO DE ÁGUAS DO PARANÁ. Outorga de Uso Recursos Hídricos. Disponível em: http://www.aguasparana.pr.gov.br/modules/conteudo/conteudo.php?conteudo=10. Acesso em 05 fev. 2012.

INSTITUTO DE ÁGUAS DO PARANÁ: Vazões Mínimas. Disponível em: http://www.aguasparana.pr.gov. br/arquivos/File/mp13.pdf. Acesso em: 8 mai. 2011.

JOWETT, I. G. Instream flow methods: A comparison of approaches. Regulated Rivers: Research and management. v. 13, p. 115-127, 1997.

KULIK. B. H. A method to refine the New England aquatic base flow policy. Rivers. v.1, n. 1, p. 8-22, 1990.

LOBODA, C. R. Práticas Socioespaciais e Espaços Públicos em Guarapuava-Pr. 2008. 352 f. Tese (Doutorado em Geografia) - Faculdade de Ciências e Tecnologia. Universidade Estadual Paulista, Presidente Prudente, 2008. 
VESTENA, L. R.; OLIVEIRA, E. D.; CUNHA, M. C.; THOMAZ, E. L. Vazão ecológica e disponibilidade hídrica na bacia das Pedras, Guarapuava-PR. Ambi-Agua, Taubaté, v. 7, n. 3, p. 212-227, 2012. (http://dx.doi.org/10.4136/ambi-agua.840)

LONGHI, E. H.; FORMIGA, K. T. M. Metodologias para determinar vazão ecológica em rios, Revista Brasileira de Ciências Ambientais, n. 20, p. 2176-9478, jun. de 2011.

MAACK, R. Geografia Física do Estado do Paraná. $3^{a}$ ed. Curitiba: Imprensa oficial, 1981, $440 \mathrm{p}$.

MEDEIROS, P. C.; SOUZA, F. A. S.; RIBEIRO, M. M. R. Aspectos conceituais sobre o regime hidrológico para a definição do hidrograma ambiental. Ambi-Agua, Taubaté, v. 6, n. 1, p. 131-147, 2011. http://dx.doi.org/10.4136/ambi-agua.179

MOLIERE, D. R.; LOWRY, J. B. C.; HUMPHREY, C. L. Classifying the flow regime of data-limited streams in the wet-dry tropical region of Australia. Journal of Hydrology, v. 367, p. 1-13, 2009. http://dx.doi.org/10.1016/j.jhydrol.2008.12.015

PARANÁ. Legislação Paranaense de Recursos Hídricos: Lei Estadual no 12.726/99 e decretos que estruturam a gestão de recurso hídrica no Estado do Paraná. São Paulo: Astúrias, 2002. 169 p.

PARANÁ. Portaria No 20/99 - SUDERHSA, de 18 de Maio de 1999. Dispõe sobre critérios para outorga de recursos hídricos superficiais. DOE. n. 5503, Curitiba, 26 mai. 1999.

PEREIRA, S. B. Evaporação no lago de Sobradinho e disponibilidade hídrica no rio São Francisco. 2004. 105 f. Tese (Doutorado em Recursos Hídricos e Ambiental) Departamento de Engenharia Agrícola, Universidade Federal de Viçosa, Viçosa - MG, 2004.

PINTO, L. C.; MACHADO, E. F. P.; DE MELlO, C. R.; SILVA, A. M. Análise de Distribuições de Probabilidades e Estimativa da $\mathrm{Q}_{7,10}$ para a Região do Rio Itabapoana, Espírito Santo/Rio De Janeiro. In: XIX CONGRESSO DE PÓS-GRADUAÇÃO DA UFLA, 19, 27 set. - 01 out. 2010. Lavras. Anais... Lavras: UFLA, 2010.

SANTOS, A. F.; KOBIYAMA, M. Contribuição potencial de cargas poluentes na bacia do Rio das Pedras, no município de Guarapuava - PR. Revista Ciências Exatas e Naturais, v. 5, n. 1, p. 33-46, Jan/Jun. 2003.

SARMENTO, R. Estado da arte da vazão ecológica no Brasil e no mundo. Unesco/ANA/CBHSF, 2007. 38 p.

SALNAKER,C.; LAMB, B. L.; HENRIKSEN,J.; BOVEE, K.; BARTHLOW, J. The Instream Flow Incremental Methodology. A Primer for IFIM. U.S. Department of Interior. National Biological Service, Washington, D. C., 1995. 47 p.

STRAHLER, A. N. Quantitative analysis of watershed Geomorphology. Am. Geophys. Union Trans., v. 38, n. 6, p. 913-920, 1957.

SUDERHSA. Superintendência de Recursos Hídricos e Saneamento Ambiental. Manual Técnico de Outorgas. Novembro/2006 - Revisão 01. Disponível em: http://www.aguasparana.pr.gov.br/arquivos/File/manual_outorgas.pdf. Acesso em 06/02/2012.

THOMAZ, E. L.; VESTENA, L. R. Aspectos Climáticos de Guarapuava-PR. Guarapuava: UNICENTRO, 2003. $106 \mathrm{p}$.

TUCCI, C. E. M. Metodologia para regionalização de vazões. Vol. II: Regionalização de vazões do Alto Paraguai. Porto Alegre: IPH/UFRGS, 1984. 
VESTENA, L. R.; OLIVEIRA, E. D.; CUNHA, M. C.; THOMAZ, E. L. Vazão ecológica e disponibilidade hídrica na bacia das Pedras, Guarapuava-PR. Ambi-Agua, Taubaté, v. 7, n. 3, p. 212-227, 2012. (http://dx.doi.org/10.4136/ambi-agua.840)

TUCCI, C. E. M. Água no Meio Urbano. In: REBOUÇAS, A. et. al, (eds.) Águas Doces do Brasil: capital ecológico, uso e conservação. 2. ed. São Paulo: Escrituras Ed., 2000, p. 475-508.

VESTENA, L. R.; THOMAZ, E. L. Avaliação de conflitos entre áreas de preservação permanentes associadas aos cursos fluviais e uso da terra na bacia do rio das pedras, Guarapuava-PR. Ambiência, v. 2, n. 1, p. 73-85, 2006 


\title{
Estudo da qualidade e quantidade da água em microbacia, afluente do rio Paraíba do Sul - São Paulo, após ações de preservação ambiental
}

(http://dx.doi.org/10.4136/ambi-agua.987)

\author{
Lívia Alves Alvarenga ${ }^{1}$; Maria Paulete Pereira Martins ${ }^{1}$; Luz Adriana Cuartas ${ }^{2}$; \\ Vinicius Alves Penteado ${ }^{3}$; Alexandra Andrade ${ }^{3}$ \\ ${ }^{1}$ Programa de Pós-Graduação em Meteorologia, \\ Instituto Nacional de Pesquisas Espaciais (INPE), São José dos Campos-SP, \\ ${ }^{2}$ Programa de Pós-Graduação em Ciência do Sistema Terrestre, \\ Instituto Nacional de Pesquisas Espaciais (INPE), São José dos Campos-SP, \\ ${ }^{3}$ Instituto Oikos de Agroecologia, Lorena-SP, \\ e-mails: livia.alvarenga@posgrad.ufla.br,maria.paulete@cptec.inpe.br, adriana.cuartas@inpe.br, \\ vinni_alves@yahoo.com.br, andrade.alexandra@uol.com.br
}

\section{RESUMO}

Cada vez mais são necessários programas de monitoramento da qualidade e quantidade da água como subsídios para avaliar as condições dos mananciais e, além disso, propiciar informações para a tomada de decisão com relação ao gerenciamento dos recursos hídricos. Este estudo analisou a qualidade e quantidade da água na microbacia do Ribeirão dos Macacos, afluente do rio Paraíba do Sul, Estado de São Paulo, por meio do monitoramento dos parâmetros: temperatura, $\mathrm{pH}$, condutividade e oxigênio dissolvido, em cinco pontos da microbacia. As medições de vazão e altura da lâmina d' água durante os períodos seco e úmido dos anos de 2010/2011, permitiram obter a "curva chave" em quatro pontos de monitoramento da qualidade da água e reconstruir as séries de vazões para estes períodos. Os resultados das análises mostraram que há um indicativo de mudanças nos parâmetros de qualidade da água devido às práticas conservacionistas adotadas. $\mathrm{O}$ parâmetro temperatura da água foi o mais influenciado pela sazonalidade da vazão. Diversos fatores físicos podem ter contribuído na correlação dos outros parâmetros com a vazão principalmente as diferentes ações de recuperação ambiental implantadas na área deste estudo e que visam uma maior sustentabilidade dos recursos hídricos.

Palavras-chave: práticas conservacionistas, sustentabilidade, parâmetros de qualidade da água e vazão.

\section{Study of the quality and quantity of waters of a tributary watershed of Paraíba do Sul river- São Paulo, after environmental preservation actions}

\begin{abstract}
Monitoring programs of water quality and quantity are necessary to provide subsidies to assess the conditions of the watersheds and for decision making regarding to the management of water resources. This study analyzed the quality and quantity of waters of the Macacos stream watershed, a tributary of the Paraíba do Sul river, in São Paulo State, by monitoring the parameters: temperature, $\mathrm{pH}$, conductivity and dissolved oxygen at five sites in the watershed. The measurements of flow and height of water depth during dry and wet seasons of 2010 and 2011 allowed the construction of the "rating curve" in four points of water quality
\end{abstract}


monitoring and to reconstruct the series of water flow in these seasons. The analysis results showed that there is indication of changes in water quality parameters due to the conservation practices adopted. The water temperature parameter was the most influenced by the seasonal variation in runoff. Several physical factors may have influenced the correlation of the other parameters with runoff, especially the different environmental recovery actions implemented in the study to achieve the sustainability of the water resources.

Keywords: conservation practices, sustainability, water quality parameters and flow.

\section{INTRODUÇÃO}

Os diferentes processos que acontecem em uma bacia dependem das características do local onde ela se insere. Flora, fauna, relevo, uso e ocupação do solo, e os fenômenos meteorológicos, interagem entre si e condicionam a resposta hidrológica na bacia. As diferentes ações antrópicas em determinadas áreas transformam as estruturas físico-químicas e biológicas dos ecossistemas naturais e alteram o ciclo hidrológico, reduzindo desta forma a oferta de água e consequentemente a queda da qualidade dos recursos hídricos. A região do Vale do Paraíba-São Paulo, de grande relevância para o Brasil por ser bastante desenvolvida economicamente, apresenta um grande contingente populacional e sérios problemas ambientais. Assim, é uma região na qual são necessárias diferentes ações voltadas para a recuperação e preservação dos recursos naturais.

Manandhar et al. (2011), em estudo feito no Nepal, centrado nas percepções e adaptações dos agricultores às mudanças climáticas, salientou que há uma necessidade de se ir além do nível individual, para planejar e fornecer apoio de tecnologias apropriadas e estratégias a fim de lidar com os esperados crescentes impactos das mudanças do clima. Desta maneira, tornase fundamental o incentivo a gestão agrícola sustentável e a difusão de práticas tecnológicas de baixo custo.

Conforme Marengo e Alves (2005), no estudo das tendências hidrológicas na bacia do rio Paraíba do Sul (para o período de 1920-2000), é evidente que o progressivo processo de industrialização e urbanização assim como a degradação ambiental no Vale do Rio Paraíba do Sul, contribuem para o intenso uso dos recursos naturais e para o comprometimento da qualidade e quantidade dos recursos hídricos hoje observados. Estes autores registraram tendências decrescentes nas vazões do rio em vários postos e dentre as possíveis causas, apontadas pelos autores, desta redução sistemática nas vazões e cotas tem-se as atividades antrópicas, que também estão associadas às mudanças gradativas no regime e distribuição de chuvas na bacia, decorrentes de mudanças climáticas regionais.

De acordo com Machado et al. (2010) torna-se de fundamental importância implementar e identificar medidas no sentido de melhorar a qualidade da água, contribuindo deste modo para a elaboração dos Planos de Gestão da Região Hidrográfica. Segundo Golla (2006), o manejo adequado (intervenção humana nos recursos naturais com a intenção de torná-los aptos à utilização agrícola e pecuária, de maneira que preservem características desejáveis às atividades destinadas ao homem) de solos e água constitui um dos grandes desafios da humanidade, estando diretamente relacionados à sustentabilidade de todas as formas de vida, bem como às atividades antrópicas.

Neary et al. (2009) relatam que a maior sustentabilidade e melhoria da qualidade de fontes de água doce do mundo se originam em ecossistemas florestais. As características químicas, biológicas e físicas dos solos florestais são particularmente adequadas para o fornecimento de água de alta qualidade em bacias hidrográficas, moderando o fluxo, e fornecendo diversos habitats aquáticos. Os sistemas radiculares sob florestas são extensos e 
relativamente profundos em relação às terras agrícolas e pastos, e tendem a criar solos com alta macroporosidade, baixa densidade, altas condutividade hidráulica saturada e taxas de infiltração. A conversão de florestas em áreas agrícolas ou urbanas quase sempre resulta na deterioração da qualidade da água. Santos et al. (2011) também reportam que a reposição florestal com matas nativas se constitui em uma das principais formas de aumentar a retenção de água (manutenção da vazão) em uma bacia hidrográfica pelo aumento da infiltração no solo e redução do escoamento superficial e erosão.

A água é um dos elementos mais importantes e fundamentais para a manutenção da vida com qualidade, para o conforto climático, e complementarmente, para o desenvolvimento econômico. Desta maneira, alguns trabalhos já foram realizados associando a qualidade das águas com os diferentes usos do solo, mostrando os efeitos na qualidade ambiental (Bolstad e Swank, 1997; Arcova e Cicco, 1999). Os trabalhos em questão foram realizados respectivamente na América do Norte e América do Sul. Os autores evidenciam os impactos da modificação da paisagem nos parâmetros de qualidade da água e enfatizam que durante eventos de tempestade no período úmido de cada um dos locais de estudo, ocorreu uma maior alteração dos parâmetros de qualidade da água analisados.

Silva et al. (2008), no estudo da influência da precipitação na qualidade da água no rio Purus (afluente do rio Amazonas) também ressaltou que o regime da precipitação em diferentes localidades pode influenciar os valores de qualidade da água. Apesar dos resultados serem preliminares, esta pesquisa mostrou que a precipitação parece ser o principal agente influenciador da qualidade da água do rio Purus, uma vez que foi observado que as variáveis monitoradas se correlacionam significativamente com o regime de chuvas do local.

Silva et al. (2009) mostraram que os seus resultados de qualidade da água no Reservatório da Usina Hidrelétrica (UHE)- Peti - MG, foram divididos em: Época de estiagem (setembro, outubro, abril e maio) e chuvosa (novembro, dezembro, janeiro, fevereiro e março) e, no geral, apresentam variação sazonal (2004-2005). Na época chuvosa, parâmetros como sólidos, turbidez e cor aumentam a concentração; sendo observada também maior variação entre as profundidades na época de estiagem, e à medida que se adentra no reservatório (montante para jusante), parâmetros como turbidez, cor e sólidos totais em suspensão diminuem em função da sua própria dinâmica.

Os autores Bueno et al. (2005) afirmam que o tipo de cobertura vegetal implica em distintos comportamentos nos atributos do solo e da água, sendo que a remoção das florestas tem causado aumento significativo dos processos que levam à degradação de áreas imensas, com prejuízos à hidrologia e à biodiversidade. Este trabalho apresenta o monitoramento da qualidade da água em duas condições de uso do solo (mata nativa e eucalipto), e foi verificado que a cobertura do solo de mata nativa foi responsável pela manutenção da temperatura da água estável, influenciando as demais variáveis da água (OD, $\mathrm{MO}$ e $\mathrm{pH})$. Assim, por meio dos plantios florestais de mata nativa os produtores rurais podem obter benefícios na conservação do solo e da água e reduzir os impactos negativos da redução das vegetações naturais remanescentes.

Neste contexto, propõe-se com este trabalho obter e analisar os dados de qualidade e quantidade de água na microbacia do Ribeirão dos Macacos em período seco e úmido nos anos de 2010 e 2011, por meio do acompanhamento de alguns parâmetros (indicativos de qualidade da água) e da vazão, enfatizando as diferentes práticas de recuperação ambiental, efetuadas pelo Instituto Oikos de Agrecologia na área de monitoramento.

Este estudo faz parte do projeto "Estudo da influência da recuperação e conservação ambiental na melhoria da qualidade e quantidade da água em microbacias do Rio Paraíba do Sul, no estado de São Paulo", financiado pelo Fundo Estadual de Recursos Hídricos 
(FEHIDRO). Este trabalho foi possível devido a uma colaboração entre o Centro de Previsão de Tempo e Estudos Climáticos (CPTEC/INPE) e o Instituto Oikos de Agroecologia.

\section{MATERIAIS E MÉTODO}

A microbacia do ribeirão dos Macacos, afluente do rio Paraíba do Sul, localiza-se no estado de São Paulo, nos municípios de Guaratinguetá e Lorena, entre as coordenadas de $22^{\circ} 38^{\prime} 56,2^{\prime \prime} \mathrm{S}, 45^{\circ} 15,2^{\prime} 2,6^{\prime}$ 'W e $22^{\circ} 42^{\prime} 45,1^{\prime}$ 'S $, 45^{\circ} 6^{\prime} 59,6^{\prime}$ ' $\mathrm{W}$, aproximadamente. A bacia hidrográfica apresenta área de aproximadamente $39 \mathrm{~km}^{2}$, o equivalente a 3900 hectares. No entanto, a área de estudo de monitoramento da qualidade e quantidade de água e acompanhamento das práticas ambientais corresponde a aproximadamente 400 hectares a jusante da microbacia do ribeirão dos Macacos (Figura 1).

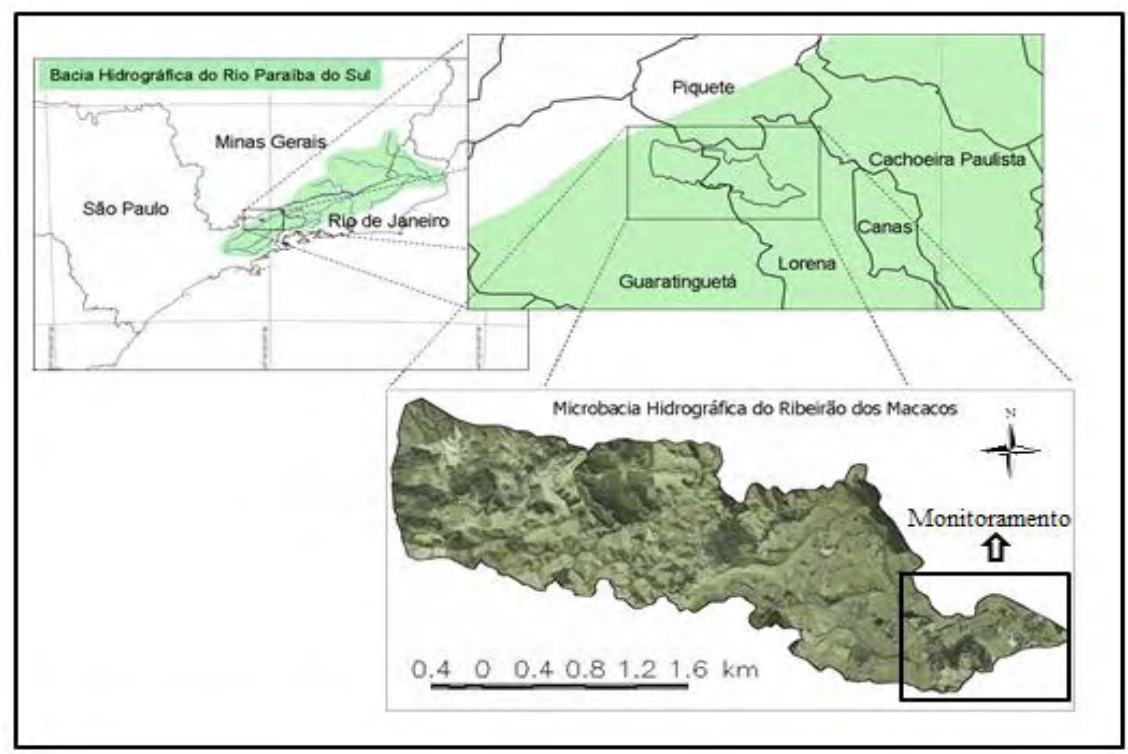

Figura 1. Localização da microbacia do ribeirão dos Macacos-SP entre as coordenadas de $22^{\circ} 38^{\prime} 56,2^{\prime}$ ' $\mathrm{S}, 45^{\circ} 15,2^{\prime} 2,6^{\prime}$ ' $\mathrm{W}$ e $22^{\circ} 42^{\prime} 45,1^{\prime}$ 'S, $45^{\circ} 6^{\prime} 59,6^{\prime}$ 'W (retângulo da parte superior direita).

Fonte: Instituto Oikos de Agroecologia [ca. 2009].

De acordo com Fisch (1995) o clima da região apresenta temperaturas elevadas - média anual de $22^{\circ} \mathrm{C}$ - e duas estações de precipitação bem definidas: uma chuvosa que corresponde ao verão, e outra seca, que corresponde ao inverno. No verão a Zona de Convergência do Atlântico Sul (ZCAS) é um dos principais mecanismos responsável pelo aumento da precipitação (Lima et al., 2010).

O padrão de uso do solo na microbacia do ribeirão dos Macacos é predominantemente rural, sendo a porcentagem de cada uso do solo apresentado na Tabela 1.

Tabela 1. Uso do solo na microbacia do ribeirão dos Macacos.

\begin{tabular}{l|c}
\hline Uso do solo na microbacia & Área (\%) \\
Pastagem & 68,0 \\
Fragmentos florestais & 21,6 \\
Plantio de eucalipto & 5,7 \\
Área urbano industrial & 3,0 \\
Plantações (capineiras e plantios de hortas) & 1,7 \\
\hline
\end{tabular}

Fonte: Instituto Oikos de Agroecologia [ca. 2009]. 
$\mathrm{Na}$ área deste estudo está localizado o Instituto Oikos de Agroecologia, que vem desenvolvendo ações de restauração ambiental, adequação de métodos produtivos, conservação dos recursos naturais (águas e biodiversidade), mobilização e capacitação de produtores rurais (educação ambiental) desde o início de 2009. Assim, por meio de parcerias entre instituições públicas e privadas juntamente com a comunidade local da microbacia do Ribeirão dos Macacos, foi possível o estudo da área e de alguns elementos desta, visando à utilização de práticas sustentáveis do uso dos recursos naturais.

O Instituto Oikos de Agroecologia a partir de questionários aplicados aos proprietários rurais da região selecionou as propriedades mais necessitadas das práticas de recuperação ambiental, com o intuito de eliminar parte das fontes pontuais de contaminação da microbacia. Na Tabela 2 apresentam-se as fazendas e as respectivas ações que ocorreram envolvendo os pontos de monitoramento da qualidade e quantidade (descritos mais adiante) e os proprietários da região (pontos de apoio utilizados).

Tabela 2. Fazendas de atuação do Instituto Oikos de Agroecologia e suas respectivas ações.

\begin{tabular}{|c|c|}
\hline Proprietários & Ações de recuperação \\
\hline Propriedade da Sra. Marisa Nunes $\left(\mathrm{P}_{1}\right)$ & $\begin{array}{l}\text { Instalação de bebedouro para o gado (início: } \\
01 / 06 / 2010 \text { ). }\end{array}$ \\
\hline Propriedade do Sr. José Fernando $\left(\mathrm{P}_{2}\right)$. & $\begin{array}{l}\text { Instalação de bebedouro para o gado (início: } \\
01 / 06 / 2010 \text { ). }\end{array}$ \\
\hline Propriedade do Sr. Fernando Miyazaki $\left(\mathrm{P}_{3}\right)$. & $\begin{array}{l}\text { Implantação de } 2,5 \text { hectares de pastejo } \\
\text { rotacionado "Voisin" (início: 08/09/2009), } \\
\text { instalação de uma chorumeira (início: } \\
\text { 23/06/2010), uma fossa séptica (início: } \\
\text { 09/04/2010) e bebedouro para o gado (início: } \\
\text { 01/06/2010). }\end{array}$ \\
\hline Propriedade do Sr. Darci de Paula $\left(\mathrm{P}_{4}\right)$. & $\begin{array}{l}\text { Implantação de } 2,5 \text { hectares de pastejo } \\
\text { rotacionado "Voisin" (início: 08/09/2009), } \\
\text { Restauração de 1,5 hectares de Áreas de } \\
\text { Preservação Permanente (início: 03/05/2010). }\end{array}$ \\
\hline $\begin{array}{l}\text { Propriedade do Sr. José Raimundo Ferreira } \\
\text { (Ponto de apoio). }\end{array}$ & $\begin{array}{l}\text { Instalação de duas fossas sépticas (início: } \\
\text { 09/04/2010), Limpeza de três fossas negras } \\
\text { (início: 09/04/2010) e instalação de } \\
\text { bebedouro para o gado (início: } 01 / 06 / 2010 \text { ). }\end{array}$ \\
\hline $\begin{array}{l}\text { Propriedade do Sr. Arnaldo Leite (Ponto de } \\
\text { apoio). }\end{array}$ & $\begin{array}{l}\text { Instalação de duas fossas sépticas (início: } \\
\text { 09/04/2010). }\end{array}$ \\
\hline Propriedade da Sra. Regina Nunes. & $\begin{array}{l}\text { Instalação de bebedouro para o gado (início: } \\
01 / 06 / 2010 \text { ). }\end{array}$ \\
\hline Propriedade da Sr. Luiz Domingos Bastos. & $\begin{array}{l}\text { Instalação de bebedouro para o gado (início: } \\
01 / 06 / 2010 \text { ). }\end{array}$ \\
\hline
\end{tabular}

Fonte: Instituto Oikos de Agroecologia [ca. 2009].

Na escolha dos pontos de monitoramento na microbacia do Ribeirão dos Macacos foi levado em consideração o uso e ocupação do solo e as ações que foram realizadas pelo Instituto Oikos de Agroecologia. Os pontos de monitoramento da qualidade da água: $\mathrm{P}_{1}, \mathrm{P}_{3} \mathrm{e}$ $\mathrm{P}_{5}$ estão localizados na entrada, na porção intermediária e na saída da região de monitoramento, respectivamente. Os pontos $\mathrm{P}_{2}$ e $\mathrm{P}_{4}$ estão localizados após nascentes que 
devem ser recuperadas futuramente. A cobertura vegetal de mata ciliar na área de monitoramento é pouco extensa, justificando a necessidade de plantio. Aliás, na área do ribeirão dos Macacos $68 \%$ da microbacia está coberta por pastagens, o que justifica ainda mais ações de recuperação ambiental.

O monitoramento, feito em campo, da qualidade e altura da lâmina d'água na microbacia segundo o Standard Methods (APHA, 1995) é realizado por meio de uma sonda multiparâmetros (YSI 6820) que mede por meio de sensores específicos as seguintes características de qualidade da água: temperatura $\left({ }^{\circ} \mathrm{C}\right)$, potencial hidrogeniônico-pH, condutividade elétrica $\left(\mu \mathrm{S} \mathrm{cm}^{-1}\right)$, oxigênio dissolvido-OD $\left(\mathrm{mg} \mathrm{L}^{-1}\right)$ e altura da lâmina d'água na microbacia $(\mathrm{m})$. Os dados provenientes da sonda multiparâmetros são armazenados em um datalogger, que consiste num sistema de acumulação de dados que se encontra ligado à sonda.

A calibração dos sensores medidores dos parâmetros de qualidade da água foi feita periodicamente no Laboratório de Instrumentação Meteorológica (LIM) do Centro de Previsão de Tempo e Estudos Climáticos (CPTEC) - INPE. As medições dos parâmetros de qualidade da água foram realizadas, em média, uma vez por semana nos cinco pontos escolhidos pelo Instituto Oikos de Agroecologia para o monitoramento. O horário da coleta dos dados foi entre 14:00 e 16:00 horas. Das cinco amostragens realizadas a cada dia, obtémse a média em cada ponto para cada parâmetro monitorado. Os dados obtidos nos anos de 2010/2011 (Junho/2010 até setembro/2011) são analisados, por meio da estatística descritiva (média e desvio padrão), levando-se em consideração o período seco e úmido destes anos.

Para as medições de vazão utilizou-se um método simples de medição indireta também conhecida como "manual". Este método foi utilizado pela falta de equipamento para a realização de medições mais acuradas. Em todos os pontos em que se fez o monitoramento da qualidade da água foram feitas as medições de vazão, exceto no ponto $\mathrm{P}_{2}$, por se tratar de um lago. Este local, muitas vezes serve para dessedentação do gado na região e no período úmido deságua na microbacia.

A velocidade média do fluxo de água foi medida utilizando-se uma bola de isopor colocada num determinado ponto, e depois solta. A distância que a bola de isopor flutuou foi pré-fixada e o tempo percorrido marcado com um cronômetro. A velocidade do fluxo de água foi obtida dividindo-se a distância percorrida pelo tempo. Este procedimento foi repetido três vezes para se obter um valor médio. Conforme Mosley e Mckerchar (1993), as medições da velocidade, por serem do fluxo superficial devem ser corrigidas, pois estas podem estar superestimadas, a velocidade média do fluxo no perfil vertical é $86 \%$ da velocidade na superfície.

Os dados de vazão foram monitorados no período úmido e seco do ano de 2011 (março/2011 até novembro/2011), em intervalos aproximados de 20 dias. Com a relação entre altura da lâmina d'água e a respectiva vazão foi possível obter a curva chave em cada ponto de monitoramento, levando-se em conta as características hidráulicas e geométricas da seção transversal de cada ponto. Com a curva chave foi possível reconstruir as vazões (nos pontos: $\mathrm{P}_{1}, \mathrm{P}_{3}, \mathrm{P}_{4}$ e $\mathrm{P}_{5}$ ) para os anos de 2010 e 2011 durante todo o período do monitoramento da qualidade da água na microbacia.

A correlação entre a vazão e os parâmetros de qualidade da água (temperatura, pH, condutividade elétrica e OD) nos diferentes pontos foi averiguada por meio do coeficiente de correlação de Pearson, que varia de +1 (correlação perfeita positiva) a -1 (correlação perfeita negativa). A correlação de Pearson é um indicador estatístico de precisão que indica o grau de dispersão dos dados estimados em relação à média (Santos, 2007). 


\section{RESULTADOS E DISCUSSÃO}

Os períodos secos deste estudo foram junho, julho e agosto (JJA) dos anos de 2010 e 2011 cuja precipitação acumulada foi de, respectivamente, 77 e $62 \mathrm{~mm}$. O período úmido foi de dezembro, janeiro, fevereiro e março (DJFM) de 2010/2011 com precipitação acumulada de $1231 \mathrm{~mm}$.

A temperatura da água foi medida somente em dois períodos JJA de 2010 e DJFM de 2010/2011, devido a problemas com o sensor da sonda multiparâmetros. Os outros parâmetros de qualidade da água (potencial hidrogeniônico, condutividade elétrica e oxigênio dissolvido) também foram medidos no período seco posterior JJA de $2011 \mathrm{com}$ a finalidade de comparações.

As médias e o desvio padrão (Desv. Pad.) da temperatura da água para os períodos, seco (JJA de 2010) e úmido (DJFM de 2010/2011) nos diferentes pontos $\left(\mathrm{P}_{1}, \mathrm{P}_{2}, \mathrm{P}_{3}, \mathrm{P}_{4}\right.$ e $\left.\mathrm{P}_{5}\right)$ encontram-se na Tabela 3.

Tabela 3. Médias e desvio padrão da temperatura da água $\left({ }^{\circ} \mathrm{C}\right)$ nos diferentes pontos e períodos de estudo.

\begin{tabular}{l|rrrrr}
\hline \multirow{2}{*}{ Pontos } & \multicolumn{5}{c}{ Temperatura $\left({ }^{\circ} \mathbf{C}\right)$} \\
\cline { 2 - 6 } & $\mathrm{P}_{1}$ & $\mathrm{P}_{2}$ & $\mathrm{P}_{3}$ & $\mathrm{P}_{4}$ & $\mathrm{P}_{5}$ \\
\hline \multirow{3}{*}{ Média } & \multicolumn{5}{c}{ Período seco (JJA de 2010) } \\
Desvio Padrão & 17,1 & 20,0 & 16,4 & 17,0 & 16,7 \\
& 1,3 & 1,0 & 1,3 & 1,0 & 1,1 \\
\hline Média & \multicolumn{5}{c}{ Período úmido (DJFM de 2010/2011) } \\
Desvio Padrão & 23,2 & 23,2 & 21,0 & 21,7 & 21,8 \\
\hline
\end{tabular}

Observando-se os dois períodos estudados, as menores temperaturas da água foram encontradas no período seco (inverno no Hemisfério Sul), como esperado. As maiores médias da temperatura da água nos dois períodos estudados foram para os pontos $\mathrm{P}_{1}$ e $\mathrm{P}_{2}$ do monitoramento. Os dados em questão, nos diferentes pontos, apresentaram baixos valores de dispersão (desvio padrão variando de 1,0 até 1,6), o que indica pouca variabilidade dos valores amostrados nos períodos, seco (JJA de 2010) e úmido (DJFM de 2010/2011) estudados.

Os pontos $\mathrm{P}_{1}$ e $\mathrm{P}_{2}$ estão localizados em áreas com menor cobertura florestal próximo as margens quando comparados com os pontos $\mathrm{P}_{3}, \mathrm{P}_{4}$ e $\mathrm{P}_{5}$. A mata ciliar na microbacia confere maior proteção contra o aquecimento da água, devido à menor exposição dos corpos d'água à luz solar direta. Torna-se importante ressaltar que além do ponto $\mathrm{P}_{1}$ outras áreas a montante da região de monitoramento apresentam total falta de mata ciliar. $O$ ponto $P_{2}$ além de apresentar pouca mata ciliar trata-se de um lago, assim, o baixo fluxo das águas faz com que as temperaturas superficiais neste ponto sejam maiores. Desta maneira, a ausência ou pequena quantidade de mata ciliar pode ser responsável pelo maior aquecimento das águas na microbacia. Outros autores como Arcova e Cicco (1999); Bueno et al. (2005) também observaram que a falta de vegetação em uma microbacia é responsável por variações na temperatura da água, podendo interferir em outras variáveis, sugerindo assim a necessidade imprescindível do manejo adequado do solo em microbacias.

Com relação à vazão, foi observado que o ponto $\mathrm{P}_{1}$ apresenta as menores medidas de altura da lâmina d'água (m) quando comparado com os pontos $\mathrm{P}_{3}, \mathrm{P}_{4}$ e $\mathrm{P}_{5}$. Em se tratando de 
largura da seção de cada ponto, em $\mathrm{P}_{1}$ (variando de 2,60 $\mathrm{m}$ até 4,40 $\mathrm{m}$ ) e $\mathrm{P}_{3}$ (variando de 4,00 $\mathrm{m}$ até $5,00 \mathrm{~m}$ ) se têm os maiores valores quando comparado com $\mathrm{P}_{4}$ (variando de $1,45 \mathrm{~m}$ até $2,00 \mathrm{~m}$ ) e $\mathrm{P}_{5}$ (variando de 3,0 $\mathrm{m}$ até 3,5 m). Maier (1987) também destacou que o aquecimento direto da água é diretamente proporcional a área da superfície e, inversamente, a altura da lâmina d'água na microbacia. O aquecimento indireto acontece por meio da insolação no solo e relaciona-se com o tipo de substrato por onde a água escoa. Sendo assim, ambos são influenciados pela fisiografia da bacia de drenagem (geologia, geomorfologia, pedologia e hidrografia), material em suspensão na água e pela vegetação marginal.

Na Tabela 4 são apresentadas as médias e o desvio padrão do potencial hidrogeniônico (pH) da água para os períodos, secos (JJA de 2010 e 2011) e úmidos (DJFM de 2010/2011) nos diferentes pontos $\left(\mathrm{P}_{1}, \mathrm{P}_{2}, \mathrm{P}_{3}, \mathrm{P}_{4}\right.$ e $\left.\mathrm{P}_{5}\right)$. A maioria dos valores de $\mathrm{pH}$ observados encontram-se dentro dos limites estipulados pela CONAMA (Brasil, 2005), que são de 6 a 9. Somente no ponto $\mathrm{P}_{2}$ algumas poucas amostragens apresentaram valores de $\mathrm{pH}$ inferiores a 6,0, o que não é considerado ideal pela CONAMA (Brasil, 2005). Na Tabela 4 as maiores médias de $\mathrm{pH}$ da água nos três períodos estudados são para o ponto $\mathrm{P}_{5}$, porém as médias nos diferentes pontos, em cada um dos períodos são bem próximas.

Tabela 4. Médias e desvio padrão dos dados de potencial hidrogeniônico da água nos diferentes pontos e períodos de estudo.

\begin{tabular}{l|ccccc}
\hline \multirow{2}{*}{\multicolumn{1}{c|}{ Pontos }} & \multicolumn{5}{|c}{ Potencial Hidrogeniônico } \\
\cline { 2 - 6 } & $\mathrm{P}_{1}$ & $\mathrm{P}_{2}$ & $\mathrm{P}_{3}$ & $\mathrm{P}_{4}$ & $\mathrm{P}_{5}$ \\
\hline \multirow{3}{*}{ Média } & \multicolumn{5}{c}{ Período seco (JJA de 2010) } \\
Desvio Padrão & 6,6 & 6,6 & 6,8 & 6,6 & 6,8 \\
\hline \multirow{3}{*}{ Média } & 0,1 & 0,1 & 0,1 & 0,2 & 0,2 \\
Desvio Padrão & \multicolumn{5}{c}{ Período úmido (DJFM de 2010/2011) } \\
\hline \multirow{3}{*}{ Média } & 6,7 & 6,4 & 6,7 & 6,9 & 6,9 \\
Desvio Padrão & 0,2 & 0,4 & 0,2 & 0,6 & 0,5 \\
\cline { 2 - 6 } & 7,3 & 7,1 & 7,4 & 7,4 & 7,5 \\
\cline { 2 - 6 } & 0,2 & 0,4 & 0,3 & 0,3 & 0,3 \\
\hline
\end{tabular}

Nos períodos, seco (JJA de 2010) e úmido (DJFM de 2010/2011) as médias do pH estão bem próximas em todos os pontos (variando de 6,4 até 6,9). Já no período seco posterior (JJA de 2011) as médias nos diferentes pontos indicam maiores valores de $\mathrm{pH}$ quando comparados com os períodos anteriores (variando de 7,1 a 7,5). Os dados em questão, nos diferentes pontos, apresentaram baixos valores de dispersão (desvio padrão variando de 0,1 até 0,6 ), o que indica pouca variabilidade nos valores amostrados nos períodos, seco (JJA de 2010), úmido (DJFM de 2010/2011) e seco (JJA de 2011) estudados.

Com relação à condutividade elétrica da água $\left(\mu \mathrm{S} \mathrm{cm}^{-1}\right)$ nos períodos, seco (JJA de 2010) e úmido (DJFM de 2010/2011) as médias foram mais aproximadas nos diferentes pontos (variando de 34,0 até $50,1 \mu \mathrm{S} \mathrm{cm} \mathrm{cm}^{-1}$ ). Já no período seco posterior (JJA de 2011) as médias nos diferentes pontos indicam valores menores quando comparados com os períodos anteriores (variando de 26,0 até 27,1 $\mu \mathrm{S} \mathrm{cm}^{-1}$ ) (Tabela 5). No período úmido (DJFM de 2010/2011), os dados nos diferentes pontos apresentam os maiores valores de dispersão (desvio padrão variando de 9,3 até 12,5), o que indica maior variabilidade nos valores amostrados neste período quando comparado aos dois períodos secos estudados. 
Tabela 5. Médias e desvio padrão dos dados de condutividade elétrica da Água $\left(\mu \mathrm{S} \mathrm{cm}^{-1}\right)$ nos diferentes pontos e períodos de estudo.

\begin{tabular}{l|ccccc}
\hline \multirow{2}{*}{ Pontos } & \multicolumn{5}{|c}{ Condutividade $(\boldsymbol{\mu S}$ cm-1) } \\
\cline { 2 - 6 } & $\mathrm{P}_{1}$ & $\mathrm{P}_{2}$ & $\mathrm{P}_{3}$ & $\mathrm{P}_{4}$ & $\mathrm{P}_{5}$ \\
\hline \multirow{2}{*}{ Média } & \multicolumn{5}{c}{ Período seco (JJA de 2010) } \\
Desvio Padrão & 42,8 & 40,4 & 42,2 & 42,4 & 41,0 \\
& 4,9 & 2,2 & 6,9 & 5,7 & 5,1 \\
\hline \multirow{3}{*}{ Média } & \multicolumn{5}{c}{ Período úmido (DJFM de 2010/2011) } \\
Desvio Padrão & 39,4 & 34,6 & 47,2 & 34,0 & 50,1 \\
\hline \multirow{2}{*}{$\begin{array}{l}\text { Média } \\
\text { Desvio Padrão }\end{array}$} & 12,5 & 11,8 & 9,3 & 11,6 & 11,5 \\
& 26,3 & 26,0 & 26,8 & 27,1 & 27,0 \\
& 4,6 & 6,3 & 4,3 & 4,5 & 4,6 \\
\hline
\end{tabular}

Segundo a CONAMA (Brasil, 2005) para águas doces de classe 2, o oxigênio dissolvido (OD) não deve ser inferior a $5 \mathrm{mg} \mathrm{L} \mathrm{L}^{-1}$. Algumas amostragens na microbacia apresentaram valores abaixo desse valor estipulado (Tabela 6), principalmente no ponto $\mathrm{P}_{5}$, apresentando os menores valores médios. Os dados em questão, nos diferentes pontos no período úmido apresentaram os maiores valores de dispersão (desvio padrão variando de 0,6 até 1,0), o que indica maior variabilidade nos valores amostrados neste período quando comparados aos dois períodos secos estudados. Observa-se também que o oxigênio dissolvido ( $\left.\mathrm{mg} \mathrm{L}^{-1}\right)$ nos diferentes pontos apresenta valores médios aproximados nos períodos, seco (JJA de 2010) e úmido (DJFM de 2010/2011) (variando de 3,6 até 5,5 $\mathrm{mg} \mathrm{L}^{-1}$ ). Já no período seco posterior (JJA de 2011) as médias de OD nos diferentes pontos indicam maiores valores quando comparados com os períodos anteriores (variando de 5,0 até $6,2 \mathrm{mg} \mathrm{L}^{-1}$ ). As médias menores no período úmido (DJFM de 2010/2011) em relação ao período seco (JJA de 2011) estudados podem ser devido ao maior total de precipitação na área, que ocasiona erosão, lixiviamento dos solos e consequentemente maior fluxo de substâncias para dentro da microbacia. Desta maneira um acréscimo do efluente agropecuário na microbacia pode ter contribuído para diminuição do OD no período úmido (DJFM de 2010/2011) em relação ao período seco (JJA de 2011).

Alguns autores verificaram a variabilidade do OD associada a outros fatores. Nos trabalhos de Arcova e Cicco (1999); Silva et al. (2009), foi observado que as menores temperaturas da água na microbacia (período seco do ano) estão diretamente ligadas as maiores concentrações de Oxigênio Dissolvido, e que menores declividades de canais podem proporcionar menores concentrações de OD na água. Maier (1987) também ressaltou que em águas correntes, sob circunstâncias normais, o conteúdo de OD é alto e varia ao longo do rio, devido a alterações em suas características ambientais e em consequência das condições climáticas. Este autor enfatizou que como a solubilidade do OD é inversamente proporcional à temperatura, seu teor tende a diminuir pelo aquecimento das águas durante o dia e aumentar pelo resfriamento no período noturno. 
Tabela 6. Médias e desvio padrão dos dados de oxigênio dissolvido da água $\left(\mathrm{mg} \mathrm{L}^{-1}\right)$ nos diferentes pontos e períodos de estudo.

\begin{tabular}{|c|c|c|c|c|c|}
\hline \multirow{2}{*}{ Pontos } & \multicolumn{5}{|c|}{ Oxigênio Dissolvido $\left(\mathrm{mg} \mathrm{L}^{-1}\right)$} \\
\hline & $\mathrm{P}_{1}$ & $\mathrm{P}_{2}$ & $\mathrm{P}_{3}$ & $\mathrm{P}_{4}$ & $\mathrm{P}_{5}$ \\
\hline & \multicolumn{5}{|c|}{ Período seco (JJA de 2010) } \\
\hline Média & 5,2 & 5,1 & 4,8 & 4,9 & 4,9 \\
\hline \multirow[t]{2}{*}{ Desvio Padrão } & 0,4 & 0,9 & 0,4 & 0,3 & 0,3 \\
\hline & \multicolumn{5}{|c|}{ Período úmido (DJFM de 2010/2011) } \\
\hline Média & 5,0 & 5,5 & 5,5 & 4,8 & 3,6 \\
\hline \multirow[t]{2}{*}{ Desvio Padrão } & 1,2 & 1,2 & 0,9 & 0,5 & 0,9 \\
\hline & \multicolumn{5}{|c|}{ Período seco (JJA de 2011) } \\
\hline Média & 6,2 & 5,4 & 5,5 & 5,8 & 5,0 \\
\hline Desvio Padrão & 0,6 & 0,9 & 0,7 & 0,6 & 1,0 \\
\hline
\end{tabular}

Por se tratar de uma área em que ações de preservação e reconstituição do ambiente natural da microbacia têm sido empregadas, desde 2009, pelo Instituo Oikos de Agroecologia, torna-se importante destacar alguns índices observados no decorrer deste estudo. Quando se compara o primeiro período seco (JJA de 2010) com o seguinte período seco (JJA de 2011), em todos os pontos estudados, pode-se observar no ano de 2011 uma leve tendência de aumento médio no potencial hidrogeniônico-pH e oxigênio dissolvido-OD ( $\left.\mathrm{mg} \mathrm{L}^{-1}\right)$, e um decaimento médio da condutividade elétrica $\left(\mu \mathrm{S} \mathrm{cm}^{-1}\right)$.

$\mathrm{Na}$ Tabela 7 se encontram as médias e o desvio padrão das vazões reconstruídas por meio das curvas chaves $\left(\mathrm{P}_{1}: \mathrm{Q}=22,07 \mathrm{~h}^{2}-11,60 \mathrm{~h}+1,858, \mathrm{R}^{2}=0,979 ; \mathrm{P}_{3}: \mathrm{Q}=0,786 \mathrm{~h}^{2}-0,276 \mathrm{~h}+0,769\right.$; $\mathrm{R}^{2}=0,808 ; \mathrm{P}_{4}: \mathrm{Q}=0,395 \mathrm{~h}^{2}+0,379 \mathrm{~h}+0,262, \mathrm{R}^{2}=0,891$ e $\mathrm{P}_{5}: \mathrm{Q}=8,56 \mathrm{~h}^{2}-2,332 \mathrm{~h}+0,179, \mathrm{R}^{2}=0,922$ ), em cada um dos pontos, nos diferentes períodos estudados. Os valores apresentam baixa dispersão (desvio padrão variando de 0,1 até 0,4 ), o que indica pouca variabilidade dos valores de vazão reconstruídos nos períodos, seco (JJA de 2010 e 2011) e úmido (DJFM de 2010/2011).

Tabela 7. Médias e desvio padrão dos dados de vazão reconstruídos $\left(\mathrm{m}^{3} \mathrm{~s}^{-1}\right)$ nos diferentes pontos e períodos de estudo.

\begin{tabular}{|c|c|c|c|c|}
\hline \multirow{2}{*}{ Pontos } & \multicolumn{4}{|c|}{$\operatorname{Vazão}\left(\mathrm{m}^{3} \mathrm{~s}^{-1}\right)$} \\
\hline & $\mathrm{P}_{1}$ & $\mathrm{P}_{3}$ & $\mathrm{P}_{4}$ & $\mathrm{P}_{5}$ \\
\hline & \multicolumn{4}{|c|}{ Período seco (JJA de 2010) } \\
\hline Média & 0,4 & 0,8 & 0,4 & 0,2 \\
\hline \multirow[t]{2}{*}{ Desvio Padrão } & 0,1 & 0,0 & 0,0 & 0,1 \\
\hline & \multicolumn{4}{|c|}{ Período úmido (DJFM de 2010/2011) } \\
\hline Média & 1,1 & 1,4 & 0,9 & 1,8 \\
\hline \multirow[t]{2}{*}{ Desvio Padrão } & 0,2 & 0,1 & 0,1 & 0,4 \\
\hline & \multicolumn{4}{|c|}{ Período seco (JJA de 2011) } \\
\hline Média & 0,4 & 0,8 & 0,5 & 0,3 \\
\hline Desvio Padrão & 0,0 & 0,0 & 0,0 & 0,1 \\
\hline
\end{tabular}


Em todo o período estudado foi feita a correlação (Coeficiente de Pearson) da vazão com os parâmetros de qualidade da água que foram medidos. Pela análise de variância (valor do teste F), observa-se que houve significância (ao nível de 1 e 5\% de probabilidade) dos modelos de regressão linear entre vazão e os parâmetros de qualidade da água em alguns pontos $(* *)$ (Tabela 8$)$.

A temperatura da água é o parâmetro mais influenciado pela sazonalidade da vazão (apresentando uma forte correlação positiva, $0,8 \leq \mathrm{r}<1$ ). A condutividade elétrica da água também apresenta maior tendência à correlação positiva (predomínio nos diferentes pontos de fraca/moderada correlação positiva, $0,1 \leq \mathrm{r}<0,5 / 0,5 \leq \mathrm{r}<0,8)$. No caso do $\mathrm{pH}$ e OD estes parâmetros apresentam maior tendência à correlação negativa (predomínio nos diferentes pontos de fraca/moderada correlação negativa, $-0,1 \leq \mathrm{r}<-0,5 /-0,5 \leq \mathrm{r}<-0,8)$. Estes resultados indicam que com um maior total precipitado (período úmido), pode-se esperar, um aumento da temperatura da água, uma leve tendência de aumento da condutividade e também uma leve tendência de diminuição do pH e OD. Este fato pode estar ligado ao aumento do escoamento superficial na área e desta maneira aumento da concentração de matéria orgânica e particulada dissolvidas no corpo d'água.

Tabela 8. Correlação de Pearson entre vazão e os parâmetros de qualidade da água (temperatura, potencial hidrogeniônico ( $\mathrm{pH}$ ), condutividade (condut.) e oxigênio dissolvido(OD)).

\begin{tabular}{l|cccc}
\hline Coeficiente de Pearson & $\mathrm{P}_{1}$ & $\mathrm{P}_{3}$ & $\mathrm{P}_{4}$ & $\mathrm{P}_{5}$ \\
Vazão x temperatura & $0,9^{* *}$ & $0,8^{* *}$ & $0,9 * *$ & $0,9 * *$ \\
Vazão x pH & $-0,5 * *$ & $-0,5^{* *}$ & 0,1 & $-0,1$ \\
Vazão x Condut. & 0,2 & $0,6 * *$ & $-0,2$ & $0,6^{* *}$ \\
Vazão x OD & $-0,4 * *$ & 0,2 & $-0,3$ & $-0,6 * *$ \\
\hline
\end{tabular}

**Significativo ao nível de 1 e $5 \%$ de probabilidade.

Reis et al. (2010) enfatizaram que bacias hidrográficas localizadas em montanhas desempenham um papel importante no transporte de sedimentos, pois possuem uma menor capacidade de armazenamento e uma resposta rápida a picos de precipitação, transportando cargas sedimentares significativas e respectivos poluentes associados, em períodos de tempo reduzidos. O escoamento da água sobre a camada superficial do solo durante os eventos pluviais é a principal fonte difusa de poluição nos mananciais hídricos (Vasco et al., 2011). Os sedimentos são componentes fundamentais do ambiente fluvial, providenciando nutrientes para os organismos vivos e atuando como depósito para muitos contaminantes de origem antrópica.

\section{CONCLUSÃO}

Com este estudo foi possível observar algumas tendências nos parâmetros de qualidade da água do Ribeirão dos Macacos. Sendo que estas tendências podem ser devido às práticas conservacionistas adotadas. Assim, foi observado aumento médio no potencial hidrogeniônico-pH e oxigênio dissolvido-OD, e no caso da condutividade elétrica um decaimento médio. Os dados observados são preliminares, mas já podem ser um indicativo da resposta da bacia às ações de recuperação iniciadas na área de monitoramento.

A temperatura da água, quando comparada aos parâmetros $\mathrm{pH}$, condutividade elétrica e OD, foi o parâmetro mais influenciado pela sazonalidade da vazão. Todavia, pode-se observar 
que em período chuvoso com o aumento do escoamento superficial e da vazão ocorreram aumento da temperatura da água, uma leve tendência de aumento da condutividade e uma leve tendência de diminuição do pH e OD.

Diante do exposto, este primeiro estudo de análise da qualidade e quantidade da água é um ponto de referência para que as ações de recuperação ambiental continuem sendo implantadas a fim de melhorar e recuperar a área, e consequentemente os corpos d' água desta região.

\section{AGRADECIMENTOS}

Agradecemos ao CNPq pela bolsa de mestrado concedida; ao CPTEC/INPE, Instituto Oikos de Agrecologia e FEHIDRO pelo apoio financeiro e disponibilização de dados para obtenção deste trabalho.

\section{REFERÊNCIAS}

AMERICAN PUBLIC HEALTH ASSOCIATION - APHA. Standard methods for examination of water and wastewater. 19. ed. Washington, 1995.

ARCOVA, F. C. S.; CICCO, V. Qualidade da água de microbacias com diferentes usos do solo na região de Cunha, Estado de São Paulo. Revista Scientia Forestalis, Piracicaba, n. 56, p. 125-134, 1999.

BOLSTAD, P. V.; SWANK, W. T. Cumulative impacts of landuse on water quality in a southern Appalachian watershed. Journal of the American Water Resources Association, Middleburg, v. 33, n. 3, p. 519-533, 1997. http://dx.doi.org/ 10.1111/j.1752-1688.1997.tb03529.x

BRASIL. Ministério do Meio Ambiente. Resolução no 357, de 17 março 2005. Dispõe sobre a classificação dos corpos de água e diretrizes ambientais para o seu enquadramento, bem como estabelece as condições e padrões de lançamento de efluentes, e dá outras providências. Brasília, 2005. 23 p.

BUENO, L. F.; GALBIATTI, J. A.; BORGES, M. J. Monitoramento de variáveis de qualidade da água do Horto Ouro Verde - Conchal - SP. Engenharia Agrícola, Jaboticabal, v. 25, n. 3, p. 742-748, set./dez. 2005. http://dx.doi.org/10.1590/S010069162005000300020

FISCH, G. Caracterização climática e balanço hídrico de Taubaté (SP). Revista Biociências, Taubaté, v. 1, n. 1, p. 81-90, 1995.

GOLLA, A. R. Meio ambiente e agricultura na microbacia hidrográfica do córrego Palmitalzinho - Regente Feijó / São Paulo. 2006. 90 f. Dissertação (Mestrado em Geografia) - Faculdade de Ciência e Tecnologia, Universidade Estadual Paulista, Presidente Prudente, 2006.

INSTITUTO OIKOS DE AGROECOLOGIA. Figuras - Lorena. [ca. 2009]. Disponível em: <http://www.institutooikos.org.br/instituto.html>. Acesso em: 10 dez. 2012.

LIMA, K. C.; SATYAMURTY, P.; FERNÁNDEZ, J. P. R. Large-scale atmospheric conditions associated with heavy rainfall episodes in southeast Brazil. Theoretical and Applied Climatology, v. 101, n. 1-2, p. 121-135, 2010. http://dx.doi.org/ 10.1007/s00704-009-0207-9 
MACHADO, A.; SILVA, M.; VALENTIM, H. Contributo para a avaliação do estado das massas de água na região norte. Revista Recursos Hídricos, Lisboa, v. 31, n.1, p. $57-$ 63, 2010.

MAIER, M. H. Ecologia da bacia do rio Jacaré-Pepira $\left(47^{\circ} 55^{\prime}-48^{\circ} 55^{\prime} \mathrm{W} ; 22^{\circ} 30^{\prime}-21^{\circ} 55^{\prime} \mathrm{S}\right.$ Brasil): qualidade da água do rio principal. Ciência e cultura, Campinas, v. 39, n. 2, p. 164-185, 1987.

MANANDHAR, S.; VOGT, D. S.; PERRET, S. R.; KAZAMA, F. Adapting cropping systems to climate change in Nepal: a cross-regional study of farmers' perception and practices. Regional Environmental Change, v. 1, n. 2, p. 335-348, 2011. http://dx.doi.org/10.1007/s10113-010-0137-1

MARENGO, J. A.; ALVES, L. M. Tendências hidrológicas da bacia do rio Paraíba do Sul. Revista Brasileira de Meteorologia, São José dos Campos, v. 20, n. 2, p. 215-226, 2005.

MOSLEY, M. P.; MCKERCHAR, A. L. Streamflow. In: MAIDMENT, D. R. Handbook of hydrology. New York: McGraw-Hill, 1993. cap. 8.

NEARY, D. G.; ICE, G. G.; JACKSON, C. R. Linkages between forest soils and water quality and quantity. Forest Ecology and Management, Elsevier, v. 258, n. 10, p. 2269-2281, 2009. http://dx.doi.org/10.1016/j.foreco.2009.05.027

REIS, A.; PARKER, A.; ALENCOÃO, A. Avaliação da qualidade de sedimentos em rios de montanha: um caso de estudo no norte de Portugal. Revista Recursos Hídricos, Lisboa, v. 31, n.1, p. 87-97, 2010.

SANTOS, C. Estatística descritiva: manual de auto-aprendizagem. 1. ed. Lisboa: Silabo, 2007. 264p.

SANTOS, A. G.; TARGA, M. S.; BATISTA, G. T.; DIAS, N. W. Florestamento compensatório com vistas à retenção de água no solo em bacias hidrográficas do município de Campos do Jordão, SP, Brasil. Revista Ambiente \& Água, Taubaté, v. 6, n. 3, 2011. http://dx.doi.org/10.4136/ambi-agua.490

SILVA, A. P. S.; DIAS, H. C. T.; BASTOS, R. K. X.; SILVA, E. Qualidade da água do Reservatório da Usina Hidrelétrica (UHE) de Peti, Minas Gerais. Revista Árvore, Viçosa, v. 33, n. 6, p. 1063-1069, 2009. http://dx.doi.org/10.1590/S010067622009000600009

SILVA, A. E. P.; ANGELIS, C. F.; MACHADO, L. A. T.; WAICHAMAN, A. V. Influência da precipitação na qualidade da água do Rio Purus. Acta Amazonica, v. 38, n. 4, p. 733-742, out./dez. 2008. http://dx.doi.org/10.1590/S0044-59672008000400017

VASCO, A. N.; BRITTO, F. B.; PEREIRA, A. P. S.; MÉLLO JÚNIOR, A. V. M.; GARCIA, C. A. B.; NOGUEIRA, L. C. Avaliação espacial e temporal da qualidade da água na sub-bacia do rio Poxim, Sergipe, Brasil. Revista Ambiente \& Água, Taubaté, v. 6, n. 1, p. 118-130, 2011. http://dx.doi.org/10.4136/ambi-agua.178 


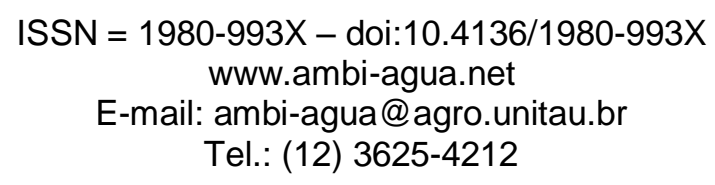

\title{
Adequação de um protocolo de avaliação rápida de rios para ser usado por estudantes do ensino fundamental
}

\author{
(http://dx.doi.org/10.4136/ambi-agua.996)
}

\author{
Ariane Guimarães ${ }^{1}$; Aline Sueli de Lima Rodrigues²; Guilherme Malafaia ${ }^{3}$ \\ ${ }^{1}$ Bolsista do Programa Institucional de Bolsas de Iniciação Científica do CNPq, \\ Curso de Ciências Biológicas /Licenciatura, Instituto Federal Goiano - Câmpus Urutaí, Urutaí, GO, \\ e-mail: arianeguimaraeslima@ hotmail.com, \\ ${ }^{2}$ Departamento de Gestão Ambiental, Instituto Federal Goiano - Câmpus Urutaí, Urutaí, GO, \\ Pesquisadora do Núcleo de Pesquisa em Ciências Ambientais e Biológicas, \\ e-mail: rodriguesasl@yahoo.com.br, \\ ${ }^{3}$ Departamento de Ciências Biológicas do Instituto Federal Goiano - Câmpus Urutaí, GO, \\ Pesquisador do Núcleo de Pesquisa em Ciências Ambientais e Biológicas, \\ e-mail: guilhermeifgoiano@gmail.com
}

\section{RESUMO}

O presente estudo teve como objetivo adequar um protocolo de avaliação rápida de rios (PAR) para utilização por crianças do ensino fundamental. O estudo foi desenvolvido em Ipameri, GO e o PAR foi adequado para avaliação de riachos do bioma Cerrado do Sudeste goiano. A partir de dois protocolos referência (um nacional e outro oriundo dos EUA), o PAR adequado abrangeu: parâmetros físicos que condicionam o funcionamento dos riachos, linguagem adaptada ao nível de escolaridade do ensino fundamental e a presença de ilustrações esquemáticas que auxiliam a aplicação do PAR em campo pelos estudantes. Para a consolidação do instrumento desenvolvido, foi oferecida uma oficina de monitoramento a 95 estudantes de duas instituições públicas de ensino e realizada uma análise e interpretação do padrão de respostas obtidas na etapa prática da oficina. Os testes Bartlett e Levene não revelaram diferenças estatísticas entre o padrão de resposta dos estudantes, possibilitando inferir que o PAR desenvolvido mostra-se compreensível por parte dos avaliadores. A aplicação do PAR foi rápida (entre 20 e 40 minutos) e os alunos relataram que o instrumento desenvolvido os aproxima das questões ambientais, que a oficina de monitoramento os ajudou a compreender o instrumento e que as ilustrações disponíveis facilitaram a avalição em campo. Em adição, os estudantes afirmaram que se sentiram sensibilizados para com as questões ligadas à preservação dos recursos hídricos e disseram que a participação na oficina de monitoramento ambiental permitiu a apropriação de conhecimentos sobre o funcionamento dos sistemas fluviais. Conclui-se que o PAR adequado mostra-se como uma ferramenta útil e interessante para ser utilizada em projetos e programas de Educação Ambiental.

Palavras-chave: avaliação ambiental; educação ambiental; aspectos físicos; estudantes.

\section{Adapting a rapid river assessment protocols to be used by elementary school children}

\section{ABSTRACT}

The present study aimed to adapt a rapid river assessment protocols (RAP) to be used by elementary school children. The study was conducted in Ipameri, GO and the RAP was 
adapted for the evaluation of streams in the Cerrado biome. Based on two protocol models, the developed RAP included: physical parameters that affect the functioning of streams, language adapted to the educational level of elementary school and the presence of drawings that could facilitate the field application of RAP by the students. For consolidation of the adapted instrument, it was offered a monitoring workshop to 95 students from two public education institutions, and developed an analysis and interpretation of the pattern of responses obtained during the practical step of the workshop. The Bartlett and Levene tests revealed no statistical differences between the response patterns of the students, allowing to infer that the developed RAP was understandable by the evaluators. The application of the RAP was fast (20 to 40 minutes) and the students reported that the developed instrument helped them to familiarize with environmental issues. In addition, the monitoring workshop helped them to understand the instrument and the available illustrations facilitated the field evaluation. In addition, the students concluded that they have become aware of the issues related to the water resource preservation and also that participation in the environmental monitoring workshop allowed the appropriation of knowledge about the river system functioning. It was concluded that adapted RAP has been proved to be a useful and interesting tool for using in environmental education projects and programs.

Keywords: environmental assessment, environmental education, physic aspects, students.

\section{INTRODUÇÃO}

Nos últimos anos a Educação Ambiental (EA) tem possibilitado cada vez mais a difusão da problemática ambiental e a promoção de práticas conservacionistas (Palhares et al., 2000). Porém, a expansão e as modificações dos processos produtivos, o crescimento populacional, a ocupação de variados nichos ecológicos, as migrações e urbanizações descontroladas, têm desestabilizado as condições de equilíbrio dos recursos naturais, dentre eles a água.

Sobre os recursos hídricos, conforme discutido por Krupek (2010), o ritmo de estudos desenvolvidos não segue a mesma velocidade de sua degradação. Levantamentos minuciosos acerca dos impactos das atividades humanas sobre o funcionamento dos ecossistemas aquáticos são necessários e importantes, porém difíceis ou até mesmo impossíveis de serem realizados quando os recursos financeiros são escassos, quando são feitos em áreas de difícil acesso e quando o tempo disponível é pequeno, o que não significa que não devam ser desenvolvidos.

Em função disso, a preocupação com o estado de degradação dos recursos hídricos tem revelado a necessidade de se estabelecer métodos de avaliação rápida de sua qualidade ambiental. Nesse contexto, uma ferramenta possível de ser utilizada como ferramenta complementar no monitoramento dos recursos hídricos, são os Protocolos de Avaliação Rápida de Rios (PARs), os quais têm a proposta de avaliar, de forma integrada, parâmetros que determinam a qualidade dos condicionantes físicos dos rios (Rodrigues e Castro, 2008a). O uso dos PARs de forma complementar a outras análises da qualidade do recurso fluvial (como análises físico-químicas e microbiológicas da água e do solo, por exemplo), com a devida cautela quando da interpretação dos resultados, permite a obtenção de informações que possibilitem o planejamento do uso e conservação dos recursos fluviais (Rodrigues e Castro, 2008a; Rodrigues et al., 2010).

Por definição, os PARs são ferramentas que reúnem procedimentos metodológicos aplicáveis à avaliação rápida, qualitativa e semi-quantitativa, de um conjunto de variáveis representativas dos principais componentes e fatores que condicionam e controlam os processos e funções ecológicas dos sistemas fluviais (Callisto et al., 2002; Rodrigues e Castro, 2008a). Nos PARs é estabelecido, em princípio, um limite considerado normal baseado em 
valores obtidos de locais minimamente perturbados, tidos como locais "referência" (Plafkin et al., 1989), partindo da premissa de que os cursos d'água pouco afetados pela ação humana exibem melhores condições biológicas (Minatti-Ferreira e Beaumord, 2006). O gradiente de estresse ambiental é definido a partir da observação destes locais e de locais com vários graus de alterações, desde os pouco alterados até os muito degradados.

No Brasil, vários estudos têm utilizado os PARs como instrumentos de avaliação da estrutura física e do funcionamento dos ecossistemas fluviais, como pode ser observado nos trabalhos de Minatti-Ferreira e Beaumord (2004, 2006), Xavier e Teixeira (2007), Dillenburg (2007), Rodrigues et al. (2008), Bergmann e Pedrozo (2008), Pimenta et al. (2009), PadovesiFonseca et al. (2010), Krupek (2010), Firmino et al. (2011), Lobo et al. (2011), Vargas e Ferreira Júnior (2012), Rodrigues et al. (2012), dentre outros.

Uma maneira de tornar esses instrumentos ainda mais úteis seria a adequação dos mesmos para fins de utilização em atividades educativas ligadas à preservação dos ambientes fluviais, conforme realizado por Bergmann e Pedrozo (2008), França et al. (2010), Callisto et al. (2010) e Carvalho (2010). Apesar da existência de estudos com essa vertente, pode-se dizer que poucos pesquisadores envolvidos com a problemática ambiental produzem e analisam materiais educativos para a população com baixo grau de instrução/escolarização, especialmente para crianças em escolarização básica (Bizerril e Faria, 2003).

$\mathrm{Na}$ literatura especializada, apenas um estudo envolvendo PARs e estudantes do ensino fundamental foram encontrados, o qual se refere ao trabalho de Guimarães et al. (2006), desenvolvido no âmbito do projeto ambiental Pampulha Limpa, criado em 2003, em Belo Horizonte, MG. Nesse estudo os autores trabalharam com a aplicação de um PAR por centenas de crianças com idade entre 11 e 13 anos de escolas localizadas na bacia hidrográfica da Pampulha. Na ocasião, foram realizadas saídas de campo para locais que apresentavam condições ambientais distintas e o PAR adequado foi aplicado pelas crianças. Desde então, o protocolo criado por Guimarães et al. (2006) vem sendo utilizado nas atividades de EA em outros projetos, como o "Manuelzão", cujo principal objetivo é a revitalização da bacia do Rio das Velhas, vinculado à Universidade Federal de Minas Gerais (UFMG) e em projetos educacionais da empresa de turismo pedagógico Estação Terra que presta assessoria em EA para escolas do ensino infantil, fundamental e médio na região metropolitana de Belo Horizonte.

Contudo, o PAR utilizado no estudo de Guimarães et al. (2006) foi adequado para ser aplicado em condições bem específicas da bacia hidrográfica da Pampulha, não podendo, dessa forma, ser utilizado em projetos de EA em outras regiões no Brasil. No Estado de Goiás, cujo bioma predominante é o Cerrado, nenhum trabalho semelhante foi desenvolvido. Assim, o presente estudo teve como objetivo adequar um PAR para uso por crianças matriculadas no ensino fundamental. Acredita-se que o PAR adequado possa ser útil na promoção da observação, sensibilização e apropriação de conceitos referentes ao funcionamento, por parte dos discentes, e na preservação dos recursos fluviais, contribuindo significativamente com o desenvolvimento da EA no nível básico da educação.

\section{MATERIAL E MÉTODOS}

\section{1. Área de estudo}

O presente estudo foi desenvolvido na cidade de Ipameri-GO cujo município está localizado entre os rios Corumbá e São Marcos, tendo como coordenadas geográficas $17^{\circ} 43^{\prime} 20^{\prime \prime}$ de latitude Sul e 48 $09^{\prime} 44^{\prime \prime}$ de longitude Oeste. Além do distrito sede, constituído hoje por 18 bairros e 17 vilas, o município conta também com mais dois distritos, Cavalheiro e Domiciano Ribeiro, e 5 povoados na zona rural: Tomazinópolis, Vila Pacheco, Vendinha, São Sebastião da União e Lago Azul (Ipameri, 2010). Demograficamente, Ipameri possui, 
GUIMARÃES, A.; RODRIGUES, A. S. L.; MALAFAIA, G. Adequação de um protocolo de avaliação rápida de rios para ser usado por estudantes do ensino fundamental. Ambi-Agua, Taubaté, v. 7, n. 3, p. 241-260, 2012. (http://dx.doi.org/10.4136/ambi-agua.996)

segundo dados do último censo realizado pelo Instituto Brasileiro de Geografia e Estatística (IBGE), aproximadamente 24.800 habitantes (IBGE, 2010).

O município está localizado sobre uma formação rochosa antiga e apresenta $45 \%$ de seu território com topografia plana, $40 \%$ ondulada e os $15 \%$ restantes montanhosa. A altitude média na quase totalidade do território municipal está na faixa de $800 \mathrm{~m}$. As principais elevações existentes são a Serra Patrona, a Serra da Arnica, a Serra da Mangaba, o Morro de São Domingos, o Morro do Brito e o Contraforte Central (Ipameri, 2010).

O clima de Ipameri pode ser considerado tipicamente tropical-úmido, com temperatura média de $25^{\circ} \mathrm{C}$. A precipitação pluviométrica anual é de $1750 \mathrm{~mm}$, com ocorrência de $80 \%$ das chuvas nos meses de dezembro, janeiro e março, e o restante se distribuindo principalmente nos meses de outubro, novembro e fevereiro (Ipameri, 2010).

\subsection{Seleção dos pontos, aplicação dos protocolos referências e adequação do novo protocolo}

Foram selecionados como referências que norteariam a adequação do PAR no presente estudo, os protocolos elaborados por Barbour et al. (1999) e Rodrigues e Castro (2008b) (versões aplicáveis a cursos d'água de baixo curso) ${ }^{1}$. Inicialmente, tais protocolos foram aplicados em 27 trechos de rios da cidade de Ipameri, GO por três avaliadores previamente treinados (Tabela 1). Os critérios de seleção destes pontos foram relacionados à distribuição espacial homogênea na rede hidrográfica da cidade e a facilidade de acesso aos mesmos.

Tabela 1. Pontos e coordenadas geográficas dos trechos de rios visitados na cidade de Ipameri, GO.

\begin{tabular}{|c|c|c|c|}
\hline Identificação & Coordenadas & Identificação & Coordenadas \\
\hline 1 & $48^{\circ} 10^{\prime} 19^{\prime \prime}$ O $17^{\circ} 42^{\prime} 19^{\prime \prime} \mathrm{S}$ & 15 & $48^{\circ} 09^{\prime} 43^{\prime \prime}$ O 17'43'03', S \\
\hline 2 & $48^{\circ} 10^{\prime} 18^{\prime \prime} \mathrm{O} 17^{\circ} 42^{\prime} 20^{\prime \prime} \mathrm{S}$ & 16 & $48^{\circ} 09^{\prime} 53^{\prime \prime} \mathrm{O} 17^{\circ} 43^{\prime} 05^{\prime \prime} \mathrm{S}$ \\
\hline 3 & $48^{\circ} 10^{\prime} 05^{\prime \prime} \mathrm{O} 17^{\circ} 42^{\prime} 58^{\prime \prime} \mathrm{S}$ & 17 & $48^{\circ} 09^{\prime} 53^{\prime \prime} \mathrm{O} 17^{\circ} 43^{\prime} 05^{\prime}, \mathrm{S}$ \\
\hline 4 & $48^{\circ} 09^{\prime} 05^{\prime \prime} \mathrm{O} 17^{\circ} 42^{\prime} 23^{\prime \prime} \mathrm{S}$ & 18 & $48^{\circ} 10^{\prime} 011^{\prime}$ O 17 $43^{\circ} 05^{\prime}, \mathrm{S}$ \\
\hline 5 & $48^{\circ} 09^{\prime} 05^{\prime \prime} \mathrm{O} 17^{\circ} 42^{\prime} 25^{\prime}, \mathrm{S}$ & 19 & $48^{\circ} 10^{\prime} 02^{\prime \prime} \mathrm{O} 17^{\circ} 43^{\prime} 05^{\prime \prime} \mathrm{S}$ \\
\hline 6 & $48^{\circ} 09^{\prime} 06^{\prime \prime} \mathrm{O} 17^{\circ} 42^{\prime} 40^{\prime \prime} \mathrm{S}$ & 20 & $48^{\circ} 09^{\prime} 55^{\prime \prime}$ O 17\%43'14', S \\
\hline 7 & 4809'09', O 17'42'40', S & 21 & $48^{\circ} 09^{\prime} 55^{\prime \prime} \mathrm{O} 17^{\circ} 43^{\prime} 15^{\prime}, \mathrm{S}$ \\
\hline 8 & $48^{\circ} 09^{\prime} 12^{\prime \prime} \mathrm{O} 17^{\circ} 42^{\prime} 42^{\prime \prime} \mathrm{S}$ & 22 & $48^{\circ} 09^{\prime} 57^{\prime \prime} \mathrm{O} 17^{\circ} 43^{\prime} 22^{\prime \prime} \mathrm{S}$ \\
\hline 9 & 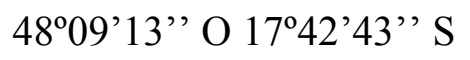 & 23 & $48^{\circ} 09^{\prime} 57^{\prime \prime} \mathrm{O} 17^{\circ} 43^{\prime} 23^{\prime \prime} \mathrm{S}$ \\
\hline 10 & $48^{\circ} 09^{\prime} 27^{\prime \prime} \mathrm{O} 17^{\circ} 42^{\prime} 57^{\prime \prime} \mathrm{S}$ & 24 & $48^{\circ} 10^{\prime} 01^{\prime \prime} \mathrm{O} 17^{\circ} 43^{\prime} 33^{\prime \prime} \mathrm{S}$ \\
\hline 11 & $48^{\circ} 09^{\prime} 28^{\prime \prime} \mathrm{O} 17^{\circ} 42^{\prime} 57^{\prime \prime} \mathrm{S}$ & 25 & $48^{\circ} 10^{\prime} 011^{\prime \prime O ~} 17^{\circ} 43^{\prime} 33^{\prime \prime} \mathrm{S}$ \\
\hline 12 & $48^{\circ} 09^{\prime} 40^{\prime \prime} \mathrm{O} 17^{\circ} 43^{\prime} 01^{\prime}, \mathrm{S}$ & 26 & $48^{\circ} 10^{\prime} 05^{\prime \prime} \mathrm{O} 17^{\circ} 43^{\prime} 47^{\prime \prime} \mathrm{S}$ \\
\hline 13 & $48^{\circ} 09^{\prime} 41^{\prime \prime} \mathrm{O} 17^{\circ} 43^{\prime} 01^{\prime}, \mathrm{S}$ & 27 & $48^{\circ} 10^{\prime} 05^{\prime \prime}$ O $17^{\circ} 43^{\prime} 48^{\prime \prime} \mathrm{S}$ \\
\hline 14 & $48^{\circ} 09^{\prime} 43^{\prime \prime} \mathrm{O} 17^{\circ} 43^{\prime} 03^{\prime \prime} \mathrm{S}$ & & \\
\hline
\end{tabular}

\footnotetext{
${ }^{1}$ O PAR de Barbour et al. (1999) é qualificado por uma compilação de vários métodos de avaliação rápida de rios existentes utilizados em várias regiões temperadas dos Estados Unidos por agências ambientais estaduais deste país. Já o PAR de Rodrigues e Castro (2008b) refere-se à proposição de um PAR desenvolvido para a avaliação de cursos d'água inseridos em campos rupestres (Cerrado) do Estado de Minas Gerais. A escolha desses dois trabalhos se deu em função de características específicas de cada um. Enquanto o primeiro trata-se do PAR que pioneiramente foi introduzido no Brasil e em outros países como proposta de avaliação integrada e complementar da qualidade dos recursos fluviais, o segundo trata-se de um protocolo que foi adaptado para uso em uma variação do bioma Cerrado, fato este que o aproxima das características fitofisionômicas de Ipameri, GO (área de estudo). O protocolo proposto por Callisto et al. (2002), muito utilizado por diversos estudos que dispuseram avaliar as condições ambientais de cursos d'água no Brasil, não foi utilizado no presente trabalho como referência uma vez que ele foi adaptado para o contexto da Mata Atlântica (Serrada Bocaina), bioma diferente do que predomina em Ipameri, GO.
} 
Em seguida, partiu-se para a adequação do novo protocolo. Tal adequação foi realizada levando-se em consideração a definição de quais parâmetros presentes nos protocolos referências seriam mantidos ou excluídos e quais poderiam ser incluídos; a definição das notas possíveis de serem atribuídas aos trechos; a categorização do gradiente de estresse ambiental condizente com as notas e essas com as possíveis condições ambientais dos trechos ("ruim", "boa" ou "ótima"); a adequação da linguagem da descrição dos parâmetros abordados no PAR, compatível ao nível de escolaridade do ensino fundamental; a elaboração de figuras que poderiam auxiliar o avaliador no momento da aplicação do PAR e, por fim, a consolidação do protocolo adequado, por meio de uma oficina de monitoramento ambiental.

\subsection{Oficina de monitoramento ambiental e consolidação do novo protocolo}

Uma oficina de monitoramento ambiental foi oferecida a 95 alunos do ensino fundamental $\left(6^{\circ}, 7^{\circ}, 8^{\circ}\right.$ e $9^{\circ}$ anos), em duas instituições de ensino de Ipameri, GO (Colégio Estadual Professor Eduardo Mancini e Escola Municipal Godofredo Perfeito). A oficina, além de oferecer aos participantes a oportunidade de ampliar seus conhecimentos específicos sobre monitoramento ambiental e funcionamento dos sistemas fluviais, também serviu para avaliar a aplicabilidade do PAR adequado, de modo a identificar possíveis falhas, adequá-las e, por fim, consolidar o protocolo desenvolvido. Tal oficina foi realizada em duas etapas.

Na primeira, adotou-se uma abordagem teórica tendo sido apresentado aos estudantes os objetivos e justificativas do presente trabalho, a relevância do monitoramento dos rios, definições de indicadores ambientais, índices de integridade ambiental, o histórico dos protocolos, bem como suas vantagens e características, além dos parâmetros a serem analisados em campo na etapa prática da oficina. $\mathrm{O}$ material de apoio didático da oficina contou com um roteiro, em forma de apostila que continha informações sobre o uso dos protocolos, instruções sobre a aplicação do PAR adequado e um guia visual composto por fotografias e exemplos de diferentes situações ambientais. Para facilitar a utilização do protocolo pelos voluntários, para cada parâmetro considerado no protocolo adequado, havia uma breve descrição do mesmo.

$\mathrm{Na}$ segunda etapa, os estudantes que participaram do treinamento teórico foram a campo aplicar - em um mesmo trecho (ponto 7 - coordenadas 48 $09^{\prime} 09^{\prime \prime} \mathrm{O} 17^{\circ} 42^{\prime} 40^{\prime}$ ' S) - o protocolo adequado. Ressalta-se que o intuito dessa etapa não foi realizar um diagnóstico das condições ambientais dos cursos d'água da cidade de Ipameri, GO a partir da avaliação dos discentes participantes da oficina de monitoramento ambiental. Neste caso, objetivou especificamente avaliar a consistência do PAR desenvolvido, o entendimento dos parâmetros e da descrição de cada um deles pelos discentes, bem como a opinião geral dos discentes quanto ao instrumento adequado.

Cada discente realizou a aplicação do protocolo individualmente. Para a consolidação do protocolo desenvolvido foi realizada uma análise e interpretação do padrão de respostas obtidas nesta etapa prática, partindo-se do pressuposto de que variações significativas entre as respostas dos estudantes poderiam indicar falhas no protocolo. Para a verificação da convergência das respostas entre os avaliadores, considerou-se a observação de um padrão de respostas similares. Para isso, os dados da aplicação do PAR pelos discentes foram submetidos à análise de igualdade de variâncias, utilizando-se os testes de Bartlett's (Snedecor e Cochran, 1989) e Levene (Brown e Forsythe, 1974), de forma semelhante à metodologia adotada por Rodrigues e Castro (2008b). Foram consideradas variações estatisticamente significativas entre as médias das respostas dos avaliadores, valores de $p$ menores que 0,05 .

Além disso, comparou-se o resultado da avaliação do trecho de rio realizada pelos discentes com os resultados obtidos do diagnóstico ambiental desse mesmo trecho realizado por Firmino et al. (2011). Partiu-se da ideia de que a consolidação do PAR necessitava se dar 
por meio de evidências que não apenas mostrassem a não variação do padrão de respostas da avaliação realizada pelos discentes, mas também que mostrassem a coerência da avaliação dos alunos do ponto de vista técnico.

Ao final desta atividade os voluntários responderam um questionário (Quadro 1) que forneceu informações que serviram para consolidação do instrumento adequado, além de permitir a identificação de opiniões dos participantes sobre a oficina de monitoramento ambiental oferecida.

Quadro 1. Questionário aplicado junto aos alunos participantes da oficina de monitoramento ambiental.

\begin{tabular}{|c|c|}
\hline 1) Nome da Escola: & 2) Série: \\
\hline 3) Sexo: ( ) masculino ( ) feminino & 4) Idade: \\
\hline $\begin{array}{l}\text { 5) Quanto tempo você gastou para aplicar } \\
\text { o protocolo? } \\
\text { ( ) Menos de } 20 \text { minutos } \\
\text { ( ) Entre } 20 \text { e } 40 \text { minutos } \\
\text { ( ) Entre } 40 \text { minutos e } 1 \text { hora } \\
\text { ( ) Mais de } 1 \text { hora }\end{array}$ & $\begin{array}{l}\text { 6) Você acredita que a utilização do protocolo é um meio } \\
\text { que o aproxima das questões ambientais? } \\
\text { ( ) Sim ( ) Não } \\
\text { Comentário: }\end{array}$ \\
\hline $\begin{array}{l}\text { 7) As instruções da oficina de } \\
\text { monitoramento ambiental ajudaram na } \\
\text { melhor compreensão do instrumento? } \\
\text { ( ) Sim ( ) Não } \\
\text { Comentário: }\end{array}$ & $\begin{array}{l}\text { 8) As ilustrações disponíveis em cada parâmetro o } \\
\text { ajudaram durante a avaliação em campo e durante a } \\
\text { atribuição de notas aos parâmetros? } \\
\text { ( ) Sim ( ) Não } \\
\text { Comentário: }\end{array}$ \\
\hline $\begin{array}{l}\text { 9) Durante a avaliação do rio com o } \\
\text { protocolo, você se sentiu um agente } \\
\text { colaborador na defesa de um rio? } \\
\text { ( ) Sim ( ) Não } \\
\text { Comentário: }\end{array}$ & $\begin{array}{l}\text { 10) A participação na oficina de monitoramento } \\
\text { ambiental permitiu a apropriação de conhecimentos } \\
\text { sobre o funcionamento dos sistemas fluviais? } \\
\text { ( ) Sim ( ) Não } \\
\text { Comentário: }\end{array}$ \\
\hline $\begin{array}{l}\text { 11) Você apresentou alguma dificuldade } \\
\text { de entendimento dos parâmetros } \\
\text { propostos no PAR? } \\
\text { ( ) Sim ( ) Não } \\
\text { Se sim, qual(is) foi(ram) esse(s) } \\
\text { parâmetro(s)? }\end{array}$ & $\begin{array}{l}\text { 12) Você acredita que qualquer pessoa previamente } \\
\text { treinada pode realizar a avaliação de um rio utilizando-se } \\
\text { este PAR? } \\
\text { ( ) Sim ( ) Não } \\
\text { Comentário: }\end{array}$ \\
\hline
\end{tabular}

\subsection{Questões éticas}

Considerando que parte desse trabalho envolveu a participação de seres humanos, o projeto que deu origem a esse estudo foi submetido para análise e parecer do Comitê de Ética em Pesquisa (CEP) do IF Goiano, sob o protocolo $n^{\circ}$ 001, tendo sido aprovado em 27 de dezembro de 2010.

\section{RESULTADOS E DISCUSSÃO}

A partir das visitas a campo e da aplicação dos PARs referências em trechos de rios da cidade de Ipameri, GO, evidenciou-se, de um modo geral, uma grande variação das pontuações atribuídas a cada parâmetro pelos três avaliadores (Figura 1). A título de demonstração dos resultados referentes a essas aplicações, foram escolhidos, aleatoriamente, os dados obtidos na avaliação de dois trechos de rios, considerando a existência de uma similaridade entre os resultados obtidos para todos os trechos visitados e a impossibilidade da utilização desses dados como condizentes com as condições ambientais locais, já que os 
protocolos aplicados foram criados para uso em um contexto fitofisionômico diferente do que se observa em Ipameri, GO.

A variação observada pode ser explicada, neste caso, pela incoerência entre a descrição das categorias estabelecidas nos PARs aplicados e as características verificadas nos trechos, o que dificultou a associação da situação real verificada a uma categoria de avaliação específica ("ótima", "sub-ótima", "razoável” e "pobre"), além do fato de que os PARs aplicados foram propostos para avaliação de rios inseridos em fitofisionomias de biomas diferentes do considerado no presente estudo (Cerrado do Sudeste goiano). O intuito dessa etapa foi verificar se os protocolos referências poderiam ter diretamente suas linguagens adequadas ao nível do ensino fundamental, ou se antes, deveriam ocorrer também adequações técnicas, no que tange aos parâmetros propostos, suas descrições, o gradiente de estresse ambiental proposto em cada documento e as categorias de condições ambientais nas quais os trechos são enquadrados.

Os Quadros 2 e 3 sumarizam os principais resultados qualitativos obtidos a partir das visitas a campo, referentes à aplicação dos PARs.
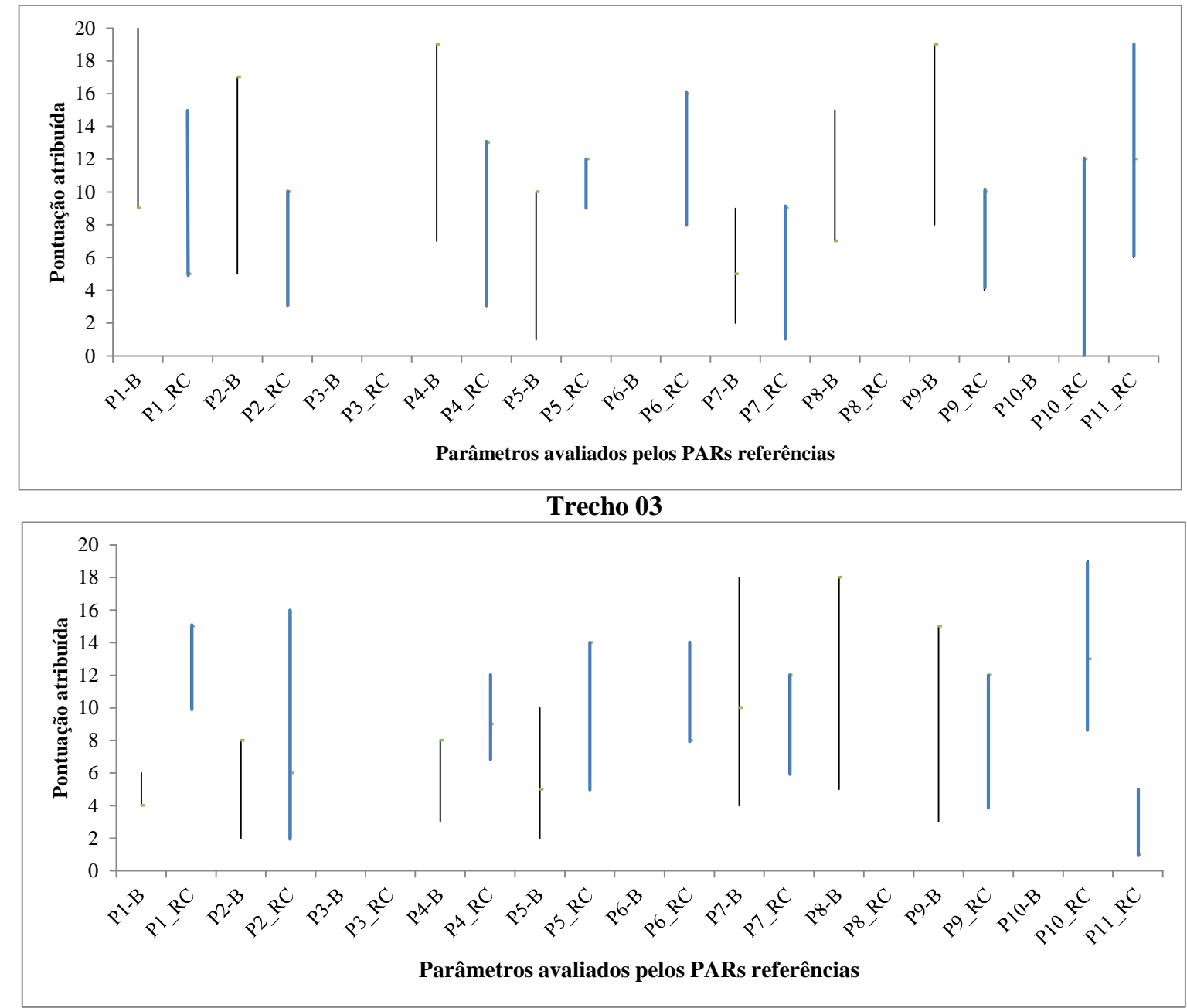

\section{Trecho 09}

Figura 1. Variação das pontuações atribuídas pelos três avaliadores aos parâmetros analisados pelos protocolos referência em dois trechos de rios localizados na cidade de Ipameri, GO. ${ }^{1,2,3,4}$

Nota: ${ }^{1}$ As barras verticais indicam a variação (máximo e mínimo) das pontuações atribuídas pelos três avaliadores. Optou-se por não apresentar a média +/- desvio padrão devido ao número pequeno de avaliações $(\mathrm{n}=3)$ realizadas no mesmo trecho de rio.

${ }^{2}$ As barras verticais pretas indicam os resultados obtidos por meio do PAR de Barbour et al. (1999) e as barras azuis os obtidos por meio do PAR de Rodrigues e Castro (2008b).

${ }^{3}$ Os parâmetros (P1, P2 (...) P11) discriminados na abscissa não são coincidentes nos PARs aplicados nos trechos de rios sob avaliação.

${ }^{4}$ A inicial "B" refere-se aos parâmetros propostos no PAR de Barbour et al. (1999) e as iniciais "RC" referem-se aos parâmetros propostos no PAR de Rodrigues e Castro (2008b). 
GUIMARÃES, A.; RODRIGUES, A. S. L.; MALAFAIA, G. Adequação de um protocolo de avaliação rápida de rios para ser usado por estudantes do ensino fundamental. Ambi-Agua, Taubaté, v. 7, n. 3, p. 241-260, 2012. (http://dx.doi.org/10.4136/ambi-agua.996)

Quadro 2 - Resultados referentes à aplicação do PAR de Barbour et al. (1999) em trechos de rios localizados na cidade de Ipameri, GO.

\begin{tabular}{|c|c|}
\hline $\begin{array}{l}\text { Parâmetros e/ou } \\
\text { características do } \\
\text { instrumento }\end{array}$ & Principais resultados/observações ${ }^{*}$ \\
\hline $\begin{array}{l}\text { O referido protocolo } \\
\text { apresenta duas versões } \\
\text { para avaliação: uma deve } \\
\text { ser aplicada em rios de } \\
\text { baixo curso e a outra em } \\
\text { rios de alto curso }\end{array}$ & $\begin{array}{l}\text { Para a área de estudo optou-se por adequar um protocolo a ser aplicado apenas } \\
\text { em rios com características de baixo curso. Os cursos d'água observados na } \\
\text { região de Ipameri, GO fazem parte de uma paisagem de baixa declividade. Via } \\
\text { de regra, possuem um perfil longitudinal de baixo gradiente, isto é, com pequeno } \\
\text { desnível entre as nascentes e a barragem. Possuem poucas corredeiras e } \\
\text { cachoeiras de modo que a velocidade do escoamento é basicamente em função de } \\
\text { seu volume, e, portanto, da dimensão da bacia de captação e do regime } \\
\text { pluviométrico regional. }\end{array}$ \\
\hline $\begin{array}{l}\text { Parâmetro 1: "Epifaunal } \\
\text { substratel Available } \\
\text { Cover" }\end{array}$ & $\begin{array}{l}\text { Apresenta descrição técnica que se baseia na distinção de percentuais subjetivos } \\
\text { que deveriam ser condizentes com a avaliação ambiental verificada in situ. Além } \\
\text { disso, conceitos como, por exemplo, os de "habitats estáveis", considerados } \\
\text { complexos ao nível de escolaridade do nível fundamental, foram evidenciados na } \\
\text { descrição das categorias de condição ambiental para esse parâmetro. }\end{array}$ \\
\hline $\begin{array}{l}\text { Parâmetro 2: "Pool } \\
\text { substrate } \\
\text { Characterization" }\end{array}$ & $\begin{array}{l}\text { O referido parâmetro apresenta conteúdo técnico considerado complexo ao nível } \\
\text { de escolaridade do nível fundamental. Além disso, a avaliação deste parâmetro } \\
\text { em determinadas épocas do ano (em virtude da cheia dos rios e do aumento da } \\
\text { turbidez das águas, por exemplo) pode ser comprometida. }\end{array}$ \\
\hline $\begin{array}{l}\text { Parâmetro 3: "Pool } \\
\text { Variability" }\end{array}$ & $\begin{array}{l}\text { Este parâmetro apresenta descrição subjetiva. A definição, por exemplo, do que } \\
\text { seriam poços largos e/ou grandes, pequenos e/ou rasos, não é muito clara, o que } \\
\text { dificulta a avaliação do trecho considerando esse parâmetro. Esse parâmetro não } \\
\text { foi aplicável utilizando-se o PAR de Barbour et al. (1999). }\end{array}$ \\
\hline $\begin{array}{l}\text { Parâmetro 4: "Sediment } \\
\text { Deposition" }\end{array}$ & $\begin{array}{l}\text { Refere-se a um parâmetro que foi incorporado ao PAR proposto no presente } \\
\text { estudo, de modo em que foi diminuída a subjetividade da descrição das } \\
\text { categorias relativas a este parâmetro e o número de categorias de condições } \\
\text { ambientais que podem ser atribuídas ao trecho referente especificamente a este } \\
\text { parâmetro. }\end{array}$ \\
\hline $\begin{array}{l}\text { Parâmetro 5: "Channel } \\
\text { Alteration" }\end{array}$ & Idem aos resultados/comentários referentes ao Parâmetro "Sediment Deposition" \\
\hline $\begin{array}{l}\text { Parâmetro 6: "Channel } \\
\text { Flow Status" }\end{array}$ & $\begin{array}{l}\text { O referido parâmetro apresenta descrição subjetiva, cuja medição pode ser } \\
\text { dificultada ou sujeita a erros utilizando-se apenas a visibilidade do avaliador. } \\
\text { Esse parâmetro não foi aplicável à área de estudo utilizando-se o PAR de } \\
\text { Barbour et al. (1999). }\end{array}$ \\
\hline $\begin{array}{l}\text { Parâmetro 7: "Channel } \\
\text { Sinuosity" }\end{array}$ & $\begin{array}{l}\text { Este parâmetro não foi considerado no PAR proposto no presente estudo em } \\
\text { função da dificuldade de avaliar o grau de sinuosidade dos rios a partir de uma } \\
\text { avaliação realizada nas margens do curso d'água (como é o caso da aplicação dos } \\
\text { PARs). Entende-se que uma avaliação mais condizente deva ser feito a partir de } \\
\text { imagens de satélite onde uma visão mais ampla é possibilitada. }\end{array}$ \\
\hline $\begin{array}{l}\text { Parâmetro 8: "Bank } \\
\text { Stability (score each } \\
\text { bank)" }\end{array}$ & Idem aos resultados/comentários referentes ao Parâmetro "Sediment Deposition" \\
\hline $\begin{array}{l}\text { Parâmetro 9: "Vegetative } \\
\text { Protection (score each } \\
\text { bank)" }\end{array}$ & Idem aos resultados/comentários referentes ao Parâmetro "Sediment Deposition" \\
\hline $\begin{array}{l}\text { Parâmetro 10: "Riparian } \\
\text { Vegetative Zone Width } \\
\text { (score each bank } \\
\text { riparian zone)" }\end{array}$ & Idem aos resultados/comentários referentes ao Parâmetro "Sediment Deposition" \\
\hline
\end{tabular}

Nota: * Os resultados/comentários apresentados neste quadro foram elaborados a partir de anotações diversas realizadas pela equipe executora do estudo durante a visitação dos 27 trechos de rios localizados na cidade de Ipameri, GO. 
GUIMARÃES, A.; RODRIGUES, A. S. L.; MALAFAIA, G. Adequação de um protocolo de avaliação rápida de rios para ser usado por estudantes do ensino fundamental. Ambi-Agua, Taubaté, v. 7, n. 3, p. 241-260, 2012. (http://dx.doi.org/10.4136/ambi-agua.996)

Quadro 3 - Resultados referentes à aplicação do PAR de Rodrigues e Castro (2008a) em trechos de rios localizados na cidade de Ipameri, GO

\begin{tabular}{|c|c|}
\hline $\begin{array}{l}\text { Parâmetros e/ou características } \\
\text { do instrumento }\end{array}$ & Principais resultados/observações* \\
\hline $\begin{array}{l}\text { O referido protocolo, assim como o } \\
\text { PAR de Barbour et al. (1999), } \\
\text { apresenta duas versões: uma deve ser } \\
\text { aplicada em rios de baixo curso e outra } \\
\text { em rios de alto curso }\end{array}$ & $\begin{array}{l}\text { Idem aos resultados/comentários apresentados, no Quadro 1, para } \\
\text { essa característica. }\end{array}$ \\
\hline $\begin{array}{l}\text { Parâmetro 1: "Substratos e/ou habitats } \\
\text { disponíveis" }\end{array}$ & $\begin{array}{l}\text { Refere-se a um parâmetro que foi incorporado ao PAR proposto no } \\
\text { presente estudo, de modo em que foi diminuída a subjetividade da } \\
\text { descrição das categorias relativas a este parâmetro (baseada na } \\
\text { distinção de percentuais referentes à quantidade de substratos e/ou } \\
\text { habitats disponíveis no trecho sob avaliação) e o número de } \\
\text { categorias de condições ambientais que podem ser atribuídas ao } \\
\text { trecho referente especificamente a este parâmetro. }\end{array}$ \\
\hline Parâmetro 2: "Substratos em poços" & $\begin{array}{l}\text { Esse parâmetro não foi considerado no PAR adequado no presente } \\
\text { estudo, pois envolve conceitos subjetivos como aqueles relativos ao } \\
\text { que seriam, para a área de estudo, "poços de vários tipos e tamanhos } \\
\text { de substratos". }\end{array}$ \\
\hline $\begin{array}{l}\text { Parâmetro 3: "Regimes de } \\
\text { velocidade/profundidade" }\end{array}$ & $\begin{array}{l}\text { O referido parâmetro apresenta descrição subjetiva, cuja medição } \\
\text { pode ser dificultada ou sujeita a erros utilizando-se apenas a } \\
\text { visibilidade do avaliador. Esse parâmetro não foi aplicável à área de } \\
\text { estudo utilizando-se o PAR de Rodrigues e Castro (2008b). }\end{array}$ \\
\hline Parâmetro 4: "Diversidade de poços" & $\begin{array}{l}\text { Esse parâmetro não foi considerado no PAR adequado no presente } \\
\text { estudo, pois apresenta conceitos subjetivos como aqueles relativos ao } \\
\text { que seriam, para a área de estudo, "diferentes tipos e tamanho de } \\
\text { poços". }\end{array}$ \\
\hline $\begin{array}{l}\text { Parâmetro 5: "Deposição de } \\
\text { sedimentos" }\end{array}$ & $\begin{array}{l}\text { Idem aos resultados/comentários referentes ao Parâmetro "Substratos } \\
\text { e/ou habitat disponíveis" }\end{array}$ \\
\hline $\begin{array}{l}\text { Parâmetro 6: "Condições de } \\
\text { escoamento do canal" }\end{array}$ & $\begin{array}{l}\text { O referido parâmetro apresenta conteúdo técnico considerado } \\
\text { complexo ao nível de escolaridade do nível fundamental e descrição } \\
\text { subjetiva, o que dificulta a sua mensuração. }\end{array}$ \\
\hline Parâmetro 7: "Alterações no canal” & $\begin{array}{l}\text { Idem aos resultados/comentários referentes ao Parâmetro "Substratos } \\
\text { e/ou habitat disponíveis" }\end{array}$ \\
\hline Parâmetro 8: "Sinuosidade do canal" & $\begin{array}{l}\text { Este parâmetro não foi considerado no PAR proposto no presente } \\
\text { estudo em função da dificuldade de avaliar o grau de sinuosidade dos } \\
\text { rios a partir de uma avaliação realizada nas margens do curso d'água } \\
\text { (como é o caso da aplicação dos PARs). Entende-se que uma } \\
\text { avaliação mais condizente deva ser feito a partir de imagens de } \\
\text { satélite onde uma visão mais ampla é possibilitada. }\end{array}$ \\
\hline $\begin{array}{l}\text { Parâmetro 9: "Estabilidade das } \\
\text { margens" }\end{array}$ & $\begin{array}{l}\text { Idem aos resultados/comentários referentes ao Parâmetro "Substratos } \\
\text { e/ou habitat disponíveis" }\end{array}$ \\
\hline $\begin{array}{l}\text { Parâmetro 10: "Proteção das margens } \\
\text { pela vegetação" }\end{array}$ & $\begin{array}{l}\text { Idem aos resultados/comentários referentes ao Parâmetro "Substratos } \\
\text { e/ou habitat disponíveis" }\end{array}$ \\
\hline $\begin{array}{l}\text { Parâmetro 11: "Estado de conservação } \\
\text { da vegetação do entorno" }\end{array}$ & $\begin{array}{l}\text { Idem aos resultados/comentários referentes ao Parâmetro "Substratos } \\
\text { e/ou habitat disponíveis" }\end{array}$ \\
\hline
\end{tabular}

Nota: * Os resultados/comentários apresentados neste quadro foram elaborados a partir de anotações diversas realizadas pela equipe executora do estudo durante a visitação dos 27 trechos de rios localizados na cidade de Ipameri, GO. 
GUIMARÃES, A.; RODRIGUES, A. S. L.; MALAFAIA, G. Adequação de um protocolo de avaliação rápida de rios para ser usado por estudantes do ensino fundamental. Ambi-Agua, Taubaté, v. 7, n. 3, p. 241-260, 2012. (http://dx.doi.org/10.4136/ambi-agua.996)

Após a constatação da necessidade de alterações nos PARs referências, além das adequações relativas à linguagem técnica, um novo PAR foi adequado. Este PAR abrange a avaliação de onze parâmetros, cujas avaliações podem refletir condições consideradas "ótimas", "boas" ou "ruins". São eles: "Características do fundo do rio", "Sedimentos no fundo do rio", "Ocupação das margens do rio", "Erosão", "Lixo", "Alterações no canal do riacho", "Esgoto doméstico ou industrial", "Oleosidade da água", "Plantas aquáticas", "Animais" e "Odor da água". O PAR adequado pode ser observado na íntegra no Quadro 4. Ressalta-se que a partir da descrição de cada parâmetro proposto é possível atribuir notas condizentes com as condições observadas no trecho de rio em avaliação.

Destaca-se que a pontuação aumenta na mesma proporção da qualidade do habitat, e pode variar de acordo com o local das observações. Para os parâmetros cuja avaliação envolve as margens do canal (esquerda e direita) a pontuação deve ser atribuída a cada margem separadamente. Neste caso, as margens poderão apresentar diferentes condições ambientais e a avaliação do trecho é obtida por meio da média aritmética das pontuações.

O resultado final do protocolo proposto é obtido a partir do somatório dos valores atribuídos a cada parâmetro avaliado. A pontuação final reflete a condição ambiental encontrada nos trechos de rios avaliados. Trechos cujas pontuações, quando somadas encontram-se no intervalo entre 71 e 110 deverão ser considerados "ótimos" (ou seja, refletem uma condição natural ou com pouca alteração antrópica), quando no intervalo entre 31 e 70 , "bons" e quando no intervalo entre 0 e 30 , "ruins".

Com o intuito de facilitar a compreensão dos aspectos que devem ser avaliados em cada parâmetro, optou-se por apresentar, junto de cada um, desenhos esquemáticos como exemplos de condições ambientais possíveis de serem identificadas em campo (Quadro 4).

Quadro 4 - Protocolo de avaliação rápida adequado.

\begin{tabular}{|c|c|c|}
\hline \multicolumn{3}{|l|}{ Nome do avaliador: } \\
\hline \multicolumn{3}{|l|}{ Nome da escola: } \\
\hline \multicolumn{2}{|l|}{ Data da avaliação: } & Turma: \\
\hline \multicolumn{3}{|c|}{$\begin{array}{l}\text { Instruções: Você agora é um pesquisador e deve avaliar a saúde de rios e riachos. Por onde começar? Este } \\
\text { protocolo é utilizado por pesquisadores em todo o mundo, e agora será utilizado por você. Leia atentamente } \\
\text { os parâmetros e depois de observado o rio ao redor, marque uma nota ( } 10,5 \text { ou } 0) \text {, de acordo com a situação } \\
\text { verificada. }\end{array}$} \\
\hline \multicolumn{3}{|c|}{ Parâmetro 1: Características do fundo do rio } \\
\hline Ótima & Boa & Ruim \\
\hline $\begin{array}{l}\text { Existem galhos ou troncos, } \\
\text { cascalhos (pedras), folhas e } \\
\text { plantas aquáticas no fundo do rio. }\end{array}$ & $\begin{array}{l}\text { Há poucos galhos ou troncos, } \\
\text { cascalhos (pedras) no fundo do rio. }\end{array}$ & $\begin{array}{l}\text { Não existem galhos ou troncos, } \\
\text { cascalhos (pedras), folhas e } \\
\text { plantas aquáticas no fundo do } \\
\text { rio. }\end{array}$ \\
\hline 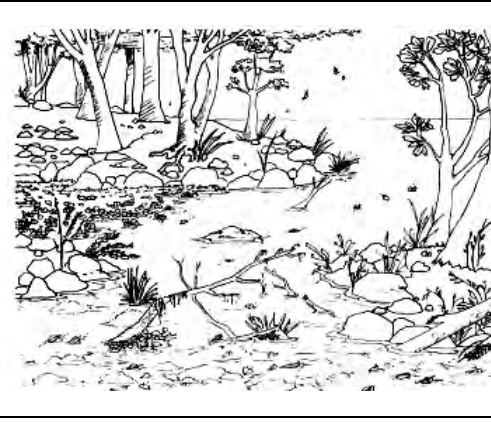 & 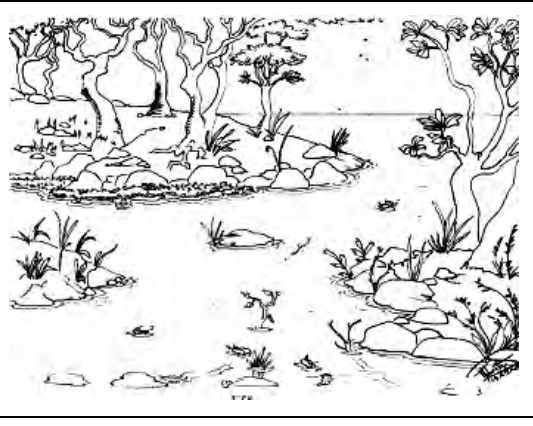 & 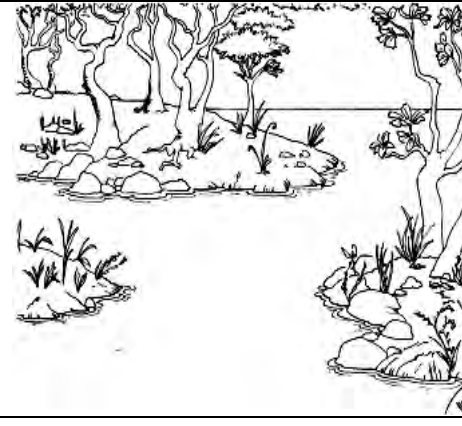 \\
\hline 10 & 5 & 0 \\
\hline
\end{tabular}


GUIMARÃES, A.; RODRIGUES, A. S. L.; MALAFAIA, G. Adequação de um protocolo de avaliação rápida de rios para ser usado por estudantes do ensino fundamental. Ambi-Agua, Taubaté, v. 7, n. 3, p. 241-260, 2012. (http://dx.doi.org/10.4136/ambi-agua.996)

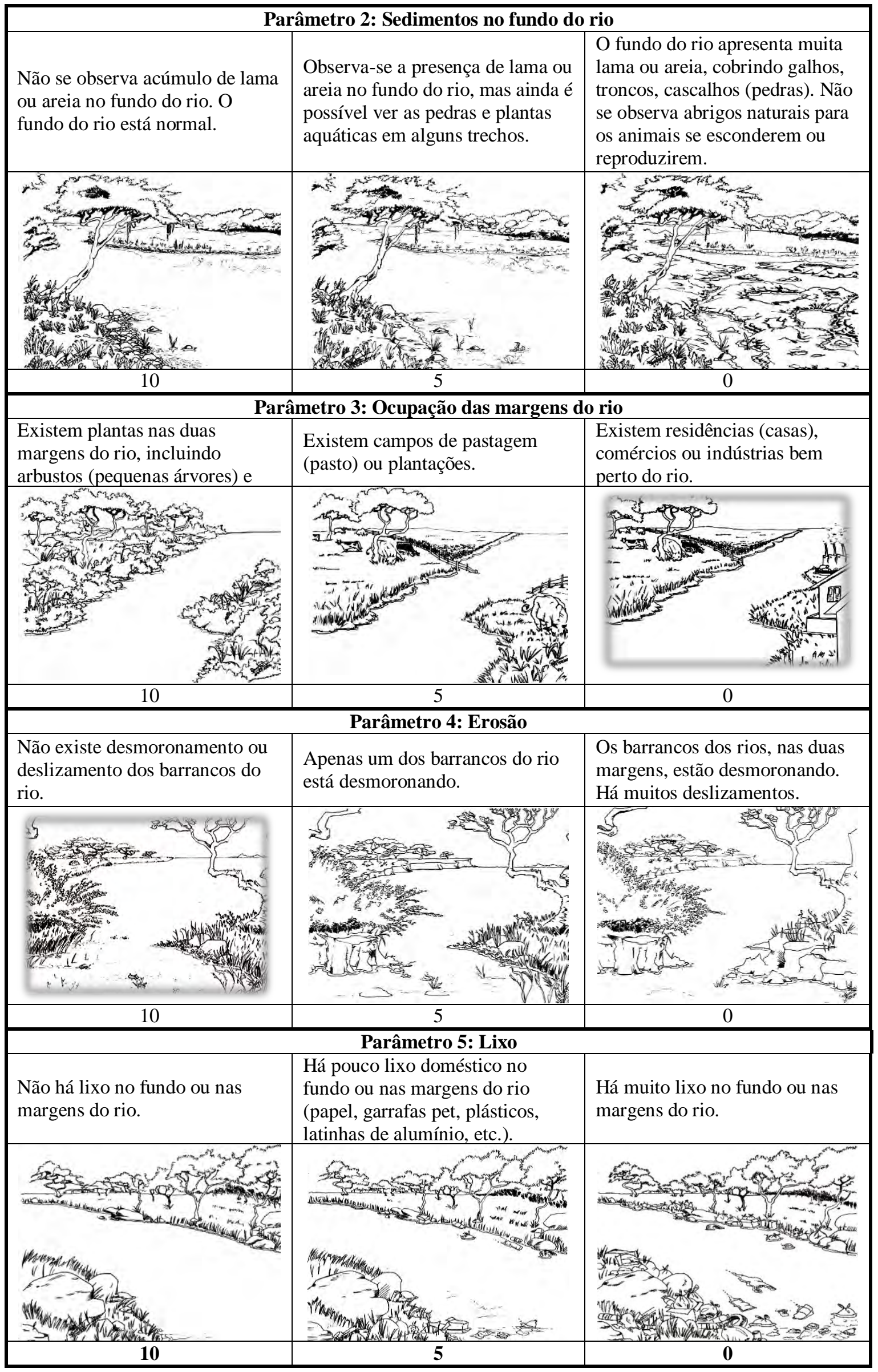


GUIMARÃES, A.; RODRIGUES, A. S. L.; MALAFAIA, G. Adequação de um protocolo de avaliação rápida de rios para ser usado por estudantes do ensino fundamental. Ambi-Agua, Taubaté, v. 7, n. 3, p. 241-260, 2012. (http://dx.doi.org/10.4136/ambi-agua.996)

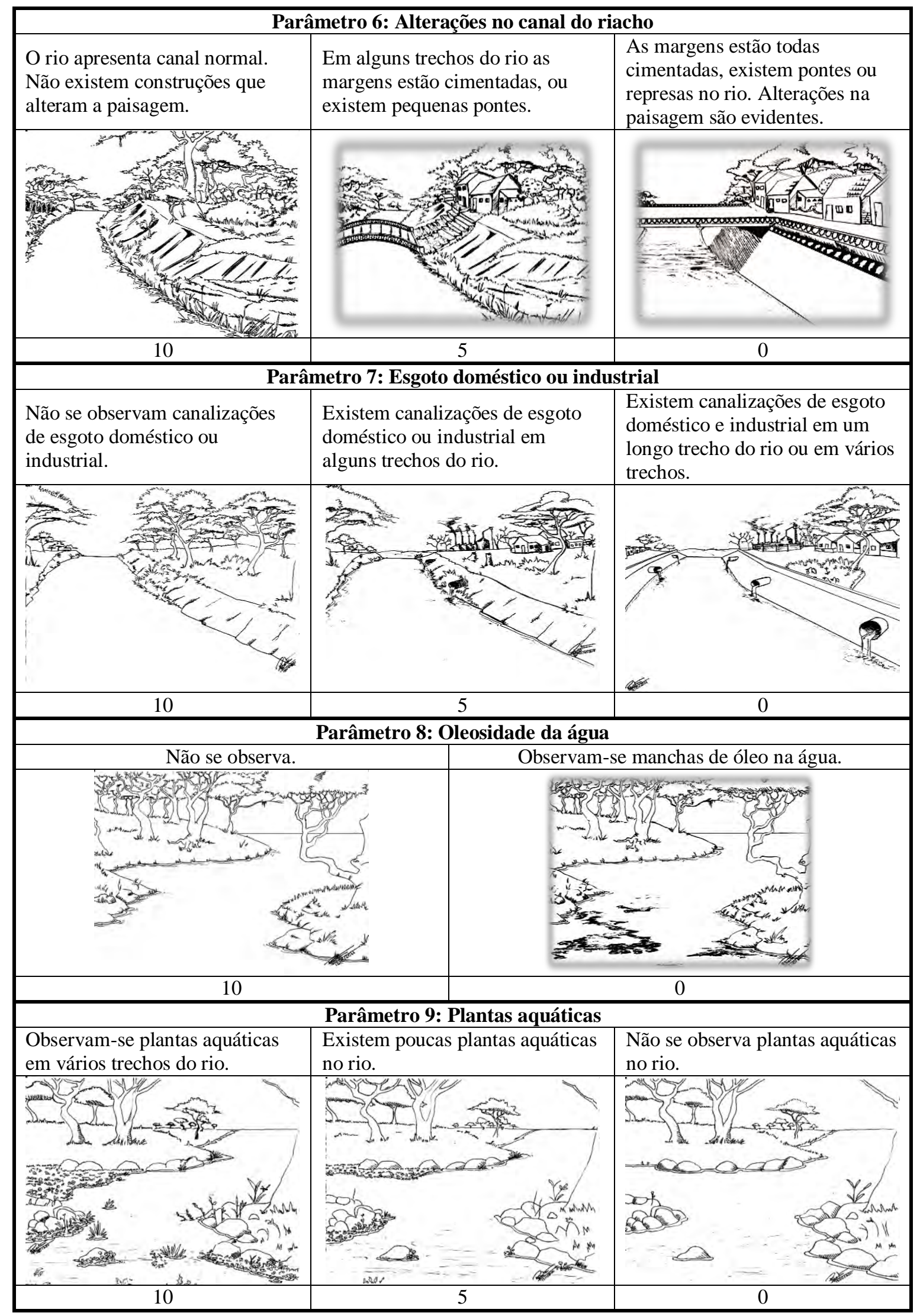


GUIMARÃES, A.; RODRIGUES, A. S. L.; MALAFAIA, G. Adequação de um protocolo de avaliação rápida de rios para ser usado por estudantes do ensino fundamental. Ambi-Agua, Taubaté, v. 7, n. 3, p. 241-260, 2012. (http://dx.doi.org/10.4136/ambi-agua.996)

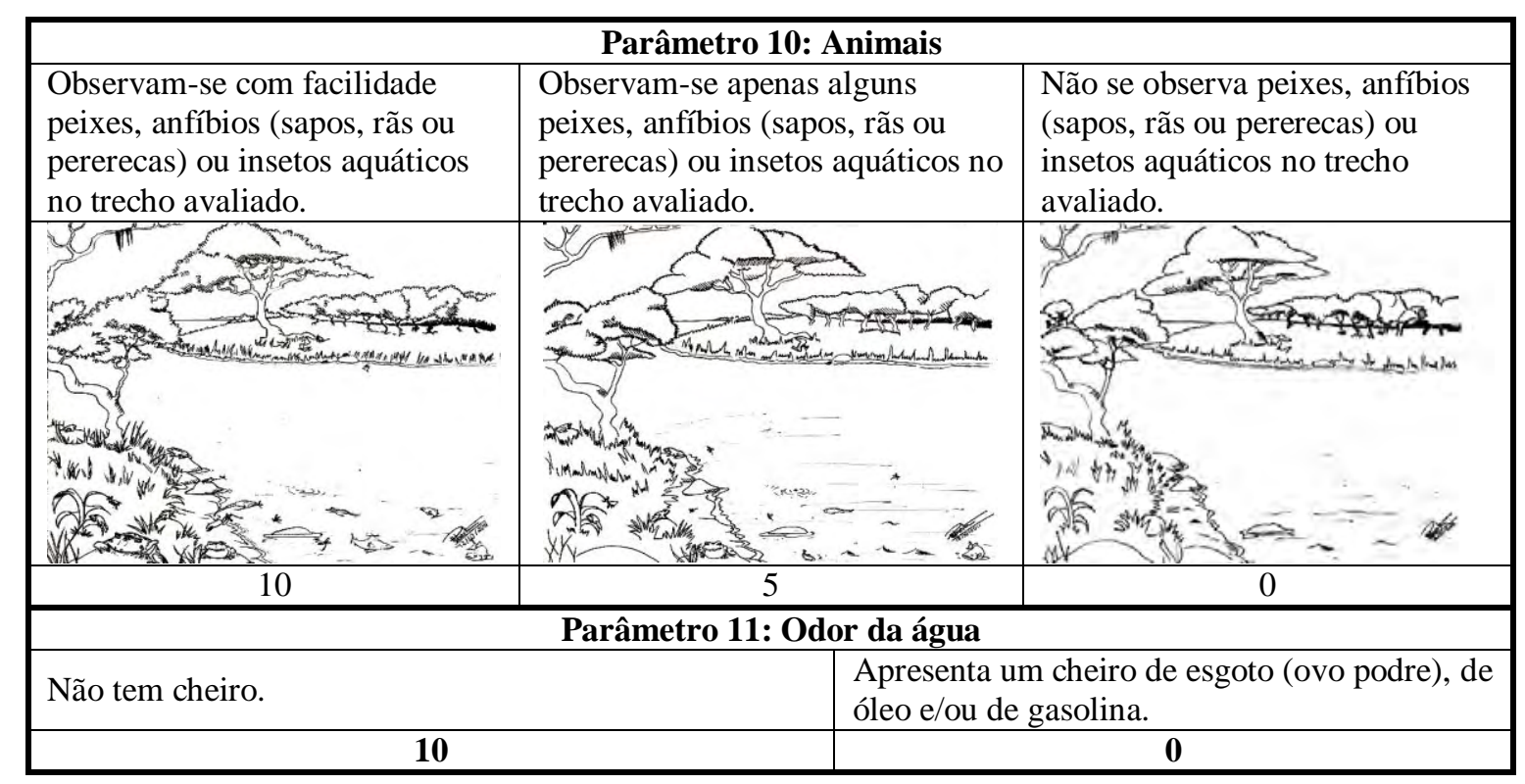

Após o desenvolvimento da parte teórica da oficina de monitoramento ambiental, os alunos foram a campo e aplicaram o PAR adequado. O tempo de aplicação do PAR pelos discentes variou entre 20 e 40 minutos, tempo este considerado relativamente pequeno. Os participantes relataram que o instrumento desenvolvido os aproxima das questões ambientais $(81 \%$ - $n=77)$, que as instruções da oficina de monitoramento ambiental ajudaram na melhor compreensão do instrumento $(75 \%$ - $n=71)$ e que as ilustrações disponíveis em cada parâmetro ajudaram significativamente durante a avaliação em campo, bem como durante a atribuição de notas aos parâmetros $(92 \%$ - $n=87)$. Além disso, $90 \%(n=86)$ dos participantes afirmaram que se sentiram sensibilizados para com as questões ligadas à preservação dos recursos hídricos, 96\% ( $\mathrm{n}=91)$ disseram que a participação na oficina de monitoramento ambiental permitiu a apropriação de conhecimentos sobre o funcionamento dos sistemas fluviais e o mesmo percentual afirmou que o PAR adequado pode ser utilizado por qualquer pessoal previamente treinada. O Quadro 5 mostra alguns comentários emitidos pelos alunos quando do preenchimento do questionário investigativo.

Conforme pode ser observado na Figura 2, os resultados da aplicação do PAR pelos discentes apresentaram variações. Porém, a análise estatística realizada revelou que essas variações não correspondem a diferenças significativas $(p=0,998$, pelo teste de Bartlett e $p=0,985$, pelo teste de Levene). Além disso, os resultados das avaliações realizadas pelos alunos no trecho de rio selecionado foram muito semelhantes aos obtidos em estudo recente também desenvolvido em Ipameri, GO. Este estudo refere-se ao trabalho de Firmino et al. (2011), no qual foi realizado um diagnóstico da integridade ambiental de trechos de rios localizados na cidade de Ipameri, por meio de um PAR (inespecífico ao nível de escolaridade do ensino fundamental). Em ambos os estudos, o mesmo trecho de rio (Figura 3) foi enquadrado numa condição "boa". A média do somatório das pontuações referentes à avaliação do trecho pelos discentes, no presente trabalho, foi de 49,1 (o que corresponde à condição "boa") e no estudo de Firmino et al. (2011) a pontuação média do referido trecho foi de 14,1, o que também corresponde à uma condição "boa", dentro de quatro possíveis categorias estabelecidas. 
GUIMARÃES, A.; RODRIGUES, A. S. L.; MALAFAIA, G. Adequação de um protocolo de avaliação rápida de rios para ser usado por estudantes do ensino fundamental. Ambi-Agua, Taubaté, v. 7, n. 3, p. 241-260, 2012. (http://dx.doi.org/10.4136/ambi-agua.996)

Quadro 5. Comentários emitidos por alguns discentes referentes a algumas questões do questionário aplicado ao final da oficina de monitoramento ambiental.

\begin{tabular}{|c|c|}
\hline Questões & Comentários \\
\hline $\begin{array}{l}\text { Durante a avaliação do rio com o } \\
\text { protocolo, você se sentiu um agente } \\
\text { colaborador na defesa de um rio? }\end{array}$ & $\begin{array}{l}\text { - "Porque às vezes a gente se senti uma pessoa } \\
\text { importante que ajuda o meio ambiente" (aluna, } 8^{\circ} \\
\text { ano). } \\
\text { - "Porque ao avaliarmos o rio percebemos o quanto } \\
\text { poluímos" (aluno, } 9^{\circ} \text { ano). } \\
\text { - "Porque eu posso saber as desvantagens de agir de } \\
\text { forma poluidora" (aluna, } 7^{\circ} \text { ano). }\end{array}$ \\
\hline $\begin{array}{l}\text { As ilustrações disponíveis em cada } \\
\text { parâmetro o ajudaram durante a avaliação } \\
\text { em campo e durante a atribuição de notas } \\
\text { aos parâmetros? }\end{array}$ & $\begin{array}{l}\text { - "Os desenhos facilitaram demais a marcação das } \\
\text { notas" (aluno, } 9^{\circ} \text { ano). } \\
\text { - "Sem a presença de algumas figuras eu não ia } \\
\text { conseguir dar nota ao rio" (aluna, } 7^{\circ} \text { ano). } \\
\text { - "As imagens ajudaram bastante" (aluno, } 9^{\circ} \text { ano). }\end{array}$ \\
\hline $\begin{array}{l}\text { A participação na oficina de } \\
\text { monitoramento ambiental permitiu a } \\
\text { apropriação de conhecimentos sobre o } \\
\text { funcionamento dos sistemas fluviais }\end{array}$ & $\begin{array}{l}\text { - "Aprendi muita coisa interessante com a oficina. Ela } \\
\text { - "A oficina acontecer mais vezes" (aluna, } 9^{\circ} \text { ano). } \\
\text { escola" (aluno, } 8^{\circ} \text { ano). } \\
\text { - "Através da oficina aprendi inúmeros aspectos do } \\
\text { funcionamento dos rios da nossa cidade" (aluno, } 9^{\circ} \\
\text { ano). }\end{array}$ \\
\hline $\begin{array}{l}\text { Você acredita que qualquer pessoa } \\
\text { previamente treinada pode realizar a } \\
\text { avaliação de um rio utilizando-se este } \\
\text { PAR? }\end{array}$ & $\begin{array}{l}\text { - "Qualquer pessoa pode aplicar o protocolo, desde } \\
\text { que ajudada por uma aula antes da aplicação" (aluno, } \\
9^{\circ} \text { ano). } \\
\text { "O protocolo foi muito fácil de utilizar. Acredito que } \\
\text { todos podem aplicar o instrumento em rios da } \\
\text { cidade" (aluna, } 8^{\circ} \text { ano). }\end{array}$ \\
\hline
\end{tabular}

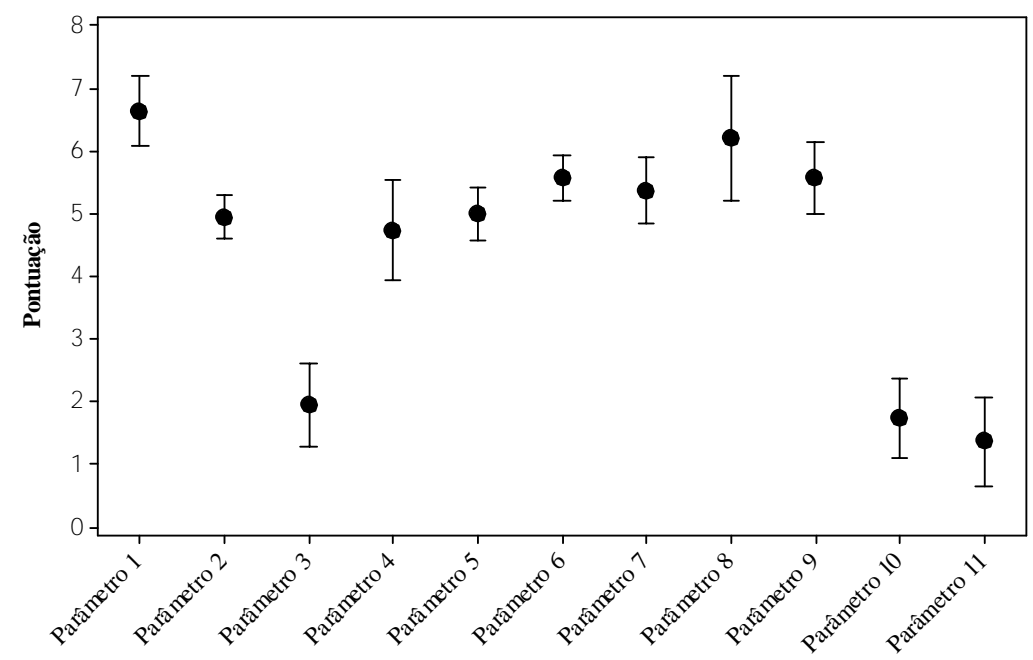

Figura 2. Variação da pontuação atribuída aos parâmetros analisados por 95 voluntários, alunos de ensino fundamental, em um trecho de rio na cidade de Ipameri, GO. Os pontos no gráfico representam a média +/- desvio padrão das pontuações atribuídas a cada parâmetro do protocolo desenvolvido.

Nota: Parâmetro 1: Características do fundo do rio; Parâmetro 2: Sedimentos no fundo do rio; Parâmetro 3: Ocupação das margens do rio; Parâmetro 4: Erosão; Parâmetro 5: Lixo; Parâmetro 6: Alterações no canal do riacho; Parâmetro 7: Esgoto doméstico ou industrial; Parâmetro 8: Oleosidade da água; Parâmetro 9: Plantas aquáticas; Parâmetro 10: Animais; Parâmetro 11: Odor da água. 


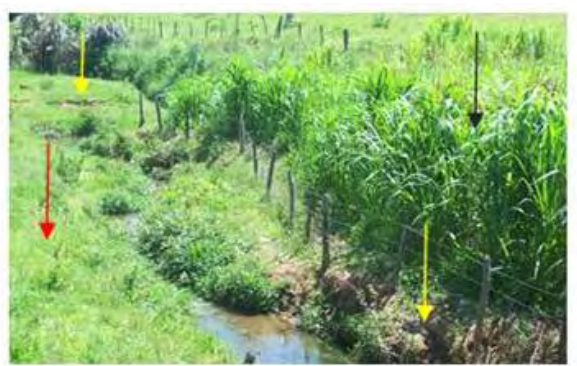

A

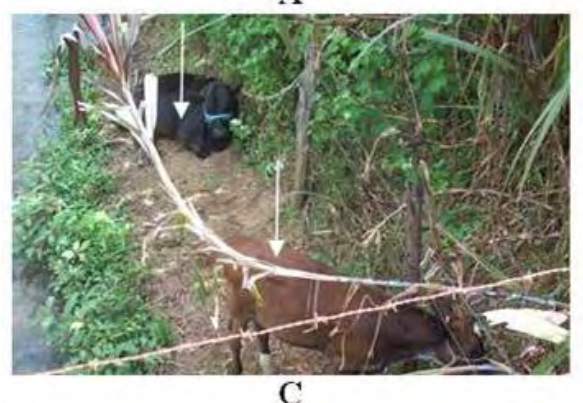

C

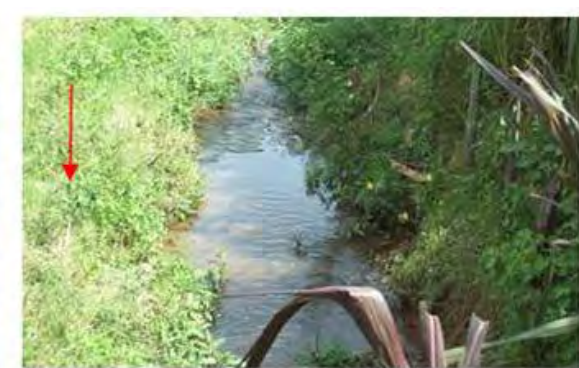

B

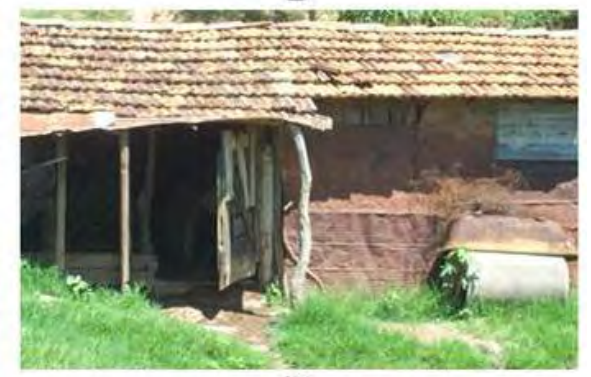

D

Figura 3. Imagens do entorno e do trecho de rio avaliado pelos discentes participantes da oficina de monitoramento ambiental, por meio do protocolo adequado no presente estudo (Ipameri, GO) ${ }^{1,2}$.

Nota: 'Esse trecho corresponde ao ponto 7 - coordenadas $48^{\circ} 09^{\prime} 09^{\prime \prime}$ O $17^{\circ} 42^{\prime} 40^{\prime \prime} \mathrm{S}$.

${ }^{2}$ As setas amarelas indicam processos erosivos em uma das margens do trecho avaliado, as setas vermelhas indicam áreas de pastagem, a seta preta área de cultivo e as setas brancas indicam animais domésticos em uma das margens do trecho.

Ao associar as características do trecho avaliado pelos discentes com as pontuações atribuídas pelos mesmos com base nas características do curso d'água apresentadas no dia da avaliação, também foi possível observar evidências que justificam o enquadramento da condição ambiental do trecho em uma condição "boa". As pontuações medianas atribuídas aos parâmetros 4, 5, 6, 7 e 8 condizem com a condição verificada no local. Para os parâmetros 4,5 e 6 as notas atribuídas não foram tão elevada possivelmente em função de uma das margens do trecho apresentar-se mais impactada que a outra. Processos erosivos na margem direita do trecho (conforme ilustrado na Figura 3A - setas amarelas) podem ser evidenciados, assim como a presença de lixo próximo à residência localizada à margem esquerda do trecho (Figura 3D). Já as alterações no canal foram mais intensas próximas à residência, mas ao longo do trecho as alterações que influenciam o curso normal do rio foram mínimas (o que justifica a nota mediana atribuída ao parâmetro). Já as baixas pontuações atribuídas aos parâmetros 3, 10 e 11 refletem a intensa ocupação das margens do rio com áreas de pastagens, criação de gado (Figura 3A, 3B e 3C - setas vermelhas e brancas) e cultivo (Figura 3A - seta preta), bem como ao odor de esgoto doméstico oriundo da residência localizada próxima ao trecho e à ausência de animais silvestres às margens do curso d'água (justificada pela ocupação humana ou ao período ou época do ano em que a avaliação do trecho foi realizada).

Nesse sentido, os resultados da oficina possibilitam inferir que o PAR adequado mostrase compreensível por parte dos avaliadores (discentes), já que não há variações significativas nas respostas dos avaliadores, bem como capaz de detectar perturbações no curso d'água que são condizentes com as características do local de avaliação.

Cabe ressaltar que apesar de não terem sido significativas estatisticamente, as variações das pontuações atribuídas aos parâmetros 4 ("Erosão") e 8 ("Oleosidade da água") foram as maiores. Tais variações podem ser explicadas possivelmente pela maior dificuldade, por parte 
dos alunos, de entender o que o parâmetro propunha mensurar ou indicar. Dentre os parâmetros que apresentaram maior dificuldade de entendimento no PAR adequado, os parâmetros 4 e 8 foram os mais mencionados pelos discentes (ambos foram mencionados por 66,7\% ( $\mathrm{n}=18)$ dos discentes que afirmaram ter dificuldade de entendimento dos parâmetros $\mathrm{n}=27$ ). Em função disso, a descrição de ambos os parâmetros sofreram modificações de modo a permitir um maior entendimento por parte dos avaliadores. A versão do PAR apresentada neste estudo (Quadro 3) refere-se àquela pós-modificação destes parâmetros em específico. Ainda assim, acredita-se é natural que ocorra variações, principalmente em função do PAR ser um instrumento que alberga certa subjetividade. Para Rodrigues e Castro (2008a), a subjetividade do método pode ser amenizada com maior treinamento do avaliador, com a realização de cursos de capacitação e com o acompanhamento parcial e de suporte de avaliadores mais experientes.

Do ponto de vista da conservação dos recursos hídricos, pode-se dizer que atualmente é indiscutível a importância do monitoramento e avaliação ambiental desses recursos, sobretudo quando se considera que a demanda e a oferta desses está cada vez mais comprometida em função das atividades poluidoras diversas. A grande quantidade de estudos sobre avaliação e monitoramento desses recursos, em diferentes contextos regionais, revela a importância que tem sido dada a essa temática (Farias et al., 2011; Vargas e Ferreira Júnior, 2012; Baldissera et al., 2011; Guedes et al., 2012; Pereira et al., 2012, Firmino et al., 2011; Rodrigues et al., 2012; Bailly et al., 2012). Porém, esses estudos têm focado análises mais técnicas, com destaque para avaliações dos parâmetros físico-químicos e microbiológicos da água, com pouca atenção aos fatores físicos condicionantes da qualidade do ecossistema fluvial, bem como aos aspectos sociais e à inserção da comunidade nessas avaliações.

No presente estudo, propõe-se uma ferramenta que permite a avaliação rápida de riachos de uma forma mais genérica e não apenas ligadas à qualidade físico-química e microbiológica da água, ao mesmo tempo em que oportuniza a inserção de jovens estudantes nessa avaliação. Nesse sentido, pode-se dizer que o instrumento aqui proposto pode contribuir enormemente para com o desenvolvimento da EA envolvendo crianças e a preservação dos recursos fluviais locais.

A utilização do PAR em projetos de EA desenvolvidos em instituições de ensino podem despertar nas crianças a atenção para as condições ambientais do recurso hídrico da localidade. Baseando-se em critérios técnicos (discriminados no próprio PAR, de forma adaptada à linguagem do ensino fundamental), oportunizou-se aos discentes a percepção e identificação de possíveis impactos que podem passar despercebidos no seu dia-a-dia, principalmente devido ao fato do impacto já ter se incorporado à realidade das pessoas e não ser enxergado como um problema ambiental. É possível então, que o PAR, aliado a análises complementares do recurso fluvial em questão, proporcione em consórcio com o desenvolvimento de atividades teórico/reflexivas o desenvolvimento de uma compreensão integrada dos recursos fluviais (incluindo aspectos ecológicos); o fortalecimento de uma consciência crítica sobre a problemática ambiental, bem como o incentivo à participação individual e coletiva na preservação do equilíbrio dos recursos hídricos, entendendo-se a defesa da qualidade ambiental como um valor inseparável do exercício da cidadania.

Estudos como os desenvolvidos por Guimarães et al. (2006) (comentado inicialmente), Bergmann e Pedrozo (2008) e por França et al. (2010), por exemplo, constituem experiências bem sucedidas ligadas à utilização de PAR por estudantes em projetos de EA, apesar de nenhum deles ter adequado um PAR especificamente para uso por estudantes do ensino fundamental. No estudo de Bergmann e Pedrozo (2008), em duas escolas públicas do município de Giruá, RS, com ênfase na sub-bacia do rio Santo Cristo, ao aplicarem um PAR durante as atividades de campo, os alunos do ensino médio puderam não apenas ter contato com uma ferramenta de avaliação ambiental, mas identificar e aprender sobre como evitar ou 
minimizar problemas ambientais da sub-bacia hidrográfica local. França et al. (2010), por meio de atividades envolvendo apresentação de vídeo, exposição interativa, jogos lúdicos, visitas a uma nascente (fonte de abastecimento de água do município) do Refúgio de Vida Silvestre (RVS) Mata do Junto (Capela, SE) e aplicação de um PAR, trabalharam EA de modo a promover a conscientização de jovens estudantes sobre a importância de seu papel na preservação dos recursos fluviais.

\section{CONCLUSÕES}

Os resultados deste estudo permitem concluir que o PAR adequado apresenta-se como ferramenta viável e útil no desenvolvimento da EA no nível fundamental da educação. Em adição, tal ferramenta foi capaz de permitir a observação, sensibilização e apropriação de conceitos referentes ao funcionamento e à preservação dos recursos fluviais locais, contribuindo significativamente com o desenvolvimento da EA no nível básico da educação.

Salienta-se que o PAR proposto neste estudo mostra-se viável para a utilização em instituições de ensino (públicas ou particulares) em projetos/programas de EA, bem como por organizações-não-governamentais relacionadas à preservação ambiental.

\section{AGRADECIMENTOS}

Os autores agradecem ao IF Goiano - Campus Urutaí pelo financiamento do projeto. Guimarães A. agradece ao CNPq pela concessão da bolsa de iniciação Científica (vigência: 2011-2012) e Rodrigues A.S.L. agradece ao IF Goiano pela concessão da bolsa produtividade (vigência: 2012)..

\section{REFERÊNCIAS}

BAILLY, D.; FERNANDES, C. A.; SILVA, V. F. B.; KASHIWAQUI, E. A. L.; DAMÁSIO, J. F.; WOLF, M. J. et al. Diagnóstico ambiental e impactos sobre a vegetação ciliar da microbacia do córrego da Ponte, Área de proteção ambiental do rio Iguatemi, MS. Revista em Agronegócios e Meio Ambiente, Maringá, v. 5, n. 2, p. 409-427, 2012.

BALDISSERA, I. T.; ZAMPIERI, S. L.; BAMPI, D. B. Monitoramento da qualidade da água na microbacia Tarumanzinho em Águas Frias, SC, Brasil. Revista de Ciências Ambientais, Canoas, v. 5, n. 2, p. 5-14, 2011.

BARBOUR, M.T.; GERRISTSEN, J.; SNYDER, B.; STRIBLING, J. Rapid bioassessment protocols for use in streams and wade able rivers: periphyton, benthic macroinvertebrates and fish. 2. ed. Washington: EPA 1999. 339p.

BERGMANN M.; PEDROZO C. S. Explorando a bacia hidrográfica na escola: contribuições à educação ambiental. Revista Ciência \& Educação, Bauru, v. 14, n. 3, p. 537-553. 2008. http://dx.doi.org/10.1590/S1516-73132008000300011

BIZERRIL, M. X. A.; FARIA, D. S. A escola e a conservação do cerrado: uma análise no ensino fundamental do Distrito Federal. Revista Eletrônica do Mestrado em Educação Ambiental, Rio Grande, v. 10, p. 19-31, 2003.

BROWN, M. B.; FORSYTHE, A. B. Robust tests for the equality of variances. Journal of the American Statistical Association, Alexandria, v. 69, n. 346, p. 1-9, 1974. http://dx.doi.org/10.1080/01621459.1974.10482955 
CALLISTO, M.; RIBEIRO, A.; SANTANA, V. B. Integração, treinamento e formação de pós-graduandos para a conservação de riachos de cabeceira. In: ENCONTRO DE RECURSOS HÍDRICOS EM SERGIPE, 3., 2010, Aracajú. Resumos expandidos... Aracajú: Embrapa Tabuleiros Costeiros, 2010. Disponível em: <http://www.cpatc. embrapa.br/publicacoes_2010/anais3_enrehse>. Acesso em: 3 jul. 2012.

CALliSTO, M.; FERREIRA, W.; MORENO, P.; GOULART, M. D. C.; PETRUCIO, M. Aplicação de um protocolo de avaliação rápida da diversidade de habitats em atividades de ensino e pesquisa (MG-RJ). Acta Limnologica Brasiliense, Sorocaba, v. 14, n. 1, p. 91-98, 2002.

CARVALHO, E. M. Estudo da utilização de um protocolo de avaliação rápida da diversidade de habitats em atividade complementar de ensino (UEMS - Dourados). In: ENCONTRO DE ENSINO DE GRADUAÇÃO, 1., 2010, Dourados. Anais... Dourados: UEMS, 2010.

DILLENBURG, A. K. A importância do monitoramento ambiental na avaliação da qualidade de um rio - estudo de caso - Mercedes, PR. Revista Urutágua - Revista Acadêmica Multidisciplinar, Maringá, n. 12, p. 1-10, 2007.

FARIAS, M. S. S.; DANTAS NETO, J.; LIMA, V. L. A. Monitoramento da qualidade da água na bacia hidrográfica do Rio Cabelo: parâmetros físico-químicos. GEPROS Gestão da Produção, Operações e Sistemas, Bauru, v. 6, n. 1, p. 161-170, 2011.

FRANÇA, J.; ANDRADE, C.; FONTENELE, A. C.; SANTOS, R. C.; RIBEIRO, A.; CALLISTO, M. Atividades de educação ambiental com comunidades do entorno do RVS Mata do Junco, Capela, SE. In: ENCONTRO DE RECURSOS HÍDRICOS EM SERGIPE, 3., 2010, Aracaju. Resumos expandidos... Aracajú: Embrapa Tabuleiros Costeiros, 2010. p. 1-4.

FIRMINO, P. F.; MALAFAIA, G.; RODRIGUES, A. S. L. Diagnóstico da integridade ambiental de trechos de rios localizados no município de Ipameri, Sudeste do Estado de Goiás, através de um protocolo de avaliação rápida. Brazilian Journal of Aquatic Science and Technology, Itajaí, v. 15, n. 2, p. 1-12, 2011.

GUEDES, H. A. S.; SILVA, D. D.; ELESBON, A. A. A.; RIBEIRO, C. B. M.; MATOS, A. T.; SOARES, J. H. P. Aplicação da análise estatística multivariada no estudo da qualidade da água do Rio Pomba, MG. Revista Brasileira de Engenharia Agrícola e Ambiental, Campina Grande, v. 16, n. 5, p. 558-563, 2012. http://dx.doi.org/10.1590/S1415-43662012000500012

GUIMARÃES, A. Q. et al. Uso de ferramentas alternativas para auxiliar saídas de campo e construção de valores conservacionistas. In: CONGRESSO IBERO-AMERICANO DE EDUCAÇÃO AMBIENTAL, 5., 2006. Joinville. Anais... Brasília: MMA, 2006.

INSTITUTO BRASILEIRO DE GEOGRAFIA E ESTATÍSTICA -IBGE. Censo 2010. Disponível em: <http://www.ibge.gov.br/home/estatistica/populacao/censo2010/ calendario.shtm>. Acesso em: 10 out. 2012.

IPAMERI. Prefeitura Municipal. Informações gerais sobre o município de Ipameri. Disponível em: <http://www.ipameri.go.gov.br/site/12/html/informacoes-gerais.html>. Acesso em: 16 de abr. 2010.

KRUPEK, R. A. Análise comparativa entre duas bacias hidrográficas utilizando um protocolo de avaliação rápida da diversidade de habitats. Ambiência, Guarapuava, v. 6, n. 1, p. 147-158, 2010. 
LOBO, E. A.; VOOS, J. G.; ABREU JÚNIOR, E. F. Utilização de um protocolo de avaliação rápida de impacto ambiental em sistemas lóticos do Sul do Brasil. Caderno de Pesquisa, Série Biologia, Santa Cruz, v. 23, n. 1, p. 18-33, 2011.

MINATTI-FERREIRA, D. D.; BEAUMORD, A. C. Avaliação rápida de integridade ambiental das sub-bacias do rio Itajaí-Mirim no Município de Brusque, SC. Revista Saúde \& Ambiente, Joinville, v. 5, n. 2, p. 21-27, 2004.

MINATTI-FERREIRA, D. D.; BEAUMORD, A. C. Adequação de um protocolo de avaliação rápida de integridade ambiental para ecossistemas de rios e riachos: aspectos físicos. Revista Saúde e Ambiente, Joinville, v. 7, n. 1, p. 39-47, 2006.

PADOVESI-FONSECA, C.; CORRÊA, A. C. G.; LEITE, G. F. M.; JOVELI, J. C.; COSTA, L. S.; PEREIRA, S. T. Diagnóstico da sub-bacia do ribeirão Mestre d'Armas por meio de dois métodos de avaliação ambiental rápida, Distrito Federal, Brasil Central. Revista Ambiente \& Água, Taubaté, v. 5, n. 1, p. 43-56, 2010. http://dx.doi.org/10.4136/1980993X

PALHARES, K.; MAYRINK, N.; MORENO, P.; GOULART, M.; MORETTI, M.; FERREIRA, W. Bioindicadores de qualidade de água: a educação ambiental como uma ferramenta de união UFMG-escolas. In: SIMPÓSIO DE ECOSSISTEMAS BRASILEIROS, 5., 2000, Vitória. Anais... São Paulo: ACIESP, 2000. V. 1. p. 182189.

PEREIRA, P. S.; FERNANDES, L. A. C.; OLIVEIRA, J. L. M.; BAPTISTA, D. F. Avaliação da integridade ecológica de rios em áreas do zoneamento ecológico econômico do complexo hidrográfico Guapiaçu-Macacu, RJ, Brasil. Revista Ambiente \& Água, Taubaté, v. 7, n. 1, p. 157-168, 2012. http://dx.doi.org/10.4136/ambi-agua.762

PIMENTA, S. M.; PENA, A. P.; GOMES, P. S. Aplicação de métodos físicos, químicos e biológicos na avaliação da qualidade das águas em áreas de aproveitamento hidroelétrico da bacia do rio São Tomás, município de Rio Verde - Goiás. Sociedade \& Natureza, Uberlândia, v. 21, n. 3, p. 393-412, 2009. http://dx.doi.org/10.1590/S198245132009000300013

PLAFKIN, J. L.; BARBOUR, M. T.; PORTER, K. D.; GROSS, S. K.; HUGHES, R. M. Rapid bioassessment protocols for use in streams and rivers: benthic macroinvertebrates and fish. Washington: EPA 440-4-89-001, 1989. 339 p.

PREFEITURA MUNICIPAL DE IPAMERI-GO (PMI). Informações gerais sobre o município de Ipameri. Disponível em: http://www.ipameri.go.gov.br/site/12/html/ informacoes-gerais.html. Acesso em: 16 de abr. 2010.

RODRIGUES, A. S. L.; CASTRO, P. T. A. Adaptation of a rapid assessment protocol for rivers on rocky meadows. Acta Limnologica Brasiliense, Sorocaba, v. 20, n. 4, p. 291$303,2008 b$.

RODRIGUES, A. S. L.; CASTRO, P. T. A. Protocolos de avaliação rápida: instrumentos complementares no monitoramento dos recursos hídricos. Revista Brasileira de Recursos Hídricos, Porto Alegre, v. 13, n. 1, p. 161-170, 2008a.

RODRIGUES, A. S. L.; CASTRO, P. T. A.; MALAFAIA, G. Utilização dos protocolos de avaliação rápida de rios como instrumentos complementares na gestão de bacias hidrográficas envolvendo aspectos da geomorfologia fluvial: uma breve discussão. Enciclopédia Biosfera, Goiânia, v. 6, n. 11, p. 1- 9, 2010. 
RODRIGUES, A. S. L.; MALAFAIA, G.; CASTRO, P. T. A. Avaliação ambiental de trechos de rios na região de Ouro Preto-MG através de um protocolo de avaliação rápida. Revista de Estudos Ambientais, Blumenau, v. 10, n. 1, p. 74-83, 2008.

RODRIGUES, A. S. L.; MALAFAIA, G.; COSTA, A. T.; NALINI-JÚNIOR, H. A. Adequação e avaliação da aplicabilidade de um Protocolo de Avaliação Rápida na bacia do rio Gualaxo do Norte, Leste-Sudeste do Quadrilátero Ferrífero, MG, Brasil. Revista Ambiente \& Água, Taubaté, v. 7, n. 2, p. 231-244, 2012. http://dx.doi.org/10.4136/ambi-agua.872

SNEDECOR, G. W.; COCHRAN, W. G. Statistical methods. 8. ed. Iowa: State University Press, 1989.

VARGAS, J. R. A.; FERREIRA JÚNIOR, P.D. Aplicação de um protocolo de avaliação rápida na caracterização da qualidade ambiental de duas microbacias do rio Guandu, Afonso Cláudio, ES. Revista Brasileira de Recursos Hídricos, Porto Alegre, v. 17, n. 1, p. 161-168, 2012.

XAVIER, A. L.; TEIXEIRA, D. A. Diagnóstico das nascentes da sub-bacia hidrográfica do rio São João em Itaúna, MG. In: CONGRESSO DE ECOLOGIA DO BRASIL, 7. 2007, Caxambu. Anais... Caxambu: SEB, 2007. p. 1-2. 


ISSN = 1980-993X - doi:10.4136/1980-993X
www.ambi-agua.net
E-mail: ambi-agua@agro.unitau.br
Tel.: (12) 3625-4212

\title{
Avaliação espaço-temporal dos parâmetros de qualidade da água do rio Santa Rita (BA) em função do lançamento de manipueira
}

(http://dx.doi.org/10.4136/ambi-agua.880)

\section{Giovanna Porto dos Santos'; Neylor Alves Calasans Rego² ${ }^{2}$ José Wildes Barbosa dos Santos $^{1}$; Franklin Delano Júnior ${ }^{3}$; Milton Ferreira da Silva Júnior ${ }^{2}$}

\author{
${ }^{1}$ Universidade Estadual do Sudoeste da Bahia - UESB, \\ e-mail: giovannaportofm@gmail.com, wildesbarbosa@yahoo.com.br, \\ ${ }^{2}$ Universidade Estadual de Santa Cruz - UESC, \\ e-mail: neylorcalasans@gmail.com, notlimf@gmail.com, \\ ${ }^{3}$ Instituto Federal de Educação, Ciência e Tecnologia da Bahia - IFBA, \\ e-mail: franklin@inside.inf.br
}

\section{RESUMO}

A bacia hidrográfica do rio Santa Rita engloba os povoados de Simão e Campinhos existindo cerca de 150 casas de farinha. O povoado de Campinhos encontra-se entre os maiores beneficiadores de mandioca na região sudoeste da Bahia, gerando muitos empregos diretos e indiretos. A manipueira é um resíduo líquido oriundo da prensagem da mandioca e apresenta alto potencial poluente decorrente da quantidade glicose e frutose, apresentando potencial poluidor 25 vezes maior do que o do esgoto doméstico. $\mathrm{O}$ trabalho teve como objetivo a avaliação dos possíveis impactos decorrentes do lançamento de manipueira na qualidade da água do rio Santa Rita. Para tanto, foi elaborado o mapa de uso do solo e realizada a caracterização fisiográfica da bacia, além de efetuadas seis campanhas de coleta de amostras de água em quatro pontos ao longo do rio. Os resultados obtidos indicaram que a bacia hidrográfica do rio Santa Rita é alongada, com baixa eficiência de drenagem e com baixa possibilidade de ocorrência de enchentes. Os dados relativos aos parâmetros de qualidade de água indicaram um forte aporte de efluentes orgânicos nos núcleos de Campinhos e Simão, comprometendo os valores de oxigênio dissolvido, condutividade elétrica, salinidade, amônia, nitrito, nitrato e zinco, sugerindo impactos decorrentes do lançamento de manipueira. A concentração de fósforo total, ferro e cobre foram superiores à jusante da Estação de Tratamento de Esgoto. A água apresentou-se salobra nos três pontos de coleta mais afetados pelo lançamento de manipueira.

Palavras-chave: bacia hidrográfica, qualidade da água, efluentes.

\section{Spatial-temporal water quality parameters evaluation of the Santa Rita river (BA) with respect to the release of manipueira}

\begin{abstract}
The watershed of the river Santa Rita includes the towns of Simão and Campinhos, where exists about 150 flour houses. Campinhos is among the largest cassava processing facilities in the region, generating many direct and indirect jobs. Manipueira is a liquid residue originating from the cassava pressing and presents high pollutant potential due to its high amount of glucose and fructose, this potential is 25 times greater than the one from
\end{abstract}


SANTOS, G. P.; REGO, N. A. C.; SANTOS, J. W. B.; DELANO JÚNIOR, F.; SILVA JÚNIOR, M. F. Avaliação espaço-temporal dos parâmetros de qualidade da água do rio Santa Rita (BA) em função do lançamento de manipueira. Ambi-Agua, Taubaté, v. 7, n. 3, p. 261-278, 2012. (http://dx.doi.org/10.4136/ambiagua.880)

domestic sewer. This work had as objective the evaluation of possible impacts of manipueira release in the water quality of Santa Rita river. For this, the land use map was elaborated and the physiographic characterization developed, besides being performed six campaigns for water samples collection in four sampling points along the river. The obtained results indicated that the watershed is elongated, with low drainage efficiency and it is not prone to flooding. Estimated water quality parameters indicated that organic effluents from Campinhos and Simão impact the values of dissolved oxygen, electrical conductivity, salinity, ammonia, nitrite, nitrate and zinc, suggesting that the water quality of the river Santa Rita is affected by manipueira release. The concentrations of total phosphorus, iron and cooper were superior downstream of the Sewer Treatment Station. The river water was saline in the three sampling points most affected by the release of manipueira.

Keywords: watershed, water quality, effluents.

\section{INTRODUÇÃO}

A água desempenha um papel vital e insubstituível em todo equilíbrio ecológico, sendo um recurso natural imprescindível à manutenção da vida na terra. $\mathrm{O}$ aumento demográfico a que se tem assistido vem provocando a crescente deterioração da água, e à medida que as civilizações aumentam, aumenta a necessidade de água e a poluição da mesma, pois, já é reconhecido que as atividades humanas degradam os sistemas aquáticos.

O Brasil se destaca mundialmente na produção de produtos alimentícios oriundos da mandioca, pois, existem milhares de unidades produtoras de farinha de mandioca distribuídas em seu território, sendo que a maior parte se localiza nas regiões Noroeste, Norte e Sul do país. As casas de farinha são a base da economia de muitas regiões do Brasil, mas também são responsáveis por boa parte da poluição produzida nessas áreas. A queima da lenha polui o ar, provocando doenças respiratórias, juntamente com o despejo da manipueira que polui as águas superficiais, causando intoxicação na população humana e dos ambientes aquáticos.

Segundo Barana (2008), no município de Vitória da Conquista (BA), notadamente nos povoados de Campinhos e Simão, a falta de tratamento adequado da manipueira tem restringido a atividade econômica, por impor limites físicos de espaço destinado à industrialização, pois em determinadas épocas do ano, muitos produtores de farinha são obrigados a reduzir suas atividades pela impossibilidade de descarte destes resíduos nas proximidades das casas de farinha.

O descarte da manipueira sem nenhum tipo de tratamento leva a poluição de uma das poucas fontes de água da região, o rio Santa Rita, e torna o local de produção insalubre pela exposição direta dos moradores ao resíduo despejado abundantemente no ambiente circundante.

A poluição ambiental proveniente da manipueira restringe fisicamente os locais de produção pela formação de enormes volumes deste líquido, provocando condições de insalubridade afetando a saúde da população e a economia desta atividade (Barana, 2008).

A manipueira que em tupi-guarani significa "o que brota da mandioca", é um resíduo líquido da prensagem da raiz de mandioca e apresenta grande potencial poluente, decorrente da quantidade de material não esgotado. Existe também o problema da toxidez, devido a presença de glicosídio característico da planta de mandioca (linamarina) potencialmente hidrolisável a ácido cianídrico (Branco, 1986), tóxico dos mais poderosos e que pode afetar células nervosas. Este glicosídio combina-se com a hemoglobina do sangue, sendo inibidor da cadeia respiratória (Cereda et al., 1981). 
SANTOS, G. P.; REGO, N. A. C.; SANTOS, J. W. B.; DELANO JÚNIOR, F.; SILVA JÚNIOR, M. F. Avaliação espaço-temporal dos parâmetros de qualidade da água do rio Santa Rita (BA) em função do lançamento de manipueira. Ambi-Agua, Taubaté, v. 7, n. 3, p. 261-278, 2012. (http://dx.doi.org/10.4136/ambiagua.880)

A importância econômica da cultura da mandioca deriva do interesse em suas raízes, ricas em amido, utilizadas na alimentação humana e animal, e de seu uso na fabricação de produtos alimentícios e de uso industrial. São exemplos as farinhas de variados tipos, fécula ou polvilho doce, polvilho azedo, amidos modificados, mandioca puba, tapioca, beiju, além das raízes minimamente processadas, congeladas, desidratadas, pré-cozidas, fritas tipo chips e dos croquetes. A mandioca pode ainda ser usada com ingrediente ou aditivo na fabricação de embutidos, chocolates, balas, bolachas, pães e sopas.

Na fabricação da farinha, devido à forma como as raízes são processadas, a concentração da manipueira em matéria orgânica e linamarina é muito elevada, correspondendo à fração aquosa da raiz. O potencial tóxico e poluente é agravado, principalmente, por ser a linamarina muito solúvel em água. Existem relatos de morte de animais que beberam da água onde ocorreram descargas da manipueira, sendo a morte de peixes fato comum. A manipueira apresenta gosto adocicado pela glicose que contém, sendo muito procurada pelos animais (Fioretto, 1987).

A manipueira "in natura", oriunda diretamente da prensagem da raiz de mandioca tem um potencial poluidor de 25 vezes a do esgoto doméstico. A manipueira de fecularia é mais diluída, conserva, porém, um potencial poluidor de aproximadamente 12,5 vezes a do esgoto doméstico (Barana, 2008). Uma tonelada de mandioca produz cerca de $300 \mathrm{~L}$ de manipueira que, quando armazenada, forma verdadeiros lagos. Dessa forma uma fecularia que utilize uma tonelada de raízes de mandioca por dia equivale à poluição ocasionada por 200 a 300 habitantes.dia-1 (Hess, 1962).

Santos (2001) coletou amostra de água do rio Santa Rita à jusante do bairro de Campinhos para análise na EMBASA (Empresa Baiana de Águas e Saneamento S.A) e concluiu que a DQO apresentou valor muito elevado, aproximadamente 30 vezes superior a DQO do esgoto doméstico indicando um consumo bastante elevado de oxigênio devido não só da atividade poluidora do despejo de manipueira, mas provavelmente a outros agentes contaminantes, sendo resultado da atividade de organismos de respiração aeróbica que metabolizam o substrato orgânico acumulando uma fração de glicose que demanda oxigênio do meio para oxidação.

Para Barana (2008), o potencial poluente da manipueira deve-se a carga orgânica que pode chegar a 100 g DQO.L-1. A maior parte da matéria orgânica da manipueira é devida à presença de açúcares solúveis. Este autor determinou os teores dos açúcares glicose e frutose em 10 lotes de manipueira da Farinheira Plaza em Santa Maria da Serra, SP. Em seus resultados a manipueira chegou a apresentar $14,7 \mathrm{~g}$ de glicose.L-1 que correspondeu a $29 \%$ da matéria orgânica presente e $22 \mathrm{~g}$ de frutose.L-1 equivalente a $42 \%$ da matéria orgânica. Por serem açúcares solúveis de fácil fermentação, degradam-se rapidamente a ácidos orgânicos. Esta característica inviabiliza o tratamento deste resíduo por processos físicos.

Segundo Helbig et al. (2008) a toxicidade dos glicosídeos presentes na mandioca foi, primeiramente, relatada no século XVII, no entanto apenas no século XIX houve o primeiro relato de uma intoxicação causada por glicosídeos cianogênicos. Desde esta época são descritos na literatura vários casos de intoxicação e morte devido à ingestão de mandioca e seus derivados.

Ramirez (2010) pesquisando na literatura os efeitos tóxicos do ácido cianídrico sobre humanos e animais, afirmou que mesmo em baixas doses são compostos letais em um tempo mínimo de exposição sendo o sistema nervoso o principal órgão alvo. Após a ingestão, inalação ou contacto os efeitos neurotóxicos são graves e fatais em humanos e animais. A exposição ocupacional produz tireoide, dores de cabeça, tontura, náusea, vômito, dermatites e a alta exposição, e num tempo curto pode levar a insuficiência respiratória e morte. Com 
SANTOS, G. P.; REGO, N. A. C.; SANTOS, J. W. B.; DELANO JÚNIOR, F.; SILVA JÚNIOR, M. F. Avaliação espaço-temporal dos parâmetros de qualidade da água do rio Santa Rita (BA) em função do lançamento de manipueira. Ambi-Agua, Taubaté, v. 7, n. 3, p. 261-278, 2012. (http://dx.doi.org/10.4136/ambiagua.880)

relação à carcinogenicidade, o cianeto é considerado no grupo D (não classificável como carcinogéneo humano).

Segundo Sant'Ana e Domene (2008) já se observou associação entre dietas ricas em mandioca pouco processada e a ocorrência de alterações neurológicas causadas pela presença de glicosídeos cianogênicos (GC) de ocorrência natural.

Neste contexto, o trabalho presente objetiva avaliar os possíveis impactos da manipueira em parâmetros de qualidade da água do rio Santa Rita.

\section{METODOLOGIA}

A bacia hidrográfica do rio Santa Rita pertencente à bacia do Rio Pardo segundo a Superintendência de Recursos Hídricos (Bahia, 2004), engloba os povoados de Simão e Campinhos (Figura 1). O bairro de Campinhos está entre os maiores beneficiadores de mandioca na região, gerando empregos diretos, além dos indiretos em função da lavoura e comercialização dos produtos oriundos da mandioca. A comunidade fica distanciada do centro da cidade de Vitória da Conquista por aproximadamente $9 \mathrm{~km}$.

Segundo Rocha e Barros (2010) a precipitação média anual varia de 800 a $1.500 \mathrm{~mm}$ e a temperatura média anual varia de $21,5^{\circ}$ a $24^{\circ} \mathrm{C}$ A população dos dois povoados é de aproximadamente 6000 habitantes existindo cerca de 150 casas de farinha na região (Rocha e Barros, 2010).

O clima da região varia de semiárido a úmido de acordo com a tipologia climática de Thornthwaite. A vegetação característica é bastante variada, ocorrendo desde floresta perenifólia (mata atlântica), floresta caducifólia e sub-caducifólia, caatinga e cerrado. Com o avanço dos processos de ocupação, observa-se uma grande predominância de áreas ocupadas com pastagens, fruticultura e culturas de subsistência, como milho, feijão e mandioca.

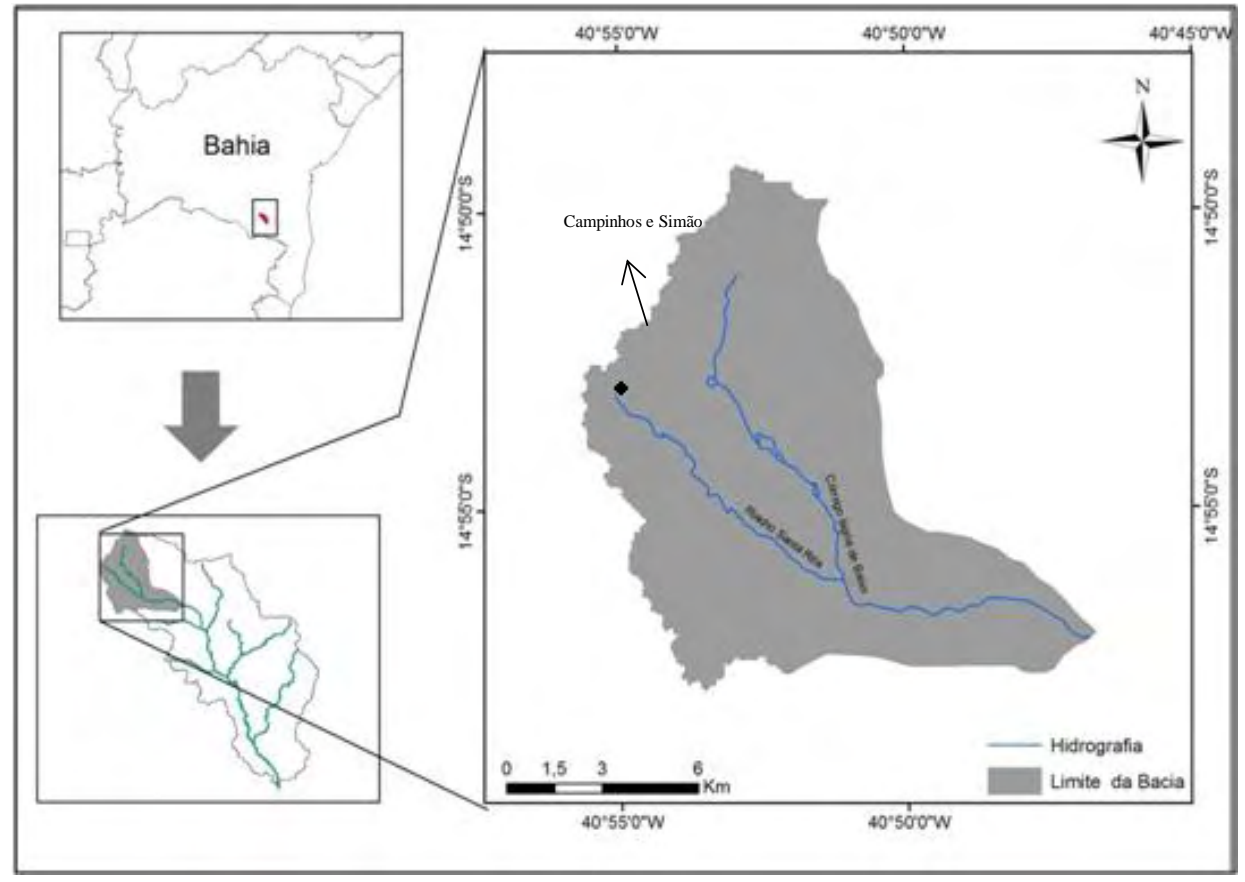

Figura 1. Bacia hidrográfica do rio Santa Rita.

O delineamento da bacia hidrográfica do rio Santa Rita e o mapa de uso do solo foram feitos utilizando-se o Sistema de Informações Geográficas (SIG) ArcView GIS versão 9.2. 
SANTOS, G. P.; REGO, N. A. C.; SANTOS, J. W. B.; DELANO JÚNIOR, F.; SILVA JÚNIOR, M. F. Avaliação espaço-temporal dos parâmetros de qualidade da água do rio Santa Rita (BA) em função do lançamento de manipueira. Ambi-Agua, Taubaté, v. 7, n. 3, p. 261-278, 2012. (http://dx.doi.org/10.4136/ambiagua.880)

A caracterização fisiográfica da bacia hidrográfica foi realizada a partir da determinação do coeficiente de compacidade (Kc), fator de forma (Kf) e densidade de drenagem (Dd), baseado em Bahia (2000).

Os pontos amostrais para as coletas de água foram escolhidos de forma a cobrir longitudinalmente o rio Santa Rita (Figura 2) sendo assim dispostos: ponto P1 localizado na nascente do rio Santa Rita e tem como comunidades ribeirinhas os núcleos comunitários de Campinhos e Simão; P2 à montante da Estação de Tratamento de Esgoto (ETE) da Embasa, logo após o Condomínio Caminho do Parque, localizado no bairro Recreio; P3 em uma área de pastagem muito próximo a rodovia e sem a presença de mata ciliar e o P4, próximo a cidade de Vitória da Conquista e do encontro com o rio Verruga.

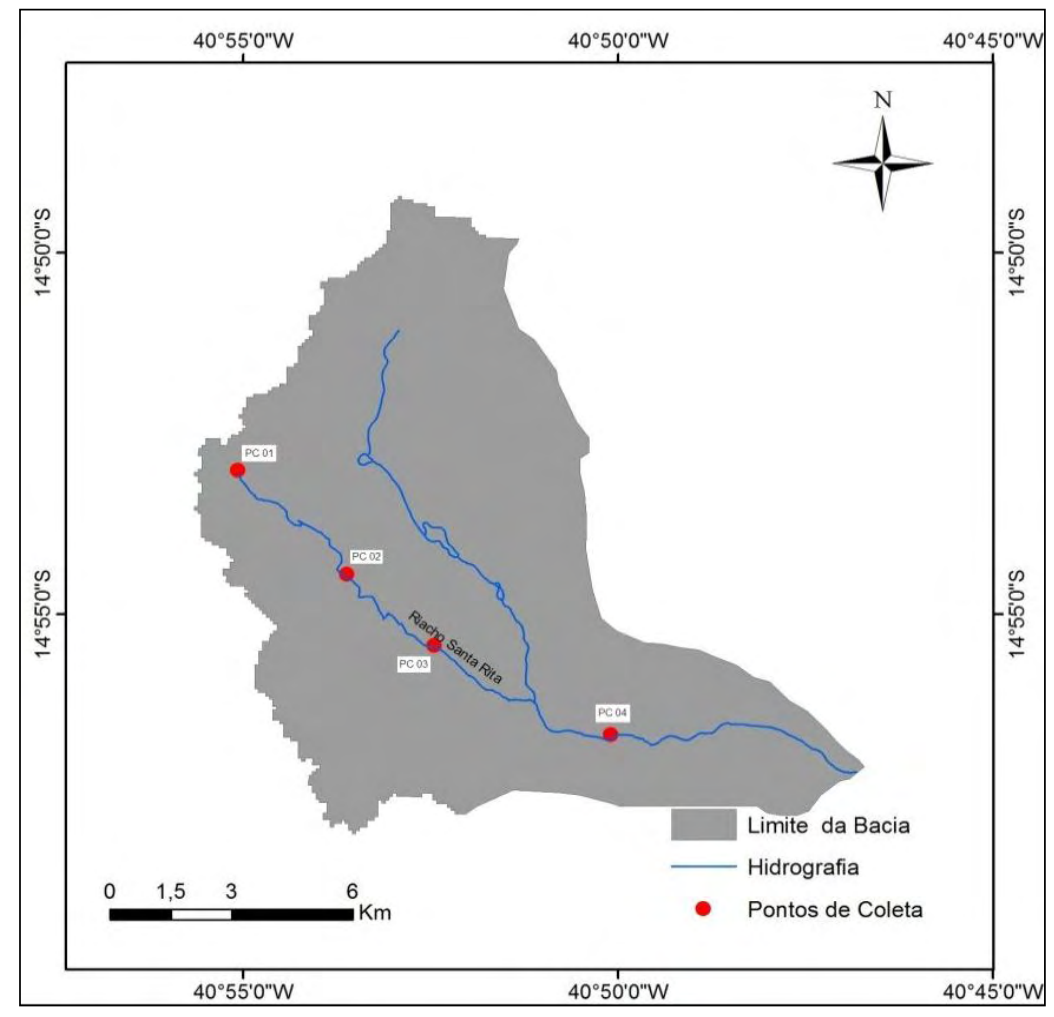

Figura 2. Localização dos pontos de coleta.

As coletas das amostras de água foram feitas utilizando-se recipientes plásticos com volume de 2 L, em 6 datas identificadas como C1 (13/07/2011), C2 (.08/08/2011), C3 (29/09/2011), C4 (24/10/2011), C5 (29/11/2011) e C6 (24/01/2012).

Foram utilizadas caixas de isopor com gelo para acondicionamento das amostras até que fossem conduzidas ao Laboratório de Análise de Água da UESC (Universidade Estadual de Santa Cruz) onde foram refrigeradas a $4^{\circ} \mathrm{C}$ e realizadas as análises num prazo não superior a 24 horas

No Laboratório de Análise de Água da UESC foram realizadas as análises das concentrações de fósforo, amônia, nitrato e nitrito utilizando-se o espectrômetro de ultravioleta. A dosagem das concentrações de ferro, zinco, cobre, manganês e sulfato foram realizadas no Laboratório de Química Analítica da UESC utilizando-se o espectrofotômetro de absorção atômica. Todas as análises foram realizadas segundo a metodologia estabelecida pelo Standard Methods for the Examination for Waterand Wastewater - 20th ed. (APHA et al., 1995). 
SANTOS, G. P.; REGO, N. A. C.; SANTOS, J. W. B.; DELANO JÚNIOR, F.; SILVA JÚNIOR, M. F. Avaliação espaço-temporal dos parâmetros de qualidade da água do rio Santa Rita (BA) em função do lançamento de manipueira. Ambi-Agua, Taubaté, v. 7, n. 3, p. 261-278, 2012. (http://dx.doi.org/10.4136/ambiagua.880)

Em cada um dos pontos selecionados foram realizadas as avaliações da salinidade, condutividade elétrica, oxigênio dissolvido e sólidos totais dissolvidos utilizando-se o equipamento multi-parâmetro HORIBA.

\section{RESULTADOS E DISCUSSÃO}

Os valores referentes às características físicas da bacia hidrográfica do rio Santa Rita, e os índices fisiográficos obtidos estão apresentados na Tabela 1.

Tabela 1. Características físicas da bacia hidrográfica do rio Santa Rita.

\begin{tabular}{l|c}
\hline \multicolumn{2}{c}{ Características físicas } \\
\hline Área de drenagem (A) & $126,84 \mathrm{~km}^{2}$ \\
Perímetro total (P) & $66,65 \mathrm{~km}$ \\
Comprimento do rio principal (L) & $19,63 \mathrm{~km}$ \\
Comprimento total dos cursos de água (Lt) & $34,3 \mathrm{~km}$ \\
Desnível máximo & $900,00 \mathrm{~m}$ \\
Coeficiente de Compacidade (Kc) & 1,65 \\
Fator de Forma (Kf) & 0,33 \\
Densidade de Drenagem (Dd) & $0,27 \mathrm{~km} \cdot \mathrm{km}^{-2}$ \\
\hline
\end{tabular}

A bacia hidrográfica do rio Santa Rita possui uma área de $126,84 \mathrm{~km}^{2}$. O comprimento do canal principal é de $19,63 \mathrm{~km}$ com uma rede de drenagem total de $34,3 \mathrm{~km}$. A densidade de drenagem é de $0,27 \mathrm{~km} / \mathrm{km}^{2}$, indicando uma baixa capacidade de drenagem, apresentando relevo pouco declivoso com rampas longas.

Christofoletti (1969) destaca que valores menores que $7,5 \mathrm{~km} / \mathrm{km}^{2}$ indicam baixa densidade de drenagem. Valores entre 7,5 e $10,0 \mathrm{~km} / \mathrm{km}^{2}$ indicam média densidade. Já valores acima de $10,0 \mathrm{~km} / \mathrm{km}^{2}$, indicam alta densidade hidrográfica.

A densidade de drenagem é um fator importante na indicação do grau de desenvolvimento do sistema de drenagem de uma bacia. Sendo assim, este índice, fornece uma indicação da eficiência da drenagem da bacia, sendo expressa pela relação entre o somatório dos comprimentos de todos os canais da rede; sejam eles perenes, intermitentes ou temporários e a área total da bacia (Antoneli e Thomaz, 2007).

Já de acordo com Villela e Mattos (1975), esse mesmo índice (densidade de drenagem), pode variar de $0,5 \mathrm{~km} / \mathrm{km}^{2}$ em bacias com drenagem pobre, a $3,5 \mathrm{~km} / \mathrm{km}^{2}$, ou mais, em bacias bem drenadas, indicando, também, que a bacia em estudo possui baixa capacidade de drenagem.

A bacia em estudo possui forma alongada, aproximando-se do retângulo com $\mathrm{Kc}>1,25 \mathrm{e}$ Kf < 0,5 (Tonello et al., 2006; Périco et al., 2011) apresentando menor possibilidade de enchentes rápidas em condições normais de precipitação.

O mapa de uso do solo é apresentado na Figura 3, no qual se pode observar que a área com ocupação urbana representa aproximadamente $32 \%$ da área total da bacia hidrográfica incluindo os povoados de Campinhos e Simão e parte da cidade de Vitória da Conquista. Observa-se também que o entorno da área com ocupação urbana sendo utilizado basicamente com pastagem e algumas áreas com solo exposto. A região abrangida pelo baixo curso do rio Santa Rita é a que apresenta o maior percentual de áreas ocupadas com florestas e onde está localizado o ponto de coleta 4. A Tabela 2 apresenta informações referentes aos diversos usos. 
SANTOS, G. P.; REGO, N. A. C.; SANTOS, J. W. B.; DELANO JÚNIOR, F.; SILVA JÚNIOR, M. F. Avaliação espaço-temporal dos parâmetros de qualidade da água do rio Santa Rita (BA) em função do lançamento de manipueira. Ambi-Agua, Taubaté, v. 7, n. 3, p. 261-278, 2012. (http://dx.doi.org/10.4136/ambiagua.880)

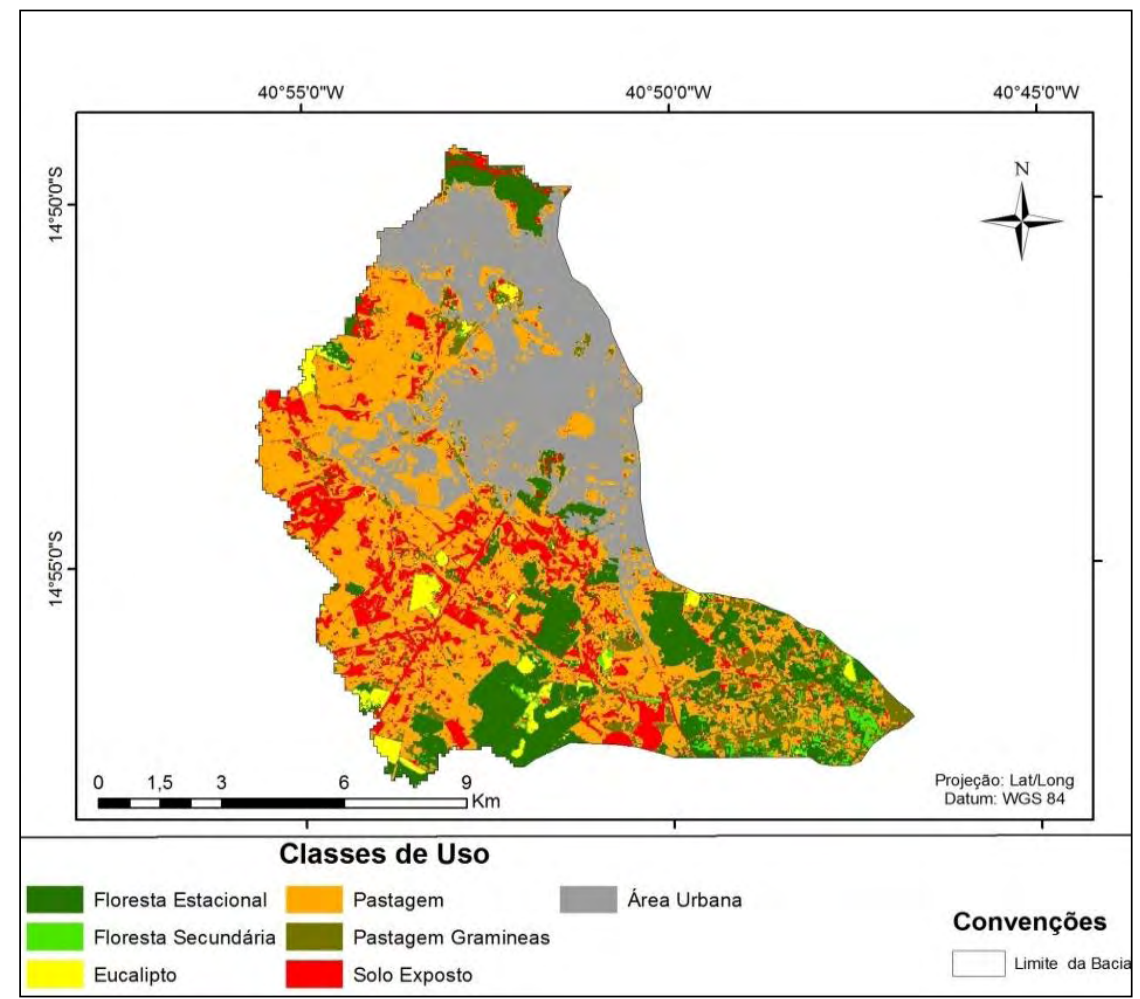

Figura 3. Mapa de uso do solo.

Tabela 2. Classes de uso do solo.

\begin{tabular}{l|cr}
\hline Classes de Uso do Solo & Área $\left(\mathbf{k m}^{\mathbf{2}}\right)$ & Área $\mathbf{( \% )}$ \\
\hline Pastagem & 47,45 & 37,41 \\
Pastagem gramínea & 7,55 & 5,95 \\
Floresta & 19,57 & 15,43 \\
Floresta secundária & 1,99 & 1,57 \\
Solo exposto & 15,29 & 12,05 \\
Eucalipto & 3,00 & 2,37 \\
Área urbana & 31,99 & 25,22 \\
\hline Total & $\mathbf{1 2 6 , 8 4}$ & $\mathbf{1 0 0 , 0 0}$ \\
\hline
\end{tabular}

A média, erro padrão e desvio padrão para os valores de oxigênio dissolvido (OD) obtidos nos pontos de coleta estão apresentados na Figura 4, na qual se pode observar que somente o ponto $\mathrm{P} 4$ apresentou valores médios superiores a $4 \mathrm{mg} . \mathrm{L}^{-1}$ e portanto podendo ser enquadrado como classe III pela Resolução CONAMA 357/2005 (Brasil, 2005).

Para os pontos de coleta P1, P2 os valores médios de OD observados indicam uma forte poluição, tais resultados estão associados ao potencial poluente da manipueira em virtude de sua carga orgânica, cuja principal origem é a atividade econômica desenvolvida nesse trecho da bacia hidrográfica, que é o beneficiamento da mandioca. Muito embora o ponto de coleta P3 tenha apresentado valores inferiores a $4 \mathrm{mg} . \mathrm{L}^{-1}$ pode-se observar que há um sensível aumento nos valores de $\mathrm{OD}$, sendo que, para o ponto de coleta $\mathrm{P} 4 \mathrm{o}$ valores observados foram superiores a $4 \mathrm{mg} . \mathrm{L}^{-1}$, sugerindo desta forma que mecanismos de aeração contribuam para a elevação dos níveis de oxigênio dissolvido. 
SANTOS, G. P.; REGO, N. A. C.; SANTOS, J. W. B.; DELANO JÚNIOR, F.; SILVA JÚNIOR, M. F. Avaliação espaço-temporal dos parâmetros de qualidade da água do rio Santa Rita (BA) em função do lançamento de manipueira. Ambi-Agua, Taubaté, v. 7, n. 3, p. 261-278, 2012. (http://dx.doi.org/10.4136/ambiagua.880)

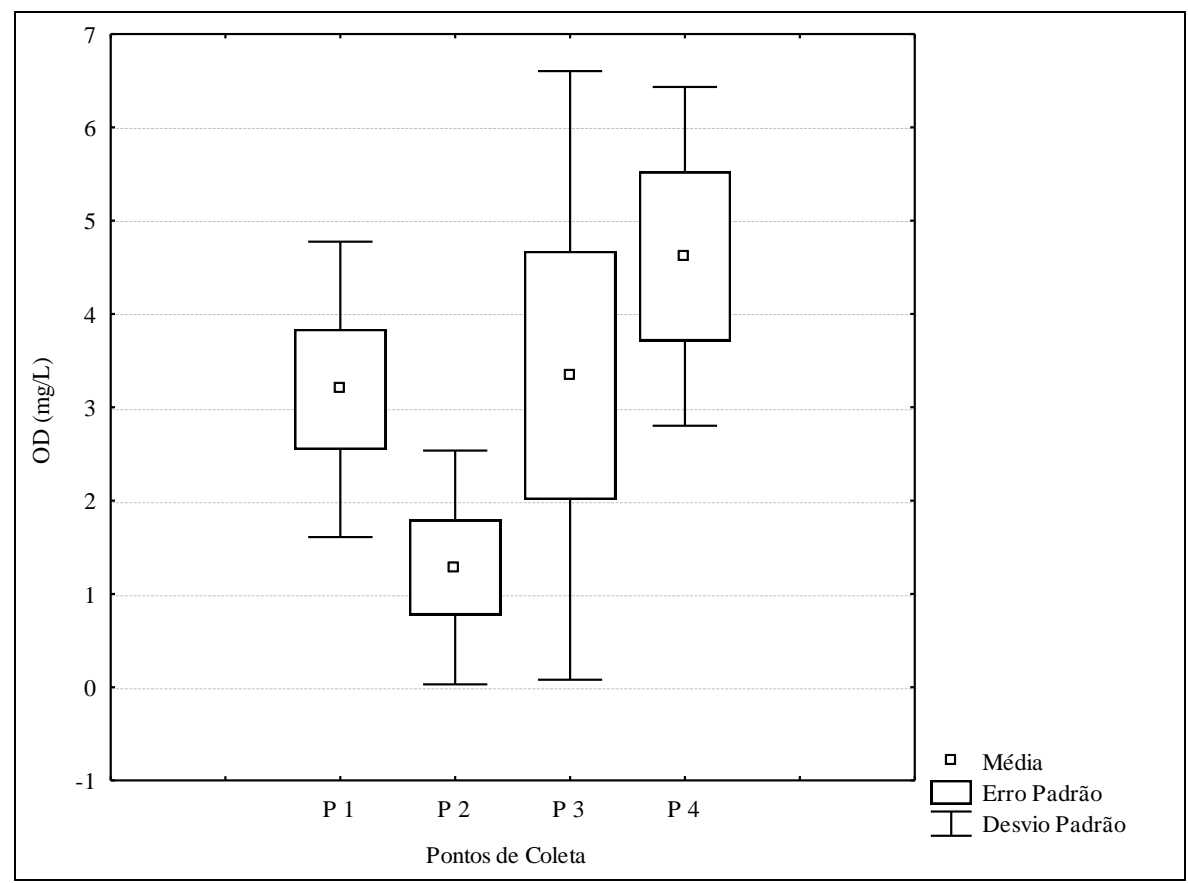

Figura 4. Média, erro padrão e desvio padrão de OD, para os diferentes pontos de coleta.

Os valores médios da condutividade elétrica (CE) obtidos nas coletas estão apresentados na Figura 5. Conforme pode ser observado, os valores médios obtidos para os pontos P1, P2 e P3 indicam um ambiente fortemente impactado (CETESB, 2007) pelo lançamento de efluentes.

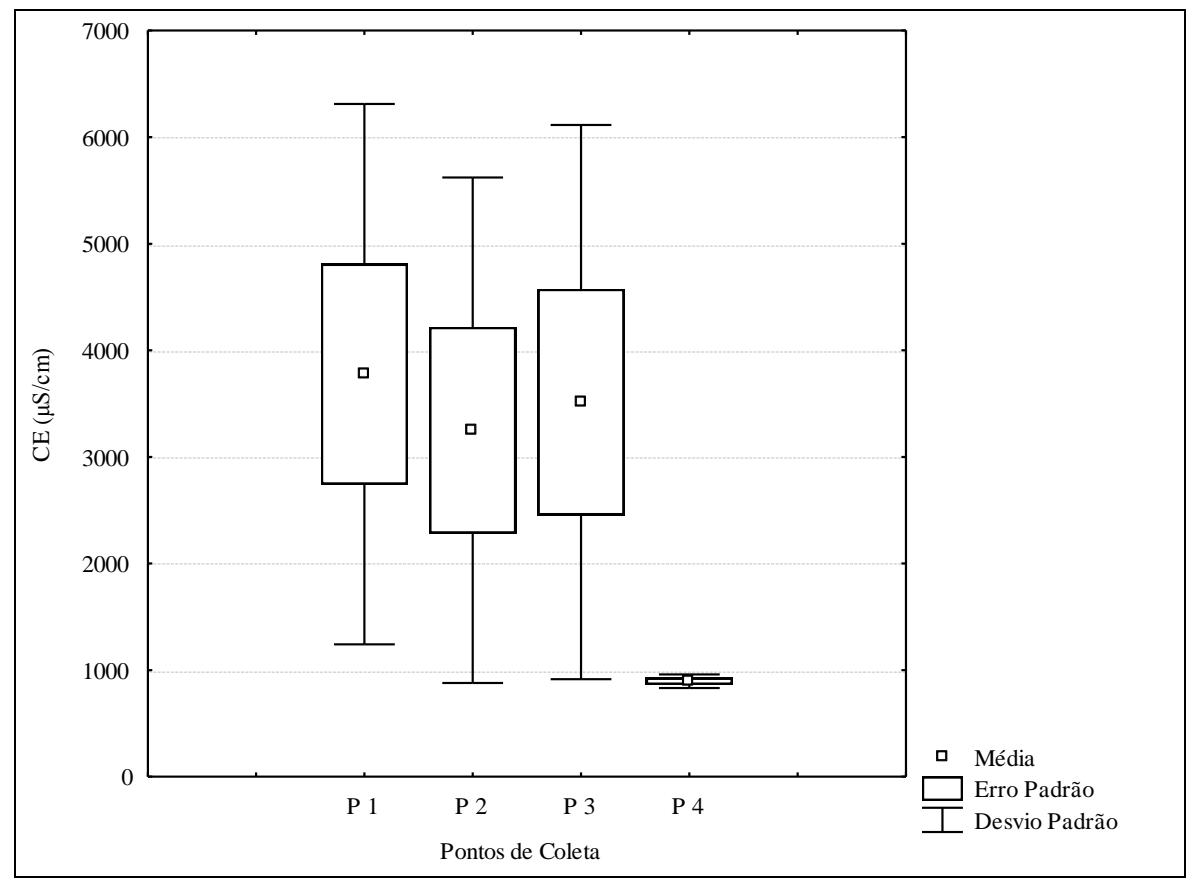

Figura 5. Média, erro padrão e desvio padrão de CE, para os diferentes pontos de coleta. 
SANTOS, G. P.; REGO, N. A. C.; SANTOS, J. W. B.; DELANO JÚNIOR, F.; SILVA JÚNIOR, M. F. Avaliação espaço-temporal dos parâmetros de qualidade da água do rio Santa Rita (BA) em função do lançamento de manipueira. Ambi-Agua, Taubaté, v. 7, n. 3, p. 261-278, 2012. (http://dx.doi.org/10.4136/ambiagua.880)

Vale ressaltar que a alta amplitude nos valores de condutividade elétrica, possivelmente estejam associados aos aspectos sazonais da atividade produtiva local, ou seja, períodos onde ocorrem uma maior intensidade no beneficiamento da mandioca potencializando uma maior carga de poluente para as águas do rio, elevando desta forma os teores de sais e matéria orgânica. Apesar de também bastante elevada, a média obtida para o ponto P4 mostra melhoria nas condições realtivas a quantidade de sais existentes na coluna d'água.

A Figura 6 mostra os valores da média, erro padrão e desvio padrão da salinidade nos 4 pontos de coleta, podendo-se observar a maior média no ponto de P1 e a menor no ponto P4. Em relação aos valores estabelecidos pela Resolução CONAMA 357/2005, a água coletada no ponto de P4 é classificada como doce e para os demais locais classificada como salobra.

Nos pontos P1, P2 e P3 a elevada salinidade pode restringir a utilização da água para abastecimento doméstico devido aos possíveis agravos à saúde da população, irrigação pela possibilidade de salinização dos solos e uso industrial pelas características de corrosão e incrustação (Rego et al., 2009)

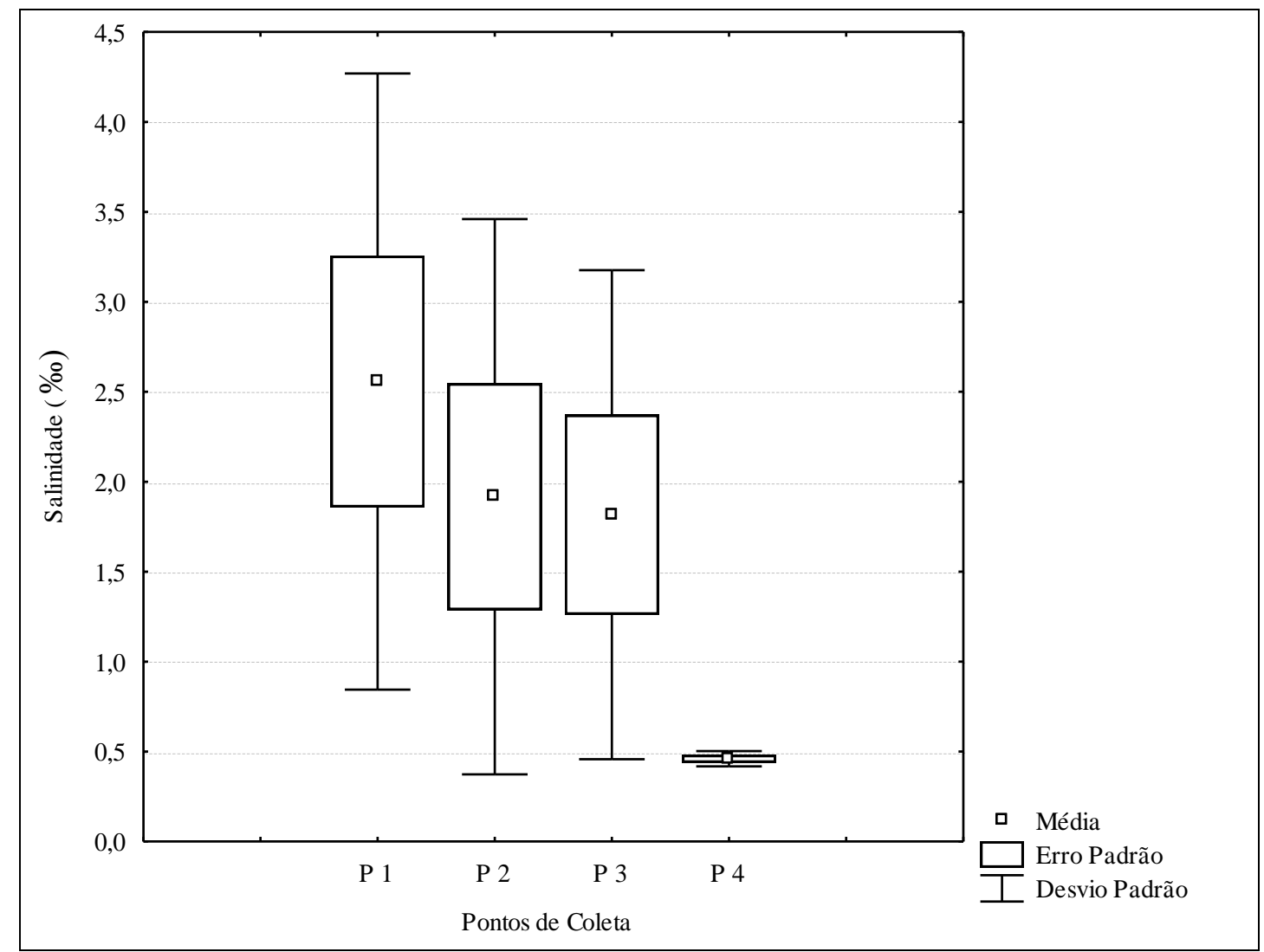

Figura 6. Média, erro padrão e desvio padrão da salinidade, para os diferentes pontos de coleta.

Em relação à concentração de sólidos totais dissolvidos (STD) e os 4 pontos amostrais, somente o ponto P4 apresentou valores médios inferiores a $500 \mathrm{mg} . \mathrm{L}^{-1}\left(469 \mathrm{mg} . \mathrm{L}^{-1}\right)$ sendo que a maior média foi a do ponto P1 e as concentrações decrescendo a jusante deste ponto (Figura 7), indicando o lançamento de efluentes neste ponto com alta carga de constituintes minerais.

De acordo com a Resolução CONAMA 357/2005, o limite máximo para o STD é de $500 \mathrm{mg} . \mathrm{L}^{-1}$ para as classes I, II e III 
SANTOS, G. P.; REGO, N. A. C.; SANTOS, J. W. B.; DELANO JÚNIOR, F.; SILVA JÚNIOR, M. F. Avaliação espaço-temporal dos parâmetros de qualidade da água do rio Santa Rita (BA) em função do lançamento de manipueira. Ambi-Agua, Taubaté, v. 7, n. 3, p. 261-278, 2012. (http://dx.doi.org/10.4136/ambiagua.880)

Os valores médios de STD e sua concentração ao longo do rio Santa Rita obtidos neste trabalho são diferentes dos apresentados por Lage (2011) que estudando o rio Piabanha (BA) encontrou valores que variaram de 78,01 a $302,29 \mathrm{mg} . \mathrm{L}^{-1}$ observando crescentes concentrações de STD ao longo do rio. Fernandes (2011), avaliando a qualidade da água no trecho urbano do rio de Contas também encontrou valores médios muito inferiores aos obtidos para o rio Santa Rita (de 219 a $296 \mathrm{mg} \cdot \mathrm{L}^{-1}$ ).

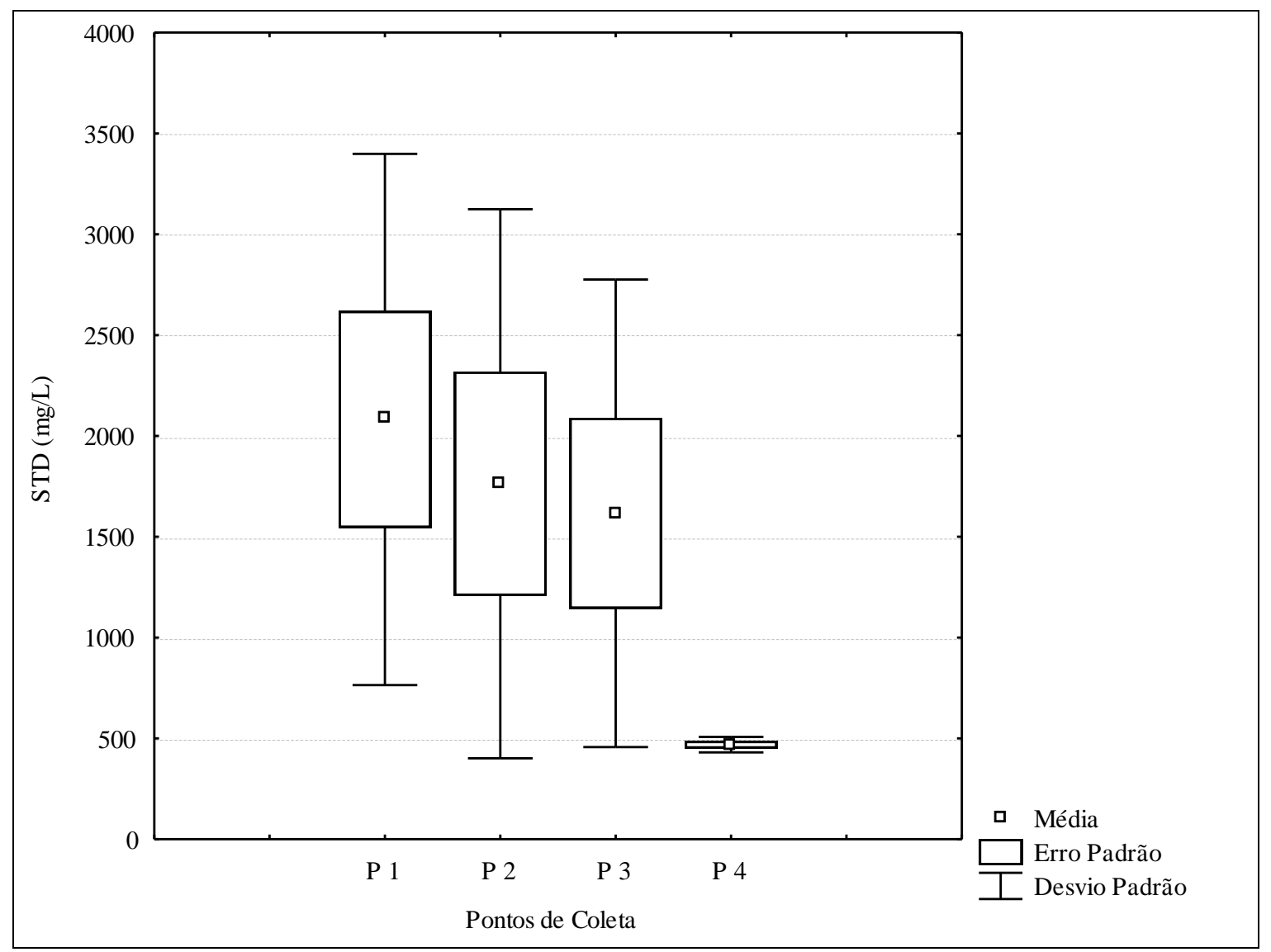

Figura 7. Média, erro padrão e desvio padrão do STD, para os diferentes Pontos de coleta.

A Figura 8 apresenta os valores obtidos para a concentração de fósforo total nos pontos de coleta, podendo-se observar as maiores médias nos pontos P1 e P3. Em face aos resultados encontrados a origem do fósforo nas águas do rio Santa Rita vêm do lançamento de efluentes ricos em matéria orgânica dos núcleos comunitários de Campinhos e Simão onde localiza-se o ponto de coleta P1 e da carga orgânica das atividade agrícolas como fertilizantes e dejetos de origem animal, pois o ponto de coleta P3 está localizado em uma região da atividade agropastoril.

Considerando a Resolução CONAMA 357/2005 e os limites estabelecidos para águas doces, os pontos P1 e P3 classificam-se como de classe IV e os pontos P2 e P4 como de classe I. Quando a salinidade da água é considerada e sendo os pontos P1, P2 e P3 classificados como de água salobra, os limites estabelecidos pela Resolução CONAMA 357/2005 indicam o ponto P1 como de classe II (limite de $0,186 \mathrm{mg} . \mathrm{L}^{-1}$ ), o ponto P2 de classe I (limite de $0,124 \mathrm{mg} . \mathrm{L}^{-1}$ ) e o ponto P3 como de Classe III (concentração média maior que $0,186 \mathrm{mg} . \mathrm{L}^{-1}$ ). 
SANTOS, G. P.; REGO, N. A. C.; SANTOS, J. W. B.; DELANO JÚNIOR, F.; SILVA JÚNIOR, M. F. Avaliação espaço-temporal dos parâmetros de qualidade da água do rio Santa Rita (BA) em função do lançamento de manipueira. Ambi-Agua, Taubaté, v. 7, n. 3, p. 261-278, 2012. (http://dx.doi.org/10.4136/ambiagua.880)

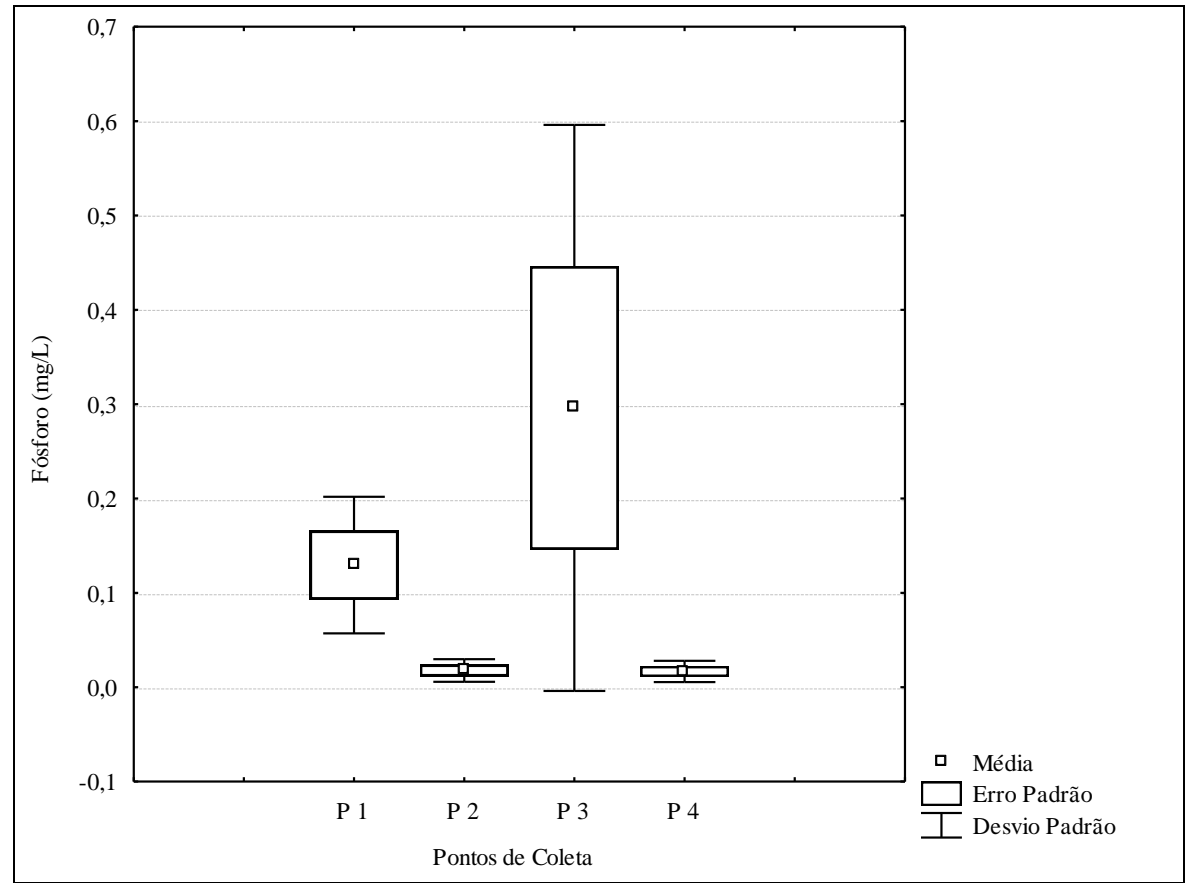

Figura 8. Média, erro padrão e desvio padrão do fósforo, para os diferentes pontos de coleta.

A Figura 9 mostra os valores médios obtidos da concentração de ferro, sendo observada para o rio Santa Rita, a maior média no ponto P3, provavelmente devido aos efeitos dos efluentes da ETE e ao escoamento de fertilizantes da área de agricultura nas proximidades do ponto de coleta (Lima et al., 2001).

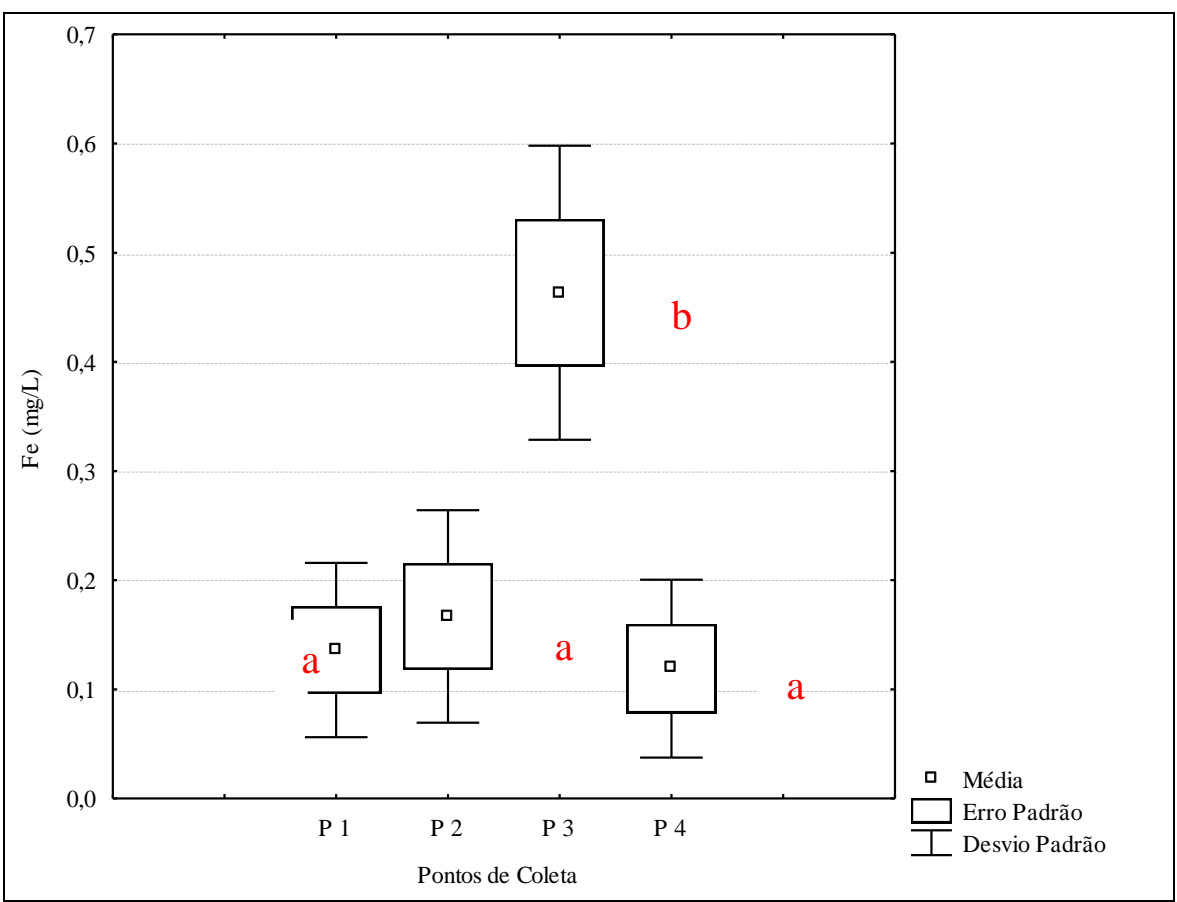

Figura 9. Média, erro padrão e desvio padrão de ferro, para os diferentes pontos de coleta.

Nota: Letras iguais significam a não existências de diferenças estatísticas significativas a $5 \%$ de probabilidade. 
SANTOS, G. P.; REGO, N. A. C.; SANTOS, J. W. B.; DELANO JÚNIOR, F.; SILVA JÚNIOR, M. F. Avaliação espaço-temporal dos parâmetros de qualidade da água do rio Santa Rita (BA) em função do lançamento de manipueira. Ambi-Agua, Taubaté, v. 7, n. 3, p. 261-278, 2012. (http://dx.doi.org/10.4136/ambiagua.880)

Considerando-se os limites da Resolução CONAMA 357/2005, somente o ponto P3 (0,46 mg. $\left.\mathrm{L}^{-1}\right)$ ultrapassa os limites da classe II, enquadrando-se na classe III.

Assim como o observado para o ferro, a Figura 10 mostra que a concentração de cobre também foi maior no ponto P3, possivelmente, esses valores tenham a mesma origem observada nos teores de fósforo, onde as atividades agrícolas estejam contribuindo para esse valor diferenciado em relação aos demais pontos de coleta, pois, escoamento de fungicidas e/ou pesticidas da área de agricultura podem contribuir para elevação de metais nas águas superficiais (Philippi et al., 2004). Como as concentrações médias em todos os pontos foram inferiores a $0,09 \mathrm{mg} . \mathrm{L}^{-1}$, todos os 4 pontos são classificados como classe I, de acordo com a Resolução CONAMA 357/2005.

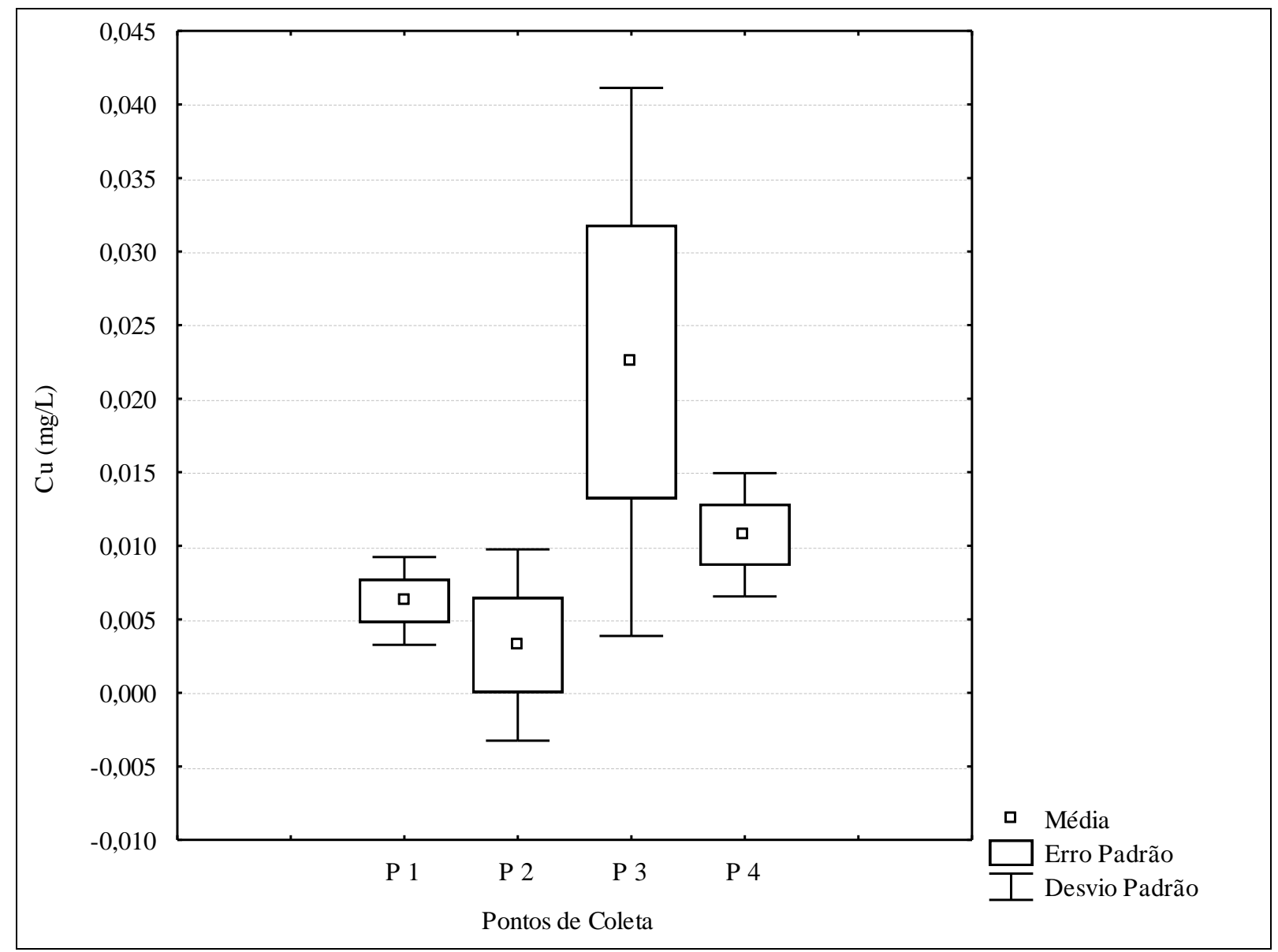

Figura 10. Média, erro padrão e desvio padrão de cobre, para os diferentes pontos de coleta.

A Figura 11 mostra as concentrações médias obtidas para o zinco nos pontos de coleta. Observa-se na referida figura as maiores médias no ponto P1 localizado próximo aos núcleos de Campinhos e Simão, provavelmente, devido a queima de madeira, lançamento de efluentes domésticos e incineração de resíduos (Philippi et al., 2004).

De acordo com a Resolução CONAMA 357/2005 todos os pontos de coleta são enquadrados como de classe I (concentração média menor que $0,18 \mathrm{mg} . \mathrm{L}^{-1}$ ). 
SANTOS, G. P.; REGO, N. A. C.; SANTOS, J. W. B.; DELANO JÚNIOR, F.; SILVA JÚNIOR, M. F. Avaliação espaço-temporal dos parâmetros de qualidade da água do rio Santa Rita (BA) em função do lançamento de manipueira. Ambi-Agua, Taubaté, v. 7, n. 3, p. 261-278, 2012. (http://dx.doi.org/10.4136/ambiagua.880)

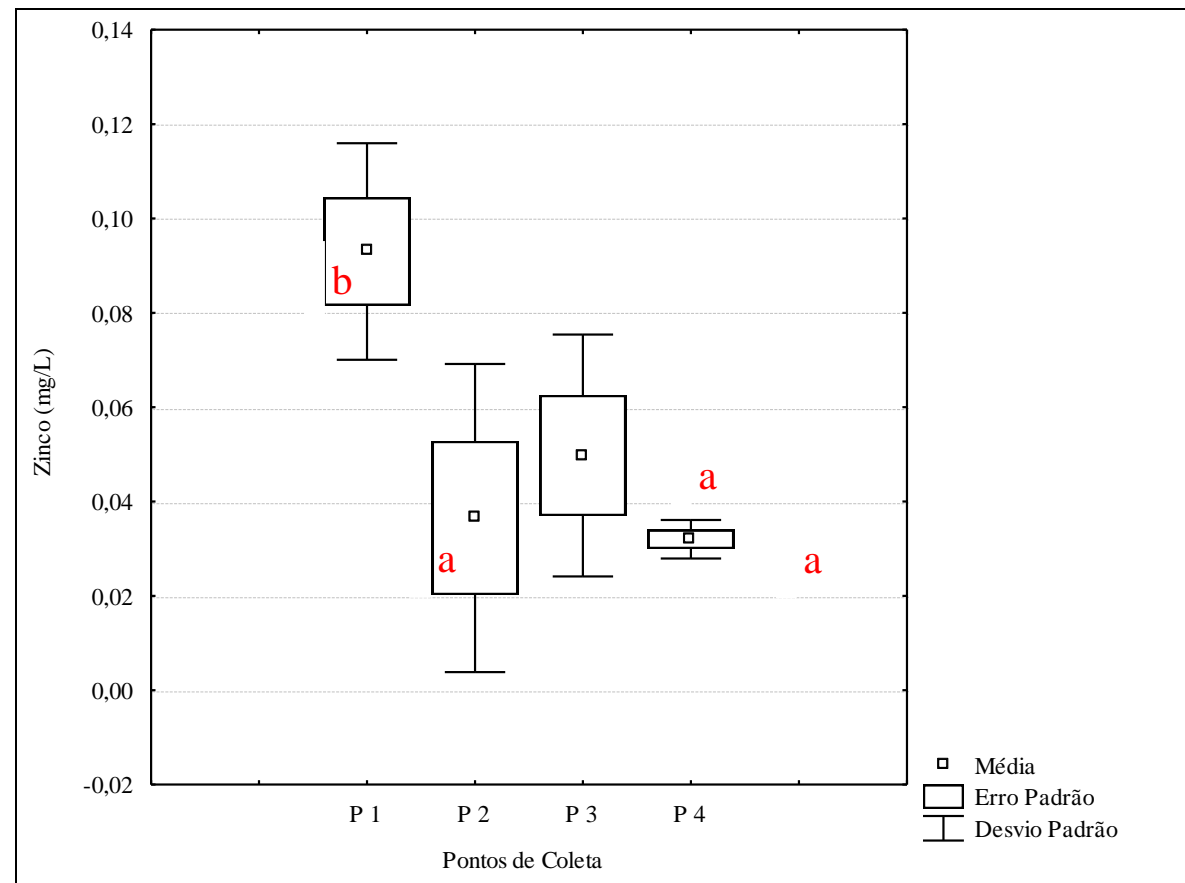

Figura 11. Média, erro padrão e desvio padrão de zinco, para os diferentes pontos de coleta.

A Figura 12 mostra a média, erro padrão e desvio padrão da concentração de amônia nos 4 pontos de coleta, podendo-se observar os maiores valores nos pontos P1 e P2 devido a degradação da matéria orgânica lançada no rio Santa Rita nestas localidades.

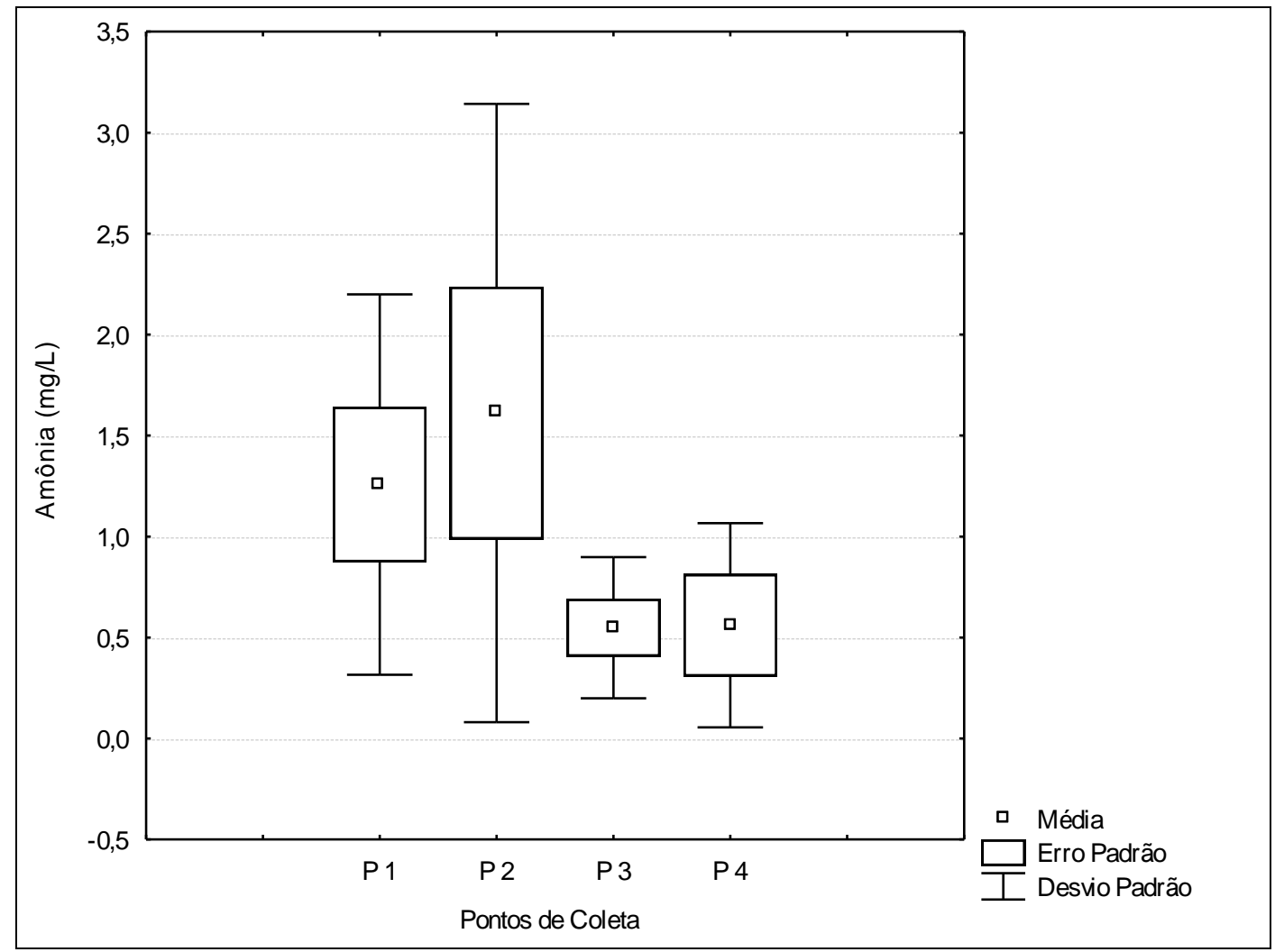

Figura 12. Média, erro padrão e desvio padrão de amônia, para os diferentes pontos de coleta. 
SANTOS, G. P.; REGO, N. A. C.; SANTOS, J. W. B.; DELANO JÚNIOR, F.; SILVA JÚNIOR, M. F. Avaliação espaço-temporal dos parâmetros de qualidade da água do rio Santa Rita (BA) em função do lançamento de manipueira. Ambi-Agua, Taubaté, v. 7, n. 3, p. 261-278, 2012. (http://dx.doi.org/10.4136/ambiagua.880)

O nitrogênio amoniacal é a forma mais reduzida do nitrogênio e é o primeiro composto produzido na degradação da matéria orgânica (A.P.H.A, 1995). Muito embora a amônia orgânica seja toxica, é mais volátil, contudo, os valores médios obervados nos pontos de coleta $\mathrm{P} 1$ e $\mathrm{P} 2$, acima de $1,0 \mathrm{mg} / \mathrm{L}$, indicam um sistema altamente poluído que associado aos níveis de oxigênio e metais pesados potencializam a sua carga tóxica.

A Figura 13 apresenta os valores da média, do erro padrão e desvio padrão das concentrações de nitrito nos 4 pontos de coleta podendo-se observar o maior valor do ponto P1 e o decréscimo nos pontos P2, P3 e P4. Este comportamento deve-se provavelmente ao fato do despejo de matéria orgânica no rio que ocorre nos núcleos de Campinhos e Simão. Concentrações elevadas de nitrito afetam a concentração dos glóbulos vermelhos do sangue reduzindo a capacidade respiratória dos organismos aquáticos podendo provocar a morte dos mesmos por asfixia.

Em relação à Resolução CONAMA 357/2005, a concentração média em todos os pontos foi inferior a $1,0 \mathrm{mg} . \mathrm{L}^{-1}$ e portanto enquadrados como classe I.

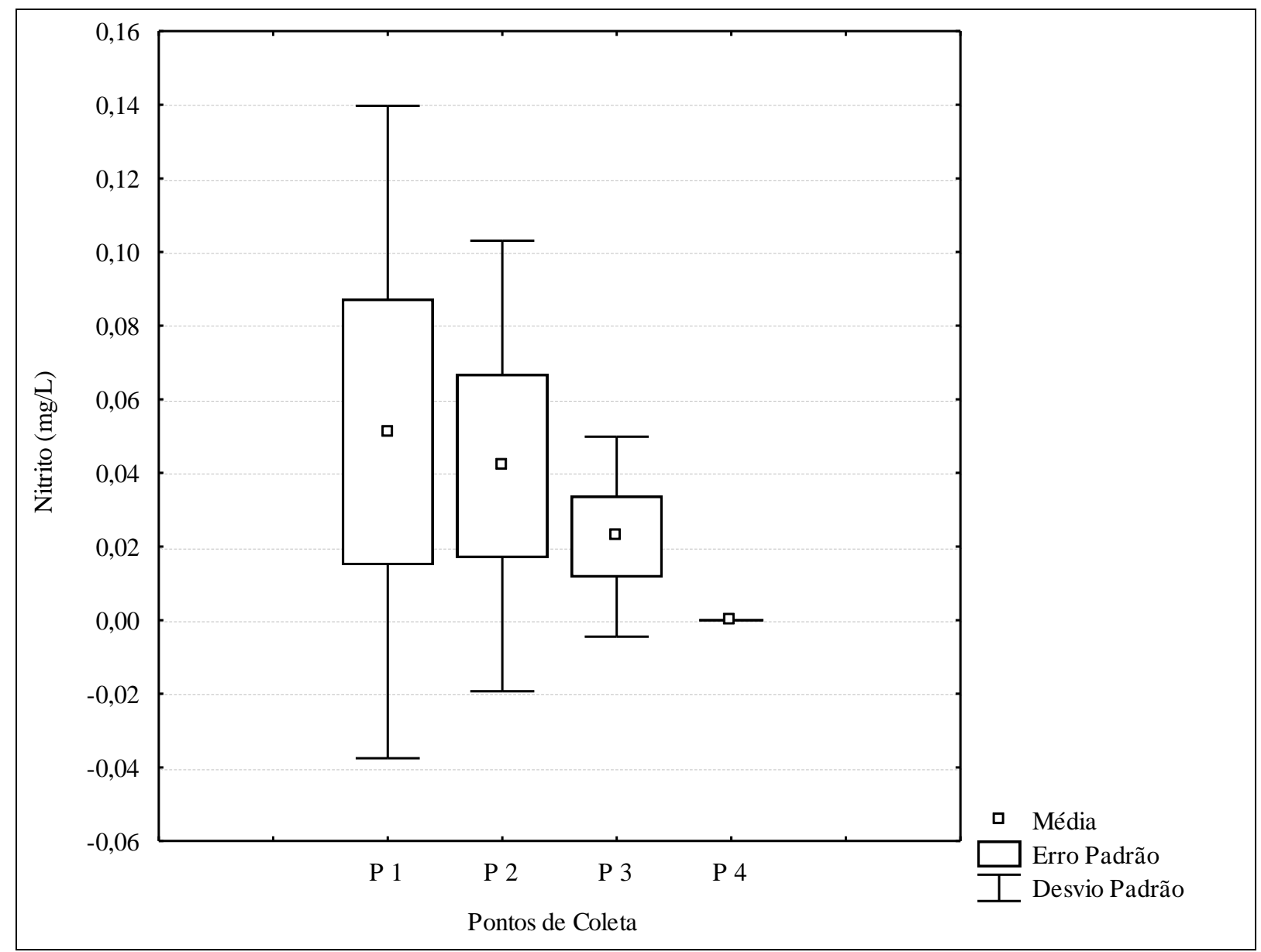

Figura 13. Média, erro padrão e desvio padrão de nitrito, para os diferentes pontos de coleta.

A Figura 14 apresenta os valores das concentrações médias de nitrato nos pontos amostrados podendo-se observar o maior valor do ponto P1 e o decréscimo nos pontos P2, P3 e ligeiro acréscimo no ponto P4. 
SANTOS, G. P.; REGO, N. A. C.; SANTOS, J. W. B.; DELANO JÚNIOR, F.; SILVA JÚNIOR, M. F. Avaliação espaço-temporal dos parâmetros de qualidade da água do rio Santa Rita (BA) em função do lançamento de manipueira. Ambi-Agua, Taubaté, v. 7, n. 3, p. 261-278, 2012. (http://dx.doi.org/10.4136/ambiagua.880)

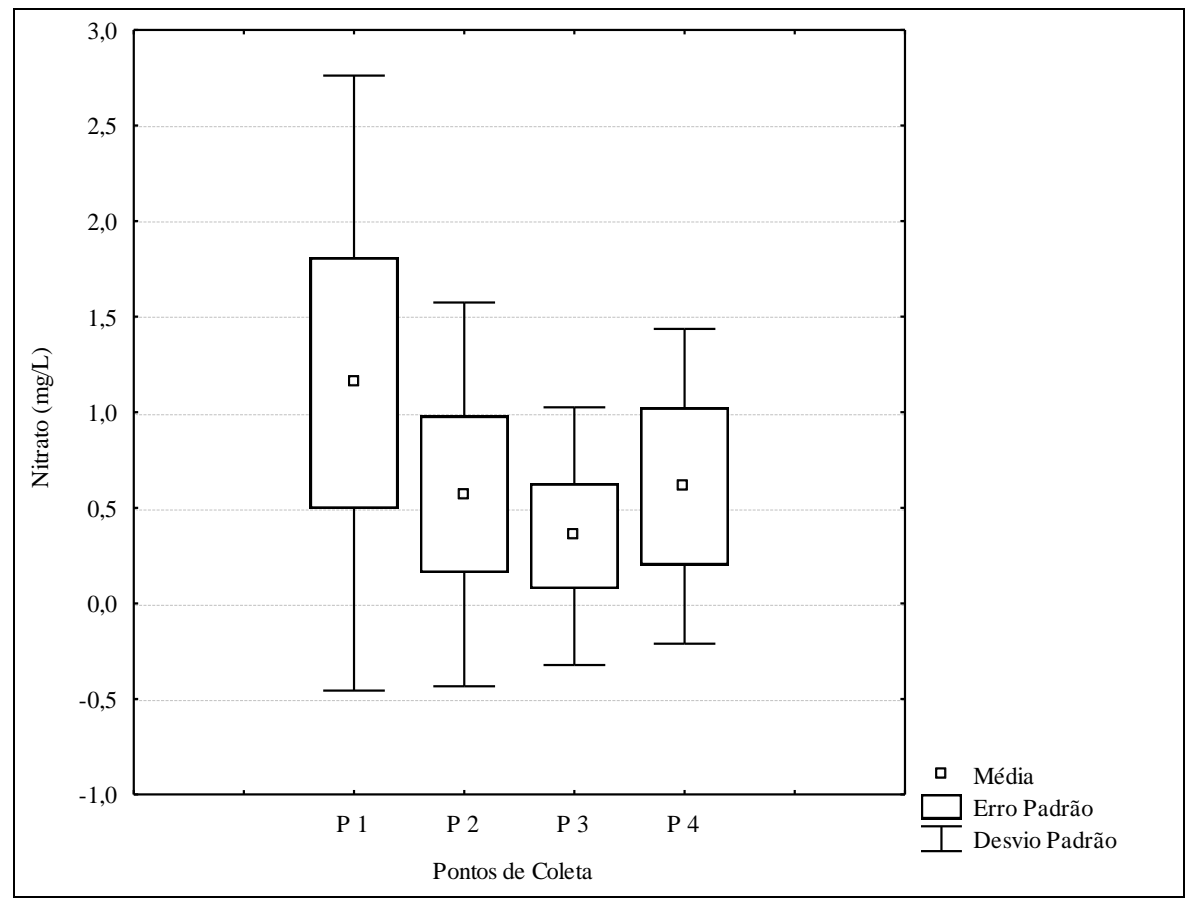

Figura 14. Média, erro padrão e desvio padrão de nitrato, para os diferentes pontos de coleta.

As menores concentrações encontradas nos pontos P2, P3 e P4 indicam que as descargas de efluentes concentram-se a montante do ponto P2 (Dias e Álvarez, 1996).

O nitrato é o composto que representa a forma mais oxidada dos compostos nitrogenados, podendo ser considerado o micronutriente responsável pelo controle de produção primária nas camadas superficiais eufóticas (Silva, 2004).

De acordo com a Resolução CONAMA 357/2005, todos os pontos analisados apresentam concentrações médias inferiores a $10 \mathrm{mg} . \mathrm{L}^{-1}$ e portanto enquadram-se como de classe I

\section{CONCLUSÕES}

Os resultados obtidos no presente trabalho mostram que os pontos de coleta de amostras de água do rio Santa Rita localizados próximos a área mais urbanizada da bacia hidrográfica apresentaram as piores condições relativas aos parâmetros avaliados.

O lançamento de manipueira que ocorre nos núcleos de Campinhos e Simão é provavelmente a causa do impacto negativo nas concentrações médias obtidas para o oxigênio dissolvido, condutividade elétrica, salinidade, amônia, nitrato, nitrito. Tais resultados indicam um forte aporte de efluentes orgânicos na cabeceira do rio, salientando-se que a água apresentou-se salobra nos três primeiros pontos de coleta.

A origem da elevada concentração de zinco no ponto de coleta $\mathrm{P} 1$ possivelmente está associado a queima de madeira, bem como lançamento de efluentes domésticos e incineração de resíduos.

As atividades agropastoris são provavelmente o causador das elevadas concentrações obtidas para o fósforo total, ferro e cobre, demonstrando desta forma, que a Bacia Hidrográfica estudada além de problemas de poluição ambiental decorrentes do beneficiamento da mandioca, apresenta um segundo aspecto de degradação dos recursos hídricos. 


\section{REFERENCIAS}

ANTONELI, V.; THOMAZ, E. L. Caracterização do meio físico da bacia do arroio Boa Vista, Guamiranga-PR. Revista Caminhos da Geografia, v. 8, n. 21, p. 46-58, 2007.

AMERICAN PUBLIC HEALTH ASSOCIATION; AMERICAN WATER WORKS ASSOCIATION; WATER ENVIRONMENT FEDERATION - APHA; AWWA; WEF. Standard methods for the examination of water and wastewater. 19th edition. Wasghington D.C., 1995.

BAHIA. Governo do Estado. Secretaria de Infraestrutura - SEINFRA. Superintendência de Recursos Hídricos - SRH. Diagnóstico das bacias hidrográficas dos rios Cachoeira e Almada: caracterização hidrológica. Vol. I. Tomo IV. Salvador, 2000.

BAHIA. Superintendência de Recursos Hídricos - SRH. Plano diretor de recursos hídricos: bacia do Recôncavo Sul. Salvador, 2004.

BARANA, A. C. Despoluição da manipueira e uso em fertilização do solo. In: SIMPÓSIO NACIONAL SOBRE A MANIPUEIRA, 1., 2008, Vitória da Conquista. Vitória da Conquista-Bahia. Anais... Vitória da Conquista: EMPBRAPA, 2008.

BRASIL. Ministério do Meio Ambiente. Resolução No 357, de 17 de março de 2005. Dispõe sobre a classificação dos corpos de água e diretrizes ambientais para o seu enquadramento, bem como estabelece as condições e padrões de lançamento de efluentes, e dá outras providências. Diário oficial [da] República Federativa do Brasil, Poder Executivo, Brasília, DF, 18 mar. 2005. Seção 1, p. 58-63.

BRANCO, S. M. Hidrologia aplicada à engenharia sanitária. 3. ed. São Paulo: CETESB/ACATESB, 1986.

CEREDA, M. P.; BRASIL, O. G.; FIORETTO, A. M. C. Microorganismos com respiração resistente ao cianeto isolados de líquido residual de fecularia. Phyton, v. 41, n. 12, p. 197-201, 1981.

CHRISTOFOLETTI, A. Análise morfométrica das bacias hidrográficas. Notícias Geomorfológicas, v. 9, n. 18, p. 19-34, 1969.

COMPANHIA DE TECNOLOGIA DE SANEAMENTO AMBIENTAL - CETESB. Qualidade das águas interiores no estado de São Paulo: índices de qualidade das águas. São Paulo, 2007.

DIAS, L. E.; ÁLVAREZ, V. H. V. Fertilidade do solo. Viçosa-MG: UFV,1996.

FERNANDES, E. S. Análise da qualidade da água do rio das Contas no município de Jequié, Bahia. 2011. Dissertação (Mestrado em Desenvolvimento Regional e Meio Ambiente) - Universidade Estadual de Santa Cruz, Ilhéus, 2011.

FIORETTO, A. M. C. Viabilidade de cultivo de Trichosporon spp. em manipueira. 1987. Dissertação (Mestrado em Agronomia) - Faculdade de Ciências Agronômicas, Universidade Estadual Júlio de Mesquita Filho, Botucatu, 1987. 
SANTOS, G. P.; REGO, N. A. C.; SANTOS, J. W. B.; DELANO JÚNIOR, F.; SILVA JÚNIOR, M. F. Avaliação espaço-temporal dos parâmetros de qualidade da água do rio Santa Rita (BA) em função do lançamento de manipueira. Ambi-Agua, Taubaté, v. 7, n. 3, p. 261-278, 2012. (http://dx.doi.org/10.4136/ambiagua.880)

HELBIG, E.; BUCHWEITZ, M. R. D. I.; GIGANTE, D. P. Análise dos teores de ácidos cianídrico e fítico em suplemento alimentar: multimistura. Revista de Nutrição, Campinas, v. 21, n. 3, p. 323-328, 2008. http://dx.doi.org/10.1590/S141552732008000300007

HESS, M. L. Tratamentos de despejos de fecularia de mandioca por oxidação biológica. Revista D.A.E., São Paulo, v. 23, n. 46. p. 29-35, 1962.

LAGE, V. F. Avaliação ambiental da qualidade da água da bacia do rio Piabanha e sua contribuição ao rio Cachoeira - Sul da Bahia. 2011. Dissertação (Mestrado em Desenvolvimento Regional e Meio Ambiente) - Universidade Estadual de Santa Cruz, Ilhéus, 2011.

LIMA, I. V.; PEDROZO, M. F. Ecotoxicologia do ferro e seus compostos. Salvador: CRA, 2001. (Cadernos de Referência Ambiental, 4).

PÉRICO, E.; CEMIN, G.; AREND, U.; REMPEL, C.; ECKHARD, R. R. Análise fisiográfica da bacia hidrográfica do Rio Forqueta, RS. In: SIMPÓSIO BRASILEIRO DE SENSORIAMENTO REMOTO - SBSR, 15., 2011, Curitiba. Anais... São José dos Campos: INPE, 2011.

PHILIPPI, A. J. R.; ROMERO, M. A.; BRUNA, G. C. Curso de gestão ambiental. Barueri: Manole, 2004.

RAMIREZ, A. V. Toxicidad del cianuro: Investigación bibliográfica de sus efectos en animales y en el hombre. Anais Faculdade de Medicina, v.71, n.1, p. 54-61, 2010.

REGO, N. A. C.; SANTOS, J. W. B.; TEIXEIRA, A. Estudos quali-quantitativos da condutividade elétrica das águas superficiais da bacia hidrográfica do Rio Cachoeira sul da Bahia. In: INSTITUTO DE GESTÃO DAS ÁGUAS E CLIMA. Gestão de recursos hídricos. Salvador, 2009. (Águas da Bahia, 3).

ROCHA, I. S.; BARROS, G. Condomínio Greenville e loteamento Vila América: um retrato da segregação sócio-espacial em Vitória da Conquista. In: COLOQUIO INTERNACIONAL DE GEOCRÍTICA, 11., 2010, Buenos Aires. La planificación territorial y el urbanismo desde el diálogo y la participación. Actas... Buenos Aires: Universidad de Buenos Aires, 2010.

SANT'ANA, A. F.; DOMENE, S. M. A. Teores de glicosídeos cianogênicos em derivados de mandioca determinados por protocolo adaptado ao laboratório de microntrientes. In: ENCONTRO DE INICIAÇÃO CIENTÍFICA, 13., 2008, Campinas. Anais... Campinas: IC-PUC, 2008.

SANTOS, A. Possibilidades e perspectivas para a sustentabilidade do cultivo da mandioca no Planalto de Conquista. 2001. Dissertação (Mestrado em Desenvolvimento Sustentável - Gestão e Políticas Ambientais) - Universidade de Brasília, Brasília, 2001.

SILVA, C. A. R. Análises físico-químicas de sistemas marginais marinhos. Rio de Janeiro: Interciências, 2004.

TONELlO, K. C.; DIAS, H. C. T.; SOUZA, A. L.; RIBEIRO, C. A. A. S.; LEITE, F. P. Morfometria da bacia hidrográfica da Cachoeira das Pombas, Guanhães-MG. Revista Árvore, v. 30, n. 5, p. 849-857, 2006. http://dx.doi.org/10.1590/S0100-67622006000500019 
SANTOS, G. P.; REGO, N. A. C.; SANTOS, J. W. B.; DElANO JÚNIOR, F.; SILVA JÚNIOR, M. F. Avaliação espaço-temporal dos parâmetros de qualidade da água do rio Santa Rita (BA) em função do lançamento de manipueira. Ambi-Agua, Taubaté, v. 7, n. 3, p. 261-278, 2012. (http://dx.doi.org/10.4136/ambiagua.880)

VILLELA, S. M.; MATTOS, A. Hidrologia aplicada. São Paulo: McGraw-Hill do Brasil, 1975. 245p. 


ISSN = 1980-993X - doi:10.4136/1980-993X
www.ambi-agua.net
E-mail: ambi-agua@agro.unitau.br
Tel.: (12) 3625-4212

\title{
Biomonitoramento do ar com Tradescantia pallida (Rose) D. R. Hunt var purpurea Boom (Commelinaceae)
}

\author{
(http://dx.doi.org/10.4136/ambi-agua.982)
}

\author{
Mateus Cabral Vasconcellos Teixeira; Agnes Barbério \\ Departamento de Biologia, Instituto Básico de Biociências \\ Universidade de Taubaté, Taubaté, SP, \\ e-mail: agnesbarberio@yahoo.com.br, mateuscabral1989@bol.com.br
}

\section{RESUMO}

O crescimento populacional e o conforto gerado pelo progresso têm contribuído amplamente para o aumento da poluição do ar, fazendo com que o ar de vários centros urbanos apresente cada vez mais substâncias nocivas aos seres vivos. O presente estudo objetivou caracterizar a qualidade do ar no município de Taubaté, Vale do Paraíba-SP utilizando biomonitoramento com Tradescantia pallida (Rose) D. R. Hunt purpurea Boom (Commelinaceae). O estudo foi desenvolvido num período de 10 meses (setembro/2010 a junho/2011) em cinco localidades (Rodovia Presidente Dutra - tráfego veicular intenso; Estiva - área residencial; Parque Aeroporto - área industrial; Campus Bom Conselho - centro da cidade com alto fluxo veicular e Agronomia - área rural. Foram realizadas semanalmente coletas das inflorescências jovens das plantas expostas. Após a coleta, as inflorescências foram fixadas em solução de etanol/ácido acético glacial (3:1) por $24 \mathrm{~h}$, transferidas para solução de etanol $70 \%$ e conservadas sob refrigeração. As mesmas foram submetidas à coloração com carmim acético e quantificado o número de micronúcleos em aproximadamente 300 tétrades para cada inflorescência, sendo confeccionadas lâminas com 10 inflorescências/semana para cada ponto estudado. As médias e desvios padrões foram: Dutra $(2,24 \pm 1,58)$, Estiva $(2,07 \pm 1,33)$, Parque Aeroporto $(1,47 \pm 1,05)$, Bom Conselho $(1,42 \pm 0,90)$ e Agronomia $(0,82 \pm 0,80)$. Apesar de Taubaté ser uma região em crescimento urbano, os dados revelaram que o ar da cidade apresentou boa qualidade. Manter esse equilíbrio é fundamental, requer esforços e aferição periódica, neste sentido, o biomonitoramento é um método rápido, barato e eficiente.

Palavras-chave: Tradescantia pallida, micronúcleo, poluição atmosférica.

\section{Biomonitoring of the air with Tradescantia pallida (Rose) D. R. Hunt var purpurea Boom (Commelinaceae)}

\begin{abstract}
The population growth and the comfort generated by progress have greatly contributed with the increase in the air pollution, making the air in several urban centers polluted by substances that are harmful to human being. This study characterized the air quality in the city of Taubaté, Vale do Paraíba-SP using biomonitoring with Tradescantia pallida (Rose) D. R. Hunt purpurea Boom (Commelinaceae). The study was developed in a period of 10 months (September/2010 to June/2011) in five locations (Rodovia Presidente Dutra - heavy vehicle traffic; Estiva - residential area; Parque Aeroporto - industrial area); Campus Bom Conselho - area in the city with heavy vehicle traffic and Agronomy - rural area. Samples were
\end{abstract}


TEIXEIRA, M. C. V.; BARBÉRIO, A. Biomonitoramento do ar com Tradescantia pallida (Rose) D. R. Hunt var purpurea Boom (Commelinaceae). Ambi-Agua, Taubaté, v. 7, n. 3, p. 279-292, 2012. (http://dx.doi.org/10.4136/ambi-agua.982)

collected on a weekly basis from young inflorescences of exposed plants. After the inflorescences were collected, they were fixed in an absolute ethanol-glacial acetic acid (3:1) solution for $24 \mathrm{~h}$, transferred to ethanol $70 \%$ solution and maintained under refrigeration. They were submitted to coloration with acetic carmine and the number of micronucleus was quantified in approximately 300 tetrads for each inflorescence, and slides were prepared with 10 inflorescences/week for each point of study. The average and standard deviations were: Dutra $(2.24 \pm 1.58)$, Estiva $(2.07 \pm 1.33)$, Parque Aeroporto $(1.47 \pm 1.05)$, Bom Conselho $(1.42 \pm 0.90)$ and Agronomy $(0.82 \pm 0.80)$. Although Taubaté area is a region experiencing urban growth, the data revealed that the air in the city has good quality. Maintaining this balance is very important, requires effort and periodic measurements, and, for this, biomonitoring is a fast, cheap and effective method.

Keywords: Tradescantia pallida, micronuclei, air pollution.

\section{INTRODUÇÃO}

O crescimento populacional e o conforto gerado pelo progresso têm contribuído amplamente para o aumento da poluição atmosférica, fazendo com que o ar de vários centros urbanos apresente cada vez mais substâncias nocivas aos seres vivos. Inúmeras são as substâncias orgânicas e inorgânicas com características aneugênicas (aquelas que podem interferir no fuso mitótico) e clastogênicas (que causam quebras cromossômicas), como benzenos, hidrocarbonetos aromáticos, materiais particulados e metais pesados (Grant, 1992; Monarca et al., 1999; Silva, 2005; Cetesb, 2006; Marcílio e Gouveia, 2007; Junior et al., 2008; Mariani et al., 2008; Carneiro et al., 2011; Habermann et al., 2011).

Entre os fatores que contribuem para o agravamento da poluição, destaca-se a emissão de gases nocivos pelas descargas de veículos automotores, podendo-se usar a intensidade do tráfego veicular como medida indireta do acúmulo desses poluentes em determinadas regiões (Monarca et al., 1999; Silva, 2005; Junior et al., 2008; Mariani et al., 2008; Yanagi, 2010; Carneiro et al., 2011; Habermann et al., 2011).

Neste contexto, o monitoramento da poluição atmosférica constitui importante ferramenta que auxilia na tomada de decisões, tais como a adoção de medidas de controle, diminuição das substâncias expelidas pelas chaminés de indústrias e controle da frota veicular (Silva, 2005). Biomonitoramento consiste em um método de análise experimental que permite avaliar poluentes em grandes extensões de áreas utilizando organismos vivos, que são denominados bioindicadores (Carneiro, 2004; Mariani et al., 2008). Tem sido uma ferramenta útil e importante para o fornecimento de informações rápidas e seguras sobre as ações dos poluentes na biota, além de promover dados que permitem comparar informações obtidas pelos métodos convencionais de monitoramento do ar atmosférico, como o medidor de partículas atmosféricas $\mathrm{PM}_{10}$ e $\mathrm{PM}_{2,5}$ (Alves et al., 2001; Carneiro, 2004; Oliveira et al., 2005; Mielli, 2008). Os biondicadores reagem ao estresse promovido por substâncias que se acumulam em seus tecidos, ocasionando modificações bioquímicas, fisiológicas e morfológicas (Klumpp et al., 2001; Carneiro, 2004). Entre os organismos mais utilizados como bioindicadores, destacam as plantas superiores que são mais sensíveis à poluição que os animais, portanto, estudos sobre os efeitos dos poluentes nas plantas fornecem subsídios importantes para os programas de controle da poluição do ar (Alves et al., 2001; Carneiro, 2004; Klumpp, 2004; Junior et al., 2008; Mariani et al., 2008). As respostas das plantas bioindicadoras aos poluentes podem ser observadas em níveis microscópicos ou macroscópicos através do aparecimento de cloroses, necroses, queda foliar ou diminuição no seu crescimento (Alves et al., 2001; Saldiva et al., 2002; Junior et al., 2008; Mariani et al., 2008). 
TEIXEIRA, M. C. V.; BARBÉRIO, A. Biomonitoramento do ar com Tradescantia pallida (Rose) D. R. Hunt var purpurea Boom (Commelinaceae). Ambi-Agua, Taubaté, v. 7, n. 3, p. 279-292, 2012. (http://dx.doi.org/10.4136/ambi-agua.982)

Dentre as plantas bioindicadoras destaca-se a Tradescantia pallida (Rose) D. R. Hunt var. purpurea Boom por apresentar fácil adaptação em qualquer ambiente, podendo se desenvolver durante todo o ano, apresentando-se como um instrumento favorável para estudos de biomonitoramento em grandes escalas (Bloching et al., 2000; Majer et al., 2001; SerranoGarcia e Montero-Montoya, 2001; Ramirez e Saldanha, 2002; Savóia, 2007; Lisbôa e Ignotti, 2008). De tamanho relativamente pequeno $(\sim 25 \mathrm{~cm})$, seis pares de grandes cromossomos e disponibilidade durante o ano todo, são características que disseminaram seu uso em estudo citogenéticos (Ma, 1981; Lorenzi e Souza, 2001; Carneiro, 2004; Lisbôa e Ignotti, 2008).

A Tradescantia tem sido utilizada experimentalmente desde os primeiros estudos que relacionavam atividade genética com a ação de compostos e agentes químicos (Ma, 1981, 1983; Grant et al., 1992; Klumpp, 2004). Praticamente todas as partes dessa planta podem compor bioensaios de mutagênese para a detecção e monitoramento da poluição ambiental: flores, pétalas, pêlos estaminais, raiz, microsporos, tubo polínico e material genético (aberrações cromossômicas mitóticas e meióticas, micronúcleos, troca entre cromátides irmãs), (Grant, 1998). Dentre esses ensaios dois são ideais na identificação da poluição atmosférica, o bioensaio de micronúcleos (Trad-MCN) e o de pêlos estaminais (Trad-SH) (Ma, 1981; Grant et al., 1992, Ma et al., 1994). O teste dos micronúcleos com Tradescantia spp. (Trad-MCN) é considerado um dos mais sensíveis e eficientes para a detecção de agentes genotóxicos no ar (Ma, 1981, 1983; Ennever et al., 1988; Rodrigues et al., 1997; Saldiva et al., 2002 Misík et al., 2007; Junior et al., 2008). Este bioensaio se baseia na formação de micronúcleos em células mãe dos grãos de pólen presentes nos botões florais de inflorescências jovens de Tradescantia expostas à poluição ambiental (Ma, 1981; Rodrigues et al., 1997; Klumpp, 2004; Klumpp et al., 2006). Os micronúcleos são estruturas resultantes de cromossomos inteiros ou de fragmentos cromossômicos que se perderam durante a divisão celular, e por isso não são incluídos nos núcleos das células filhas, permanecendo no citoplasma das células interfásicas (Majer et al., 2001; Carrard et al., 2007). Embora os mecanismos de reparo celular sejam eficientes, a sua formação se deve a alterações cromossômicas, muitas vezes ligadas a fatores ambientais (Majer et al., 2001). Neste sentido, a ocorrência tanto de danos estruturais, quanto de aneuploidia, permitem detectar a ação de agentes clastogênicos e aneugênicos (Marcilio e Gouveia, 2007; Junior et al., 2008; Carneiro et al., 2011; Habermann et al., 2011).

As grandes concentrações de poluentes atmosféricos representam um risco eminente para a saúde humana e para o bem estar de outros organismos (Klumpp et al., 2006; Marcílio e Gouveia, 2007). Embora os resultados dessas exposições não sejam completamente conhecidos, diversos estudos epidemiológicos relatam correlações significativas entre diferentes níveis de poluições do ar e seus efeitos agudos, crônicos e deletérios aos organismos, principalmente aos seres humanos (Saldiva et al., 1994, 1995; WHO, 2000, 2005; Branco e Murgel, 2004; Molina e Molina, 2004; Wilson et al., 2004; Cançado et al., 2006; Nascimento et al., 2006; Marcilio e Gouveia, 2007).

Considerando Taubaté uma importante cidade do Vale do Paraíba, situada entre o eixo Rio-São Paulo, o presente estudo objetivou caracterizar a qualidade do ar desta região, utilizando o bioensaio com Tradescantia pallida (Rose) D. R. Hunt var. purpurea Boom (Commelinaceae).

\section{MÉTODO}

Para o estudo foram utilizadas plantas jovens de T. pallida provenientes de um mesmo clone, onde foram cultivadas e propagadas no Campus Ciências Agrárias da Universidade de Taubaté (UNITAU) localizado na Estrada Municipal Dr. José Luis Cembranelli. 
TEIXEIRA, M. C. V.; BARBÉRIO, A. Biomonitoramento do ar com Tradescantia pallida (Rose) D. R. Hunt var purpurea Boom (Commelinaceae). Ambi-Agua, Taubaté, v. 7, n. 3, p. 279-292, 2012. (http://dx.doi.org/10.4136/ambi-agua.982)

O estudo foi realizado no município de Taubaté (23 01' 33” S $45^{\circ} 33^{\prime} 31^{\prime \prime} \mathrm{O}$ ), Vale do Paraíba, São Paulo, durante dez meses (setembro de 2010 a junho de 2011). Taubaté possui área total de $625,9 \mathrm{~km}^{2}$, que abriga uma população de mais de 278.686 habitantes (IBGE, 2010). Apresenta clima tropical com inverno seco, tendo no verão, médias acima de $22{ }^{\circ} \mathrm{C}$ e no inverno $18{ }^{\circ} \mathrm{C}$; a média pluviométrica é de $1.344 \mathrm{~mm} / \mathrm{ano}$. Em sua frota veicular constam mais de 3.115 caminhões, 8.728 caminhonetes, 801 microônibus, 39.462 motos, 816 ônibus, 103.437 automóveis e outros tipos de veículos, totalizando 167.614 veículos (DENATRAN, 2012) e possui aproximadamente 424 indústrias (SEADE, 2012).

Foram monitoradas cinco localidades de acordo com a intensidade de tráfego veicular: 1) Rodovia Presidente Dutra, altura do Km 109 sentido São Paulo, região que apresenta alto fluxo veicular, com diversos tipos de veículos, moradias e indústrias nas proximidades; 2) Campus Bom Conselho (UNITAU), cercado por árvores, prédios e avenidas com intenso fluxo veicular; 3) Campus Ciências Agrárias (UNITAU), localidade distante do centro da cidade cerca de $10 \mathrm{~km}$, com áreas verdes e baixo fluxo de veículos; 4) Escola Municipal Prof. Ernesto de Oliveira Filho, localizada no Bairro Parque Aeroporto, situada em uma área industrial, entretanto aberta, com boa ventilação e baixo fluxo veicular; e 5) Escola Municipal Monsenhor Evaristo Campista Cesar, situada no Bairro Estiva, considerada uma área residencial com elevado fluxo veicular.

Foram colocadas duas floreiras com 30 espécimes de $T$. pallida em cada uma dessas localidades. As floreiras receberam o mesmo substrato e posicionadas no local de melhor ventilação.

As coletas das inflorescências jovens foram realizadas semanalmente nos cinco pontos amostrais, o termo inflorescência refere-se à fase de pré-floração da espécie (botão). Após a coleta as mesmas foram fixadas em solução de etanol/ácido acético glacial (3:1) por $24 \mathrm{~h}$, transferidas para solução de etanol $70 \%$ e mantidas sob refrigeração até o momento da análise. Para a análise citológica foi realizada a contagem de micronúcleos (MCN), onde se procedeu ao esmagamento das anteras e quando verificada a fase de tétrades, as mesmas foram submetidas à coloração com carmim acético, de acordo com o protocolo estabelecido por Ma (1981). Para cada local estudado foram confeccionadas 10 lâminas por semana. As frequências de micronúcleos foram registradas em cada lâmina (número de micronúcleos em 300 tétrades) e expressas em termos de número de micronúcleos por 100 tétrades (Campos Junior e Kerr, 2009). Esta etapa foi desenvolvida no Laboratório de Biologia da Universidade de Taubaté (IBB - UNITAU).

A frequência de micronúcleos foi analisada pelo teste de Kruskal-Wallis e ANOVA um critério, e comparadas entre os diferentes locais de exposição pelo teste de Dunn, $p<0,05$.

\section{RESULTADOS E DISCUSSÃO}

As coletas foram feitas em intervalos semanais no período de dez meses de exposição, abrangendo assim três estações consecutivas: primavera, verão e outono. De acordo com a análise de variância Kruskal-Wallis como pré-teste, houve diferença significativa entre os cinco pontos estudados quando comparados entre si $(K=203,6 ; p=0,001)$. Em uma segunda análise utilizou-se o método de Dunn como pós-teste, demonstrando quais as localidades foram diferentes entre si (Tabela 1).

A dinâmica da poluição demonstrada pela frequência de $\mathrm{MCN}$ nas diferentes localidades sugere o seguinte gradiente de poluição: Dutra > Estiva > Bom Conselho > Parque Aeroporto e Agronomia.

A média da frequência de micronúcleos nas cinco localidades variou de $0,8 \%$ a 2,3\%. Estudo realizado por Savóia (2007) em cinco localidades na cidade de Santo André/SP foram observadas frequências de micronúcleos que variaram de 0,5 a 4,8\%, sendo que o menor valor 
TEIXEIRA, M. C. V.; BARBÉRIO, A. Biomonitoramento do ar com Tradescantia pallida (Rose) D. R. Hunt var purpurea Boom (Commelinaceae). Ambi-Agua, Taubaté, v. 7, n. 3, p. 279-292, 2012. (http://dx.doi.org/10.4136/ambi-agua.982)

foi observado na área controle caracterizada pelo baixo índice de tráfego veicular localizada em área de manancial próximo a um parque. Nos estudos realizados por Guimarães et al. (2000), Alves et al. (2003) e Sant'Anna (2003) em regiões consideradas controles, com baixos níveis de poluição atmosférica, foi observada média de 2,3\% de MCN. Junior et al. (2008) com estudo realizado na cidade de Senhor de Bonfim, Bahia e Mariani et al. (2008) com estudos em São José dos Campos, Vale do Paraíba-SP, também demonstraram que regiões com baixo tráfego veicular apresentaram frequência média baixa de micronúcleos. Neste sentido, os resultados obtidos na presente pesquisa estão de acordo com a literatura pertinente.

Tabela 1. Frequência de micronúcleos em $T$. pallida exposta a cinco localidades na cidade de Taubaté/SP no período de setembro/2010 a junho/2011.

\begin{tabular}{l|cc}
\hline Localidade & Média \pm Desvio Padrão & $\begin{array}{c}\mathbf{N}^{\mathbf{0}} \text { Total de Inflorescências } \\
\text { Analisadas }\end{array}$ \\
\hline Dutra & $2,24 \pm 1,58^{\mathrm{a}}$ & 352 \\
Estiva & $2,07 \pm 1,33^{\mathrm{a}}$ & 253 \\
Bom Conselho & $1,47 \pm 1,05^{\mathrm{ba}}$ & 285 \\
Pq. Aeroporto & $1,42 \pm 0,90^{\mathrm{ba}}$ & 208 \\
Agronomia & $0,82 \pm 0,80^{\mathrm{b}}$ & 373 \\
\hline
\end{tabular}

Nota: Frequências médias indicadas com letras distintas são significativamente diferentes (Kruskal-Wallis, Dunn sendo $p<0,01$ ).

A distribuição temporal para os cinco pontos de coleta pode ser observada na Figura 1.

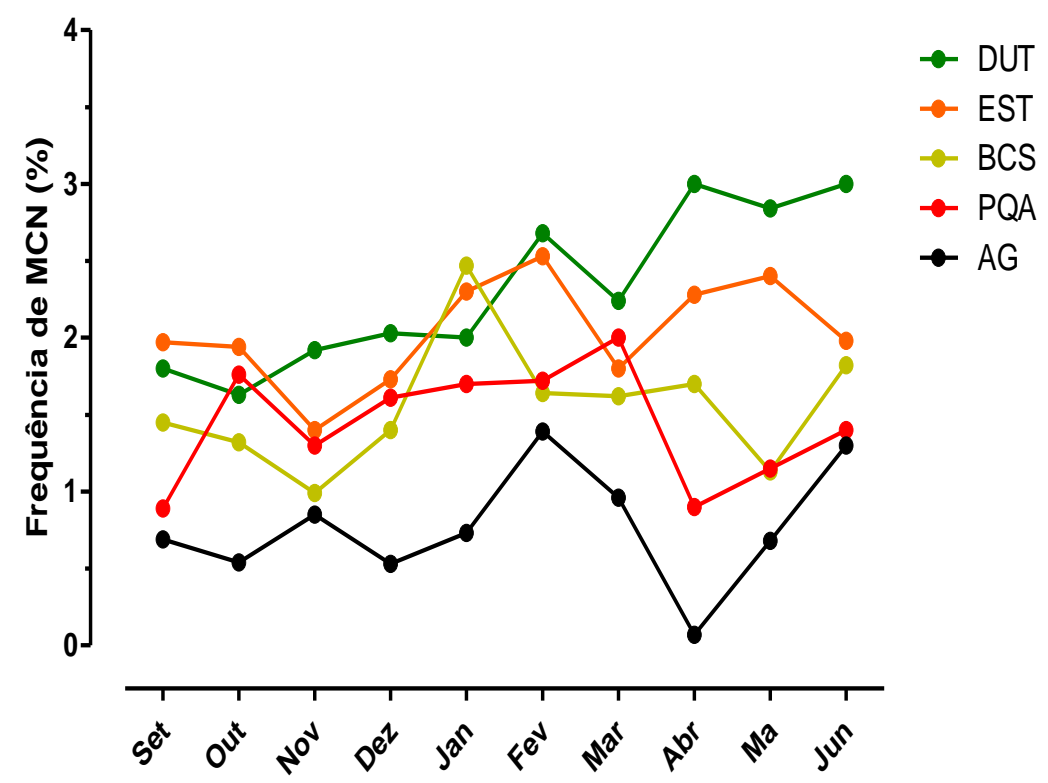

Figura 1. Frequência média de MCN obtidas a partir de inflorescências de $T$. pallida expostas cinco localidades: DUT Dutra, EST - Estiva, BCS - Bom Conselho, PQA - Parque Aeroporto e AG - Agronomia.

Em uma segunda abordagem foram realizadas análises estatísticas para cada ponto comparando os meses entre si. Dutra $(\mathrm{F}=4,183 ; \mathrm{p}<0,001)$, Estiva $(\mathrm{F}=126,8 ; \mathrm{p}<0,001)$, Bom Conselho $(F=3,029 ; p<0,001)$, Parque Aeroporto $(F=4,715 ; p<0,001)$ e Agronomia $(\mathrm{F}=5,753 ; \mathrm{p}<0,001)$. 
TEIXEIRA, M. C. V.; BARBÉRIO, A. Biomonitoramento do ar com Tradescantia pallida (Rose) D. R. Hunt var purpurea Boom (Commelinaceae). Ambi-Agua, Taubaté, v. 7, n. 3, p. 279-292, 2012. (http://dx.doi.org/10.4136/ambi-agua.982)

A frequência de MCN foi mais alta nos meses de abril e junho de 2011 na Dutra, apresentou picos de até 3,00\%, em fevereiro na Estiva com 2,53\%, janeiro com 2,47\% no Bom Conselho, em março no Pq. Aeroporto (2,00\%) e Agronomia 1,39\% e 1,30\% apresentaram os maiores valores nos meses de fevereiro e junho, respectivamente. Isso mostra um comportamento sazonal esperado, com maiores concentrações depois do período chuvoso.

Dutra foi o ponto que apresentou a maior frequência de micronúcleo. Por ser uma importante Rodovia que liga as duas maiores cidades do Brasil, São Paulo e Rio de Janeiro, apresenta alto tráfego veicular, em média 45,5 veículos por minuto, com uma frota variada de veículos, desde carros de passeio, ônibus, entre outros, até caminhões de transporte de carga. Devido a essa grande variedade e intensidade de fluxo de veículos automotores, são liberados diversos poluentes na atmosfera como hidrocarbonetos policíclicos aromáticos, metais pesados, benzenos, cuja atividade genotóxica pode causar danos aos seres vivos. Além do alto tráfego veicular, também existem em suas margens, indústrias de diversos ramos como automobilística, usinagem, alimentícias, etc, que também contribuem com a liberação de diversas substâncias orgânicas e inorgânicas para a atmosfera.

No bairro Estiva, foi registrada a segunda maior frequência de micronúcleo em todo o período estudado. Este resultado se justifica pelo fato da região apresentar alto tráfego veicular com carros de passeios e de transporte coletivo, além de ser cortada pela Estrada do Pinhão, uma importante via que liga a região rural ao centro da cidade; é rodeada por avenidas importantes como Avenida Charles Schnneider, Avenida Vito e Benedito Sergio, trata-se de uma região rodeada por casas, escolas, pouca vegetação, muitos semáforos, fator que promove maior descarga de poluentes, já que quando os veículos "arrancam" eles tendem a emitir uma carga maior de poluentes. Em adição a esses fatores, é uma região desfavorável à dispersão dos poluentes atmosféricos.

Esses dados estão de acordo com os realizados por Monarca et al. (1999) que também observaram maior frequência de micronúcleos em plantas expostas a amostras de ar coletadas em uma rua com intenso tráfego de veículos. Para Guimarães et al. (2000) em estudo realizado na cidade de São Paulo em duas regiões que possuem diversas avenidas com intenso tráfego veicular com diversos tipos de veículos, Congonhas e Cerqueira Cesar, a frequência de MCN observada foi em torno de 5,7\% e 3,9\%, respectivamente.

Prajapati e Tripathi (2008) avaliaram a interferência de indústrias e fluxo veicular em uma cidade na Índia, nos resultados observados a frequência de MCN foi de 3,6\%. Ferreira et al. (2003) relataram associações entre o fluxo veicular e danos genéticos que promoveram mutações em pêlos estaminais de Tradescantia. Valverde e Brandeburgo (2009) também encontraram associações positivas entre o fluxo veicular (movidos por diferentes tipos de combustíveis) e aborto polínico em Tradescantia.

O ponto do Parque Aeroporto seguido de Agronomia, foram os que apresentaram menor frequência de micronúcleo. Ambas as localidades estão distante do centro da cidade, cerca de $10 \mathrm{~km}$ da área urbana, com baixo fluxo veicular, em torno de 3 carros por minuto, apresentam características típicas de locais com baixo índice de poluição atmosférica, sendo ambas as regiões consideradas de "campo aberto". Essas características favorecem a dispersão dos poluentes (Branco e Murgel, 2004). A direção dos ventos é outro fator importante para a dispersão dos poluentes, podendo diminuir a ocorrência de danos genéticos (Alves et al., 2003).

O ponto do Bom Conselho foi a localidade que apresentou média intermediária entre os demais pontos, em desacordo com o esperado. A região está inserida no centro da cidade, rodeada por casas e prédios altos e cortada por uma avenida com médio tráfego veicular (Avenida Tiradentes). É possível que a presença de árvores de grande porte em toda a sua extensão, somando à presença de prédios altos, possa favorecer a formação de correntes e ar dissipando os poluentes liberados pelos veículos que transitam pela região. Contudo, se faz 
TEIXEIRA, M. C. V.; BARBÉRIO, A. Biomonitoramento do ar com Tradescantia pallida (Rose) D. R. Hunt var purpurea Boom (Commelinaceae). Ambi-Agua, Taubaté, v. 7, n. 3, p. 279-292, 2012. (http://dx.doi.org/10.4136/ambi-agua.982)

necessário dar continuidade ao biomonitoramento nessa localidade a fim de verificar a possibilidade de um evento esporádico ou a relação com a dispersão dos poluentes por meio da direção e intensidade dos ventos nesta localidade.

Variações climáticas como precipitação e temperatura tem influência na qualidade da atmosfera (Branco e Murgel, 2004), porém no presente estudo não foi observada nenhuma relação entre as variações climáticas e a frequência de micronúcleos durante o período estudado.

De acordo com os resultados de precipitação no período referente à coleta de inflorescências, os meses de maior pluviosidade foram entre dezembro e abril (Figura 2), entretanto, Dutra e Estiva apresentaram valores altos na frequência de $\mathrm{MCN}$, que se mantiveram nos meses de maio e junho, onde a precipitação foi baixa. Nesse contexto, a precipitação não foi um fator limitante na frequência de MCN. Os valores de precipitação foram obtidos na estação meteorológica da Universidade de Taubaté.

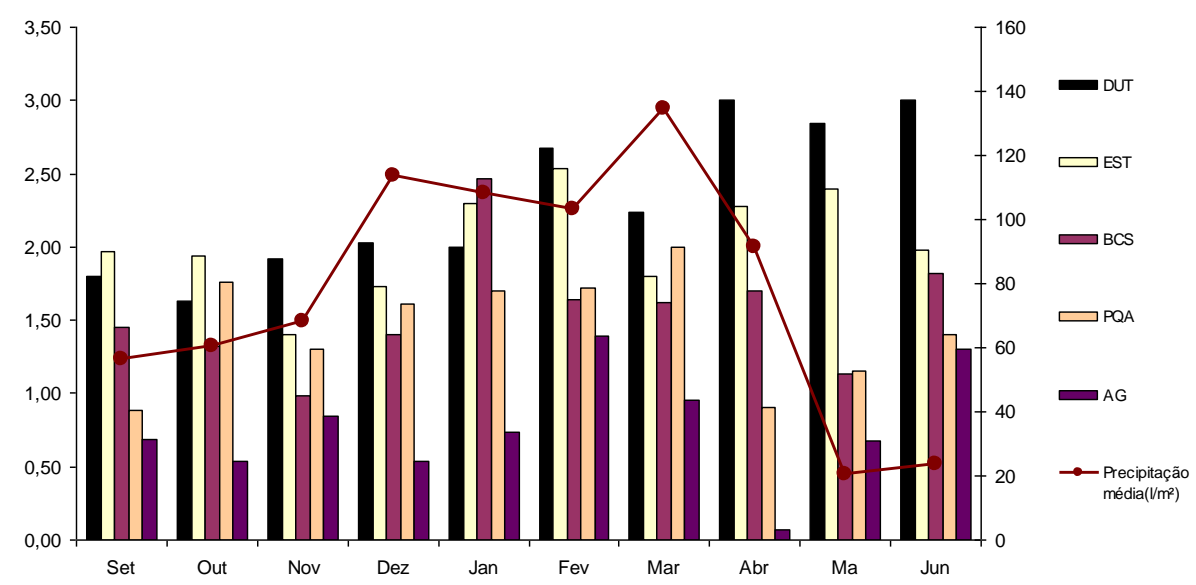

Figura 2. Relação entre a frequência média de $\mathrm{MCN}$ e a precipitação média durante o período de estudo (setembro de 2010 a junho de 2011).

Nota: DUT - Dutra, EST - Estiva, BCS - Bom Conselho, PQA - Parque Aeroporto e AG - Agronomia.

Do mesmo modo, não foi observada associação entre a temperatura e a frequência de MCN nas plantas durante o período de exposição (Figura 3), embora o local Dutra apresente um discreto aumento de MCN nos mês de outono.

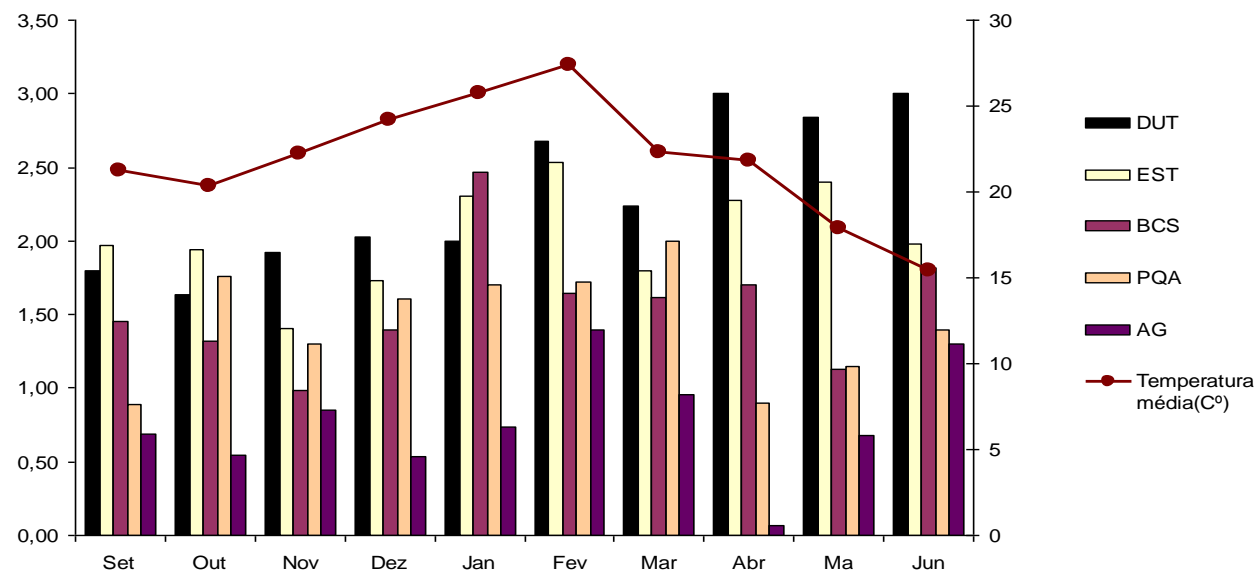

Figura 3. Relação entre a frequência de $\mathrm{MCN}$ e a temperatura durante o período de exposição dos exemplares de T. pallida. DUT - Dutra, EST - Estiva, BCS Bom Conselho, PQA - Parque Aeroporto e AG - Agronomia. 
TEIXEIRA, M. C. V.; BARBÉRIO, A. Biomonitoramento do ar com Tradescantia pallida (Rose) D. R. Hunt var purpurea Boom (Commelinaceae). Ambi-Agua, Taubaté, v. 7, n. 3, p. 279-292, 2012. (http://dx.doi.org/10.4136/ambi-agua.982)

Estudos realizados por Isidori et al. (2003), utilizaram a Tradescantia como ferramenta de monitoramento atmosférico em 17 locais de uma cidade na Itália, em duas estações do ano (verão e inverno), demonstraram que a frequência de MCN foi significantemente mais alta em plantas dos pontos experimentais selecionados, quando comparada à das plantas controles, sendo a frequência de MCN maior no inverno do que no verão. Ferreira et al. (2003) afirmaram que o período chuvoso, é mais propício à dispersão dos poluentes atmosféricos.

Os efeitos da poluição do ar se caracterizam tanto pela alteração de condições consideradas normais como pelo aumento de problemas já existentes; podem ocorrer em nível local, regional e global e podem se manifestar na saúde humana comprometendo o bem estar da população, também afetando os ecossistemas, flora e fauna, podem ainda danificar edificações históricas, exercer interferência sobre as propriedades da atmosfera passando pela redução da visibilidade, alteração da acidez das águas da chuva (chuva ácida), aumento da temperatura da Terra (efeito estufa) e modificação da intensidade da radiação solar (aumento da incidência de radiação ultravioleta, causado pela redução da camada de ozônio), etc.

Em relação à saúde, os efeitos vão desde um simples desconforto respiratório até as doenças crônicas, além do aumento da procura pelo sistema de saúde (centros de saúde, hospitais e pronto socorro).

A qualidade do ar interfere diretamente na vida das pessoas, sendo importante manter controladas as fontes de emissão de poluentes. Bioensaios são eficientes, rápidos e baratos podendo ser uma ferramenta bastante útil na detecção e biomonitoramento desses poluentes. Neste estudo, foi possível atestar a boa qualidade do ar na cidade de Taubaté através do bioensaio TRAD-MCN, de modo que se mantenham análises periódicas para manter este cenário.

Alguns estudos mencionados neste trabalho encontraram níveis de poluição atmosférica considerados significantemente baixos, cuja média de frequência observada variou entre $0,5 \%$ e 2,3\% micronúcleos. Neste estudo, frequências maiores foram observadas em locais com intenso tráfego veicular, corroborando o esperado. Por outro lado, a menor frequência observada foi de $0,8 \%$ micronúcleos. A princípio, uma frequência abaixo de $1 \%$ parece razoável, entretanto, é necessário que se façam outros estudos de modo a abordar uma frequência significantemente baixa obtida em sítio controle nos quais estudos posteriores possam se basear.

A cidade de Taubaté encontra-se em franco desenvolvimento, cenário representado pelo crescimento populacional e industrial a cada ano. Na cidade, a CETESB realiza monitoramento manual para fumaça, que é um tipo de poluente atmosférico originado a partir de processos de combustão da indústria ou de veículos automotores, da poeira na rua, queima de biomassa, entre outros. Seus efeitos na saúde humana são diversos, além de causar danos na vegetação e contaminar a água e o solo (CETEB, 2011). Apesar disso, o biomonitoramento através do bioensaio Trad-MCN é uma ferramenta mais abrangente no sentido biológico e geográfico, no primeiro porque detecta mutação em exemplares expostos in vivo, no segundo, porque esses exemplares podem ser facilmente distribuídos em vários locais permitindo uma maior cobertura de aferição. Além de ser, rápido, barato e eficiente. Diante do exposto, propõe-se que esta metodologia seja utilizada no biomonitoramento da região, em especial Taubaté, como método complementar às análises químicas realizadas pela CETESB.

\section{CONCLUSÃO}

A qualidade do ar atmosférico da cidade de Taubaté encontra-se predominantemente dentro dos padrões de normalidade preconizados. Estudos subsequentes não necessários para delimitar valores de referência em sítios controles de modo que se permitam delimitar uma distância de segurança dos locais onde a poluição atmosférica é intensa. 
TEIXEIRA, M. C. V.; BARBÉRIO, A. Biomonitoramento do ar com Tradescantia pallida (Rose) D. R. Hunt var purpurea Boom (Commelinaceae). Ambi-Agua, Taubaté, v. 7, n. 3, p. 279-292, 2012. (http://dx.doi.org/10.4136/ambi-agua.982)

\section{AGRADECIMENTOS}

Agradecemos à Universidade de Taubaté pelo fomento concedido pela bolsa PIBIC e por disponibilizar espaço, equipamentos e soluções. Também à Profa. Dra. Maria Cecília Barbosa de Toledo pelas sugestões e auxílio nas análises estatísticas.

\section{REFERENCIAS}

ALVES, E. S.; GIUSTI, P. M.; DOMINGOS, M.; SALDIVA, P. H. N.; GUIMARÃES, E. T.; LOBO, D. J. A. Estudo anatômico foliar do clone híbrido 4430 de Tradescantia: alterações decorrentes da poluição aérea urbana. Revista Brasileira Botânica, São Paulo, v. 24, n. 4, p. 561-566, 2001.

ALVES, E. S., PEDROSO, A. N. V., GUIMARÃES, E. T., DOMINGOS, M.; SALDIVA, P. H. N. Biomonitoramento indoor do potencial mutagênico do ar em laboratórios e herbário do Instituto de Botânica por meio do bioensaio Trad-MCN. Hoehnea, São Paulo, v. 30, n. 2, p. 89-94, 2003.

BLOCHING, M.; HOFMANN, A.; LAUTENSCHLÄGER, C. H.; BERGHAUS, A.; GRUMMIT, T. Exfoliative Cytology of Normal Buccal Mucosa to Predict the Relative Risk of Cancer in the Upper Aerodigestive Tract Using the MN-assay. Oral Oncology, Amsterdam, v. 36, n. 6, p. 550-555, 2000.

BRANCO, S. M.; MURGEL, E. Poluição do ar. Série Polêmica. 2 ed., Ed. Moderna, 2004. 112p.

CANÇADO, J. E. D.; BRAGA, A.; PEREIRA, L. A. A.; ARBEX, M. A.; SALDIVA, P. H. N.; SANTOS, U. P. Repercussões clínicas da exposição à poluição atmosférica. Jornal Brasileiro de Pneumologia, Brasília, v. 32, n. 2, p. 5-11, 2006.

CAMPOS JUNIOR, E. O.; KERR, W. E. Teste de micronúcleo com Tradescantia pallida aplicado ao Biomonitoramento da qualidade do ar da cidade de Uberlândia. Horizonte Científico, Uberlândia, v. 3, n. 2, p. 1-18, 2009.

CARNEIRO, R. M. A. Bioindicadores vegetais de poluição atmosférica: uma contribuição para a saúde da comunidade, 2004 Dissertação (Mestrado em Enfermagem em Saúde Pública), Escola de Enfermagem de Ribeirão Preto da Universidade de São Paulo, São Paulo, 2004.

CARNEIRO, M. F. H.; RIBEIRO, F. Q.; FERNANDES-FILHO, F. N.; LOBO, D. J. A.; BARBOSA JR., F.; RHODEN, C. R.; MAUAD, T.; SALDIVA P. H. N.; CARVALHO-OLIVEIRA, R. Pollen abortion rates, nitrogen dioxide by passive diffusive tubes and bioaccumulation in tree barks are effective in the characterization of air pollution. Environmental and Experimental Botany, Oxford, v. 75, n. 2, p. 272-277, 2011.

CARRARD, V. C.; COSTA, C. H.; FERREIRA, L. A.; LAUXEN, I. S.; RADOS, P. V. Teste dos Micronúcleos - Um Biomarcador de Dano Genotóxico em Células Descamadas da Mucosa. Revista da Faculdade de Odontologia de Porto Alegre, Porto Alegre, v. 48, n. 1/3, p. 77-81, 2007. 
TEIXEIRA, M. C. V.; BARBÉRIO, A. Biomonitoramento do ar com Tradescantia pallida (Rose) D. R. Hunt var purpurea Boom (Commelinaceae). Ambi-Agua, Taubaté, v. 7, n. 3, p. 279-292, 2012. (http://dx.doi.org/10.4136/ambi-agua.982)

CETESB Relatório da Qualidade do Ar no Estado de São. In: Companhia de tecnologia de saneamento ambiental. São Paulo, 2006, Disponível em: http://www.cetesb.sp. gov.br/ agua/aguas-superficiais/35-publicacoes-/-relatorios Acesso em: 8 mar. 2011.

DENATRAN - Departamento Nacional de Trânsito. Frota de Veículos - Frota 2012. Disponível em: http://www.denatran.gov.br/frota.htm. Acesso: 07 abr. 2012.

ENNEVER, F. K.; ANDREANO, G.; ROSENKRANZ, H. S. The ability of plant genotoxicity assay to predict carcinogenicity. Mutation Research, Amsterdam, v. 205, n. 1-4, p. 99-105, 1988.

FERREIRA, M. I.; RODRIGUES, G. S.; DOMINGOS, M.; SALDIVA, P. H. N. In situ monitoring of mutagenicity of air pollutants in São Paulo City using Tradescantia-SHM bioassay. Brazilian Archives of Biology and Technology, Curitiba, v. 46, n. 2, p. 253-258, 2003.

GRANT, W. F. Higher Plant Assays for the Detection of Genotoxicity in Air Polluted Environments. Ecosystem Health, New York, v. 4, n. 4, p. 210-229, 1998. http://dx.doi.org/10.1016/S0027-5107(99)00050-0I

GUIMARÃES, E. T.; DOMINGOS, M.; ALVES, E. S.; CALDINI Jr, N.; LOBO, D. J. A.; LICHTENFELS, A. J. F. C.; SALDIVA, P. H. N. Detection of genotoxicity of air pollutants in and around the city of São Paulo (Brazil) with the Tradescantiamicronucleus (Trad-MCM) assay. Environmental and Experimental Botany, Oxford, v. 44, n. 1, p. 1-8, 2000.

HABERMANN, M.; MEDEIROS, A. P. P.; GOUVEIA, N. Tráfego veicular como método de avaliação da exposição à poluição atmosférica nas grandes metrópoles. Revista Brasileira de Epidemiologia, São Paulo, v. 14, n. 1, p. 120-30, 2011.

IBGE - Instituto Brasileiro de Geografia e Estatística. IBGE Cidades - Censo Demográfico 2010. Disponível em: http://www.ibge.gov.br/cidadesat/topwindow. htm?1. Acesso em: 7 abr. 2012.

ISIDORI, M.; FERRARA, M.; LAVORGNA, M.; NARDELLI, A.; PARRELLA A. In situ monitoring of urban air in Southern Italy with the Tradescantia micronucleus bioassay and semipermeable membrane devices (SPMDs). Chemosphere, Oxford, v. 52, n. 1, p. 121-6, 2003.

JÚNIOR, J. A. S.; JÚNIOR, J. C. S. S.; OLIVEIRA, J. L.; CERQUEIRA, E. M. M.; MEIRELES, J. R. C. Micronúcleos em tétrades de Tradescantia pallida (Rose) Hunt. cv. purpurea Boom: alterações genéticas decorrentes de poluição aérea urbana. Acta Scientiarum Biological Sciences, Maringá, v. 30, n. 3, p. 295-301, 2008 .

KLUMPP, A.; ANSEL, W.; KLUMPP, G.; FOMIN, A. Um novo conceito de monitoramento e comunicação ambiental: a rede européia para a avaliação da qualidade do ar usando plantas bioindicadoras (EuroBionet). Revista Brasileira de Botânica, São Paulo, v. 24, n. 4, p. 511-518, 2001. 
TEIXEIRA, M. C. V.; BARBÉRIO, A. Biomonitoramento do ar com Tradescantia pallida (Rose) D. R. Hunt var purpurea Boom (Commelinaceae). Ambi-Agua, Taubaté, v. 7, n. 3, p. 279-292, 2012. (http://dx.doi.org/10.4136/ambi-agua.982)

KLUMPP, A. European network for the assessment of air quality by the use of bioindicator plants - Final report In: University of Hohenheim, Germany, 2004. Disponível em: http://ec.europa.eu/environment/life/project/Projects/index.cfm? fuseaction $=$ search.dspPage\&n_proj_id=1050\&docType=pdf. Acesso em: 15 abr. 2011.

KLUMPP, A.; ANSEL, W.; KLUMPP, G.; CALATAYUD, V.; GARREC, J. P.; HE, S.; PEÑUELAS, J.; RIBAS, A.; RO-POULSEN, H.; RASMUSSEN, S.; SANZ, M. J.; VERGNE, P. Tradescantia micronucleus test indicates genotoxic potential of traffic emissions in European cities. Environmental Pollution, Barking, v. 139, n. 3, p. 515-522, 2006.

LISBÔA, M. F.; IGNOTTI, E. Viabilidade polínica de Tradescantia pallida (Rose) D. R. Hunt var. purpurea ocorrente no município de Alta Floresta (Commelinaceae). In: $1^{\text {a }}$ Jornada cientifica de Unemat, Cárceres, Mato Grosso, 2008. Disponível em: http://www.unemat.br/eventos/jornada2008/resumos_conic/Expandido_00764.pdf Acesso em: 3 mar. 2011.

LORENZI, H.; SOUZA, H. M. Plantas Ornamentais do Brasil. Ed. Nova Odessa: Plantarum, p. 1088, 2001.

MA, T. H. Tradescantia micronucleus bioassay and pollen tube chromatid aberration test for in situ monitoring and mutagen screening. Environmental Health Perspectives, Estados Unidos, v. 37, p. 85-90, 1981.

MA, T. H. Tradescantia micronuclei (Trad-MN) test for environmental clastogens. In: Kolber, A. R.; Wong, T. K.; Grant, Lester D.; DeWoskin, Robert S. \& Hughes, J. $\mathrm{T}$. In vitro toxicity testing of environmental agents. Ed. Plenum Publishing Corporation, New York, 1983, p. 1191-214.

MA, T. H.; CABRERA, G. L.; CEBULSKA-WASILEWSKA, A.; CHEN, R.; LOARCA, F.; VANDENBER, A. L.; SALAMONE, M. F. Tradescantia stamen hair mutation bioassay. Mutation Research, Amsterdam, v. 310, n. 2, p. 211-220, 1994.

MAJER, B. J.; LAKY, B.; KNASMÜLLER, S.; KASSIE, F. Use of the Micronucleus Assay with Exfoliated Epithelial Cells as a Biomarker for Monitoring Individuals at Elevated Risk of Genetic Damage and in Chemoprevention Trials. Mutation Research, Amsterdam, v. 489, n. 2-3, p. 147-172, 2001.

MARCILIO, I.; GOUVEIA, N. Quantifying the impact of air pollution on the urban population of Brazil. Caderno de Saúde Pública, Rio de Janeiro, v. 23, n. 4, p. 529-536, 2007.

MARIANI, R. L.; JORGE, M. P. M.; PEREIRA, S. S. Caracterização da qualidade do ar em São José Dos Campos-SP, utilizando Tradescantia pallida (Trad-MCN). Revista Brasileira de Geoquímica, Goiás, v. 22, n. 1, p. 27-33, 2008.

MIELLI, A. C. Avaliação da atividade genotóxica de logo de esgoto tratado do estado de São Paulo com o teste de micronúcleo em células germinativa de Tradescantia (Trad-MN), 2008 Tese (Doutorado em Patologia), Faculdade de Medicina da Universidade de São Paulo, São Paulo. 2008. 
TEIXEIRA, M. C. V.; BARBÉRIO, A. Biomonitoramento do ar com Tradescantia pallida (Rose) D. R. Hunt var purpurea Boom (Commelinaceae). Ambi-Agua, Taubaté, v. 7, n. 3, p. 279-292, 2012. (http://dx.doi.org/10.4136/ambi-agua.982)

MISÍK, M.; MICIETA, K.; SOLENSKÁ, M.; MISÍKOVÁ, K.; PISARCÍKOVÁ, H.; KNASMÜLLER, S. In situ biomonitoring of the genotoxic effects of mixed industrial emissions using the Tradescantia micronucleus and pollen abortion tests with wild life plants: Demonstration of the efficacy of emission controls in an eastern European city. Environmental Pollution, Barking, v. 145, n. 2, p. 459466, 2007.

MOLINA, M. J.; MOLINA, L. T. Megacities and atmospheric pollution. Journal of the Air and Waste Management Association, Pittsburgh, v. 54, n. 6, p. 644-680, 2004.

MONARCA, S.; FERRETI, D.; ZANARDINI, A.; FALISTOCCO, E.; NARDI, G. Monitoring of mutagens in urban air sample. Mutation Research, Amsterdam, v. 426, n. 2, p. 189-192, 1999.

NASCIMENTO, L. F. C.; PEREIRA, L. A. A.; BRAGA, A. L. F.; MÓDOLO, M. C. C.; CARVALHO, J. R. J. A. Efeitos da poluição atmosférica na saúde infantil em São José dos Campos, SP. Revista de Saúde Pública, São Paulo, v. 40, n. 1, p. 77-82, 2006.

OLIVEIRA, D. S.; CRNKOVIC, P. M.; PAGLIUSO, J. D. Avaliação da mutagenicidade causada por poluentes presentes na exaustão de motor diesel por meio de bioindicador vegetal. In: VII Congresso de Ecologia do Brasil, 2005, Caxambú, MG. Disponível em: http://www.seb-ecologia.org.br/viiceb/resumos/ 489a.pdf. Acesso: 25 mai. 2011.

PRAJAPATI, S. K.; TRIPATHI, B. D. Assessing the genotoxicity of urban air pollutants in Varanasi City using Tradescantia micronucleus (Trad-MCN) bioassay. Environment International, Elmsford, v. 34, n. 4, p. 1092-1096, 2008.

RAMIREZ, A.; SALDANHA, P. H. Micronucleus investigation of alcoholic patients with oral carcinomas. Genetics and Molecular Research, Ribeirão Preto, v 1, n. 3, p. 246-260, 2002.

RODRIGUES, G. S.; MA, T. H.; PIMENTEL, D.; WEINSTEIN, L. H.; ICHIKAWA, $\mathrm{S}$. Tradescantia bioassays as monitoring systems for environmental mutagenesis a review. Critical Reviews in Plant Sciences, Boca Raton, v. 16, n. 4, p. 325359, 1997.

SALDIVA, P. H. N.; LICHTENFELS, A. J. F. C.; PAIVA, P. S. O.; BARONE, I. A.; MARTINS, M. A.; MASSAD, E.; PEREIRA, J. C. R.; XAVIER, V. P.; SINGER, J. M.; BÖHM, G. M. Association between air pollution and mortality due to respiratory diseases in children in São Paulo: a preliminary report. Environmental Research, Hauppauge, v. 65, n. 2, p. 218-225, 1994.

SALDIVA, P. H. N.; POPE, C. A.; SCHWARTZ, J.; DOCKEY, D.; LICHTENFELS, A. J. F. C.; SALGE, J. M.; BARONE, I. A.; BÖHM, G. M. Air pollution and mortality in elderly people: a times series in São Paulo. Archives of Environmental Health, Chicago, v. 50, n. 2, p. 159-163, 1995. 
TEIXEIRA, M. C. V.; BARBÉRIO, A. Biomonitoramento do ar com Tradescantia pallida (Rose) D. R. Hunt var purpurea Boom (Commelinaceae). Ambi-Agua, Taubaté, v. 7, n. 3, p. 279-292, 2012. (http://dx.doi.org/10.4136/ambi-agua.982)

SALDIVA, P. H. N.; CLARKE, R. W.; COULL, B. A.; STEARNS, R. C.; LAWRENCE, J.; MURTHY, G. G. K.; DIAZ, E.; KOUTRAKIS, P.; SUH, H.; TSUDA, A.; GODLESKI, J. J. Lung inflammation induced by concentrated ambient air particles is related to particle composition. American Journal of Respiratory and Critical Care Medicine, New York, v. 165, n. 12, p. 16101617, 2002.

SANT'ANNA, E. T. G. Poluição atmosférica urbana na cidade de São Paulo e mutagênese: avaliação de riscos utilizando-se bioindicadores vegetais do gênero Tradescantia. 2003 Tese (Doutorado em Fisiopatologia Experimental), Universidade de São Paulo, São Paulo - SP. 2003.

SAVÓIA, E. J. L. Potencial de Tradescantia pallida cv. Purpurea para biomonitoramento da poluição aérea de Santo André - São Paulo, por meio do bioensaio Trad - MCN e do acúmulo foliar de elementos tóxicos, 2007 Dissertação (Mestrado em Fisiopatologia Experimental), Universidade de São Paulo, São Paulo - SP. 2007.

SEADE - Sistema Estadual de Análise de Dados (Secretaria de Planejamento e Desenvolvimento Regional - Governo do Estado de São Paulo). Informação dos Municípios Paulistas - IMP: Número de indústrias em Taubaté (código da localidade 3554102). Disponível em: http://www.seade.gov.br/produtos/imp/ index.php?page $=$ consulta\&action $=$ var_list $\&$ tema $=1 \&$ tabs $=1 \&$ aba $=$ tabela 1 . Aces so: 08 mai. 2012.

SERRANO-GARCÍA, L.; MONTERO-MONTOYA, R. Micronuclei and chromatid buds are the result of related genotoxic events. Environmental and Molecular Mutagenesis, New York, v. 38, n. 1, p. 38-45, 2001.

SILVA, J. S. Efeitos genotoxicos em tétrades de Tradescantia pallida (Rose) D. R. Hunt Var. purpurea induzidos por poluentes atmosféricos na cidade de Salvador-BA. 2005 Monografia (Graduação em Ciências Biológicas), Universidade Federal de Feira de Santana, Feira de Santana-BA. 2005.

VALVERDE, B. T.; BRANDEBURGO, M. A. M. Ensaio do aborto de pólen aplicado ao biomonitoramento da qualidade do ar da cidade de Uberlândia. In: IX Encontro Interno \& XIII Seminário de Iniciação Cientifica, Universidade Federal de Uberlândia, 2009. Disponível em: https://ss14799.websiteseguro.com/ swge5/seg/cd2009/PDF/IC2009-0146.pdf. Acesso em: 25 mai. 2011.

WILSON, A. M.; SALLOWAY, J. C.; WAKE, C. P.; KELLY, T. Air pollution and the demand for hospital services: a review. Environment International, Elmsford, v. 30, n. 8, p. 1109-1118, 2004.

WHO. Evaluation and use of epidemiological evidence for environmental health risk assessment - Guideline document. In: World Health Organization Regional Office for Europe, Copenhagen, Denmark, 2000. Disponível em: http://www.euro.who.int/_data/assets/pdf_file/0006/74733/E68940.pdf. Acesso em: 4 de jun. 2011.

WHO The effects of air pollution on children's health and development: a review of the evidence. In: World Health Organization Regional Office for Europe, Copenhagen, Denmark, 2005. Disponível em: http://www.euro.who.int/_data/ assets/ pdf_file/0010/74728/E86575.pdf. Acesso em: 4 de jun. 2011. 
TEIXEIRA, M. C. V.; BARBÉRIO, A. Biomonitoramento do ar com Tradescantia pallida (Rose) D. R. Hunt var purpurea Boom (Commelinaceae). Ambi-Agua, Taubaté, v. 7, n. 3, p. 279-292, 2012. (http://dx.doi.org/10.4136/ambi-agua.982)

YANAGI, Y. Estudo da influência do material particulado na incidência e mortalidade por câncer na cidade de São Paulo, 2010 Dissertação (Mestrado em Saúde Ambiental), Faculdade de Saúde Publica da Universidade de São Paulo, São Paulo. 2010. 